\title{
POINT 2011: ENDF/B-VII.1 Beta2 Temperature Dependent Cross Section Library
}

U.S. Department of Energy

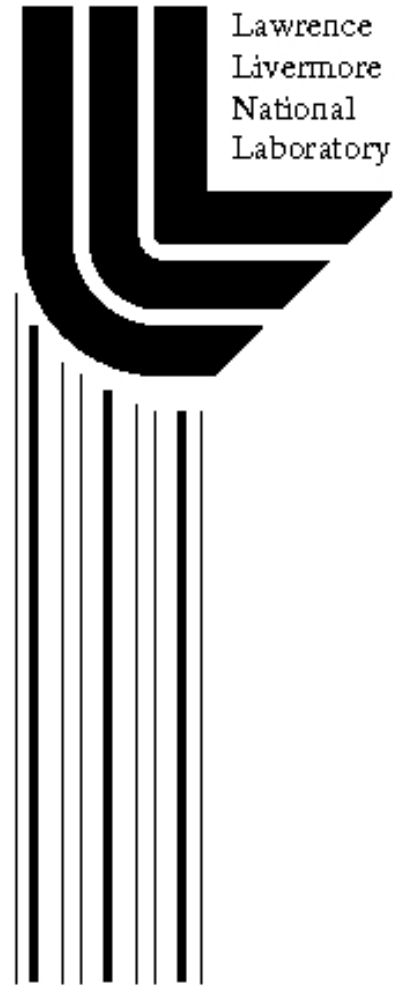

\author{
by \\ Dermott E. Cullen \\ University of California \\ Lawrence Livermore National Laboratory \\ P.O.Box 808/L-198 \\ Livermore, CA 94550
}

April 1, 2011

Approved for public release; further dissemination unlimited 


\section{DISCLAIMER}

This document was prepared as an account of work sponsored by an agency of the United States government. Neither the United States government nor Lawrence Livermore National Security, LLC, nor any of their employees makes any warranty, expressed or implied, or assumes any legal liability or responsibility for the accuracy, completeness, or usefulness of any information, apparatus, product, or process disclosed, or represents that its use would not infringe privately owned rights. Reference herein to any specific commercial product, process, or service by trade name, trademark, manufacturer, or otherwise does not necessarily constitute or imply its endorsement, recommendation, or favoring by the United States government or Lawrence Livermore National Security, LLC. The views and opinions of authors expressed herein do not necessarily state or reflect those of the United States government or Lawrence Livermore National Security, LLC, and shall not be used for advertising or product endorsement purposes.

This work performed under the auspices of the U.S. Department of Energy by Lawrence Livermore National Laboratory under Contract DE-AC52-07NA27344.

This report has been reproduced directly from the best available copy.

Available to DOE and DOE contractors from the Office of Scientific and Technical Information

P.O. Box 62, Oak Ridge, TN 37831

Prices available from (423) 576-8401

http://apollo.osti.gov/bridge/

Available to the public from the National Technical Information Service

U.S. Department of Commerce 5285 Port Royal Rd., Springfield, VA 22161 http://www.ntis.gov/

OR

Lawrence Livermore National Laboratory Technical Information Department's Digital Library http://www.llnl.gov/tid/Library.html 


\title{
POINT 2011: ENDF/B-VII.1 Beta2 \\ Temperature Dependent Cross Section Library
}

\author{
by \\ Dermott E. Cullen \\ University of California \\ Lawrence Livermore National Laboratory \\ P.O.Box 808/L-198 \\ Livermore, CA 94550
}

April 1, 2011

1) Overview

Table of Contents

2) Introduction

3) POINT 2011: ENDF/B-VII.1

4) Summary of VII.1

5) PREPRO 2010 Codes

6) Data Processing

7) Accuracy of Results

8) Contents of the Library

9) Requesting POINT 2011 Data

10) Installation and Use of POINT 2011

11) Acknowledgments

12) References

13) Appendix A: Contents of ENDF/B-VII.1

14) Appendix B: Contents of ENDF/B-VII.0

15) Appendix C: Elemental vs. Isotopic Evaluations

16) Appendix D: Completeness of VII.1

17) Appendix E: Summary of $\langle v(E)>$ for all isotopes in ENDF/B-VII.1

18) Appendix F: An Initial Comparison of VII.1 versus VII.0

19) Appendix G: Earlier Versions of ENDF/B

20) Appendix H: The Effects of Temperature and Doppler Broadening

21) Appendix I: MF/MT Differences between VII.1 and VII.0 


\section{Overview}

This report is one in the series of "POINT" reports that over the years have presented temperature dependent cross sections for the then current version of ENDF/B. In each case I have used my personal computer at home and publicly available data and codes :

1) publicly available nuclear data (the current ENDF/B data, available on-line at the National Nuclear Data Center, Brookhaven National Laboratory http://www.nndc.bnl.gov/) and,

2) publicly available computer codes (the current PREPRO codes, available on-line at the Nuclear Data Section, IAEA, Vienna, Austria http://wwwnds.iaea.or.at/ndspub/endf/prepro/) and,

3) My own personal computer located in my home.

I have used these in combination to produce the temperature dependent cross sections used in applications and presented in this report. I should mention that today anyone with a personal computer can produce these results.

\section{Introduction}

The latest ENDF/B-VII.1 beta2 data library was recently and is now freely available through the National Nuclear Data Center (NNDC), Brookhaven National Laboratory. This release completely supersedes all preceding releases of ENDF/B.

WARNING: The final ENDF/B-VII.1 will not be distributed until about the end of 2011; this will give us time to test the present VII.1 beta2 library. Throughout the remainder of this report I will refer to VII.1, as shorthand in actually referring to VI.1 beta2 - I can only hope this does not result in any confusion.

As distributed the ENDF/B-VII.1 data includes cross sections represented in the form of a combination of resonance parameters and/or tabulated energy dependent cross sections, nominally at 0 Kelvin temperature.

For use in our applications the ENDF/B-VII.1 library has been processed into cross sections at eight neutron reactor like temperatures, between 0 and 2100 Kelvin, in steps of 300 Kelvin (the exception being 293.6 Kelvin, for exact room temperature at 20 Celsius). It has also been processed to five astrophysics like temperatures, 1, 10, $100 \mathrm{eV}$, 1 and $10 \mathrm{keV}$. For reference purposes, 300 Kelvin is approximately $1 / 40 \mathrm{eV}$, so that 1 $\mathrm{eV}$ is approximately 12,000 Kelvin. At each temperature the cross sections are tabulated and linearly interpolable in energy.

All results are in the computer independent ENDF-6 character format [R2], which allows the data to be easily transported between computers. In its processed form the POINT 2011 library is approximately 16 gigabyte in size and is distributed on one compressed DVDs (see, below for the details of the contents of each DVD). 


\section{POINT 2011: ENDF/B-VII.1 beta2}

ENDF/B-VII.1 beta2 (I will refer to it as VII.1) which was recently released by CSEWG, includes 418 evaluations, compared to the earlier VII.0 which included 393 evaluations. Of the 418 evaluations in VII.1, 391 are evaluations for the same materials as in VII.0, and 27 are new evaluations. Two VII.0 evaluation for naturally occurring elemental mixtures that were included in VII.0, namely, 23-V - Nat and 30-Zn-Nat, have been dropped and replaced by isotopic evaluations in VII.1.

For use in applications it is obviously useful to know what has and has not changed between the older ENDF/B-VII.0 and newer VII.1 data libraries; below is a summary. Based on my character-by-character comparisons of VII.1 and VII.0 for all MF=1 through 6 data (data that can directly affect neutron transport), I have found (see Appendix D for details),

New evaluation.................... 27

Identical........................... 153

Modified............................ 238

Total. .418

New evaluations in VII.1, not in VII.0 (27 Evaluations); note isotopes of V and Zn

\begin{tabular}{|c|c|c|c|c|}
\hline $\begin{array}{l}23-V-50 \\
23-V-51 \\
30-Z n-64 \\
30-Z n-65 \\
30-Z n-66 \\
30-Z n-67\end{array}$ & $\begin{array}{l}30-Z n-68 \\
30-Z n-70 \\
73-T a-180 \\
74-W-180 \\
90-T h-231 \\
91-P a-229\end{array}$ & $\begin{array}{l}91-\mathrm{Pa}-230 \\
92-\mathrm{U}-230 \\
92-\mathrm{U}-231 \\
93-\mathrm{Np}-234 \\
95-\mathrm{Am}-240 \\
96-\mathrm{Cm}-240\end{array}$ & $\begin{array}{l}97-\mathrm{Bk}-245 \\
97-\mathrm{Bk}-246 \\
97-\mathrm{Bk}-247 \\
97-\mathrm{Bk}-248 \\
98-\mathrm{Cf}-246 \\
98-\mathrm{Cf}-248\end{array}$ & $\begin{array}{c}99-E S-251 \\
99-E S-252 \\
99-E S-254 m\end{array}$ \\
\hline
\end{tabular}

Evaluations that are Identical in VII.0 and VII.1 (153 Evaluations)

\begin{tabular}{|l|l|l|l|l|l|}
\hline $1-\mathrm{H}-2$ & $28-\mathrm{Ni}-59$ & $39-\mathrm{Y}-90$ & $49-\mathrm{In}-113$ & $54-\mathrm{Xe}-128$ & $63-\mathrm{Eu}-151$ \\
$2-\mathrm{He}-3$ & $31-\mathrm{Ga}-71$ & $39-\mathrm{Y}-91$ & $49-\mathrm{In}-115$ & $54-\mathrm{Xe}-129$ & $63-\mathrm{Eu}-154$ \\
$3-\mathrm{Li}-7$ & $32-\mathrm{Ge}-70$ & $40-\mathrm{Zr}-95$ & $50-\mathrm{Sn}-112$ & $54-\mathrm{Xe}-132$ & $63-\mathrm{Eu}-155$ \\
$5-\mathrm{B}-10$ & $32-\mathrm{Ge}-72$ & $41-\mathrm{Nb}-94$ & $50-\mathrm{Sn}-114$ & $54-\mathrm{Xe}-133$ & $63-\mathrm{Eu}-156$ \\
$5-\mathrm{B}-11$ & $32-\mathrm{Ge}-73$ & $41-\mathrm{Nb}-95$ & $50-\mathrm{Sn}-116$ & $54-\mathrm{Xe}-135$ & $65-\mathrm{Tb}-159$ \\
$6-\mathrm{C}-\mathrm{Nat}$ & $32-\mathrm{Ge}-74$ & $42-\mathrm{Mo}-98$ & $50-\mathrm{Sn}-117$ & $54-\mathrm{Xe}-136$ & $66-\mathrm{Dy}-161$ \\
$7-\mathrm{N}-15$ & $32-\mathrm{Ge}-76$ & $42-\mathrm{Mo}-99$ & $50-\mathrm{Sn}-118$ & $55-\mathrm{Cs}-134$ & $68-\mathrm{Er}-162$ \\
$8-\mathrm{O}-17$ & $34-\mathrm{Se}-74$ & $42-\mathrm{Mo}-100$ & $50-\mathrm{Sn}-119$ & $55-\mathrm{Cs}-135$ & $68-\mathrm{Er}-164$ \\
$12-\mathrm{Mg}-24$ & $34-\mathrm{Se}-76$ & $44-\mathrm{Ru}-96$ & $50-\mathrm{Sn}-120$ & $55-\mathrm{Cs}-136$ & $68-\mathrm{Er}-167$ \\
$12-\mathrm{Mg}-25$ & $34-\mathrm{Se}-77$ & $44-\mathrm{Ru}-98$ & $50-\mathrm{Sn}-122$ & $55-\mathrm{Cs}-137$ & $68-\mathrm{Er}-168$ \\
$12-\mathrm{Mg}-26$ & $34-\mathrm{Se}-78$ & $44-\mathrm{Ru}-99$ & $50-\mathrm{Sn}-123$ & $56-\mathrm{Ba}-130$ & $71-\mathrm{Lu}-175$ \\
$13-\mathrm{Al}-27$ & $34-\mathrm{Se}-79$ & $44-\mathrm{Ru}-102$ & $50-\mathrm{Sn}-124$ & $56-\mathrm{Ba}-132$ & $71-\mathrm{Lu}-176$ \\
$14-\mathrm{Si}-28$ & $34-\mathrm{Se}-80$ & $44-\mathrm{Ru}-103$ & $50-\mathrm{Sn}-126$ & $56-\mathrm{Ba}-134$ & $73-\mathrm{Ta}-182$ \\
$18-\mathrm{Ar}-36$ & $34-\mathrm{Se}-82$ & $44-\mathrm{Ru}-104$ & $51-\mathrm{Sb}-121$ & $56-\mathrm{Ba}-135$ & $79-\mathrm{Au}-197$ \\
$18-\mathrm{Ar}-38$ & $35-\mathrm{Br}-79$ & $44-\mathrm{Ru}-105$ & $51-\mathrm{Sb}-124$ & $56-\mathrm{Ba}-136$ & $82-\mathrm{Pb}-204$ \\
$19-\mathrm{K}-40$ & $35-\mathrm{Br}-81$ & $44-\mathrm{Ru}-106$ & $51-\mathrm{Sb}-125$ & $56-\mathrm{Ba}-138$ & $83-\mathrm{Bi}-209$ \\
$20-\mathrm{Ca}-40$ & $36-\mathrm{Kr}-80$ & $45-\mathrm{Rh}-105$ & $52-\mathrm{Te}-120$ & $56-\mathrm{Ba}-140$ & $88-\mathrm{Ra}-223$ \\
$20-\mathrm{Ca}-42$ & $36-\mathrm{Kr}-82$ & $46-\mathrm{Pd}-102$ & $52-\mathrm{Te}-122$ & $57-\mathrm{La}-138$ & $88-\mathrm{Ra}-224$ \\
$20-\mathrm{Ca}-43$ & $36-\mathrm{Kr}-83$ & $46-\mathrm{Pd}-104$ & $52-\mathrm{Te}-123$ & $58-\mathrm{Ce}-142$ & $88-\mathrm{Ra}-225$ \\
$20-\mathrm{Ca}-44$ & $36-\mathrm{Kr}-84$ & $46-\mathrm{Pd}-105$ & $52-\mathrm{Te}-125$ & $58-\mathrm{Ce}-144$ & $88-\mathrm{Ra}-226$ \\
$20-\mathrm{Ca}-46$ & $37-\mathrm{Rb}-85$ & $46-\mathrm{Pd}-106$ & $52-\mathrm{Te}-127 \mathrm{~m}$ & $59-\mathrm{Pr}-141$ & $94-\mathrm{Pu}-241$ \\
$20-\mathrm{Ca}-48$ & $38-\mathrm{Sr}-86$ & $46-\mathrm{Pd}-107$ & $52-\mathrm{Te}-128$ & $59-\mathrm{Pr}-142$ & $94-\mathrm{Pu}-243$ \\
$26-\mathrm{Fe}-58$ & $38-\mathrm{Sr}-87$ & $46-\mathrm{Pd}-108$ & $52-\mathrm{Te}-129 \mathrm{~m}$ & $59-\mathrm{Pr}-143$ & $95-\mathrm{Am}-243$ \\
$27-\mathrm{Co}-58$ & $38-\mathrm{Sr}-88$ & $46-\mathrm{Pd}-110$ & $52-\mathrm{Te}-130$ & $61-\mathrm{Pm}-148 \mathrm{~m}$ & \\
$27-\mathrm{Co}-58 \mathrm{~m}$ & $38-\mathrm{Sr}-89$ & $47-\mathrm{Ag}-107$ & $53-\mathrm{I}-129$ & $62-\mathrm{Sm}-151$ & \\
$27-\mathrm{Co}-59$ & $38-\mathrm{Sr}-90$ & $47-\mathrm{Ag}-110 \mathrm{~m}$ & $53-\mathrm{I}-131$ & $62-\mathrm{Sm}-152$ & \\
\hline
\end{tabular}


Evaluations Modified between VII.0 and VII.1 (238 Evaluations)

\begin{tabular}{|c|c|c|c|c|c|}
\hline & & & & & \\
\hline $1-H-1$ & $28-\mathrm{Ni}-62$ & $48-\mathrm{cd}-116$ & 61-Pm-151 & $74-W-186$ & 93-Np-237 \\
\hline $1-H-3$ & $28-N i-64$ & $50-S n-113$ & $62-\mathrm{Sm}-144$ & $75-\mathrm{Re}-185$ & $93-N p-238$ \\
\hline $2-\mathrm{He}-4$ & $29-\mathrm{Cu}-63$ & $50-S n-115$ & $62-\mathrm{Sm}-147$ & $75-\operatorname{Re}-187$ & $93-N p-239$ \\
\hline 3-Li-6 & $29-\mathrm{Cu}-65$ & $50-S n-125$ & $62-\mathrm{Sm}-148$ & $77-\operatorname{Ir}-191$ & $94-\mathrm{Pu}-236$ \\
\hline $4-\mathrm{Be}-7$ & $31-\mathrm{Ga}-69$ & $51-\mathrm{Sb}-123$ & $62-\mathrm{Sm}-149$ & $77-\mathrm{Ir}-193$ & $94-\mathrm{Pu}-237$ \\
\hline $4-\mathrm{Be}-9$ & $33-A s-74$ & $51-S b-126$ & $62-\mathrm{Sm}-150$ & $80-\mathrm{Hg}-196$ & $94-P u-238$ \\
\hline $7-N-14$ & $33-A s-75$ & $52-\mathrm{Te}-124$ & $62-\mathrm{Sm}-153$ & $80-\mathrm{Hg}-198$ & $94-\mathrm{Pu}-239$ \\
\hline $8-0-16$ & $36-\mathrm{Kr}-78$ & $52-\mathrm{Te}-126$ & $62-\mathrm{Sm}-154$ & $80-\mathrm{Hg}-199$ & $94-\mathrm{Pu}-240$ \\
\hline $9-F-19$ & $36-\mathrm{Kr}-85$ & $52-\mathrm{Te}-132$ & $63-\mathrm{Eu}-152$ & $8 \odot-\mathrm{Hg}-20 \odot$ & $94-\mathrm{Pu}-242$ \\
\hline $11-\mathrm{Na}-22$ & $36-\mathrm{Kr}-86$ & $53-I-127$ & $63-E u-153$ & $8 \odot-\mathrm{Hg}-201$ & $94-\mathrm{Pu}-244$ \\
\hline $11-\mathrm{Na}-23$ & $37-R b-86$ & $53-I-130$ & $63-\mathrm{Eu}-157$ & $80-\mathrm{Hg}-202$ & $94-\mathrm{Pu}-246$ \\
\hline 14-Si-29 & $37-\mathrm{Rb}-87$ & $53-I-135$ & $64-G d-152$ & $8 \odot-\mathrm{Hg}-2 \odot 4$ & $95-A m-241$ \\
\hline 14-Si-30 & $38-S r-84$ & $54-X e-123$ & $64-G d-153$ & $82-P b-206$ & $95-A m-242$ \\
\hline $15-P-31$ & $39-Y-89$ & $54-\mathrm{Xe}-124$ & $64-G d-154$ & $82-P b-207$ & $95-A m-242 m$ \\
\hline $16-s-32$ & $4 \odot-Z r-9 \odot$ & $54-\mathrm{Xe}-126$ & $64-G d-155$ & $82-P b-208$ & $95-\mathrm{Am}-244$ \\
\hline $16-S-33$ & $40-Z r-91$ & $54-X e-130$ & $64-G d-156$ & $89-A c-225$ & $95-A m-244 m$ \\
\hline $16-5-34$ & $4 \odot-Z r-92$ & $54-\mathrm{Xe}-131$ & $64-G d-157$ & $89-A c-226$ & $96-\mathrm{Cm}-241$ \\
\hline $16-S-36$ & $4 \odot-Z r-93$ & $54-X e-134$ & $64-G d-158$ & $89-A c-227$ & $96-\mathrm{Cm}-242$ \\
\hline $17-\mathrm{Cl}-35$ & $40-Z r-94$ & $55-\mathrm{Cs}-133$ & $64-G d-160$ & $90-$ Th -227 & $96-\mathrm{Cm}-243$ \\
\hline $17-\mathrm{Cl}-37$ & $40-Z r-96$ & $56-\mathrm{Ba}-133$ & $65-\mathrm{Tb}-16 \odot$ & $90-\mathrm{Th}-228$ & $96-\mathrm{Cm}-244$ \\
\hline $18-\mathrm{Ar}-40$ & $41-N b-93$ & $56-\mathrm{Ba}-137$ & $66-\mathrm{Dy}-156$ & $90-T h-229$ & $96-\mathrm{cm}-245$ \\
\hline $19-\mathrm{K}-39$ & $42-$ Мо -92 & 57-La-139 & $66-\mathrm{Dy}-158$ & $90-\mathrm{Th}-230$ & $96-\mathrm{Cm}-246$ \\
\hline $19-\mathrm{K}-41$ & $42-\mathrm{Mo}-94$ & $57-\mathrm{La}-140$ & $66-\mathrm{Dy}-160$ & $9 \odot-\mathrm{Th}-232$ & $96-\mathrm{Cm}-247$ \\
\hline $21-S c-45$ & 42-Mo- 95 & $58-\mathrm{Ce}-136$ & $66-$ Dy -162 & $90-\mathrm{Th}-233$ & $96-\mathrm{Cm}-248$ \\
\hline $22-T i-46$ & $42-$ Мо -96 & $58-\mathrm{Ce}-138$ & $66-\mathrm{Dy}-163$ & $90-\mathrm{Th}-234$ & $96-\mathrm{Cm}-249$ \\
\hline $22-\mathrm{T}-47$ & 42-Mo- 97 & $58-\mathrm{Ce}-139$ & $66-$ Dy-164 & $91-\mathrm{Pa}-231$ & $96-\mathrm{Cm}-250$ \\
\hline $22-\mathrm{Ti}-48$ & $43-$-TC- 99 & $58-\mathrm{Ce}-140$ & $67-\mathrm{Ho}-165$ & $91-\mathrm{Pa}-232$ & $97-\mathrm{Bk}-249$ \\
\hline $22-T i-49$ & $44-\mathrm{Ru}-10 \odot$ & $58-\mathrm{Ce}-141$ & $67-\mathrm{Ho}-166 \mathrm{~m}$ & $91-\mathrm{Pa}-233$ & $97-\mathrm{Bk}-250$ \\
\hline $22-\mathrm{Ti}-50$ & $44-\mathrm{Ru}-101$ & $58-\mathrm{Ce}-143$ & $68-E r-166$ & $92-U-232$ & $98-C f-249$ \\
\hline $24-\mathrm{Cr}-50$ & $45-\mathrm{Rh}-103$ & $60-\mathrm{Nd}-142$ & $68-\mathrm{Er}-170$ & $92-U-233$ & $98-\mathrm{Cf}-250$ \\
\hline $24-\mathrm{Cr}-52$ & $47-A g-109$ & $60-\mathrm{Nd}-143$ & $72-\mathrm{HF}-174$ & $92-U-234$ & $98-C f-251$ \\
\hline $24-\mathrm{Cr}-53$ & $47-\mathrm{Ag}-111$ & $6 \odot-\mathrm{Nd}-144$ & $72-\mathrm{Hf}-176$ & $92-U-235$ & $98-C f-252$ \\
\hline $24-\mathrm{Cr}-54$ & $48-c d-106$ & $60-\mathrm{Nd}-145$ & $72-\mathrm{Hf}-177$ & $92-U-236$ & $98-C f-253$ \\
\hline $25-M n-55$ & $48-C d-108$ & $60-\mathrm{Nd}-146$ & $72-\mathrm{HF}-178$ & $92-U-237$ & $98-C f-254$ \\
\hline $26-\mathrm{Fe}-54$ & $48-\mathrm{Cd}-110$ & $60-\mathrm{Nd}-147$ & 72-Hf-179 & $92-U-238$ & $99-E s-253$ \\
\hline $26-\mathrm{Fe}-56$ & $48-c d-111$ & $60-\mathrm{Nd}-148$ & $72-\mathrm{Hf}-180$ & $92-U-239$ & 99 -Es- 254 \\
\hline $26-\mathrm{Fe}-57$ & $48-\mathrm{Cd}-112$ & $6 \odot-\mathrm{Nd}-15 \odot$ & $73-\mathrm{Ta}-181$ & $92-U-240$ & $99-E s-255$ \\
\hline $28-\mathrm{Ni}-58$ & $48-C d-113$ & $61-\mathrm{Pm}-147$ & $74-W-182$ & $92-U-241$ & $100-F m-255$ \\
\hline $28-N i-60$ & $48-c d-114$ & $61-P m-148$ & $74-W-183$ & $93-\mathrm{Np}-235$ & \\
\hline $28-\mathrm{Ni}-61$ & $48-C d-115 m$ & $61-P m-149$ & $74-W-184$ & $93-N p-236$ & \\
\hline
\end{tabular}

For details of VII.1 versus VII.0 see the below tables in Appendices B and D.

\section{PREPRO 2010 Codes}

In addition to the changes in the ENDF/B-VII.1 evaluations, it should be noted that between the last version of this report, where the PREPRO 2007 codes were used, and the current version, where the PREPRO 2010 codes were used, there have been major improvements in the ENDF/B Pre-processing codes (PREPRO). The major improvements were both in terms of improving the basic methods used by the codes and in terms of incorporating the latest ENDF-6 Formats and Procedures used by the current evaluations. The result is more accurate cross section data throughout the POINT 2011 library.

WARNING - due to recent changes in ENDF-6 Formats and Procedures only the latest version of the ENDF/B Pre-processing codes, namely PREPRO 2010, can be used to accurately process all current ENDF/B-VII evaluations. If you fail to heed this warning and you use any earlier versions of these codes the results will be inaccurate.

The PREPRO 2010 codes run on virtually any computer, and will soon be available FREE on-line from the Nuclear Data Section, IAEA, Vienna, Austria, website at, 
http://www-nds.iaea.or.at/ndspub/endf/prepro/

\section{Data Processing}

As distributed the original evaluated data includes cross sections represented in the form of a combination of resonance parameters and/or tabulated energy dependent cross sections, nominally at 0 Kelvin temperature. For use in applications, this data has been processed using the 2010 version of the ENDF/B Pre-processing codes (PREPRO 2010) to produce temperature dependent, linearly interpolable in energy, tabulated cross sections, in the ENDF-6 format.

For use in applications this library has been processed into the form of temperature dependent cross sections at eight neutron reactor like temperatures, between 0 and 2100 Kelvin, in steps of 300 Kelvin (the exception being 293.6 Kelvin, for exact room temperature at 20 Celsius). It has also been processed to five astrophysics like temperatures, 1, 10, $100 \mathrm{eV}, 1$ and $10 \mathrm{keV}$. For reference purposes, 300 Kelvin is approximately $1 / 40 \mathrm{eV}$, so that $1 \mathrm{eV}$ is approximately 12,000 Kelvin. At each temperature the cross sections are tabulated and linearly interpolable in energy.

The steps required and codes used to produce room temperature, linearly interpolable tabulated cross sections, in the ENDF-6 format, are described below (the name of each code in given in parenthesis; for details of each code see reference [R3]).

Here are the steps, and PREPRO 2010 codes, used to process the data, in the order in which the codes were used.

1) Linearly interpolable, tabulated cross sections (LINEAR)

2) Including the resonance contribution (RECENT)

3) Doppler broaden all cross sections to temperature (SIGMA1)

4) Check data, define redundant cross sections by summation (FIXUP)

5) Update evaluation dictionary in MF/MT=1/451 (DICTIN)

For the "cold" (0 Kelvin) data steps 1), 2) and 4), 5) were used (no Doppler broadening). For the data at other temperatures, after steps 1) and 2), the data was Doppler broadened to each temperature using step 3), and the results were then made consistent with the ENDF/B formats and conventions using steps 4) and 5), to produce the final distributed data.

The result is linearly interpolable in energy, tabulated, temperature dependent cross sections, in the ENDF-6 format, ready to be used in applications.

Note - this processing only involved the energy dependent neutron cross sections. All other data in the evaluations, e.g., angular and energy distributions, was not affected by this processing, and is identical in all versions of the final results, i.e., it is the same in all of the directories, ORIGINAL, as well as K0 through K2100, and 1ev through 10kev, on the DVDs. 


\section{Accuracy of Results}

Each of the codes described above that was used to process data to obtain tabulated, linearly interpolable in energy cross sections, processed the data to within a user defined accuracy, or allowable uncertainty. The ENDF/B Pre-processing codes (PREPRO 2010) are self-documenting, in the sense that the ENDF/B formatted output data that each code produces includes comments at the beginning of each evaluation defining the accuracy to which the cross sections were calculated. The combination of comments added by all of the codes defines the sequence and accuracy used by all of them. The accuracy is the same for all evaluations. Therefore, for exact details of the accuracy of the data, see the comments at the beginning of any evaluation. For use in POINT 2011 all cross sections were reconstructed to within an accuracy of $0.01 \%$ in the thermal range, and $0.1 \%$ at all other energies and temperatures; this is beyond the accuracy to which this data in known, so that I assume that the data processing does not add any significant additional error to the inherent error of the data.

\section{Contents of the Library}

This library contains all of the evaluations in the ENDF/B-VI.1 general purpose library. A table in the appendix summarizes the contents of the ENDF/B-VII.1 general purpose library. This library contains evaluations for 418 materials (isotopes or naturally occurring elemental mixtures of isotopes).

This library does not contain data from special purpose ENDF/B-VII libraries, such as fission products, thermal scattering, photon interaction data. To obtain any of these special purpose libraries contact the National Nuclear Data Center, Brookhaven National Laboratory,

\section{ENDF@bnlnd2.dne.bnl.gov}

In the POINT 2011 library each evaluation is stored as a separate file. The following table defines each material and the corresponding filename. The entire library is in the computer independent ENDF-6 character format, which allows the data to be easily transported between computers. The entire library requires approximately 16 gigabyte of storage and is distributed on one DVD compressed; see below for details of the DVD.

This library contains data for some metastable materials, which are indicated by an "M" at the end of their descriptions.

The majority of these evaluations are complete, in the sense that they include all cross sections over the energy range $10^{-5} \mathrm{eV}$ to at least $20 \mathrm{MeV}$. See the appendix for a list of all evaluations, plus a separate list of incomplete evaluations; there are now only a few.

The DVD single is compressed; when uncompressed you will find a single directory named POINT2011 containing fifteen (15) sub-directories, 
DOCUMENT - A copy of this report in MSWord and PDF formats.

ORIGINAL - The original ENDF/B data before it was processed.

K0 $\quad-0$ Kelvin cross sections

K293.6 - 293.6 Kelvin cross sections

K600 - 600 Kelvin cross sections

K900 - -900 Kelvin cross sections

K1200 - $\quad 1200$ Kelvin cross sections

K1500 - 1500 Kelvin cross sections

K1800 - 1800 Kelvin cross sections

K2100 - 2100 Kelvin cross sections

$1 \mathrm{eV} \quad-1 \mathrm{eV}$ cross sections

$10 \mathrm{eV} \quad-10 \mathrm{eV}$ cross sections

$100 \mathrm{eV} \quad-100 \mathrm{eV}$ cross sections

$1 \mathrm{keV} \quad-1 \mathrm{keV}$ cross sections

$10 \mathrm{keV} \quad-10 \mathrm{keV}$ cross sections

With the exception of DOCUMENT, each of these directories contains 419 files, one file for each of the 418 evaluation, plus one HTML file to allow interactive data retrieval. Each file is a complete ENDF/B "tape" [R2], including a starting "tape" identification line, and ending with a "tape" end line [R2]. In this form, each file can be used by a wide variety of available computer codes that treat data in the ENDF/B format, e.g., all of the PREPRO codes.

\section{Requesting POINT 2011 Data}

Please do not contact the author of this report to request this data; I do not have the resources necessary to directly respond to requests for this data. This data has been distributed and is Internationally available from nuclear data/code centers throughout the World,

1) Within the United States: contact the National Nuclear Data Center, Brookhaven National Laboratory, Mike Herman at, services@bnlnd2.dne.bnl.gov

2) Within Western Europe: contact the OECD Nuclear Energy Agency/ Data Bank (NEA/DB), Paris, France, programs@nea.fr

3) Otherwise: contact the Nuclear Data Section, International Atomic Energy Agency, Vienna, Austria, Alberto Mengoni at, A.Mengoni@iaea.org 


\section{Installation and Use of POINT 2011}

I recommend that you,

1) Copy the single file from the POINT 2011 DVD to your computer,

2) Uncompress and un-tar the file; then delete the compressed and tar files.

3) You should then have one directory named POINT2011 containing all of the data

4) To random access the data execute (double click) POINT2011.htm.

The main POINT2011 directory will contain the fifteen (15) sub-directories, described above. These POINT 2011 directories include HTML routines to allow interactive retrieval of the data. The result will be a directory of about 16 gigabytes. To put that in perspective, today it costs less than \$0.10 U.S. to purchase, install, and maintain on-line one gigabyte of disk storage. Therefore the cost of maintaining this 16 gigabyte library on-line is trivial.

\section{Acknowledgments}

I thank Said Mughabghab for his detailed explanation of the use of his newly published resonance parameters [R4] in ENDF/B-VII.0 evaluations. I thank Ramon E. Arcilla, Jr., of the National Nuclear Data Center (NNDC), Brookhaven National Laboratory, for supplying the original ENDF/B-VII.0, used in this project. I thank Liam Costello, of the Nuclear Data Section, International Atomic Energy Agency, for supplying the ENDF/B Pre-processing codes, PREPRO 2010, used in this project. I thank Nancy Larsen, Bob MacFarlane, Maurice Greene, and Mike Dunn, for their comparison of their cross section processing codes (SAMMY, NJOY and AMPX) against the PREPRO codes. These comparisons have led to significant improvements in the accuracy and reliability of the results produced by all four codes (SAMMY, NJOY, AMPX, PREPRO). I thank Andre Trkov, Skip Kahler, Robert MacFarlane, Mike Herman and Dave Heinrichs for proofreading the draft of this report and making many helpful corrections and improvements, which I incorporated in the final report. 


\section{References}

[R1a] "POINT 2009: A Temperature Dependent ENDF/B-VII.0 data Cross Section Library, June 6, 2009.

[R1b] "POINT 2007: A Temperature Dependent ENDF/B-VII.0 data Cross Section Library", Lawrence Livermore National Laboratory, UCRL-TR228089, February 2007.

[R2] Data Formats and Procedures for the Evaluated Nuclear Data File ENDF-6, BNLNCS-44945, Rev. 11/95, edited by V. McLane, et al. National Nuclear Data Center, Brookhaven National Lab. http://www.nndc.bnl.gov/nndcscr/documents/endf/endf102/

[R3] "PREPRO 2010: The 2010 ENDF/B Pre-Processing Codes," by D.E. Cullen, Nuclear Data Section, International Atomic Energy Agency, Vienna, Austria, IAEANDS-39, Rev. 14, Oct. 31, 2010; PREPRO 2010 is now publicly available. http://wwwnds.iaea.or.at/ndspub/endf/prepro/

[R4] "Atlas of Nuclear Resonances”, by S.F. Mughabghab, National Nuclear Data Center, Brookhaven National Laboratory, published by Elsevier, March 2006.

[R5] "Exact Doppler Broadening of Tabulated Cross Sections," by D.E. Cullen and C.R. Weisbin, Nuclear Science and Engineering 60, p. 199 (1975)

[R6] "THERMAL: A Routine Designed to Calculate Neutron Thermal Scattering," by D.E. Cullen, Lawrence Livermore National Laboratory, UCRL-ID-120560-Rev-1, Sept. 1995.

http://home.comcast.net/ redcullen1

[R7] "Verification of High Temperature Free Atom Thermal Scattering in MERCURY Compared to TART", by D.E. Cullen, Scott McKinley and Christian Hagmann, Lawrence Livermore National Laboratory, UCRL-TR-226340, August 1, 2006.

[R8] "TART2005: A Coupled Neutron-Photon 3-D, Time Dependent, Combinatorial Geometry Monte Carlo Transport Code," by D.E. Cullen, Lawrence Livermore National Laboratory, UCRL-SM-218009, Nov. 22, 2005. 
Appendix A: Contents of ENDF/B-VII.1 (27 new + 391 old = 418 total evaluations)

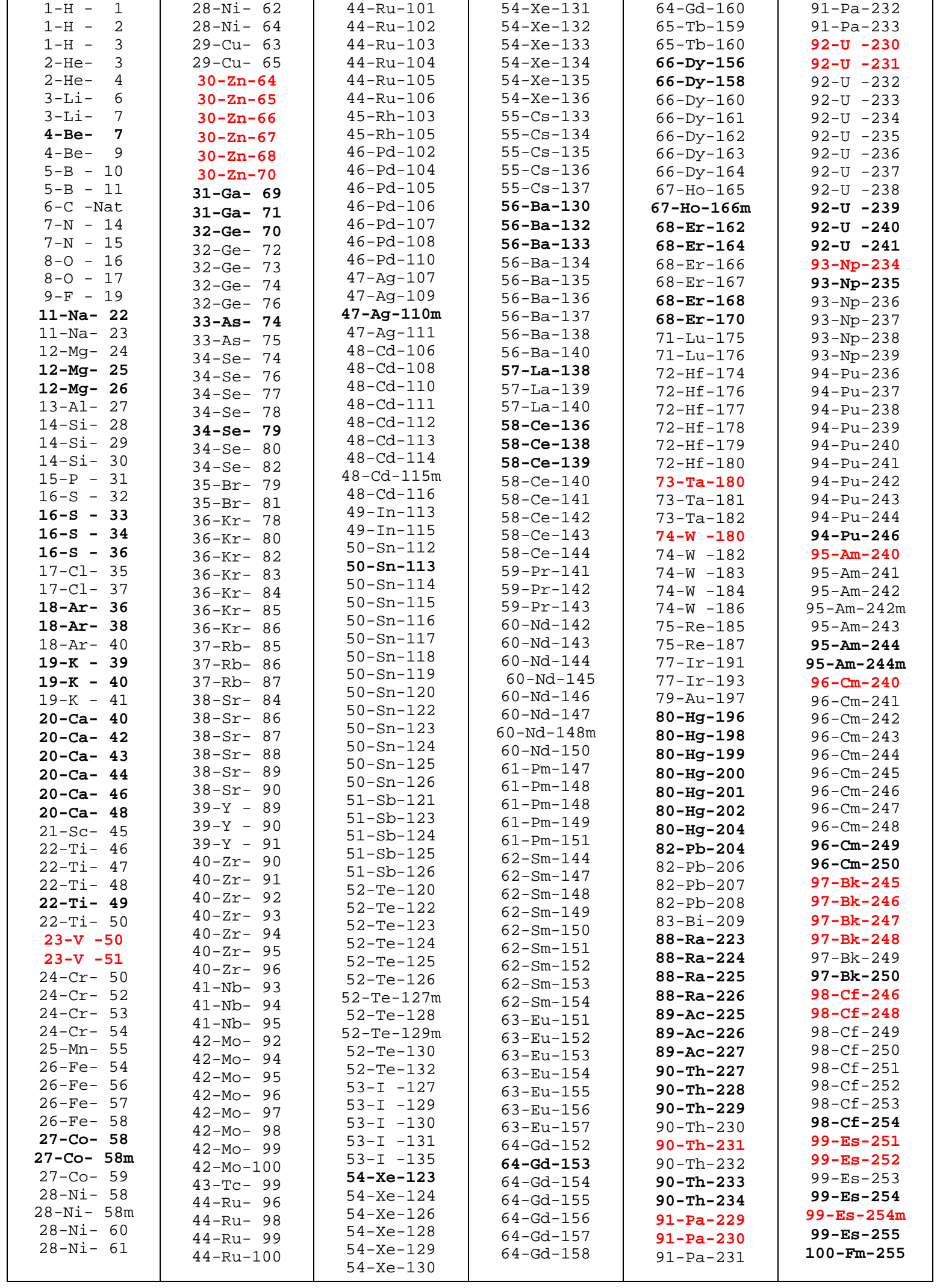



Appendix B: Contents of ENDF/B-VII.0 (78 new + 315 old = 393 total evaluations)

\begin{tabular}{|c|c|c|c|c|c|}
\hline $1-\mathrm{H}-$ & $28-\mathrm{Ni}-61$ & $44-R u-101$ & $54-\mathrm{Xe}-124$ & $63-\mathrm{Eu}-156$ & $90-T h-228$ \\
\hline $1-\mathrm{H}-$ & $28-\mathrm{Ni}-62$ & $44-R u-102$ & $54-X e-126$ & 63-Eu-157 & $90-\mathrm{Th}-229$ \\
\hline $2-\mathrm{He}-$ & $28-\mathrm{Ni}-64$ & $44-R u-103$ & $54-X e-128$ & $64-G d-152$ & $90-\mathrm{Th}-230$ \\
\hline $2-\mathrm{He}-$ & $29-\mathrm{Cu}-63$ & $44-R u-104$ & $54-X e-129$ & $64-G d-153$ & $90-T h-232$ \\
\hline 3-Li- & $29-\mathrm{Cu}-65$ & $44-R u-105$ & $54-X e-130$ & $64-G d-154$ & $90-\mathrm{Th}-233$ \\
\hline 3-Li- & $30-\mathrm{Zn}-\mathrm{Nat}$ & $44-R u-106$ & $54-X e-131$ & $64-G d-155$ & $90-T h-234$ \\
\hline 4-Be- & 31-Ga- 69 & $45-R h-103$ & $54-X e-132$ & $64-G d-156$ & $91-\mathrm{Pa}-231$ \\
\hline $4-\mathrm{Be}-$ & 31-Ga- 71 & $45-R h-105$ & $54-X e-133$ & $64-G d-157$ & $91-\mathrm{Pa}-232$ \\
\hline $5-B-10$ & $32-\mathrm{Ge}-70$ & $46-P d-102$ & $54-X e-134$ & $64-G d-158$ & $91-\mathrm{Pa}-233$ \\
\hline $5-B-11$ & $32-\mathrm{Ge}-72$ & $46-P d-104$ & $54-X e-135$ & $64-G d-160$ & $92-U-232$ \\
\hline 6-C - Nat & $32-\mathrm{Ge}-73$ & $46-P d-105$ & $54-X e-136$ & $65-\mathrm{Tb}-159$ & $92-U-233$ \\
\hline $7-N-14$ & $32-\mathrm{Ge}-74$ & $46-P d-106$ & $55-C s-133$ & $65-\mathrm{Tb}-160$ & $92-U-234$ \\
\hline $7-N-15$ & $32-\mathrm{Ge}-76$ & $46-\mathrm{Pd}-107$ & $55-C s-134$ & 66-Dy-156 & $92-U-235$ \\
\hline $8-0-16$ & 33-As- 74 & $46-P d-108$ & $55-C s-135$ & 66 -Dy-158 & $92-U-236$ \\
\hline $8-0-17$ & $33-A s-75$ & $46-P d-110$ & $55-C s-136$ & $66-\mathrm{Dy}-160$ & $92-U-237$ \\
\hline $9-F-19$ & $34-\mathrm{Se}-74$ & $47-\mathrm{Ag}-107$ & $55-C s-137$ & 66 -Dy-161 & $92-U-238$ \\
\hline $11-\mathrm{Na}-22$ & $34-\mathrm{Se}-76$ & $47-\mathrm{Ag}-109$ & $56-\mathrm{Ba}-130$ & 66-Dy-162 & $92-U-239$ \\
\hline $11-\mathrm{Na}-23$ & $34-\mathrm{Se}-77$ & $47-\mathrm{Ag}-110 \mathrm{~m}$ & 56-Ba-132 & $66-\mathrm{Dy}-163$ & $92-U-240$ \\
\hline $12-\mathrm{Mg}-24$ & $34-\mathrm{Se}-78$ & $47-\mathrm{Ag}-111$ & $56-\mathrm{Ba}-133$ & $66-\mathrm{Dy}-164$ & $92-U-241$ \\
\hline $12-\mathrm{Mg}-25$ & $34-\mathrm{Se}-79$ & $48-c d-106$ & $56-\mathrm{Ba}-134$ & 67-Ho-165 & $93-\mathrm{Np}-235$ \\
\hline $12-\mathrm{Mg}-26$ & $34-\mathrm{Se}-80$ & $48-c d-108$ & $56-\mathrm{Ba}-135$ & $67-\mathrm{Ho}-166 \mathrm{~m}$ & $93-N p-236$ \\
\hline 13-Al- 27 & $34-\mathrm{Se}-82$ & $48-\mathrm{Cd}-110$ & $56-\mathrm{Ba}-136$ & $68-E r-162$ & $93-N p-237$ \\
\hline 14-Si- 28 & $35-\mathrm{Br}-79$ & 48-Cd-111 & $56-\mathrm{Ba}-137$ & $68-E r-164$ & $93-N p-238$ \\
\hline 14-Si- 29 & $35-\mathrm{Br}-81$ & $48-\mathrm{Cd}-112$ & $56-\mathrm{Ba}-138$ & $68-E r-166$ & $93-N p-239$ \\
\hline $14-\mathrm{Si}-30$ & $36-\mathrm{Kr}-78$ & $48-\mathrm{Cd}-113$ & $56-\mathrm{Ba}-140$ & $68-E r-167$ & $94-\mathrm{Pu}-236$ \\
\hline $15-P-31$ & $36-\mathrm{Kr}-80$ & $48-C d-114$ & 57-La- 138 & $68-E r-168$ & $94-\mathrm{Pu}-237$ \\
\hline $16-S-32$ & $36-\mathrm{Kr}-82$ & $48-c d-115 m$ & 57-La-139 & $68-E r-170$ & $94-\mathrm{Pu}-238$ \\
\hline $16-s-33$ & $36-K r-83$ & $48-C d-116$ & $57-\mathrm{La}-14 \odot$ & $71-\mathrm{Lu}-175$ & $94-\mathrm{Pu}-239$ \\
\hline $16-S-34$ & $36-\mathrm{Kr}-84$ & $49-$ In -113 & $58-\mathrm{Ce}-136$ & $71-\mathrm{Lu}-176$ & $94-\mathrm{Pu}-240$ \\
\hline $16-S-36$ & $36-\mathrm{Kr}-85$ & $49-$ In- 115 & 58-Ce-138 & $72-\mathrm{Hf}-174$ & $94-\mathrm{Pu}-241$ \\
\hline $17-\mathrm{Cl}-35$ & $36-\mathrm{Kr}-86$ & $50-S n-112$ & 58-Ce-139 & $72-\mathrm{Hf}-176$ & $94-\mathrm{Pu}-242$ \\
\hline 17-Cl- 37 & $37-R b-85$ & $50-S n-113$ & $58-\mathrm{Ce}-140$ & 72-Hf-177 & $94-\mathrm{Pu}-243$ \\
\hline $18-A r-36$ & $37-R b-86$ & $50-S n-114$ & $58-\mathrm{Ce}-141$ & $72-\mathrm{Hf}-178$ & $94-\mathrm{Pu}-244$ \\
\hline $18-A r-38$ & $37-\mathrm{Rb}-87$ & $50-S n-115$ & $58-\mathrm{Ce}-142$ & 72-Hf-179 & $94-\mathrm{Pu}-246$ \\
\hline $18-\mathrm{Ar}-40$ & $38-\mathrm{Sr}-84$ & $50-S n-116$ & $58-\mathrm{Ce}-143$ & $72-\mathrm{Hf}-180$ & $95-\mathrm{Am}-241$ \\
\hline $19-\mathrm{K}-39$ & $38-S r-86$ & $50-S n-117$ & $58-\mathrm{Ce}-144$ & 73-Та-181 & $95-A m-242$ \\
\hline $19-\mathrm{K}-40$ & $38-\mathrm{Sr}-87$ & $50-S n-118$ & $59-\operatorname{Pr}-141$ & 73-Та-182 & $95-A m-242 m$ \\
\hline $19-K-41$ & $38-\mathrm{Sr}-88$ & $50-S n-119$ & $59-\mathrm{Pr}-142$ & $74-W-182$ & $95-A m-243$ \\
\hline $20-\mathrm{Ca}-40$ & $38-S r-89$ & $50-S n-120$ & $59-\operatorname{Pr}-143$ & $74-W-183$ & $95-A m-244$ \\
\hline 20-Ca- 42 & $38-\mathrm{Sr}-90$ & $50-S n-122$ & $60-\mathrm{Nd}-142$ & $74-W-184$ & $95-A m-244 m$ \\
\hline $20-\mathrm{Ca}-43$ & $39-Y-89$ & $50-S n-123$ & $60-\mathrm{Nd}-143$ & $74-W-186$ & $96-\mathrm{cm}-241$ \\
\hline $20-\mathrm{Ca}-44$ & $39-Y-90$ & $50-S n-124$ & $60-\mathrm{Nd}-144$ & $75-\mathrm{Re}-185$ & $96-\mathrm{Cm}-242$ \\
\hline $20-\mathrm{Ca}-46$ & $39-Y-91$ & $50-S n-125$ & $60-\mathrm{Nd}-145$ & $75-\operatorname{Re}-187$ & $96-\mathrm{Cm}-243$ \\
\hline 20-Ca- 48 & $40-Z r-90$ & $50-S n-126$ & $60-\mathrm{Nd}-146$ & $77-$ Ir -191 & $96-\mathrm{Cm}-244$ \\
\hline $21-S c-45$ & $40-Z r-91$ & $51-S b-121$ & $60-\mathrm{Nd}-147$ & $77-$ Ir -193 & $96-\mathrm{Cm}-245$ \\
\hline $22-\mathrm{Ti}-46$ & $40-Z r-92$ & $51-S b-123$ & $60-N d-148 m$ & $79-\mathrm{Au}-197$ & $96-\mathrm{Cm}-246$ \\
\hline $22-\mathrm{Ti}-47$ & $40-Z r-93$ & $51-\mathrm{Sb}-124$ & $60-\mathrm{Nd}-15 \odot$ & $80-\mathrm{Hg}-196$ & $96-\mathrm{Cm}-247$ \\
\hline $22-T i-48$ & $40-Z r-94$ & $51-S b-125$ & $61-\mathrm{Pm}-147$ & $80-\mathrm{Hg}-198$ & $96-\mathrm{Cm}-248$ \\
\hline 22-Ti- 49 & $40-Z r-95$ & $51-\mathrm{Sb}-126$ & $61-\mathrm{Pm}-148$ & $80-\mathrm{Hg}-199$ & $96-\mathrm{Cm}-249$ \\
\hline $22-T i-50$ & $40-Z r-96$ & $52-\mathrm{Te}-120$ & $61-P m-148$ & $80-\mathrm{Hg}-200$ & $96-\mathrm{Cm}-250$ \\
\hline $23-V$ - Nat & $41-N b-93$ & $52-\mathrm{Te}-122$ & $61-P m-149$ & $80-\mathrm{Hg}-201$ & $97-B k-249$ \\
\hline $24-\mathrm{Cr}-50$ & $41-\mathrm{Nb}-94$ & $52-\mathrm{Te}-123$ & $61-P m-151$ & $80-\mathrm{Hg}-202$ & $97-B k-250$ \\
\hline $24-\mathrm{Cr}-52$ & $41-\mathrm{Nb}-95$ & $52-\mathrm{Te}-124$ & $62-\mathrm{Sm}-144$ & $80-\mathrm{Hg}-204$ & $98-C f-249$ \\
\hline $24-\mathrm{Cr}-53$ & $\begin{array}{l}42-M O-92 \\
42-M o-94\end{array}$ & $52-\mathrm{Te}-125$ & $62-\mathrm{Sm}-147$ & $82-P b-204$ & $98-C f-250$ \\
\hline $24-\mathrm{Cr}-54$ & 42-Mo- 95 & $\begin{array}{c}52-\mathrm{Te}-126 \\
52-\mathrm{Te}-127 \mathrm{~m}\end{array}$ & $62-\mathrm{Sm}-148$ & $82-\mathrm{Pb}-206$ & $98-C f-251$ \\
\hline $25-M n-55$ & $42-$ Мо -96 & $\begin{array}{c}52-\mathrm{Te}-127 \mathrm{~m} \\
52-\mathrm{Te}-128\end{array}$ & 62-Sm-149 & $82-\mathrm{Pb}-207$ & $98-C f-252$ \\
\hline $26-\mathrm{Fe}-54$ & 42-Mo - 97 & $52-\mathrm{Te}-129 \mathrm{~m}$ & $62-\mathrm{Sm}-150$ & $82-\mathrm{Pb}-208$ & $98-C f-253$ \\
\hline $26-\mathrm{Fe}-56$ & 42-Mo- 98 & $52-\mathrm{Te}-130$ & $\begin{array}{l}62-\mathrm{Sm}-151 \\
62-\mathrm{Sm}-152\end{array}$ & $83-B i-209$ & 98 -Cf-254 \\
\hline $26-\mathrm{Fe}-57$ & 42-Mo- 99 & $52-\mathrm{Te}-132$ & $\begin{array}{l}62-\mathrm{Sm}-152 \\
62-\mathrm{Sm}-153\end{array}$ & $88-R a-223$ & $99-E s-253$ \\
\hline $26-\mathrm{Fe}-58$ & $42-\mathrm{Mo}-10 \odot$ & $53-I-127$ & $\begin{array}{l}02-5 \mathrm{II}-153 \\
62-\mathrm{Sm}-154\end{array}$ & 88-Ra-224 & 99 -Es - 254 \\
\hline 27-Co- 58 & $43-\mathrm{TC}-99$ & $53-I-129$ & $63-\mathrm{Eu}-151$ & $88-R a-225$ & $99-E s-255$ \\
\hline 27-Co- 58m & $44-\mathrm{Ru}-96$ & $53-I \quad-130$ & $63-\mathrm{Eu}-152$ & $88-R a-226$ & $100-F m-255$ \\
\hline 27-Co- 59 & 44-Ru- 98 & $53-I-131$ & $63-E u-153$ & $89-A C-225$ & \\
\hline $28-\mathrm{Ni}-58$ & 44-Ru- 99 & $53-I-135$ & $63-\mathrm{Eu}-154$ & $89-A C-226$ & \\
\hline $28-N i-58 m$ & & & & $89-A c-227$ & \\
\hline
\end{tabular}




\section{Appendix C: Elemental vs. Isotopic Evaluations}

Successive versions of ENDF/B have replaced elemental evaluations by isotopic evaluations. Between ENDF/B-VI and VII 13 elemental evaluations were deleted, i.e., included in ENDF/BVI, but not included in ENDF/B-VII, with VII.0 only including elemental evaluations for three elements: 6-C, 23-V, and 30-Zn. Between VII.0 and VII.1 2 elemental evaluations were deleted (23-V, and 30-Zn), and replaced by isotopic evaluations, leaving only 6-C: 6-C-12 98.93\%/ 6C-13 1.07\% missing

All of these isotopes in VII.1 are complete, in the sense that they include major cross sections (elastic, capture, inelastic) over the energy range $10^{-5} \mathrm{eV}$ up to at least $20 \mathrm{MeV}$.

WARNING: Be aware that evaluating isotopes is difficult and the quality of minor isotopes may be poor. To my knowledge as yet the summing these isotopes to define equivalent elemental evaluations has not been verified against experimental measurements.

\begin{tabular}{|c|c|c|c|c|c|}
\hline \multicolumn{6}{|c|}{ Elemental Evaluations Replaced by Isotopic evaluations in VII.1 (23 new, 19 old) } \\
\hline Element & Isotope & Element & Isotope & Element & Isotope \\
\hline \multirow{3}{*}{$12-\mathrm{Mg}-\mathrm{Nat}$} & $12-\mathrm{Mg}-24$ & 22-Ti-Nat & $22-T i-46$ & 42-Mo-Nat & $42-$ Мo- 92 \\
\hline & $12-M g-25$ & & 22-Ti- 47 & & 42-Мо- 94 \\
\hline & $12-M g-26$ & & $22-\mathrm{Ti}-48$ & & 42-Mo- 95 \\
\hline \multirow{3}{*}{ 14-Si-Nat } & 14-Si- 28 & & 22-Ti- 49 & & 42-Мo- 96 \\
\hline & $14-S i-29$ & & $22-T i-50$ & & 42-Мo- 97 \\
\hline & $14-\mathrm{Si}-30$ & 23-V -Nat & $23-v-50$ & & 42-Mo- 98 \\
\hline \multirow[t]{4}{*}{$16-\mathrm{S}-\mathrm{Nat}$} & $16-s-32$ & & $23-V-51$ & & 42-Mo- 99 \\
\hline & $16-s-33$ & 30-Zn-Nat & $30-Z n-64$ & & 42-Mo-100 \\
\hline & $16-s-34$ & & $30-\mathrm{Zn}-65$ & 49-In-Nat & $49-\operatorname{In}-113$ \\
\hline & $16-S-36$ & & $30-Z n-66$ & & $49-\mathrm{In}-115$ \\
\hline \multirow[t]{2}{*}{ 17-Cl-Nat } & $17-\mathrm{Cl}-35$ & & $30-Z n-67$ & 72-Hf-Nat & $72-\mathrm{Hf}-174$ \\
\hline & $17-\mathrm{Cl}-37$ & & $30-Z n-68$ & & 72-Hf-176 \\
\hline \multirow[t]{3}{*}{$19-\mathrm{K}-\mathrm{Nat}$} & $19-K-39$ & & $30-Z n-70$ & & 72-Hf-177 \\
\hline & $19-\mathrm{K}-40$ & 31-Ga-Nat & $31-\mathrm{Ga}-69$ & & $72-\mathrm{Hf}-178$ \\
\hline & $19-K-41$ & & 31-Ga- 71 & & $72-\mathrm{Hf}-179$ \\
\hline \multirow[t]{6}{*}{ 20-Ca-Nat } & $20-\mathrm{Ca}-40$ & $40-\mathrm{Zr}-\mathrm{Nat}$ & $40-Z r-9 \Theta$ & 74-Wl -Nat & 72-H†-180 \\
\hline & 20-Ca- 42 & & $40-Z r-91$ & $14-\mathrm{W}-\mathrm{Nad}$ & $\begin{array}{ll}74-W & -182 \\
74-W & -183\end{array}$ \\
\hline & $20-\mathrm{Ca}-43$ & & $4 \odot-Z r-92$ & & $\begin{array}{ll}74-W & -183 \\
74-W & -184\end{array}$ \\
\hline & $20-\mathrm{Ca}-44$ & & $40-Z r-93$ & & 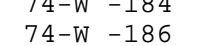 \\
\hline & $20-\mathrm{Ca}-46$ & & $40-Z r-94$ & & \\
\hline & 20-Ca- 48 & & $\begin{array}{l}40-Z r-95 \\
40-Z r-96\end{array}$ & & \\
\hline
\end{tabular}




\section{Appendix D: Completeness of VII.1}

Here I present the results of simple tests to see how complete VII.1 is. The results presented here should not be interpreted as indicating ERRORS, but rather as WARNINGS, that we should check the indicated data; in many cases I judge the data to be o.k. 4-Be-7 is a partial evaluations that failed almost all tests, and should never have been included in a ENDF/B general purpose library. Here I checked,

1) $\mathrm{MT}=2,102,18,4,16$ (elastic, capture, fission, inelastic, $n, 2 n$ ).

2) $\mathrm{MF}=3,4,5,6$ (cross sections, angular, energy, double differential)

For these test I assume,

1) Every evaluation includes $M T=2,102,4$ and 16; this is obviously not true for some light isotopes, but for completeness they are included here, i.e., this is merely to inform users. Of the heavier isotopes only 28-Ni-59 appears to be a problem with no inelastic data.

2) All cross sections are positive ( $>0$ ) above their threshold up to at least $20 \mathrm{MeV}$. This is not true for some reactions which do not extend up to at least $20 \mathrm{MeV}$, particularly high energy capture, which is o.k. There are also a number of cases where the elastic is negative due to the resonance contribution.

3) No isotopes with $\mathrm{Z}<90$ include $\mathrm{MT}=18$ (fission). This test found the obvious ERROR in 43-Tc-99, which has positive fission widths = NONSENSE!!! It also flagged isotopes of 88Ra and 89-Ac, which are questionable.

4) I included many more completeness tests, but these were the only ones that failed.

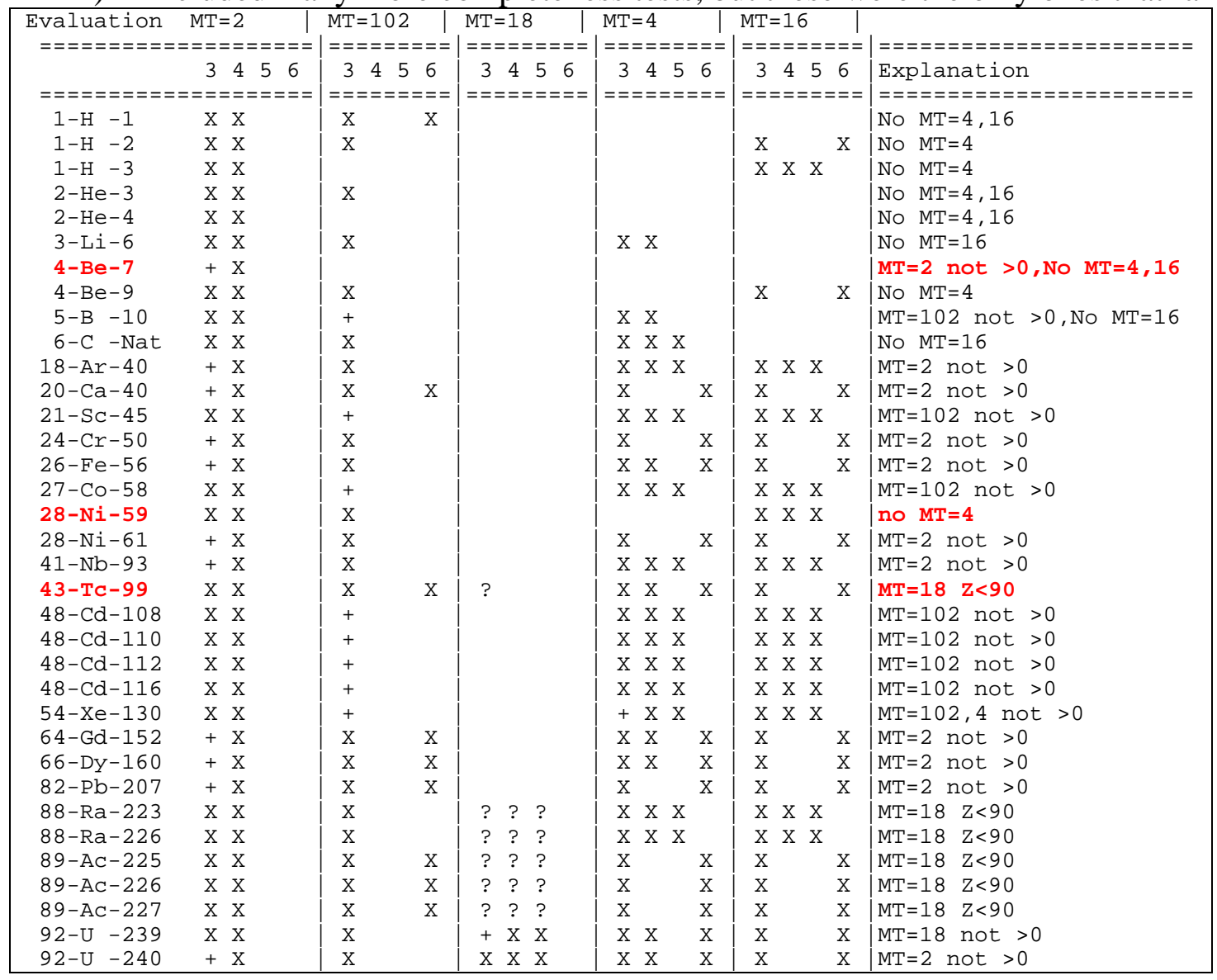


To see the improvements in VII.1 vs. VII.0, the below tables shows the results from running exactly the same tests on VII.0, here without the annotation explaining what's wrong in each case; hopefully from the above table you can figure this out. By comparing the above and below tables you can see the improvements in VII.1 compare to VII.0.

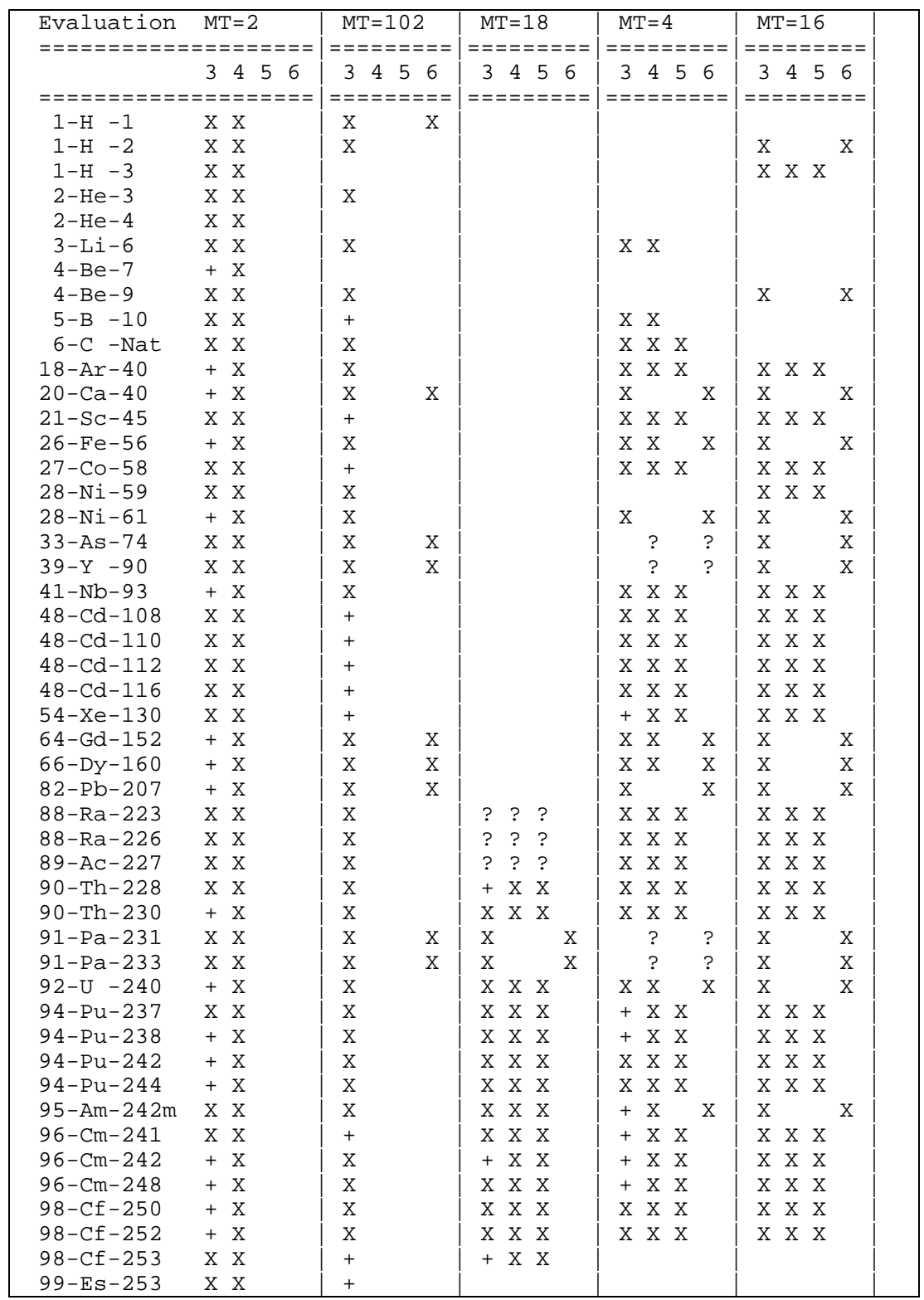


Appendix E: Summary of $\langle v(E)>$ for all fissile/fertile isotopes in ENDF/B-VII.1

For applications involving fission (F) I require both prompt and delayed neutrons per fission. In the ENDF/B format the evaluator can optionally include: Total (T), Delayed (D) and/or Prompt (P); given any two of these three we can uniquely define the third. Below is a summary of all fissile/fertile materials in ENDF/B-VII.1, indicating the neutrons per fission data included for each isotope. This table indicates each evaluation that includes,

$$
\begin{array}{lc}
\mathrm{F}=\text { Fission cross section } & (\mathrm{MF} / \mathrm{MT}=3 / 18) \\
\mathrm{T}=\text { Total }\langle v(E)> & (\mathrm{MF} / \mathrm{MT}=1 / 452) \\
\mathrm{D}=\text { Delayed }\langle v(E)> & (\mathrm{MF} / \mathrm{MT}=1 / 455) \\
\mathrm{P}=\text { Prompt }\langle v(E)\rangle & (\mathrm{MF} / \mathrm{MT}=1 / 456)
\end{array}
$$

This table indicates VII.1 is in much better shape than VII.0, where more evaluations only included total $(\mathrm{T})<v(E)>$. There is one glaring ERROR: 43-Tc-99 which is obviously non-fissile/fertile, but includes fission widths. In addition there are three evaluations that only include total $(\mathrm{T})<v(E)>$ : 88-Ra-233, 88-Ra-226, and 94-Pu-243. It is also questionable whether or not 88-Ra and 89-Ac should be identified as fissile/fertile.

Summary of all fissile/fertile isotopes in ENDF/B-VII.1 $<v(E)>$ (86 evaluations)

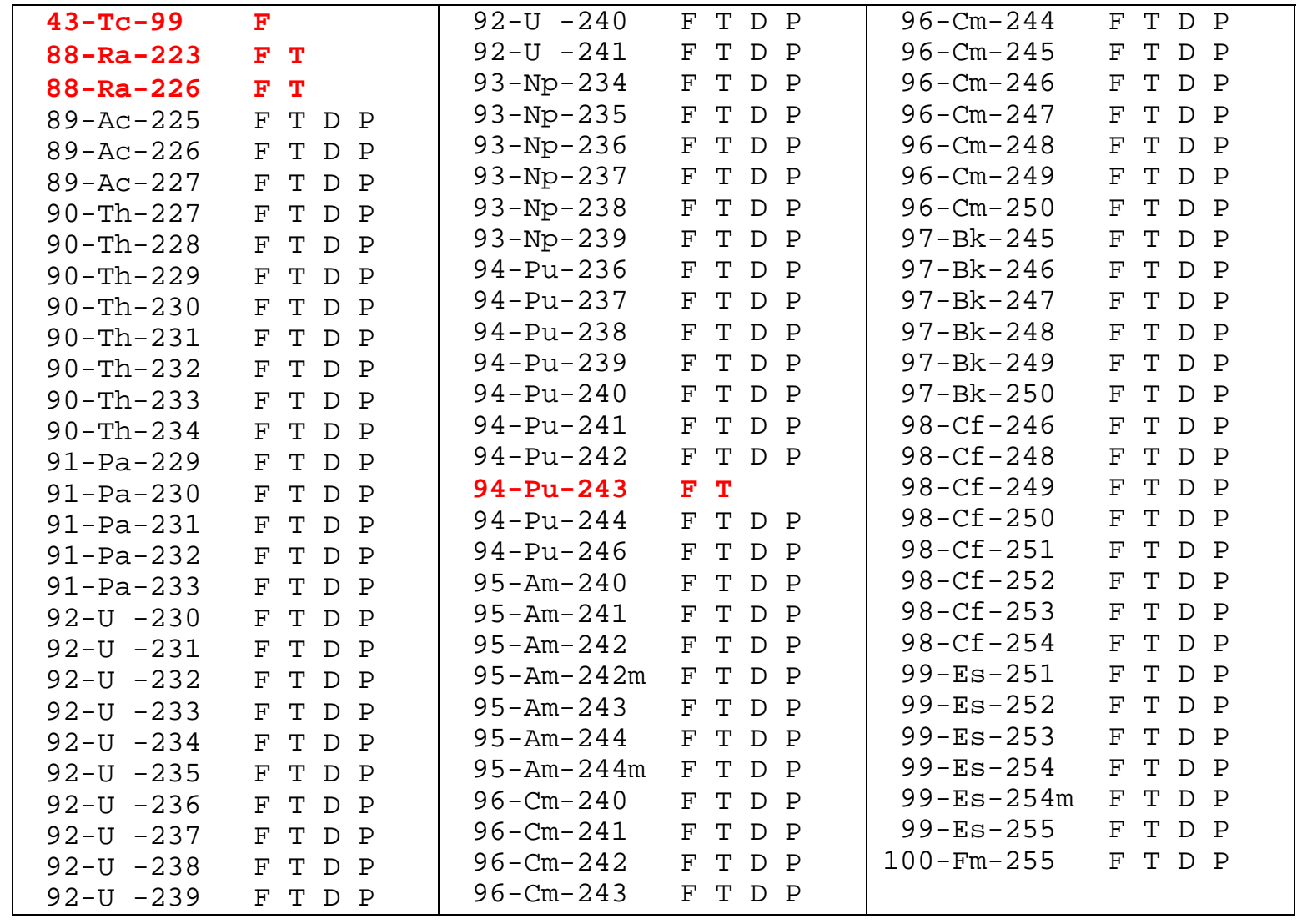




\section{Summary of all fissile/fertile isotopes in ENDF/B-VII.0 $<v(E)>$ (65 evaluations)}

Comparing the above table for VII.1 and the below table for VII.0 we can see that the $\langle v(E)>$ data is much improved, but VII.1 still has the same three deficiencies that we found in VII.0, namely evaluations that only include total $(\mathrm{T})<v(E)>$ : 88-Ra-233, 88Ra-226, and 94-Pu-243.

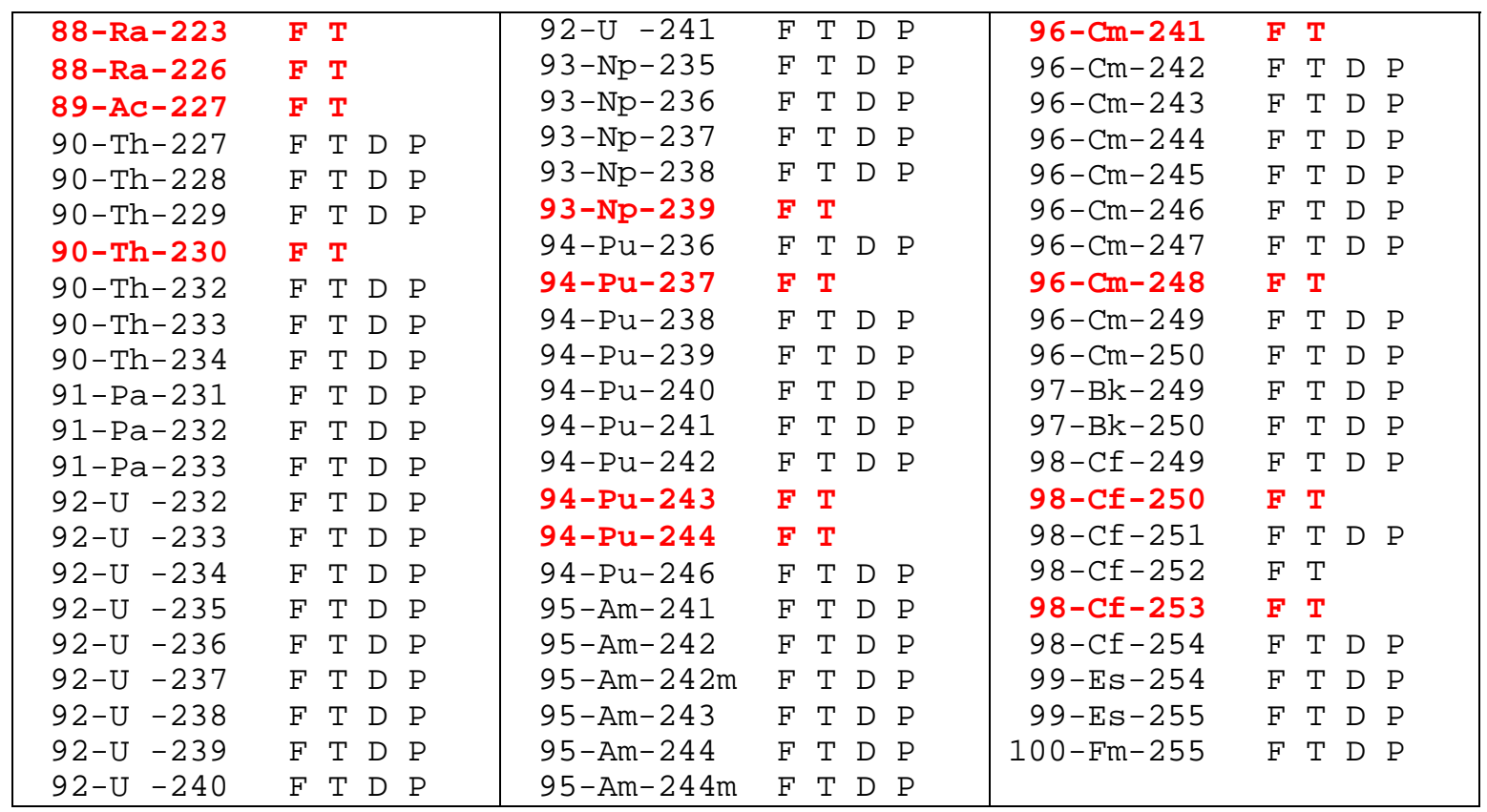




\section{Appendix F: An Initial Comparison of VII.1 versus VII.0}

So far I have only had time to check the major cross sections (MT=1, 2, 18, 102; total, elastic, fission and capture) for completeness: defined $10^{-5} \mathrm{eV}$ up to at least $20 \mathrm{MeV}$. WARNING: Many evaluations have cross sections above $20 \mathrm{MeV}$, but capture (MT=102) only up to $20 \mathrm{MeV}$, so there is a discontinuity at $20 \mathrm{MeV}$. For purposes of the below table this is not considered to be ERROR and as such is not indicated in this table, but users should be aware of this if you use the data above $20 \mathrm{MeV}$. The following table only includes those evaluations for which I "saw" problems; all other evaluations a judge to be o.k.

For the 238 Evaluations where I found “differences”, these differences may or may not be in the major cross sections that I have "seen. For details of which sections (that is which MF/MT) I found differences see the appendix D.

I used the PREPRO graphics codes EVALPLOT and COMPLOT to view ALL 418 evaluations in VII.1; for details of the following summary you can use these codes to "see" the cross sections.

For compactness in the below tables I use the following shorthand,

1) "Up to" means up to this \% difference between VII.0 and VII.1

2) “BIG” or "ENORMOUS" means BIG or ENORMOUS Differences

3) “Reg. region” means Res. Region; "Unresolved” mean unresolved Reg. region

4) “Horrible” means no resonances; can probably be improved using Mughabghab’s resonance parameters [R4].

5) "VII.0 BETTER" means VII.1 includes no or few resonances, while VII.0 includes many resonances and certainly appears to be physically BETTER

\section{New Evaluations in VII.1, not in VII.0 (27 Evaluations)}

Most of these were adopted from JENDL-4. The isotopes of $\mathrm{V}$ and $\mathrm{Zn}$ look o.k. The others are very crude; that's the bad news. The good news is that they are all minor isotopes which generally will have little effect on our applications.

\section{Identical in VII.0 and VII.1 (153 Evaluations)}

\begin{tabular}{|cl|ll|}
$5-\mathrm{B}-10$ & MT=102 $(\mathrm{n}, \mathrm{g})=0$ above $1 \mathrm{MeV}$ & $50-\mathrm{Sn}-123$ & Very crude; only unres. \\
$20-\mathrm{Ca}-46$ & No resonances & $50-\mathrm{Sn}-126$ & Very crude; only unres. \\
$27-\mathrm{Co}-58$ & Very crude; weird capture & $51-\mathrm{Sb}-124$ & Very crude; only unres. \\
$27-\mathrm{Co}-58 \mathrm{~m}$ & Very crude; weird capture & $51-\mathrm{Sb}-125$ & Very crude; only unres. \\
$34-\mathrm{Se}-79$ & Very crude; only unres. & $52-\mathrm{Te}-120$ & Very crude; only unres. \\
$36-\mathrm{Kr}-82$ & Very crude & $52-\mathrm{Te}-127 \mathrm{~m}$ & Very crude; only unres. \\
$36-\mathrm{Kr}-83$ & Very crude & $52-\mathrm{Te}-129 \mathrm{~m}$ & Very crude; only unres. \\
$38-\mathrm{Sr}-89$ & Very crude & $54-\mathrm{Xe}-133$ & Very crude; only unres. \\
$38-\mathrm{Sr}-90$ & Very crude & $55-\mathrm{Cs}-136$ & Very crude; only unres. \\
$39-\mathrm{Y}-91$ & Very crude; only unres. & $55-\mathrm{Cs}-137$ & Very crude; only unres. \\
$40-\mathrm{Zr}-95$ & Very crude; only unres. & $56-\mathrm{Ba}-132$ & Crude \\
$41-\mathrm{Nb}-95$ & Crude & $58-\mathrm{Ce}-144$ & Very crude; only unres. \\
$42-\mathrm{Mo}-98$ & Very crude; only unres. & $59-\mathrm{Pr}-143$ & Crude \\
$42-\mathrm{Mo}-99$ & Very crude; only unres. & $63-\mathrm{Eu}-156$ & Very crude; only unres. \\
$44-\mathrm{Ru}-96$ & Very crude; only unres. & $88-\mathrm{Ra}-223$ & Very crude; no resonances \\
$44-\mathrm{Ru}-98$ & Very crude; only unres. & $88-\mathrm{Ra}-224$ & Very crude; no resonances \\
$44-\mathrm{Ru}-105$ & Very crude; no resonances & $88-\mathrm{Ra}-225$ & Very crude; no resonances \\
$44-\mathrm{Ru}-106$ & Very crude; only unres. & & \\
$45-\mathrm{Rh}-105$ & Very crude & &
\end{tabular}




\section{Different in VII.0 and VII.1 (238 Evaluations)}

\begin{tabular}{|c|c|c|c|}
\hline $2-\mathrm{He}-4$ & Up to $2 \%$ & $74-W-183$ & Larger Res. region \\
\hline 3-Li-6 & Uo to $18 \%$ at low Energy & $74-W-184$ & Larger Res. region \\
\hline $4-\mathrm{Be}-9$ & Up to $8 \%$ & $74-W-186$ & Larger Res: region \\
\hline $8-0-16$ & BIG in Res. region & $75-\mathrm{Re}-185$ & ENOORMOUS in Res. region \\
\hline $9-F \quad-19$ & BIG in Res. region & $75-\operatorname{Re}-187$ & ENOORMOUS in Res. region \\
\hline $11-\mathrm{Na}-23$ & BIG in Res. region & $77-\operatorname{Ir}-193$ & Very crude \\
\hline $15-P-31$ & Up to $18 \%$ Capture & $80-\mathrm{Hg}-202$ & Very crude \\
\hline $17-C 1-35$ & BIG in Res. region & $80-\mathrm{Hg}-204$ & VERY crude \\
\hline $17-\mathrm{Cl}-37$ & BIG in Res: region & $89-A c-225$ & HORRIBLE \\
\hline $19-K-39$ & ENOORMOUS in Res. region & $89-A c-226$ & HORRIBLE \\
\hline $19-\mathrm{K}-41$ & ENOORMOUS in Res. region & $89-A c-227$ & HORRIBLE \\
\hline $22-\mathrm{Ti}-46$ & ENOORMOUS in Res. region & $90-$ Th -227 & HORRIBLE \\
\hline $22-\mathrm{Ti}-47$ & ENOORMOUS in Res. region & $90-T h-228$ & BIG; $(n, f)=0,2$ to $400 \mathrm{keV}$ \\
\hline $22-\mathrm{Ti}-48$ & ENOORMOUS in Res. region & $90-$ Th -229 & ENOORMOUS in Res, region \\
\hline $22-\mathrm{Ti}-49$ & ENOORMOUS in Res, region & $90-T h-230$ & ENOORMOUS in Res. region \\
\hline $22-T i-50$ & ENOORMOUS in Res. region & $90-$ Th -232 & Up to $2 \%$ in unresolved \\
\hline $24-\mathrm{Cr}-50$ & ENOORMOUS in Res, region & $90-$ Th- 233 & HORRIBLE \\
\hline $24-C r-52$ & ENOORMOUS in Res. region & $90-T h-234$ & HORRIBLE \\
\hline $24-\mathrm{Cr}-53$ & ENOORMOUS in Res. region & $91-\mathrm{Pa}-232$ & BIG in Res. region \\
\hline $24-\mathrm{Cr}-54$ & ENOORMOUS in Res. region & $92-U-232$ & BIG in Res. region \\
\hline $25-M n-55$ & ENOORMOUS in Res, region & $92-U-235$ & Up to $9 \%$ \\
\hline $28-\mathrm{Ni}-58$ & ENOORMOUS in Res. region & $92-U-237$ & ENOORMOUS in Res. Region \\
\hline $28-\mathrm{Ni}-60$ & ENOORMOUS in Res. region & $92-U-239$ & ENOORMOUS in Res, region \\
\hline $28-N i-61$ & ENOORMOUS in Res. region & $93-N p-235$ & HORRIBLE \\
\hline $28-N i-62$ & ENOORMOUS in Res, region & $93-N p-236$ & ENOORMOUS in Res, region \\
\hline $28-\mathrm{Ni}-64$ & ENOORMOUS in Res, region & $93-N p-237$ & Up to $13 \%$ at low energy \\
\hline $33-A s-74$ & Crude & $93-N p-238$ & ENOORMOUS in Res. region \\
\hline $33-A s-75$ & Up to $11 \%$ & $93-N p-239$ & HORRIBLE \\
\hline $36-\mathrm{Kr}-78$ & Up to $83 \%$ & $94-\mathrm{Pu}-236$ & Up to $150 \%$ in unresolved \\
\hline $39-Y-89$ & Much Smaller Res: region & $94-P u-237$ & HORRIBLE \\
\hline $40-Z r-90$ & Smaller Res. region & $94-P u-238$ & ENOORMOUS in Res. region \\
\hline $40-Z r-91$ & BIG in Res. region & $94-P u-239$ & Up to $1 \%$ in unresolved \\
\hline $42-$ Mo- 95 & Differences in Res, region & $94-\mathrm{Pu}-240$ & ENOORMOUS in Res. capture? \\
\hline $43-$ Tc -99 & Differences in Res. region & $94-P u-242$ & ENOORMOUS in Res. region \\
\hline $45-\mathrm{Rh}-103$ & Differences in Res. region & $94-\mathrm{Pu}-244$ & ENOORMOUS in Res. region \\
\hline $47-\mathrm{Ag}-109$ & ENOORMOUS in Res. region & $94-\mathrm{Pu}-246$ & HORRIBLE \\
\hline $48-C d-106$ & ENOORMOUS in Res, region & $95-\mathrm{Am}-241$ & BIG in Res. region \\
\hline $48-C d-108$ & ENOORMOUS in Res. region & $95-\mathrm{Am}-244$ & HORRIBLE \\
\hline $48-C d-110$ & ENOORMOUS in Res, region & $95-A m-244 m$ & HORRIBLE \\
\hline $48-C d-111$ & ENOORMOUS in Res, region & $96-\mathrm{Cm}-241$ & HORRIBLE \\
\hline $48-C d-112$ & ENOORMOUS in Res: region & $96-\mathrm{Cm}-242$ & BIG in Res. region \\
\hline $48-\mathrm{Cd}-113$ & Smaller Res. region & $96-\mathrm{Cm}-243$ & ENOORMOUS in Res. region \\
\hline $48-C d-114$ & ENOORMOUS in Res. region & $96-\mathrm{Cm}-244$ & ENOORMOUS in Res. region \\
\hline $48-C d-116$ & BIG in Res. region & $96-\mathrm{Cm}-245$ & BIG in Res. region \\
\hline $53-I-135$ & Very crude; no Res. region & $96-\mathrm{Cm}-246$ & ENOORMOUS in Res, region \\
\hline $54-\mathrm{Xe}-123$ & HORRIBLE; nonphysical & $96-\mathrm{Cm}-247$ & ENOORMOUS in Res. region \\
\hline $54-\mathrm{Xe}-124$ & Up to $50 \%$ & $96-\mathrm{Cm}-248$ & Smaller resolved region \\
\hline $54-\mathrm{Xe}-130$ & MT $=102(n, g)=\odot$ above $11 \mathrm{MeV}$ & $96-\mathrm{Cm}-249$ & HORRIBLE； VII.0 BETTER \\
\hline $55-C s-133$ & ENOORMOUS in Res. region & $96-\mathrm{Cm}-250$ & ENOORMOUS in Res. region \\
\hline $6 \odot-\mathrm{Nd}-145$ & ENOORMOUS in Res. region & $97-B k-249$ & BIG in Res. region \\
\hline $61-\mathrm{Pm}-148$ & Crude; only unresolved & $97-\mathrm{Bk}-250$ & HORRIBLE； VII.O BETTER \\
\hline $61-\mathrm{Pm}-149$ & Crude; only unresolved & $98-C f-249$ & Larger Res. region \\
\hline $62-\mathrm{Sm}-153$ & Up to $10 \%$ at low energy & $98-C f-250$ & Smaller res.: VII.O BETTER \\
\hline $64-\mathrm{Gd}-157$ & Up to $16 \%$ & $98-C f-251$ & Smaller res.: VII.0 BETTER \\
\hline $72-\mathrm{Hf}-174$ & ENOORMOUS in Res, region & $98-C f-252$ & ENOORMOUS \\
\hline $72-\mathrm{Hf}-176$ & BIG in Res. region & $98-C f-253$ & HORRIBLE; VII.๑ incomplete \\
\hline $72-\mathrm{Hf}-177$ & ENOORMOUS in Res, region & $98-C f-254$ & HORRIBLE \\
\hline $72-\mathrm{Hf}-178$ & BIG in Res, region & $99-E s-253$ & Smaller res.: VII.0 BETTER \\
\hline $72-\mathrm{Hf}-179$ & ENOORMOUS in Res, region & $99-E s-254$ & HORRIBLE \\
\hline $72-\mathrm{Hf}-180$ & Smaller Res. region & $99-E S-255$ & HORRIBLE \\
\hline $73-\mathrm{Ta}-181$ & Up to $62 \%$ & $100-F m-255$ & HORRIBLE \\
\hline $74-W-182$ & ENOORMOUS in Res. Region & & \\
\hline
\end{tabular}




\section{Appendix G: Earlier Versions of ENDF/B}

\section{POINT 2009: ENDF/B-VII.0}

ENDF/B-VII.0 was released by CSEWG in November 2006 and is to be frozen for three years until November 2009. As such the Original data included in POINT 2009 [R1a] is identical to that included in the earlier release POINT 2007 [R1b]. However, the processed, temperature dependent results may differ because of improvements in the PREPRO ENDF/B Preprocessing Codes, particularly with regard to accuracy, and correct interpretation of the ENDF/B rules as defined by the ENDF/B formats and procedures manual, ENDF-102.

\section{POINT 2007: ENDF/B-VI.8}

The version of ENDF/B, preceding ENDF/B-VII.0, namely ENDFV/B-VI, Release 8, contained 328 evaluations [R2]; of the evaluations 13 elemental evaluations are not included in ENDF/B-VII.0 (these have been replaced by isotopic evaluations).

ENDF/B-VII.0 includes 315 evaluations from ENDF/B-VI and 78 evaluations for new isotopes, for a total of 393 evaluations in ENDF/B-VII.0. The contents of ENDF/BVII.0 are defined in the Appendix A. The appendix includes a variety of what I hope are useful summaries of the VII.0, including,

1) Contents of ENDF/B-VII.0 (78 new + 315 old = 393 total evaluations)

2) Elemental Evaluations Replaced by Isotopic evaluations (16 new, 19 old)

3) New Evaluations for ENDF/B-VII.0 (78 new)

4) Summary of $\langle v(E)\rangle$ for all 65 fissile/fertile isotopes in ENDF/B-VII.0

5) Completeness of ENDF/B-VII.0 Evaluations

6) Same Evaluations in ENDF/B-VI and VII (315)

\section{Deficiencies and Proposed Updates}

ENDF/B-VII.0 was released by CSEWG in November 2006 and is to be frozen for three years until November 2009. All recognized ENDF/B-VII.0 deficiencies and proposed updates can be viewed at,

http://www.nndc.bnl.gov/exfor/4web/VII.0-deficiencies.html

These data will be reviewed by CSEWG and these data will serve as the basis for the next version of ENDF/B, namely ENDF/B-VII.1. 


\section{New Evaluations for ENDF/B-VII.0 (78 new)}

After six versions of ENDF/B over almost 40 years, most of the important isotopes have already been evaluated and included in earlier versions of ENDF/B. The new ENDF/BVII evaluations were difficult to do, since usually there is little experimental data for rarer isotopes. Most of the new evaluations are complete in the sense that they include major cross sections from $10^{-5} \mathrm{eV}$ to $20 \mathrm{MeV}$. The except is 4-Be-7 which only extends up to $8.1 \mathrm{MeV}$, and only includes elastic and charged particle reactions; this is a theoretical evaluation that should not have been included in ENDF/B-VII.0. Many of the other evaluations are pretty bad; better than nothing, but crude, so CAVEAT EMPTOR!

The below table includes a list of all 78 new evaluations. If there are no comments, I judge the evaluation to be o.k.

\section{New Evaluations}

\begin{tabular}{|c|c|c|c|}
\hline Material & Comments & Material & Comments \\
\hline $4-\mathrm{Be}-\quad 7$ & Useless partial & $67-\mathrm{Ho}-166 \mathrm{M}$ & Crude \\
\hline $11-\mathrm{Na}-22$ & Crude & $68-E r-162$ & \\
\hline $12-M g-25$ & & $68-E r-164$ & \\
\hline $12-M g-26$ & & $68-E r-168$ & \\
\hline $16-s-33$ & & $68-E r-170$ & \\
\hline $16-s-34$ & & $80-\mathrm{Hg}-196$ & Crude \\
\hline $16-s-36$ & Crude & $80-\mathrm{Hg}-198$ & Crude, check capture \\
\hline $18-A r-36$ & Crude & $80-\mathrm{Hg}-199$ & \\
\hline $18-A r-38$ & Crude & $80-\mathrm{Hg}-200$ & \\
\hline $19-K-39$ & & $80-\mathrm{Hg}-201$ & \\
\hline $19-K-40$ & Very crude & $80-\mathrm{Hg}-202$ & Crude, check capture \\
\hline $20-\mathrm{Ca}-40$ & $\mathrm{o}_{i} \mathrm{k}$. to $20 \mathrm{MeV}$ & $80-\mathrm{Hg}-204$ & Very crude \\
\hline $20-\mathrm{Ca}-42$ & & $82-P \vec{b}-204$ & Horesorud \\
\hline $20-\mathrm{Ca}-43$ & “ & $88-\mathrm{Ra}-223$ & Very crude \\
\hline $20-\mathrm{Ca}-44$ & " & $88-\mathrm{Ra}-224$ & Very crude \\
\hline $20-\mathrm{Ca}-46$ & Very crude & $88-\mathrm{Ra}-225$ & Very crude \\
\hline $20-\mathrm{Ca}-48$ & o.k. to $20 \mathrm{MeV}$ & $88-\mathrm{Ra}-226$ & \\
\hline $22-\mathrm{Ti}-49$ & & $89-A c-225$ & Very crude \\
\hline $27-\mathrm{Co}-58$ & Very crude & $89-A C-226$ & Very crude \\
\hline $27-\mathrm{Co}-58 \mathrm{M}$ & Very crude & $89-\mathrm{Ac}-227$ & Very crude \\
\hline $30-\mathrm{Zn}-\mathrm{Nat}$ & Check ( $n$, alpha) & $9 \odot-\mathrm{Th}-227$ & Very crude \\
\hline $31-\mathrm{Ga}-69$ & & $90-T h-228$ & Rubbish \\
\hline $31-\mathrm{Ga}-71$ & & $90-T h-229$ & $\left(n, n^{\prime}\right)$ down to $100 \mathrm{eV}$ \\
\hline $32-\mathrm{Ge}-70$ & & $90-$ Th -233 & Very crude \\
\hline $33-A s-74$ & & $9 \odot-T h-234$ & Rubbish \\
\hline $34-\mathrm{Se}-79$ & Crude & $92-U-239$ & Weird resonances \\
\hline $47-\mathrm{Ag}-110 \mathrm{M}$ & $\left(n, n^{\prime}\right)$ down to $3 \mathrm{eV}$ ? & $92-U-240$ & \\
\hline $50-5 n-113$ & Resonance gap & $92-U-241$ & Weird resonances \\
\hline $54-X e-123$ & Very crude & $93-N p-235$ & Very crude \\
\hline $56-\mathrm{Ba}-130$ & & $94-\mathrm{Pu}-246$ & Very crude \\
\hline $56-B a-132$ & Crude & $95-A m-244$ & Very crude \\
\hline $56-\mathrm{Ba}-133$ & & $95-\mathrm{Am}-244 \mathrm{M}$ & Very crude \\
\hline $57-\mathrm{La}-138$ & & $96-\mathrm{Cm}-249$ & \\
\hline $58-\mathrm{Ce}-136$ & & $96-\mathrm{Cm}-250$ & Crude \\
\hline $58-\mathrm{Ce}-138$ & & $97-\mathrm{Bk}-250$ & Weird resonances \\
\hline $58-\mathrm{Ce}-139$ & & $98-C f-254$ & Very crude \\
\hline $64-G d-153$ & & $99-E s-254$ & Very crude \\
\hline $66-$ Dy -156 & & $99-E s-255$ & Very crude \\
\hline $66-$ Dy- 158 & & $100-F m-255$ & Very crude \\
\hline
\end{tabular}




\section{Completeness of ENDF/B-VII.0 Evaluations}

For ENDF/B-VI.8, I judged that only about half of the 328 evaluations were complete and physically acceptable enough to be used in neutron transport calculations. In contrast in ENDF/B-VII.0, only a few evaluations are incomplete $\left(10^{-5} \mathrm{eV}\right.$ to $\left.20 \mathrm{MeV}\right)$ or physically unacceptable (negative cross sections). Below is a summary (no comment $=$ o.k.)

\begin{tabular}{|c|c|c|c|c|c|}
\hline Material & & & & & Comments \\
\hline ZA001001 & $\mathrm{mt}=$ & 50 & no inelastic & & \\
\hline ZA००10०2 & $\mathrm{mt}=$ & 50 & no inelastic & & \\
\hline ZA001003 & $\mathrm{mt}=$ & 102 & no capture & & \\
\hline ZA००1003 & $\mathrm{mt}=$ & 50 & no inelastic & & \\
\hline $\mathrm{ZA} \odot \odot 2003$ & $\mathrm{mt}=$ & 50 & no inelastic & & \\
\hline ZA०02004 & $\mathrm{mt}=$ & 102 & no capture & & \\
\hline ZA००2004 & $m t=$ & 50 & no inelastic & & \\
\hline ZA004007 & $m t=$ & 2 & cross section ends & 8.1000D+06 eV & Incomplete only elastic \\
\hline ZA004007 & $m t=$ & 1 & no total & & up to $8.1 \mathrm{MeV}$ \\
\hline ZA004007 & $\mathrm{mt}=$ & 102 & no capture & & \\
\hline ZA004007 & $\mathrm{mt}=$ & 50 & no inelastic & & \\
\hline ZA००4००9 & $\mathrm{mt}=$ & 50 & no inelastic & & \\
\hline ZA005010 & $m t=$ & 102 & cross section ends & $5.0000 \mathrm{D}+05 \mathrm{eV}$ & \\
\hline ZA017035 & $\mathrm{mt}=$ & 2 & cross section start & 1.4519D-05 eV & Negative elastic \\
\hline ZA018040 & $\mathrm{mt}=$ & 2 & cross section $<=0$ & $9.7825 \mathrm{D}+05 \mathrm{eV}$ & Negative elastic \\
\hline ZA020040 & $m t=$ & 2 & cross section $<=0$ & $5.0000 \mathrm{D}+05 \mathrm{eV}$ & Negative elastic \\
\hline ZA०21045 & $\mathrm{mt}=$ & 102 & cross section ends & $5 . \odot \odot \odot \odot D+\odot 6 \mathrm{eV}$ & \\
\hline ZA०26056 & $m t=$ & 2 & cross section $<=0$ & 1.1971D+06 eV & Negative elastic \\
\hline ZA027058 & $\mathrm{mt}=$ & 102 & cross section $<=0$ & $8.4925 \mathrm{D}+0 \odot \mathrm{eV}$ & Negative capture \\
\hline ZA027058.M & $\mathrm{mt}=$ & 51 & level energy > $\odot$ & $2.4900 \mathrm{D}+\odot 4 \mathrm{eV}$ & \\
\hline ZA028059 & $m t=$ & 50 & no inelastic & & Incomplete \\
\hline ZA028061 & $\mathrm{mt}=$ & 2 & cross section $<=0$ & $7.4355 \mathrm{D}+05 \mathrm{eV}$ & Negative elastic \\
\hline ZA०41093 & $m t=$ & 2 & cross section $<=0$ & $2.3344 \mathrm{D}+03 \mathrm{eV}$ & Negative elastic \\
\hline ZA०47110.M & $\mathrm{mt}=$ & 51 & level energy > $\odot$ & $1.1760 \mathrm{D}+05 \mathrm{eV}$ & \\
\hline ZA047110.M & $\mathrm{mt}=$ & 52 & level energy $>0$ & $1.0660 \mathrm{D}+05 \mathrm{eV}$ & \\
\hline ZA०48108 & $m t=$ & 102 & cross section ends & $1.0000 \mathrm{D}+07 \mathrm{eV}$ & \\
\hline $\mathrm{ZA} \odot 48110$ & $\mathrm{mt}=$ & 102 & cross section ends & $1.0000 \mathrm{D}+07 \mathrm{eV}$ & \\
\hline $\mathrm{ZA} \odot 48112$ & $\mathrm{mt}=$ & 102 & cross section ends & 1.00००D+07 eV & \\
\hline ZA०48115.M & $\mathrm{mt}=$ & 51 & level energy $>0$ & $1.8100 \mathrm{D}+05 \mathrm{eV}$ & \\
\hline ZA०48116 & $\mathrm{mt}=$ & 102 & cross section ends & $1 . \odot \odot \odot \odot D+\odot 7 \mathrm{eV}$ & \\
\hline ZA052127. M & $\mathrm{mt}=$ & 51 & level energy $>0$ & $8.8260 \mathrm{D}+04 \mathrm{eV}$ & \\
\hline ZA052127. M & $m t=$ & 52 & level energy $>0$ & $2.7140 \mathrm{D}+04 \mathrm{eV}$ & \\
\hline ZA052129. M & $\mathrm{mt}=$ & 51 & level energy > $>$ & $1.0550 \mathrm{D}+05 \mathrm{eV}$ & \\
\hline ZA054130 & $\mathrm{mt}=$ & 102 & cross section ends & $1.0000 \mathrm{D}+07 \mathrm{eV}$ & \\
\hline ZA061148.M & $\mathrm{mt}=$ & 51 & level energy $>0$ & $1.3790 \mathrm{D}+05 \mathrm{eV}$ & \\
\hline ZA061148.M & $m t=$ & 52 & level energy $>0$ & $6.2200 \mathrm{D}+\odot 4 \mathrm{eV}$ & \\
\hline ZA०64152 & $\mathrm{mt}=$ & 2 & cross section $<=0$ & $3.3186 \mathrm{D}+01 \mathrm{eV}$ & Negative elastic \\
\hline ZA066160 & $\mathrm{mt}=$ & 2 & cross section $<=0$ & $3.3293 \mathrm{D}+02 \mathrm{eV}$ & Negative elastic \\
\hline ZA067166.M & $m t=$ & 51 & level energy > $>$ & $5.9850 \mathrm{D}+03 \mathrm{eV}$ & \\
\hline ZA०82207 & $\mathrm{mt}=$ & 2 & cross section $<=0$ & $4.7500 \mathrm{D}+05 \mathrm{eV}$ & Negative elastic \\
\hline ZA090228 & $\mathrm{mt}=$ & 18 & cross section $<=0$ & $3.0000 \mathrm{D}+03 \mathrm{eV}$ & \\
\hline ZA090230 & $m t=$ & 2 & cross section $<=0$ & $1.2856 \mathrm{D}+0 \odot \mathrm{eV}$ & Negative elastic \\
\hline ZA०92240 & $\mathrm{mt}=$ & 2 & cross section $<=0$ & $2.2262 \mathrm{D}+\odot \odot \mathrm{eV}$ & Negative elastic \\
\hline ZA094238 & $\mathrm{mt}=$ & 2 & cross section $<=0$ & $5.9743 \mathrm{D}+01 \mathrm{eV}$ & Negative elastic \\
\hline ZA094242 & $\mathrm{mt}=$ & 2 & cross section $<=0$ & $2.2348 \mathrm{D}+0 \odot \mathrm{eV}$ & Negative elastic \\
\hline ZA094244 & $\mathrm{mt}=$ & 2 & cross section $<=0$ & $2.0770 \mathrm{D}+01 \mathrm{eV}$ & Negative elastic \\
\hline ZA095242.M & $m t=$ & 51 & level energy $>\odot$ & $4.8600 \mathrm{D}+04 \mathrm{eV}$ & \\
\hline ZA095242. M & $\mathrm{mt}=$ & 52 & level energy $>0$ & $4.5000 \mathrm{D}+03 \mathrm{eV}$ & \\
\hline ZA095244. M & $\mathrm{mt}=$ & 51 & level energy $>0$ & $8.8000 \mathrm{D}+04 \mathrm{eV}$ & \\
\hline ZA०96241 & $\mathrm{mt}=$ & 102 & cross section ends & $4 . \odot \odot \odot \odot D+\odot 6 \mathrm{eV}$ & \\
\hline ZA०96242 & $\mathrm{mt}=$ & 2 & cross section $<=0$ & $1.3448 \mathrm{D}+01 \mathrm{eV}$ & Negative elastic \\
\hline ZA०96242 & $\mathrm{mt}=$ & 18 & cross section $<=0$ & $2.7600 \mathrm{D}+02 \mathrm{eV}$ & \\
\hline ZA०96248 & $\mathrm{mt}=$ & 2 & cross section $<=0$ & $6.8142 \mathrm{D}+00 \mathrm{eV}$ & Negative elastic \\
\hline ZA०98250 & $\mathrm{mt}=$ & 2 & cross section $<=0$ & $1.4329 \mathrm{D}+01 \mathrm{eV}$ & Negative elastic \\
\hline ZA०98252 & $\mathrm{mt}=$ & 2 & cross section $<=0$ & $1.6674 \mathrm{D}+01 \mathrm{eV}$ & Negative elastic \\
\hline ZA098253 & $\mathrm{mt}=$ & 18 & cross section ends & $1.1000 \mathrm{D}+04 \mathrm{eV}$ & Incomplete only to $11 \mathrm{keV}$ \\
\hline ZA098253 & $\mathrm{mt}=$ & 102 & cross section ends & $1.1000 \mathrm{D}+04 \mathrm{eV}$ & \\
\hline ZA098253 & $\mathrm{mt}=$ & 50 & no inelastic & & \\
\hline ZA099253 & $\mathrm{mt}=$ & 102 & cross section ends & $1.1000 \mathrm{D}+04 \mathrm{eV}$ & Incomplete only to $11 \mathrm{keV}$ \\
\hline ZA099253 & $m t=$ & 50 & no inelastic & & \\
\hline
\end{tabular}





\section{Same Evaluations in ENDF/B-VI and VII (315)}

Above I stated that ENDF/B-VII.0 includes 315 evaluations from ENDF/B-VI. By this I mean that there are evaluations for the same 315 elements or isotopes in both VI and VII. The contents of these evaluations may be identical to ENDF/B-VI, or completely different. Below I provide a brief, one line summary comparing the contents of ENDF/BVII.0 to VI.8. These summaries are based only on my comparing major cross sections (total, elastic, capture and fission) for the 315 same evaluations. For more details of any given evaluation the reader can use the COMPLOT code [R3] to "see" comparisons.

The intent here is to hopefully save users time and effort by telling them which evaluations have or have not changed. For example, many metals and fissile isotopes have not changed. There are also materials where the cross sections are what I call "similar", but which I mean similar resonance structure, but actual cross section values may be quite different.

1) Many single level Breit-Wigner (SLBW) resonances have been changed to multilevel (MLBW). In many case this eliminates negative elastic cross sections, and results in what I identify in the following table as "similar". WARNING because of the use of non-physical average $J$ values, switching from SLBW to MLBW does not always eliminate negative cross sections. WARNING "similar" here means similar resonance structure; the actual energy dependent cross sections may be very different.

2) Many incomplete ENDF/B-VI evaluations have now been extended up to $20 \mathrm{MeV}$ and are now complete in VII.0. Also the high energy range of many other evaluations were re-done using nuclear model code calculations; this has changed some high energy cross sections by 10 to $20 \%$.

3) Many evaluations now include resonance parameters from the latest 2006 version of the atlas of nuclear resonances, BNL-325 [R4]; this has allowed many resonance regions to be extended to higher energies. However, in many cases no additional evaluation was performed to eliminate resonance gaps in the experimentally measured resonance parameters, and many isotopes do not included an unresolved resonance energy range.

4) I try to identify evaluations where the major cross sections differ substantially; roughly speaking my criteria was differences of at least $\sim 1 \%$.

5) I also compared $<$ nu $>$, where smaller differences can be important. For the major fuel, U-233, U-235, and Pu-235, there have been minor $~ 0.5 \%$ changes in $<$ nu $>$ which may be reflected in calculated integral parameters, such as K-eff. Some minor fissile/fertile have changes in $<$ nu $>$ of 5 to $10 \%$ 
315 Same Materials (1-H - 1 to 44-Ru- 99)

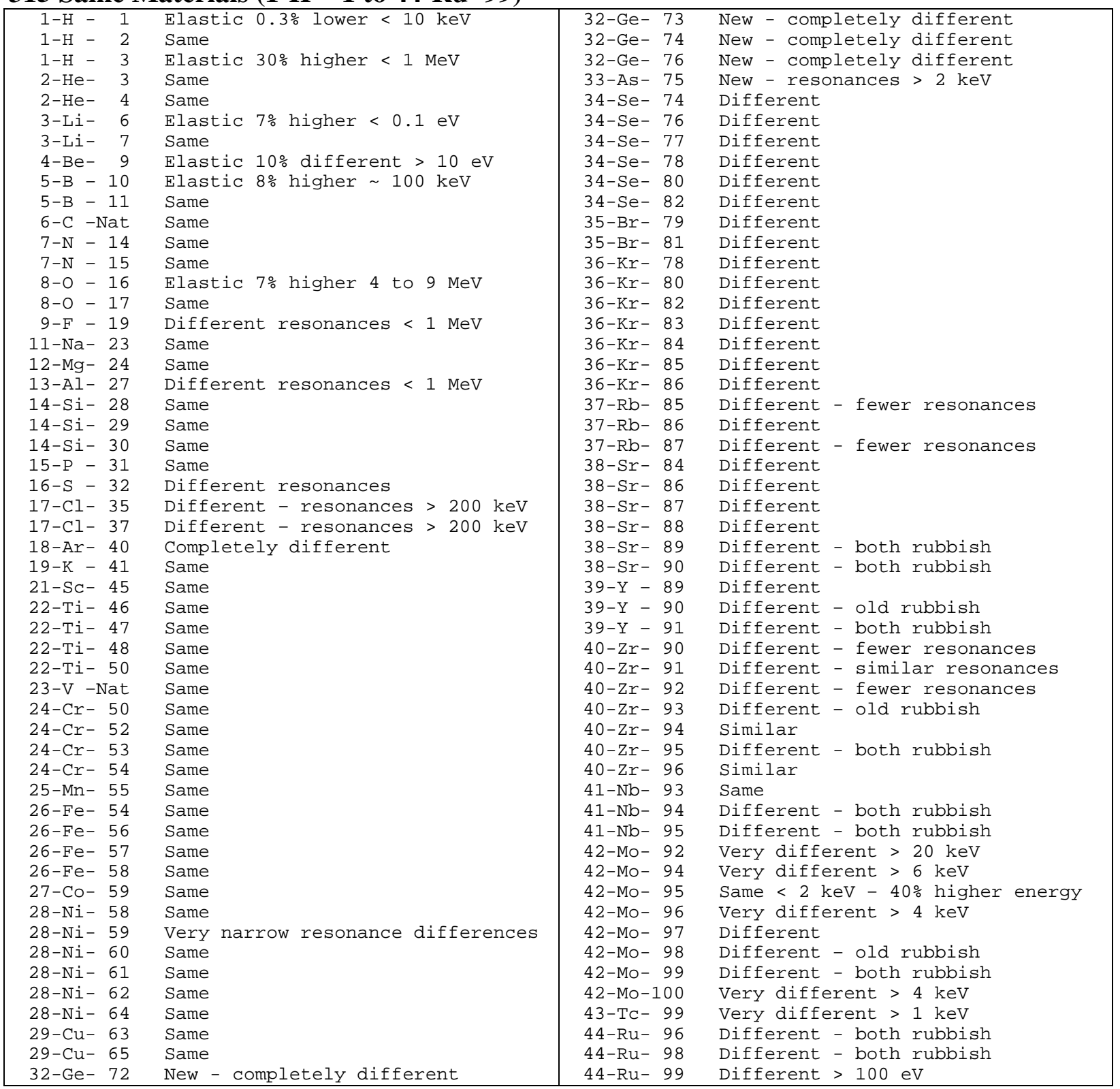




\section{Same Materials (44-Ru- 100 to 61-Pm-148)}

\begin{tabular}{|c|c|c|c|}
\hline $44-\mathrm{Ru}-100$ & Old Rubbish - new poor & $52-\mathrm{Te}-126$ & More resonances $>6 \mathrm{keV}$ \\
\hline $44-\mathrm{Ru}-101$ & $50 \%$ higher $<10$ eV & $52-\mathrm{Te}-127 \mathrm{M}$ & Different - both rubbish \\
\hline $44-\mathrm{Ru}-102$ & Similar & $52-\mathrm{Te}-128$ & More resonances $>3.5 \mathrm{keV}$ \\
\hline $44-\mathrm{Ru}-103$ & Old Rubbish - new poor & $52-\mathrm{Te}-129 \mathrm{M}$ & Different - both rubbish \\
\hline $44-\mathrm{Ru}-104$ & Different $>1 \mathrm{keV}$ & $52-\mathrm{Te}-130$ & Different \\
\hline $44-\mathrm{Ru}-105$ & Similar - both rubbish & $52-\mathrm{Te}-132$ & Different - old rubbish \\
\hline $44-\mathrm{Ru}-106$ & Similar - both rubbish & $53-I-127$ & More resonances $>1 \mathrm{keV}$ \\
\hline $45-\mathrm{Rh}-103$ & Same $<4 \mathrm{keV}-40 \%$ higher energy & $53-I-129$ & More resonances $>150 \mathrm{eV}$ \\
\hline $45-\mathrm{Rh}-105$ & Different - both rubbish & $53-I-130$ & Different - old rubbish \\
\hline $46-P d-102$ & old rubbish - new poor & $53-I-131$ & Different - both rubbish \\
\hline $46-P d-104$ & Different - old rubbish & $53-I-135$ & Different - both rubbish \\
\hline $46-P d-105$ & Same $<2 \mathrm{keV}-20 \%$ higher energy & $54-\mathrm{Xe}-124$ & Similar \\
\hline $46-P d-106$ & Different - old rubbish & $54-\mathrm{Xe}-126$ & Different resonances \\
\hline $46-P d-107$ & Very similar & $54-\mathrm{Xe}-128$ & Similar \\
\hline $46-P d-108$ & Different - old poor & $54-\mathrm{Xe}-129$ & Similar \\
\hline $46-P d-110$ & Different - old rubbish & $54-\mathrm{Xe}-130$ & Similar \\
\hline $47-\mathrm{Ag}-107$ & Different $>3 \mathrm{keV}$ & $54-\mathrm{Xe}-131$ & Same \\
\hline $47-\mathrm{Ag}-109$ & Similar $<5 \mathrm{keV}-7 \%>100 \mathrm{keV}$ & $54-\mathrm{Xe}-132$ & Different \\
\hline $47-\mathrm{Ag}-111$ & New - old rubbish & $54-\mathrm{Xe}-133$ & Different - both rubbish \\
\hline $48-\mathrm{cd}-106$ & No resonances $600 \mathrm{eV}-3 \mathrm{keV}$ & $54-\mathrm{Xe}-134$ & Different \\
\hline $48-C d-108$ & No resonances $350 \mathrm{eV}-2.6 \mathrm{keV}$ & $54-\mathrm{Xe}-135$ & Same - both rubbish $>10 \mathrm{eV}$ \\
\hline $48-\mathrm{Cd}-110$ & Similar & $54-\mathrm{Xe}-136$ & New - old rubbish \\
\hline $48-\mathrm{Cd}-111$ & Different $<1$ eV $60 \%$ lower & $55-C s-133$ & Same $-14 \%>100 \mathrm{keV}$ \\
\hline $48-\mathrm{Cd}-112$ & Similar $<2 \mathrm{keV}$ & $55-C s-134$ & Similar \\
\hline $48-\mathrm{Cd}-113$ & Similar $<2 \mathrm{keV}$ & $55-C s-135$ & Similar \\
\hline $48-\mathrm{Cd}-114$ & Same & $55-C s-136$ & Different - new rubbish \\
\hline $48-C d-115 M$ & New - old rubbish & $55-C s-137$ & Similar - both rubbish \\
\hline $48-C d-116$ & Similar & $56-\mathrm{Ba}-134$ & Similar $<10 \mathrm{keV}$ \\
\hline $49-\operatorname{In}-113$ & New resonances $>50 \mathrm{eV}$ & $56-\mathrm{Ba}-135$ & Similar $<1 \mathrm{keV}$ \\
\hline $49-\operatorname{In}-115$ & Very different $>1 \mathrm{keV}$ & $56-\mathrm{Ba}-136$ & Different \\
\hline $50-\mathrm{Sn}-112$ & Similar & $56-\mathrm{Ba}-137$ & Different \\
\hline $50-\mathrm{Sn}-114$ & Different - more resonances & $56-\mathrm{Ba}-138$ & Similar \\
\hline $50-S n-115$ & Different - both poor & $56-\mathrm{Ba}-140$ & Different - old rubbish \\
\hline $50-S n-116$ & New resonances $>2 \mathrm{keV}$ & $57-\mathrm{La}-139$ & Different \\
\hline $50-\mathrm{Sn}-117$ & Different & $57-\mathrm{La}-140$ & Different - old rubbish \\
\hline $50-S n-118$ & Similar & $58-\mathrm{Ce}-140$ & Different - old rubbish \\
\hline $50-S n-119$ & Different & $58-\mathrm{Ce}-141$ & Different - old rubbish \\
\hline $50-S n-120$ & New resonances $>15 \mathrm{keV}$ & $58-\mathrm{Ce}-142$ & Different - old rubbish \\
\hline $50-S n-122$ & New resonances $>900 \mathrm{eV}$ & $58-\mathrm{Ce}-143$ & Different - old rubbish \\
\hline $50-S n-123$ & Different - both rubbish & $58-\mathrm{Ce}-144$ & Different - both rubbish \\
\hline $50-S n-124$ & New resonances $>700 \mathrm{eV}$ & $59-\mathrm{Pr}-141$ & Similar \\
\hline $50-S n-125$ & New - old rubbish & $59-\mathrm{Pr}-142$ & Different - old rubbish \\
\hline $50-S n-126$ & Different - both rubbish & $59-\operatorname{Pr}-143$ & Different - old rubbish \\
\hline $51-S b-121$ & More resonances $>2.5 \mathrm{keV}$ & $60-\mathrm{Nd}-142$ & Different \\
\hline $51-S b-123$ & More resonances $>2.5 \mathrm{keV}$ & $60-\mathrm{Nd}-143$ & Similar $-4 \%>100 \mathrm{keV}$ \\
\hline $51-\mathrm{Sb}-124$ & Different - both rubbish & $60-\mathrm{Nd}-144$ & Different \\
\hline $51-\mathrm{Sb}-125$ & Different - both rubbish & $60-\mathrm{Nd}-145$ & Similar - 5\% > $100 \mathrm{keV}$ \\
\hline $51-S b-126$ & Different - old rubbish & $60-\mathrm{Nd}-146$ & Different \\
\hline $52-\mathrm{Te}-120$ & Different - both rubbish & $60-\mathrm{Nd}-147$ & Different $>30 \mathrm{eV}$ \\
\hline $52-\mathrm{Te}-122$ & More resonances $>4 \mathrm{keV}$ & $60-\mathrm{Nd}-148$ & Different \\
\hline $52-\mathrm{Te}-123$ & More resonances $>500 \mathrm{eV}$ & $60-\mathrm{Nd}-150$ & Different \\
\hline $52-\mathrm{Te}-124$ & More resonances $>6 \mathrm{keV}$ & $61-\mathrm{Pm}-147$ & No resonances $>100 \mathrm{eV}$ \\
\hline $52-\mathrm{Te}-125$ & More resonances $>1 \mathrm{keV}$ & $61-\mathrm{Pm}-148$ & Different - both rubbish \\
\hline
\end{tabular}




\section{Same Materials (61-Pm-148M to 99-Es-293)}

\begin{tabular}{|c|c|c|c|}
\hline $61-\mathrm{Pm}-148 \mathrm{M}$ & Similar - not great & $77-$ Ir -191 & Very similar \\
\hline $61-P m-149$ & Different - both rubbish & $77-\operatorname{Ir}-193$ & Similar \\
\hline $61-P m-151$ & Different - old rubbish & $79-\mathrm{Au}-197$ & Similar \\
\hline $62-\mathrm{Sm}-144$ & Similar & $82-P b-206$ & Very similar \\
\hline $62-\mathrm{Sm}-147$ & Similar > $10 \mathrm{eV}$ & $82-\mathrm{Pb}-207$ & Very similar, $3 \% \sim 10 \mathrm{MeV}$ \\
\hline $62-\mathrm{Sm}-148$ & Different - old rubbish & $82-\mathrm{Pb}-208$ & Very similar \\
\hline $62-\mathrm{Sm}-149$ & Very similar & $83-\mathrm{Bi}-209$ & Same \\
\hline $62-\mathrm{Sm}-150$ & Very similar & $90-\mathrm{Th}-230$ & Same \\
\hline $62-S m-151$ & Same $-15 \%>10 \mathrm{keV}$ & $90-T h-232$ & Different resonances \\
\hline $62-S m-152$ & Similar & $91-\mathrm{Pa}-231$ & Similar $<15 \mathrm{eV}$ \\
\hline $62-\mathrm{Sm}-153$ & Different - old rubbish & $91-\mathrm{Pa}-232$ & Similar \\
\hline $62-S m-154$ & Different & $91-\mathrm{Pa}-233$ & Similar $<40 \mathrm{eV}$ \\
\hline $63-E u-151$ & Very similar & $92-U-232$ & Same $<200 \mathrm{eV}$ \\
\hline $63-E u-152$ & Same $-30 \%>100 \mathrm{eV}$ & $92-U-233$ & Similar to $60 \mathrm{eV}$ \\
\hline $63-E u-153$ & Same $-16 \%>100 \mathrm{eV}$ & $92-U-234$ & Similar \\
\hline $63-\mathrm{Eu}-154$ & Different & $92-U-235$ & Same $-1 \% \sim 20 \mathrm{MeV}$ \\
\hline $63-E u-155$ & Very similar & $92-U-236$ & Same $-6 \% \sim 500 \mathrm{keV}$ \\
\hline $63-E u-156$ & Different - both rubbish & $92-U-237$ & Similar - weird resonances \\
\hline $63-\mathrm{Eu}-157$ & Different - old rubbish & $92-U-238$ & Similar < $10 \mathrm{keV}$ \\
\hline $64-G d-152$ & Different $<10 \mathrm{eV}$ & $93-N p-236$ & Same \\
\hline $64-G d-154$ & Similar & $93-N p-237$ & Similar $<150 \mathrm{eV}$ \\
\hline $64-G d-155$ & Same $-20 \%>200 \mathrm{eV}$ & $93-N p-238$ & Different - both rubbish \\
\hline $64-G d-156$ & Different & $93-N p-239$ & Same - both rubbish \\
\hline $64-G d-157$ & Similar, same < $400 \mathrm{eV}$ & $94-P u-236$ & Same \\
\hline $64-G d-158$ & Different & $94-\mathrm{Pu}-237$ & Same \\
\hline $64-G d-160$ & Different & $94-\mathrm{Pu}-238$ & Same \\
\hline $65-T b-159$ & Different $>100 \mathrm{eV}$ & $94-\mathrm{Pu}-239$ & Same \\
\hline $65-\mathrm{Tb}-160$ & Different - old rubbish & $94-\mathrm{Pu}-240$ & Same \\
\hline $66-$ Dy -160 & Very similar & $94-\mathrm{Pu}-241$ & Same \\
\hline $66-$ Dy-161 & Similar & $94-\mathrm{Pu}-242$ & Same \\
\hline $66-$ Dy -162 & Similar $<5 \mathrm{keV}$ & $94-\mathrm{Pu}-243$ & Same \\
\hline $66-\mathrm{Dy}-163$ & Similar & $94-\mathrm{Pu}-244$ & Same \\
\hline $66-\mathrm{Dy}-164$ & Similar $<7 \mathrm{keV}$ & $95-A m-241$ & Same \\
\hline $67-\mathrm{Ho}-165$ & Different & $95-\mathrm{Am}-242$ & Different - old bad \\
\hline $68-E r-166$ & Similar $<2 \mathrm{keV}$ & $95-A m-242 M$ & Similar $-30 \%>3.5 \mathrm{eV}$ \\
\hline $68-E r-167$ & Similar $<500 \mathrm{eV}$ & $95-A m-243$ & Same \\
\hline $71-\mathrm{Lu}-175$ & Same & $96-\mathrm{Cm}-241$ & Same \\
\hline $71-\mathrm{Lu}-176$ & Same & $96-\mathrm{Cm}-242$ & Same \\
\hline $72-\mathrm{Hf}-174$ & Same & $96-\mathrm{Cm}-243$ & Same \\
\hline $72-\mathrm{Hf}-176$ & Same & $96-\mathrm{Cm}-244$ & Similar $<500 \mathrm{eV}$ \\
\hline $72-\mathrm{Hf}-177$ & Same & $96-\mathrm{Cm}-245$ & Same \\
\hline $72-\mathrm{Hf}-178$ & Same & $96-\mathrm{Cm}-246$ & Same \\
\hline $72-\mathrm{Hf}-179$ & Same & $96-\mathrm{Cm}-247$ & Different \\
\hline $72-\mathrm{Hf}-180$ & Same & $96-\mathrm{Cm}-248$ & Same - negative elastic \\
\hline $73-\mathrm{Ta}-181$ & Same & $97-\mathrm{Bk}-249$ & Same \\
\hline $73-\mathrm{Ta}-182$ & Same & $98-C f-249$ & Same \\
\hline $74-W-182$ & Same & $98-C f-250$ & Same \\
\hline $74-W-183$ & Same & $98-C f-251$ & Same \\
\hline $74-W-184$ & Same & $98-C f-252$ & Same \\
\hline $74-W-186$ & Same & $98-C f-253$ & Same - partial to $11 \mathrm{keV}$ \\
\hline $75-\operatorname{Re}-185$ & Same & $99-E s-253$ & Same - partial to $11 \mathrm{keV}$ \\
\hline $75-\operatorname{Re}-187$ & Same & & \\
\hline
\end{tabular}




\section{Appendix H: The Effects of Temperature and Doppler Broadening}

For those readers who are not familiar with the effects of temperature and Doppler broadening on neutron cross sections and transport, for details I suggest that you read references [R5] and [R6], listed below. Here I will give a brief description of these effects. Users of neutron cross sections should be aware that there are several important effects of temperature and Doppler broadening,

1) There is the well known effect in the neutron resonance region, where as the temperature increases resonances become broader, hence the name Doppler broadening. Figure 1 below illustrates the effect of temperature on the $\mathrm{U}^{238}$ capture cross section for neutron reactor like temperatures, and figure 2 illustrates this effect for astrophysical like temperatures. These figures each contain four sub-figures, with each sub-figure comparing cross sections at two progressively higher temperatures. In both figure 1 and 2 each sub-figure shows exactly the same energy and cross section range. From these figures we can see that as temperature increases the peaks of the resonances become lower, and the minima between resonances become higher. At extremely high temperature the entire resonance structure disappears and the cross section approaches a simple $1 / v$ shape (where $v$ is the neutron speed). This temperature effect will have a very important effect on resonance self-shielding in any neutron transport calculation. You should note from these figures that due to the large resonance spacing in $U^{238}$ the resonance structure can still be seen up to very high temperatures.

To understand the importance of considering temperature we should consider reaction rates, such as captures/second, in various systems. In optically thin systems (few mean free paths dimensions) the flux will be unshielded, and our reaction rates will be defined by a simple cross section average,

Unshielded Capture $=\int_{E 1}^{E 2}[\Sigma c(E) \phi(E)] d E=$ capture cross section times neutron flux

In optically thick systems (many mean free paths dimensions) the flux will be shielded (the flux is suppressed by the total cross section) and our reaction rates must include the effect of self-shielding on the cross section average,

Shielded Capture $=\int_{E 1}^{E 2}[\Sigma c(E) \phi(E) / \Sigma t(E)] d E=$ including one over total cross section

Consider for example the U238 capture cross section between 1 and $10 \mathrm{keV}$ as shown in fig. 1 and 2. If we calculate the unshielded and shielded average capture cross section for the energy interval over the range of temperatures shown in figs. 1 and 2, we obtain the results shown below in table 1 .

What we see from these results is that the unshielded average capture cross section is virtually independent of temperature, being about 1 barn over the entire temperature 
range. In contrast the shielded average cross section varying by over a factor of three between the $0 \mathrm{~K}$ average ( 0.293 barns) and the $10 \mathrm{keV}$ average ( 0.939 barns). The point to learn from this is that without including the effect of self-shielding in multi-group calculations, temperature has very little effect on the average cross sections, which is quite simply wrong for optically thick systems.

$\begin{array}{rll}\text { Table 1: Effect of Temperature on Average Cross Sections } \\ \text { Temp. } & \begin{array}{l}\text { Unshielded } \\ \text { (barns) }\end{array} & \begin{array}{l}\text { Shielded } \\ \text { (barns) }\end{array} \\ 0 \mathrm{~K} & 0.996 & 0.293 \\ 293.6 \mathrm{~K} & 0.966 & 0.526 \\ 600 \mathrm{~K} & 0.996 & 0.576 \\ 1,200 \mathrm{~K} & 0.996 & 0.630 \\ 12,000 \mathrm{~K}(1 \mathrm{eV}) & 0.996 & 0.799 \\ 10 \mathrm{eV} & 0.998 & 0.905 \\ 100 \mathrm{eV} & 1.000 & 0.933 \\ 1 \mathrm{keV} & 1.004 & 0.935 \\ 10 \mathrm{keV} & 1.007 & 0.939\end{array}$

2) Another, less well known, effect of Doppler broadening is at lower energies where as temperature increases the low energy constant scattering cross section increases and at very low energies approaches a simple $1 / \mathrm{v}$ shape (where $\mathrm{v}$ is the neutron speed); this effect is explained in detail in ref [R5]. Figure 3 illustrates the effect of temperature on the hydrogen total cross section. From this figure we can see that starting from a "cold" (0 Kelvin) cross section that is constant at about 20 barns, as temperature increases the cross section increases. Compared to the "cold" 20 barn cross section, at thermal energy the Doppler broadened cross section is about 30 barns, i.e., 50 \% higher. Note also from this figure that this effect extends well above thermal energy. For example, at 293.6 Kelvin the thermal energy is $0.0253 \mathrm{eV}$, but we can see this effect up to about $1 \mathrm{eV}$; a factor of 400 higher in energy. From the lower half of figure 2 we can see that at very low energy the cross section approaches a simple $1 / \mathrm{v}$ shape (where $\mathrm{v}$ is the neutron speed) and the cross sections at various temperatures become proportional to one another. This effect on the cross sections at low energy is very important for thermal and low energy neutron systems.

3) Yet another important effect of temperature is that at lower energies neutrons do not slow down in energy as quickly and neutron scatter can even result in the upscatter of neutrons, i.e., when neutrons scatter they can gain, rather than lose, energy. This is a well known effect at low energies, where thermal scattering law data or a free gas model is used to model the interaction of neutrons with target atoms that are moving about with thermal motion. Figure 4 illustrates the effect of temperature on the neutron spectrum over a wide range of temperatures [R7]. This effect can also be important at higher energies, particularly near narrow resonances, where thermal motion of the target atoms can cause neutrons to slightly upscatter, but even slight upscatter can cause a neutron to scatter from below to above the energy of a very narrow resonance. See reference [R6], below for a routine designed to be used in conjunction with the SIGMA1 method of Doppler broadening [R5], to handle neutron thermal scattering. This routine [R6] is completely compatible for use with the cross sections included here, since these cross sections were Doppler broadened using the SIGMA1 method [R5]. The combination of 
SIGMA1 [R5] Doppler broadened cross sections and THERMAL [R6] to handle thermal scattering, is currently used in the TART Monte Carlo transport code [R8].

Fig.1: Effect of Doppler Broadening on Resonance Cross Sections
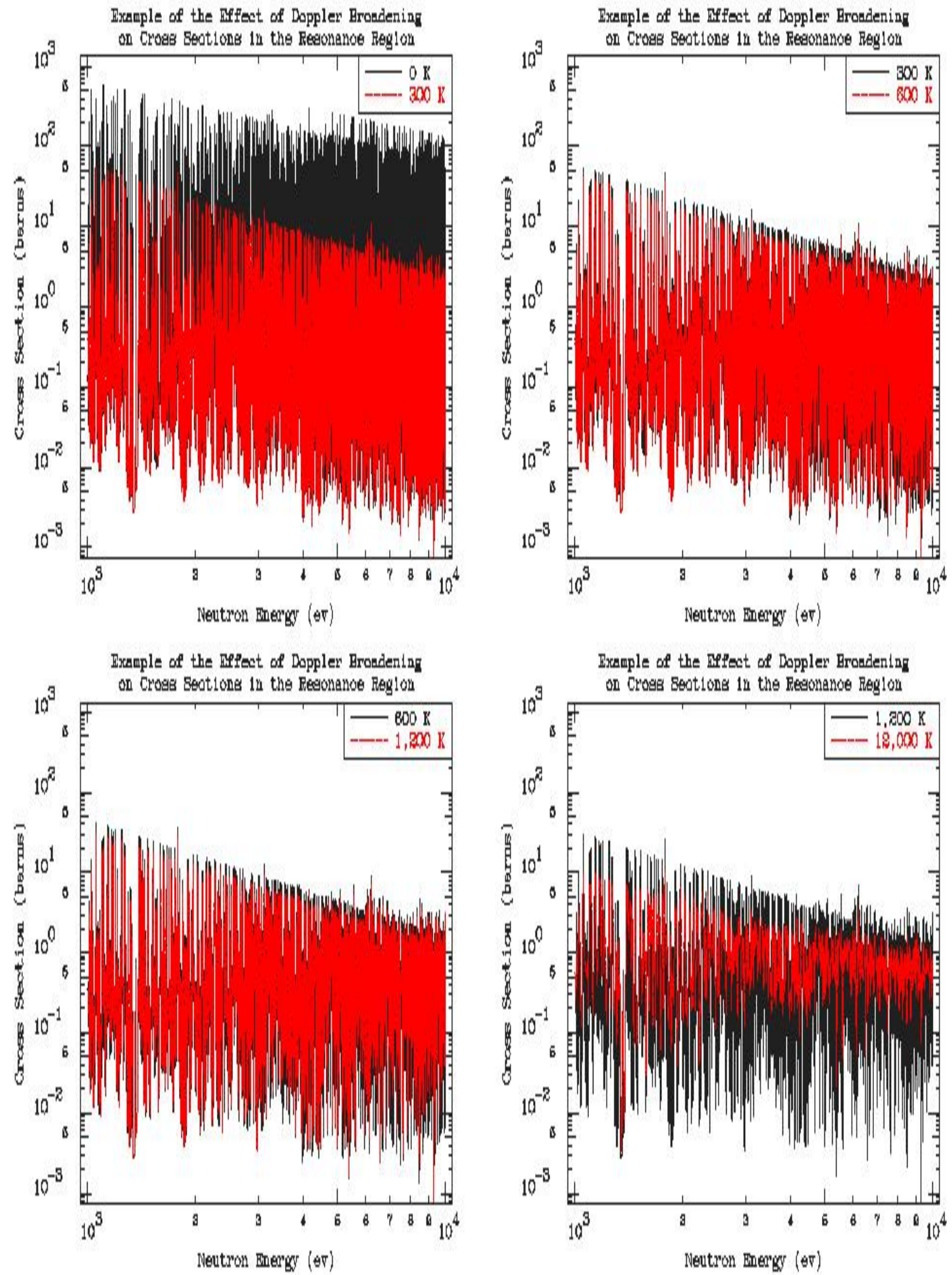
Fig.2: Effect of Doppler Broadening on Resonance Cross Sections
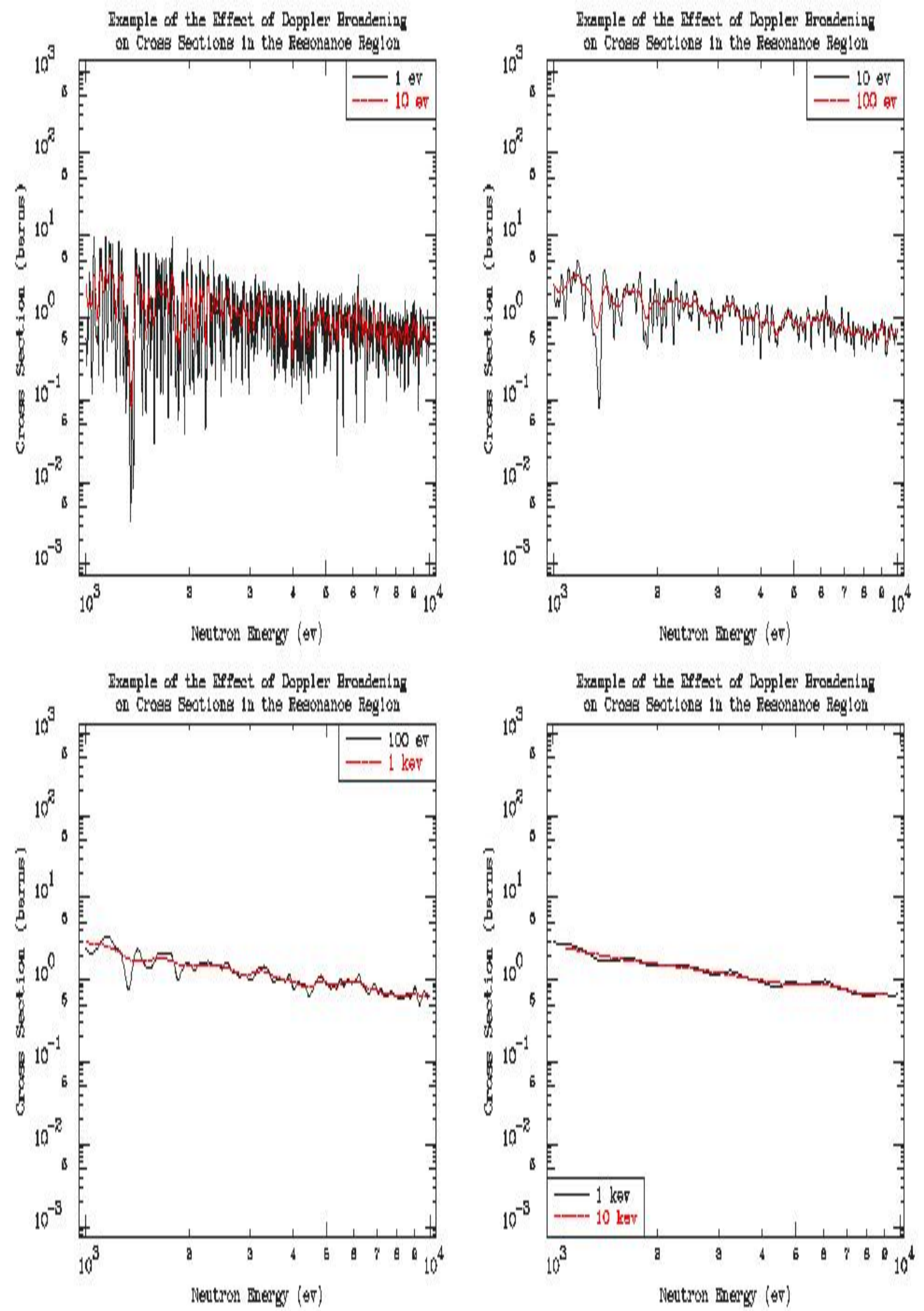
Fig.3: Effect of Doppler Broadening on Low Energy Cross Sections
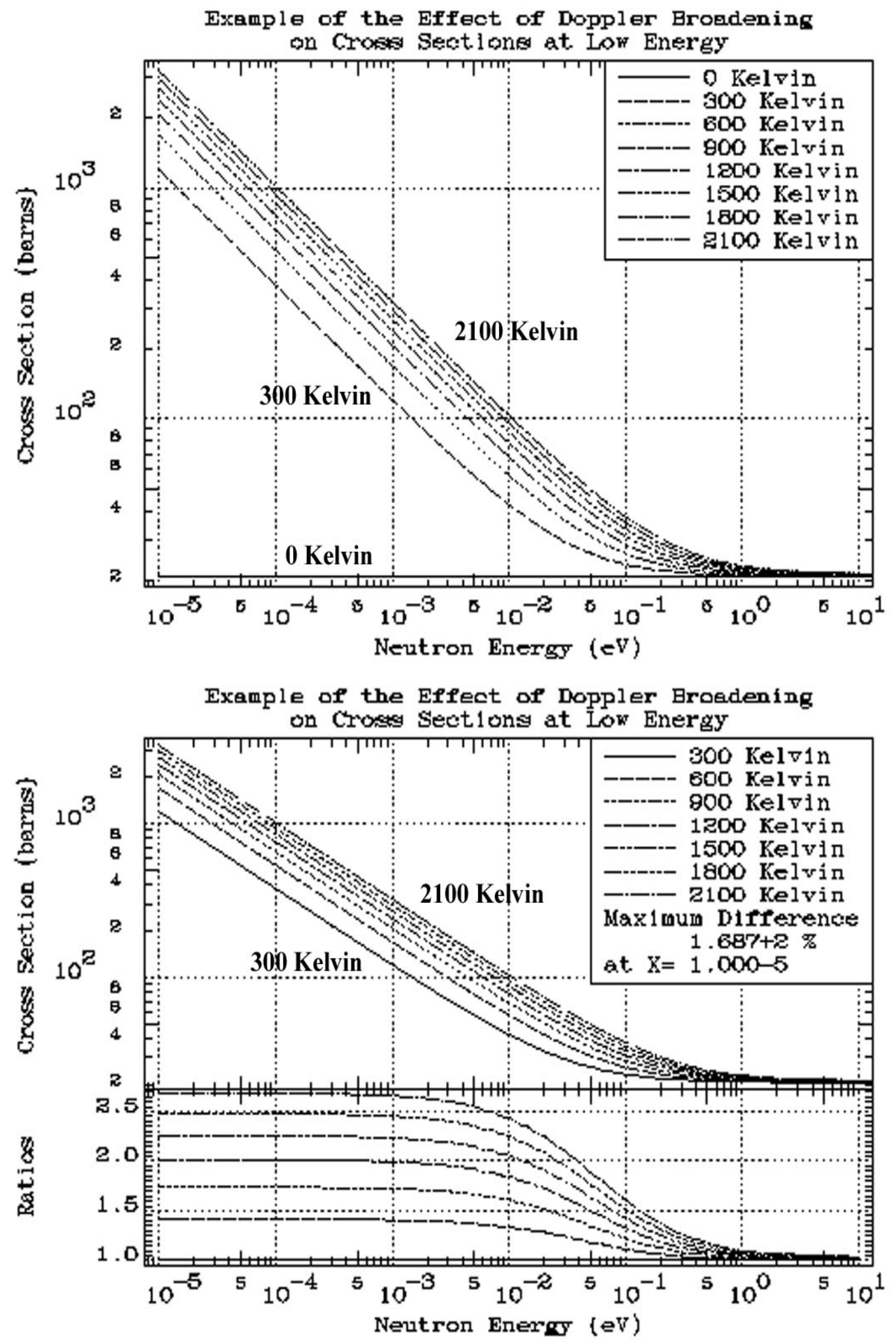
Fig.4: Effect of Doppler Broadening on Neutron Spectrum

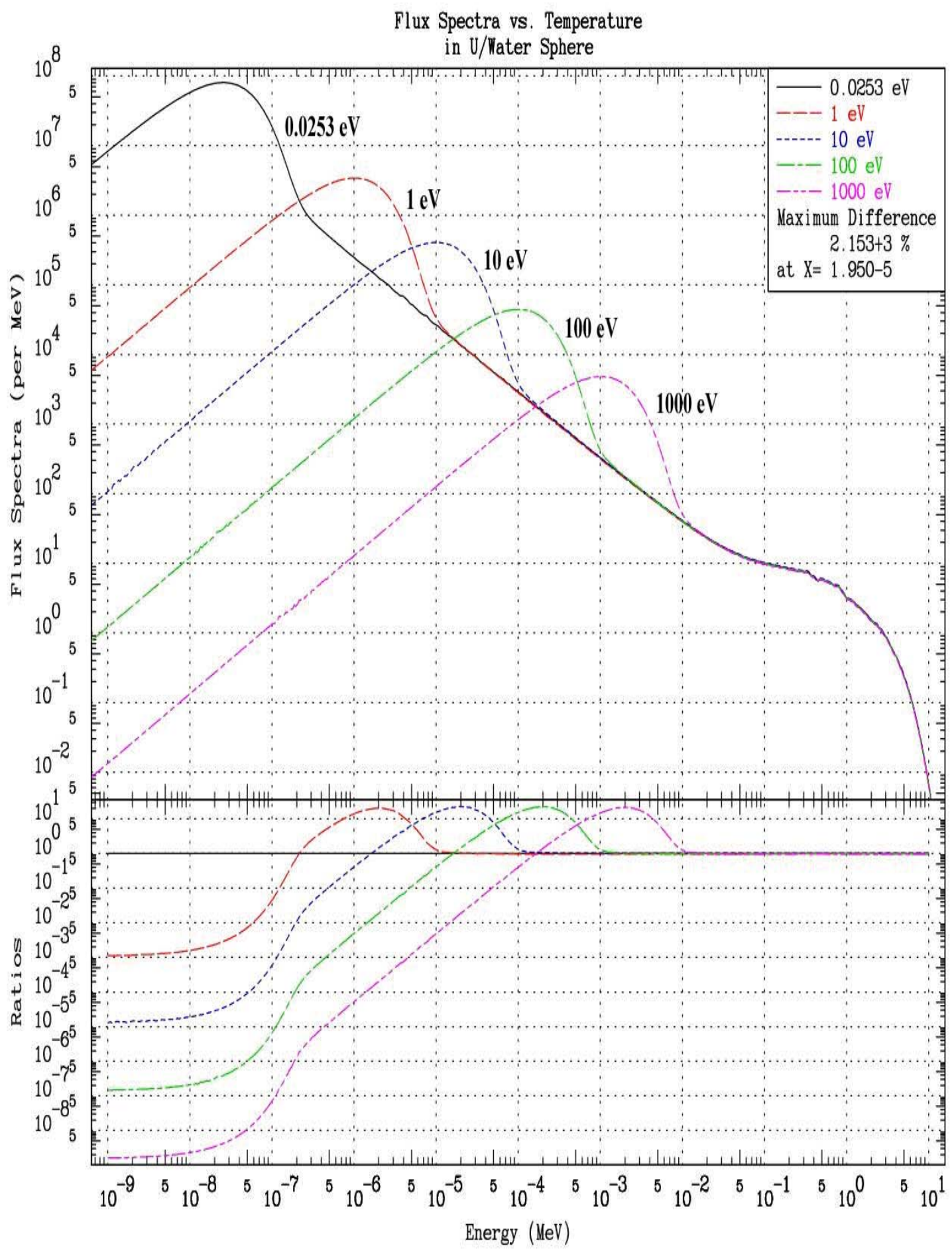




\section{Appendix I: MF/MT Differences between VII.1 and VII.0}

I have crudely compared the entire contents of VII.1 and VII.0, character by character looking for differences between the two libraries. My comparisons are summarized below based on,

1) Ignoring Tape Labels (TPID)

2) Ignoring the comment section, $\mathrm{MF} / \mathrm{MT}=1 / 451$.

3) I only compare $\mathrm{MF}=1$ through 6; these can directly affect neutron transport

4) Ignoring the first line of each section (MF/MT); these may include minor differences, particularly in the atomic weight.

5) When I find differences in any section (MF/MT) I only list the first line

6) I identify ALL sections (MF/MT) that only appear in one library

Here I make no attempt to determine how important these differences may be; the following is intended solely to allow you to "see" which sections differ and allow you to look in more detail at the actual data to determine whether or not these differences may be important for YOUR APPLICATIONS.

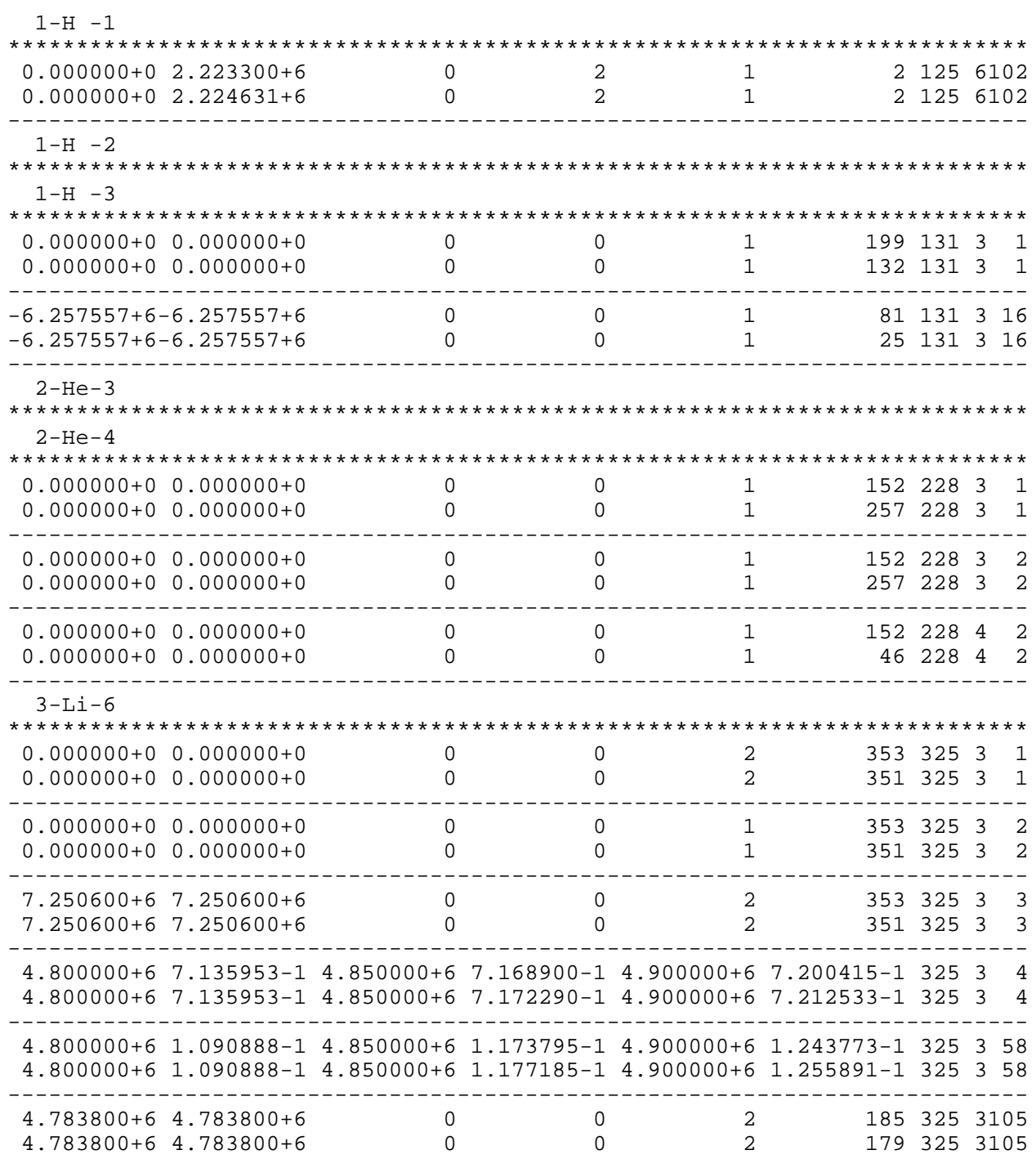



8.228500-2 2.953700-2 $1.424200-2 \quad 4.543600-3 \quad 9.996100-4 \quad 4.870900-532542$

$3-\mathrm{Li}-7$

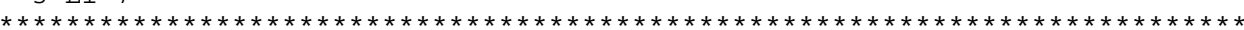

$4-\mathrm{Be}-7$

$2.389000-3$ 1.026600-1 $2.062300-2$ 9.809301-4 4196650

$2.389000-3 \quad 1.026600-12.062300-2 \quad 9.809300-4 \quad 4196650$

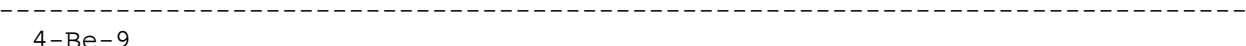

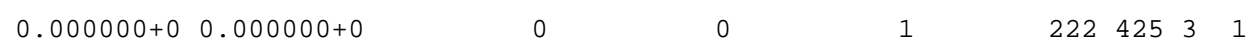

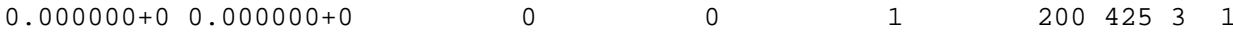

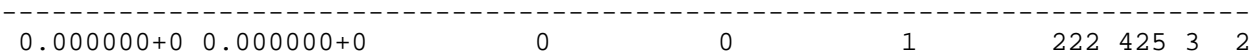

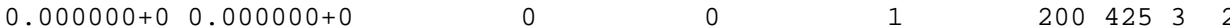

.

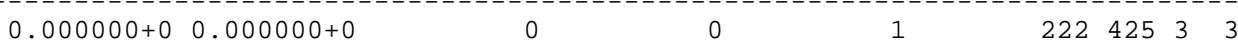

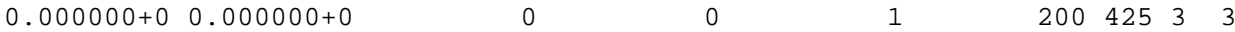

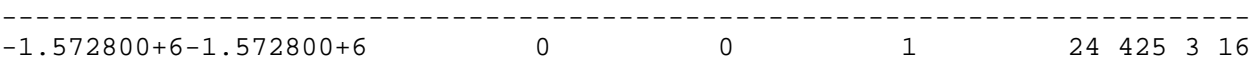

(1) 10

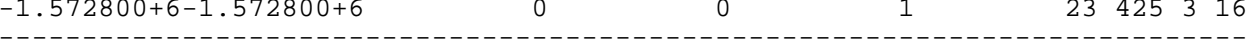

$\begin{array}{llllll}6.812380+6 & 6.812380+6 & 0 & 0 & 1 & 124253102\end{array}$

$\begin{array}{rrrrrr}6.812380+6 & 6.812380+6 & 0 & 0 & 1 & 694253102\end{array}$

(-

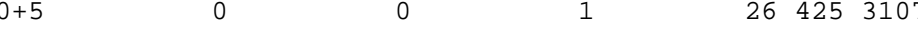

$-6.000000+5-6.000000+5 \quad 00000$

$-6.000000+5-6.000000+5 \quad 0001254253800$

(

$5-B-10$

$* * * * * * * * * * *+1$

$5-\mathrm{B}-11$
$* * * * * * * * * * * *$

6-C - Nat

$7-\mathrm{N}-14$
$* * * * * * * * * * * *$

$0.000000+\odot \quad 0.000000+0$

$0.000000+\odot \quad 0.000000+\odot$

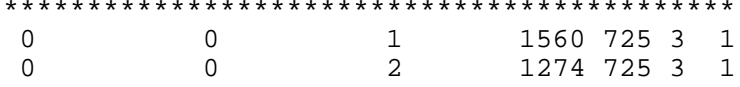

等

$7-\mathrm{N}-15$

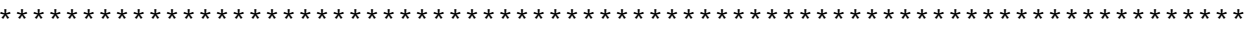

$8-0-16$

\begin{tabular}{|c|c|c|c|c|c|c|}
\hline 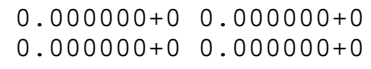 & $\begin{array}{l}\odot \\
\odot\end{array}$ & $\begin{array}{l}\odot \\
\odot\end{array}$ & $\begin{array}{l}1 \\
1\end{array}$ & $\begin{array}{l}2938 \\
2316\end{array}$ & $\begin{array}{l}825 \\
825\end{array}$ & $\begin{array}{ll}3 & 1 \\
3 & 1\end{array}$ \\
\hline 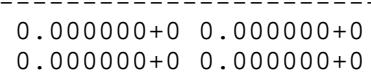 & $\begin{array}{l}\odot \\
\ominus\end{array}$ & $\begin{array}{l}\odot \\
\ominus\end{array}$ & $\begin{array}{l}1 \\
1\end{array}$ & $\begin{array}{l}2938 \\
2316\end{array}$ & $\begin{array}{l}825 \\
825\end{array}$ & $\begin{array}{ll}3 & 2 \\
3 & 2\end{array}$ \\
\hline $\begin{array}{ll}\odot .00000 \odot+\odot & \odot .000000+\odot \\
\odot .0000 \odot \odot+\odot & \odot .00000 \odot+\odot\end{array}$ & $\begin{array}{l}\odot \\
\odot\end{array}$ & $\begin{array}{l}\odot \\
\odot\end{array}$ & $\begin{array}{l}1 \\
1\end{array}$ & $\begin{array}{l}2938 \\
2316\end{array}$ & $\begin{array}{l}825 \\
825\end{array}$ & $\begin{array}{l}3 \\
3\end{array}$ \\
\hline $\begin{array}{l}-2.214300+6-2.214300+6 \\
-2.214300+6-2.214300+6\end{array}$ & $\begin{array}{l}0 \\
\odot\end{array}$ & $\begin{array}{l}\odot \\
\odot\end{array}$ & $\begin{array}{l}1 \\
3\end{array}$ & $\begin{array}{l}1674 \\
1208\end{array}$ & $\begin{array}{l}825 \\
825\end{array}$ & $\begin{array}{l}3107 \\
3107\end{array}$ \\
\hline
\end{tabular}

$-2.214300+6-2.214300+6$

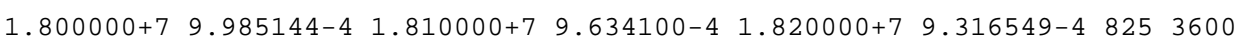
$1.800000+7 \quad 9.985143-4 \quad 1.810000+7 \quad 9.634100-4 \quad 1.820000+7 \quad 9.316549-48253600$

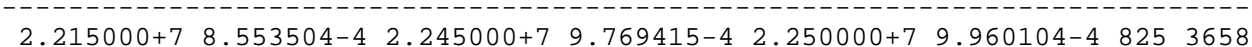
$2.215000+7 \quad 8.553504-4 \quad 2.245000+7 \quad 9.769416-4 \quad 2.2500000+7 \quad 9.960104-4 \quad 8253658$

$2.500000+7 \quad 9.763200-4 \quad 2.515000+7 \quad 9.828137-4 \quad 2.545000+7 \quad 9.978088-4 \quad 8253659$ $2.500000+7 \quad 9.763200-4 \quad 2.515000+7 \quad 9.828136-4 \quad 2.545000+7 \quad 9.978088-4 \quad 8253659$

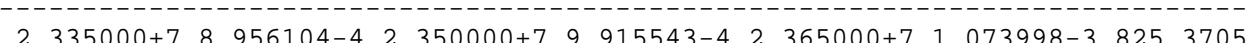
$2.335000+7 \quad 8.956104-4 \quad 2.350000+7 \quad 9.915544-4 \quad 2.365000+7 \quad 1.073998-3 \quad 8253705$

(2.

$\begin{array}{lllll}-2.214300+6-2.214300+6 & 0 & 0 & 1 & 16748253800\end{array}$




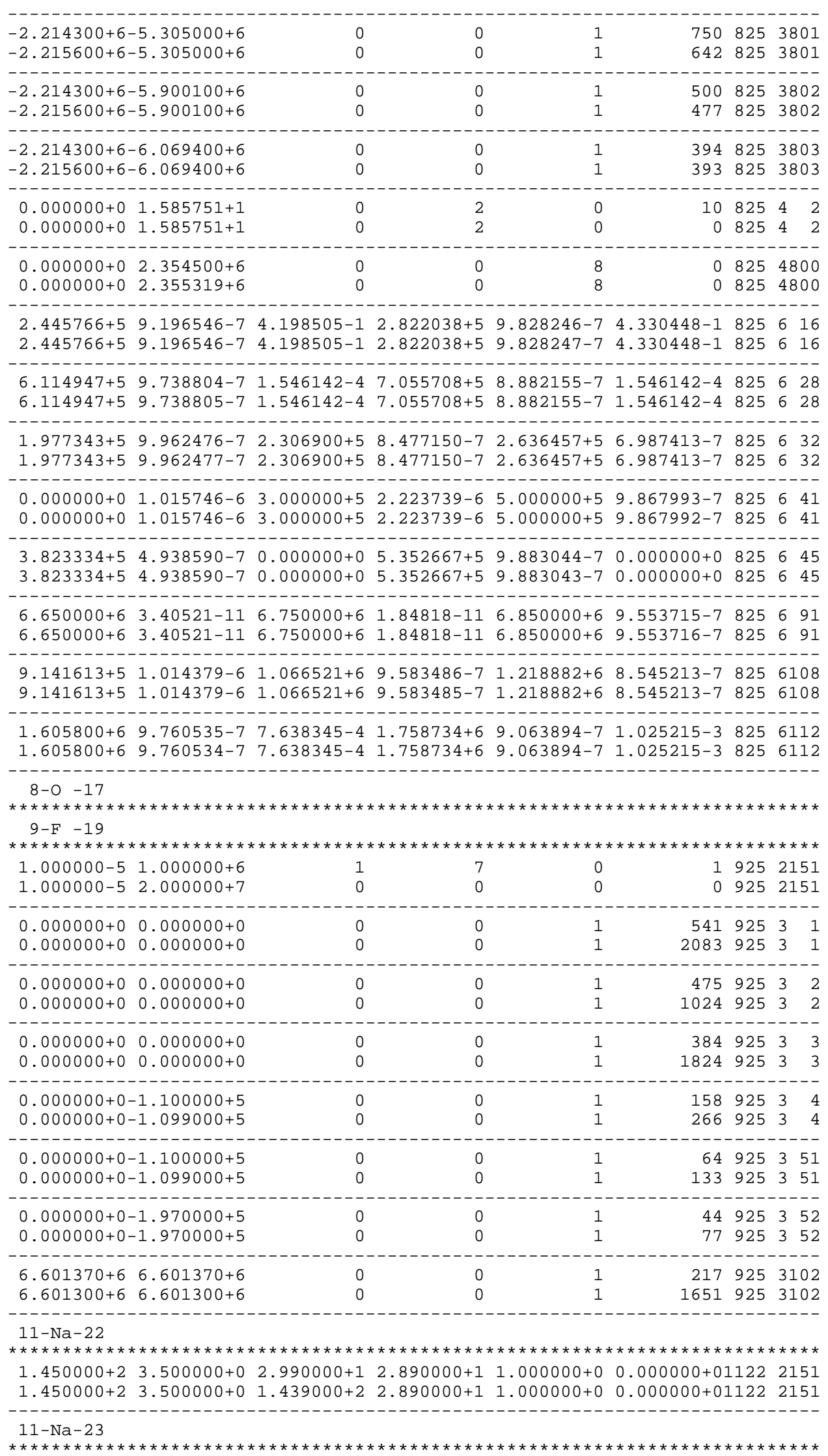




\begin{tabular}{|c|c|c|c|c|c|c|c|}
\hline $\begin{array}{ll}1.00000 \odot-5 & 5.00000 \odot+5 \\
6.000 \odot \odot \odot+2 ~ & 5.0000 \odot \odot+5\end{array}$ & $\begin{array}{l}1 \\
1\end{array}$ & $\begin{array}{l}2 \\
2\end{array}$ & $\begin{array}{l}\odot \\
\odot\end{array}$ & $\begin{array}{l}01125 \\
01125\end{array}$ & $\begin{array}{l}215 \\
215\end{array}$ & & \\
\hline 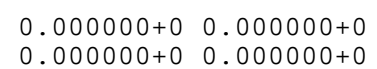 & $\begin{array}{l}\odot \\
\odot\end{array}$ & $\begin{array}{l}\odot \\
\odot\end{array}$ & $\begin{array}{l}1 \\
2\end{array}$ & $\begin{array}{r}7031125 \\
14071125\end{array}$ & $\begin{array}{l}3 \\
3\end{array}$ & $\begin{array}{l}1 \\
1\end{array}$ & \\
\hline 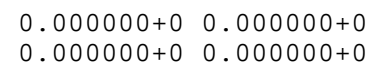 & $\begin{array}{l}\odot \\
\odot\end{array}$ & $\begin{array}{l}\odot \\
\odot\end{array}$ & $\begin{array}{l}1 \\
1\end{array}$ & $\begin{array}{l}18151125 \\
14071125\end{array}$ & $\begin{array}{l}3 \\
3\end{array}$ & $\begin{array}{l}2 \\
2\end{array}$ & \\
\hline $\begin{array}{l}\odot .00000 \odot+\odot-4.399910+5 \\
\odot .00000 \odot+\odot-4.400000+5\end{array}$ & $\begin{array}{l}\odot \\
\odot\end{array}$ & $\begin{array}{l}\odot \\
\odot\end{array}$ & $\begin{array}{l}1 \\
1\end{array}$ & $\begin{array}{r}1125 \\
14011125 \\
3251125\end{array}$ & $\begin{array}{l}-\cdot- \\
3 \\
3 \\
3\end{array}$ & $\begin{array}{l}- \\
3 \\
4 \\
4\end{array}$ & Only in VII.0 \\
\hline $\begin{array}{l}-1.241800+7-1.241800+7 \\
-1.241400+7-1.241400+7\end{array}$ & $\begin{array}{l}\odot \\
\odot\end{array}$ & $\begin{array}{l}\odot \\
\odot\end{array}$ & $\begin{array}{l}1 \\
1\end{array}$ & $\begin{array}{l}111125 \\
121125\end{array}$ & $\begin{array}{ll}3 & 1 \\
3 & 1\end{array}$ & 16 & \\
\hline $\begin{array}{l}\odot .00000 \odot+\odot-4.399910+5 \\
0.00000 \odot+\odot-4.400000+5\end{array}$ & $\begin{array}{l}\odot \\
\odot\end{array}$ & $\begin{array}{l}\odot \\
\odot \\
\odot\end{array}$ & $\begin{array}{l}1 \\
1\end{array}$ & $\begin{array}{r}1125 \\
1125 \\
11651125 \\
1621125\end{array}$ & $\begin{array}{ll}3 & 2 \\
3 & 2 \\
3 & 5 \\
3 & 5\end{array}$ & $\begin{array}{l}2- \\
22 \\
28 \\
51 \\
51\end{array}$ & $\begin{array}{l}\text { Only in VII.1 } \\
\text { Only in VII.1 }\end{array}$ \\
\hline $\begin{array}{l}\odot . \odot \odot \odot \odot \odot \odot+\odot-2 . \odot 76 \odot 1 \odot+6 \\
\odot . \odot \odot \odot \odot \odot \odot+\odot-2 . ๑ 78 \odot \odot \odot+6\end{array}$ & $\begin{array}{l}\odot \\
\odot\end{array}$ & $\begin{array}{l}\odot \\
\odot\end{array}$ & $\begin{array}{l}1 \\
1\end{array}$ & $\begin{array}{l}941125 \\
471125\end{array}$ & $\begin{array}{ll}3 & 5 \\
3 & 5\end{array}$ & $\begin{array}{l}-- \\
52 \\
52\end{array}$ & \\
\hline $\begin{array}{l}\odot .00 \odot \odot \odot \odot+\odot-2.390730+6 \\
\odot .00 \odot \odot \odot \odot+\odot-2.3930 \odot \odot+6\end{array}$ & $\begin{array}{l}\odot \\
\odot\end{array}$ & $\begin{array}{l}\odot \\
\odot\end{array}$ & $\begin{array}{l}1 \\
1\end{array}$ & $\begin{array}{r}1071125 \\
461125\end{array}$ & $\begin{array}{ll}3 & 5 \\
3 & 5\end{array}$ & $\begin{array}{l}53 \\
53\end{array}$ & \\
\hline $\begin{array}{l}\odot .0000 \odot \odot+\odot-2.639860+6 \\
\odot .000000+\odot-2.640000+6\end{array}$ & $\begin{array}{l}\odot \\
\odot\end{array}$ & $\begin{array}{l}\odot \\
\odot\end{array}$ & $\begin{array}{l}1 \\
1\end{array}$ & $\begin{array}{l}951125 \\
271125\end{array}$ & $\begin{array}{ll}3 & 5 \\
3 & 5\end{array}$ & $\begin{array}{l}54 \\
54\end{array}$ & \\
\hline $\begin{array}{l}\odot .000 \odot \odot \odot+\odot-2.703500+6 \\
\odot .00 \odot \odot \odot \odot+\odot-2.7050 \odot \odot+6\end{array}$ & $\begin{array}{l}\odot \\
\odot\end{array}$ & $\begin{array}{l}\odot \\
\odot\end{array}$ & $\begin{array}{l}1 \\
1\end{array}$ & $\begin{array}{l}911125 \\
201125\end{array}$ & $\begin{array}{ll}3 & 5 \\
3 & 5\end{array}$ & $\begin{array}{l}55 \\
55\end{array}$ & \\
\hline 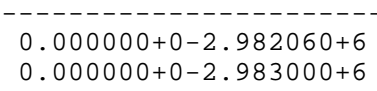 & $\begin{array}{l}\odot \\
\odot\end{array}$ & $\begin{array}{l}\odot \\
\odot\end{array}$ & $\begin{array}{l}1 \\
1\end{array}$ & $\begin{array}{l}991125 \\
141125\end{array}$ & $\begin{array}{ll}3 & 5 \\
3 & 5\end{array}$ & $\begin{array}{l}-- \\
56 \\
56\end{array}$ & \\
\hline $\begin{array}{l}\odot .0000 \odot \odot+\odot-3.677600+6 \\
\odot .0000 \odot \odot+\odot-3.6800 \odot \odot+6\end{array}$ & $\begin{array}{l}0 \\
\odot\end{array}$ & $\begin{array}{l}\odot \\
\odot\end{array}$ & $\begin{array}{l}1 \\
1\end{array}$ & $\begin{array}{l}811125 \\
161125\end{array}$ & $\begin{array}{ll}3 & 5 \\
3 & 5\end{array}$ & $\begin{array}{l}57 \\
57\end{array}$ & \\
\hline $\begin{array}{l}\odot .0 \odot \odot \odot \odot \odot+\odot-3.848 \odot 7 \odot+6 \\
\odot .0 \odot \odot \odot \odot \odot+\odot-3.880 \odot \odot \odot+6\end{array}$ & $\begin{array}{l}\odot \\
\odot\end{array}$ & $\begin{array}{l}\odot \\
\odot\end{array}$ & $\begin{array}{l}1 \\
1\end{array}$ & $\begin{array}{l}841125 \\
161125\end{array}$ & $\begin{array}{ll}3 & 5 \\
3 & 5\end{array}$ & $\begin{array}{l}-- \\
58 \\
58\end{array}$ & \\
\hline $\begin{array}{l}\odot .00 \odot \odot \odot \odot+\odot-3.914240+6 \\
\odot . \odot \odot \odot \odot \odot \odot+\odot-4.43 \odot \odot \odot \odot+6\end{array}$ & $\begin{array}{l}\odot \\
\odot\end{array}$ & $\begin{array}{l}\odot \\
\odot\end{array}$ & $\begin{array}{l}1 \\
1\end{array}$ & $\begin{array}{l}811125 \\
121125\end{array}$ & $\begin{array}{ll}3 & 5 \\
3 & 5\end{array}$ & $\begin{array}{l}-- \\
59 \\
59\end{array}$ & \\
\hline $\begin{array}{l}\odot .0 \odot \odot \odot \odot \odot+\odot-4.429630+6 \\
\odot .0 \odot \odot \odot \odot \odot+\odot-4.7700 \odot \odot+6\end{array}$ & $\begin{array}{l}- \\
0\end{array}$ & $\begin{array}{l}\odot \\
\odot\end{array}$ & $\begin{array}{l}1 \\
1\end{array}$ & $\begin{array}{l}771125 \\
121125\end{array}$ & 36 & $\begin{array}{l}-- \\
6 \odot \\
6 \odot\end{array}$ & \\
\hline $\begin{array}{l}\odot .00 \odot \odot \odot \odot+\odot-4.774610+6 \\
\odot .00 \odot \odot \odot \odot+\odot-5.380 \odot \odot \odot+6\end{array}$ & $\begin{array}{l}\odot \\
\odot\end{array}$ & $\begin{array}{l}\odot \\
\odot\end{array}$ & $\begin{array}{l}1 \\
1\end{array}$ & $\begin{array}{l}731125 \\
121125\end{array}$ & 36 & $\begin{array}{l}-- \\
61 \\
61\end{array}$ & \\
\hline $\begin{array}{l}\odot .00000 \odot+\odot-5.378560+6 \\
\odot .000000+\odot-5.53000 \odot+6\end{array}$ & $\begin{array}{l}\odot \\
\odot\end{array}$ & $\begin{array}{l}\odot \\
\odot\end{array}$ & $\begin{array}{l}1 \\
1\end{array}$ & $\begin{array}{l}581125 \\
101125\end{array}$ & $\begin{array}{ll}3 & 6 \\
3 & 6\end{array}$ & $\begin{array}{l}62 \\
62\end{array}$ & \\
\hline $\begin{array}{l}\odot .00000 \odot+\Theta-5.5340 \odot \odot+6 \\
\odot .0000 \odot \odot+\odot-5.7600 \odot \odot+6\end{array}$ & $\begin{array}{l}\odot \\
\odot\end{array}$ & $\begin{array}{l}\odot \\
\odot\end{array}$ & $\begin{array}{l}1 \\
1\end{array}$ & $\begin{array}{r}621125 \\
81125\end{array}$ & 36 & $\begin{array}{l}-\overline{6} \\
63 \\
63\end{array}$ & \\
\hline $\begin{array}{l}\odot .000000+\odot-5.741800+6 \\
\odot .000000+\odot-5.955000+6\end{array}$ & $\begin{array}{l}\odot \\
\odot\end{array}$ & $\begin{array}{l}\odot \\
\odot\end{array}$ & $\begin{array}{l}1 \\
1\end{array}$ & $\begin{array}{r}721125 \\
81125\end{array}$ & 36 & $\begin{array}{l}64 \\
64\end{array}$ & \\
\hline $\begin{array}{l}\odot .00 \odot \odot \odot \odot+\odot-5.766030+6 \\
\odot .0 \odot \odot \odot \odot \odot+\odot-6.0785 \odot \odot+6\end{array}$ & $\begin{array}{l}\odot \\
\odot\end{array}$ & $\begin{array}{l}\odot \\
\odot\end{array}$ & $\begin{array}{l}1 \\
1\end{array}$ & $\begin{array}{r}711125 \\
91125\end{array}$ & 36 & $\begin{array}{l}65 \\
65\end{array}$ & \\
\hline $\begin{array}{l}\odot . \odot \odot \odot \odot \odot \odot+\odot-5.778 \odot \odot \odot+6 \\
\odot . \odot \odot \odot \odot \odot \odot+\odot-6.27 \odot \odot \odot \odot+6\end{array}$ & $\begin{array}{l}\odot \\
\odot\end{array}$ & $\begin{array}{l}\odot \\
\odot\end{array}$ & $\begin{array}{l}1 \\
1\end{array}$ & $\begin{array}{l}701125 \\
101125\end{array}$ & $\begin{array}{ll}36 \\
3 & 6\end{array}$ & $\begin{array}{l}66 \\
66\end{array}$ & \\
\hline $\begin{array}{l}\odot .000 \odot \odot \odot+\odot-5.926800+6 \\
\odot .00 \odot \odot \odot \odot+\odot-7.1100 \odot \odot+6\end{array}$ & $\begin{array}{l}\odot \\
\odot\end{array}$ & $\begin{array}{l}\odot \\
\odot\end{array}$ & $\begin{array}{l}1 \\
1\end{array}$ & $\begin{array}{r}641125 \\
81125\end{array}$ & 36 & $\begin{array}{l}67 \\
67\end{array}$ & \\
\hline $\begin{array}{l}\odot .00 \odot \odot \odot \odot+\odot-5.9644 \odot \odot+6 \\
\odot .0 \odot \odot \odot \odot \odot+\odot-7.790 \odot \odot \odot+6\end{array}$ & $\begin{array}{l}\odot \\
\odot\end{array}$ & $\begin{array}{l}\odot \\
\odot\end{array}$ & $\begin{array}{l}1 \\
1\end{array}$ & $\begin{array}{l}651125 \\
101125\end{array}$ & 36 & $\begin{array}{l}68 \\
68\end{array}$ & \\
\hline & & & & $\begin{array}{l}1125 \\
1125 \\
1125\end{array}$ & $\begin{array}{ll}3 & 6 \\
3 & 7 \\
3 & 7\end{array}$ & $\begin{array}{l}-- \\
69 \\
70 \\
71\end{array}$ & $\begin{array}{l}\text { Only in VII.1 } \\
\text { Only in VII.1 } \\
\text { Only in VII.1 }\end{array}$ \\
\hline
\end{tabular}


1125372 only in VII.1 1125373 only in VII.1 1125374 only in VII.1 1125375 Only in VII.1 1125376 Only in VII.1 1125377 only in VII.1 1125378 only in VII.1 1125379 only in VII.1 1125380 only in VII.1 1125381 Only in VII.1 1125382 only in VII.1 1125383 Only in VII.1 1125384 only in VII.1 1125385 only in VII.1 1125386 only in VII.1 1125387 Only in VII.1 1125388 only in VII.1 1125389 Only in VII.1 $\odot .000000+\odot-5.367790+6$
$\odot .000000+\odot-5.843600+6$

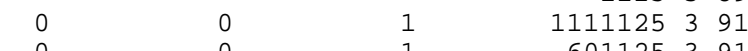

$6.960000+6 \quad 6.960000+6$ $6.959490+6 \quad 6.959490+6$

$0.000000+\odot \quad 0.000000+\odot$ $-3.597000+6-3.597000+6$

$\odot .000000+\odot$ ๑. $0000 \odot \odot+\odot$ $-3.866000+6-3.866000+6$

- - - - -

$0.000000+0 \quad 2.279230+1$

$0.000000+0 \quad 2.279200+1$

(......

(-

$\begin{array}{cc}\odot .000000+\odot & 2.279230+1 \\ \odot .0000 \odot \odot+\odot & 2.279200+1\end{array}$

$\odot .000000+\odot \quad 2.279230+1$ $0.000000+\odot \quad 2.279200+1$

$0.000000+0 \quad 2.279230+1$ $\odot .000000+\odot \quad 2.279200+1$

$0.000000+02.279230+1$ $0.000000+\odot \quad 2.279200+1$

$0.000000+\odot 2.279230+1$ $\odot .000000+\odot \quad 2.279200+1$

$\odot .000000+\odot 2.279230+1$ $0.000000+02.279200+$

$0.000000+0 \quad 2.279230+1$ $0.0000000+0 \quad 2.279200+1$

$0.000000+0 \quad 2.279230+1$

$0.000000+\odot 2.279200+1$

$0.000000+0 \quad 2.279230+1$ $0.000000+02.279200+1$

(1)

$0.000000+0 \quad 2.279230+1$ $0.000000+0 \quad 2.279200+1$

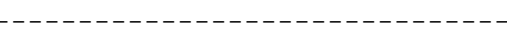

$\odot .000000+\odot \quad 2.279230+1$ $\odot .000000+\odot \quad 2.279200+1$

0

$0.000000+\odot \quad 2.279200+1$

$0.000000+0 \quad 2.279230+1$

$0.000000+\odot \quad 2.279200+1$

$0.000000+0 \quad 2.279230+1$ $0.000000+\odot 2.279200+1$

. . . . . . . . .

$\begin{array}{rrrrr}0 & 0 & 1 & 12031125 & 3102 \\ 0 & 0 & 4 & 341125 & 3102\end{array}$

$\odot \quad 0 \quad 110911253103$

$0 \quad 0 \quad 10211253103$

$0 \quad 0011911253107$

$\begin{array}{llll}0 & 0 & 1 & 10111253107\end{array}$

$0 \quad 2 \quad 0 \quad 0112542$

$\begin{array}{lllll}0 & 2 & 0 & 011254 & 4\end{array}$

管

1125416 only in VII. 0 $01125 \quad 451$ $01125 \quad 4 \quad 51$

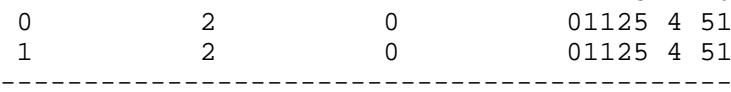

$0 \quad 201125452$

$\begin{array}{llll}1 & 2 & 0 & 01125452\end{array}$

$0 \quad 01125453$

$\begin{array}{llll}0 & 2 & 0 & 01125453\end{array}$

$\odot \quad 2 \quad 0 \quad 01125454$

$\begin{array}{llll}0 & 2 & 0 & 01125454\end{array}$

$0 \quad 2501125455$

$\begin{array}{llll}1 & 2 & 0 & 01125455\end{array}$

$0 \quad 0 \quad 01125456$

$\begin{array}{llll}0 & 2 & 0 & 01125456\end{array}$

$0 \quad 0 \quad 01125457$

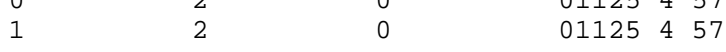

(2.

$\begin{array}{lllll}0 & 2 & 0 & 01125458\end{array}$

$1 \quad 2 \quad 0 \quad 01125458$

$\begin{array}{llll}0 & 2 & 0 & 01125459\end{array}$

101001125459

$0 \quad 201125460$

$\begin{array}{rrrr}1 & 2 & 0 & 01125460\end{array}$

$0 \quad 0 \quad 01125461$

$\begin{array}{lllll}1 & 2 & 0 & 01125 & 4\end{array}$

$0 \quad 0 \quad 01125462$

$\begin{array}{rrrr}1 & 2 & 0 & 01125462\end{array}$

$\begin{array}{llll}0 & 2 & 0 & 0 \\ 1 & 2 & 0 & 01125463\end{array}$

001125464

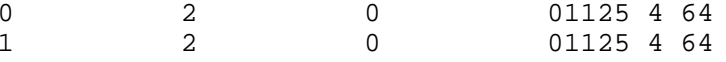




\begin{tabular}{|c|c|c|c|c|c|c|c|c|c|}
\hline $\begin{array}{ll}\odot . \odot \odot \odot \odot \odot \odot+\odot ~ & 2.27923 \odot+1 \\
\odot . \odot \odot \odot \odot \odot \odot+\odot ~ & 2.2792 \odot \odot+1\end{array}$ & $\begin{array}{l}\odot \\
1\end{array}$ & $\begin{array}{l}2 \\
2\end{array}$ & $\begin{array}{l}\odot \\
\odot\end{array}$ & $\begin{array}{l}01125 \\
01125\end{array}$ & $\begin{array}{l}4 \\
4\end{array}$ & $\begin{array}{l}65 \\
65\end{array}$ & & & \\
\hline \multicolumn{10}{|c|}{ 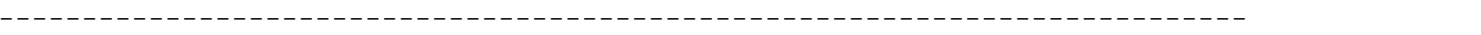 } \\
\hline$\odot .0 \odot \odot \odot \odot \odot+\odot \quad 2.279230+1$ & $\odot$ & 2 & $\odot$ & 01125 & 4 & 66 & & & \\
\hline $0.000000+0 \quad 2.279200+1$ & 1 & 2 & $\odot$ & 01125 & 4 & 66 & & & \\
\hline \multicolumn{10}{|c|}{ 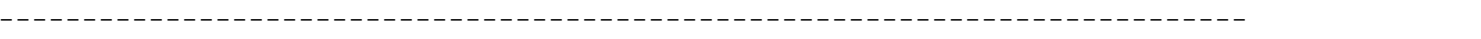 } \\
\hline $0.000000+0 \quad 2.279230+1$ & $\odot$ & 2 & $\odot$ & 01125 & 4 & 67 & & & \\
\hline$\odot .000000+0 \quad 2.279200+1$ & 1 & 2 & $\odot$ & 01125 & 4 & 67 & & & \\
\hline \multicolumn{10}{|c|}{ 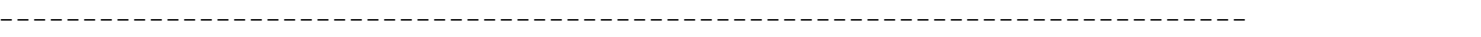 } \\
\hline$\odot .0 \odot \odot \odot \odot \odot+\odot \quad 2.279230+1$ & $\odot$ & 2 & $\odot$ & 01125 & 4 & 68 & & & \\
\hline $0.000000+0 \quad 2.279200+1$ & 1 & 2 & $\odot$ & 01125 & 4 & 68 & & & \\
\hline \multicolumn{10}{|c|}{ 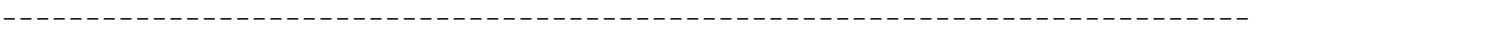 } \\
\hline & & & & 1125 & 4 & 69 & Only & in & VII.1 \\
\hline & & & & 1125 & 4 & 70 & Only & in & VII.1 \\
\hline & & & & 1125 & 4 & 71 & only & in & VII. 1 \\
\hline & & & & 1125 & 4 & 72 & Only & in & VII . 1 \\
\hline & & & & 1125 & 4 & 73 & only & in & VII.1 \\
\hline & & & & 1125 & 4 & 74 & Only & in & VII. 1 \\
\hline & & & & 1125 & 4 & 75 & only & in & VII. 1 \\
\hline & & & & 1125 & 4 & 76 & Only & in & VII. 1 \\
\hline & & & & 1125 & 4 & 77 & Only & in & VII. 1 \\
\hline & & & & 1125 & 4 & 78 & only & in & VII.1 \\
\hline & & & & 1125 & 4 & 79 & Only & in & VII. 1 \\
\hline & & & & 1125 & 4 & 80 & only & in & VII. 1 \\
\hline & & & & 1125 & 4 & 81 & Only & in & VII. 1 \\
\hline & & & & 1125 & 4 & 82 & Only & in & VII. 1 \\
\hline & & & & 1125 & 4 & 83 & Only & in & VII. 1 \\
\hline & & & & 1125 & 4 & 84 & only & in & VII. 1 \\
\hline & & & & 1125 & 4 & 85 & only & in & VII. 1 \\
\hline & & & & 1125 & 4 & 86 & Only & in & VII. 1 \\
\hline & & & & 1125 & 4 & 87 & Only & in & VII. 1 \\
\hline & & & & 1125 & 4 & 88 & Only & in & VII. 1 \\
\hline & & & & 1125 & 4 & 89 & Only & in & VII.1 \\
\hline & & & & 1125 & 4 & 91 & only & in & VII. $\odot$ \\
\hline & & & & 1125 & 5 & 16 & Only & in & VII. $\odot$ \\
\hline & & & & 1125 & 5 & 91 & Only & in & VII. $\odot$ \\
\hline & & & & 1125 & 6 & 16 & Only & in & VII.1 \\
\hline & & & & 1125 & 6 & 22 & Only & in & VII.1 \\
\hline & & & & 1125 & 6 & 28 & only & in & VII.1 \\
\hline & & & & 1125 & 6 & 91 & Only & in & VII.1 \\
\hline & & & & 1125 & 6 & 102 & only & in & VII. 1 \\
\hline
\end{tabular}

$12-M g-24$

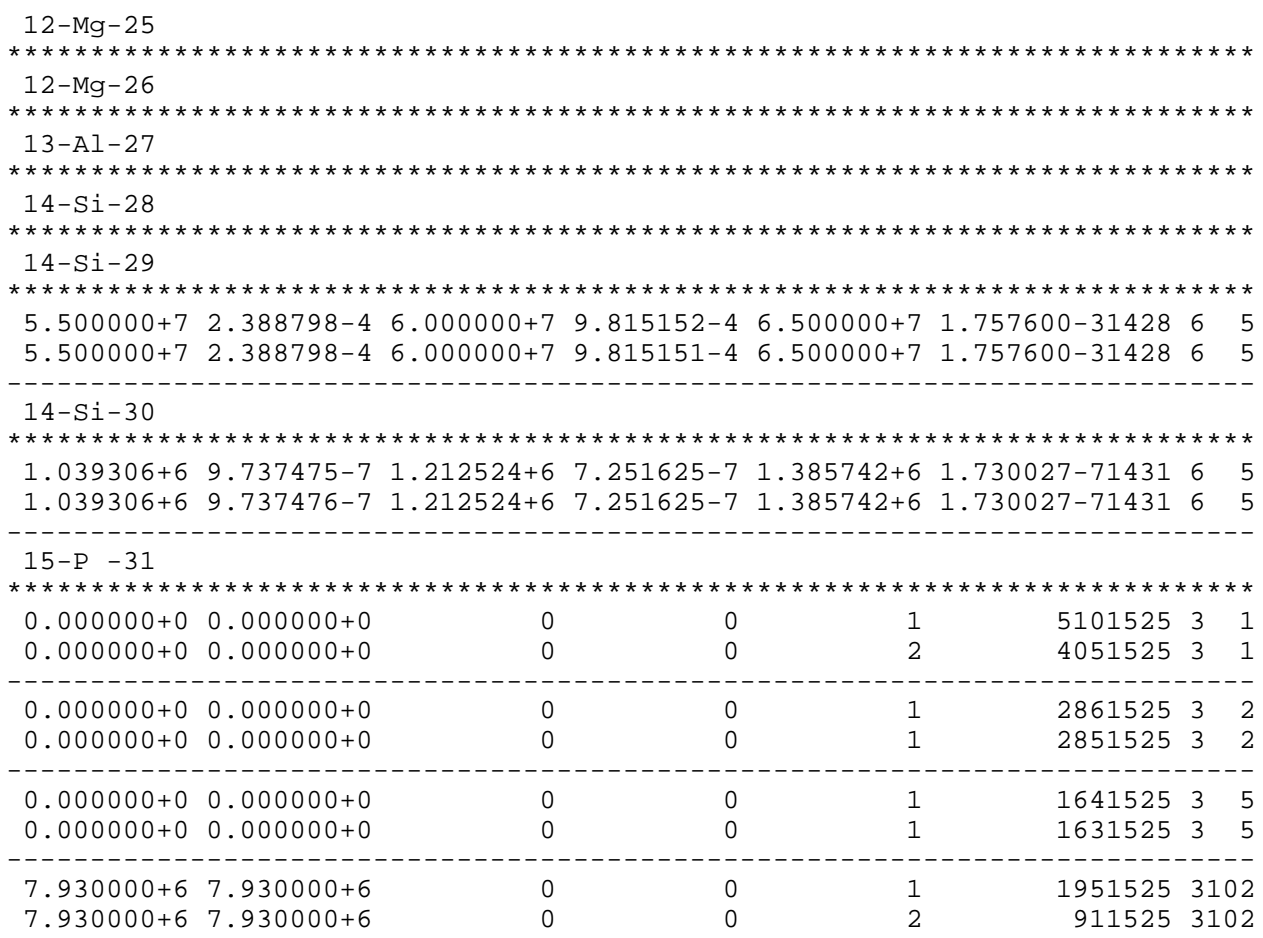


$6.948146+5 \quad 8.370184-7 \quad 9.264195+5 \quad 9.708006-7 \quad 1.158024+6 \quad 9.552120-71525 \quad 6 \quad 5$ $\begin{array}{llllllll}6.948146+5 & 8.370184-7 & 9.264195+5 & 9.708005-7 & 1.158024+6 & 9.552121-71525 & 6 & 5\end{array}$

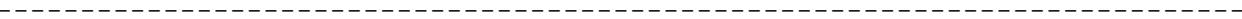

$16-S-32$

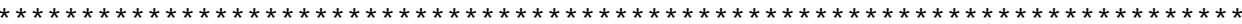

8.468570-2-9.955131-4-4.789310-2 2.259380-4 8.620540-3-1.526400-31625 456 8.468570-2-9.955130-4-4.789310-2 2.259380-4 8.620540-3-1.526400-31625 4 56

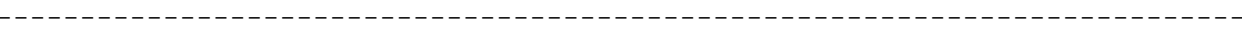

$16-\mathrm{S}-33$

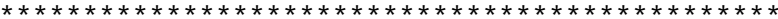

$9.708079-7$ 8.318220-8 6.959210-9 4.43943-10

$9.708080-7 \quad 8.318220-8 \quad 6.959210-9 \quad 4.43943-10 \quad 162842$

$16-5-34$

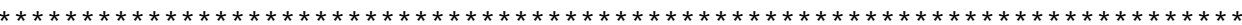

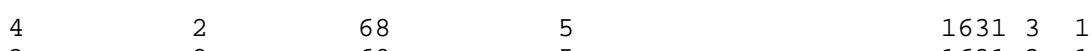

$\begin{array}{rrrrrr}3 & 2 & 68 & 5 & 1631 & 3\end{array}$

2.993170-4 5.444440-5 $6.873360-6 \quad 9.945539-7 \quad 1.306950-7 \quad 1.268380-81631442$ 2.993170-4 5.444440-5 $6.873360-6 \quad 9.945540-7 \quad 1.306950-7 \quad 1.268380-81631442$

2. $16-S-36$

$\begin{array}{llllll}1.340760+7 & 0.000000+\odot & 1.500000+7 & 4.607200-5 & 1.600000+7 & 9.939451-41637328\end{array}$ $1.340760+7 \quad 0.000000+0 \quad 1.500000+7 \quad 4.607200-5 \quad 1.600000+7 \quad 9.939450-41637328$

$17-\mathrm{Cl}-35$ -
$17-\mathrm{Cl}-35$

\begin{tabular}{|c|c|c|c|c|c|}
\hline 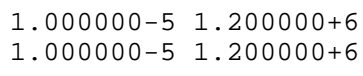 & $\begin{array}{l}1 \\
0\end{array}$ & $\begin{array}{l}7 \\
\odot\end{array}$ & $\begin{array}{l}\odot \\
\odot\end{array}$ & $\begin{array}{l}01725 \\
01725\end{array}$ & $\begin{array}{l}2151 \\
2151\end{array}$ \\
\hline 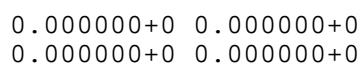 & $\begin{array}{l}\odot \\
\odot\end{array}$ & $\begin{array}{l}\odot \\
\odot\end{array}$ & $\begin{array}{l}2 \\
1\end{array}$ & $\begin{array}{r}2341725 \\
283511725\end{array}$ & $\begin{array}{l}3 \\
3\end{array}$ \\
\hline 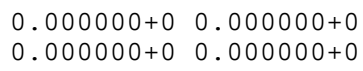 & $\begin{array}{l}\odot \\
\odot\end{array}$ & $\begin{array}{l}\odot \\
\odot\end{array}$ & $\begin{array}{l}1 \\
1\end{array}$ & $\begin{array}{r}2311725 \\
200391725\end{array}$ & $\begin{array}{l}3 \\
3\end{array}$ \\
\hline $\begin{array}{ll}8.579770+6 & 8.579770+6 \\
8.579770+6 & 8.579770+6\end{array}$ & $\begin{array}{l}\odot \\
\odot\end{array}$ & $\begin{array}{l}\odot \\
\odot\end{array}$ & $\begin{array}{l}2 \\
1\end{array}$ & $\begin{array}{r}561725 \\
200391725\end{array}$ & $\begin{array}{l}31 \\
31\end{array}$ \\
\hline $\begin{array}{ll}6.15220 \odot+5 & 6.15220 \ominus+5 \\
6.152200+5 & 6.152200+5\end{array}$ & $\begin{array}{l}\odot \\
\odot\end{array}$ & $\stackrel{\odot}{\odot}$ & $\begin{array}{l}1 \\
1\end{array}$ & $\begin{array}{r}801725 \\
60311725\end{array}$ & 31 \\
\hline 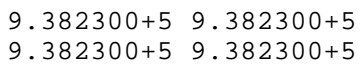 & $\begin{array}{l}\odot \\
\odot\end{array}$ & $\begin{array}{l}\odot \\
\odot\end{array}$ & $\begin{array}{l}1 \\
1\end{array}$ & $\begin{array}{l}24971725 \\
86291725\end{array}$ & $\begin{array}{l}31 \\
31\end{array}$ \\
\hline $\begin{array}{ll}6.15220 \ominus+5 & 6.15220 \ominus+5 \\
6.152200+5 & 6.152200+5\end{array}$ & $\begin{array}{l}\odot \\
\odot\end{array}$ & $\begin{array}{l}\odot \\
\odot\end{array}$ & $\begin{array}{l}1 \\
1\end{array}$ & $\begin{array}{r}801725 \\
54131725\end{array}$ & 36 \\
\hline
\end{tabular}
$\begin{array}{lllllll}9.844741+5 & 7.51750-23 & 9.848862+5 & 3.38607-22 & 9.850922+5 & 4.70291-221725 & 3601\end{array}$ (1)

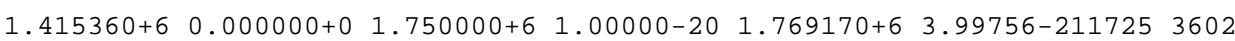
$1.415360+6 \quad 1.00000-201.750000+6 \quad 1.00000-201.769170+6 \quad 3.99756-2117253602$

$1.781940+6 \quad 0.000000+\odot \quad 1.814008+6 \quad 1.47068-21 \quad 1.858577+6 \quad 3.51455-2117253603$ $1.781940+6 \quad 1.47064-21 \quad 1.814008+6 \quad 1.47068-21 \quad 1.858577+6 \quad 3.51455-2117253603$

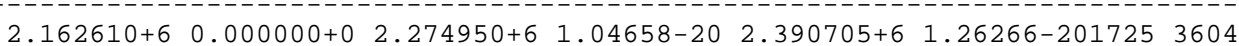
$2.162610+6 \quad 1.00000-20 \quad 2.274950+6 \quad 1.04658-20 \quad 2.390705+6 \quad 1.26266-2017253604$

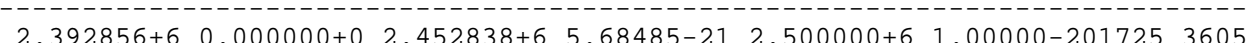
$2.392856+6 \quad 1.96717-22 \quad 2.452838+6 \quad 5.68485-21 \quad 2.500000+6 \quad 1.00000-2017253605$

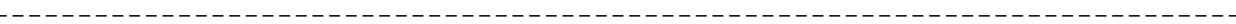

$2.886400+6 \quad 0.000000+0 \quad 3.000000+6 \quad 1.00000-20 \quad 3.026624+6 \quad 1.17666-2017253606$ $2.886400+6 \quad 4.81738-21 \quad 3.000000+6 \quad 1.00000-20 \quad 3.026624+6 \quad 1.17666-2017253606$

3.

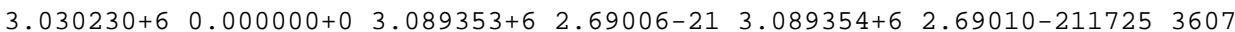
$3.030230+6 \quad 1.69472-21 \quad 3.089353+6 \quad 2.69006-21 \quad 3.089354+6 \quad 2.69010-2117253607$

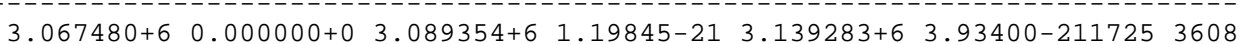
$3.067480+6 \quad 1.19840-21 \quad 3.089354+6 \quad 1.19845-21 \quad 3.139283+6 \quad 3.93400-2117253608$ $3.147730+6 \quad 0.000000+\odot \quad 3.157392+6 \quad 9.44754-22 \quad 3.250000+6 \quad 1.00000-201725 \quad 3609$ 
$3.147730+6 \quad 9.44656-22 \quad 3.157392+6 \quad 9.44754-22 \quad 3.250000+6 \quad 1.00000-201725 \quad 3609$ $3.278600+6 \quad 0.000000+\odot \quad 3.364815+6 \quad 3.89414-21 \quad 3.386421+6 \quad 4.87001-2117253610$ 3.278600+6 $7.38837-22 \quad 3.364815+6 \quad 3.89414-21 \quad 3.386421+6 \quad 4.87001-2117253610$

$3.294960+6 \quad 0.000000+\odot \quad 3.386421+6 \quad 4.46075-21 \quad 3.500000+6 \quad 1.00000-2017253611$ $3.294960+6 \quad 3.40702-21 \quad 3.386421+6 \quad 4.46075-21 \quad 3.500000+6 \quad 1.00000-2017253611$

$3.364810+6 \quad 0.000000+\odot \quad 3.500000+6 \quad 1.00000-20 \quad 3.504738+6 \quad 1.03355-2017253612$ $3.364810+6 \quad 1.59825-21 \quad 3.500000+6 \quad 1.00000-20 \quad 3.504738+6 \quad 1.03355-2017253612$

$3.386420+6 \quad 0.000000+\odot \quad 3.504738+6 \quad 1.0400 \odot-20 \quad 3.510911+6 \quad 1.08711-2017253613$ $3.386420+6 \quad 1.00000-20 \quad 3.504738+6 \quad 1.04000-20 \quad 3.510911+6 \quad 1.08711-2017253613$

$3.504740+60.000 \odot 0 \odot+\Theta \quad 3.592190+6 \quad 3.56566-21 \quad 3.667295+6 \quad 6.62789-2117253614$ $3.504740+6 \quad 2.51690-22 \quad 3.592190+6 \quad 3.56566-21 \quad 3.667295+6 \quad 6.62789-211725 \quad 3614$ $3.510910+60.000000+03.667295+6 \quad 6.54083-21 \quad 3.677481+6 \quad 6.96686-2117253615$ 3.510910+6 3.39953-21 3.667295+6 6.54083-21 3.677481+6 6.96686-211725 3615 $3.592100+60.000000+03.677481+$ $3.592190+6 \quad 4.75920-21 \quad 3.677481+6 \quad 5.40466-21 \quad 3.750000+6 \quad 1.00000-2017253616$ (3. $3.667300+6 \quad 0.000000+0 \quad 3.750000+6 \quad 1.00000-20 \quad 3.792814+6 \quad 1.34818-2017253617$ $3.667300+6 \quad 1.23161-21 \quad 3.750000+6 \quad 1.00000-20 \quad 3.792814+6 \quad 1.34818-2017253617$

$3.677480+6 \quad 0.000000+\odot \quad 3.792814+6 \quad 1.39903-20 \quad 3.975949+6 \quad 1.00797-2017253618$ $3.677480+6 \quad 1.00000-20 \quad 3.792814+6 \quad 1.39903-20 \quad 3.975949+6 \quad 1.00797-2017253618$

$3.792810+6 \quad 0.000 \odot \odot \odot+\odot \quad 4.000 \odot \odot \odot+6 \quad 1.00 \odot \odot \odot-204.031507+6 \quad 1.05071-2017253619$ 3.792810+6 $8.83916-21 \quad 4.000000+6 \quad 1.00000-20 \quad 4.031507+6 \quad 1.05071-2017253619$ $3.975950+6 \quad 0.000000+\odot \quad 4.031507+6 \quad 1.64108-20 \quad 4.031508+6 \quad 1.64110-2017253620$ $3.975950+6 \quad 1.00000-20 \quad 4.031507+6 \quad 1.64108-20 \quad 4.031508+6 \quad 1.64110-2017253620$

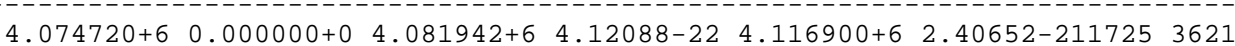
4.074720+6 4.12031-22 4.081942+6 4.12088-22 4.116900+6 2.40652-211725 3621

$4.116900+60000000+04.176287+64046179-214.231947+68.64364-2117253622$ $4.116900+6 \quad 4.46172-21 \quad 4.176287+6 \quad 4.46179-21 \quad 4.231947+6 \quad 8.64364-211725 \quad 3622$

(1) $4.345300+6 \quad 0.000000+\odot \quad 4.473086+6 \quad 8.26020-21 \quad 4.473108+6 \quad 8.26162-211725 \quad 3623$ $4.345300+6 \quad 4.28970-21 \quad 4.473086+6 \quad 8.26020-21 \quad 4.473108+6 \quad 8.26162-2117253623$ $4.411660+60.000000+04.473108+60.95571-214.473109+60.95582-2117253624$ $4.411660+6 \quad 6.95322-21 \quad 4.473108+6 \quad 6.95571-21 \quad 4.473109+6 \quad 6.95582-2117253624$ (1) $4.473108+6 \quad 0.000000+\odot \quad 4.473109+6 \quad 8.17449-24 \quad 4.500000+6 \quad 1.00000-2017253625$ 4.473108+6 7.80292-24 4.473109+6 8.17449-24 4.5000९९+6 $1.000000-2017253625$ $4.500660+6 \quad 0.000000+\odot \quad 4.641611+6 \quad 5.65298-21 \quad 4.750000+6 \quad 1.00000-2017253626$ $4.500660+6 \quad 2.80588-21 \quad 4.641611+6 \quad 5.65298-21 \quad 4.750000+6 \quad 1.00000-2017253626$ $4.570620+6 \quad 0.00000 \odot+\odot \quad 4.750000+6 \quad 1.000 \odot \odot-20 \quad 4.757789+6 \quad 1.01042-2017253627$ $\begin{array}{lllllll}4.570620+6 & 3.95754-21 & 4.750000+6 & 1.00000-20 & 4.757789+6 & 1.01042-201725 & 3627\end{array}$ $4.641610+6 \quad 0.000 \odot \odot \odot+\odot \quad 4.757789+6 \quad 1.01671-20 \quad 4.757790+6 \quad 1.01671-2017253628$ $4.641610+6 \quad 1.00000-20 \quad 4.757789+6 \quad 1.01671-20 \quad 4.757790+6 \quad 1.01671-2017253628$

$4.265400+6 \quad 0.000000+\odot \quad 4.293833+6 \quad 1.21198-21 \quad 4.298410+6 \quad 1.40708-2117253650$ $4.265400+6 \quad 1.21194-21 \quad 4.293833+6 \quad 1.21198-21 \quad 4.298410+6 \quad 1.40708-2117253650$

$6.454060+60.000000+06.554151+61.661158-66.554153+61.661219-617253651$ $6.454060+6 \quad 1.00000-20 \quad 6.554151+6 \quad 1.661158-6 \quad 6.554153+6 \quad 1.661219-617253651$ $7.663880+60000000+07.927969+67.85699-218.000000+61.00000-2017253652$

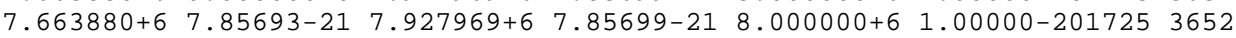
$8.292500+6 \quad 0.000000+\odot \quad 8.498168+6 \quad 9.91171-21 \quad 8.5000000+6 \quad 1.00000-2017253653$ $8.292500+6 \quad 7.92345-21 \quad 8.498168+6 \quad 9.91171-21 \quad 8.500000+6 \quad 1.000000-2017253653$ $8.456910+6 \quad 0.00000 \odot+\odot \quad 8.500000+6 \quad 1.00000-20 \quad 9.0000 \odot 0+6 \quad 8.224970-917253654$ $8.456910+6 \quad 9.57482-21 \quad 8.500000+6 \quad 1.00000-20 \quad 9.000000+6 \quad 8.224970-917253654$ $8.500000+6 \quad 0.000000+\odot \quad 9.000000+6 \quad 1.00000-2 \odot \quad 9.021439+6 \quad 1.691144-917253655$ 
$8.500000+6 \quad 1.00000-20 \quad 9.000000+6 \quad 1.00000-20 \quad 9.021439+6 \quad 1.691144-917253655$ $9.021440+6 \quad 0.000000+\odot \quad 9.281839+6 \quad 5.44131-21 \quad 9.295729+6 \quad 5.73156-2117253656$ $9.021440+6 \quad 1.39527-21 \quad 9.281839+6 \quad 5.44131-21 \quad 9.295729+6 \quad 5.73156-2117253656$ $9.088210+6 \quad 0.000000+\odot \quad 9.295729+6 \quad 5.03943-21 \quad 9.500000+6 \quad 1.00000-2017253657$ $9.088210+6 \quad 4.70212-21 \quad 9.295729+6 \quad 5.03943-21 \quad 9.500000+6 \quad 1.000000-2017253657$

$9.281840+6 \quad 0.000000+\odot \quad 9.500000+6 \quad 1.00000-20 \quad 9.574061+6 \quad 9.515951-917253658$ $9.281840+6 \quad 6.36686-22 \quad 9.500000+6 \quad 1.00000-20 \quad 9.574061+6 \quad 9.515951-917253658$

$9.295730+6 \quad 0.000000+\odot \quad 9.574061+6 \quad 5.998988-9 \quad 9.643170+6 \quad 1.159686-817253659$ $9.295730+6 \quad 1.00000-20 \quad 9.574061+6 \quad 5.998988-9 \quad 9.643170+6 \quad 1.159686-817253659$

$9.643170+6 \quad 0.000000+\odot 9.802641+6 \quad 4.46910-21 \quad 1.000000+7 \quad 1.0000 \odot-2017253660$ $9.643170+6 \quad 2.62380-21 \quad 9.802641+6 \quad 4.46910-21 \quad 1.000000+7 \quad 1.00000-2017253660$

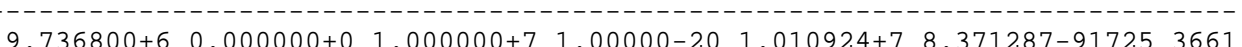
$9.736800+6 \quad 2.50170-21 \quad 1.000000+7 \quad 1.00000-201.010924+7 \quad 8.371287-917253661$

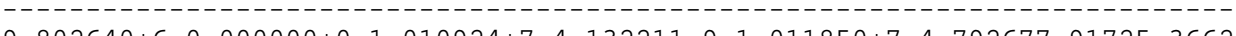

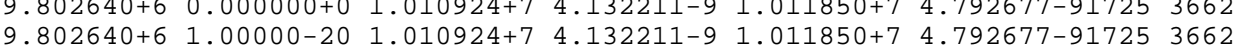
1.011850+7 0.00000001010

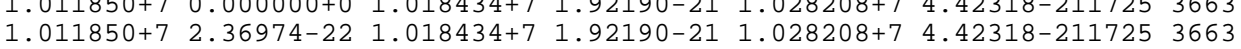
1. $\begin{array}{lllllll}1.011850+7 & 0.000000+\odot & 1.028208+7 & 4.28781-21 & 1.043126+7 & 8.19817-211725 & 3664\end{array}$ $1.011850+7 \quad 1.72582-21 \quad 1.028208+7 \quad 4.28781-21 \quad 1.043126+7 \quad 8.19817-2117253664$ $1.018430+7 \quad 0.000000+\odot \quad 1.043126+7 \quad 7.82234-21 \quad 1.043924+7 \quad 8.07514-2117253665$ $1.018430+7 \quad 3.09637-21 \quad 1.043126+7 \quad 7.82234-21 \quad 1.043924+7 \quad 8.07514-2117253665$ $1.028210+7 \quad 0.000000+\odot \quad 1.043924+7 \quad 7.21182-21 \quad 1.050000+7 \quad 1.00000-2017253666$ $1.028210+7 \quad 6.84563-21 \quad 1.043924+7 \quad 7.21182-21 \quad 1.050000+7 \quad 1.00000-2017253666$

$1.043924+7 \quad 0.000000+\odot \quad 1.050000+7 \quad 1.0000 \odot-201.056193+7 \quad 3.00686-1017253667$ $1.043924+7 \quad 1.15961-21 \quad 1.050000+7 \quad 1.00000-201.056193+7 \quad 3.00686-1017253667$

$1.061700+70.000000+\Theta 1.069671+72.08182-211.079342+74.60669-2117253669$ $\begin{array}{llllllll}1.061700+7 & 2.05493-21 & 1.069671+7 & 2.08182-21 & 1.079342+7 & 4.60669-211725 & 3669\end{array}$

$1.093230+70.00000 \odot+\odot 1.100000+7 \quad 1.00000-201.109590+7 \quad 2.68349-1017253674$ $\begin{array}{lllllll}1.093230+7 & 8.05584-21 & 1.100000+7 & 1.00000-20 & 1.109590+7 & 2.68349-101725 & 3674\end{array}$

1.09860+7 $000000+01.109590+71.43718-101211417+72.92924-1017253675$

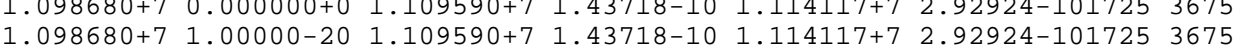

1.

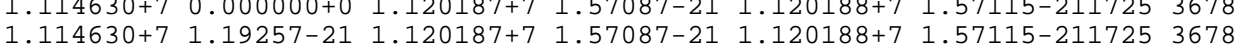

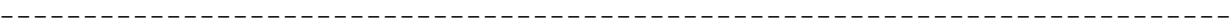
$\begin{array}{lllllll}1.118850+7 & 0.000000+\odot & 1.120188+7 & 4.29842-22 & 1.127893+7 & 2.90328-211725 & 3679\end{array}$ $\begin{array}{lllllll}1.118850+7 & 4.29521-22 & 1.120188+7 & 4.29842-22 & 1.127893+7 & 2.90328-211725 & 3679\end{array}$ $9.574060+6 \quad 0.000000+\odot \quad 9.736795+6 \quad 3.82058-21 \quad 9.802641+6 \quad 5.36649-211725 \quad 3700$ $9.574060+6 \quad 1.62249-21 \quad 9.736795+6 \quad 3.82058-21 \quad 9.802641+6 \quad 5.36649-2117253700$

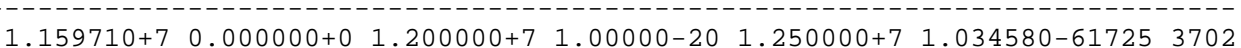
$\begin{array}{llllllll}1.159710+7 & 8.83997-21 & 1.200000+7 & 1.000000-20 & 1.250000+7 & 1.034580-61725 & 3702\end{array}$

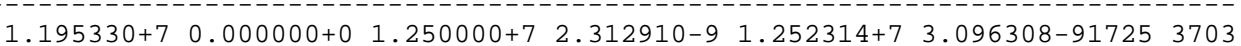
$\begin{array}{lllllll}1.195330+7 & 1.00000-20 & 1.250000+7 & 2.312910-9 & 1.252314+7 & 3.096308-91725 & 3703\end{array}$

$1.252130+70.000000+01.262829+72.20505-211.288684+77.62698-2117253704$ $1.252130+7 \quad 1.45200-21 \quad 1.262829+7 \quad 2.20505-21 \quad 1.288684+7 \quad 7.62698-2117253704$

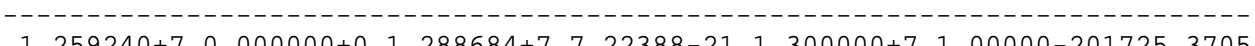
$1.259240+7 \quad 8.80968-22 \quad 1.288684+7 \quad 7.22388-21 \quad 1.300000+7 \quad 1.00000-2017253705$ (1.262830+7

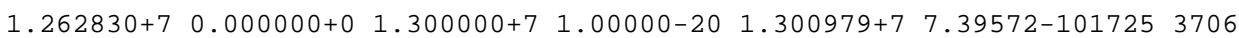
$\begin{array}{lllllll}1.262830+7 & 6.95569-21 & 1.3000000+7 & 1.000000-20 & 1.3000979+7 & 7.39572-101725 & 3706\end{array}$

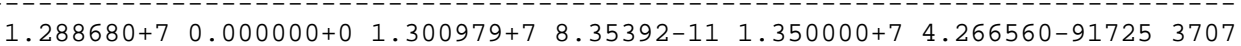
$\begin{array}{lllllll}1.288680+7 & 1.000000-20 & 1.300979+7 & 8.35392-11 & 1.350000+7 & 4.266560-91725 & 3707\end{array}$

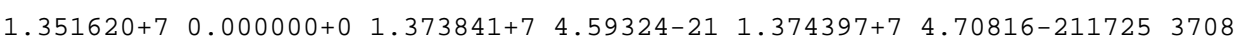


$\begin{array}{lllllll}1.351620+7 & 2.19028-21 & 1.373841+7 & 4.59324-21 & 1.374397+7 & 4.70816-211725 & 3708\end{array}$ $\begin{array}{lllllll}1.362220+7 & 0.000000+\odot & 1.374397+7 & 3.22403-21 & 1.378615+7 & 4.34035-211725 & 3709\end{array}$ $\begin{array}{llllllll}1.362220+7 & 3.07688-21 & 1.374397+7 & 3.22403-21 & 1.378615+7 & 4.34035-211725 & 3709\end{array}$ $\begin{array}{lllllll}1.374397+7 & 0.000 \odot \odot \odot+\odot & 1.378615+7 & 1.82499-21 & 1.383728+7 & 3.77958-211725 & 3710\end{array}$ $\begin{array}{llllllll}1.374397+7 & 2.12546-22 & 1.378615+7 & 1.82499-21 & 1.383728+7 & 3.77958-211725 & 3710\end{array}$

$\begin{array}{llllllll}1.374400+7 & \odot .000000+\odot & 1.383728+7 & 3.64449-21 & 1.390591+7 & 6.32504-211725 & 3711\end{array}$ $1.374400+7 \quad 1.64746-21 \quad 1.383728+7 \quad 3.64449-21 \quad 1.390591+7 \quad 6.32504-2117253711$

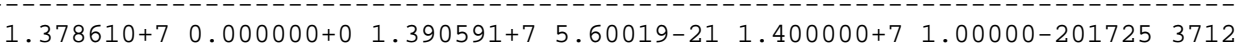
$\begin{array}{llllllll}1.378610+7 & 2.39093-21 & 1.390591+7 & 5.60019-21 & 1.400000+7 & 1.00000-201725 & 3712\end{array}$

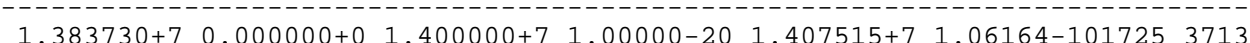
$\begin{array}{llllllll}1.383730+7 & 4.21767-21 & 1.400000+7 & 1.00000-20 & 1.407515+7 & 1.06164-101725 & 3713\end{array}$

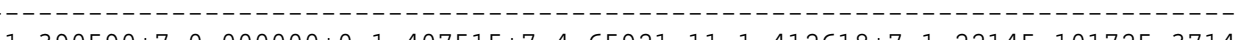
$\begin{array}{lllllll}1.390590+7 & 1.00000-20 & 1.407515+7 & 4.65921-11 & 1.412618+7 & 1.22145-101725 & 3714\end{array}$

1.407520+7

$1.407520+7 \quad 0.000000+\odot \quad 1.443988+7 \quad 8.58491-21 \quad 1.445696+7 \quad 8.98694-2117253715$ $1.407520+7 \quad 1.20113-21 \quad 1.443988+7 \quad 8.58491-21 \quad 1.445696+7 \quad 8.98694-2117253715$

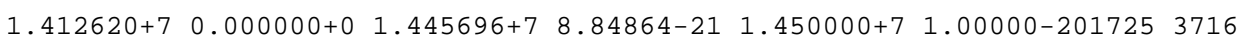
$\begin{array}{lllllll}1.412620+7 & 8.39174-21 & 1.445696+7 & 8.84864-21 & 1.450000+7 & 1.00000-201725 & 3716\end{array}$ $\begin{array}{lllllll}1.443990+7 & 0.000000+\odot & 1.450000+7 & 1.00000-20 & 1.458011+7 & 9.81345-111725 & 3717\end{array}$ $\begin{array}{llllllll}1.443990+7 & 2.84098-21 & 1.450000+7 & 1.00000-20 & 1.458011+7 & 9.81345-111725 & 3717\end{array}$

$1.458010+7 \quad 0.000000+\odot 1.465861+7 \quad 1.86954-21 \quad 1.490039+7 \quad 7.62771-2117253719$ $1.458010+7 \quad 1.30606-21 \quad 1.465861+7 \quad 1.86954-21 \quad 1.490039+7 \quad 7.62771-2117253719$

$1.463490+7 \quad 0.000000+\odot \quad 1.490039+7 \quad 7.27133-21 \quad 1.493331+7 \quad 8.17313-2117253720$ $1.463490+7 \quad 6.48130-22 \quad 1.490039+7 \quad 7.27133-21 \quad 1.493331+7 \quad 8.17313-2117253720$

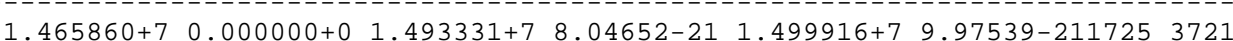
$1.465860+7 \quad 7.08222-21 \quad 1.493331+7 \quad 8.04652-21 \quad 1.499916+7 \quad 9.97539-2117253721$

$1.490040+7$ ( $900000+01.499916+7901567-211500000+7100000-2017253722$ $1.490040+7 \quad 3.30489-21 \quad 1.499916+7 \quad 9.91567-21 \quad 1.500000+7 \quad 1.00000-2017253722$

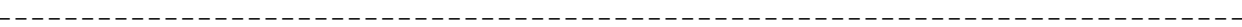

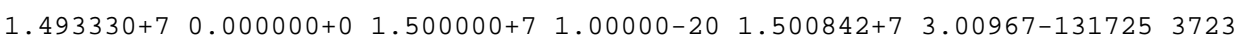
$1.493330+7 \quad 9.87404-21 \quad 1.500000+7 \quad 1.00000-201.500842+7 \quad 3.00967-1317253723$

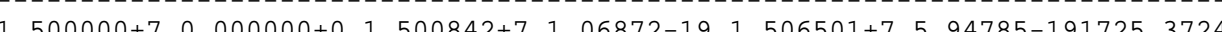
$\begin{array}{lllllll}1.500000+7 & 1.00000-20 & 1.500842+7 & 1.06872-19 & 1.506501+7 & 5.94785-191725 & 3724\end{array}$

(1.500

$1.500840+7 \quad 0.000000+\odot \quad 1.507529+7 \quad 1.36031-21 \quad 1.512468+7 \quad 2.36503-2117253725$ $\begin{array}{lllllll}1.500840+7 & 1.15119-21 & 1.507529+7 & 1.36031-21 & 1.512468+7 & 2.36503-211725 & 3725\end{array}$

$1.507530+7 \quad 0.000000+\odot \quad 1.520709+7 \quad 3.10329-21 \quad 1.533250+7 \quad 6.05613-2117253727$ $\begin{array}{lllllll}1.507530+7 & 1.16291-21 & 1.520709+7 & 3.10329-21 & 1.533250+7 & 6.05613-211725 & 3727\end{array}$

$1.512470+7 \quad 0.000 \odot \odot \odot+\odot \quad 1.533250+7 \quad 5.53714-21 \quad 1.533251+7 \quad 5.53741-2117253728$ $\begin{array}{llllllll}1.512470+7 & 2.19573-21 & 1.533250+7 & 5.53714-21 & 1.533251+7 & 5.53741-211725 & 3728\end{array}$

.

$\begin{array}{lllllll}1.520710+7 & 0.000000+\odot & 1.533251+7 & 4.28186-21 & 1.550000+7 & 1.00000-201725 & 3729\end{array}$ $1.520710+7 \quad 4.28152-21 \quad 1.533251+7 \quad 4.28186-21 \quad 1.550000+7 \quad 1.00000-2017253729$

\begin{tabular}{|c|c|c|c|c|}
\hline $\begin{array}{ll}9.382300+5 & 9.382300+5 \\
9.382300+5 & 9.382300+5\end{array}$ & $\begin{array}{l}\odot \\
\odot\end{array}$ & $\begin{array}{l}\odot \\
\odot\end{array}$ & $\begin{array}{l}1 \\
1\end{array}$ & $\begin{array}{l}24871725380 \odot \\
86191725380 \odot\end{array}$ \\
\hline $\begin{array}{ll}9.382300+5 & 8.601300+5 \\
9.382300+5 & 8.601300+5\end{array}$ & $\begin{array}{l}\odot \\
\odot\end{array}$ & $\begin{array}{l}\odot \\
\odot\end{array}$ & $\begin{array}{l}1 \\
1\end{array}$ & $\begin{array}{rr}8761725 & 3801 \\
86191725 & 3801\end{array}$ \\
\hline $\begin{array}{ll}9.382300+5 & 4.252300+5 \\
9.382300+5 & 4.252300+5\end{array}$ & $\begin{array}{l}\odot \\
\odot\end{array}$ & $\begin{array}{l}\odot \\
\odot\end{array}$ & $\begin{array}{l}1 \\
1\end{array}$ & $\begin{array}{r}30917253802 \\
861917253802\end{array}$ \\
\hline $\begin{array}{l}9.382300+5-2 \cdot 114700+5 \\
9.382300+5-2.114700+5\end{array}$ & $\begin{array}{l}\odot \\
\odot\end{array}$ & $\begin{array}{l}\odot \\
\odot\end{array}$ & $\begin{array}{l}1 \\
1\end{array}$ & $\begin{array}{r}63117253803 \\
522417253803\end{array}$ \\
\hline $\begin{array}{l}9.382300+5-3.849700+5 \\
9.382300+5-3.849700+5\end{array}$ & $\begin{array}{l}\odot \\
\odot\end{array}$ & $\begin{array}{l}\odot \\
\odot\end{array}$ & $\begin{array}{l}1 \\
1\end{array}$ & $\begin{array}{rr}8781725 & 3804 \\
38901725 & 3804\end{array}$ \\
\hline $9.382300+5-8.162700+5$ & $\odot$ & $\odot$ & 1 & 82917253805 \\
\hline
\end{tabular}


$1.328820+6 \quad 0.000000+\odot \quad 1.415360+6 \quad 5.05532-21 \quad 1.500000+6 \quad 1.00000-2017253808$ $1.328820+6 \quad 4.15799-21 \quad 1.415360+6 \quad 5.05532-21 \quad 1.500000+6 \quad 1.00000-2017253808$

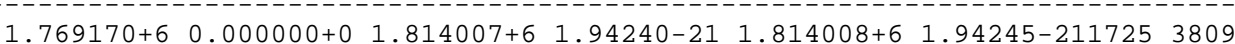
$1.769170+6 \quad 5.53053-22 \quad 1.814007+6 \quad 1.94240-21 \quad 1.814008+6 \quad 1.94245-2117253809$

$1.858580+6$ 0 $000000+02.126180+61.00000-202.162610+61.00000-2017253810$

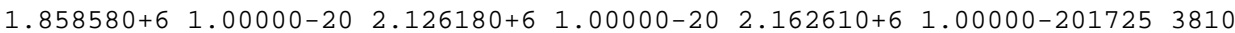

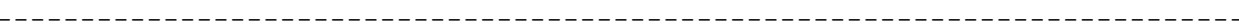

$2.126180+6 \quad 0.000000+\odot \quad 2.250000+6 \quad 1.00000-20 \quad 2.274950+6 \quad 1.00000-2017253811$ $2.126180+6 \quad 2.94212-21 \quad 2.250000+6 \quad 1.00000-20 \quad 2.274950+6 \quad 1.000 \odot \odot-2017253811$ $2.274950+6 \quad 5.14352-21 \quad 2.392856+6 \quad 5.23910-21 \quad 2.452838+6 \quad 7.90438-2117253812$

2.

$2.392860+6 \quad 0.0000000+\odot \quad 2.5000000+6 \quad 1.00000-20 \quad 2.577019+6 \quad 1.00000-2017253813$ $2.392860+6 \quad 5.59826-21 \quad 2.5000000+6 \quad 1.00000-20 \quad 2.577019+6 \quad 1.000000-2017253813$

$2.452840+6 \quad 0.000000+\odot \quad 2.577019+6 \quad 1.00000-20 \quad 2.579077+6 \quad 1.00000-2017253814$ $2.452840+6 \quad 1.00000-20 \quad 2.577019+6 \quad 1.00000-20 \quad 2.579077+6 \quad 1.00000-2017253814$

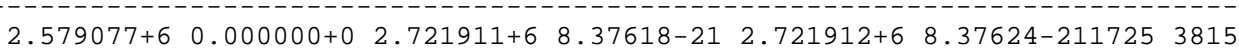
$\begin{array}{lllllll}2.579077+6 & 1.18973-22 & 2.721911+6 & 8.37618-21 & 2.721912+6 & 8.37624-211725 & 3815\end{array}$

$2.579080+6 \quad 0.0000 \odot \odot+\odot \quad 2.721912+6 \quad 8.35668-21 \quad 2.750000+6 \quad 1.00000-2017253816$ $\begin{array}{llllllll}2.579080+6 & 8.35662-21 & 2.721912+6 & 8.35668-21 & 2.750000+6 & 1.00000-201725 & 3816\end{array}$

$2.941130+6 \quad 0.000 \odot \odot \odot+\odot \quad 3.026624+6 \quad 1.000 \odot \odot-20 \quad 3.030235+6 \quad 1.0000 \odot-2017253817$ $2.941130+6 \quad 1.00000-20 \quad 3.026624+6 \quad 1.00000-20 \quad 3.030235+6 \quad 1.00000-2017253817$

$3.026620+60000000+03.067479+61.82895-213.089353+62.80821-2117253818$ $3.026620+6 \quad 1.61612-22 \quad 3.067479+6 \quad 1.82895-21 \quad 3.089353+6 \quad 2.80821-2117253818$

3. $3.139280+6 \quad 0.000000+\odot \quad 3.157391+6 \quad 1.63552-21 \quad 3.157392+6 \quad 1.63561-2117253819$ $\begin{array}{lllllll}3.139280+6 & 7.62797-22 & 3.157391+6 & 1.63552-21 & 3.157392+6 & 1.63561-211725 & 3819\end{array}$

- - - - - - - - - - - - - - - - - - - - - - - - - - - - - $17-\mathrm{Cl}-37$

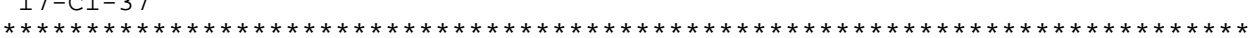

$\begin{array}{cccccc}1.703700+4 & 1.000000+\odot & 0 & 1 & 1 & 017312151\end{array}$

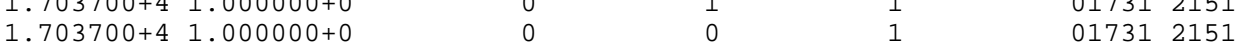

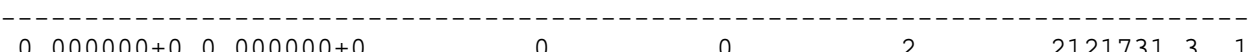

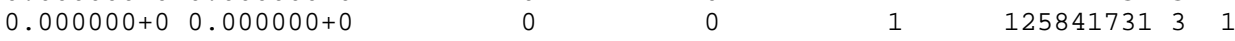

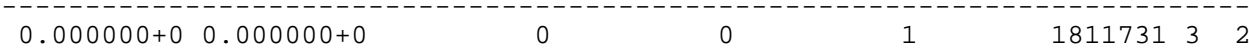

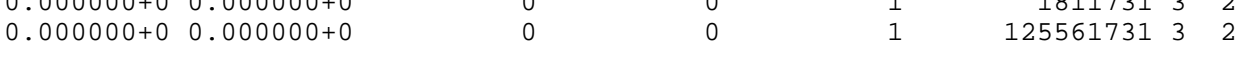

$1.285228+71.096897-31.300000+7 \quad 1.021640-31.308003+79.934485-41731366$ $\begin{array}{llllllll}1.285228+7 & 1.096897-3 & 1.300000+7 & 1.021640-3 & 1.308003+7 & 9.934486-41731 & 3 & 66\end{array}$ 6. $\begin{array}{rrrrrr}6.107830+6 & 6.107830+6 & 0 & 0 & 2 & 18417313102\end{array}$ $\begin{array}{rrrrrr}6.107830+6 & 6.107830+6 & 0 & 0 & 1 & 1255617313102\end{array}$

$1.357498+7 \quad 9.766011-4 \quad 1.371859+7 \quad 1.105391-3 \quad 1.375578+7 \quad 1.138743-317313652$ $\begin{array}{llllllll}1.357498+7 & 9.766012-4 & 1.371859+7 & 1.105391-3 & 1.375578+7 & 1.138743-31731 & 3652\end{array}$

$18-\mathrm{Ar}-36$ $* * * * * * * * * * * * * * * * * * * * * * * * * * * * * * * * * * * * * * * * * * * * * * * * * * * * * * * * * * * * * * * * * * * * * * * * * * *$ $18-\mathrm{Ar}-38$

$* * * * * * * * * *$
$18-\mathrm{Ar}-40$

18 - Ar -40

$\begin{array}{llllllll}1.700000+7 & 2.082740-6 & 1.800000+7 & 9.694151-7 & 1.900000+7 & 4.672320-71837 & 3 & 72\end{array}$ $\begin{array}{lllllllll}1.700000+7 & 2.082740-6 & 1.800000+7 & 9.694150-7 & 1.900000+7 & 4.672320-71837 & 3 & 72\end{array}$

$5.002670-39.847870-41.103430-41.318250-59.725391-72.816520-818374$ $\begin{array}{lllllll}5.002670-3 & 9.847870-4 & 1.103430-4 & 1.318250-5 & 9.725390-7 & 2.816520-81837 & 4\end{array}$ 


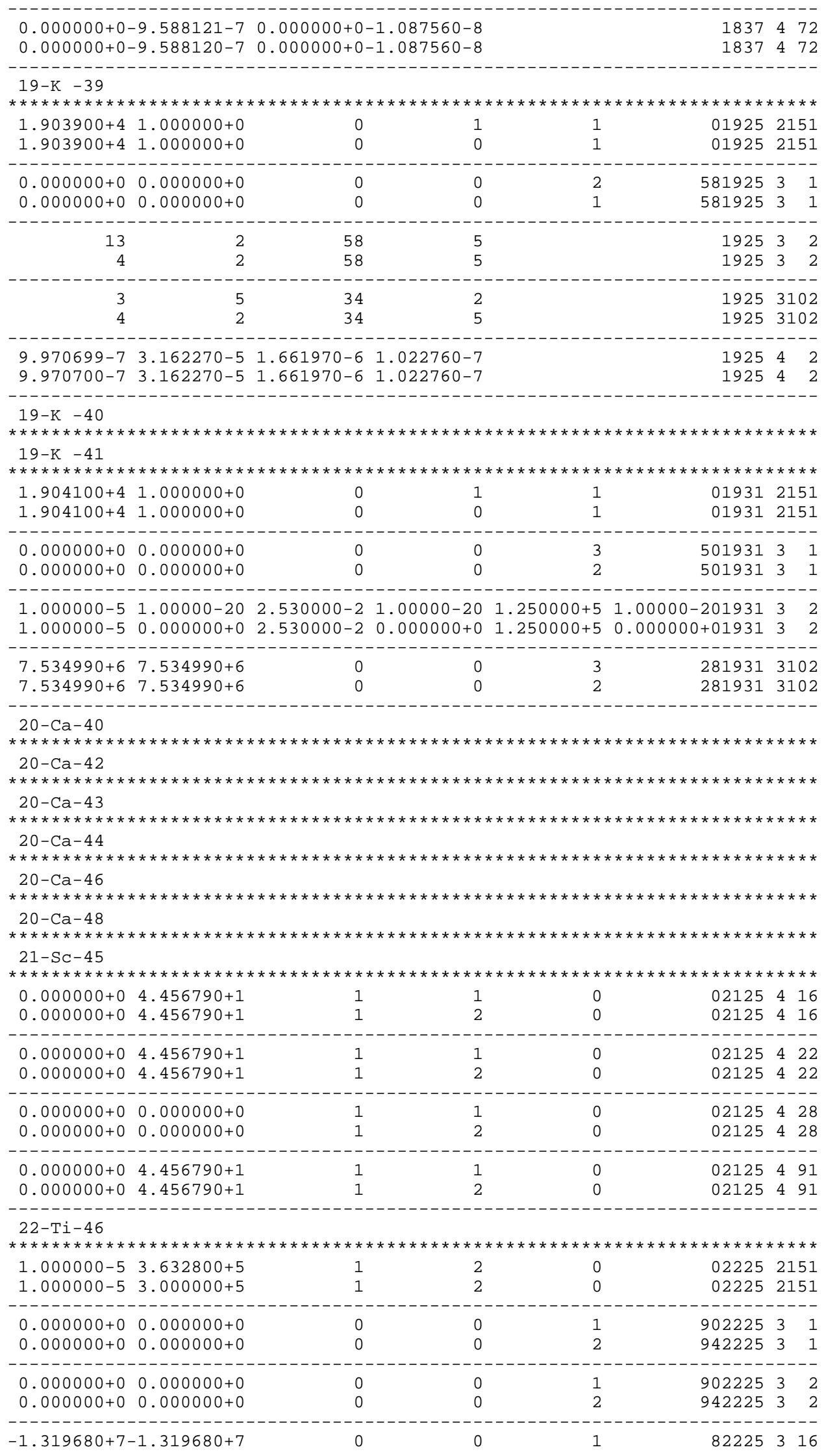




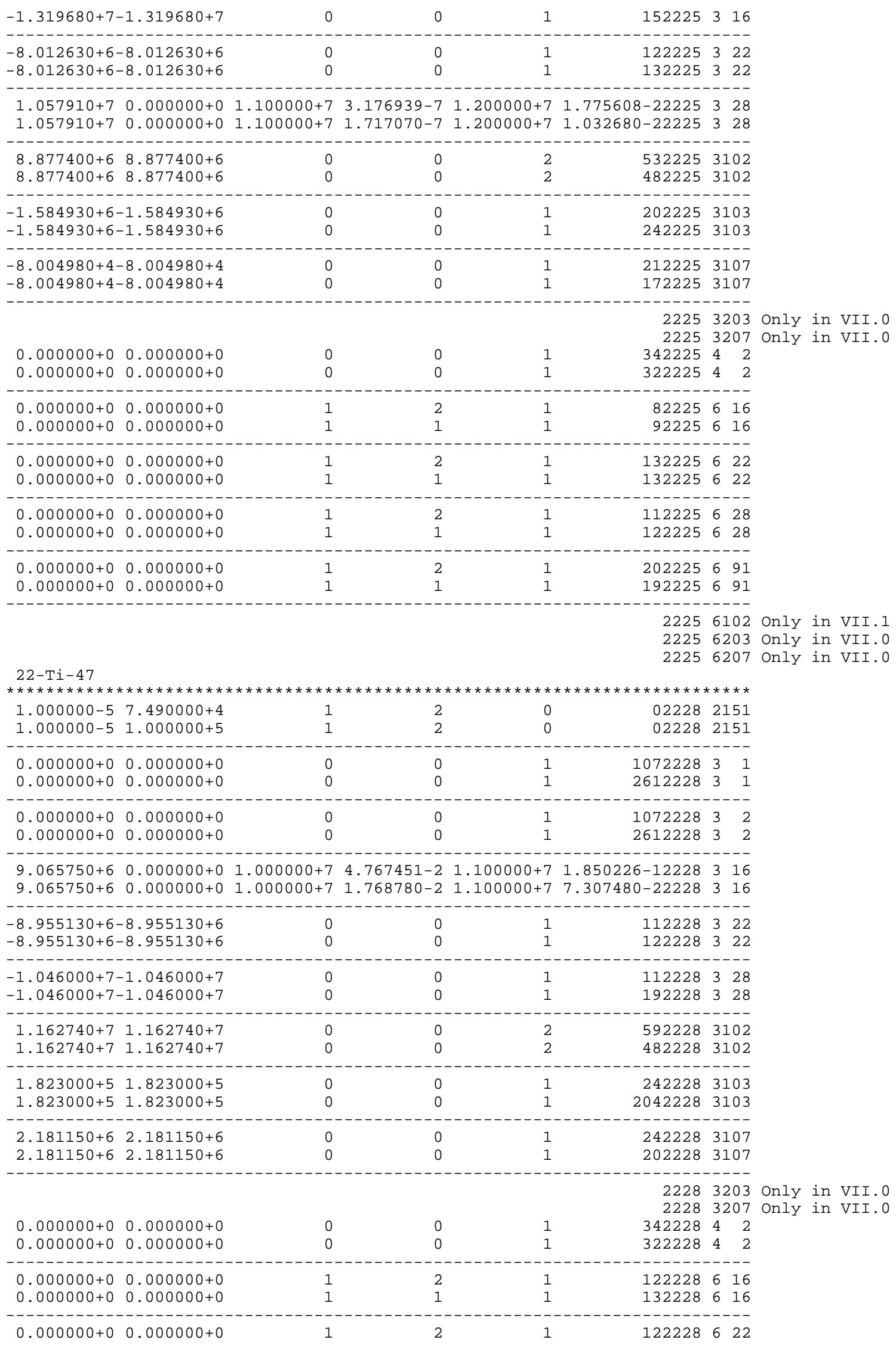




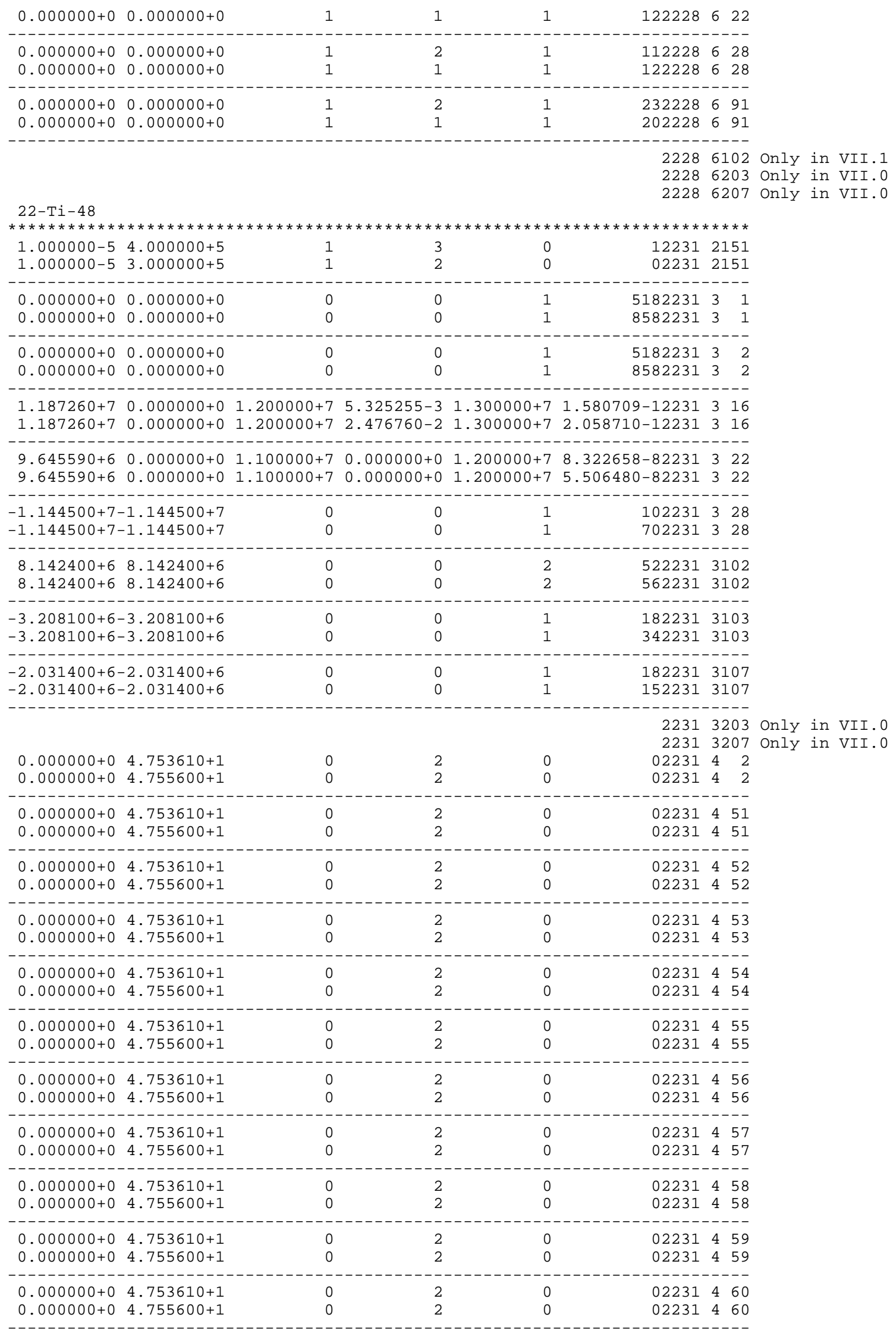




\begin{tabular}{|c|c|c|c|c|c|c|}
\hline $\begin{array}{l}\odot . \odot \odot \odot \odot \odot \odot+\odot ~ \\
\odot . \odot \odot \odot \odot \odot \odot+\odot\end{array}$ & $\begin{array}{l}4.753610+1 \\
4.755600+1\end{array}$ & $\begin{array}{l}\odot \\
\odot\end{array}$ & $\begin{array}{l}2 \\
2\end{array}$ & $\begin{array}{l}\odot \\
\odot\end{array}$ & $\begin{array}{lll}02231 & 4 & 61 \\
02231 & 4 & 61\end{array}$ & \\
\hline $\begin{array}{l}\odot . \odot \odot \odot \odot \odot \odot+\odot \\
\odot . \odot \odot \odot \odot \odot \odot+\odot\end{array}$ & $\begin{array}{l}4.753610+1 \\
4.755600+1\end{array}$ & $\stackrel{\odot}{\odot}$ & $\begin{array}{l}2 \\
2\end{array}$ & $\begin{array}{l}\odot \\
\odot\end{array}$ & $\begin{array}{lll}02231 & 4 & 62 \\
02231 & 4 & 62\end{array}$ & \\
\hline $\begin{array}{l}\odot .0 \odot \odot \odot \odot \odot+\odot \\
\odot .0 \odot \odot \odot \odot \odot+\odot\end{array}$ & $\begin{array}{l}4.753610+1 \\
4.755600+1\end{array}$ & $\begin{array}{l}\odot \\
\odot\end{array}$ & $\begin{array}{l}2 \\
2\end{array}$ & $\begin{array}{l}\odot \\
\odot\end{array}$ & $\begin{array}{lll}02231 & 4 & 63 \\
02231 & 4 & 63\end{array}$ & \\
\hline $\begin{array}{l}\odot . \odot \odot \odot \odot \odot \odot+\odot \\
\odot . \diamond \odot \odot \odot \odot \odot+\odot\end{array}$ & $\begin{array}{l}4.753610+1 \\
4.755600+1\end{array}$ & $\begin{array}{l}\odot \\
\odot\end{array}$ & $\begin{array}{l}2 \\
2\end{array}$ & $\begin{array}{l}\odot \\
\odot\end{array}$ & $\begin{array}{lll}02231 & 4 & 64 \\
02231 & 4 & 64\end{array}$ & \\
\hline $\begin{array}{l}\odot .0 \odot \odot \odot \odot \odot+\odot \\
\odot .0 \odot \odot \odot \odot \odot+\odot\end{array}$ & $\begin{array}{l}4.753610+1 \\
4.755600+1\end{array}$ & $\begin{array}{l}\odot \\
\odot\end{array}$ & $\begin{array}{l}2 \\
2\end{array}$ & $\begin{array}{l}\odot \\
\odot\end{array}$ & $\begin{array}{l}022314 \\
022314\end{array}$ & \\
\hline 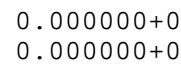 & $\begin{array}{l}4.753610+1 \\
4.755600+1\end{array}$ & $\begin{array}{l}\odot \\
\odot\end{array}$ & $\begin{array}{l}2 \\
2\end{array}$ & $\begin{array}{l}\odot \\
\odot\end{array}$ & $\begin{array}{lll}02231 & 4 & 66 \\
02231 & 4 & 66\end{array}$ & \\
\hline $\begin{array}{l}\odot .0 \odot \odot \odot \odot \odot+\odot \\
\odot .0 \odot \odot \odot \odot \odot+\odot\end{array}$ & $\begin{array}{l}4.753610+1 \\
4.755600+1\end{array}$ & $\begin{array}{l}\odot \\
\odot\end{array}$ & $\begin{array}{l}2 \\
2\end{array}$ & $\begin{array}{l}\odot \\
\odot\end{array}$ & $\begin{array}{lll}02231 & 4 & 67 \\
02231 & 4 & 67\end{array}$ & \\
\hline $\begin{array}{l}\odot .00 \odot \odot \odot \odot+\odot \\
\odot .0 \odot \odot \odot \odot \odot+\odot\end{array}$ & $\begin{array}{l}4.753610+1 \\
4.755600+1\end{array}$ & $\begin{array}{l}\ominus \\
\odot\end{array}$ & $\begin{array}{l}2 \\
2\end{array}$ & $\begin{array}{l}\odot \\
\odot\end{array}$ & $\begin{array}{lll}02231 & 4 & 68 \\
02231 & 4 & 68\end{array}$ & \\
\hline $\begin{array}{l}\odot .0 \odot \odot \odot \odot \odot+\odot \\
\odot . \odot \odot \odot \odot \odot \odot+\odot\end{array}$ & $\begin{array}{l}\odot .0 \odot \odot \odot \odot \odot+\odot \\
\odot . \diamond \odot \odot \odot \odot \odot+\odot\end{array}$ & $\begin{array}{l}1 \\
1\end{array}$ & $\begin{array}{l}2 \\
1\end{array}$ & $\begin{array}{l}1 \\
1\end{array}$ & $\begin{array}{lll}102231 & 6 & 16 \\
102231 & 6 & 16\end{array}$ & \\
\hline $\begin{array}{l}\odot .0 \odot \odot \odot \odot \odot+\odot \\
\odot .0 \odot \odot \odot \odot \odot+\odot\end{array}$ & $\begin{array}{l}\odot . \odot \odot \odot \odot \odot \odot+\odot \\
\odot . \odot \odot \odot \odot \odot \odot+\odot\end{array}$ & $\begin{array}{l}1 \\
1\end{array}$ & $\begin{array}{l}2 \\
1\end{array}$ & $\begin{array}{l}1 \\
1\end{array}$ & $\begin{array}{lll}122231 & 6 & 22 \\
112231 & 6 & 22\end{array}$ & \\
\hline $\begin{array}{l}0.0 \odot \odot \odot \odot \odot+\odot \\
\odot . \diamond \odot \odot \odot \odot \odot+\odot\end{array}$ & $\begin{array}{l}\odot . \diamond \odot \odot \odot \odot \odot+\odot \\
\odot . \diamond \odot \odot \odot \odot \odot+\odot\end{array}$ & $\begin{array}{l}- \\
1 \\
1\end{array}$ & $\begin{array}{l}2 \\
1\end{array}$ & 1 & $\begin{array}{lll}102231 & 6 & 28 \\
112231 & 6 & 28\end{array}$ & \\
\hline $\begin{array}{l}3.933030+6 \\
3.933000+6\end{array}$ & $\begin{array}{l}1.0 \odot \odot \odot \odot \odot+\odot \\
1 . \diamond \odot \odot \odot \odot \odot+\odot\end{array}$ & $\begin{array}{l}2.0 \odot \odot \odot \odot \odot+7 \\
2.0 \odot \odot \odot \odot \odot+7\end{array}$ & $\begin{array}{l}1.00 \odot \odot \odot \odot+\odot \\
1.0 \odot \odot \odot \odot \odot+\odot\end{array}$ & & $\begin{array}{lll}2231 & 6 & 91 \\
2231 & 6 & 91\end{array}$ & \\
\hline - - - - - - - - & - - - - - - - - - & ------ & - - - - - - & & 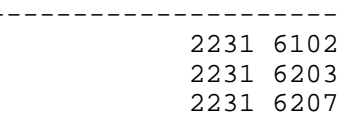 & $\begin{array}{l}\text { Only in VII.1 } \\
\text { Only in VII.0 } \\
\text { Only in VII.0 }\end{array}$ \\
\hline $\begin{array}{l}22-\mathrm{Ti}-49 \\
* * * * * * * * *\end{array}$ & $\star * * * * * * * *$ & $\star * * * *$ & $\star * * * * * * * * *$ & $* * * * * * *$ & 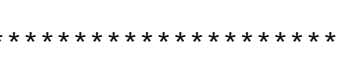 & \\
\hline $\begin{array}{l}1.000 \odot \odot \odot-5 \\
1.0 \odot \odot \odot \odot \odot-5\end{array}$ & $\begin{array}{l}2.41100 \odot+5 \\
1.800 \odot \odot \odot+5\end{array}$ & $\begin{array}{l}1 \\
1\end{array}$ & $\begin{array}{l}2 \\
2\end{array}$ & $\begin{array}{l}\odot \\
\odot\end{array}$ & $\begin{array}{ll}02234 & 2151 \\
02234 & 2151\end{array}$ & \\
\hline $\begin{array}{l}\odot .000 \odot \odot \odot+\odot \\
\odot .0 \odot \odot \odot \odot \odot+\odot\end{array}$ & $\begin{array}{l}\odot .0 \odot \odot \odot \odot \odot+\odot \\
\odot . \odot \odot \odot \odot \odot \odot+\odot\end{array}$ & $\begin{array}{l}\odot \\
\odot\end{array}$ & $\begin{array}{l}\ominus \\
\odot\end{array}$ & $\begin{array}{l}1 \\
2\end{array}$ & $\begin{array}{lll}1012234 & 3 & 1 \\
1012234 & 3 & 1\end{array}$ & \\
\hline $\begin{array}{l}\odot . \odot \odot \odot \odot \odot \odot+\odot \\
\odot . \diamond \odot \odot \odot \odot \odot+\odot\end{array}$ & $\begin{array}{l}\odot . \diamond \odot \odot \odot \odot \odot+\odot \\
\odot . \diamond \odot \odot \odot \odot \odot+\odot\end{array}$ & $\begin{array}{l}\odot \\
\odot\end{array}$ & $\begin{array}{l}\ominus \\
\odot\end{array}$ & $\begin{array}{l}1 \\
2\end{array}$ & $\begin{array}{lll}1012234 & 3 & 2 \\
1012234 & 3 & 2\end{array}$ & \\
\hline $\begin{array}{l}8.311200+6 \\
8.311200+6\end{array}$ & $\begin{array}{l}\odot .00000 \odot+\odot \\
\odot .000 \odot \odot \odot+\odot\end{array}$ & $\begin{array}{l}9.000000+6 \\
9.000000+6\end{array}$ & $\begin{array}{l}2.522955-2 \\
6.723230-2\end{array}$ & $\begin{array}{l}1.000000+7 \\
1.0000000+7\end{array}$ & $\begin{array}{lll}1.304847-12234 & 3 & 16 \\
1.794180-12234 & 3 & 16\end{array}$ & \\
\hline $\begin{array}{l}-1.017590+7 \\
-1.017590+7\end{array}$ & $\begin{array}{l}-1.017590+7 \\
-1.017590+7\end{array}$ & $\stackrel{\odot}{\odot}$ & $\begin{array}{l}0 \\
0\end{array}$ & $\begin{array}{l}1 \\
1\end{array}$ & 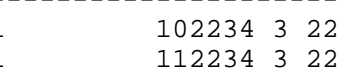 & \\
\hline $\begin{array}{l}1.158540+7 \\
1.158540+7\end{array}$ & $\begin{array}{l}0.000000+\odot \\
0.0000 \odot \odot+\odot\end{array}$ & $\begin{array}{l}1.200 \odot \odot \odot+7 \\
1.20 \odot \odot \odot \odot+7\end{array}$ & $\begin{array}{ll}7 & 0.00 \odot \odot \odot \odot+\odot ~ \\
7 & 3.06662-10\end{array}$ & $\begin{array}{l}1.30 \odot \odot \odot \odot+7 \\
1.300 \odot \odot \odot+7\end{array}$ & $\begin{array}{lll}1.299876-52234 & 3 & 28 \\
2.153490-52234 & 3 & 28\end{array}$ & \\
\hline $\begin{array}{l}1.093940+7 \\
1.093940+7\end{array}$ & $\begin{array}{l}1.093940+7 \\
1.093940+7\end{array}$ & $\begin{array}{l}- \\
\odot \\
\odot\end{array}$ & $\begin{array}{l}\odot \\
\ominus\end{array}$ & 2 & $\begin{array}{ll}562234 & 3102 \\
532234 & 3102\end{array}$ & \\
\hline$-1.222830+6$ & $-1.222830+6$ & $\begin{array}{l}\odot \\
\odot\end{array}$ & 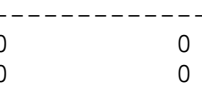 & $\begin{array}{l}- \\
1 \\
1\end{array}$ & 2022343103 & \\
\hline $\begin{array}{l}2.274500+5 \\
2.274500+5\end{array}$ & $\begin{array}{l}2.274500+5 \\
2.274500+5\end{array}$ & $\begin{array}{l}\odot \\
\odot\end{array}$ & $\begin{array}{l}\odot \\
\odot\end{array}$ & $\begin{array}{l}1 \\
1\end{array}$ & $\begin{array}{ll}212234 & 3107 \\
182234 & 3107\end{array}$ & \\
\hline & & & & & $\begin{array}{r}22343203 \\
22343207\end{array}$ & $\begin{array}{l}\text { Only in VII.0 } \\
\text { Only in VII.e }\end{array}$ \\
\hline $\begin{array}{l}\odot . \odot \odot \odot \odot \odot \odot+\odot \\
\odot . \odot \odot \odot \odot \odot \odot+\odot\end{array}$ & $\begin{array}{l}\odot .0 \odot \odot \odot \odot \odot+\odot \\
\odot .00 \odot \odot \odot \odot+\odot\end{array}$ & $\begin{array}{l}\odot \\
\odot\end{array}$ & $\begin{array}{l}\odot \\
\odot\end{array}$ & $\begin{array}{l}1 \\
1\end{array}$ & $\begin{array}{lll}342234 & 4 & 2 \\
322234 & 4 & 2\end{array}$ & \\
\hline $\begin{array}{l}\odot .00000 \odot+\odot \\
\odot .0 \odot \odot \odot \odot \odot+\odot\end{array}$ & $\begin{array}{l}\odot .000000+\odot \\
\odot .000000+\odot\end{array}$ & $\begin{array}{l}1 \\
1\end{array}$ & $\begin{array}{l}2 \\
1\end{array}$ & $\begin{array}{l}1 \\
1\end{array}$ & $\begin{array}{lll}132234 & 6 & 16 \\
142234 & 6 & 16\end{array}$ & \\
\hline
\end{tabular}




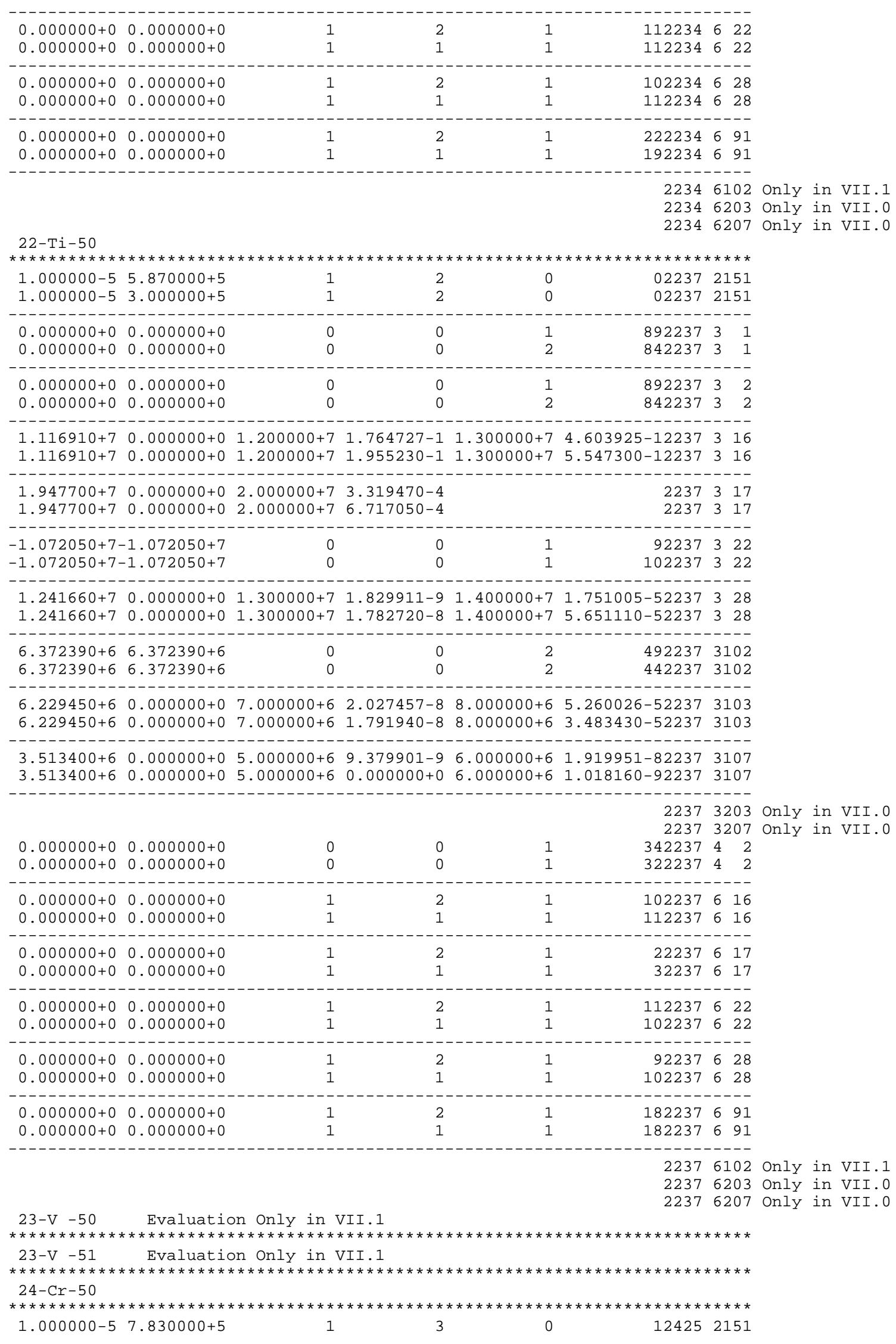




\begin{tabular}{|c|c|c|c|c|c|}
\hline $1.0000 \odot \odot-5 \quad 7.920000+5$ & 1 & 3 & $\odot$ & 12425 & \\
\hline$\odot .000 \odot \odot \odot+\odot \quad \odot .00 \odot \odot \odot \odot+\odot$ & $\odot$ & $\odot$ & 1 & 13702425 & 3 \\
\hline $0.000000+\odot \quad 0.000000+0$ & $\odot$ & $\odot$ & 1 & 23762425 & 3 \\
\hline$\odot . \odot \odot \odot \odot \odot \odot+\odot \quad \odot . \odot \odot \odot \odot \odot \odot+\odot$ & $\odot$ & $\odot$ & 1 & 13702425 & 3 \\
\hline$\odot . \odot \odot \odot \odot \odot \odot+\odot \quad \odot . \odot \odot \odot \odot \odot \odot+\odot$ & $\odot$ & $\odot$ & 1 & 23762425 & 3 \\
\hline
\end{tabular}

$1.326254+7 \quad 0.000000+\odot \quad 1.368000+7 \quad 1.730000-3 \quad 1.378000+7 \quad 4.210000-324253 \quad 16$ $1.326300+7 \quad 0.0000000+\odot \quad 1.368000+7 \quad 1.730000-3 \quad 1.378000+7 \quad 4.210000-32425316$

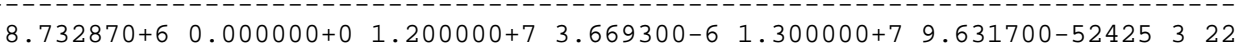
$8.732900+6 \quad 0.000000+\odot \quad 1.200000+7 \quad 3.669300-6 \quad 1.300000+7 \quad 9.631700-52425322$

$9.783671+60.000000+\Theta \quad 1.100000+7 \quad 7.727000-4 \quad 1.200000+7 \quad 6.708700-22425328$ $9.783700+6 \quad 0.000000+\odot \quad 1.100000+7 \quad 7.727000-4 \quad 1.200000+7 \quad 6.708700-22425328$

(1)

$1.918987+6 \quad 0.0000000+\odot \quad 2.000000+6 \quad 1.467900-2 \quad 2.2500000+6 \quad 5.297700-22425352$ $1.919000+6 \quad 0.000000+\odot \quad 2.0000000+6 \quad 1.467900-2 \quad 2.2500000+6 \quad 5.297700-22425352$

$2.984071+6 \quad 0.000000+03.000000+67.559700-2 \quad 3.500000+6 \quad 1.753400-12425353$ $2.984100+6 \quad 0.000000+0 \quad 3.000000+6 \quad 7.559700-2 \quad 3.500000+6 \quad 1.753400-12425353$

$3.227897+6 \quad 0.0000000+\odot \quad 3.5000000+6 \quad 9.140000-4 \quad 4.0000000+6 \quad 6.573400-32425355$ $3.227900+6 \quad 0.000000+\odot \quad 3.500000+6 \quad 9.140000-4 \quad 4.000000+6 \quad 6.573400-32425355$ $\begin{array}{lllllll}1.000000-5 & 0.000000+\odot & 3.250000+5 & 0.0000000+\odot & 3.250000+5 & 4.600000-32425 & 3102\end{array}$

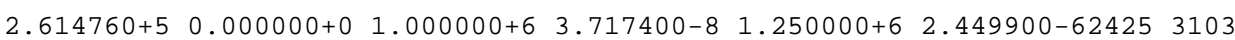
$2.614800+5 \quad 0.000000+\odot \quad 1.000000+6 \quad 3.717400-8 \quad 1.250000+6 \quad 2.449900-624253103$

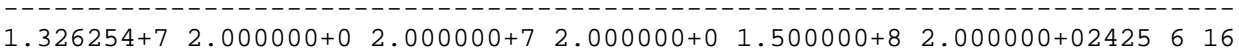
$\begin{array}{llllllllll}1.326300+7 & 2.000000++\odot & 2.0000000+7 & 2.0000000+\odot & 1.5000000+8 & 2.0000000+02425 & 6 & 16\end{array}$

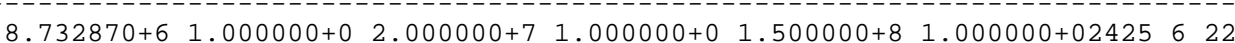
$8.732900+6 \quad 1.000000+\odot \quad 2.000000+7 \quad 1.0000000+0 \quad 1.500000+8 \quad 1.0000000+02425622$

$9.783671+61.000000+02.000000+71.000000+01500000+81.000000+02425628$ $9.783700+6 \quad 1.000000+0 \quad 2.000000+7 \quad 1.0000000+0 \quad 1.500000+8 \quad 1.000000+02425628$

_. . - .

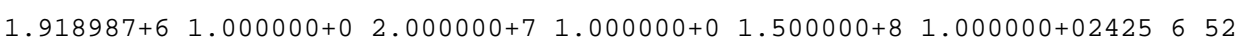

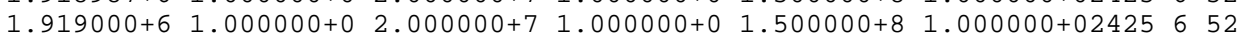

$2.984071+61.000000+02.000000+71.000000+01.500000+81.000000+02425$

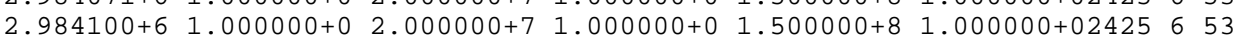

(2.

$\begin{array}{llllllll}3.227897+6 & 1.000000+0 & 2.000000+7 & 1.000000+0 & 1.500000+8 & 1.000000+02425 & 655\end{array}$

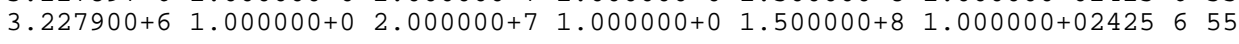

$2.614760+5 \quad 1.000000+\odot \quad 2.000000+7 \quad 1.000000+\odot \quad 1.500000+8 \quad 1.000000+024256103$ $2.614800+5 \quad 1.000000+0 \quad 2.000000+7 \quad 1.000000+01.500000+8 \quad 1.000000+024256103$

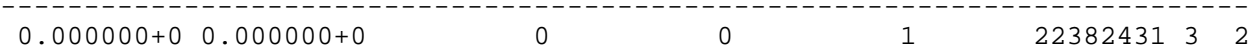

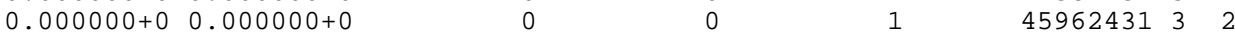

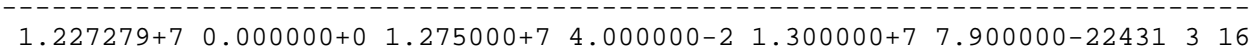
$1.227300+7 \quad 0.000000+0 \quad 1.275000+7 \quad 4.000000-2 \quad 1.300000+7 \quad 7.900000-22431316$

$2.821754+6 \quad 0.00000 \odot+\odot \quad 3.000000+6 \quad 3.489500-2 \quad 3.500000+6 \quad 8.986300-22431354$ $2.821800+6 \quad 0.000000+0 \quad 3.000000+6 \quad 3.489500-2 \quad 3.500000+6 \quad 8.986300-22431354$

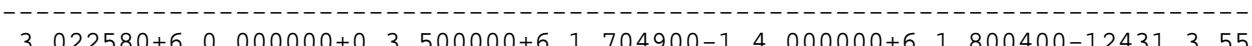
$3.022600+6 \quad 0.000000+\odot \quad 3.500000+6 \quad 1.704900-1 \quad 4.0000000+6 \quad 1.800400-12431355$

3. $3.174473+6 \quad 0.000000+\odot \quad 3.5000000+6 \quad 1.739000-3 \quad 4.000000+6 \quad 7.636700-32431356$ $3.174500+6 \quad 0.000000+0 \quad 3.500000+6 \quad 1.739000-3 \quad 4.000000+6 \quad 7.636700-32431356$ 


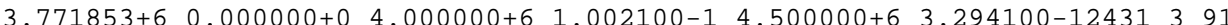

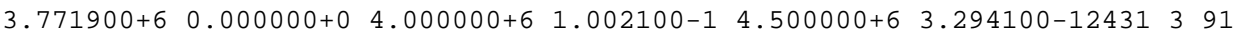

$\begin{array}{rrrrrr}7.939230+6 & 7.939230+6 & 0 & 0 & 1 & 1924313102\end{array}$

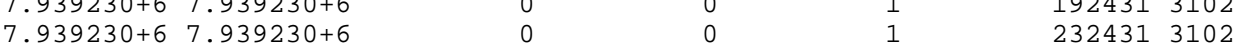

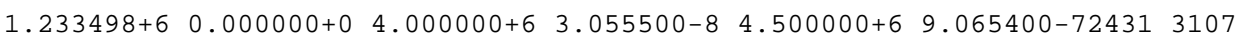
$1.233500+6 \quad 0.000000+\odot \quad 4.0000000+6 \quad 3.055500-8 \quad 4.5000000+6 \quad 9.065400-724313107$

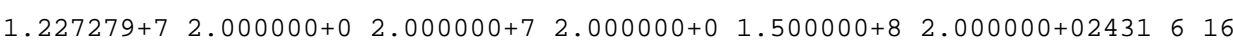
$\begin{array}{lllllllllll}1.227300+7 & 2.000000+0 & 2.000000+7 & 2.000000+0 & 1.500000+8 & 2.000000+02431 & 6 & 16\end{array}$

$2.821754+6 \quad 1.000 \odot \odot \odot+\odot \quad 2.000 \odot \odot \odot+7 \quad 1.000 \odot \odot \odot+\odot \quad 1.50 \odot \odot \odot \odot+8 \quad 1.00 \odot \odot \odot \odot+02431654$ $\begin{array}{llllllll}2.821800+6 & 1.000000+\odot & 2.000000+7 & 1.000000+\odot & 1.500000+8 & 1.0000000+02431 & 6 & 54\end{array}$

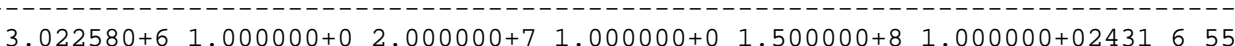
$3.022600+6 \quad 1.0000000+\odot \quad 2.0000000+7 \quad 1.0000000+\odot \quad 1.500000+8 \quad 1.000000+02431655$

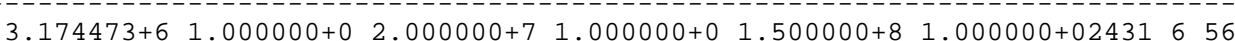
$3.174500+6 \quad 1.000000+0 \quad 2.000000+7 \quad 1.0000000+0 \quad 1.500000+8 \quad 1.0000000+02431656$

$3.771853+61.000000+022.000000+71.000000+01500000+81.000000+02431691$

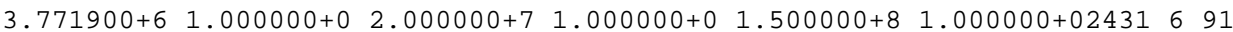

1.233498+6 $1.000000+0200000$

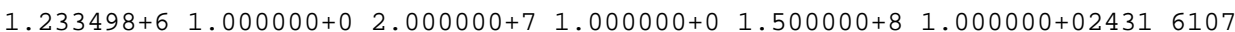
$\begin{array}{lllllllll}1.233500+6 & 1.000000+\odot & 2.0000000+7 & 1.0000000+\odot & 1.500000+8 & 1.000000+02431 & 6107\end{array}$

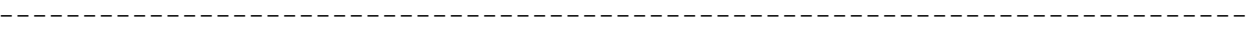

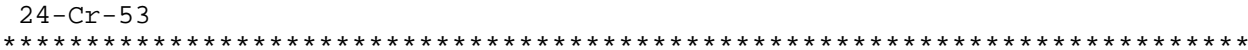

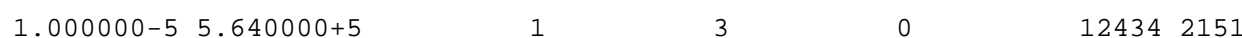

$3=0$

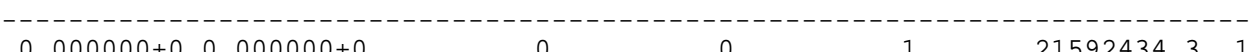

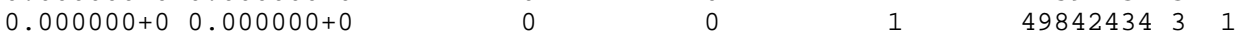

$\odot . \odot \odot \odot \odot \odot \odot+\odot \odot ., \odot \odot \odot \odot \odot \ominus+\Theta$

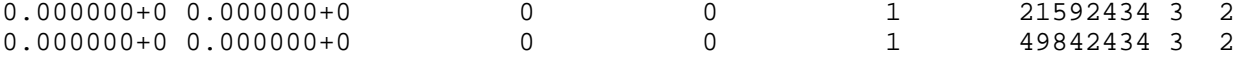

$5.7474575000000+08000000+5128$ $5.747500+5 \quad 0.000000+0 \quad 8.000000+5 \quad 1.887400-1 \quad 9.000000+5 \quad 2.202000-1243434$

$8.091279+60.000000+08.500000+62.209300-20.000000+67.086500-22434316$ $8.091300+6 \quad 0.000000+\odot \quad 8.500000+6 \quad 2.209300-2 \quad 9.000000+6 \quad 7.986500-22434316$

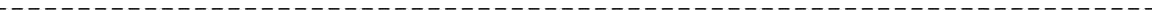
$5.747457+5 \quad 0.000000+\odot \quad 8.000000+5 \quad 1.887400-1 \quad 9.0000000+5 \quad 2.202000-12434351$

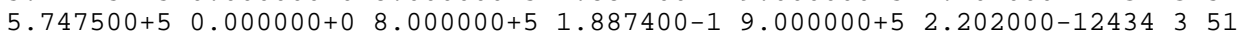
$1.025167+6 \quad 0.00000 \odot+\odot \quad 1.250000+6 \quad 3.308700-1 \quad 1.500000+6 \quad 3.732200-12434352$ $1.025200+6 \quad 0.000000+\odot \quad 1.250000+6 \quad 3.308700-1 \quad 1.500000+6 \quad 3.732200-12434352$

$1.314578+60.000000+\Theta 1.500000+6 \quad 2.245800-1 \quad 1.750000+6 \quad 2.452500-12434353$ $\begin{array}{llllllll}1.314600+6 & 0.000000+0 & 1.500000+6 & 2.245800-1 & 1.750000+6 & 2.452500-12434 & 3 & 53\end{array}$

$1.56628+60.000000+01.750000+61.419600-12000000+61.776100-12434354$ $1.566300+6 \quad 0.000000+\odot \quad 1.750000+6 \quad 1.419600-1 \quad 2.000000+6 \quad 1.776100-12434354$

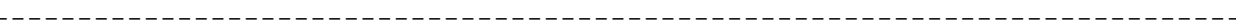

$2.213383+6 \quad 0.000000+0 \quad 2.250000+6 \quad 5.217400-3 \quad 2.500000+6 \quad 1.492300-22434356$ $2.213400+6 \quad 0.000000+0 \quad 2.250000+6 \quad 5.217400-3 \quad 2.500000+6 \quad 1.492300-22434356$

$2.720871+60.000000+02.750000+61.508000-23.000000+62.672900-22434361$

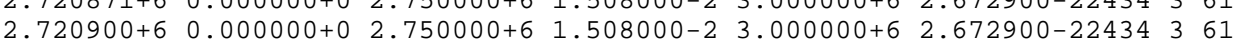

2.757557600

$2.757557+6 \quad 0.000000+0 \quad 3.000000+6 \quad 9.002700-3 \quad 3.500000+6 \quad 2.059200-22434362$ $2.757600+6 \quad 0.00000 \odot+\odot \quad 3.0000 \odot \odot \odot+6 \quad 9.002700-3 \quad 3.5000 \odot \odot \odot+6 \quad 2.059200-22434362$ $2.758576+6 \quad 0.000000+\odot \quad 3.000000+6 \quad 4.534000-2 \quad 3.500000+6 \quad 5.911100-22434363$ $2.758600+6 \quad 0.000000+\odot \quad 3.000000+6 \quad 4.534000-2 \quad 3.500000+6 \quad 5.911100-22434363$

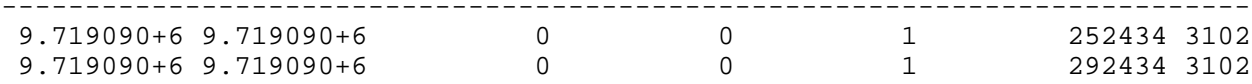

$2.690299+6 \quad 0.0000 \odot \odot+\odot \quad 3.500000+6 \quad 1.794900-7 \quad 4.000 \odot \odot \odot+6 \quad 2.886900-524343103$ $2.690300+6 \quad 0.000000+0 \quad 3.500000+6 \quad 1.794900-7 \quad 4.000000+6 \quad 2.886900-524343103$ 
$\begin{array}{lllllllllll}8.091279+6 & 2.000000+0 & 2.0000000+7 & 2.000000+0 & 1.500000+8 & 2.000000+02434 & 6 & 16\end{array}$ $8.091300+6 \quad 2.000000+0 \quad 2.000000+7 \quad 2.0000000++0 \quad 1.5000000+8 \quad 2.0000000++02434616$

$5.747457+5 \quad 1.000000+\odot \quad 2.000000+7 \quad 1.000000++\odot \quad 1.5000000+8 \quad 1.000000+02434 \quad 651$ $5.747500+5 \quad 1.000000+0 \quad 2.000000+7 \quad 1.0000000+0 \quad 1.500000+8 \quad 1.0000000+02434651$

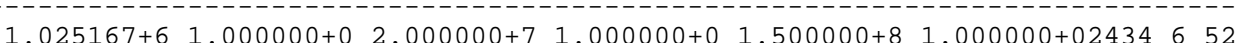

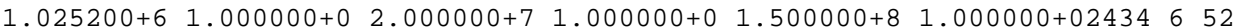

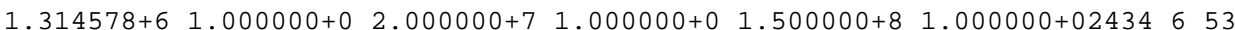

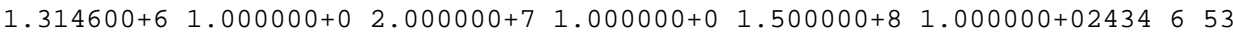

$1.566284+61.000000+02.000000+7 \quad 1.000000+0 \quad 1.500000+81.000000+02434654$

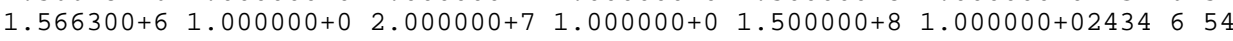

$2.213383+6 \quad 1.000000+0 \quad 2.000000+7 \quad 1.0000000+0 \quad 1.500000+8 \quad 1.000000+02434656$ $2.213400+6 \quad 1.000000+0 \quad 2.000000+7 \quad 1.000000+01.500000+8 \quad 1.000000+02434656$ $2.720871+6 \quad 1.000000+0 \quad 2.000000+7 \quad 1.000000+0 \quad 1.5000000+8 \quad 1.0000000+02434661$

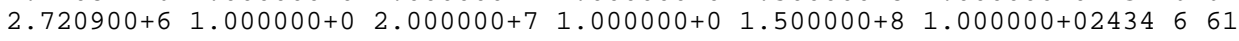
0
0 $2.757557+6 \quad 1.000000+\odot \quad 2.000000+7 \quad 1.000000++\odot \quad 1.500000+8 \quad 1.000000+02434662$ $2.757600+6 \quad 1.000000+0 \quad 2.000000+7 \quad 1.000000+0 \quad 1.500000+8 \quad 1.000000+02434662$

$2.758576+6 \quad 1.000000+\odot \quad 2.000000+7 \quad 1.000000+\odot \quad 1.500000+8 \quad 1.000000+02434663$ $2.758600+6 \quad 1.0000000+0 \quad 2.000000+7 \quad 1.0000000+0 \quad 1.500000+8 \quad 1.000000+02434663$

$2.690299+6 \quad 1.000 \odot \odot \odot+\odot \quad 2.000 \odot \odot \odot+7 \quad 1.000 \odot \odot \odot+\odot \quad 1.50 \odot \odot \odot \odot+8 \quad 1.00 \odot \odot \odot \odot+\odot 24346103$ $2.690300+6 \quad 1.0000000+0 \quad 2.000000+7 \quad 1.000000+0 \quad 1.500000+8 \quad 1.000000+024346103$

(24 $24-\mathrm{Cr}-54$

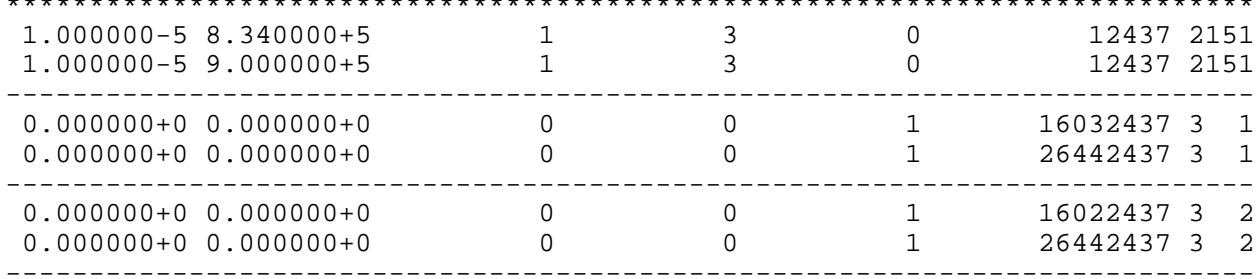

$2.668994+6 \quad 0.000000+\Theta \quad 2.7500000+6 \quad 1.290400-1 \quad 3.000000+6 \quad 1.927700-12437 \quad 3 \quad 53$ $2.669000+6 \quad 0.000000+0 \quad 2.750000+6 \quad 1.290400-1 \quad 3.000000+6 \quad 1.927700-12437353$

(-1

$3.131484+6 \quad 0.000000+0 \quad 3.5000000+61.368100-144.000000+6 \quad 4.029500-12437391$ $3.131500+6 \quad 0.000000+\odot \quad 3.5000 \odot \odot \odot+6 \quad 1.368100-1 \quad 4.0000 \odot \odot+6 \quad 4.029500-12437391$

$\begin{array}{llcccc}6.246350+6 & 6.246350+6 & 0 & \odot & 1 & 7924373102 \\ 6.246350+6 & 6.246350+6 & 0 & 0 & 1 & 1824373102\end{array}$

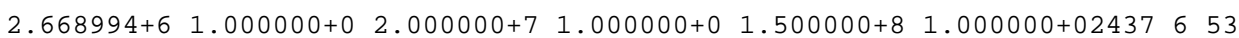

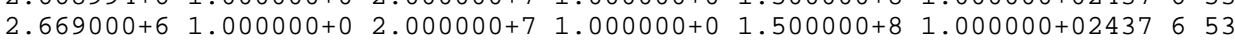
(1.6.

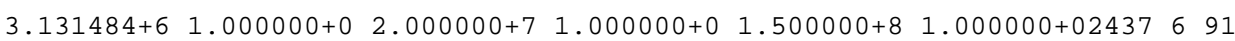
$\begin{array}{lllllllll}3.131500+6 & 1.000000+0 & 2.0000000+7 & 1.000000+0 & 1.500000+8 & 1.000000+02437 & 6 & 91\end{array}$

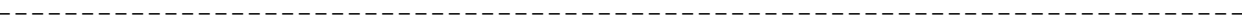
$25-\mathrm{Mn}-55$

\begin{tabular}{|c|c|c|c|c|c|c|}
\hline $\begin{array}{ll}2.50550 \odot+4 & 1.000 \odot \odot \odot+\odot \\
2.505500+4 & 1.000 \odot \odot \odot+\odot\end{array}$ & $\begin{array}{l}\odot \\
\odot\end{array}$ & $\begin{array}{l}\odot \\
\odot\end{array}$ & $\begin{array}{l}2 \\
1\end{array}$ & $\begin{array}{l}02525 \\
02525\end{array}$ & $\begin{array}{l}2151 \\
2151\end{array}$ & \\
\hline 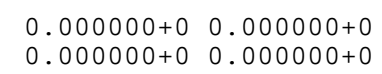 & $\begin{array}{l}\odot \\
\odot\end{array}$ & $\begin{array}{l}\odot \\
\odot\end{array}$ & $\begin{array}{l}1 \\
1\end{array}$ & $\begin{array}{l}2242525 \\
7322525\end{array}$ & $\begin{array}{ll}3 & 1 \\
3 & 1\end{array}$ & \\
\hline 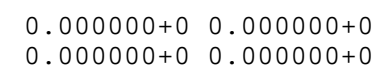 & $\begin{array}{l}\odot \\
\odot\end{array}$ & $\begin{array}{l}\odot \\
\odot\end{array}$ & $\begin{array}{l}1 \\
1\end{array}$ & $\begin{array}{r}842525 \\
7322525\end{array}$ & $\begin{array}{ll}3 & 2 \\
3 & 2\end{array}$ & \\
\hline $\begin{array}{l}\odot .000 \odot \odot \odot+\odot-1.25950 \odot+5 \\
\odot .000 \odot \odot \odot+\odot-1.2600 \odot \odot+5\end{array}$ & $\begin{array}{l}\odot \\
\odot\end{array}$ & $\begin{array}{l}0 \\
\odot\end{array}$ & $\begin{array}{l}1 \\
1\end{array}$ & $\begin{array}{r}2525 \\
1212525 \\
672525\end{array}$ & $\begin{array}{ll}3 & 3 \\
3 & 4 \\
3 & 4\end{array}$ & Only in VII.O \\
\hline $\begin{array}{l}-1.022600+7-1.02260 \odot+7 \\
-1.022700+7-1.022700+7\end{array}$ & $\begin{array}{l}0 \\
0\end{array}$ & $\begin{array}{l}\odot \\
\odot\end{array}$ & $\begin{array}{l}1 \\
1\end{array}$ & $\begin{array}{r}2525 \\
382525 \\
122525\end{array}$ & $\begin{array}{rr}3 & 5 \\
3 & 16 \\
3 & 16\end{array}$ & Only in VII.1 \\
\hline
\end{tabular}




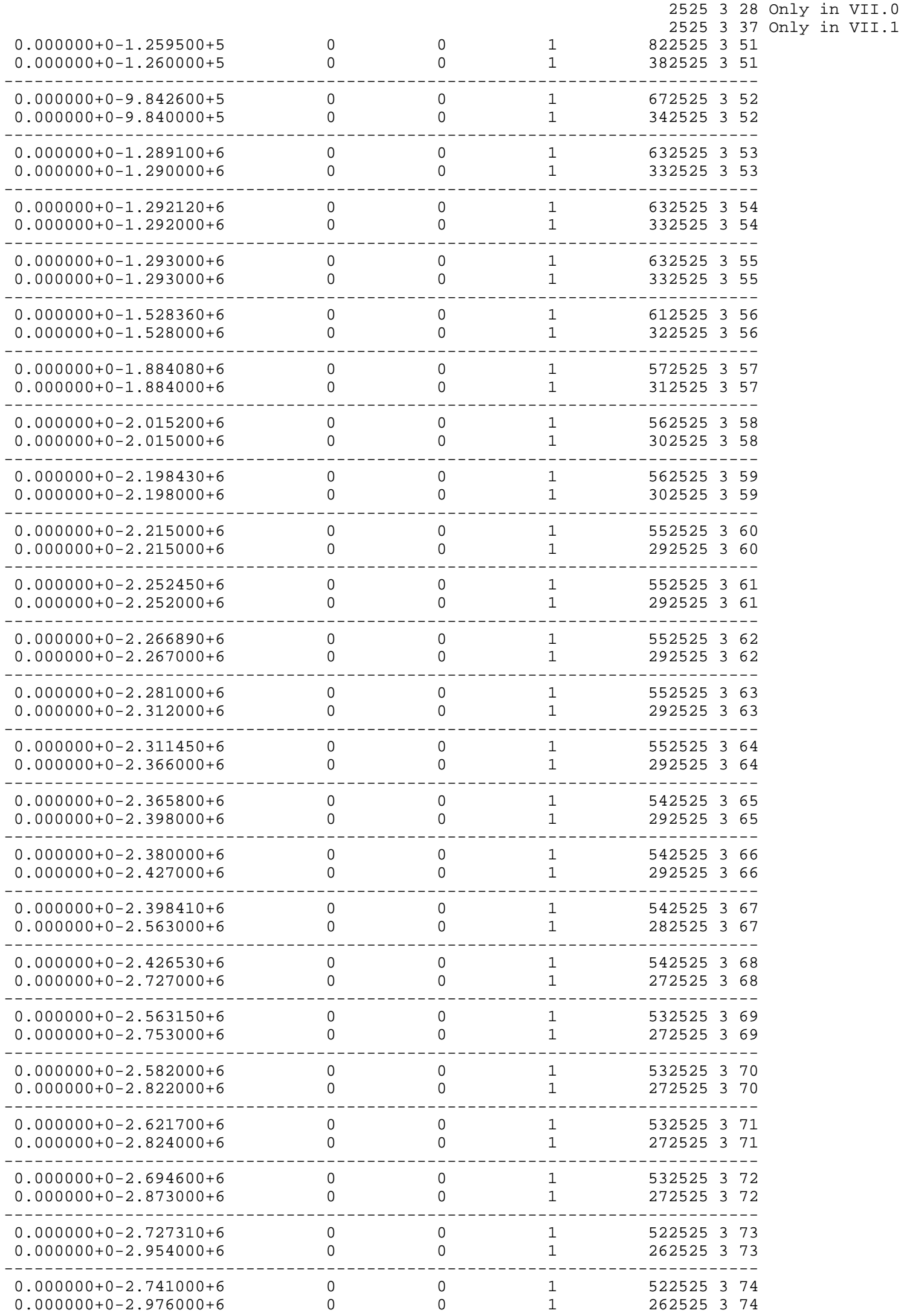




\begin{tabular}{|c|c|c|c|c|}
\hline 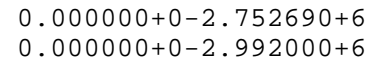 & 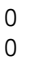 & $\begin{array}{l}\odot \\
\odot\end{array}$ & $\begin{array}{l}1 \\
1\end{array}$ & $\begin{array}{lll}522525 & 3 & 7 \\
262525 & 3 & 7\end{array}$ \\
\hline \multicolumn{5}{|c|}{ 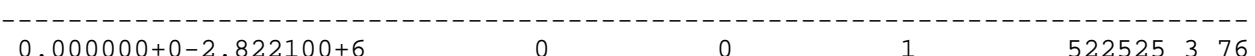 } \\
\hline$\odot .000000+0-2.822100+6$ & $\odot$ & $\odot$ & 1 & 52252537 \\
\hline $0.000000+0-3.006000+6$ & $\odot$ & $\odot$ & 1 & 2625253 \\
\hline \multirow{2}{*}{\multicolumn{5}{|c|}{$\begin{array}{ccccc}0.000000+0-2.823650+6 & 0 & 0 & 1 & 52252537\end{array}$}} \\
\hline & & & & \\
\hline$\odot .000000+\odot-3.036000+6$ & $\odot$ & $\odot$ & 1 & 2625253 \\
\hline \multirow{2}{*}{\multicolumn{5}{|c|}{$\begin{array}{ccccc}0.000000+0-2.828440+6 & 0 & 0 & 1 & 0\end{array}$}} \\
\hline & & & & \\
\hline$\odot .000 \odot \odot \odot+\odot-3.03800 \odot+6$ & $\odot$ & $\odot$ & 1 & 2625253 \\
\hline \multicolumn{5}{|l|}{ - - - - - - - - - - - - - } \\
\hline$\odot .000 \odot \odot \odot+\odot-2.873280+6$ & $\odot$ & 0 & 1 & 5125253 \\
\hline$\odot .0000 \odot \odot+\odot-3.0400 \odot \odot+6$ & $\odot$ & $\odot$ & 1 & 2625253 \\
\hline
\end{tabular}
2525381 only in VII.1 2525382 only in VII.1 2525383 Only in VII.1 2525384 Only in VII.1 2525385 only in VII.1 2525386 only in VII.1 2525387 only in VII.1 2525388 only in VII.1 2525389 0nly in VII.1

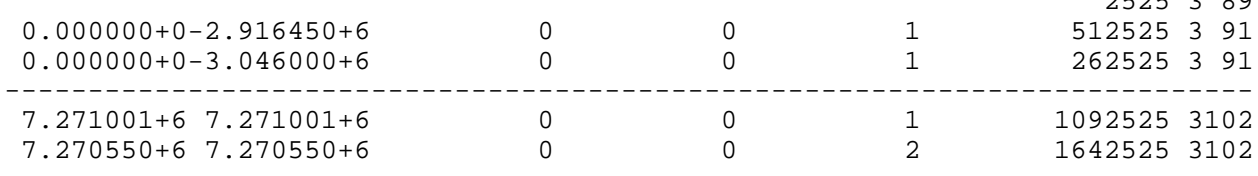
25253104 only in VII.0 25253105 only in VII.0 25253106 only in VII.0 25253107 only in VII.0 25253600 only in VII.1 25253601 Only in VII.1 25253602 only in VII.1 25253603 only in VII.1 25253604 only in VII.1 25253605 only in VII.1 25253606 only in VII.1 25253607 only in VII.1 25253608 Only in VII.1 25253609 only in VII.1 25253610 only in VII.1 25253611 only in VII.1 25253612 only in VII.1 25253613 only in VII.1 25253614 Only in VII.1 25253615 only in VII.1 25253616 only in VII.1 25253617 only in VII.1 25253618 only in VII.1 25253619 only in VII.1 25253620 only in VII.1 25253621 only in VII.1 25253622 only in VII.1 25253623 only in VII.1 25253624 Only in VII.1 25253625 only in VII.1 25253626 only in VII.1 25253627 only in VII.1 25253628 only in VII.1 25253629 only in VII.1 25253630 Only in VII.1 25253631 only in VII.1 25253632 only in VII.1 25253649 only in VII.1 25253800 only in VII.1 25253801 Only in VII.1 25253802 only in VII.1 25253803 Only in VII.1 


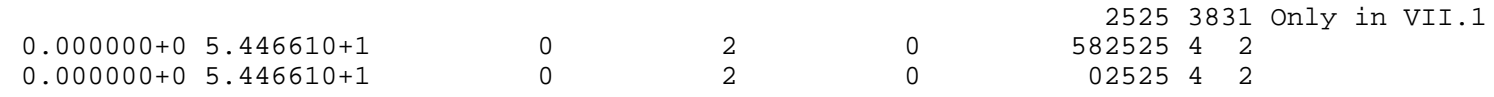
0.

2525451 only in VII.1 2525452 only in VII.1 2525453 Only in VII.1 2525454 only in VII.1 2525455 only in VII.1 2525456 only in VII.1 2525457 only in VII.1 2525458 only in VII.1 2525459 only in VII.1 2525460 only in VII.1 2525461 only in VII.1 2525462 only in VII.1 2525463 only in VII.1 2525464 only in VII.1 2525465 only in VII.1 2525466 only in VII.1 2525467 Only in VII.1 2525468 only in VII.1 2525469 Only in VII.1 2525470 Only in VII.1 2525471 Only in VII.1 2525472 only in VII.1 2525473 only in VII.1 2525474 Only in VII.1 2525475 Only in VII.1 2525476 only in VII.1 2525477 only in VII.1 2525478 only in VII.1 2525479 only in VII.1 2525480 Only in VII.1 2525481 only in VII.1 2525482 Only in VII.1 2525483 only in VII.1 2525484 only in VII.1 2525485 only in VII.1 2525486 only in VII.1 2525487 only in VII.1 2525488 Only in VII.1 2525489 only in VII.1 25254600 only in VII.1 25254601 only in VII.1 25254602 only in VII.1 25254603 Only in VII.1 25254604 only in VII.1 
$2.969996+6 \quad 1.000000+0 \quad 6.000000+7 \quad 1.000000+0$ $3.101920+6 \quad 1.000000+0 \quad 2.000000+7 \quad 1.000000+0$
2525658 only in VII.0 2525659 only in VII.0 2525660 only in VII.0 2525661 only in VII.0 2525662 only in VII.0 2525663 only in VII.0 2525664 only in VII.0 2525665 only in VII.0 2525666 only in VII.0 2525667 only in VII.0 2525668 only in VII.0 2525669 only in VII.0 2525670 only in VII.0 2525671 only in VII.0 2525672 only in VII.0 2525673 only in VII.0 2525674 only in VII.0 2525675 only in VII.0 2525676 only in VII.0 2525677 only in VII.0 2525678 only in VII.0 2525679 only in VII.0 $\begin{array}{lll}2525 & 6 & 91\end{array}$ $2525 \quad 6 \quad 91$ - - - - - - - .

25256102 0nly in VII.1 25256103 only in VII.0 25256107 only in VII.0 25256649 only in VII.1

\section{$26-\mathrm{Fe}-54$}

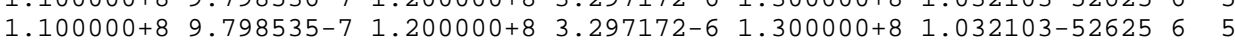

$26-\mathrm{Fe}-56$

****************************************************************************

$9.427423+5$ 9.717345-7 $1.099866+6 \quad 9.837624-7 \quad 1.256990+6 \quad 1.005964-6263166$

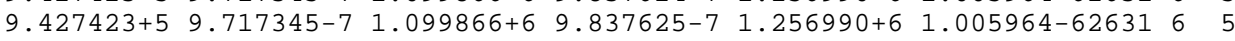

$26-\mathrm{Fe}-57$

****************************************************************************

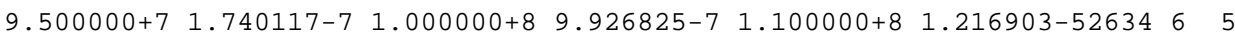
$\begin{array}{llllllll}9.500000+7 & 1.740117-7 & 1.0000000+8 & 9.926826-7 & 1.100000+8 & 1.216903-52634 & 6 & 5\end{array}$

- - - - - - -

$26-\mathrm{Fe}-58$

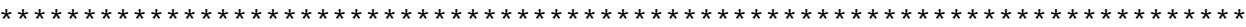

$27-\mathrm{Co}-58$

$27-\mathrm{Co}-58 \mathrm{~m}$

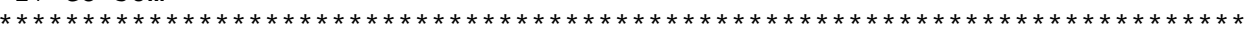

$27-$ Co -59

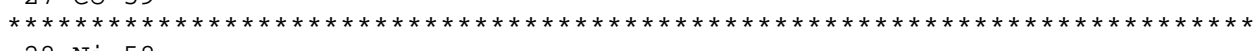

$28-\mathrm{Ni}-58$

$* * * * * * * * * * * * * * * * * * * * * * * * * * * * * * * * * * * * * * * * * * * * * * * * * * * * * * * * * * * * * * * * * * * * * * * * * * *$

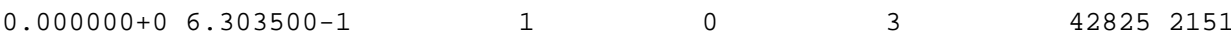

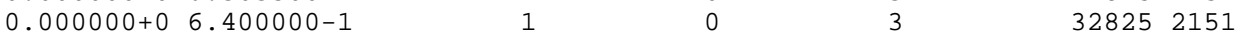

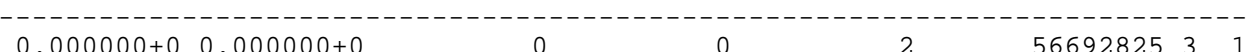

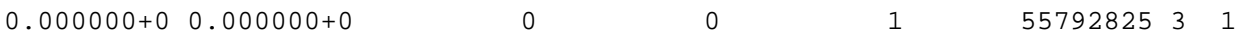

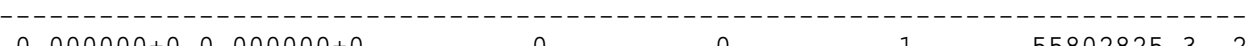

$\begin{array}{cccc}0 & 0 & 1 & 55802\end{array}$

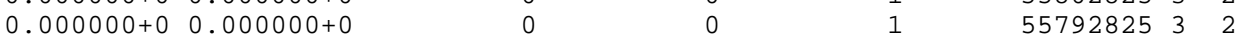

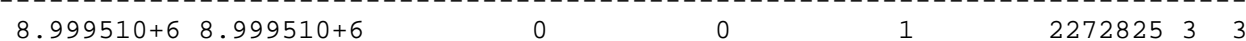

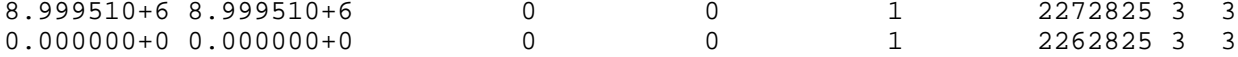

-

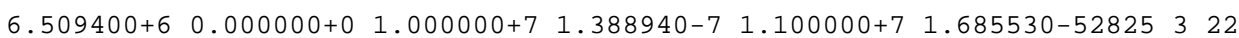
$\begin{array}{llllllll}6.509400+6 & 0.000000+0 & 1.000000+7 & 8.394700-8 & 1.100000+7 & 2.235000-52825 & 3 & 22\end{array}$

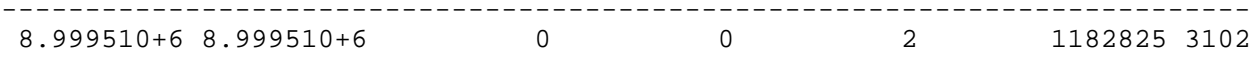

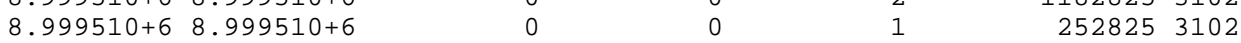

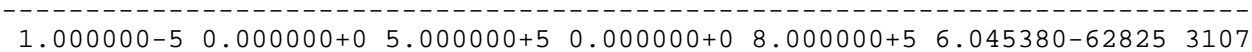
$1.000000-5 \quad 0.000000+0 \quad 5.0000000+5 \quad 0.000000+0 \quad 8.000000+5 \quad 1.149200-528253107$ 


\begin{tabular}{|c|c|c|c|c|c|}
\hline 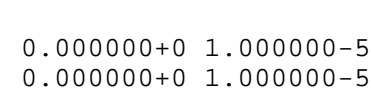 & $\begin{array}{l}\odot \\
\ominus\end{array}$ & $\begin{array}{l}\odot \\
\odot\end{array}$ & $\begin{array}{l}2 \\
1\end{array}$ & $\begin{array}{r}2825 \\
02825 \\
02825\end{array}$ & $\begin{array}{lr}3112 \\
4 & 2 \\
4 & 2\end{array}$ \\
\hline 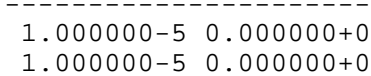 & $\begin{array}{l}2.0 \odot \odot \odot \odot \odot+7 \\
2.00 \odot \odot \odot \odot+7\end{array}$ & $\begin{array}{l}\odot .0 \odot \odot \odot \odot \odot+\odot \\
\odot . \odot \odot \odot \odot \odot \odot+\odot\end{array}$ & $\begin{array}{l}2.00 \odot \odot \odot 1+7 \\
2.00 \odot \odot \odot 1+7\end{array}$ & $\begin{array}{l}1.022532-12825 \\
9.758364-22825\end{array}$ & $\begin{array}{ll}6 & 5 \\
6 & 5\end{array}$ \\
\hline 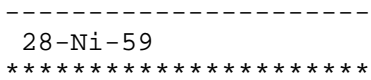 & 月 & & & & \\
\hline $\begin{array}{l}28-\mathrm{Ni}-6 \odot \\
* * * * * * * * * * * * * * * * * * * *\end{array}$ & & & & 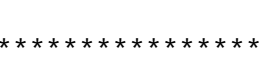 & \\
\hline 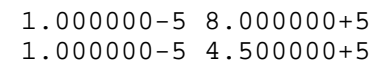 & $\begin{array}{l}1 \\
1\end{array}$ & $\begin{array}{l}3 \\
3\end{array}$ & $\begin{array}{l}\odot \\
\odot\end{array}$ & $\begin{array}{l}12831 \\
12831\end{array}$ & $\begin{array}{l}2151 \\
2151\end{array}$ \\
\hline 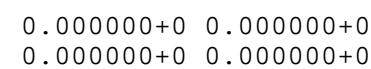 & $\begin{array}{l}\odot \\
\odot\end{array}$ & $\begin{array}{l}\odot \\
\odot\end{array}$ & $\begin{array}{l}3 \\
1\end{array}$ & $\begin{array}{l}10742831 \\
17282831\end{array}$ & $\begin{array}{l}3 \\
3\end{array}$ \\
\hline 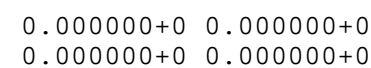 & $\begin{array}{l}\odot \\
\odot\end{array}$ & $\begin{array}{l}\odot \\
\odot\end{array}$ & $\begin{array}{l}1 \\
1\end{array}$ & $\begin{array}{l}10382831 \\
17282831\end{array}$ & $\begin{array}{ll}3 & 2 \\
3 & 2\end{array}$ \\
\hline 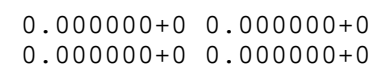 & $\begin{array}{l}\odot \\
\odot\end{array}$ & $\begin{array}{l}\odot \\
\odot\end{array}$ & $\begin{array}{l}3 \\
1\end{array}$ & $\begin{array}{l}2572831 \\
2242831\end{array}$ & \\
\hline $\begin{array}{ll}7.820050+6 & 7.820050+6 \\
7.820050+6 & 7.820050+6\end{array}$ & $\begin{array}{l}\odot \\
\odot\end{array}$ & $\begin{array}{l}\odot \\
\odot\end{array}$ & $\begin{array}{l}3 \\
1\end{array}$ & $\begin{array}{l}592831 \\
262831\end{array}$ & $\begin{array}{l}3102 \\
3102\end{array}$ \\
\hline 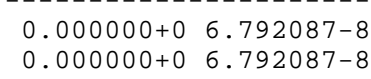 & $\begin{array}{l}6.028654+5 \\
6.028654+5\end{array}$ & $\begin{array}{l}9.995852-7 \\
9.995851-7\end{array}$ & $\begin{array}{l}1.205731+6 \\
1.205731+6\end{array}$ & $\begin{array}{l}5.773278-72831 \\
5.773278-72831\end{array}$ & $\begin{array}{l}6 \\
6\end{array}$ \\
\hline 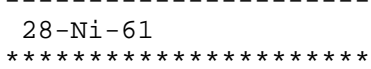 & $\approx * * * * * * *$ & $\star * \star * \star * *$ & $\pm \star$ & ** & \\
\hline 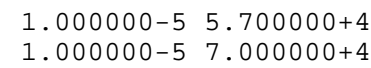 & $\begin{array}{l}1 \\
1\end{array}$ & $\begin{array}{l}2 \\
3\end{array}$ & $\begin{array}{l}\odot \\
\odot\end{array}$ & $\begin{array}{l}02834 \\
12834\end{array}$ & $\begin{array}{l}2151 \\
2151\end{array}$ \\
\hline 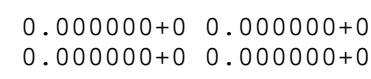 & $\stackrel{\odot}{\odot}$ & $\stackrel{\odot}{\odot}$ & $\begin{array}{l}1 \\
1\end{array}$ & $\begin{array}{l}36492834 \\
27082834\end{array}$ & $\begin{array}{l}3 \\
3\end{array}$ \\
\hline 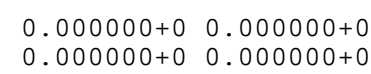 & $\begin{array}{l}\odot \\
\odot\end{array}$ & $\begin{array}{l}\odot \\
\odot\end{array}$ & $\begin{array}{l}1 \\
1\end{array}$ & $\begin{array}{l}36492834 \\
27082834\end{array}$ & $\begin{array}{l}3 \\
3\end{array}$ \\
\hline
\end{tabular}

(2.

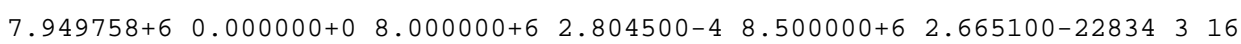
$7.949800+6 \quad 0.0000000+\odot \quad 8.0000000+6 \quad 2.804500-4 \quad 8.500000+6 \quad 2.665100-22834316$

$6.668595+5 \quad 0.000000+\odot \quad 8.000000+5 \quad 7.857700-2 \quad 9.000000+5 \quad 9.850700-22834353$ $6.668600+50.0000000+\odot \quad 8.0000000+5 \quad 7.857700-2 \quad 9.000000+5 \quad 9.850700-22834353$

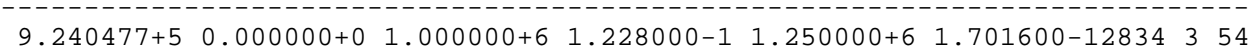
$9.240500+5 \quad 0.000000+01.000000+6 \quad 1.228000-1 \quad 1.250000+6 \quad 1.701600-12834354$

$1.479086+60.000000+01.500000+6 \quad 3.028500-3 \quad 1.750000+69.293100-22834391$ $1.479100+6 \quad 0.0000000+\odot \quad 1.500000+6 \quad 3.028500-3 \quad 1.7500000+6 \quad 9.293100-22834391$

1.

$\begin{array}{rrrrrr}1.059733+7 & 1.059733+7 & 0 & 0 & 1 & 9672834 \\ 1.059733+7 & 1.059733+7 & 0 & 0 & 1 & 2528343102\end{array}$

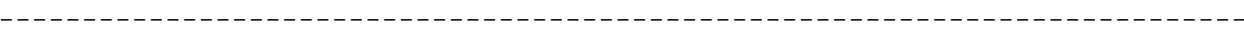

$9.442466+6 \quad 0.000000+\odot \quad 1.200000+7 \quad 2.80400-10 \quad 1.300000+7 \quad 9.923000-828343111$ $\begin{array}{llllllll}9.442500+6 & 0.000000+0 & 1.200000+7 & 2.80400-10 & 1.300000+7 & 9.923000-82834 & 3111\end{array}$

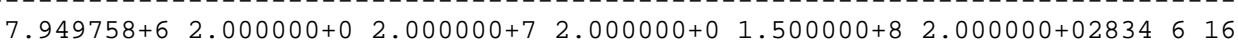

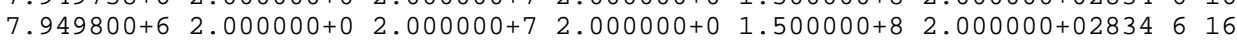
-

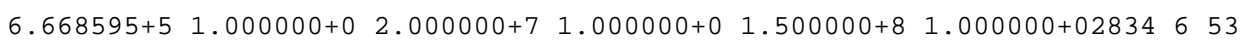
$\begin{array}{lllllllll}6.668600+5 & 1.000000+0 & 2.000000+7 & 1.000000+0 & 1.500000+8 & 1.000000+02834 & 63\end{array}$

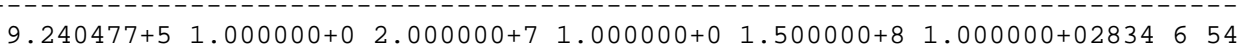
$9.240500+5 \quad 1.000000+\odot \quad 2.000000+7 \quad 1.000000+0 \quad 1.500000+8 \quad 1.0000000+02834654$

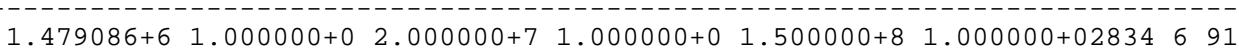
$\begin{array}{lllllllll}1.479100+6 & 1.000000+0 & 2.000000+7 & 1.000000+0 & 1.500000+8 & 1.000000+02834 & 6 & 91\end{array}$ 28-Ni-62 $28-\mathrm{Ni}-62$

$\begin{array}{llllll}2.806200+4 & 1.000000+\odot & 0 & 0 & 2 & 028372151 \\ 2.806200+4 & 1.000000+0 & 0 & 0 & 1 & 028372151\end{array}$




\begin{tabular}{|c|c|c|c|c|}
\hline 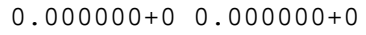 & 0 & $\odot$ & 1 & 17092837 \\
\hline 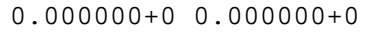 & 0 & 0 & 1 & 26782837 \\
\hline$\odot . \odot \odot \odot \odot \odot \odot+\odot \quad \odot . ๑ \odot \odot \odot \odot \odot+\odot$ & $\odot$ & $\odot$ & 1 & 17092837 \\
\hline$\odot .00 \odot \odot \odot \odot+\odot$ & 0 & 0 & 1 & 26722837 \\
\hline
\end{tabular}

$\begin{array}{lllllllll}1.077062+7 & 0.000000+\odot & 1.100000+7 & 1.768800-2 & 1.200000+7 & 2.885200-12837 & 3 & 16\end{array}$ $1.077100+7 \quad 0.000000+\odot \quad 1.100000+7 \quad 1.768800-2 \quad 1.200000+7 \quad 2.885200-12837316$

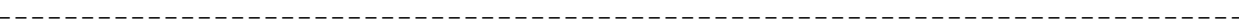

$2.082374+6 \quad 0.000000+\odot \quad 2.250000+6 \quad 5.961000-2 \quad 2.500000+6 \quad 8.846500-22837352$ $2.082400+6 \quad 0.00000 \odot+\odot \quad 2.250000+6 \quad 5.961000-2 \quad 2.5000 \odot \odot+6 \quad 8.846500-22837352$

$2.339494+6 \quad 0.000000+0 \quad 2.500000+6 \quad 1.573900-12.750000+62.293500-12837353$ $2.339500+6 \quad 0.000000+\odot \quad 2.500000+6 \quad 1.573900-1 \quad 2.750000+6 \quad 2.293500-12837353$

$2.938088+60.000000+03.000000+61.328700-2 \quad 3.500000+62.940900-12837301$ $2.938100+6 \quad 0.000000+0 \quad 3.000000+6 \quad 1.328700-2 \quad 3.500000+6 \quad 2.940900-12837391$

$\begin{array}{cccccc}6.837910+6 & 6.837910+6 & 0 & 0 & 1 & 2028373102\end{array}$

$\begin{array}{rrrrrr}6.837910+6 & 6.837910+6 & 0 & 0 & 1 & 3128373102\end{array}$

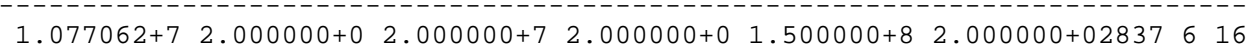
$\begin{array}{llllllllll}1.077100+7 & 2.000000+\odot & 2.0000000+7 & 2.000000+\odot & 1.500000+8 & 2.0000000+02837 & 6 & 16\end{array}$

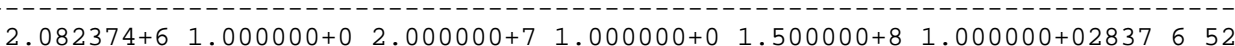
$2.082400+6 \quad 1.000000+0 \quad 2.000000+7 \quad 1.0000000+0 \quad 1.500000+8 \quad 1.000000+02837 \quad 652$

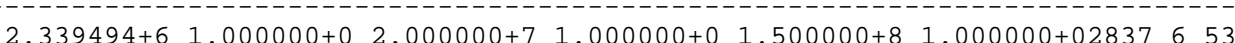
$2.339500+6 \quad 1.0000000+0 \quad 2.0000000+7 \quad 1.0000000+0 \quad 1.500000+8 \quad 1.000000+02837 \quad 6 \quad 53$

$2.938088+61.000000+02.000000+71.000000+01500000+81.000000+02837691$

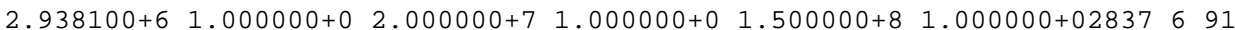

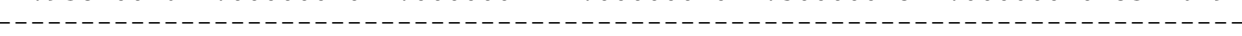
$28-\mathrm{Ni}-64$

$* * * * * * * * * * * * * * * * * * * * * * * * * * * * * * * * * * * * * * * * * * * * * * * * * * * * * * * * * * * * * * * * * * * * * * * * * * *$

$\begin{array}{cccccc}1.000000-5 & 5.530000+5 & 1 & 2 & 0 & 028432151\end{array}$

.

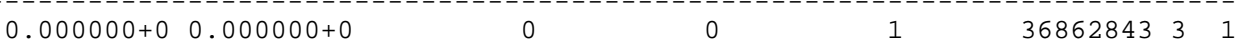

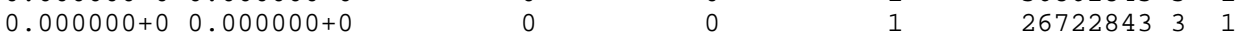

(0.0000000

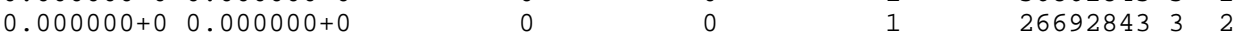

$9.809369+60.000000+01.000000+71.378500-21.100000+73.419200-12843316$ $\begin{array}{lllllllll}9.809400+6 & 0.000000+\odot & 1.000000+7 & 1.378500-2 & 1.100000+7 & 3.419200-12843 & 3 & 16\end{array}$

$1.273379+7 \quad 0.000000+01.450000+7 \quad 1.634200-7 \quad 1.600000+7 \quad 1.041900-42843328$

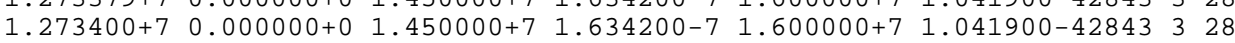

$\begin{array}{cccccc}6.098061+6 & 6.098061+6 & 0 & 0 & 1 & 104328433102\end{array}$ $\begin{array}{rrrrrr}6.098060+6 & 6.098060+6 & 0 & 0 & 1 & 2928433102\end{array}$

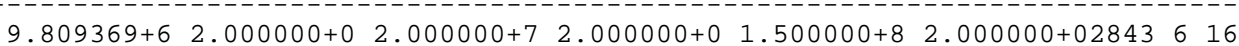
$\begin{array}{llllllllll}9.809400+6 & 2.000000+\odot & 2.000000+7 & 2.000000+\odot & 1.500000+8 & 2.0000000+02843 & 6 & 16\end{array}$

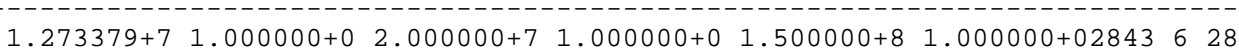
$1.273400+7 \quad 1.000000+0 \quad 2.000000+7 \quad 1.000000+0 \quad 1.500000+8 \quad 1.000000+02843628$

$29-\mathrm{Cu}-63$
$29-\mathrm{Cu}-63$

$9.945868+5 \quad 9.704225-7 \quad 1.326116+6 \quad 4.663644-7 \quad 1.657645+6 \quad 5.521164-8292566$ $9.945868+5 \quad 9.704226-7 \quad 1.326116+6 \quad 4.663644-7 \quad 1.657645+6 \quad 5.521164-829256 \quad 5$ 


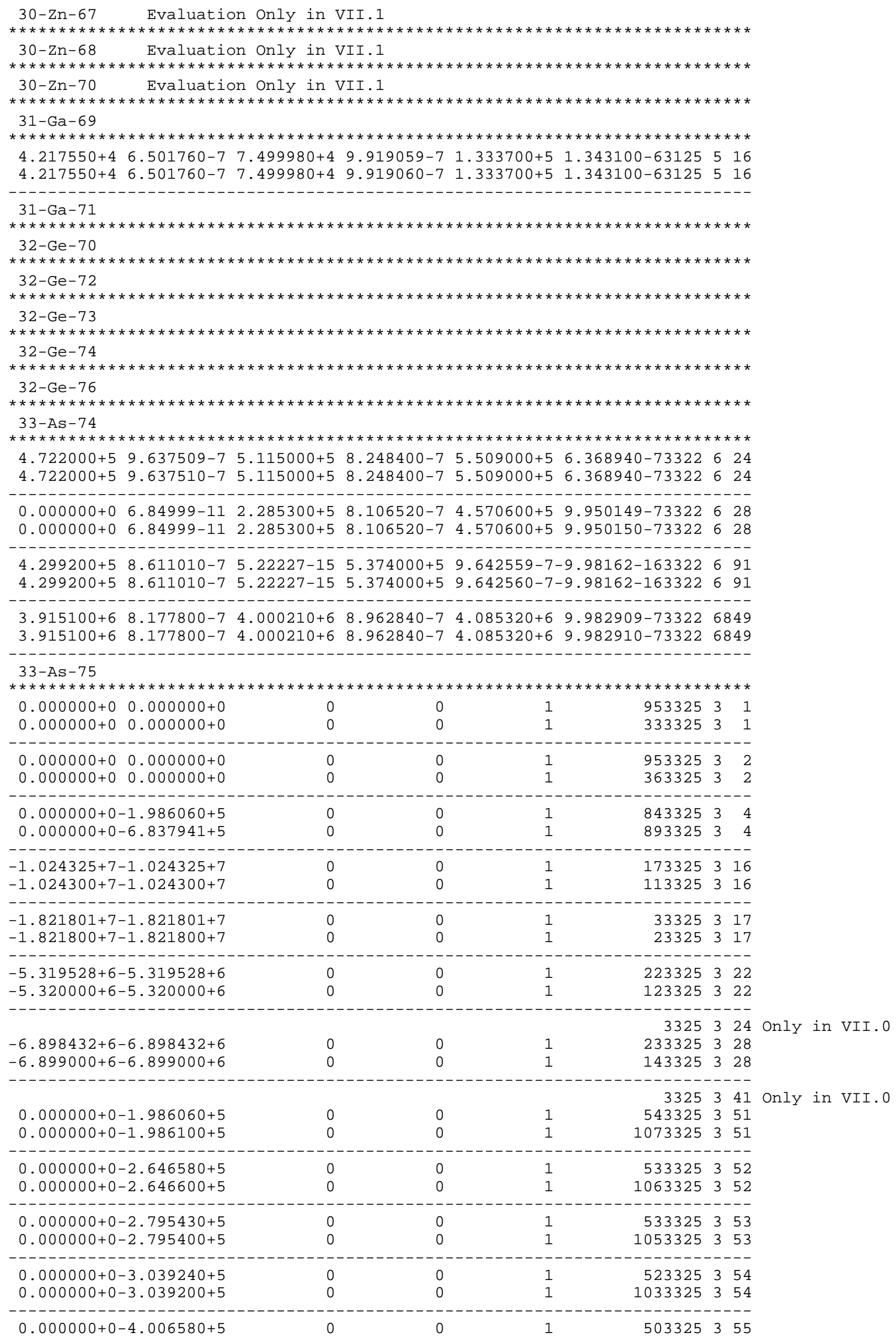




\begin{tabular}{|c|c|c|c|c|}
\hline$\odot .0 \odot \odot \odot \odot \odot+\odot-4.0 \odot 66 \odot \odot+5$ & 0 & 0 & 1 & 1003325355 \\
\hline$\odot .0 \odot \odot \odot \odot \odot+\odot-4.68750 \odot+5$ & $\odot$ & $\odot$ & 1 & 493325356 \\
\hline$\odot .000000+\odot-4.686000+5$ & 0 & 0 & 1 & 983325356 \\
\hline$\odot . \odot \odot \odot \odot \odot \odot+\odot-5.7237 \odot \odot+5$ & $\odot$ & $\odot$ & 1 & $483325 \quad 3 \quad 57$ \\
\hline$\odot .0 \odot \odot \odot \odot \odot+\odot-5.722200+5$ & $\odot$ & $\odot$ & 1 & 953325357 \\
\hline$\odot .000000+\odot-5.8500 \odot \odot+5$ & $\odot$ & $\odot$ & 1 & 483325358 \\
\hline$\odot .00 \odot \odot \odot \odot+\odot-5.850 \odot \odot \odot+5$ & $\odot$ & $\odot$ & 1 & 943325358 \\
\hline $0.0000000+0-6.176900+5$ & $\odot$ & 0 & 1 & 473325359 \\
\hline$\odot .000000+\odot-6.177000+5$ & $\odot$ & 0 & 1 & 913325359 \\
\hline$\odot . \odot \odot \odot \odot \odot \odot+\odot-8.2166 \odot \odot+5$ & $\odot$ & $\odot$ & 1 & 453325360 \\
\hline$\odot .000 \odot 0 \odot+\odot-8.215600+5$ & 0 & 0 & 1 & 853325360 \\
\hline$\odot .000000+\odot-8.598000+5$ & $\odot$ & $\odot$ & 1 & 453325361 \\
\hline 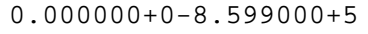 & $\odot$ & $\odot$ & 1 & 833325361 \\
\hline $0.000000+0-8.650000+5$ & $\odot$ & $\odot$ & 1 & 453325362 \\
\hline$\odot . \odot \odot \odot \odot \odot \odot+\odot-8.6480 \odot \odot+5$ & $\odot$ & 0 & 1 & 823325362 \\
\hline$\odot . \odot \odot \odot \odot \odot \odot+\odot-8.864 \odot \odot \odot+5$ & 0 & $\odot$ & 1 & 453325363 \\
\hline$\odot . ๑ \odot \odot \odot \odot \odot+\odot-8.860 \odot \odot \odot+5$ & 0 & 0 & 1 & 813325363 \\
\hline$\odot .0 \odot \odot \odot \odot \odot+\odot-1.0434 \odot \odot+6$ & 0 & $\theta$ & 1 & $433325 \quad 3 \quad 64$ \\
\hline$\odot .00 \odot \odot \odot \odot+\odot-1.04180 \odot+6$ & $\odot$ & 0 & 1 & 763325364 \\
\hline$\odot .00000 \odot+\odot-1.063300+6$ & 0 & $\odot$ & 1 & 433325365 \\
\hline$\odot . \odot \odot \odot \odot \odot \odot+\odot-1.06430 \odot+6$ & 0 & 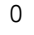 & 1 & 743325365 \\
\hline$\odot .00 \odot \odot \odot \odot+\odot-1.07450 \odot+6$ & 0 & 0 & 1 & 433325366 \\
\hline$\odot .000000+\odot-1.075600+6$ & 0 & $\odot$ & 1 & 723325366 \\
\hline$\odot . \odot \odot \odot \odot \odot \odot+\odot-1.08 \odot 8 \odot \odot+6$ & $\odot$ & $\odot$ & 1 & $433325 \quad 3 \quad 67$ \\
\hline$\odot .00 \odot \odot \odot \odot+\odot-1 . \odot 8 \odot 40 \odot+6$ & 0 & 0 & 1 & 713325367 \\
\hline$\odot .0000 \odot \odot+\odot-1.09630 \odot+6$ & 0 & $\odot$ & 1 & 423325368 \\
\hline$\odot .0000 \odot \odot+\odot-1.095500+6$ & 0 & 0 & 1 & 693325368 \\
\hline$\odot .0000 \odot \odot+\odot-1.10 \odot 20 \odot+6$ & $\odot$ & $\odot$ & 1 & 423325369 \\
\hline$\odot .0000 \odot \odot+\odot-1.101000+6$ & 0 & $\theta$ & 1 & 683325369 \\
\hline$\odot .0 \odot 0 \odot \odot \odot+\odot-1.126700+6$ & $\odot$ & $\odot$ & 1 & $423325 \quad 3 \quad 70$ \\
\hline$\odot .00 \odot \odot \odot \odot+\odot-1.12770 \odot+6$ & 0 & $\theta$ & 1 & 673325370 \\
\hline$\odot . \odot \odot \odot \odot \odot \odot+\odot-1.12890 \odot+6$ & $\odot$ & $\odot$ & 1 & $423325 \quad 3 \quad 71$ \\
\hline$\odot .0000 \odot \odot+\odot-1.1280 \odot \odot+6$ & 0 & $\theta$ & 1 & 663325371 \\
\hline$\odot .00000 \odot+\odot-1.17200 \odot+6$ & 0 & $\odot$ & 1 & $423325 \quad 3 \quad 72$ \\
\hline $0.000000+\odot-1.171600+6$ & 0 & 0 & 1 & 643325372 \\
\hline$\odot .000000+\odot-1.203600+6$ & $\odot$ & $\odot$ & 1 & $413325 \quad 3 \quad 73$ \\
\hline$\odot . \odot \odot \odot \odot \odot \odot+\odot-1.2 \odot 45 \odot \odot+6$ & $\odot$ & 0 & 1 & 623325373 \\
\hline$\odot . \odot \odot \odot \odot \odot \odot+\odot-1.264 \odot \odot \odot+6$ & 0 & $\Theta$ & 1 & $413325 \quad 3 \quad 74$ \\
\hline$\odot .000000+\odot-1.263100+6$ & 0 & 0 & 1 & 603325374 \\
\hline$\odot .0 \odot \odot \odot \odot \odot+\odot-1.3 \odot 23 \odot \odot+6$ & 0 & $\Theta$ & 1 & 403325375 \\
\hline $0.000000+0-1.301200+6$ & $\odot$ & $\Theta$ & 1 & 563325375 \\
\hline$\odot .0 \odot \odot \odot \odot \odot+\odot-1.3094 \odot \odot+6$ & 0 & $\theta$ & 1 & 403325376 \\
\hline $0.000000+0-1.309000+6$ & $\odot$ & $\odot$ & 1 & 553325376 \\
\hline$\odot .0 \odot \odot \odot \odot \odot+\odot-1.34930 \odot+6$ & 0 & $\odot$ & 1 & 403325377 \\
\hline$\odot .000000+\odot-1.349300+6$ & 0 & $\odot$ & 1 & 543325377 \\
\hline$\odot .00000 \odot+\odot-1.370800+6$ & 0 & $\theta$ & 1 & 403325378 \\
\hline $0.00000 \odot+\odot-1.370000+6$ & 0 & $\theta$ & 1 & 533325378 \\
\hline$\odot .0 \odot \odot \odot \odot \odot+\odot-1.42 \odot 2 \odot \odot+6$ & 0 & $\odot$ & 1 & 393325379 \\
\hline$\odot .0 \odot \odot \odot \odot \odot+\odot-1.41980 \odot+6$ & $\odot$ & $\odot$ & 1 & 513325379 \\
\hline $0.000000+0-1.430500+6$ & 0 & $\odot$ & 1 & 393325380 \\
\hline
\end{tabular}




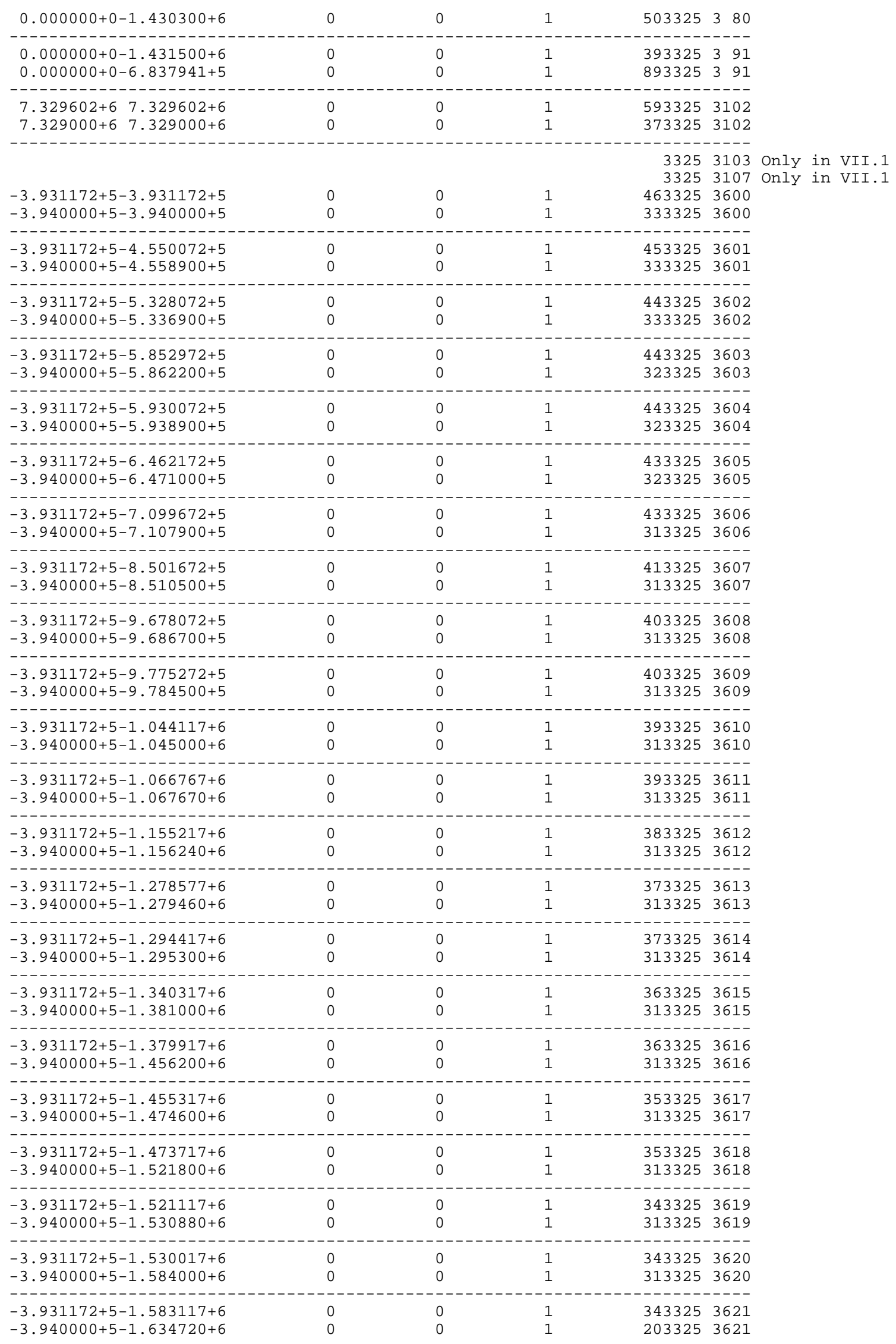




$\begin{array}{lc}-3.931172+5-1.616017+6 & 0 \\ -3.940000+5-1.651000+6 & 0 \\ -3.931172+5-1.633847+6 & \odot \\ -3.940000+5-1.729200+6 & 0 \\ -3.931172+5-1.650117+6 & \odot \\ -3.940000+5-1.788410+6 & \odot\end{array}$

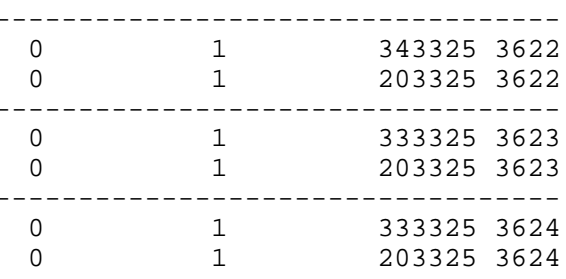

33253625

33253626 only in VII.0

33253627 only in VII.0

33253628 only in VII.0

33253629 only in VII.0

33253630 only in VII.0

33253631 only in VII.0

33253632 only in VII.0

33253633 only in VII. $\odot$

33253634 only in VII.0

33253635 only in VII.0

33253636 only in VII.O

33253637 only in VII.0

33253638 only in VII.0

33253639 only in VII.0

$-3.931172+5-1.651117+6$

$-3.940000+5-2.397927+6$

1. $201572+61.201572+6$

1. $20100 \odot+6$ 1. $20100 \odot+6$

1. $201572+61.185132+6$

1. $201000+6$ 1.184560+6

1. $201572+61.081912+6$

1. $201000+61.081340+6$

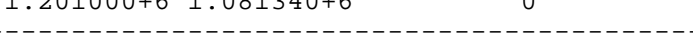

$\begin{array}{lll}1.201572+6 & 1.072782+6 & \odot \\ 1.201000+6 & 1.072210+6 & \odot\end{array}$

$1.201572+61.040042+6$

$1.201000+61.039470+6$

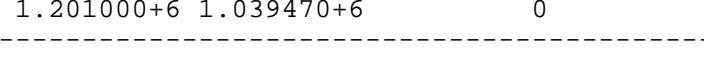

$\begin{array}{lll}1.201572+6 & 1.036062+6 & 0 \\ 1.201000+6 & 1.035490+6 & 0\end{array}$

- -

$1.201572+61.003632+6 \quad 000633253806$

$1.201000+61.003060+6 \quad 0 \quad 0 \quad 1 \quad 2233253806$

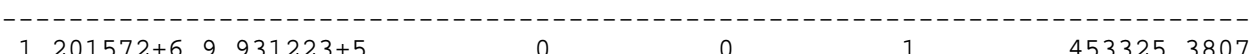

$\begin{array}{llllll}9.931223+5 & 0 & 0 & 1 & 453325 & 3807\end{array}$

$1.201000+6 \quad 9.925500+5 \quad 00012233253807$

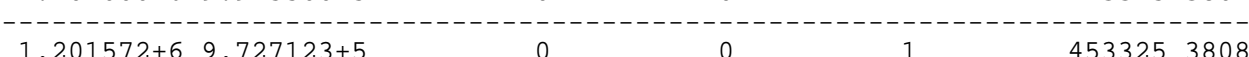

$\begin{array}{ccccc}1.201572+6 & 9.727123+5 & 0 & 0 & 1\end{array}$

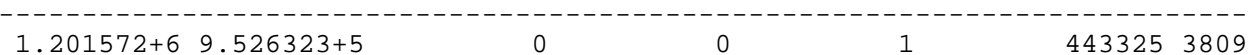

$\begin{array}{llllll}1.201572+6 & 9.526323+5 & 0 & 0 & 1 & 4433253809 \\ 1.201000+6 & 9.520600+5 & 0 & 0 & 1 & 2233253809\end{array}$

$1.201000+6 \quad 9.520600+5$

33253810 only in VII.0

33253811 only in VII.0

33253812 only in VII.0

33253813 only in VII.0

33253814 only in VII.0

33253815 only in VII.0

33253816 only in VII.0

33253817 only in VII.0

33253818 only in VII.0

33253819 only in VII.0

33253820 only in VII.0

33253821 only in VII.0

33253822 only in VII.0

33253823 only in VII.0

33253824 only in VII.0

33253825 only in VII.0

33253826 only in VII. $\odot$ 


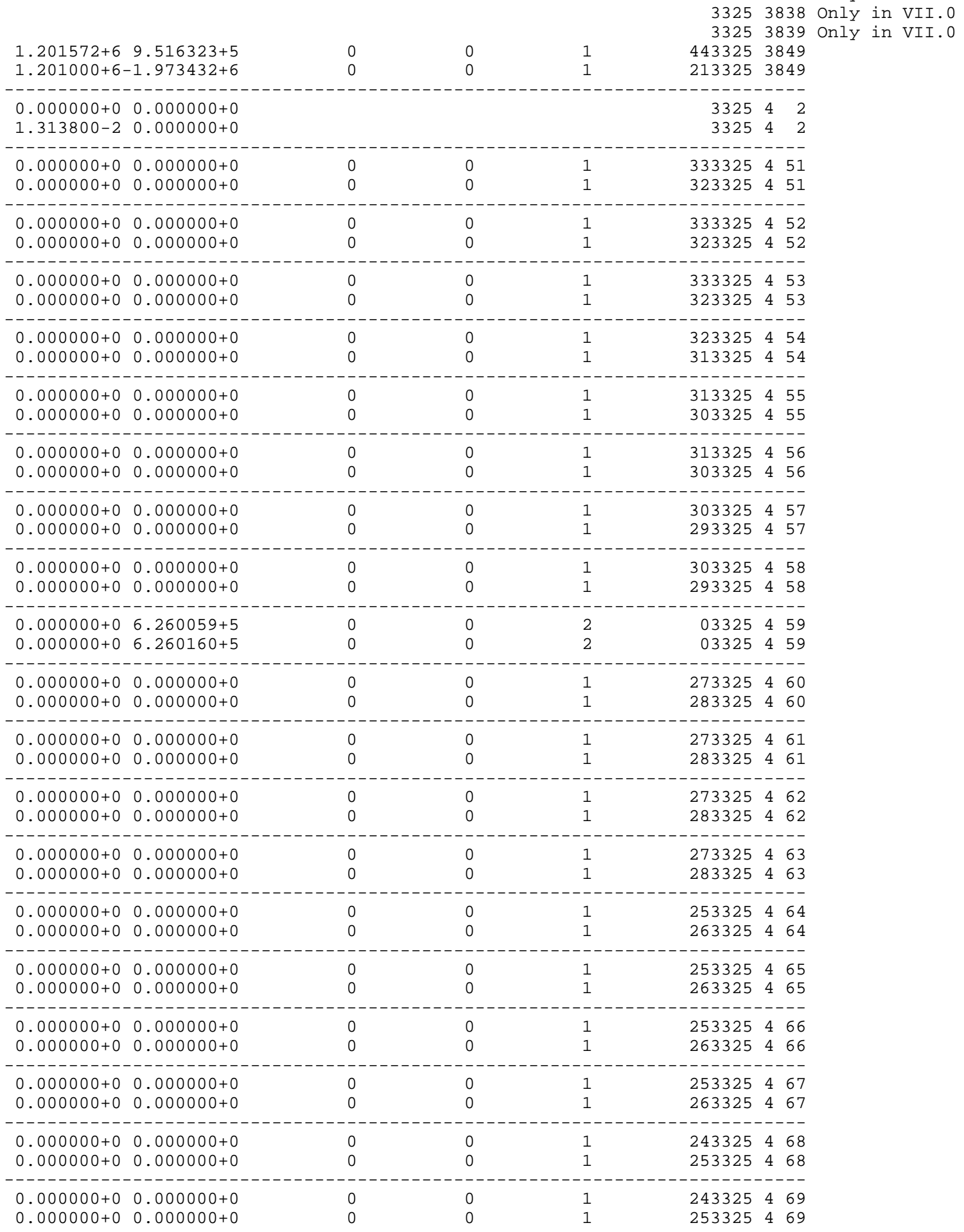




\begin{tabular}{|c|c|c|c|c|}
\hline 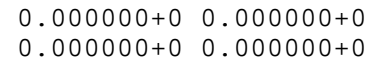 & $\begin{array}{l}\odot \\
\odot\end{array}$ & $\begin{array}{l}\odot \\
\odot\end{array}$ & $\begin{array}{l}1 \\
1\end{array}$ & $\begin{array}{lll}243325 & 4 & 70 \\
253325 & 4 & 70\end{array}$ \\
\hline - - - - - - - - - - - - - - - - & -- & - & - & 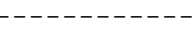 \\
\hline $0.000000+0 \quad 0.000000+0$ & 0 & $\odot$ & 1 & $243325 \quad 471$ \\
\hline $0.000000+0 \quad 0.000000+0$ & $\odot$ & 0 & 1 & $253325 \quad 471$ \\
\hline 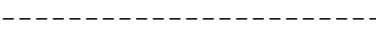 & - & - & - & 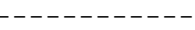 \\
\hline $0.000000+0 \quad 0.000000+0$ & $\odot$ & $\odot$ & 1 & 243325472 \\
\hline$\odot .000000+\odot \quad \odot .000000+\odot$ & 0 & $\odot$ & 1 & 253325472 \\
\hline----------------1 & - & - & -- & 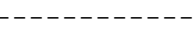 \\
\hline $0.000000+0 \quad 0.000000+0$ & $\odot$ & $\odot$ & 1 & 233325473 \\
\hline $0.000000+0 \quad 0.000000+0$ & $\odot$ & 0 & 1 & 243325473 \\
\hline - - - - - - - - - - - - - - - - - - & -- & - & -- & - - - - - - - - - \\
\hline$\odot .000000+\odot \quad 0.000000+\odot$ & 0 & $\odot$ & 1 & $233325 \quad 4 \quad 74$ \\
\hline$\odot .000000+\odot \quad \odot .000000+\odot$ & 0 & 0 & 1 & $243325 \quad 474$ \\
\hline 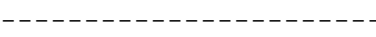 & -- & - & - & 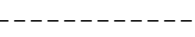 \\
\hline$\odot . \odot \odot \odot \odot \odot \odot+\odot 1.319833+6$ & $\odot$ & $\odot$ & 2 & 03325475 \\
\hline $0.000000+01.318718+6$ & 0 & $\odot$ & 2 & 03325475 \\
\hline------------------ & --- & - & -- & ---------- \\
\hline$\odot .000000+\odot \quad 1.327028+6$ & $\odot$ & ○ & 2 & 03325476 \\
\hline$\odot . \odot \odot \odot \odot \odot \odot+\odot 1.326620+6$ & $\odot$ & $\odot$ & 2 & 03325476 \\
\hline------------------ & --- & -- & -- & --------- \\
\hline $0.000000+01.367466+6$ & $\odot$ & $\odot$ & 2 & 03325477 \\
\hline$\odot .0 \odot \odot \odot \odot \odot+\odot 1.367465+6$ & $\odot$ & $\odot$ & 2 & 03325477 \\
\hline 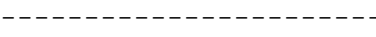 & --- & -- & -- & ---------- \\
\hline $0.000000+\odot 1.389255+6$ & $\odot$ & $\odot$ & 2 & 03325478 \\
\hline$\odot . \odot \odot \odot \odot \odot \odot+\odot 1.388440+6$ & $\odot$ & $\odot$ & 2 & 03325478 \\
\hline------------------- & -- & -- & -- & --------- \\
\hline $0.000000+0 \quad 0.000000+0$ & $\odot$ & $\odot$ & 1 & 223325479 \\
\hline$\odot .000000+\odot \quad 0.000000+0$ & $\odot$ & $\odot$ & 1 & 233325479 \\
\hline 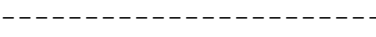 & --- & -- & -- & ---------- \\
\hline $0.000000+0 \quad 0.000000+0$ & $\odot$ & $\odot$ & 1 & 223325480 \\
\hline $0.000000+\odot \quad 0.000000+\odot$ & $\odot$ & 0 & 1 & 233325480 \\
\hline------------- & -- - & -- & -- & ---------- \\
\hline$\odot .000000+0 \quad 0.000000+0$ & $\odot$ & $\odot$ & 1 & 2833254600 \\
\hline$\odot .000000+\odot \quad 0.00000 \odot+\odot$ & $\odot$ & 0 & 1 & 2233254600 \\
\hline - - - - - - - - - - - - - - - & & - & -- & $-2--1--1$ \\
\hline$\odot . \odot \odot \odot \odot \odot \odot+\odot ~ \odot . \diamond \odot \odot \odot \odot \odot+\odot$ & $\odot$ & $\odot$ & 1 & 2733254601 \\
\hline $0.000000+0 \quad 0.000000+0$ & $\odot$ & $\odot$ & 1 & 2233254601 \\
\hline - - - - - - - - - - - - - - - & & - & - & - - - - - - - - \\
\hline 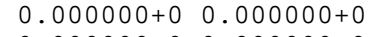 & $\odot$ & $\odot$ & 1 & 2633254602 \\
\hline$\odot .000000+\odot \quad \odot .000000+\odot$ & $\odot$ & $\odot$ & 1 & 2233254602 \\
\hline - - - - - - - - - - - - - - - - - - & -- & - & - & -------2 \\
\hline $0.000000+0 \quad 0.000000+0$ & $\odot$ & $\odot$ & 1 & 2633254603 \\
\hline$\odot .000000+0 \quad 0.000000+0$ & $\odot$ & $\odot$ & 1 & 2233254603 \\
\hline - - - - - - - - - - - - - - - & -- & - & - & ------1 \\
\hline$\odot . \diamond \odot \odot \odot \odot \odot+\odot \quad \odot . \diamond \odot \odot \odot \odot \odot+\odot$ & $\odot$ & $\odot$ & 1 & 2633254604 \\
\hline$\odot . \odot \odot \odot \odot \odot \odot+\odot ~ \odot . \diamond \odot \odot \odot \odot \odot+\odot$ & $\odot$ & $\odot$ & 1 & 2233254604 \\
\hline - - - - - - - - - - - - - - - - - - & - & - & -- & --------- \\
\hline$\odot .000000+0 \quad \odot .000000+\odot$ & $\odot$ & $\odot$ & 1 & 2533254605 \\
\hline$\odot . \odot \odot \odot \odot \odot \odot+\odot ~ \odot . ० \odot \odot \odot \odot \odot+\odot ~$ & $\odot$ & $\odot$ & 1 & 2233254605 \\
\hline------------------- & -- & -- & -- & --------- \\
\hline $0.000000+0 \quad 0.000000+0$ & $\odot$ & $\odot$ & 1 & 2533254606 \\
\hline 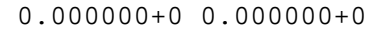 & $\odot$ & $\odot$ & 1 & 2233254606 \\
\hline------------------- & -- & -- & -- & --------- \\
\hline$\odot . \diamond \odot \odot \odot \odot \odot+\odot \quad \odot . \diamond \odot \odot \odot \odot \odot+\odot$ & $\odot$ & $\odot$ & 1 & 2333254607 \\
\hline$\odot . \odot \odot \odot \odot \odot \odot+\odot ~ \odot . ๑ \odot \odot \odot \odot \odot+\odot ~$ & $\odot$ & $\odot$ & 1 & 2233254607 \\
\hline----------------- & -- & -- & -- & ---------- \\
\hline $0.000000+0 \quad 0.000000+0$ & $\odot$ & $\odot$ & 1 & 2333254608 \\
\hline $0.000000+0 \quad 0.000000+0$ & $\odot$ & $\odot$ & 1 & 2233254608 \\
\hline------------------ & & -- & & ------- \\
\hline$\odot .000000+\odot \quad \odot .000000+0$ & $\odot$ & $\odot$ & 1 & 2333254609 \\
\hline $0.000000+0 \quad 0.000000+0$ & $\odot$ & $\odot$ & 1 & 2233254609 \\
\hline 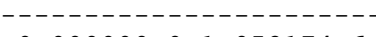 & & & & \\
\hline $0.000000+01.058174+6$ & $\odot$ & $\odot$ & 2 & 033254610 \\
\hline $0.000000+01.059069+6$ & $\odot$ & $\odot$ & 2 & 033254610 \\
\hline - & & & & --- \\
\hline $0.000000+01.081129+6$ & $\odot$ & $\odot$ & 2 & 033254611 \\
\hline $0.000000+01.082044+6$ & $\odot$ & $\odot$ & 2 & 033254611 \\
\hline-- & & -- & & --- \\
\hline $0.000000+01.170770+6$ & $\odot$ & $\odot$ & 2 & 033254612 \\
\hline$\odot .000000+\odot 1.171806+6$ & $\odot$ & $\odot$ & 2 & 033254612 \\
\hline 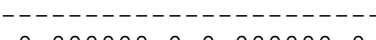 & & & & 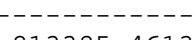 \\
\hline$\odot . \odot \odot \odot \odot \odot \odot+\odot \quad \odot . \odot \odot \odot \odot \odot \odot+\odot$ & $\odot$ & $\odot$ & 1 & 2133254613 \\
\hline$\odot . \odot \odot \odot \odot \odot \odot+\odot ~ \odot . \odot \odot \odot \odot \odot \odot+\odot ~$ & $\odot$ & $\odot$ & 1 & 2233254613 \\
\hline
\end{tabular}




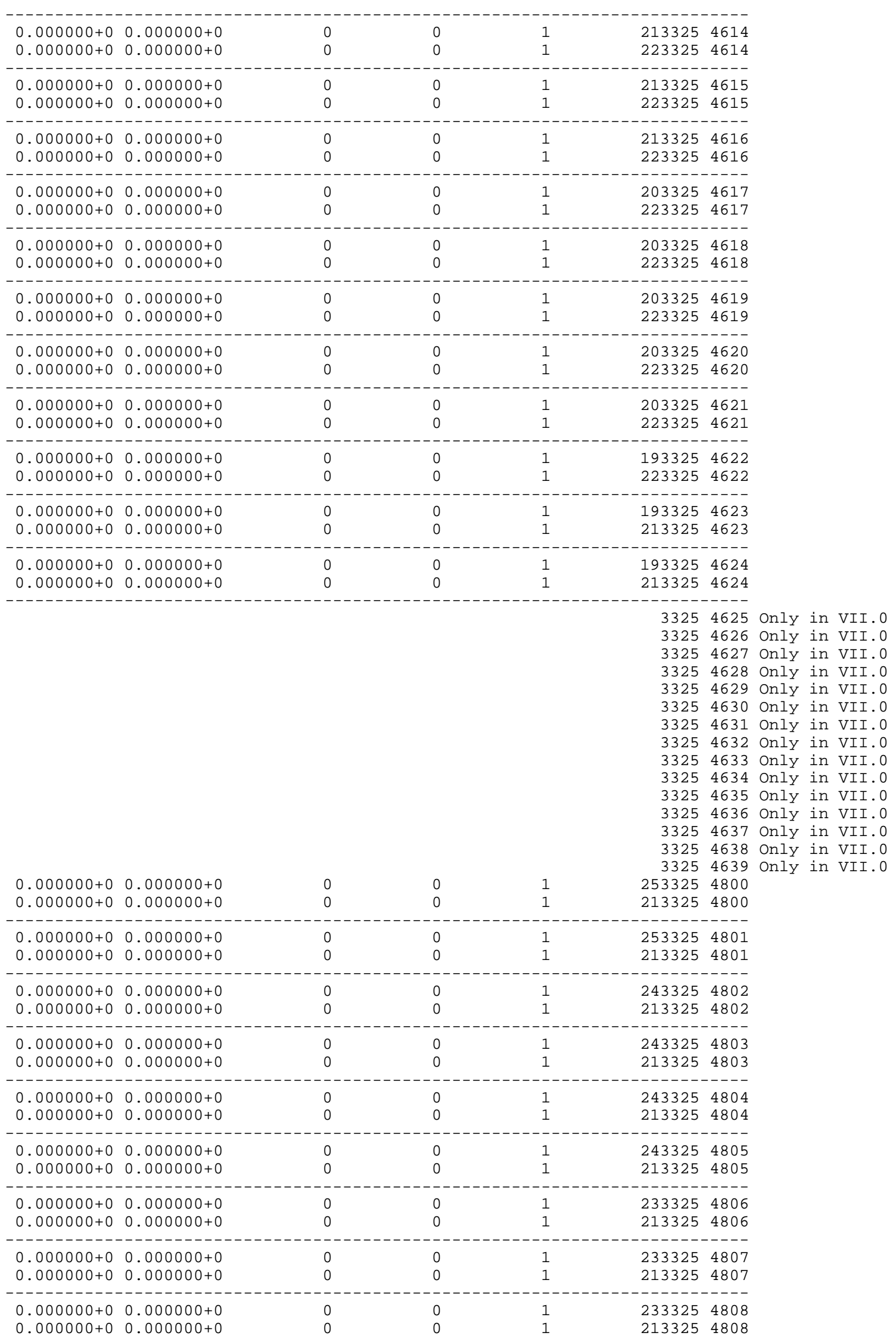




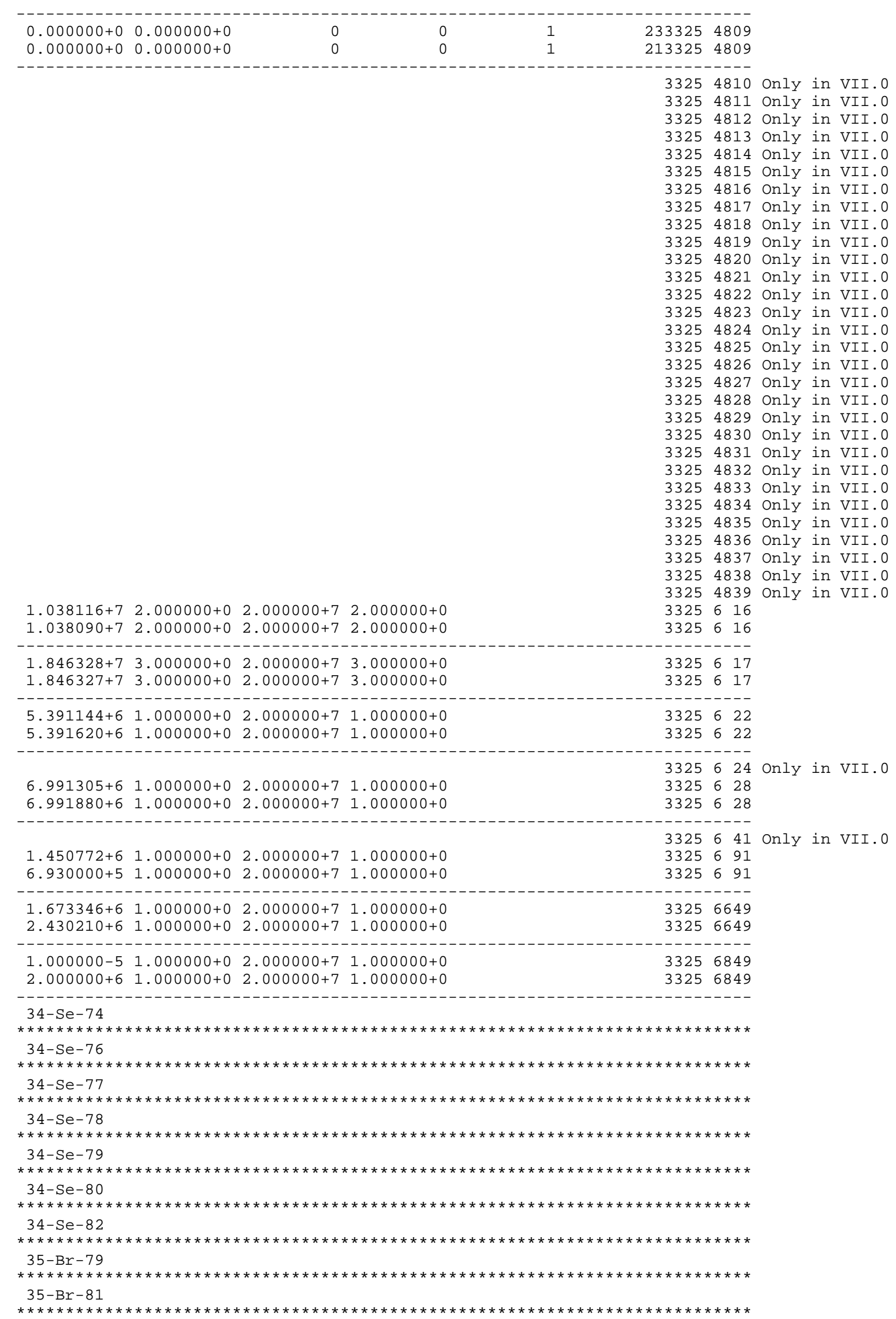




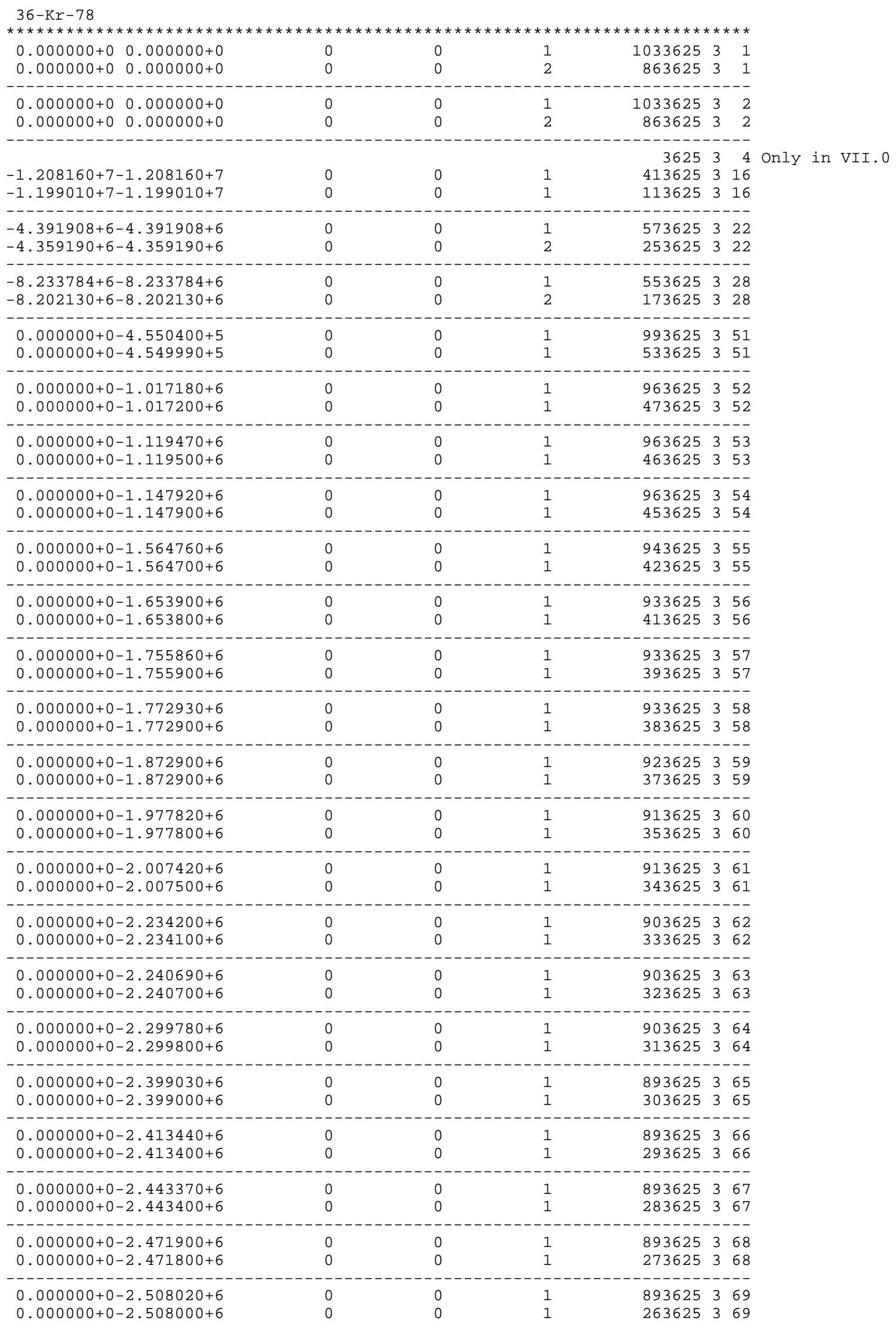


$\odot .000000+\odot-2.573350+6$ $0.000000+0-2.573300+6$ $\begin{array}{rrrrr}\odot & 0 & 1 & 8836253 & 30 \\ 0 & 0 & 1 & 2536253 & 30\end{array}$

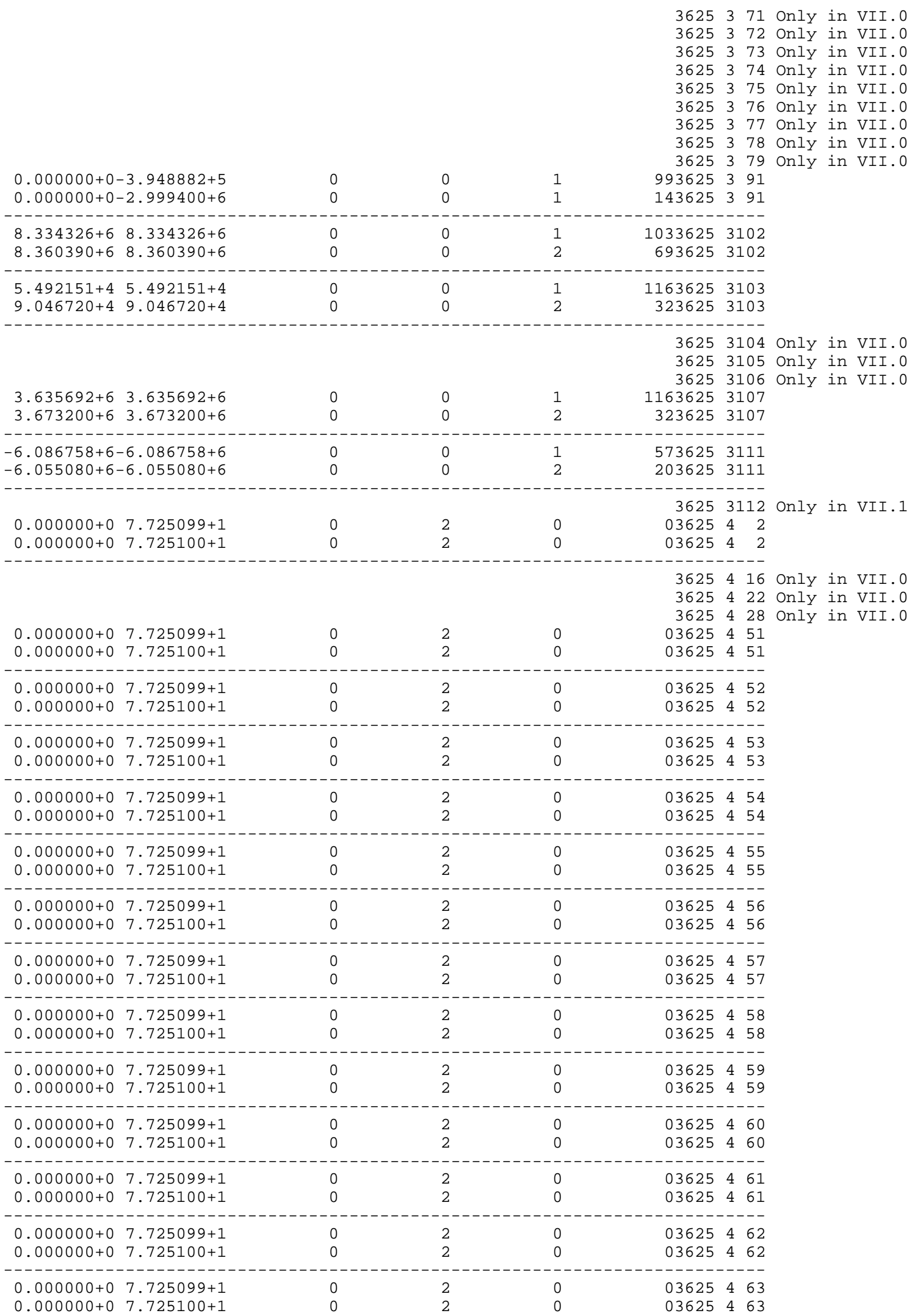




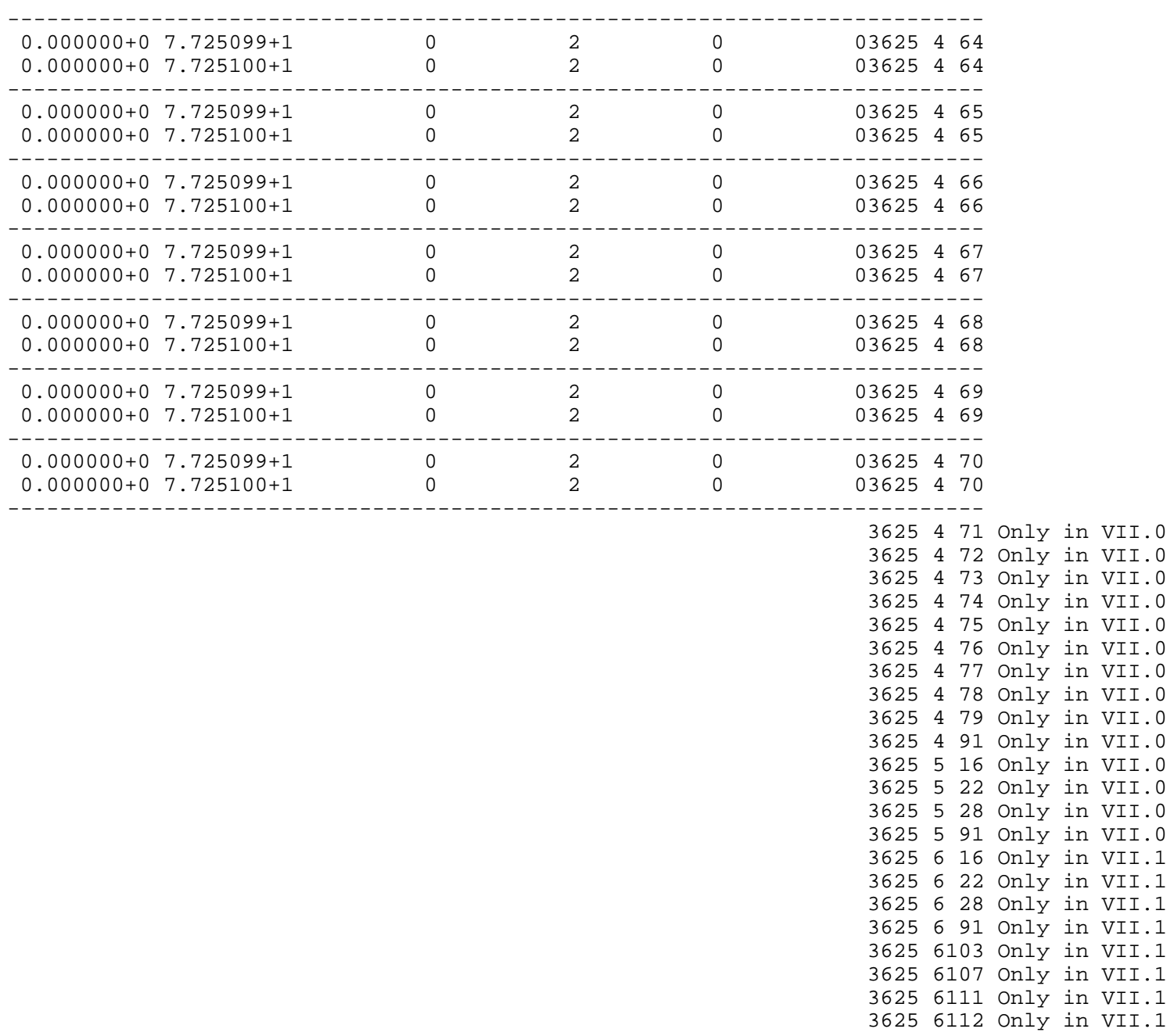

$36-\mathrm{Kr}-80$

$36-\mathrm{Kr}-82$

$36-\mathrm{Kr}-83$

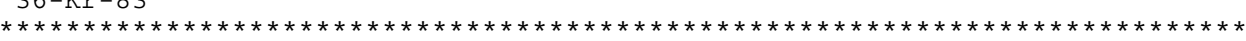

$36-\mathrm{Kr}-84$

$* * * * * * * *$
$36-\mathrm{Kr}-85$

$36-\mathrm{Kr}-85$
$* * * * * * * * * * *$

4.062800+5 9.830489-7 1.70875-14 6.094200+5 4.184170-7-4.12103-133646 622 $4.062800+5$ 9.830490-7 $1.70875-14 \quad 6.094200+5 \quad 4.184170-7-4.12103-133646622$

- -

$2.983730-8 \quad 4.024000-9 \quad 3.457650+6 \quad 9.605239-7 \quad 4.420200-8 \quad 6.034690-93646 \quad 6649$ $2.983730-8 \quad 4.024000-9 \quad 3.457650+6 \quad 9.605240-7 \quad 4.420200-8 \quad 6.034690-93646 \quad 6649$

6. $385710+6 \quad 1.471380-6 \quad 6.534210+6 \quad 9.563699-7 \quad 6.682720+6 \quad 4.95074-123646 \quad 6849$ $6.385710+6 \quad 1.471380-6 \quad 6.534210+6 \quad 9.563700-7 \quad 6.682720+6 \quad 4.95074-123646 \quad 6849$

$36-\mathrm{Kr}-86$

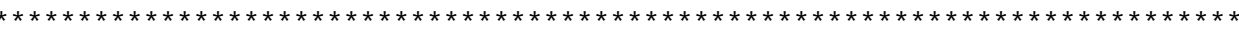

$2.274390+6 \quad 1.688790-3 \quad 2.377190+6 \quad 1.157120-3 \quad 2.765090+6 \quad 9.803399-436493102$

$2.274390+6 \quad 1.688790-3 \quad 2.377190+6 \quad 1.157120-3 \quad 2.765090+6 \quad 9.803400-43649 \quad 3102$

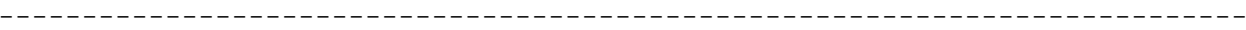

$37-\mathrm{Rb}-85$

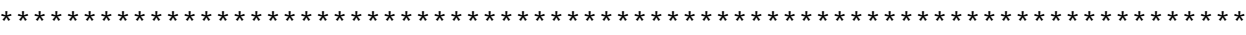

$37-\mathrm{Rb}-86$

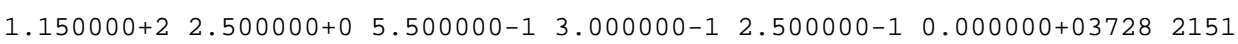

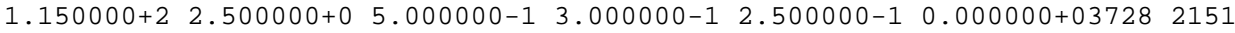




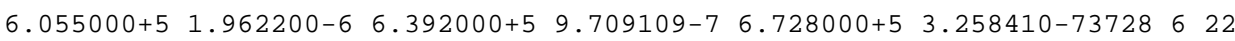
$6.055000+5 \quad 1.962200-6 \quad 6.392000+5 \quad 9.709110-7 \quad 6.728000+5 \quad 3.258410-73728622$

-5.00491-11 $\odot .000000+\odot 1.997800+5$ 9.599351-7-6.83934-11 $0.0000000+03728691$

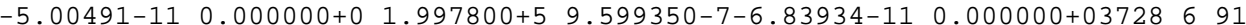

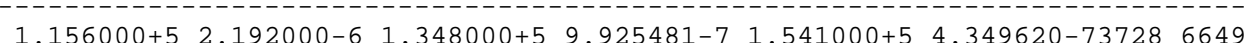
$1.156000+5 \quad 2.192000-6 \quad 1.348000+5 \quad 9.925480-7 \quad 1.541000+5 \quad 4.349620-737286649$

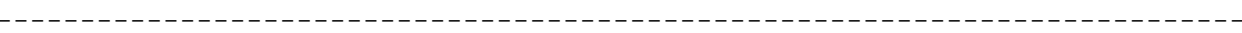

$37-\mathrm{Rb}-87$

$* * * * * * * * * * * * * * * * * * * * * * * * * * * * * * * * * * * * * * * * * * * * * * * * * * * * * * * * * * * * * * * * * * * * * * * * * * * *$

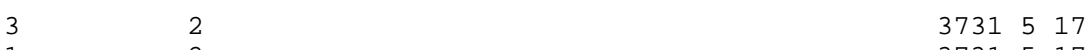

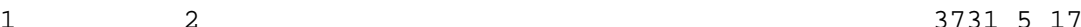

$38-\mathrm{Sr}-84$

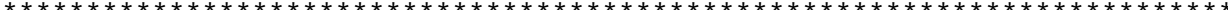

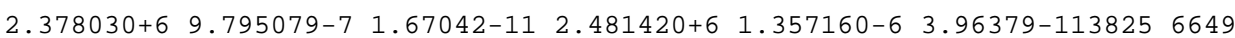
$2.378030+6 \quad 9.795080-7 \quad 1.67042-11 \quad 2.481420+6 \quad 1.357160-6 \quad 3.96379-1138256649$

$38-\mathrm{Sr}-86$

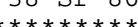

$38-\mathrm{Sr}-87$

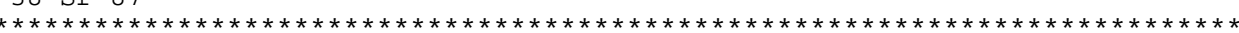

$38-\mathrm{Sr}-88$

***************************************************************************

$38-\mathrm{Sr}-89$

$* * * * * * * * * * *$
$38-\mathrm{Sr}-90$

$39-Y-89$

$1.0000000-5,4.5000000+4$

100

(1.

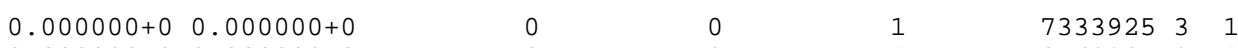

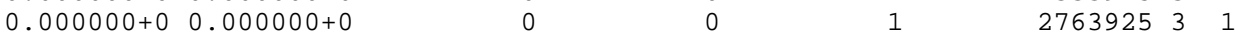

(-

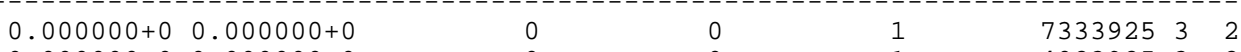

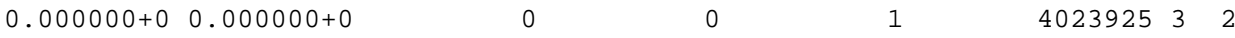

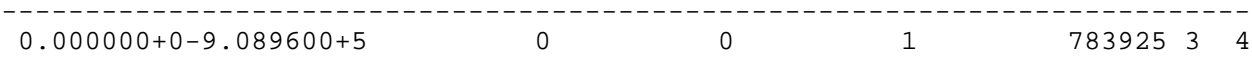

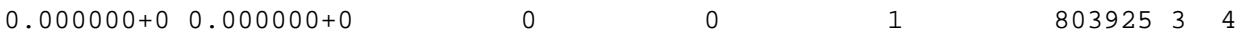

$\begin{array}{llllllll}1.160670+7 & 0.000000+0 & 1.200000+7 & 7.250379-2 & 1.250000+7 & 2.733047-13925 & 3 & 16\end{array}$ $1.160620+7 \quad 0.000000+0 \quad 1.200000+7 \quad 7.250400-2 \quad 1.250000+7 \quad 2.733100-13925316$

$9.192724+5 \quad 0.000000+\odot \quad 1.000000+6 \quad 5.901300-2 \quad 1.100000+6 \quad 8.690040-23925351$ $9.192724+5 \quad 0.000000+\odot \quad 1.000000+6 \quad 5.353590-2 \quad 1.100000+6 \quad 8.167970-23925351$

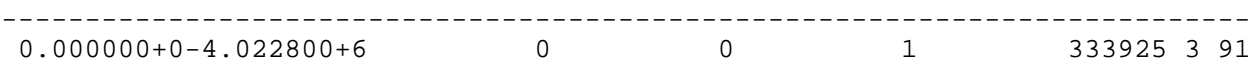

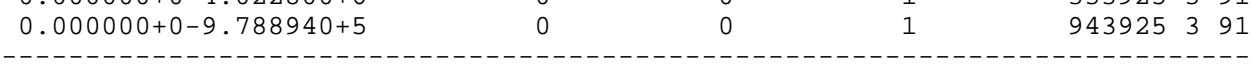

$\begin{array}{llllll}6.857000+6 & 6.857000+6 & 0 & 0 & 1 & 6039253102\end{array}$

$\begin{array}{rrrrrr}6.857000+6 & 6.857000+6 & 0 & 0 & 1 & 5539253102\end{array}$

\begin{tabular}{|c|}
\hline $130000+5-7.130000+5$ \\
\hline
\end{tabular}

$\begin{array}{rrrrr}-7.130000+5-7.130000+5 & 0 & 0 & 1 & 8039253103 \\ 0.000000+\odot-0.000000+\odot & 0 & 0 & 1 & 8139253103\end{array}$

-

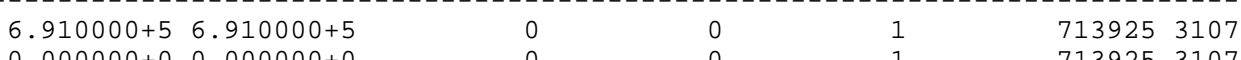

$\begin{array}{rlrrrr}0.000000+\odot & 0.000000+\odot & 0 & 0 & 1 & 7139253107\end{array}$

-

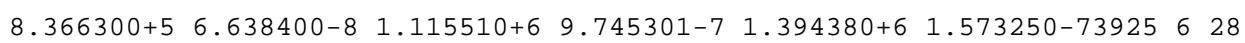
$8.366300+5 \quad 6.638400-8 \quad 1.115510+6 \quad 9.745300-7 \quad 1.394380+6 \quad 1.573250-73925628$

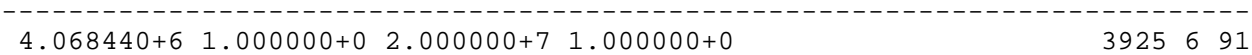
$9.899999+51.000000+02.000000+7 \quad 1.000000+0 \quad 3925691$

$39-Y-9 \odot$
$* * * * * * * * * * * * * * * * * * * * * * * * * * * * * * * * * * * * * * * * * * * * * * * * * * * * * * * * * * * * * * * * * * * * * * * * *$

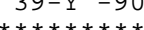

$39-Y-91$

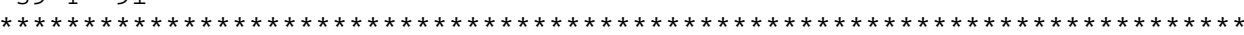

$40-\mathrm{Zr}-90$

\begin{tabular}{|c|c|c|c|c|}
\hline 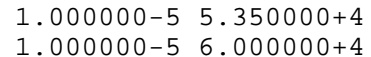 & $\begin{array}{l}1 \\
1\end{array}$ & 2 & $\stackrel{\odot}{\odot}$ & $\begin{array}{ll}04025 & 2151 \\
04025 & 2151\end{array}$ \\
\hline
\end{tabular}




\begin{tabular}{|c|c|c|c|c|c|c|}
\hline $\begin{array}{l}\odot .000 \odot \odot \odot+\odot \\
\odot .00 \odot \odot \odot \odot+\odot\end{array}$ & $\begin{array}{l}\odot .0000 \odot \odot+\odot \\
\odot .0 \odot \odot ० \odot \odot+\odot\end{array}$ & $\begin{array}{l}\odot \\
\odot\end{array}$ & $\begin{array}{l}\odot \\
\odot\end{array}$ & $\begin{array}{l}1 \\
1\end{array}$ & $\begin{array}{l}364025 \\
404025\end{array}$ & $\begin{array}{ll}3 & 1 \\
3 & 1\end{array}$ \\
\hline$\odot . \odot \odot \odot \odot \odot \diamond+\odot$ & $\odot . \odot \odot \odot \odot \odot \odot+\odot$ & $\odot$ & $\odot$ & 1 & 1104025 & 3 \\
\hline $0.000000+0$ & $\odot .000000+\odot$ & $\odot$ & $\odot$ & 1 & 1134025 & 3 \\
\hline$\odot . \odot \odot \odot \odot \odot \odot+\odot$ & $\odot .00000 \odot+\odot$ & $\odot$ & $\odot$ & 1 & 494025 & 3 \\
\hline $0.000000+0$ & $\odot .000000+0$ & $\odot$ & $\odot$ & & 504025 & 3 \\
\hline $1.500000+7$ & $8.468020-2$ & $1.600000+7$ & $1.053760-1$ & $1.7000 \odot \odot+7$ & $71.181900-14025$ & 328 \\
\hline $1.500000+7$ & $8.468020-2$ & $1.600000+7$ & $1.113760-1$ & $1.700000+7$ & $71.181900-14025$ & 328 \\
\hline $7.194000+6$ & $7.194000+6$ & $\odot$ & $\odot$ & 1 & 444025 & 3102 \\
\hline $7.194000+6$ & $7.194000+6$ & $\odot$ & $\odot$ & 1 & 484025 & 3102 \\
\hline $0.000000+0$ & $0.000000+0$ & 0 & 0 & 1 & 484025 & 3103 \\
\hline $0.000000+0$ & $0.000000+0$ & $\odot$ & $\odot$ & 1 & 494025 & 3103 \\
\hline$\odot .000000+\odot$ & $\odot .000000+\odot$ & $\odot$ & $\odot$ & 1 & 684025 & 3107 \\
\hline $0.000000+0$ & $0.000000+0$ & $\odot$ & $\odot$ & 1 & 694025 & 3107 \\
\hline $1.756000+6$ & $1.756000+6$ & 0 & 0 & 1 & 354025 & 3800 \\
\hline $1.756000+6$ & $1.756000+6$ & $\theta$ & $\theta$ & & 364025 & 3800 \\
\hline & & & & & & \\
\hline $\begin{array}{l}1.756000+6 \\
1.756000+6\end{array}$ & $\begin{array}{l}1.367470+6 \\
1.367470+6\end{array}$ & $\begin{array}{l}\ominus \\
\ominus\end{array}$ & $\begin{array}{l}\ominus \\
\ominus\end{array}$ & $\begin{array}{l}1 \\
1\end{array}$ & $\begin{array}{l}334025 \\
344025\end{array}$ & $\begin{array}{l}3801 \\
3801\end{array}$ \\
\hline & & & & & & \\
\hline $1.756000+6$ & $8.826600+5$ & 0 & 0 & 1 & 314025 & 3802 \\
\hline $1.756000+6$ & $8.826600+5$ & 0 & 0 & & 324025 & 3802 \\
\hline $1.756000+6$ & $5.275800+5$ & $\odot$ & 0 & 1 & 304025 & 3803 \\
\hline $1.756000+6$ & $5.275800+5$ & $\odot$ & $\Theta$ & & 314025 & 3803 \\
\hline $1.756000+6$ & $5.020600+5$ & $\odot$ & $\odot$ & 1 & 304025 & 3804 \\
\hline $1.756000+6$ & $5.020600+5$ & $\odot$ & $\odot$ & & 314025 & 3804 \\
\hline 1.756 & $1.6000 \odot \odot+4$ & $\odot$ & $\odot$ & 1 & 264025 & 3805 \\
\hline $1.756000+6$ & $1.600000+4$ & $\odot$ & $\odot$ & & 274025 & 3805 \\
\hline 1.756 & $1.400000+4$ & $\odot$ & $\odot$ & 1 & 264025 & 3806 \\
\hline $1.756000+6$ & $1.400000+4$ & $\odot$ & $\odot$ & 1 & 274025 & 3806 \\
\hline 1.286 & $9.330550-1$ & $\odot \odot \odot+6$ & $9.423740-1$ & $-1.500000+6$ & $69.279840-14025$ & \\
\hline $1.286999+6$ & $1.376280+0$ & $1.300000+6$ & $1.007370+0$ & $1.500000+6$ & $61.002450+04025$ & \\
\hline 4. $000 \odot \odot \odot+5$ & $1.296860+0$ & $5.000000+5$ & $1.389520+0$ & $7.000000+5$ & $51.718870+04025$ & 6102 \\
\hline $4.000000+5$ & $1.296860+\odot$ & $5.000000+5$ & $1.385600+0$ & $7.000000+5$ & & 6102 \\
\hline $2.897000+5$ & $1.731810-7$ & $3.091000+5$ & $4.356770-7$ & $000+5$ & $6129-74025$ & 6849 \\
\hline & $1.731810-7$ & & & & $59.836130-74025$ & 6849 \\
\hline $\begin{array}{c}40-\mathrm{Zr}-91 \\
\star * * * * * * * * *\end{array}$ & & $\star * * * *$ & $* \star \star \star \star \star \star * \star * \star * \star$ & $\star \star * * * * * * * *$ & 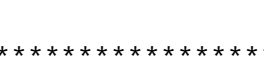 & \\
\hline $\begin{array}{l}-1 \cdot 180000+3 \\
-1.179500+3\end{array}$ & $\begin{array}{l}2.000000+\odot \\
2.000000+\odot\end{array}$ & $\begin{array}{l}6.126350+1 \\
3.200600+1\end{array}$ & $\begin{array}{l}6.112440+1 \\
3.187200+1\end{array}$ & $\begin{array}{l}1.391000-1 \\
1.340000-1\end{array}$ & 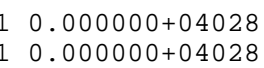 & $\begin{array}{l}2151 \\
2151\end{array}$ \\
\hline $\begin{array}{l}1.400000+7 \\
1.400000+7\end{array}$ & $\begin{array}{l}1.002000-3 \\
1.002000-3\end{array}$ & $\begin{array}{l}1.500000+7 \\
1.500000+7\end{array}$ & $\begin{array}{l}9.841440-4 \\
9.841440-4\end{array}$ & $\begin{array}{l}1.600000+7 \\
1.600000+7\end{array}$ & $\begin{array}{l}79.831639-44028 \\
79.831640-44028\end{array}$ & $\begin{array}{l}3102 \\
3102\end{array}$ \\
\hline & & & & & & \\
\hline$-9.861481-7$ & $-1.904180-7$ & & & & 4028 & 463 \\
\hline-9.861 & $1.904180-7$ & & & & 4028 & 463 \\
\hline $\begin{array}{c}40-Z r-92 \\
* * * * * * * * *\end{array}$ & * * & $* * * * * * ;$ & 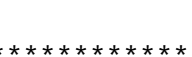 & $* \star \star * \star * \star$ & $\star \star * \star * \star * \star * \star$ & \\
\hline $2.303200-2$ & $9.185300-3$ & $5.515400-3$ & $2.502100-3$ & $9.807301-4$ & $43.522600-44031$ & \\
\hline $2.303200-2$ & $9.185300-3$ & $5.515400-3$ & $2.502100-3$ & $9.807300-4$ & $43.522600-44031$ & \\
\hline & & & & & & \\
\hline & & & & & 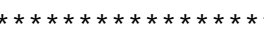 & \\
\hline $1.500000+7$ & $9.786889-4$ & $1.600000+7$ & $9.747370-4$ & & $79.851620-44034$ & 3102 \\
\hline $1.500000+7$ & $9.786890-4$ & $1.600000+7$ & $9.747370-4$ & $1.700000+7$ & $79.851620-44034$ & 3102 \\
\hline$\odot .0 \odot \odot$ & $9.826761-4$ & $\odot . \odot \odot \odot \odot \odot \odot+\odot$ & $-6.126880-3$ & $\odot .000000+\odot$ & $0-3.234990-44034$ & 460 \\
\hline$\odot .0 \odot \odot \odot \odot \odot+\odot$ & $9.826760-4$ & $0.000000+0$ & $-6.126880-3$ & $0.000000+\odot$ & $0-3.234990-44034$ & 460 \\
\hline
\end{tabular}


$4 \odot-Z r-94$

$\odot .000000+\odot-3.804930-3 \quad 0.000000+\odot-9.915431-4 \quad 0.000000+0-5.042840-54037458$ $\odot .0000 \odot \odot+\odot-3.804930-3 \quad 0.0000000+\odot-9.915430-4 \quad 0.00 \odot \odot \odot \odot+\odot-5.042840-54037458$

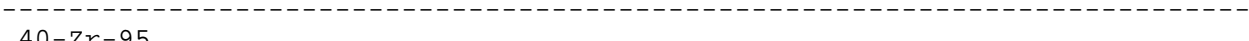
$40-Z r-95$

$40-\mathrm{Zr}-96$

$\begin{array}{llllll}1.200000+7 & 7.883900-4 & 1.300000+7 & 9.838491-4 & 1.400000+7 & 1.000530-34043 \quad 3102\end{array}$ $\begin{array}{lllllll}1.200000+7 & 7.883900-4 & 1.300000+7 & 9.838490-4 & 1.400000+7 & 1.000530-34043 & 3102\end{array}$

$\begin{array}{lllllll}2.500000+5 & 9.543199-7 & 1.863010-9 & 2.201160-8 & 6.49679-11 & 8.22708-104043 \quad 6 \quad 17\end{array}$ $2.500000+5$ 9.543200-7 $1.863010-9 \quad 2.201160-8 \quad 6.49679-11 \quad 8.22708-104043617$

$\begin{array}{lllllll}6.120150+6 & 2.234700-7 & 9.999660-1 & 6.609760+6 & 2.919190-7 & 0.999998+04043 & 6204\end{array}$ $\begin{array}{lllllllll}6.120150+6 & 2.234700-7 & 9.999660-1 & 6.609760+6 & 2.919190-7 & 0.999998+14043 \quad 6204\end{array}$

$41-\mathrm{Nb}-93$ -
$41-\mathrm{Nb}-93$

$\begin{array}{llllllll}1.667793+6 & 9.613437-7 & 2.223723+6 & 2.242543-7 & 2.779654+6 & 5.367876-84125 & 6 & 5\end{array}$ $\begin{array}{lllllllll}1.667793+6 & 9.613438-7 & 2.223723+6 & 2.242543-7 & 2.779654+6 & 5.367876-84125 & 6 & 5\end{array}$

(1)

$41-\mathrm{Nb}-94$

$* * * * * * * * * * * * * * * * * * * * * * * * * * * * * * * * * * * * * * * * * * * * * * * * * * * * * * * * * * * * * * * * * * * * * * * * * * *$

$41-\mathrm{Nb}-95$

$42-$ Mo -92

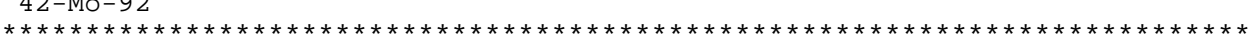

$\begin{array}{lllllll}1.700000+7 & 9.789560-4 & 1.800000+7 & 9.955449-4 & 1.9000000+7 & 1.023700-34225 & 3102\end{array}$ $\begin{array}{lllllll}1.700000+7 & 9.789560-4 & 1.800000+7 & 9.955450-4 & 1.900000+7 & 1.023700-34225 & 3102\end{array}$

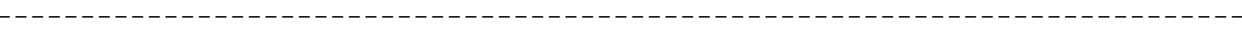
$42-$ Mo- 94

$* \star * * * * * * * * * * * * * * * *$

$\begin{array}{llllllll}4.976340+4 & 9.733971-7 & 2.903960-9 & 2.188130-8 & 9.95301-11 & 8.05128-104231 & 6 & 28\end{array}$ $4.976340+4$ 9.733970-7 2.903960-9 2.188130-8 9.95301-11 8.05128-104231 628 42-Mo-95

$9.409060+10.000000+\odot \quad 000138 \quad 001542342151$

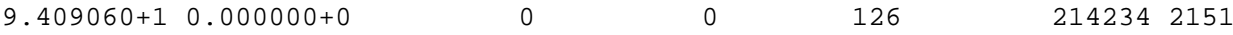

$2.100000+5 \quad 1.215620-6 \quad 2.333000+5 \quad 9.537119-7 \quad 2.566000+5 \quad 7.355840-74234 \quad 691$ $2.100000+5 \quad 1.215620-6 \quad 2.333000+5 \quad 9.537120-7 \quad 2.566000+5 \quad 7.355840-74234691$ $42-$ Mo-96 Mo- 96

9.957409-7 $1.388850-7 \quad 2.117540-8 \quad 3.11672-10 \quad 2.166470-9-1.620620-94237451$ 9.957410-7 1.388850-7 2.117540-8 3.11672-10 2.166470-9-1.620620-94237 451

$0.000000+0-5.083260-2 \quad 0.000000+\odot \quad 4.312350-3 \quad 0.000000+\odot \quad 9.864341-44237463$ $0.000000+0-5.083260-2 \quad 0.000000+0 \quad 4.312350-3 \quad 0.000000+0 \quad 9.864340-44237463$

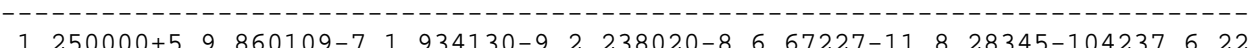
$1.250000+5$ 9.860110-7 $1.934130-9 \quad 2.238020-8 \quad 6.67227-11 \quad 8.28345-104237622$

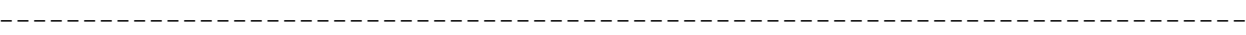
$42-$ Mo -97

$\begin{array}{lllllll}1.250000+5 & 9.780561-7 & 2.001600-9 & 2.219960-8 & 6.90503-11 & 8.21662-1042406 & 62\end{array}$ $1.250000+5$ 9.780560-7 2.001600-9 2.219960-8 6.90503-11 8.21662-104240622

$1.250000+5.9 .780560-72.001600-92.219960-86.90503-118.21662-104240622$

$\begin{array}{llllll}1.573730+7 & 1.558500-8 & 0.999998+0 & 1.600180+7 & 0.000000+\odot & 0.000000+04240 \quad 6203\end{array}$ $\begin{array}{lllllllll}1.573730+7 & 1.558500-8 & 0.999998+1 & 1.600180+7 & 0.000000+\Theta & 0.000000+042406203\end{array}$

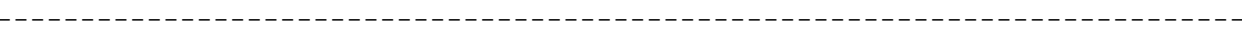
$42-$ Mo -98

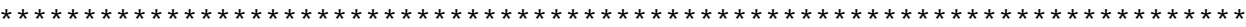
$42-$ Mo- 99

$42-\mathrm{Mo}-100$

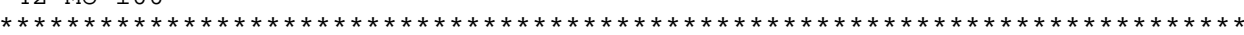
$43-$ TC -99 4.

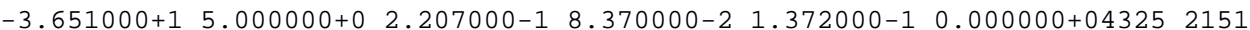

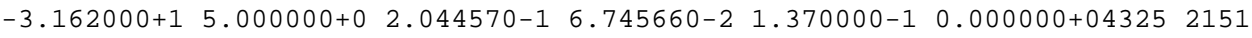


$\begin{array}{llllllll}1.000000-5 & 0.000000+0 & 1.403400+5 & 0.000000+0 & 1.403400+5 & 9.730000+04325 & 3 & 1\end{array}$

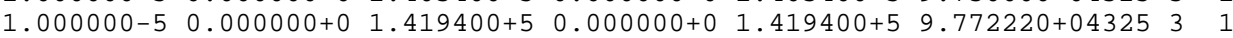

.

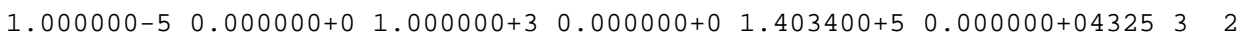
$\begin{array}{lllllll}1.000000-5 & 0.000000+0 & 1.000000+3 & 0.000000+0 & 1.419400+5 & 0.000000+043253 & 2\end{array}$

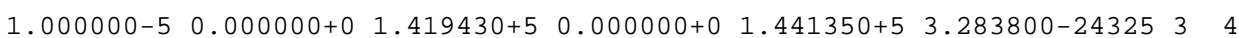

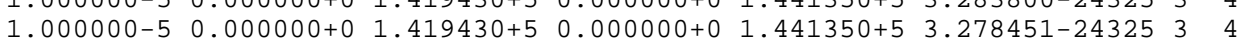

$1.000000-5 \quad \odot .000000+\odot \quad 1.403400+5 \quad 0.000000+\odot \quad 1.403400+5 \quad 3.775000-143253102$ $\begin{array}{llllll}1.000000-5 & 0.000000+0 & 1.419400+5 & 0.000000+0 & 1.419400+5 & 3.728027-143253102\end{array}$

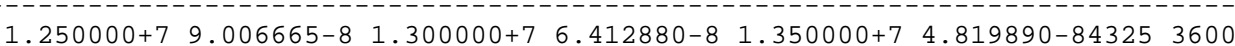
$\begin{array}{lllllll}1.250000+7 & 9.006650-8 & 1.300000+7 & 6.412880-8 & 1.350000+7 & 4.819890-84325 & 3600\end{array}$

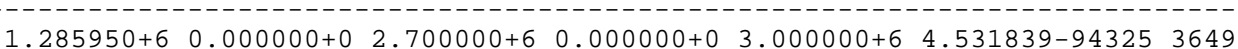
$1.285950+6 \quad 0.000000+0 \quad 2.7000000+6 \quad 0.000000+0 \quad 3.000000+6 \quad 4.531840-943253649$

$1.100000+6 \quad 3.790880-9 \quad 1.2000000+6 \quad 5.302141-9 \quad 1.500000+6 \quad 1.215270-843253800$ $1.100000+6 \quad 3.790880-9 \quad 1.200000+6 \quad 5.302140-9 \quad 1.500000+6 \quad 1.215270-843253800$

$2.200000+61.204650-92.500000+63.619270-92.700000+67.048959-943253849$ $2.200000+6 \quad 1.204650-9 \quad 2.500000+6 \quad 3.619270-9 \quad 2.700000+6 \quad 7.048960-943253849$

7.

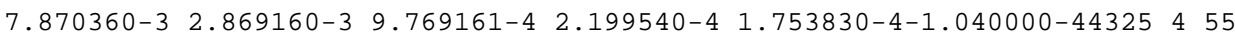
7.870360-3 2.869160-3 9.769160-4 2.199540-4 1.753830-4-1.040000-44325 455

$7.870360-32.869160-39.769161-42.199540-41.753830-4-1.040000-44325456$ 7.870360-3 2.869160-3 9.769160-4 2.199540-4 1.753830-4-1.0400९९-44325 456

$3.642160-11.684870-10.847831-4-2.282160-2-5.735480-2-2.582740-24325466$ $3.642160-1$ 1.684870-1 9.847830-4-2.282160-2-5.735480-2-2.582740-24325 466 3.642160-1 $1.684870-10.847830-4-2.282160-2-5.735$

2.391800-3 9.968519-4 4.718870-4 1.512340-4 2.900290-7-1.282850-44325 467 2.391800-3 9.968520-4 4.718870-4 1.512340-4 2.900290-7-1.282850-44325 467 2. $2.391800-3 \quad 9.968519-4 \quad 4.718870-4 \quad 1.512340-4 \quad 2.900290-7-1.282850-44325468$ 2.391800-3 9.968520-4 4.718870-4 1.512340-4 2.900290-7-1.282850-44325 468

$2.391800-3 \quad 9.968519-4 \quad 4.718870-4 \quad 1.512340-4 \quad 2.900290-7-1.282850-44325469$ 2.391800-3 9.968520-4 4.718870-4 1.512340-4 2.900290-7-1.282850-44325 469

$2.391800-39.968519-44.718870-4 \quad 1.512340-4 \quad 2.900290-7-1.282850-44325470$ 2.391800-3 9.968520-4 4.718870-4 1.512340-4 2.900290-7-1.282850-44325 470

$2.116000+5 \quad 4.797110-62.351000+5 \quad 9.931360-7 \quad 3.057000+59.902139-74325624$ $2.116000+5 \quad 4.797110-6 \quad 2.351000+5 \quad 9.931360-7 \quad 3.057000+5 \quad 9.902140-74325624$ $1.222000+59.923029-7 \quad 1.357000+5 \quad 8.051440-7 \quad 1.493000+56.635360-74325691$

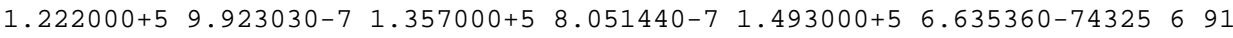
(-10

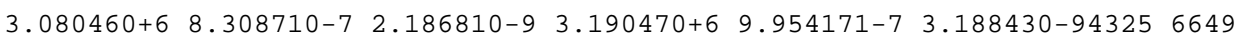
$\begin{array}{lllllll}3.080460+6 & 8.308710-7 & 2.186810-9 & 3.190470+6 & 9.954170-7 & 3.188430-94325 & 6649\end{array}$ $44-\mathrm{Ru}-96$

$* * * * * * * * * * * * * * * * * * * * * * * * * * * * * * * * * * * * * * * * * * * * * * * * * * * * * * * * * * * * * * * * * * * * * * * * * * *$

$44-\mathrm{Ru}-98$

$* * * * * * * * * * * * * * * * * * * * * * * * * * * * * * * * * * * * * * * * * * * * * * * * * * * * * * * * * * * * * * * * * * * * * * * * * * *$ $44-\mathrm{Ru}-99$

$44-\mathrm{Ru}-100$

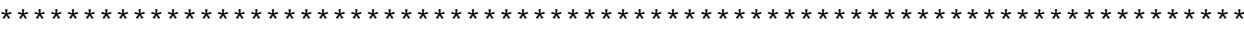

$0.000000+\odot \quad 0.000000+\odot \quad 1.250000+5 \quad 9.539619-7 \quad 2.5000000+5 \quad 1.186470-64437528$

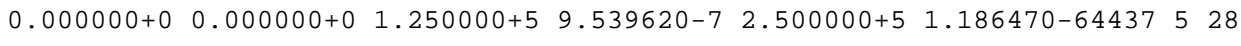
$44-\mathrm{Ru}-101$

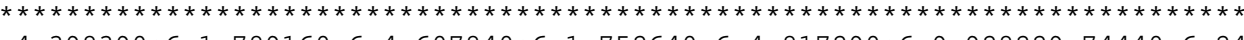

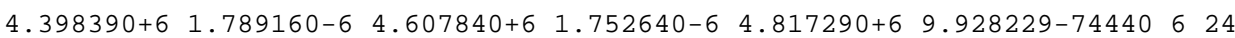
$4.398390+6 \quad 1.789160-6 \quad 4.607840+6 \quad 1.752640-6 \quad 4.817290+6 \quad 9.928230-74440 \quad 6 \quad 24$ $3.690600+59.832530-7 \quad 1.77209-14 \quad 4.613300+5 \quad 9.689981-7-1.22967-144440691$ $3.690600+59.832530-7 \quad 1.77209-14 \quad 4.613300+5$ 9.689980-7-1.22967-144440 691 $44-\mathrm{Ru}-102$ 


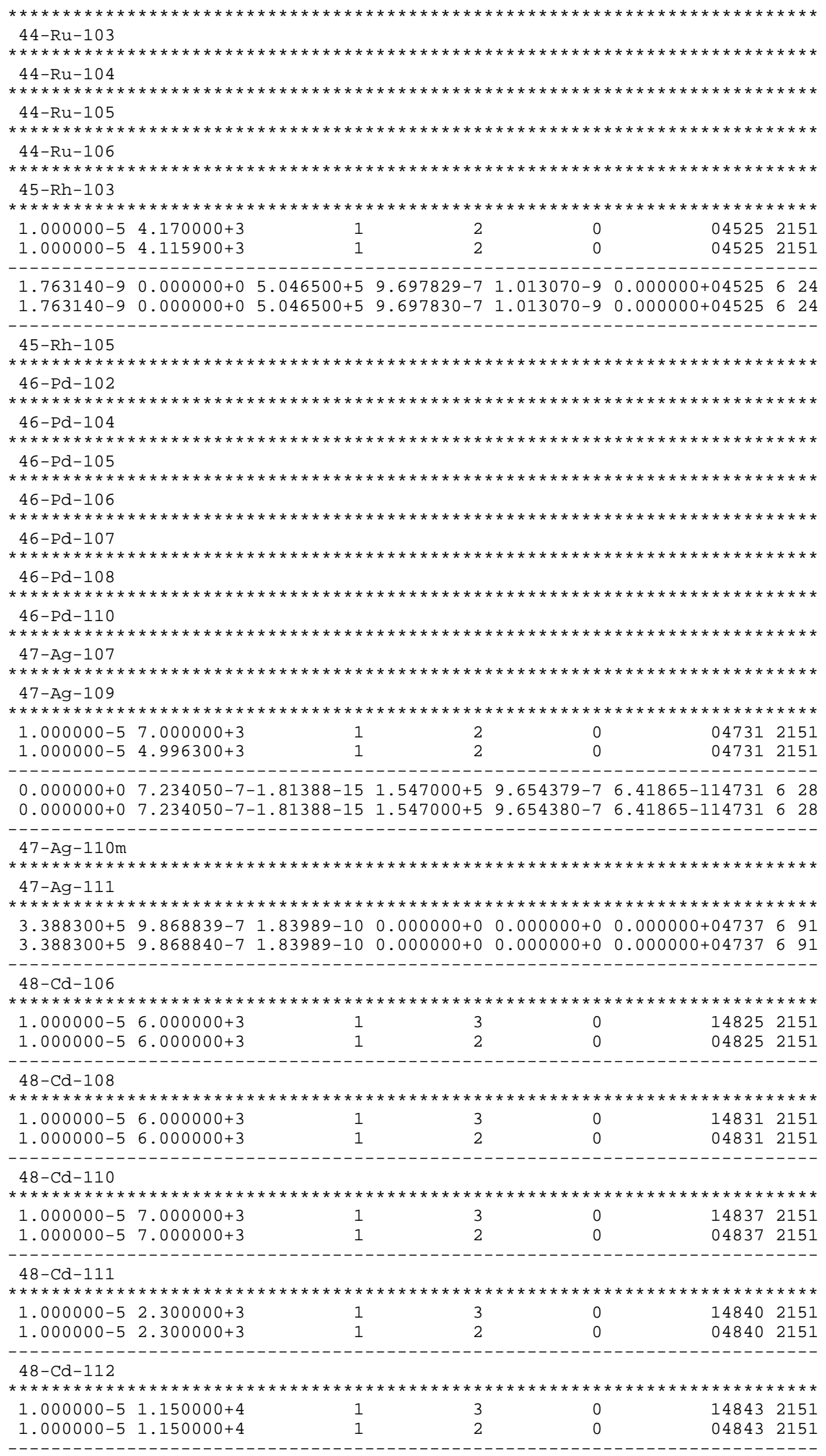


$48-\mathrm{Cd}-113$

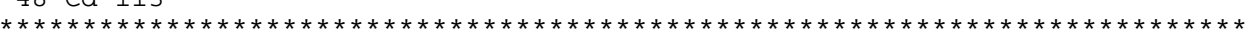

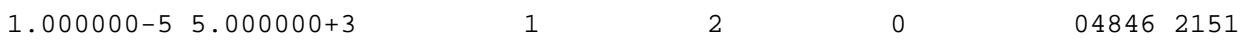

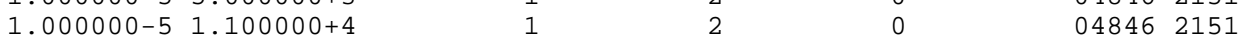

$48-\mathrm{Cd}-114$

$* \star * * * * * * * * * * * * * * * * * * * * * * * * * * * * * * * * * * * * * * * * * * * * * * * * * * * * * * * * * * * * * * * * * * * * * * * * *$

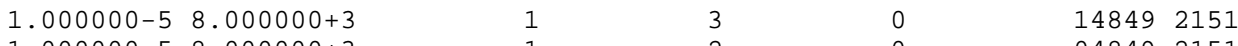

$1.000000-58.000000+3 \quad 11000048492151$

48-Cd-115m

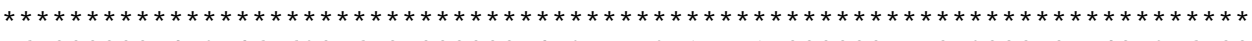

$6.000000+4 \quad 9.465630-6 \quad 8.000000+4 \quad 9.557371-7 \quad 1.000000+5 \quad 2.308270-74853628$ $6.000 \odot \odot \odot+4 \quad 9.465630-6 \quad 8.00000 \odot+4 \quad 9.557370-7 \quad 1.0000 \odot \odot+5 \quad 2.308270-74853 \quad 6 \quad 28$

$48-\mathrm{Cd}-116$

\begin{tabular}{|c|c|c|c|c|}
\hline 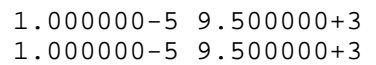 & $\begin{array}{l}1 \\
1\end{array}$ & $\begin{array}{l}3 \\
2\end{array}$ & $\begin{array}{l}\odot \\
\odot\end{array}$ & $\begin{array}{ll}14855 & 2151 \\
04855 & 2151\end{array}$ \\
\hline
\end{tabular}

$49-\operatorname{In}-113$
$* * * * * * * * * * * * * * * * * * * * * * * * * * * * * * * * * * * * * * * * * * * * * * * * * * * * * * * * * * * * * * * * * * * * * * * * * *$

$49-\mathrm{In}-115$

*

$50-\mathrm{Sn}-112$

$50-\mathrm{Sn}-113$

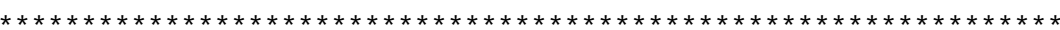

$0.000000+\odot \quad 9.556099-7 \quad 2.14353-14 \quad 1.918100+5 \quad 1.218530-6 \quad 4.76731-115028622$ $0.000000+\odot \quad 9.556100-7 \quad 2.14353-14 \quad 1.918100+5 \quad 1.218530-6 \quad 4.76731-115028622$

$1.560000+59.624069-71.820000+51.223840-62.080000+51.436140-65028624$ $\begin{array}{llllllll}1.560000+5 & 9.624070-7 & 1.820000+5 & 1.223840-6 & 2.080000+5 & 1.436140-65028 & 24\end{array}$

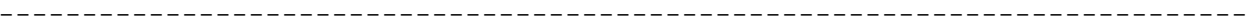

$\bullet .000000+\odot \quad 2.315400+5 \quad 9.632651-7 \quad 1.81886-11 \quad 0.0000 \odot \odot+\odot \quad 0.00000 \odot+05028 \quad 628$ $\odot .0 \odot \odot \odot \odot \odot+\odot ~ 2.31540 \odot+5 \quad 9.632650-7 \quad 1.81886-11 \quad 0.00 \odot \odot \odot \odot+\odot \quad 0.0 \odot \odot \odot \odot \odot+05 \odot 28628$ $1.120000+5 \quad 1.638990-6 \quad 1.307000+5 \quad 9.884139-7 \quad 1.493000+5 \quad 7.349770-750286649$ $1.120000+5 \quad 1.638990-6 \quad 1.307000+5 \quad 9.884140-7 \quad 1.493000+5 \quad 7.349770-75028 \quad 6649$

$50-\mathrm{Sn}-114$

$* * * * * * * * * * * * * * * * * * * * * * * * * * * * * * * * * * * * * * * * * * * * * * * * * * * * * * * * * * * * * * * * * * * * * * * * * * *$ $50-\mathrm{Sn}-115$

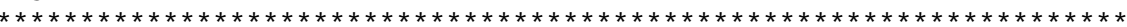

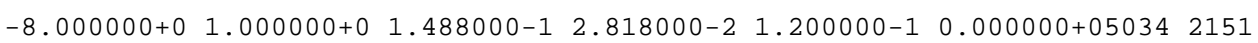

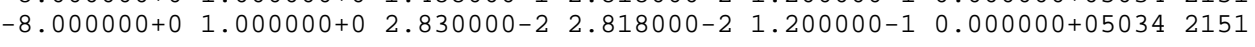

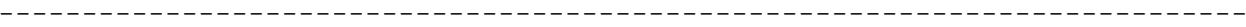

$50-\mathrm{Sn}-116$

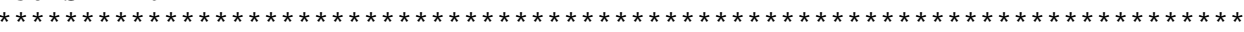

$50-\mathrm{Sn}-117$

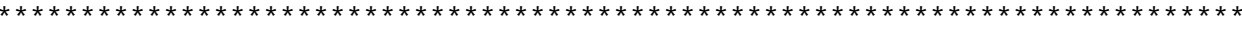

$50-\mathrm{Sn}-118$

$50-\mathrm{Sn}-119$

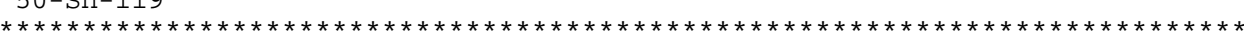

$50-\mathrm{Sn}-120$

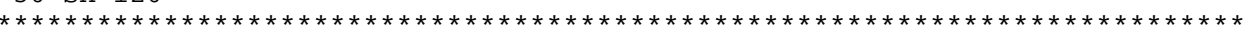

$50-\mathrm{Sn}-122$

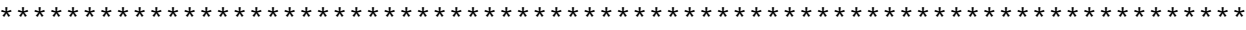

$50-\mathrm{Sn}-123$

$50-\mathrm{Sn}-124$

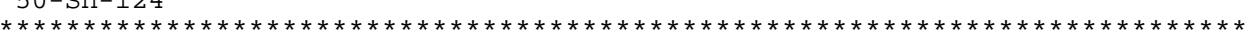

$50-\mathrm{Sn}-125$

$2.77200 \odot+4 \quad 5.790200+\odot \quad 2.772210+4 \quad 5.79020 \odot+\odot \quad 5.0 \odot \odot \odot \odot \odot+4 \quad 5.71030 \odot+05064322$ $2.772000+4 \quad 5.700144+0 \quad 2.772210+4 \quad 5.700138+0 \quad 5.000000+4 \quad 5.634612+05064 \quad 3 \quad 2$

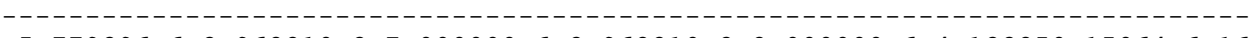

$5.779296+6 \quad 2.968810-2 \quad 7.000000+6 \quad 2.968810-2 \quad 8.000000+6 \quad 4.122850-15064616$ $5.779296+6 \quad 1.56530 \odot+5 \quad 7.0 \odot \odot \odot \odot \odot+6 \quad 2.968810-2 \quad 8.0 \odot \odot \odot \odot \odot+6 \quad 4.122850-15 \odot 64 \quad 6 \quad 16$

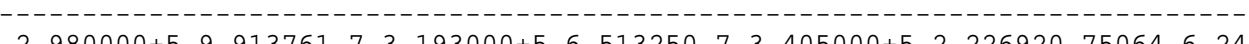
$\begin{array}{llllllll}2.980000+5 & 9.913760-7 & 3.193000+5 & 6.513250-7 & 3.405000+5 & 2.226920-75064 & 64\end{array}$ 50-Sn-126 
$53-\mathrm{I}-131$

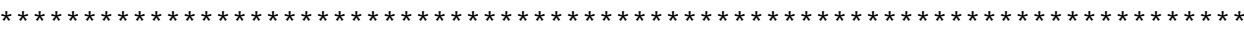

$53-\mathrm{I}-135$

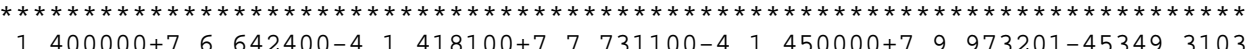

$1.400000+7 \quad 6.642400-4 \quad 1.418100+7 \quad 7.731100-4 \quad 1.450000+7 \quad 9.973200-453493103$

(6)

$54-\mathrm{Xe}-123$

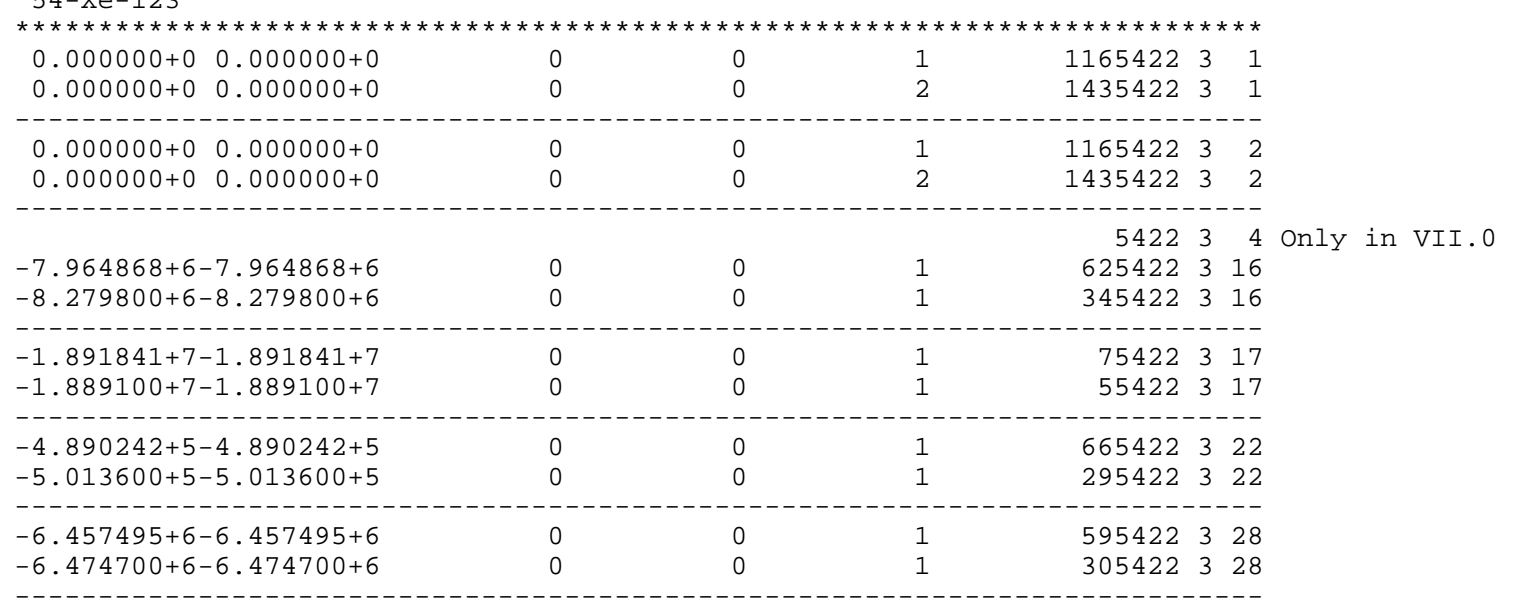

5422341
34

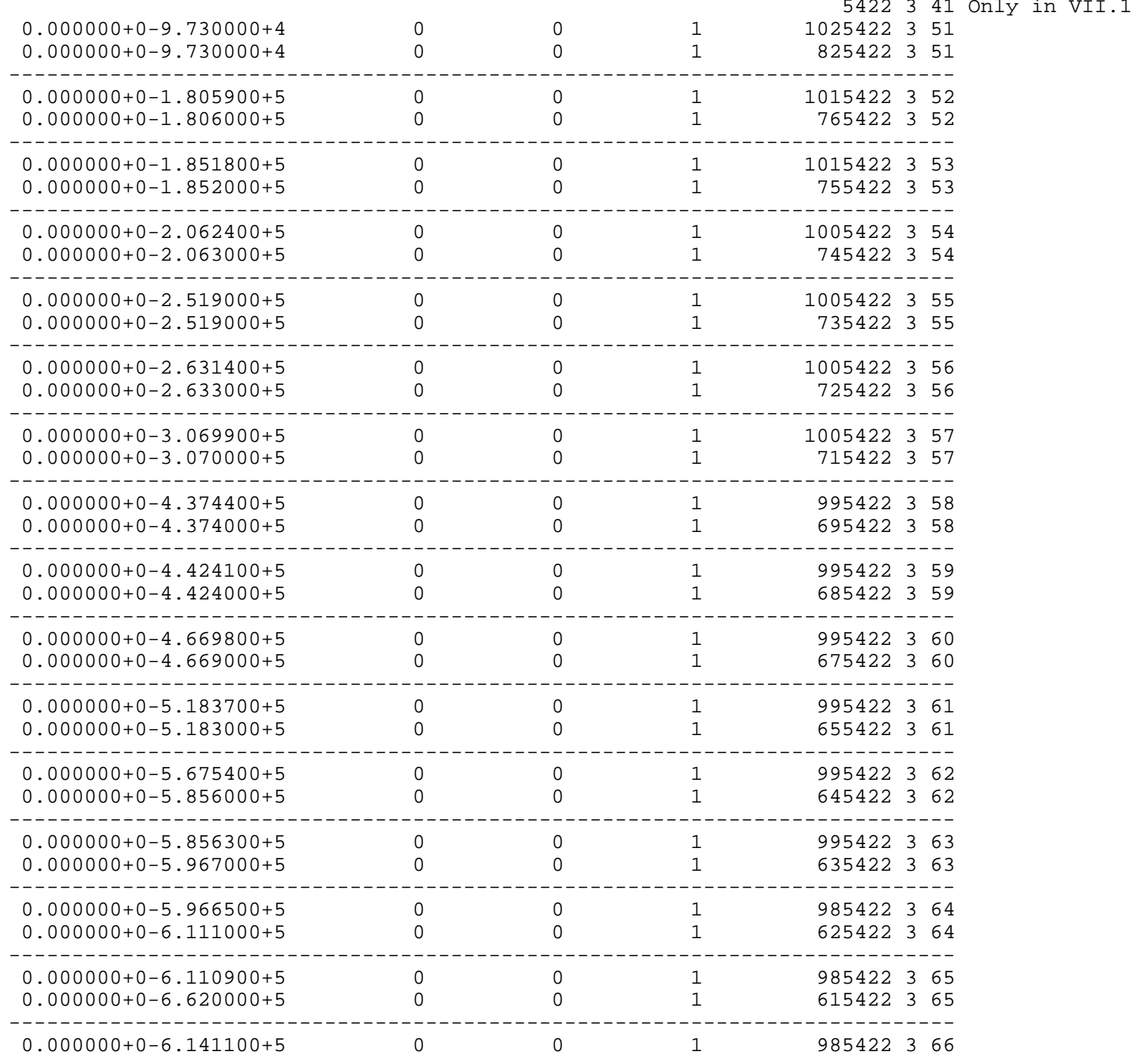




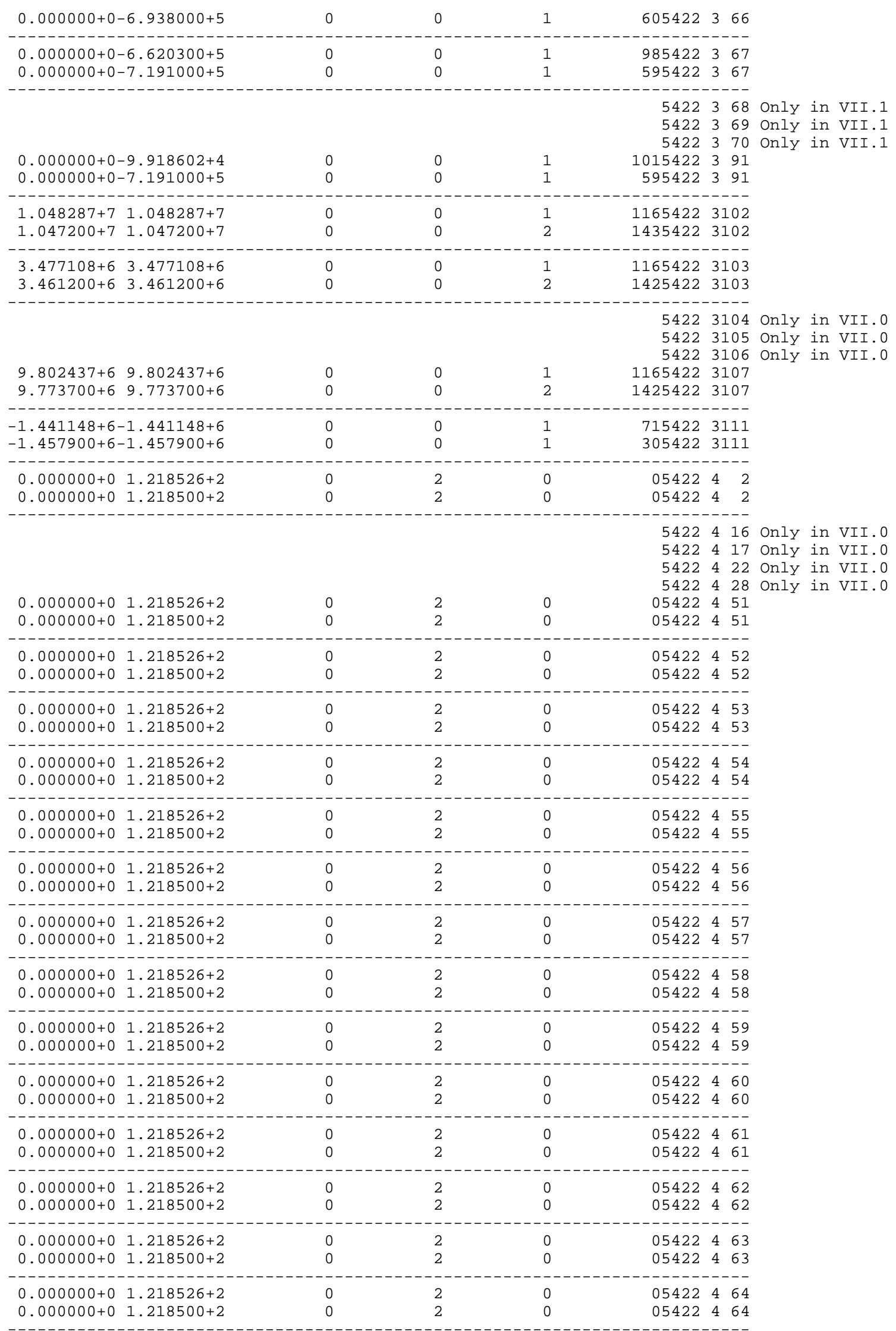




\begin{tabular}{|c|c|c|c|c|c|c|}
\hline 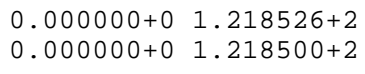 & $\begin{array}{l}\odot \\
\odot\end{array}$ & $\begin{array}{l}2 \\
2\end{array}$ & $\begin{array}{l}\odot \\
\odot\end{array}$ & $\begin{array}{l}05422 \\
05422\end{array}$ & $\begin{array}{ll}4 & 65 \\
4 & 65\end{array}$ & \\
\hline 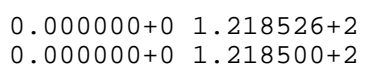 & $\begin{array}{l}\odot \\
\odot\end{array}$ & $\begin{array}{l}2 \\
2\end{array}$ & $\begin{array}{l}\odot \\
\odot\end{array}$ & $\begin{array}{l}05422 \\
05422\end{array}$ & $\begin{array}{l}466 \\
466\end{array}$ & \\
\hline 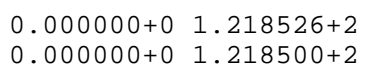 & $\begin{array}{l}\odot \\
\odot\end{array}$ & $\begin{array}{l}2 \\
2\end{array}$ & $\begin{array}{l}\odot \\
\odot\end{array}$ & $\begin{array}{l}05422 \\
05422\end{array}$ & $\begin{array}{l}467 \\
467\end{array}$ & \\
\hline & & & & $\begin{array}{l}5422 \\
5422 \\
5422 \\
5422 \\
5422 \\
5422 \\
5422 \\
5422 \\
5422 \\
5422 \\
5422 \\
5422 \\
5422 \\
5422 \\
5422 \\
5422 \\
5422 \\
5422\end{array}$ & $\begin{array}{ll}4 & 68 \\
4 & 69 \\
4 & 70 \\
4 & 91 \\
5 & 16 \\
5 & 17 \\
5 & 22 \\
5 & 28 \\
5 & 91 \\
6 & 16 \\
6 & 17 \\
6 & 22 \\
6 & 28 \\
6 & 41 \\
6 & 91 \\
6103 \\
6107 \\
6111\end{array}$ & $\begin{array}{l}\text { Only in VII.1 } \\
\text { Only in VII.1 } \\
\text { Only in VII.1 } \\
\text { Only in VII.0 } \\
\text { Only in VII.0 } \\
\text { Only in VII.0 } \\
\text { Only in VII.0 } \\
\text { Only in VII.0 } \\
\text { Only in VII.0 } \\
\text { Only in VII.1 } \\
\text { Only in VII.1 } \\
\text { Only in VII.1 } \\
\text { Only in VII.1 } \\
\text { Only in VII.1 } \\
\text { Only in VII.1 } \\
\text { Only in VII.1 } \\
\text { Only in VII.1 } \\
\text { Only in VII.1 }\end{array}$ \\
\hline
\end{tabular}

\begin{tabular}{|c|c|c|c|c|c|}
\hline 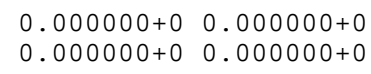 & $\begin{array}{l}\odot \\
\odot\end{array}$ & $\begin{array}{l}\odot \\
\odot\end{array}$ & $\begin{array}{l}1 \\
1\end{array}$ & $\begin{array}{rrr}1035425 & 3 & 1 \\
845425 & 3 & 1\end{array}$ & \\
\hline 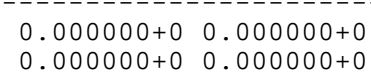 & $\begin{array}{l}\odot \\
\odot\end{array}$ & $\begin{array}{l}\odot \\
\odot\end{array}$ & $\begin{array}{l}1 \\
1\end{array}$ & $\begin{array}{rrr}1035425 & 3 & 2 \\
845425 & 3 & 2\end{array}$ & \\
\hline $\begin{array}{l}-1.048287+7-1.048287+7 \\
-1.047200+7-1.047200+7\end{array}$ & $\begin{array}{l}\odot \\
\odot\end{array}$ & $\begin{array}{l}\odot \\
\odot\end{array}$ & $\begin{array}{l}1 \\
1\end{array}$ & $\begin{array}{rrr}5425 & 3 & 4 \\
495425 & 3 & 16 \\
325425 & 3 & 16\end{array}$ & only in VII. \\
\hline $\begin{array}{l}-1.844774+7-1.844774+7 \\
-1.875100+7-1.875100+7\end{array}$ & $\begin{array}{l}\odot \\
\odot\end{array}$ & $\begin{array}{l}\odot \\
\odot\end{array}$ & $\begin{array}{l}1 \\
1\end{array}$ & $\begin{array}{lll}95425 & 3 & 17 \\
65425 & 3 & 17\end{array}$ & \\
\hline $\begin{array}{l}-6.804314+5-6.804314+5 \\
-6.979000+5-6.979000+5\end{array}$ & $\begin{array}{l}0 \\
\odot\end{array}$ & $\begin{array}{l}\ominus \\
\odot\end{array}$ & $\begin{array}{l}1 \\
1\end{array}$ & $\begin{array}{lll}655425 & 3 & 22 \\
385425 & 3 & 22\end{array}$ & \\
\hline $\begin{array}{l}-7.005761+6-7.005761+6 \\
-7.010300+6-7.010300+6\end{array}$ & $\begin{array}{l}0 \\
\odot\end{array}$ & $\begin{array}{l}\odot \\
\odot\end{array}$ & $\begin{array}{l}1 \\
1\end{array}$ & $\begin{array}{lll}575425 & 3 & 28 \\
365425 & 3 & 28\end{array}$ & \\
\hline $\begin{array}{l}0.0000 \odot \odot+\odot-3.541400+5 \\
0.0000 \odot \odot+\odot-3.541000+5\end{array}$ & $\begin{array}{l}0 \\
0\end{array}$ & $\begin{array}{l}\odot \\
\odot\end{array}$ & $\begin{array}{l}1 \\
1\end{array}$ & $\begin{array}{rrr}1005425 & 3 & 51 \\
775425 & 3 & 51\end{array}$ & \\
\hline $\begin{array}{l}\odot .000000+0-8.466100+5 \\
\odot .000000+0-8.466000+5\end{array}$ & $\begin{array}{l}0 \\
0\end{array}$ & $\begin{array}{l}\odot \\
\odot\end{array}$ & $\begin{array}{l}1 \\
1\end{array}$ & $\begin{array}{lll}975425 & 3 & 52 \\
705425 & 3 & 52\end{array}$ & \\
\hline 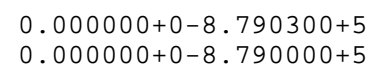 & $\begin{array}{l}\odot \\
\odot\end{array}$ & $\begin{array}{l}\odot \\
\odot\end{array}$ & $\begin{array}{l}1 \\
1\end{array}$ & $\begin{array}{lll}975425 & 3 & 53 \\
685425 & 3 & 53\end{array}$ & \\
\hline $\begin{array}{l}\odot .0000 \odot \odot+\odot-1.248040+6 \\
\odot .0000 \odot \odot+\odot-1.2480 \odot \odot+6\end{array}$ & $\begin{array}{l}0 \\
0\end{array}$ & $\begin{array}{l}\odot \\
\odot\end{array}$ & $\begin{array}{l}1 \\
1\end{array}$ & $\begin{array}{lll}955425 & 3 & 54 \\
665425 & 3 & 54\end{array}$ & \\
\hline $\begin{array}{l}\odot .000 \odot \odot \odot+\odot-1.268950+6 \\
\odot .000 \odot \odot \odot+\odot-1.268900+6\end{array}$ & $\begin{array}{l}\odot \\
0\end{array}$ & $\begin{array}{l}\odot \\
\odot\end{array}$ & $\begin{array}{l}1 \\
1\end{array}$ & $\begin{array}{lll}955425 & 3 & 55 \\
655425 & 3 & 55\end{array}$ & \\
\hline $\begin{array}{l}0.00 \odot \odot \odot \odot+\odot-1.438050+6 \\
0.0000 \odot \odot+\odot-1.438100+6\end{array}$ & $\begin{array}{l}0 \\
\odot\end{array}$ & $\begin{array}{l}\odot \\
\odot\end{array}$ & $\begin{array}{l}1 \\
1\end{array}$ & $\begin{array}{lll}945425 & 3 & 56 \\
645425 & 3 & 56\end{array}$ & \\
\hline$\odot . \odot \odot \odot \odot \odot \odot+\odot-1.548780+6$ & $\begin{array}{l}\odot \\
\odot\end{array}$ & $\begin{array}{l}\odot \\
\odot\end{array}$ & $\begin{array}{l}1 \\
1\end{array}$ & $\begin{array}{lll}945425 & 3 & 57 \\
635425 & 3 & 57\end{array}$ & \\
\hline $\begin{array}{l}\odot . \odot \odot \odot \odot \odot \odot+\odot-1.62869 \odot+6 \\
\odot .0 \odot \odot \odot \odot \odot+\odot-1.62870 \odot+6\end{array}$ & $\begin{array}{l}0 \\
\odot\end{array}$ & $\begin{array}{l}\odot \\
\odot\end{array}$ & $\begin{array}{l}1 \\
1\end{array}$ & $\begin{array}{lll}935425 & 3 & 58 \\
625425 & 3 & 58\end{array}$ & \\
\hline $\begin{array}{l}\odot .0000 \odot \odot+\odot-1.690020+6 \\
\odot .0000 \odot \odot+\odot-1.69000 \odot+6\end{array}$ & $\begin{array}{l}0 \\
\odot\end{array}$ & $\begin{array}{l}\odot \\
\odot\end{array}$ & $\begin{array}{l}1 \\
1\end{array}$ & $\begin{array}{lll}935425 & 3 & 59 \\
615425 & 3 & 59\end{array}$ & \\
\hline
\end{tabular}




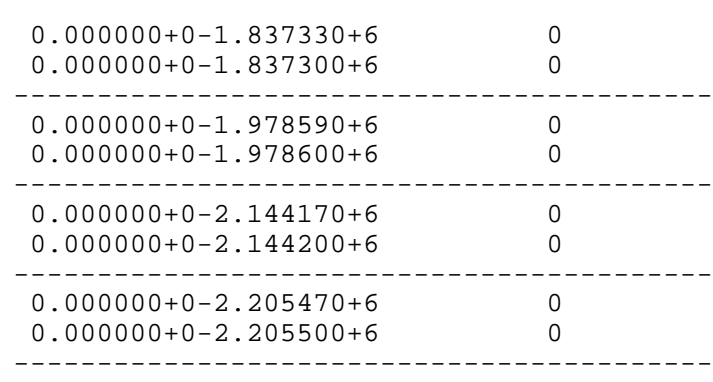

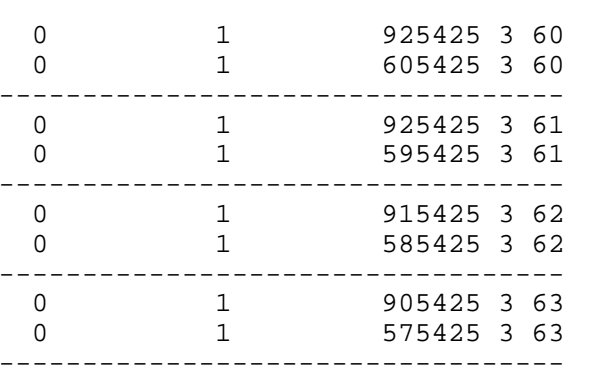

5425364 only in VII.1 5425365 only in VII.1 5425366 only in VII.1 5425367 only in VII.1 5425368 only in VII.1 5425369 only in VII.1

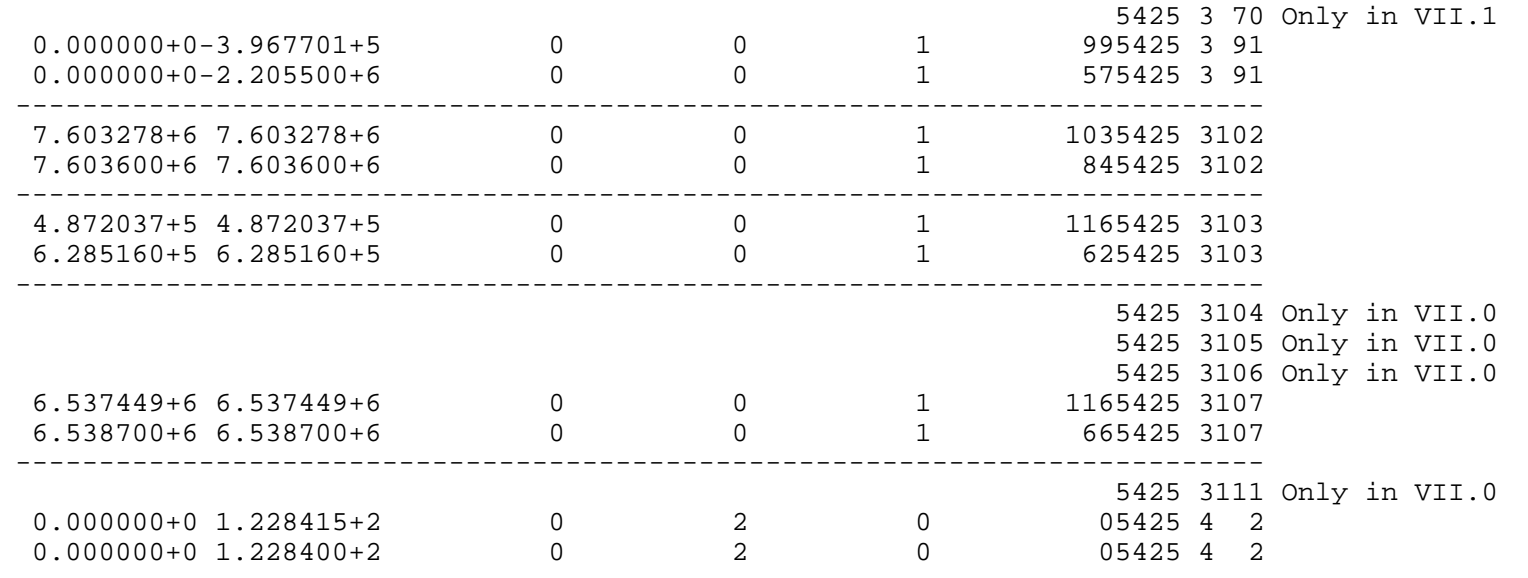

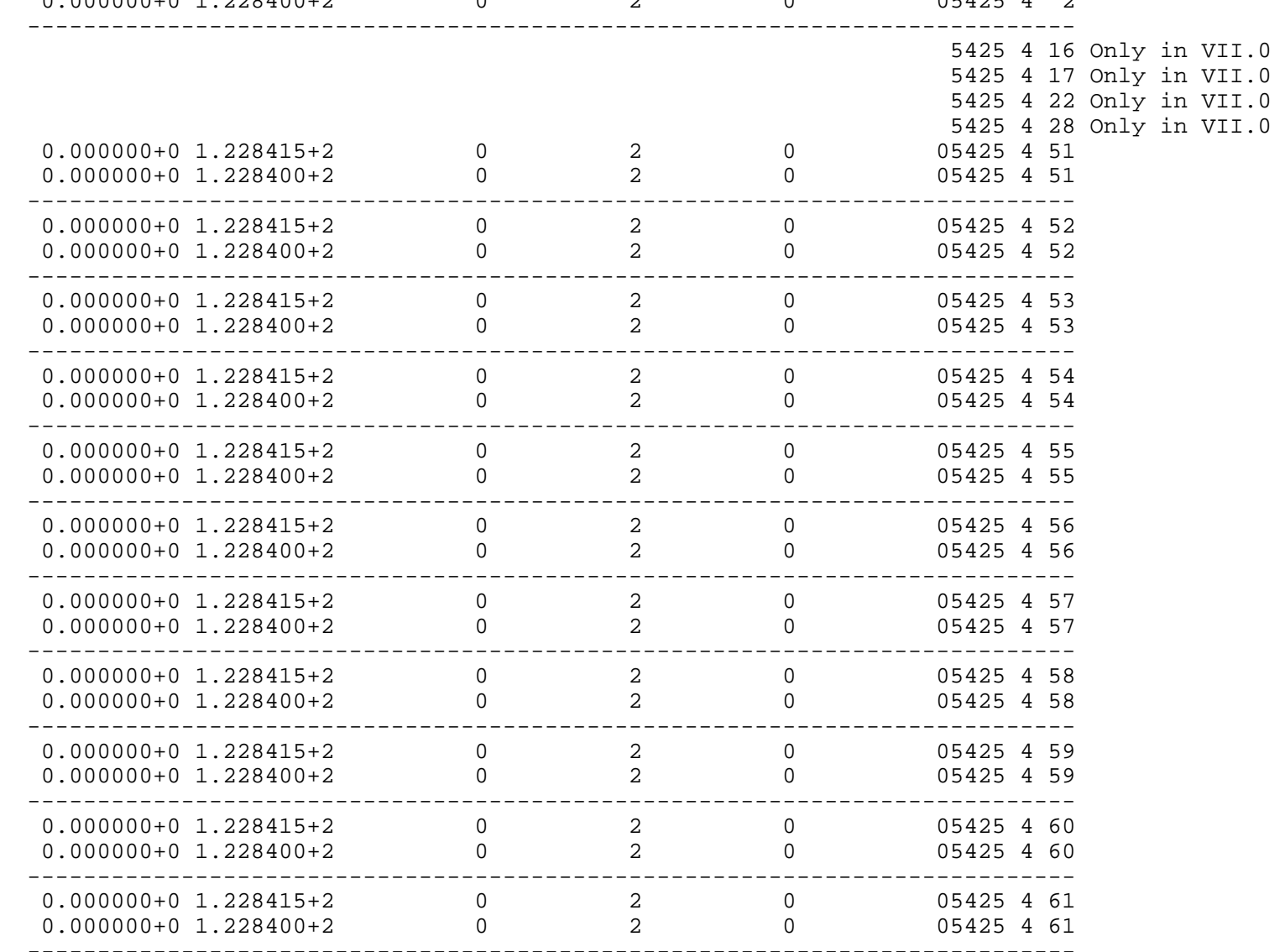




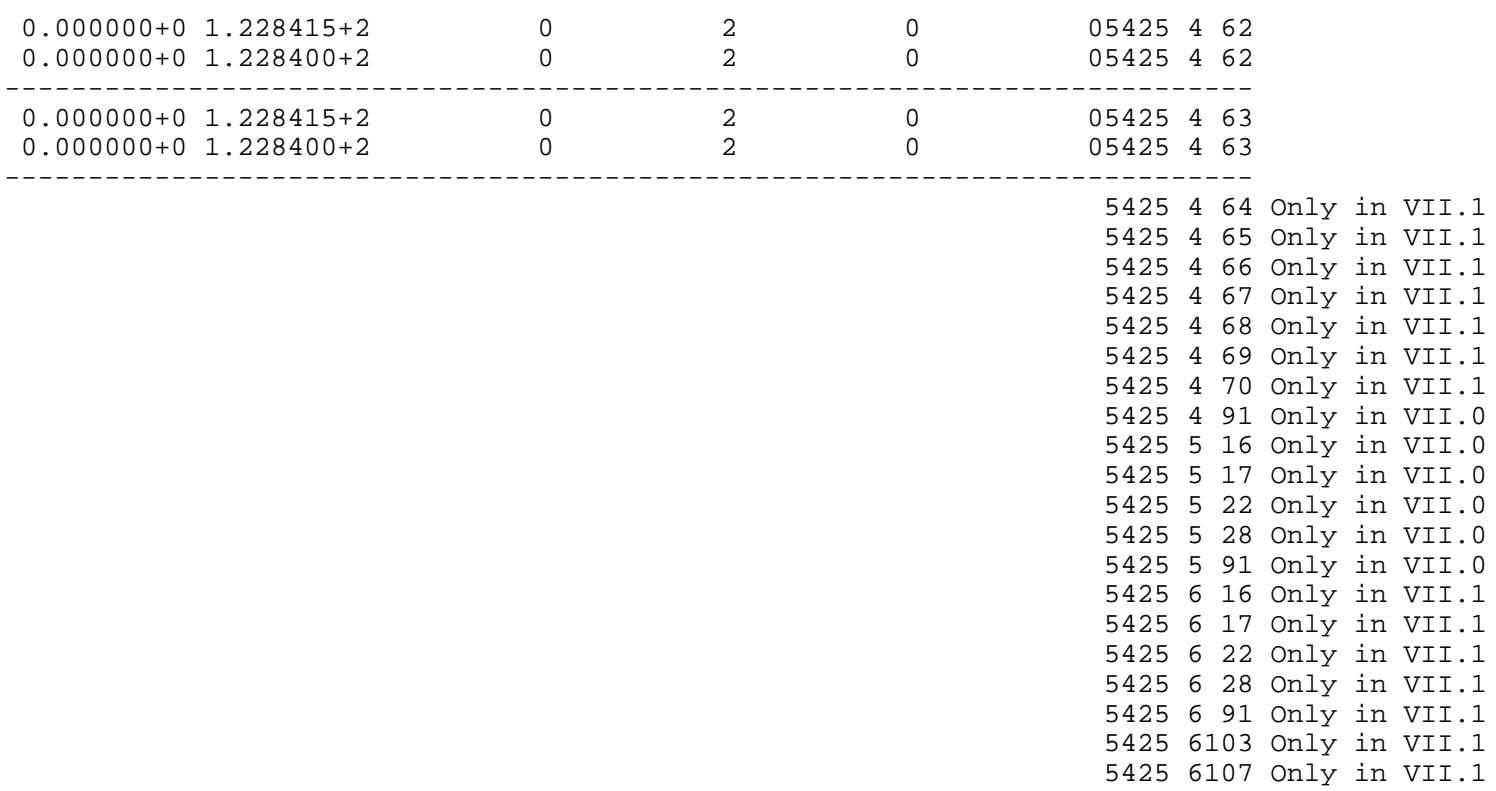

$54-\mathrm{Xe}-126$

$9.797740-40.794621-43.059650-5$

9.797740-4 9.794620-4 3.059650-5 $3.432840-6 \quad 1.104380-7 \quad 1.640590-95431442$

$54-\mathrm{Xe}-128$

$* * * * * * * * * * * * * * * * * * * * * * * * * * * * * * * * * * * * * * * * * * * * * * * * * * * * * * * * * * * * * * * * * * * * * * * * * * *$

$54-\mathrm{Xe}-129$

$* * * * * * * * * * * * * * * * * * * * * * * * * * * * * * * * * * * * * * * * * * * * * * * * * * * * * * * * * * * * * * * * * * * * * * * * * * *$

$54-\mathrm{Xe}-130$

$-9.645501-7-1.118900-6 \quad 544342$

$-9.645500-7-1.118900-6 \quad 544342$

$54-\mathrm{Xe}-131$

$\begin{array}{llllllll}4.578500+5 & 9.976030-7 & 8.76563-10 & 6.867700+5 & 9.921361-7 & 9.12219-105446 & 6 & 24\end{array}$ $\begin{array}{llllllll}4.578500+5 & 9.976030-7 & 8.76563-10 & 6.867700+5 & 9.921360-7 & 9.12219-105446 & 6 & 24\end{array}$

(5)

$55-\mathrm{Cs}-134$

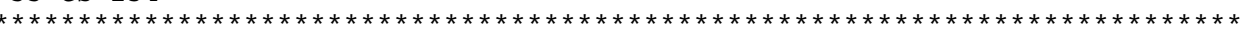

$55-$ Cs -135

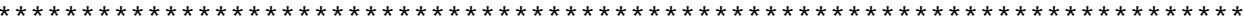

55 - Cs -136

$55-\mathrm{Cs}-137$

$* * * * * * * * * * * * * * * * * * * * * * * * * * * * * * * * * * * * * * * * * * * * * * * * * * * * * * * * * * * * * * * * * * * * * * * * * * *$

$56-\mathrm{Ba}-130$

$* * * * * * * * * * * * * * * * * * * * * * * * * * * * * * * * * * * * * * * * * * * * * * * * * * * * * * * * * * * * * * * * * * * * * * * * * * * * *$

$56-\mathrm{Ba}-132$

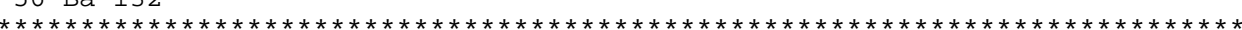


$56-\mathrm{Ba}-133$

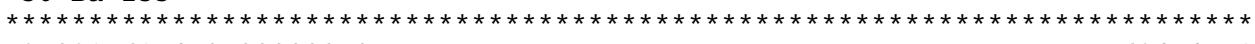

$-9.831761-40.000000+\odot \quad 5634451$

$-9.831760-40.000000+\odot \quad 5634451$

$\begin{array}{llllllll}5.444400+5 & 9.815529-7 & 2.03305-10 & 8.166700+5 & 4.819580-7 & 1.29712-105634 & 6 & 41\end{array}$ $5.444400+5 \quad 9.815530-7 \quad 2.03305-10 \quad 8.166700+5 \quad 4.819580-7 \quad 1.29712-105634 \quad 641$

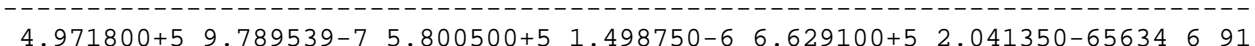
$4.971800+5 \quad 9.789540-7 \quad 5.800500+5 \quad 1.498750-6 \quad 6.629100+5 \quad 2.041350-65634 \quad 691$ $56-\mathrm{Ba}-134$

$56-\mathrm{Ba}-135$

$* * * * * * * * * * * * * * * * * * * * * * * * * * * * * * * * * * * * * * * * * * * * * * * * * * * * * * * * * * * * * * * * * * * * * * * * * * *$

$56-\mathrm{Ba}-136$

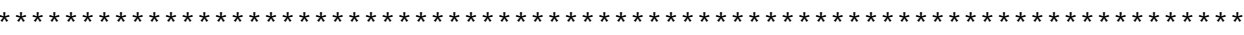

$56-\mathrm{Ba}-137$

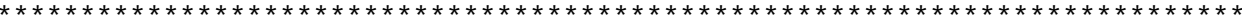

$8.485280+6 \quad 1.024070-3 \quad 9.000000+6 \quad 1.030470-3 \quad 1.000000+7 \quad 9.989931-456463102$ $8.485280+6 \quad 1.024070-3 \quad 9.000000+6 \quad 1.030470-3 \quad 1.000000+7 \quad 9.989930-456463102$ 56-Ba-138

$56-\mathrm{Ba}-140$

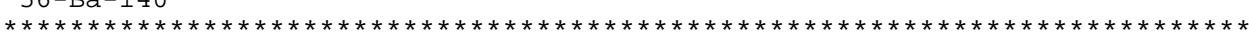
$57-\mathrm{La}-138$

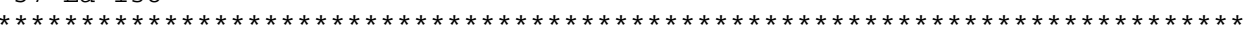

$57-$ La -139

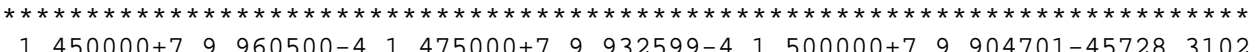
$1.450000+7 \quad 9.960500-4 \quad 1.475000+7 \quad 9.932600-4 \quad 1.500000+7 \quad 9.904700-457283102$

6. 6.503900-4 9.969300-4 6.318900-5 9.014300-6 $7.826100-7 \quad 7.331300-85728442$

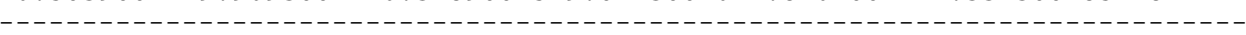

$57-\mathrm{La}-140$

$* * * * * * * * * * * * * * * * * * * * * * * * * * * * * * * * * * * * * * * * * * * * * * * * * * * * * * * * * * * * * * * * * * * * * * * * * * *$

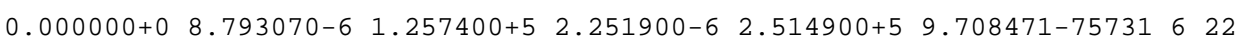
$0.000000+\odot \quad 8.793070-6 \quad 1.257400+5 \quad 2.251900-6 \quad 2.514900+5 \quad 9.708470-75731622$

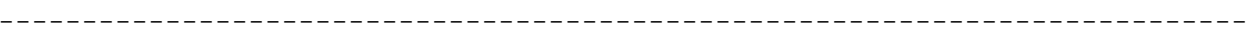
$58-\mathrm{Ce}-136$

$* * * * * * * * * * * * * * * * * * * * * * * * * * * * * * * * * * * * * * * * * * * * * * * * * * * * * * * * * * * * * * * * * * * * * * * * * * * *$

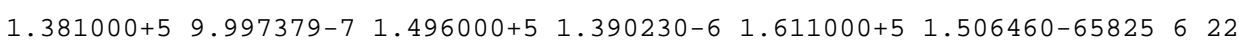
$1.381000+5 \quad 9.997380-7 \quad 1.496000+5 \quad 1.390230-6 \quad 1.611000+5 \quad 1.506460-65825622$ 58-Ce-138 Ce-138

-1.950180-6 $9.758051-7 \quad 4.866210-6 \quad 6.953170-6 \quad 1.012650-5 \quad 1.208540-55831451$ -1.950180-6 9.758050-7 4.866210-6 6.953170-6 $1.012650-5$ 1.208540-55831 451

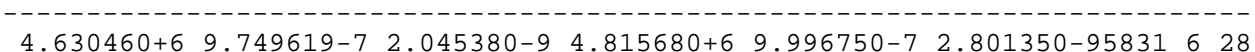
$\begin{array}{llllllll}4.630460+6 & 9.749620-7 & 2.045380-9 & 4.815680+6 & 9.996750-7 & 2.801350-95831 & 6 & 28\end{array}$ $6.381600+51.198730-6 \quad 7.445200+5 \quad 9.779779-7 \quad 8.508800+5 \quad 7.797550-758316649$ $6.381600+5 \quad 1.198730-6 \quad 7.445200+5 \quad 9.779780-7 \quad 8.508800+5 \quad 7.797550-758316649$

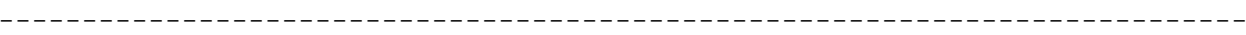
$58-\mathrm{Ce}-139$

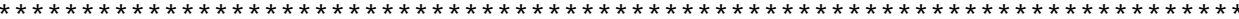

6.262330-3 1.963680-3 5.131870-3 2.447670-3 9.897019-4 4.556340-45834 455 $6.262330-3$ 1.963680-3 5.131870-3 2.447670-3 9.897020-4 4.556340-45834 455

$6.263150-31.963700-35.132310-3 \quad 2.448080-39.896239-4 \quad 4.555990-45834456$ $6.263150-3 \quad 1.963700-3 \quad 5.132310-3 \quad 2.448080-3 \quad 9.896240-4 \quad 4.555990-45834456$

$7.397900+51.960720-79.863800+59.698661-79232980+67.660690-75834616$ $\begin{array}{llllllll}7.397900+5 & 1.960720-7 & 9.863800+5 & 9.698660-7 & 1.232980+6 & 7.660690-75834 & 6 & 16\end{array}$ 1. $\begin{array}{llllllll}1.097000+5 & 2.235010-6 & 1.280000+5 & 9.777521-7 & 1.462000+5 & 7.189340-75834 & 6 & 28\end{array}$ $\begin{array}{llllll}1.097000+5 & 2.235010-6 & 1.280000+5 & 9.777520-7 & 1.462000+5 & 7.189340-75834 \\ 6 & 28\end{array}$ $\odot .0 \odot \odot \odot \odot \odot+\odot ~ 9.724901-7-6.29609-15 \quad 7.716000+4 \quad 2.552690-6 \quad 3.48698-145834691$ $0.000000+09.724900-7-6.29609-15 \quad 7.716000+4 \quad 2.552690-6 \quad 3.48698-145834691$ $2.571000+5 \quad 9.632751-7 \quad 2.769000+5 \quad 7.239150-7 \quad 2.966000+5 \quad 5.127830-758346649$ 
$2.571000+5 \quad 9.632750-7 \quad 2.769000+5 \quad 7.239150-7 \quad 2.966000+5 \quad 5.127830-758346649$ $58-\mathrm{Ce}-140$

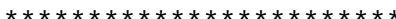

$0.000000+0-3.467580-4 \quad 0.000000+0-2.728410-4 \quad 0.000000+\odot-9.874479-75837454$ $0.000000+\odot-3.467580-4 \quad 0.000000+0-2.728410-4 \quad 0.000000+0-9.874480-75837454$ $\odot .000000+\odot-2.620740-3 \quad 0.000000+0-2.434110-3 \quad 0.000000+0-9.838320-45837464$ $58-\mathrm{Ce}-141$

5

$3.350000+2 \quad 3.0000000+\odot \quad 1.082857+0 \quad 9.828570-1 \quad 1.000000-1 \quad 0.000000+058402151$ $3.350000+2 \quad 3.000000+01.082857-1 \quad 9.828570-1 \quad 1.000000-1 \quad 0.000000+058402151$

$58-\mathrm{Ce}-142$

$* * * * * * * * * * * * * * * * * * * * * * * * * * * * * * * * * * * * * * * * * * * * * * * * * * * * * * * * * * * * * * * * * * * * * * * * * * *$

$58-\mathrm{Ce}-143$

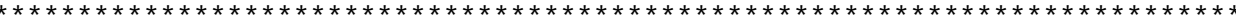

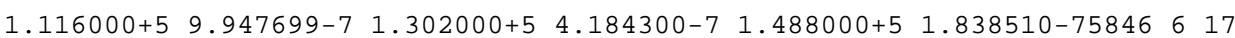
$1.116000+5 \quad 9.947700-7 \quad 1.302000+5 \quad 4.184300-7 \quad 1.488000+5 \quad 1.838510-75846 \quad 6 \quad 17$

$3.706700+59.943529-7-6.41340-154.324500+57.258800-76.80303-165846691$ $3.706700+5$ 9.943530-7-6.41340-15 4.324500+5 7.258800-7 6.80303-165846 691

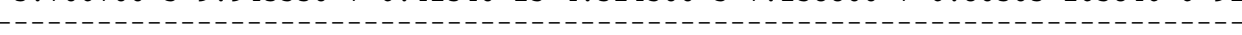

$58-\mathrm{Ce}-144$

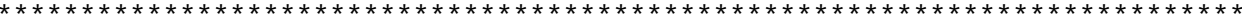

$59-\operatorname{Pr}-141$

$59-\operatorname{Pr}-142$

$* * * * * * * * * * * * * * * * * * * * * * * * * * * * * * * * * * * * * * * * * * * * * * * * * * * * * * * * * * * * * * * * * * * * * * * * * * *$

$59-\mathrm{Pr}-143$

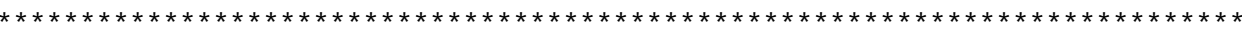

$60-\mathrm{Nd}-142$

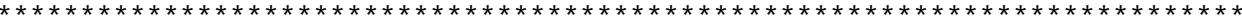

$2.524000+5 \quad 1.119510-6 \quad 2.692000+5 \quad 9.952799-7 \quad 2.861000+5 \quad 8.584830-76025628$ $2.524000+5 \quad 1.119510-6 \quad 2.692000+5 \quad 9.952800-7 \quad 2.861000+5 \quad 8.584830-76025628$

$5.420500+5 \quad 9.837599-7 \quad 1.47261-14 \quad 6.022800+5 \quad 9.721490-7 \quad 1.09859-156025691$ $\begin{array}{lllllllll}5.420500+5 & 9.837600-7 & 1.47261-14 & 6.022800+5 & 9.721490-7 & 1.09859-156025 & 6 & 91\end{array}$

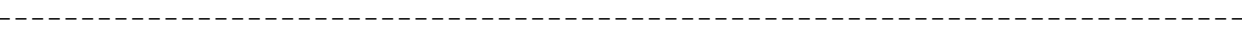
$60-\mathrm{Nd}-143$

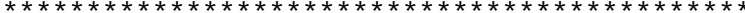

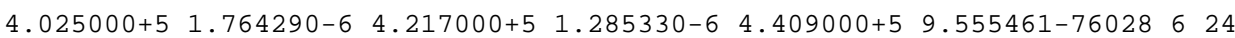
$4.025000+5 \quad 1.764290-6 \quad 4.217000+5 \quad 1.285330-6 \quad 4.409000+5 \quad 9.555460-76028624$ _. _ _ _ _ _ _ _. $4.955900+5 \quad 9.764721-7-7.28759-15 \quad 5.575400+5 \quad 7.706030-7-1.47853-146028691$ $4.955900+59.764720-7-7.28759-15 \quad 5.575400+5 \quad 7.706030-7-1.47853-146028691$

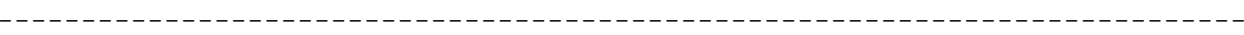
$60-\mathrm{Nd}-144$

$9.830000+4 \quad 1.169130-6 \quad 1.147000+5 \quad 9.809841-7 \quad 1.311000+5 \quad 9.029900-76031616$ $9.830000+4 \quad 1.169130-6 \quad 1.147000+5 \quad 9.809840-7 \quad 1.311000+5 \quad 9.029900-76031616$

$5.341900+51.096380-6590557-155.935400+59.928279-7-2.91084-156031691$ $5.341900+5$ 1.096380-6 5.90557-15 5.935400+5 9.928280-7-2.91084-156031 691

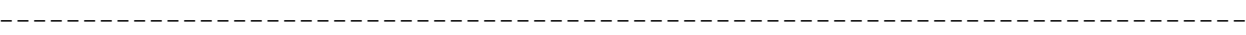
$60-\mathrm{Nd}-145$

$* * * * * * * * * * * * * * * * * * * * * * * * * * * * * * * * * * * * * * * * * * * * * * * * * * * * * * * * * * * * * * * * * * * * * * * * * * * * * * *$

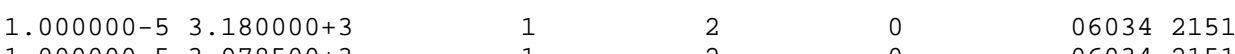

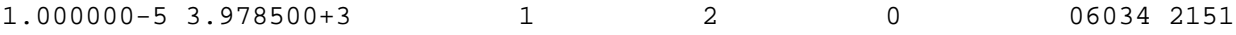

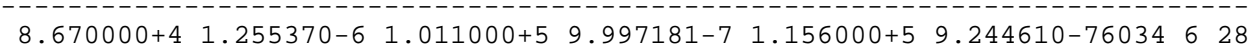
$8.670000+4 \quad 1.255370-6 \quad 1.011000+5 \quad 9.997180-7 \quad 1.156000+5 \quad 9.244610-76034 \quad 628$

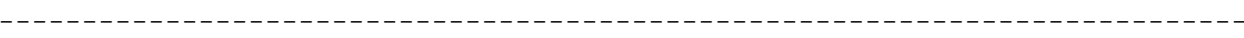
$60-\mathrm{Nd}-146$

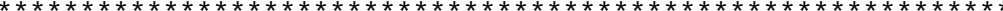

$\begin{array}{lllllll}5.164600+6 & 9.600529-7 & 1.192980-7 & 1.426010-8 & 5.304190+6 & 5.431390-76037 & 6649\end{array}$ $5.164600+6 \quad 9.600530-7 \quad 1.192980-7 \quad 1.426010-8 \quad 5.304190+6 \quad 5.431390-76037 \quad 6649$ 60-12-147 $60-\mathrm{Nd}-147$

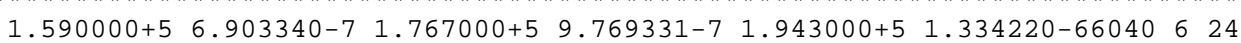
$1.590000+5 \quad 6.903340-7 \quad 1.767000+5 \quad 9.769330-7 \quad 1.943000+5 \quad 1.334220-66040624$ 


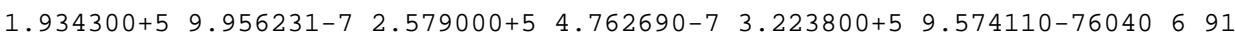
$1.934300+5 \quad 9.956230-7 \quad 2.579000+5 \quad 4.762690-7 \quad 3.223800+5 \quad 9.574110-76040691$ $60-\mathrm{Nd}-148$

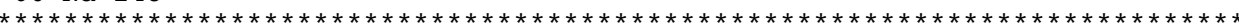
$\odot .000000+\odot \quad \odot .000000+\odot \quad 2.436600+5 \quad 9.670611-7 \quad 1.69996-15 \quad 0.000000+06043628$ $\odot .000000+\odot \quad 0.0000000+\odot \quad 2.436600+5 \quad 9.670610-7 \quad 1.69996-15 \quad 0.000000+06043 \quad 628$

$60-\mathrm{Nd}-150$

$* * * * * * * * * * * * * * * * * * * * * * * * * * * * * * * * * * * * * * * * * * * * * * * * * * * * * * * * * * * * * * * * * * * * * * * * * * * * * *$

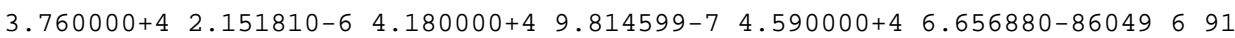
3.760000+4 2.151810-6 $4.180000+4 \quad 9.814600-7 \quad 4.590000+4 \quad 6.656880-86049691$

$61-\mathrm{Pm}-147$

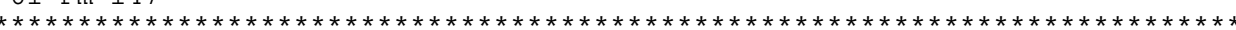

$\begin{array}{lllllll}1.400000+7 & 9.981249-4 & 1.401780+7 & 1.013990-3 & 1.450000+7 & 1.534330-36149 & 3104\end{array}$

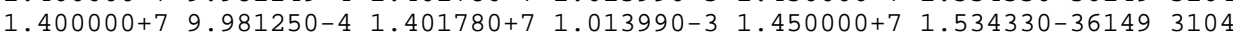

$0.000000+0 \quad 0.000000+0 \quad 1.250000+5 \quad 6.941960-7 \quad 2.500000+5 \quad 9.796699-76149 \quad 5 \quad 17$ $0.000000+\odot \quad 0.000000+\odot \quad 1.250000+5 \quad 6.941960-7 \quad 2.500000+5 \quad 9.796700-76149517$

$\odot .000000+\odot \quad 0.000000+\odot \quad 1.250000+5 \quad 1.109710-6 \quad 2.500000+5 \quad 9.825191-76149528$ $0.000000+\odot \quad 0.000000+0 \quad 1.250000+5 \quad 1.109710-6 \quad 2.500000+5 \quad 9.825190-76149528$

$61-\mathrm{Pm}-148$

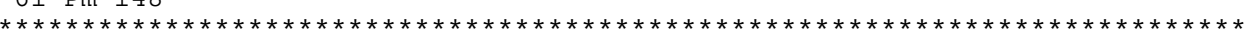

2.614140-1 1.039110-1-2.615020-3 $1.228630-3 \quad 2.107490-7 \quad 9.610141-76152442$ 2.614140-1 1.039110-1-2.615020-3 $1.228630-3 \quad 2.107490-7 \quad 9.610140-76152442$

$61-\mathrm{Pm}-148 \mathrm{~m}$

$* * * * * * * * * * * * * * * * * * * * * * * * * * * * * * * * * * * * * * * * * * * * * * * * * * * * * * * * * * * * * * * * * * * * * * * * * * *$ $61-\mathrm{Pm}-149$

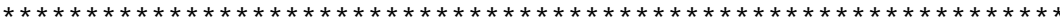

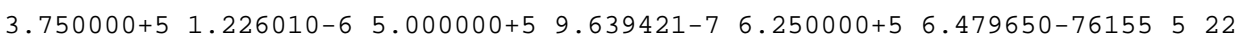
$3.750000+5 \quad 1.226010-6 \quad 5.000000+5 \quad 9.639420-7 \quad 6.250000+5 \quad 6.479650-76155522$

-

$61-\mathrm{Pm}-151$

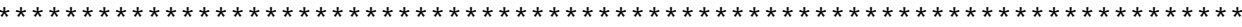

$9.760000+4 \quad 8.708460-8 \quad 1.058000+5 \quad 7.305680-7 \quad 1.139000+5 \quad 9.586749-76161622$ $9.760000+4 \quad 8.708460-8 \quad 1.058000+5 \quad 7.305680-7 \quad 1.139000+5 \quad 9.586750-76161622$

$3.121300+5$ 1.785190-6 $1.066960-9 \quad 4.682000+5 \quad 9.803710-7 \quad 5.53040-106161628$

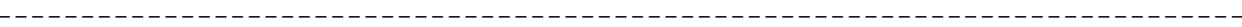

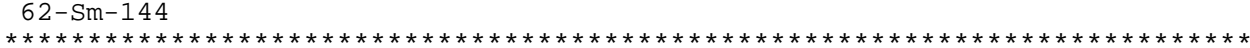

$9.326400+5 \quad 1.019010-6 \quad 1.98448-14 \quad 1.017420+6 \quad 9.668159-7 \quad 2.62158-146225691$ $9.326400+5 \quad 1.019010-6 \quad 1.98448-14 \quad 1.017420+6 \quad 9.668160-7 \quad 2.62158-146225691$

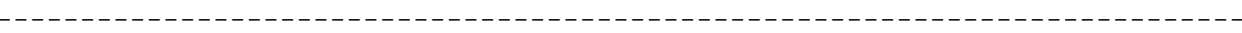
$62-\mathrm{Sm}-147$

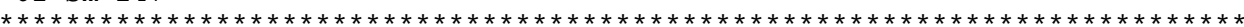

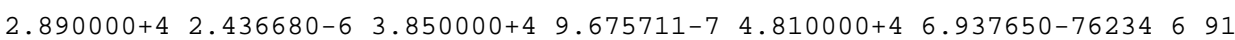
$2.890000+4 \quad 2.436680-6 \quad 3.850000+4 \quad 9.675710-7 \quad 4.810000+4 \quad 6.937650-76234691$

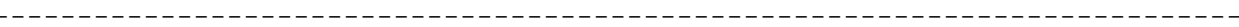
$62-\mathrm{Sm}-148$

$* * * * * * * * * * * * * * * * * * * * * * * * * * * * * * * * * * * * * * * * * * * * * * * * * * * * * * * * * * * * * * * * * * * * * * * * * * *$

$-4.355610-41.254870-31.365730-3-9.981419-4 \quad 6237458$ $-4.355610-41.254870-31.365730-3-9.981420-4 \quad 6237458$

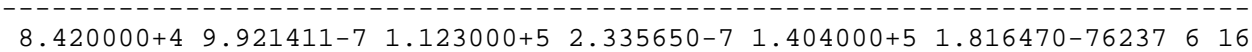
$8.420000+4 \quad 9.921410-7 \quad 1.123000+5 \quad 2.335650-7 \quad 1.404000+5 \quad 1.816470-76237 \quad 6 \quad 16$

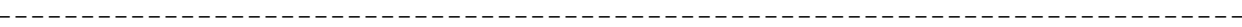

$2.783000+5 \quad 5.799850-6 \quad 3.092000+5 \quad 1.121050-6 \quad 3.401000+5 \quad 9.865259-76237624$ $2.783000+5 \quad 5.799850-6 \quad 3.092000+5 \quad 1.121050-6 \quad 3.401000+5 \quad 9.865260-76237 \quad 6 \quad 24$

2. $399000+59.893701-72.665000+57521800-72.932000+55.615800-76237691$ $\begin{array}{llllllll}2.399000+5 & 9.893700-7 & 2.665000+5 & 7.521800-7 & 2.932000+5 & 5.615800-76237 & 6 & 91\end{array}$

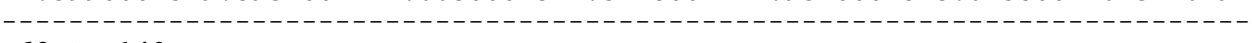

$62-\mathrm{Sm}-149$

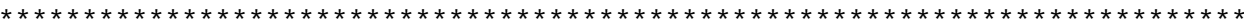

$0.000000+\odot \quad 0.0000000+\odot \quad 0.0000000+\odot \quad 0.0000000+\odot \quad 4.4890000+5 \quad 9.801551-76240628$ $\odot .00000 \odot+\odot \quad 0.00000 \odot+\odot \quad \odot .0000000+\odot \quad 0.000000+\odot \quad 4.489000+5 \quad 9.801550-76240628$

5.340000+4 $1.096830-5 \quad 7.120000+4 \quad 3.664950-6 \quad 8.900000+4 \quad 9.671641-76240641$ $5.340000+4 \quad 1.096830-5 \quad 7.120000+4 \quad 3.664950-6 \quad 8.900000+4 \quad 9.671640-76240 \quad 6 \quad 41$ 
$1.716000+5 \quad 1.810990-6 \quad 1.859000+5 \quad 1.543600-6 \quad 2.145000+5 \quad 9.899341-76240601$ $\begin{array}{llllllll}1.716000+5 & 1.810990-6 & 1.859000+5 & 1.543600-6 & 2.145000+5 & 9.899340-76240 & 6 & 91\end{array}$ $0.000000+0 \quad 5.29382-102.159500+5 \quad 7.557770-7 \quad 4.319000+5 \quad 9.618580-762406649$ $62-\mathrm{Sm}-150$

每

$\begin{array}{lllllll}1.095000+5 & 9.737749-7 & 1.217000+5 & 9.680350-7 & 1.338000+5 & 9.527930-762436 & 64\end{array}$ $\begin{array}{llllllll}1.095000+5 & 9.737750-7 & 1.217000+5 & 9.680350-7 & 1.338000+5 & 9.527930-76243 \quad 6 & 24\end{array}$

-

$62-\mathrm{Sm}-151$

$* * * * * * * * * * * * * * * * * * * * * * * * * * * * * * * * * * * * * * * * * * * * * * * * * * * * * * * * * * * * * * * * * * * * * * * * * * *$

$62-\mathrm{Sm}-152$

等

$62-\mathrm{Sm}-153$ $1.752000+5 \quad 1.950450-6 \quad 1.898000+5 \quad 1.437260-6 \quad 2.044000+5 \quad 9.934210-762526649$ $62-\mathrm{Sm}-154$

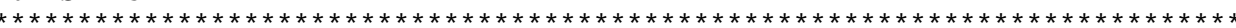

3.917340-2-1.222060-5 6.054040-3-9.783501-4-1.690800-3 0.000000+06255 451 3.917340-2-1.222060-5 6.054040-3-9.783500-4-1.690800-3 $0.000000+06255451$ $4.903300+59.790421-7-2.03340-14 \quad 0.000000+\odot \quad 0.000000+\odot \quad 0.000000+06255628$ 4.903300+5 9.790420-7-2.03340-14 $0.000000+\odot \quad 0.0000 \odot \odot+\odot \quad 0.000000+06255628$

$63-\mathrm{Eu}-151$

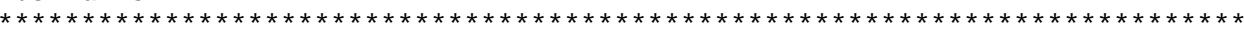
$63-\mathrm{Eu}-152$

$3.00000 \odot+4 \quad 4.4859000+\odot \quad \odot .00000 \odot+\odot \quad 9.868901-4 \quad 1.60000 \odot-1 \quad 0.0000 \odot \odot \odot+063282151$ $3.000000+4 \quad 4.485900+\odot \quad 0.000000+0 \quad 9.868900-4 \quad 1.600000-1 \quad 0.0000000+063282151$

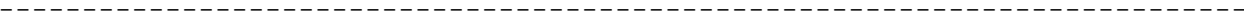

$\odot .000000+\odot \quad \odot .000000+\odot \quad 1.250000+5 \quad 8.612070-7 \quad 2.500000+5 \quad 9.608571-76328522$ $0.000000+\odot \quad 0.000000+\odot \quad 1.250000+5 \quad 8.612070-7 \quad 2.500000++5 \quad 9.608570-76328522$

$63-\mathrm{Eu}-153$

\begin{tabular}{|c|c|c|c|c|}
\hline 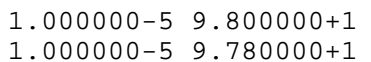 & $\begin{array}{l}1 \\
1\end{array}$ & $\begin{array}{l}2 \\
2\end{array}$ & $\begin{array}{l}\odot \\
\odot\end{array}$ & $\begin{array}{ll}06331 & 2151 \\
06331 & 2151\end{array}$ \\
\hline
\end{tabular}

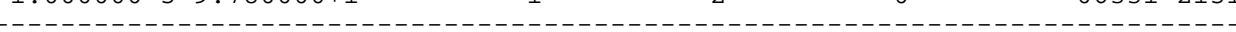

$63-\mathrm{Eu}-154$
$* * * * * * * * * * * * * * * * * * * * * * * * * * * * * * * * * * * * * * * * * * * * * * * * * * * * * * * * * * * * * * * * * * * * * * * * * *$

$63-\mathrm{Eu}-155$

$63-\mathrm{Eu}-156$

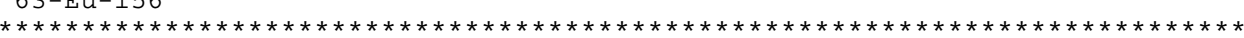

$63-\mathrm{Eu}-157$

*******************t.

$0.000000+0 \quad 3.928740-7 \quad 1.88371-15 \quad 1.979100+5 \quad 9.999439-7 \quad 7.67853-116343617$ ๑. $000000+\odot \quad 3.928740-7 \quad 1.88371-15 \quad 1.979100+5 \quad 9.999440-7 \quad 7.67853-116343617$

$9.616861-71.12027-120.000000+00$.

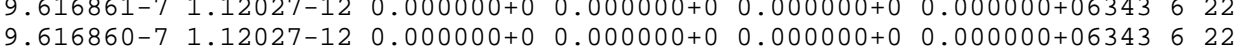

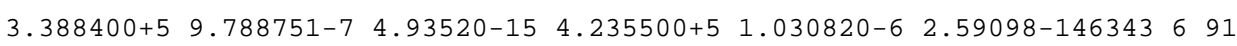
$\begin{array}{lllllll}3.388400+5 & 9.788750-7 & 4.93520-15 & 4.235500+5 & 1.030820-6 & 2.59098-146343 & 6\end{array}$

64-Gd-152

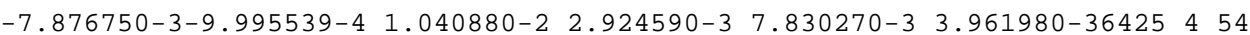

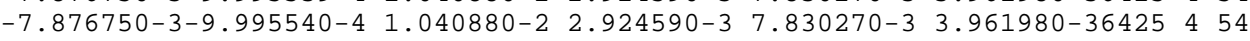
$64-\mathrm{Gd}-153$

(5)

$\begin{array}{llllllll}4.873700+5 & 9.887279-7 & 9.00119-10 & 7.310500+5 & 3.597220-7 & 3.75925-106428 & 6 & 24\end{array}$ $4.873700+5 \quad 9.887280-7 \quad 9.00119-10 \quad 7.310500+5 \quad 3.597220-7 \quad 3.75925-106428624$

$2.811750+60.716611-75.69256-143.124170+61.488850-6-1.75920-14642864$ $2.811750+6 \quad 9.716610-7 \quad 5.69256-14 \quad 3.124170+6 \quad 1.488850-6-1.75920-146428 \quad 6 \quad 41$ $64-\mathrm{Gd}-154$ 
$* * * * * * * * * * * * * * * * * * * * * * * * * * * * * * * * * * * * * * * * * * * * * * * * * * * * * * * * * * * * * * * * * * * * * * * * * * *$ $\begin{array}{lllllll}2.208000+5 & 9.884239-7 & 2.392000+5 & 5.819140-7 & 2.576000+5 & 2.719030-764316649\end{array}$ $\begin{array}{llllllll}2.208000+5 & 9.884240-7 & 2.392000+5 & 5.819140-7 & 2.576000+5 & 2.719030-764316649\end{array}$ $64-\mathrm{Gd}-155$

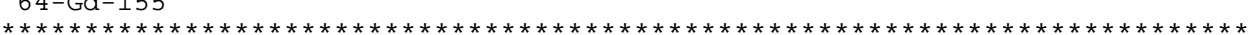

$\begin{array}{llllll}7.800000+6 & 9.573019-7 & 8.000000+6 & 7.043740-7 & 8.5000000+6 & 3.348030-76434365\end{array}$ $7.800000+6 \quad 9.573020-7 \quad 8.0000000+6 \quad 7.043740-7 \quad 8.500000+6 \quad 3.348030-76434365$

$\odot .000000+\odot \quad 1.781480-7 \quad 2.81344-16 \quad 3.128500+5 \quad 9.964861-7 \quad 2.01016-106434 \quad 6 \quad 17$

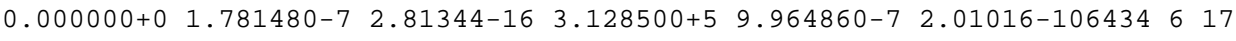

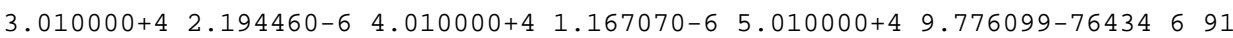
$3.010000+4 \quad 2.194460-6 \quad 4.010000+4 \quad 1.167070-6 \quad 5.010000+4 \quad 9.776100-76434 \quad 691$ $64-G d-156$

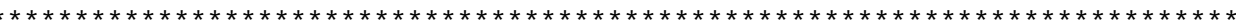

$2.797430-1$ 6.646970-2 2.183240-2-2.341120-2-9.774721-4-2.186820-264374 52 $2.797430-1$ 6.646970-2 2.183240-2-2.341120-2-9.774720-4-2.186820-26437 452

0.000000

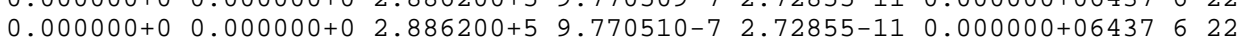

$6.299500+51.010650-6-1.20581-147.349400+50.655849-7-9.68713-15643761$ $6.299500+51.010650-6-1.20581-14 \quad 7.349400+5$ 9.655850-7-9.68713-156437 691 $64-\mathrm{Gd}-157$

$* * * * * * * * * * * * * * * * * * * * * * * * * * * * * * * * * * * * * * * * * * * * * * * * * * * * * * * * * * * * * * * * * * * * * * * * * * *$

$\begin{array}{llllll}3.140000-2 & 2.000000+\odot & 4.374700-4 & 1.060000-1 & 0.000000++\odot & 0.0000000+064402151\end{array}$

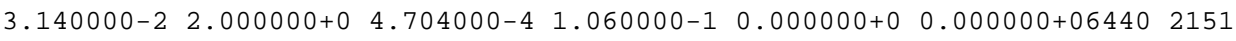

1. $000000+1-1.207630-8 \quad 1.258925+1-2.217340-81.584803+1-9.657271-7644033$

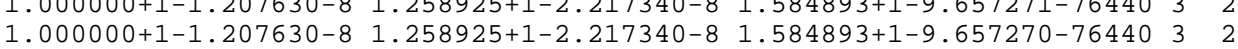

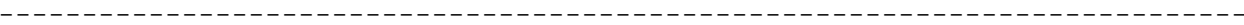

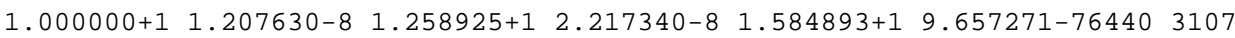
$\begin{array}{llllllll}1.000000+1 & 1.207630-8 & 1.258925+1 & 2.217340-8 & 1.584893+1 & 9.657270-76440 & 3107\end{array}$

$2.634620-39.927161-4 \quad 6440451$

$2.634620-39.927160-4 \quad 6440451$

2.

$2.481000+5 \quad 9.532600-7 \quad 2.605000+5 \quad 9.563699-7 \quad 2.729000+5 \quad 9.018220-76440 \quad 6 \quad 22$ $2.481000+5 \quad 9.532600-7 \quad 2.605000+5 \quad 9.563700-7 \quad 2.729000+5 \quad 9.018220-76440622$

$2.089700+5 \quad 8.995850-7 \quad 2.52282-14 \quad 3.134600+5 \quad 9.966679-7 \quad 7.84240-156440691$ $2.089700+5 \quad 8.995850-7 \quad 2.52282-14 \quad 3.134600+5 \quad 9.966680-7 \quad 7.84240-156440691$

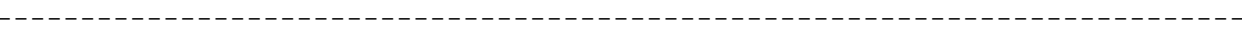
$64-\mathrm{Gd}-158$

$\odot .000000+\odot \quad 1.375310-7-6.82073-16 \quad 2.919600+5 \quad 9.871781-7 \quad 2.53966-116443624$ $0.000000+\odot 1.375310-7-6.82073-16 \quad 2.919600+5 \quad 9.871780-7 \quad 2.53966-116443624$

$4.148340+6 \quad 9.867661-7 \quad 4.056630-9 \quad 4.307890+6 \quad 1.114760-6 \quad 5.234410-964436649$ $4.148340+6 \quad 9.867660-7 \quad 4.056630-9 \quad 4.307890+6 \quad 1.114760-6 \quad 5.234410-964436649$

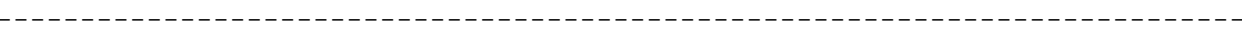
$64-\mathrm{Gd}-160$

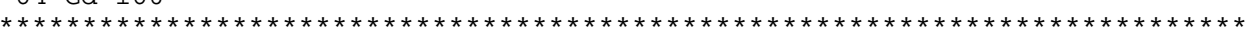

$2.501840+6 \quad 1.014900-6-1.15336-14 \quad 2.814570+6 \quad 9.656829-7 \quad 1.24388-146449641$ $2.501840+6 \quad 1.014900-6-1.15336-14 \quad 2.814570+6 \quad 9.656830-7 \quad 1.24388-146449641$

$\odot .000000+09.829261-7-5.00844-15 \quad 7.852000+4 \quad 3.309290-6 \quad 6.88051-146449691$ $\odot .000000+\odot$ 9.829260-7-5.00844-15 7.852000+4 $3.309290-6 \quad 6.88051-146449691$

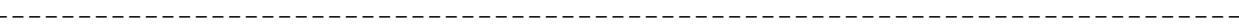
$65-\mathrm{Tb}-159$ $65-\mathrm{Tb}-160$

$* * * * * * * * * * * * * * * * * * * * * * * * * * * * * * * * * * * * * * * * * * * * * * * * * * * * * * * * * * * * * * * * * * * * * * * * * * *$

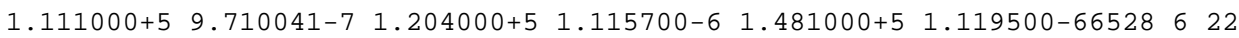

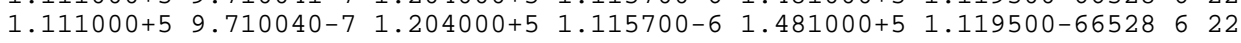
$66-$ Dy -156

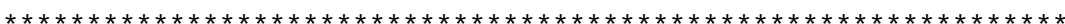

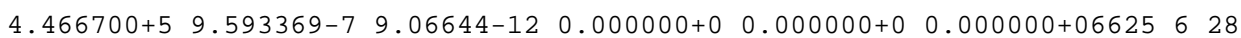

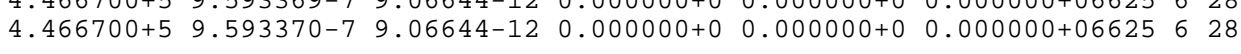
$66-$ Dy -158 


\begin{tabular}{|c|c|}
\hline 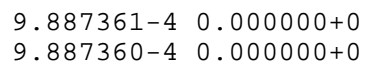 & $\begin{array}{lll}6631 & 4 & 58 \\
6631 & 4 & 58\end{array}$ \\
\hline
\end{tabular}

$66-\mathrm{Dy}-160$

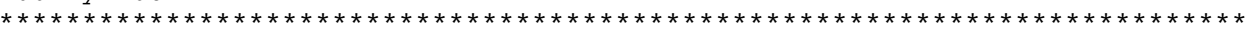

5.554200+5 1.004710-6 2.39768-15 6.479900+5 9.620099-7-2.03041-146637 691 $5.554200+5 \quad 1.004710-6 \quad 2.39768-15 \quad 6.479900+5 \quad 9.620100-7-2.03041-146637691$

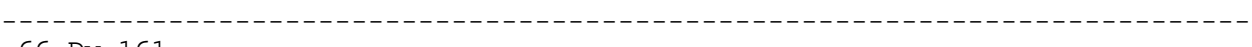

$66-$ Dy -161

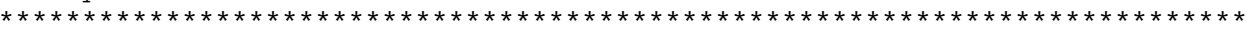
$66-\mathrm{Dy}-162$

$3.883300+5$ 9.572771-7-1.67215-14 4.530600+5 6.368730-7 1.70717-146643 691 $3.883300+59.572770-7-1.67215-14 \quad 4.530600+5 \quad 6.368730-7 \quad 1.70717-146643691$

$0.000000+0 \quad 4.072990-7 \quad 2.066600+5 \quad 9.390560-7 \quad 4.133200+5 \quad 9.901941-766436649$

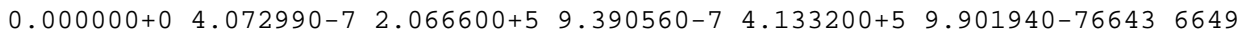

66-Dy-163 **************t.

$2.969800+5$ 1.351860-6 4.30625-11 4.454700+5 9.733139-7 4.55079-116646 622 $2.969800+5 \quad 1.351860-6 \quad 4.30625-11 \quad 4.454700+5 \quad 9.733140-7 \quad 4.55079-116646 \quad 622$

$0.000000+0 \quad 4.219390-8 \quad 2.197000+5 \quad 6.891320-7 \quad 4.394000+5 \quad 9.608131-76646 \quad 691$

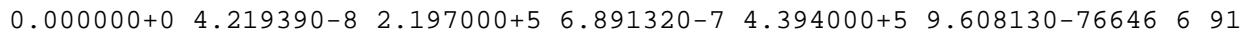
- 66 - Dy-164

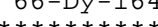

$2.077060-1$ 1.112350-1 3.778870-2 1.241820-2 3.120290-2-9.935719-46649 452 2.077060-1 1.112350-1 3.778870-2 1.241820-2 3.120290-2-9.935720-46649 452

-

$9.826639-4-7.577080-4 \quad 6649465$ $9.826640-4-7.577080-4 \quad 6649465$

4. 266560-8 5.071220-9 5.158900+6 $9.720829-7 \quad 7.208380-8 \quad 8.717750-9664966$ 4.266560-8 5.071220-9 5.158900+6 9.720830-7 $7.208380-8 \quad 8.717750-96649628$

$1.332800+6 \quad 1.108250-6 \quad 1.402950+6 \quad 1.087710-6 \quad 1.473090+69.750401-76649691$ $1.332800+6 \quad 1.108250-6 \quad 1.402950+6 \quad 1.087710-6 \quad 1.473090+6 \quad 9.750400-76649691$

$5.024340+6 \quad 8.251370-7 \quad 1.298440-7 \quad 1.551920-8 \quad 5.167890+6 \quad 9.578120-766496649$

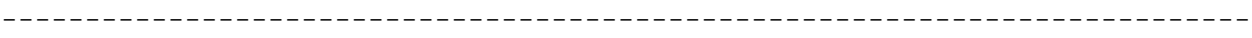

$67-\mathrm{Ho}-165$

$* * * * * * * * * * * * * * * * * * * * * * * * * * * * * * * * * * * * * * * * * * * * * * * * * * * * * * * * * * * * * * * * * * * * * * * * * * *$

$4.000000+6 \quad 2.519800-3 \quad 4.500000+6 \quad 1.507400-3 \quad 5.000000+6 \quad 9.903701-46725363$ $\begin{array}{lllllll}4.000000+6 & 2.519800-3 & 4.500000+6 & 1.507400-3 & 5.000000+6 & 9.903700-46725 & 363\end{array}$

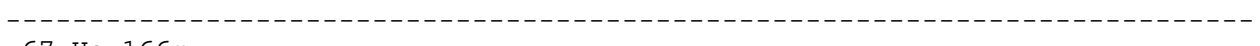

$67-\mathrm{Ho}-166 \mathrm{~m}$

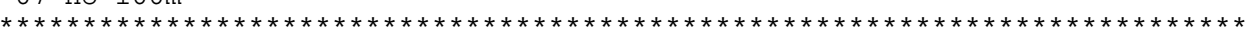

$3.298000+5 \quad 1.524700-6 \quad 3.448000+5 \quad 9.997819-7 \quad 3.598000+5 \quad 6.569480-76729624$ 3. $298000+5 \quad 1.524700-6 \quad 3.448000+5 \quad 9.997820-7 \quad 3.598000+5 \quad 6.569480-76729624$

$4.947600+59.738091-7 \quad 7.17351-127.421400+5 \quad 4.006520-7 \quad 5.37691-126729641$ $4.947600+5 \quad 9.738090-7 \quad 7.17351-12 \quad 7.421400+5 \quad 4.006520-7 \quad 5.37691-126729641$

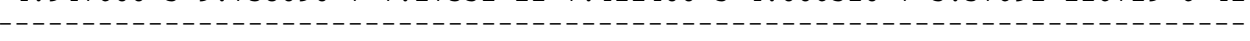
$68-\mathrm{Er}-162$

$* * * * * * * * * * * * * * * * * * * * * * * * * * * * * * * * * * * * * * * * * * * * * * * * * * * * * * * * * * * * * * * * * * * * * * * * * * *$

$68-\mathrm{Er}-164$

$68-\mathrm{Er}-166$

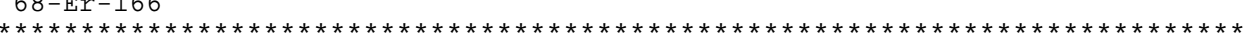

$2.500000+6 \quad 1.448530-3 \quad 3.000000+6 \quad 9.930501-4 \quad 4.000000+6 \quad 5.475800-46837374$ $2.50 \odot \odot \odot \odot+6 \quad 1.448530-3 \quad 3.0 \odot \odot \odot \odot \odot+6 \quad 9.9305 \odot \odot-4 \quad 4.0 \odot \odot \odot \odot \odot+6 \quad 5.47580 \odot-46837374$ $68-\mathrm{Er}-167$

$* * * * * * * * * * * * * * * * * * * * * * * * * * * * * * * * * * * * * * * * * * * * * * * * * * * * * * * * * * * * * * * * * * * * * * * * * * *$

$68-\mathrm{Er}-168$
$* * * * * * * * * * * * * * * * * * * * * * * * * * * * * * * * * * * * * * * * * * * * * * * * * * * * * * * * * * * * * * * * * * * * * * * * * * *$

$68-\mathrm{Er}-170$

$6.102360+2 \quad 9.730589-7 \quad 9.671590+2 \quad 1.530850-6 \quad 1.532840+3 \quad 2.398020-66849522$ $6.102360+29.730590-7 \quad 9.671590+2 \quad 1.530850-6 \quad 1.532840+32.398020-66849522$ 


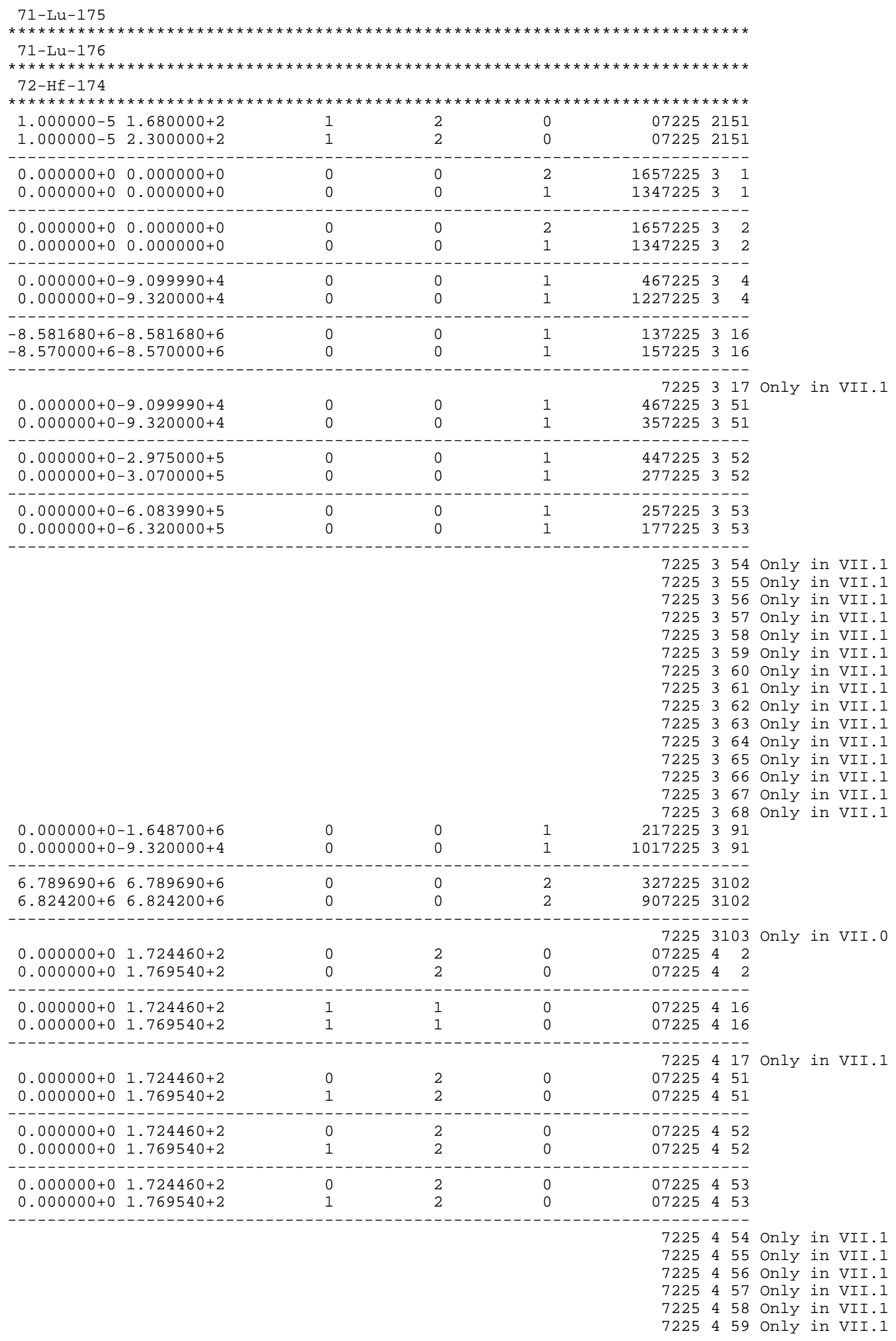




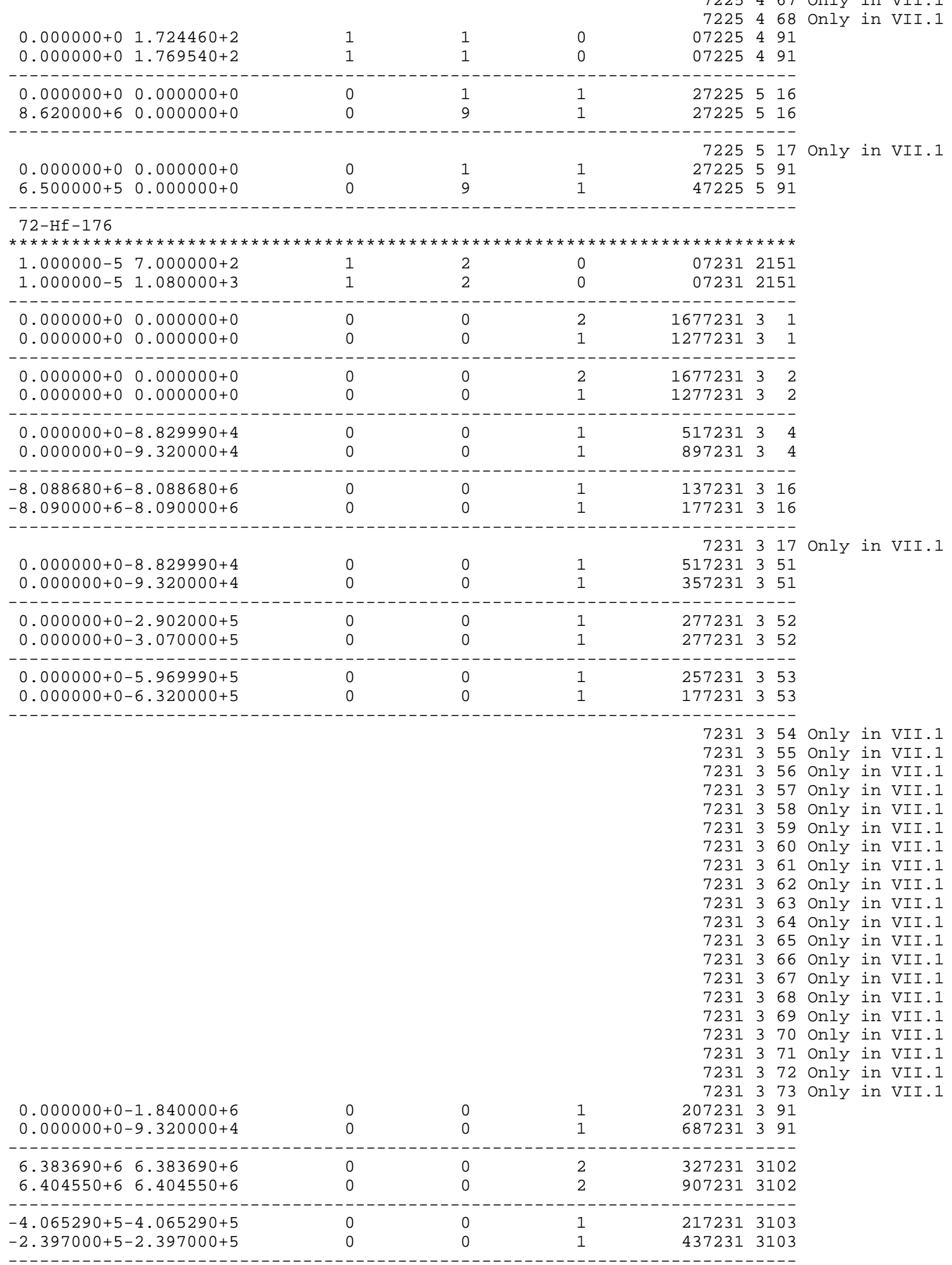




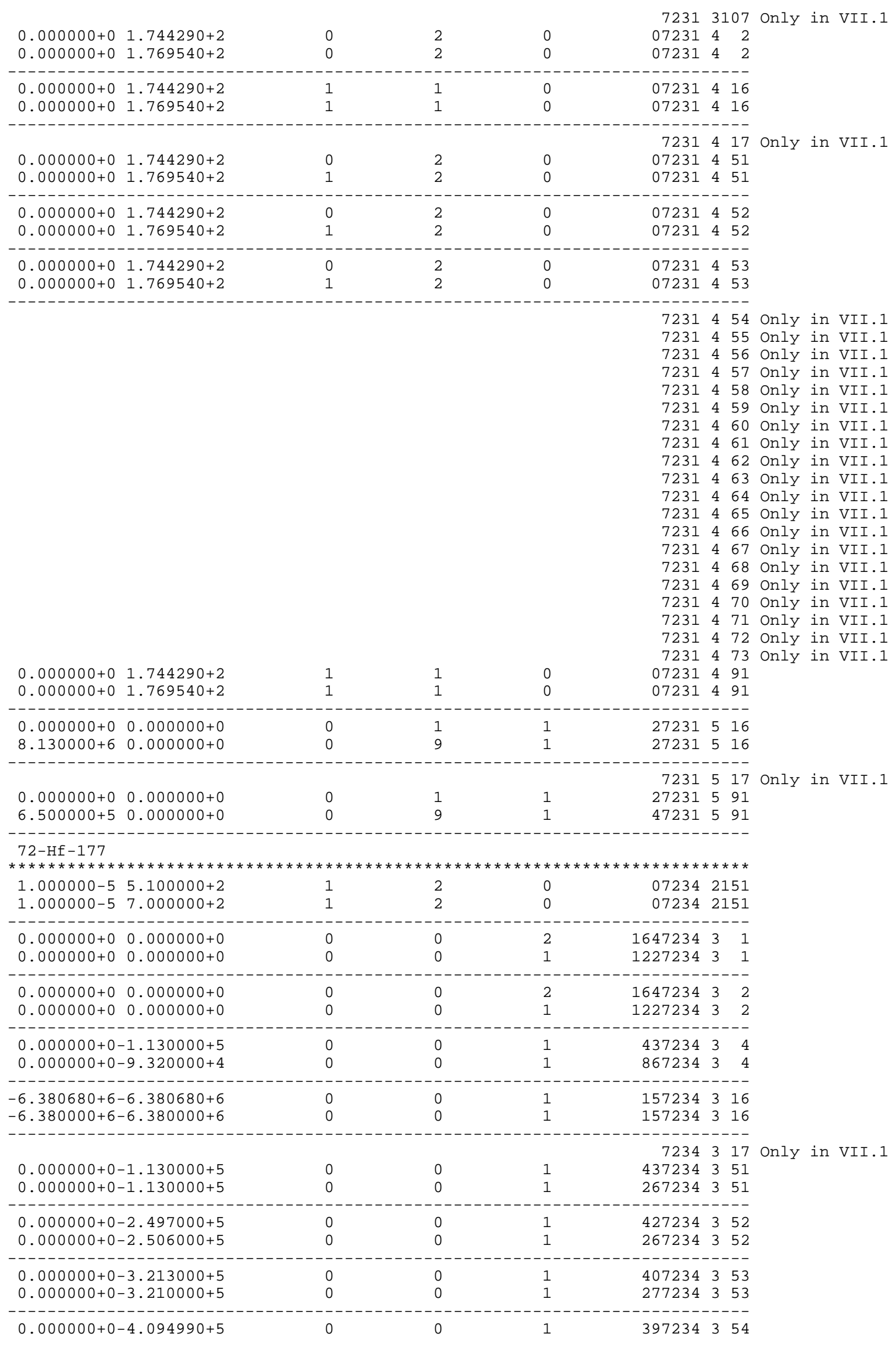




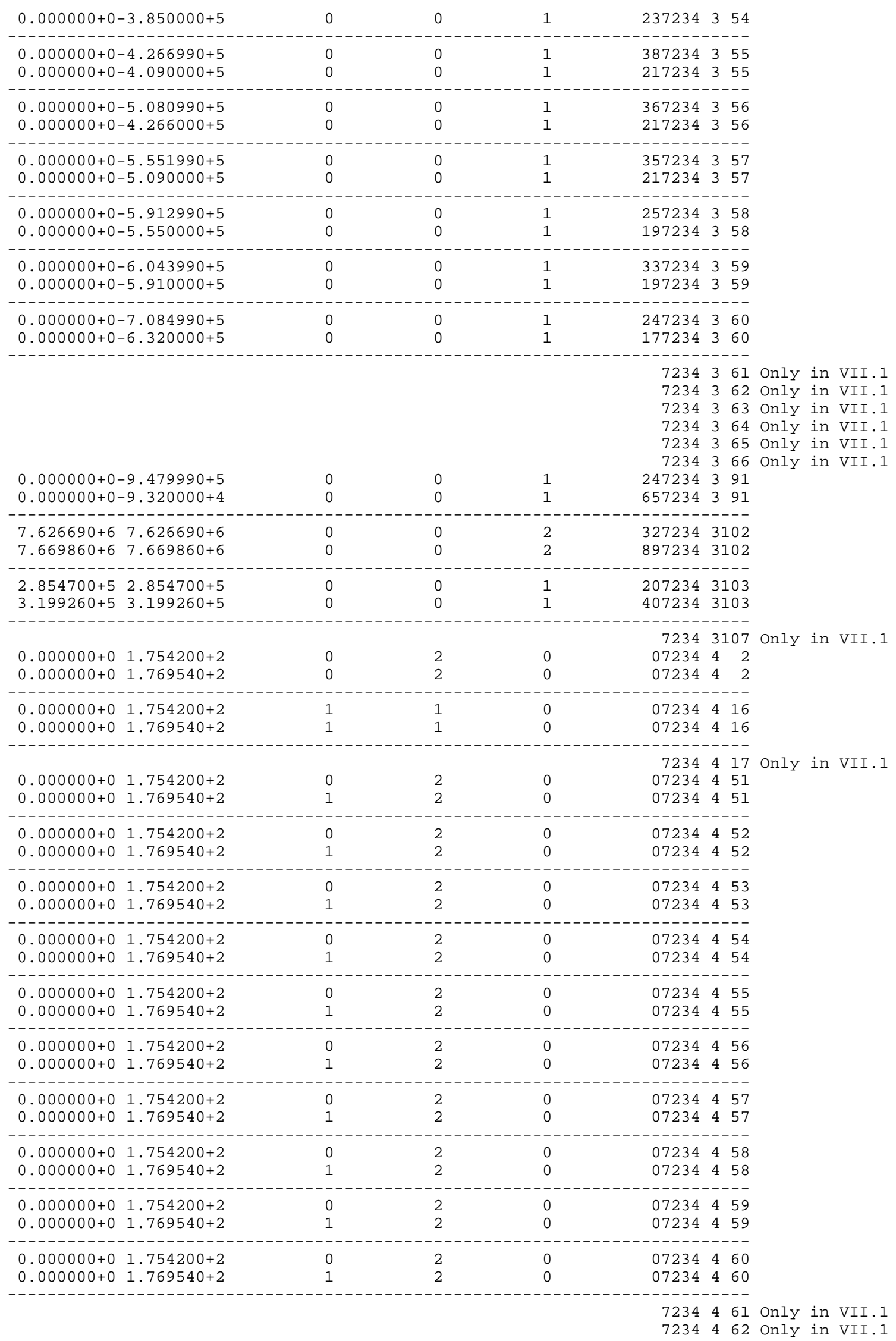




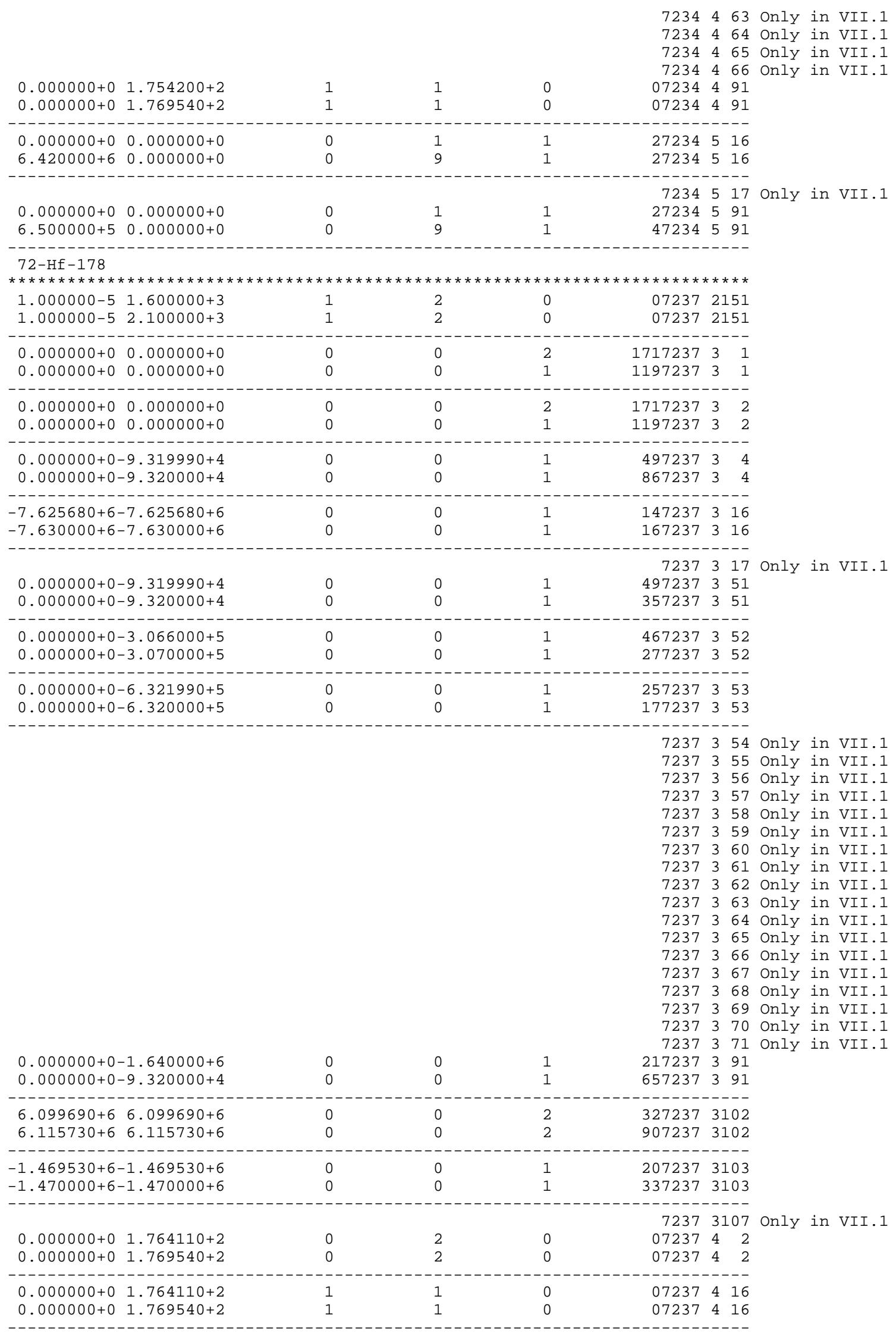




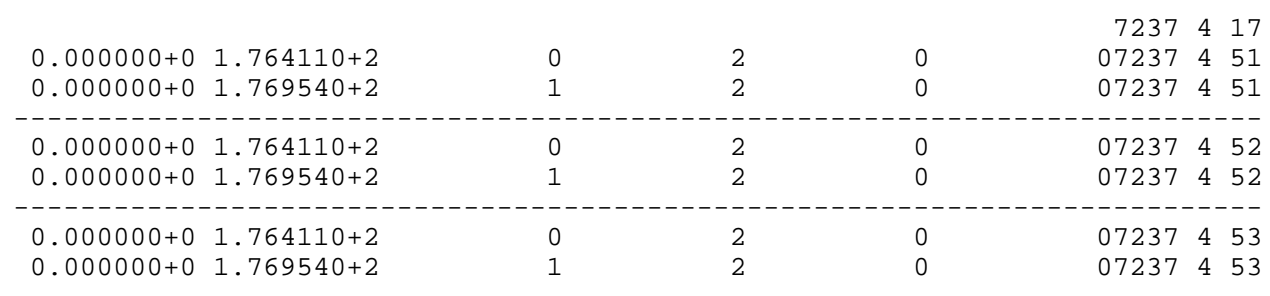

7237454 only in VII.1 7237455 Only in VII.1 7237456 only in VII.1 7237457 only in VII.1 7237458 only in VII.1 7237459 Only in VII.1 7237460 only in VII.1 7237461 Only in VII.1 7237462 only in VII.1 7237463 only in VII.1 7237464 only in VII.1 7237465 only in VII.1 7237466 only in VII.1 7237467 only in VII.1 7237468 only in VII.1 7237469 0nly in VII.1 7237470 Only in VII.1 7237471 only in VII.1

$0.000000+\odot \quad 1.764110+2$ $\odot .000000+\odot 1.769540+2$ $0.000000+0 \quad 0.000000+0$ $7.670000+6 \quad 0.000000+\odot$

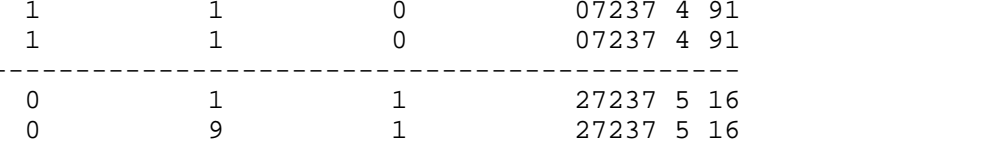

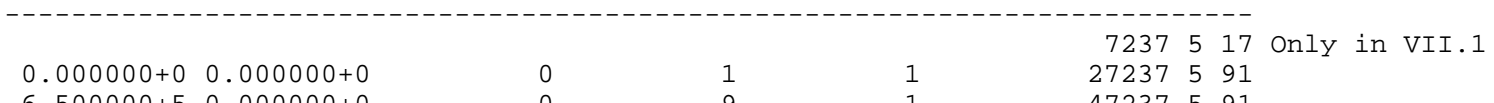
$6.500000+5$. $000000+0$

$0 \quad 9$

\section{$72-\mathrm{Hf}-179$}

$* * * * * * * * * * * * * * * * * * * * * * * * * * * * * * * *$

1. $0000000-55.100000+2$ 1. $000000-5$ 4.500000+2

1
1

$\odot .000000+\odot \quad 0.000000+\odot$ $0.000000+0 \quad 0.000000+0$

$\odot .0000 \odot \odot+\odot \odot .00000 \odot+$ $0.000000+\odot \quad 0.000000+\odot$

(2)

$\odot .0 \odot \odot \odot \odot \odot+\odot-1.22700 \odot+5$
$\odot .0 \odot \odot \odot \odot \odot+\odot-9.3200 \odot \odot+4$

-

$-6.099680+6-6.099680+6$ $-6.100000+6-6.100000+6$

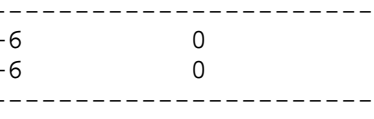

$0.000000+0-1.227000+5$ $0.0000000+0-1.130000+5$

\begin{tabular}{ll}
5 & 0 \\
5 & 0 \\
\hline 5 & 0 \\
5 & 0 \\
\hline 5 & 0 \\
5 & 0 \\
-5 & 0 \\
5 & 0 \\
\hline
\end{tabular}

$* * * * * * * * * * t *$
2
2
0
0
0
0
0
0
0
0

- . - . . . . - .

$2 \quad 1627240331$

$11127240 \quad 3 \quad 1$

$2 \quad 16272403 \quad 3$

$1 \quad 11272403 \quad 2$

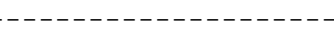

$1 \quad 3972403 \quad 3$

$1 \quad 8672403 \quad 3$

$1+157240316$

$1 \quad 127240316$

7240317 only in VII.1 397240351 267240351

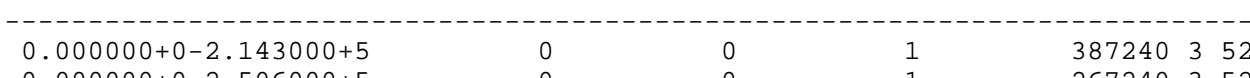

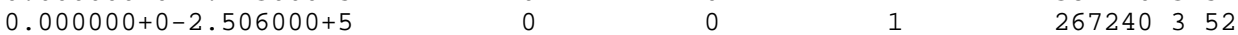

$0.000000+0-2.688000+50000377240353$

$0.000000+0-3.070000+5 \quad 00010277240353$

$0.000000+0-3.377000+5 \quad 000357240354$

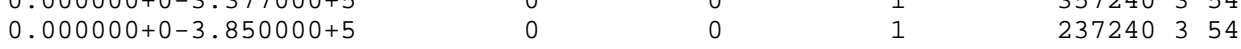

(2.

7240355 only in VII.1

7240356 only in VII. 1

7240357 only in VII.1

7240358 only in VII.1

7240359 only in VII.1

7240360 only in VII.1

7240361 only in VII.1 


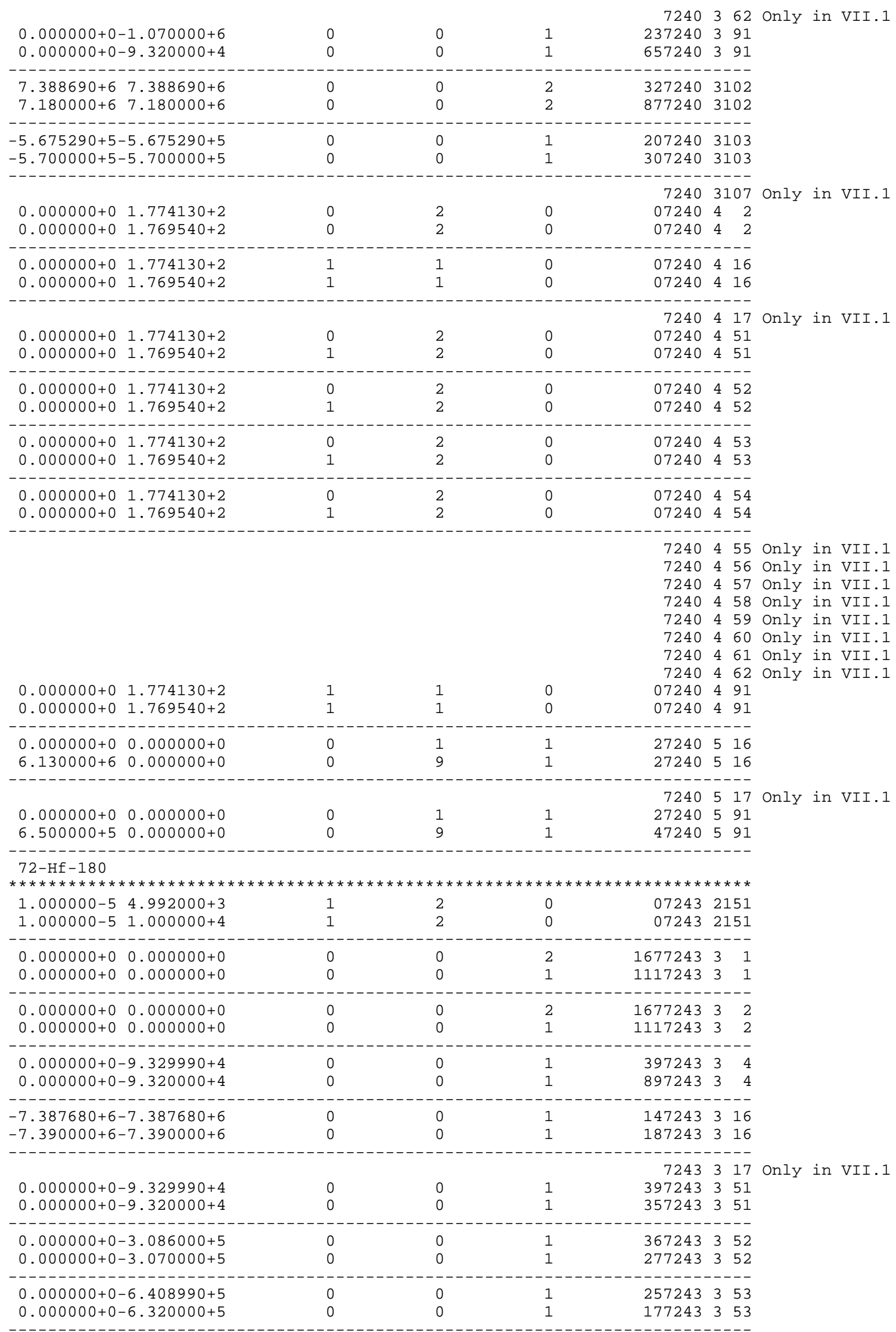




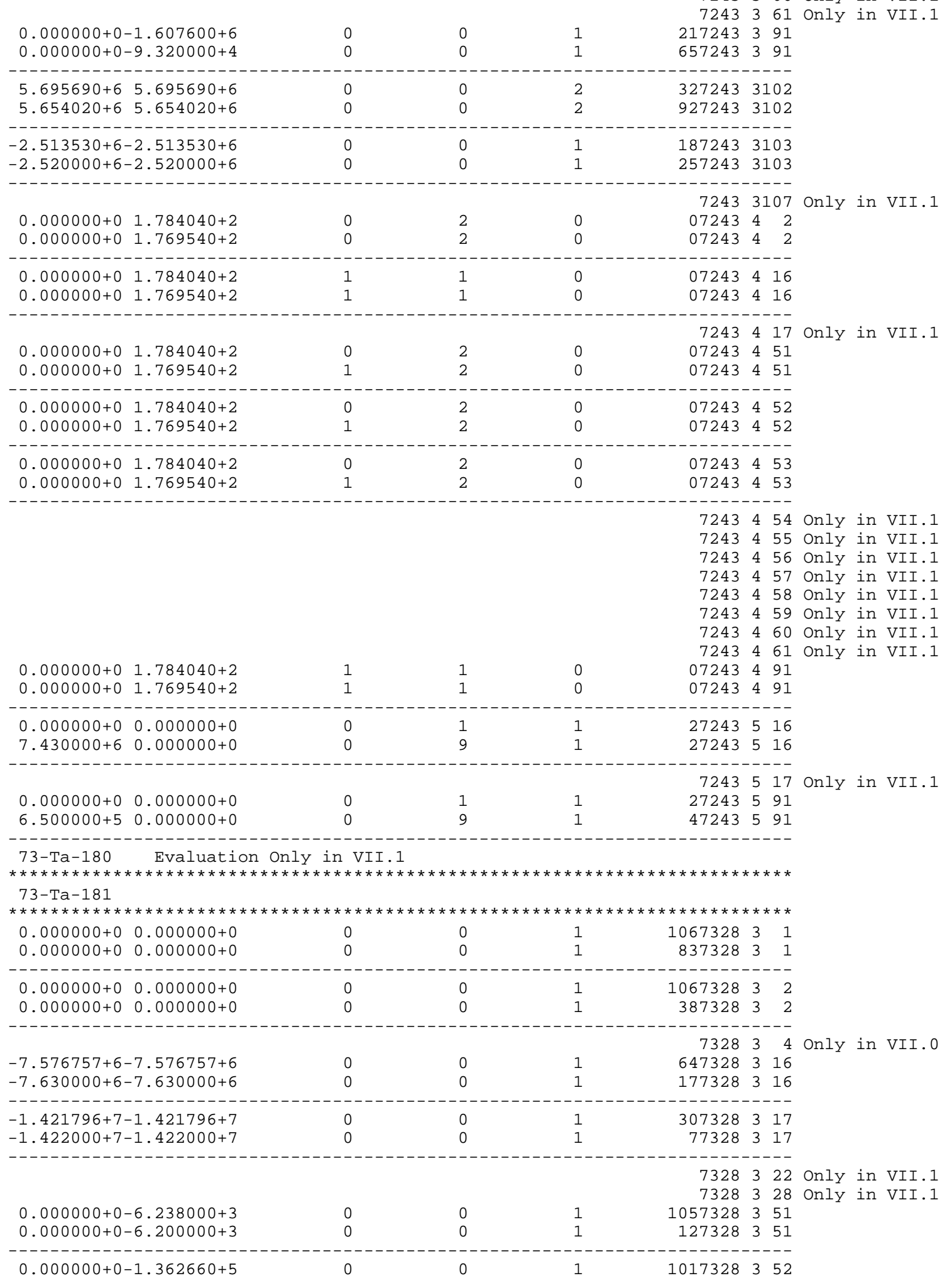




\begin{tabular}{|c|c|c|c|c|}
\hline$\odot .0000 \odot \odot+\odot-1.3610 \odot \odot+5$ & 0 & $\odot$ & 1 & 127328352 \\
\hline$\odot .0000 \odot \odot+\odot-1.58550 \odot+5$ & 0 & $\odot$ & 1 & 1017328353 \\
\hline$\odot .00000 \odot+\odot-1.586000+5$ & 0 & $\odot$ & 1 & $127328 \quad 353$ \\
\hline$\odot .0000000+\odot-3.016200+5$ & 0 & 0 & 1 & $1007328 \quad 3 \quad 54$ \\
\hline$\odot . \odot \odot \odot \odot \odot \odot+\odot-3.015 \odot \odot \odot+5$ & 0 & $\odot$ & 1 & 1073283 \\
\hline$\odot . \odot \odot \odot \odot \odot \odot+\odot-3.3754 \odot \odot+5$ & 0 & $\odot$ & 1 & $1007328 \quad 3 \quad 55$ \\
\hline$\odot .000000+\odot-3.375000+5$ & $\odot$ & $\odot$ & 1 & 107328355 \\
\hline $0.000000+0-4.821820+5$ & 0 & 0 & 1 & $997328 \quad 356$ \\
\hline$\odot .00000 \odot+\odot-4.822000+5$ & 0 & $\Theta$ & 1 & 107328356 \\
\hline $0.000000+0-4.951900+5$ & 0 & 0 & 1 & $997328 \quad 3 \quad 57$ \\
\hline$\odot .000000+\odot-4.950000+5$ & 0 & $\theta$ & 1 & $107328 \quad 3 \quad 57$ \\
\hline & & & & \\
\hline$\odot .0 \odot 0 \odot \odot \odot+\odot-5.425100+5$ & 0 & 0 & 1 & $997328 \quad 358$ \\
\hline$\odot .00 \odot \odot \odot \odot+\odot-6.200 \odot \odot \odot+5$ & 0 & $\odot$ & 1 & 107328358 \\
\hline $0.000000+0-6.152100+5$ & 0 & 0 & 1 & $987328 \quad 3 \quad 59$ \\
\hline $0.000000+0-7.200000+5$ & 0 & $\odot$ & 1 & 973283 \\
\hline & & & & \\
\hline$\odot .0000 \bullet \odot+\odot-6.190500+5$ & 0 & 0 & 1 & 987328360 \\
\hline$\odot .000000+\odot-9.250000+5$ & 0 & 0 & 1 & 773283 \\
\hline
\end{tabular}

7328361 only in VII.1 7328362 only in VII.1 7328363 only in VII.1 7328364 only in VII.1 7328365 Only in VII.1 7328366 only in VII.1 7328367 only in VII.1 7328368 only in VII.1 7328369 only in VII.1 7328370 only in VII.1

$\odot .000000+0-1.392239+6$ $0.000000+0-1.193000+6$

0
0

$6.062936+6 \quad 6.062936+6$ $6.070000+6 \quad 6.070000+6$

$\odot \quad 0 \quad 10673283102$

- . . . . . . . . . . . . . . . .

$\odot$

$-2.474042+5-2.474042+5$ $-2.387000+5-2.387000+5$

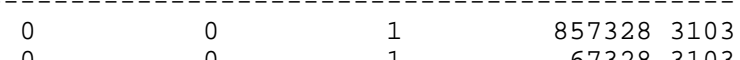

$\begin{array}{lllll}67328 & 0 & 1 & 1\end{array}$

73283104 only in VII.1 73283105 only in VII.1 73283107 only in VII.1

$0.000000+\odot \quad 1.793936+2$ $\odot .000000+\odot 1.794000+2$

0

$07328 \quad 4 \quad 2$

$07328 \quad 4 \quad 2$

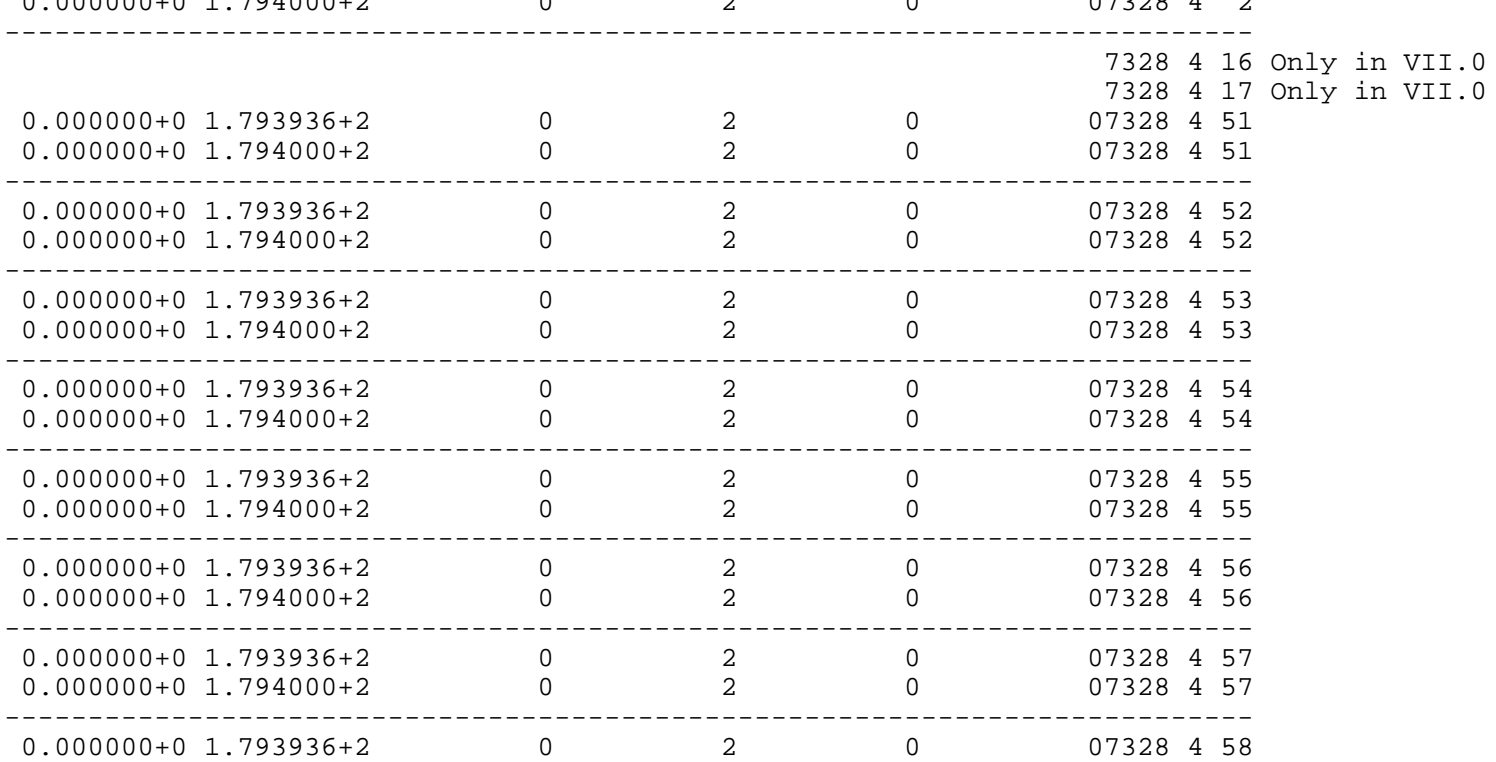




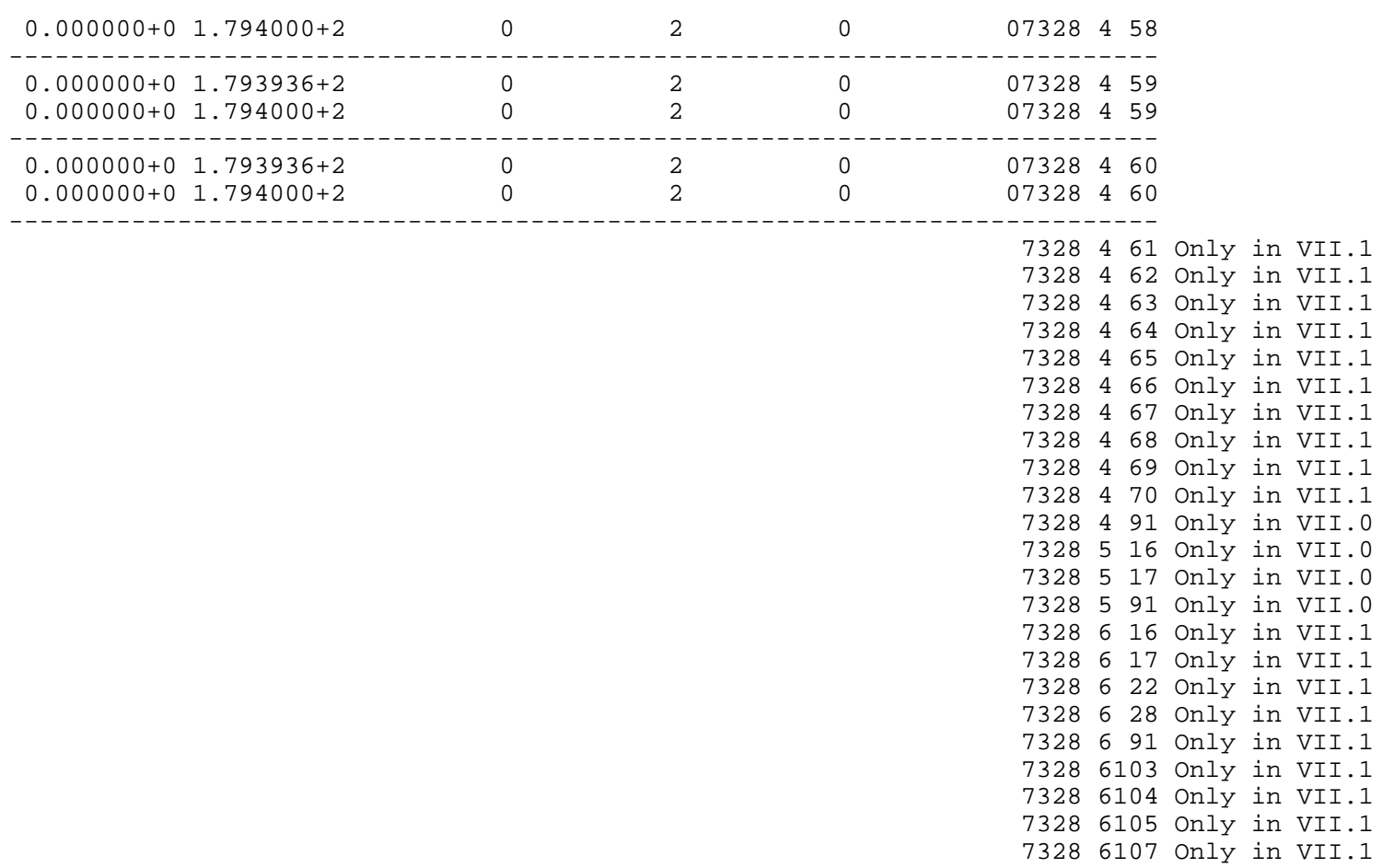

73-Ta-182

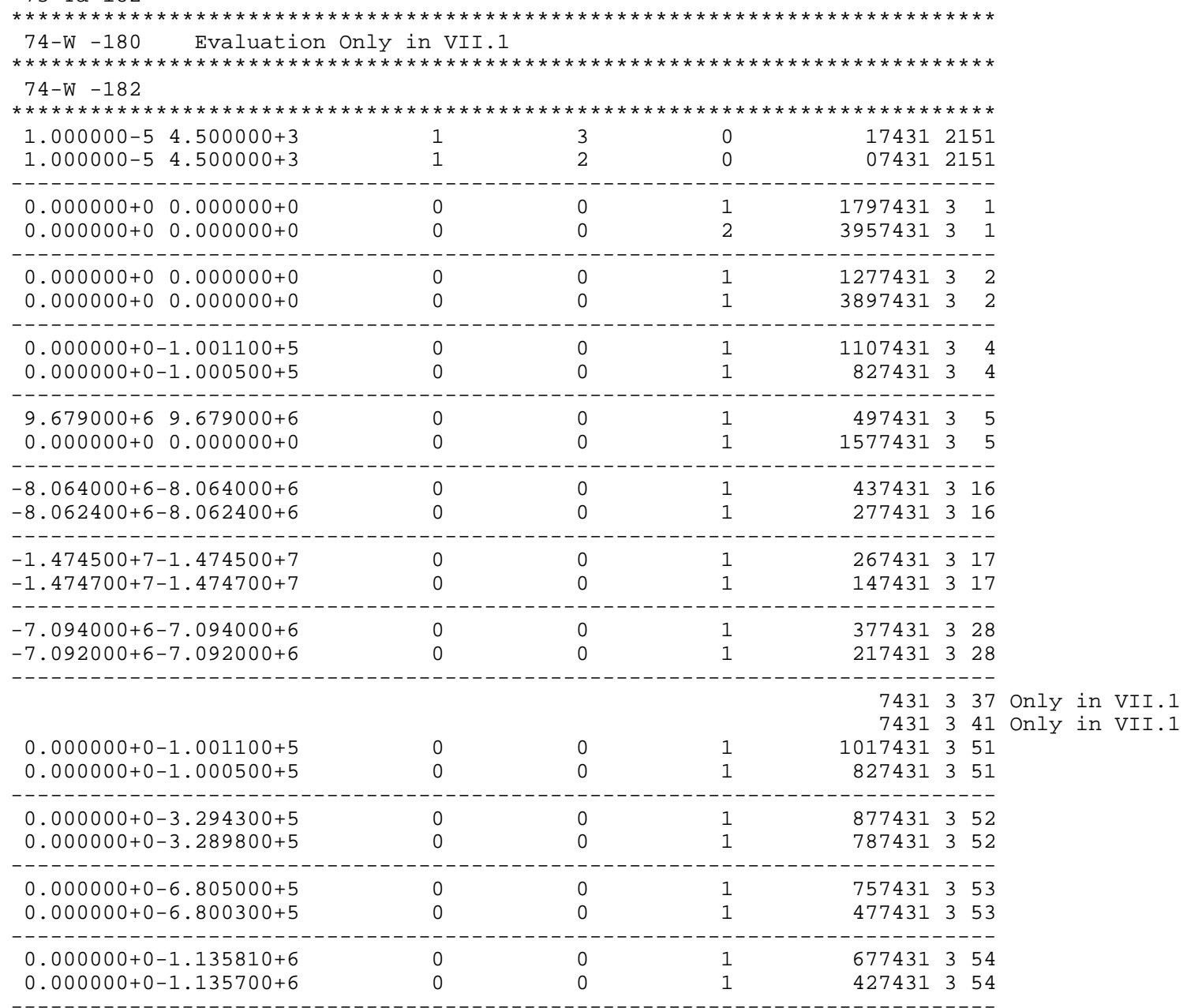




\begin{tabular}{|c|c|c|c|c|}
\hline $\begin{array}{l}\odot .0 \odot \odot \odot \odot \odot+\odot-1.1444 \odot \odot+6 \\
\odot . \odot \odot \odot \odot \odot \odot+\odot-1.1445 \odot \odot+6\end{array}$ & $\begin{array}{l}\odot \\
\odot\end{array}$ & $\begin{array}{l}\odot \\
\odot\end{array}$ & $\begin{array}{l}1 \\
1\end{array}$ & $\begin{array}{lll}667431 & 3 & 55 \\
417431 & 3 & 55\end{array}$ \\
\hline \multicolumn{5}{|c|}{$\begin{array}{l}- \\
-\end{array}$} \\
\hline $0.000000+0-1.221410+6$ & $\odot$ & $\odot$ & 1 & 657431356 \\
\hline $0.000000+0-1.222200+6$ & $\odot$ & $\odot$ & 1 & 397431356 \\
\hline \multicolumn{5}{|c|}{ 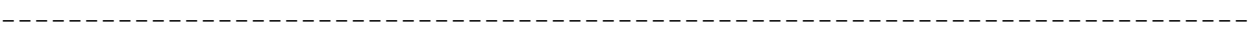 } \\
\hline $0.000000+0-1.257420+6$ & $\odot$ & ○ & 1 & 647431357 \\
\hline $0.000000+0-1.258000+6$ & $\odot$ & $\odot$ & 1 & 387431357 \\
\hline \multicolumn{5}{|c|}{ 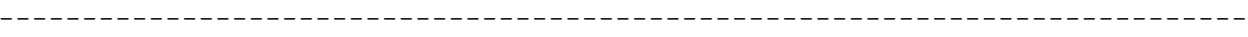 } \\
\hline $0.000000+0-1.289160+6$ & $\odot$ & $\odot$ & 1 & 647431358 \\
\hline $0.000000+0-1.288900+6$ & $\odot$ & $\odot$ & 1 & 377431358 \\
\hline \multicolumn{5}{|c|}{ 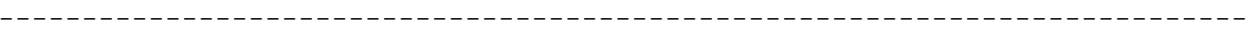 } \\
\hline $0.000000+0-1.331130+6$ & $\odot$ & 0 & 1 & 637431359 \\
\hline $0.000000+0-1.330600+6$ & $\odot$ & 0 & 1 & 36743135 \\
\hline
\end{tabular}

7431360 only in VII. 0

7431361 only in VII.0

7431362 only in VII.0

7431363 Only in VII.0

7431364 only in VII.0

7431365 only in VII.0

7431366 only in VII.0

7431367 only in VII.0

7431368 only in VII.0

7431369 only in VII.0

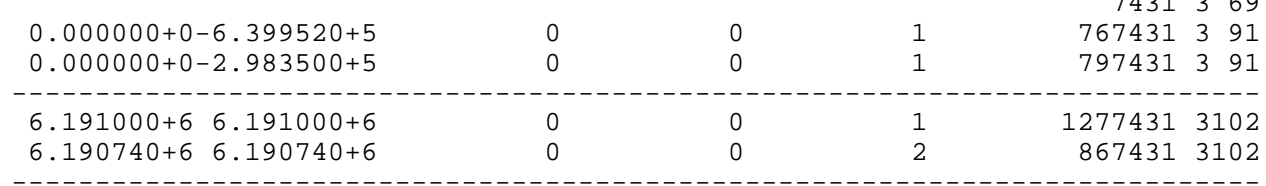

$6.190740+6 \quad 6.190740+6$

74313103 only in VII.0

74313107 only in VII.0

74313600 0nly in VII.1

74313601 only in VII.1

74313602 only in VII.1

74313603 only in VII.1

74313604 only in VII.1

74313605 only in VII.1

74313606 only in VII.1

74313607 only in VII.1

74313608 only in VII.1

74313609 only in VII.1

74313610 only in VII.1

74313611 only in VII.1

74313612 only in VII.1

74313613 only in VII.1

74313614 only in VII.1

74313615 only in VII.1

74313616 only in VII.1

74313617 only in VII.1

74313618 only in VII.1

74313619 only in VII.1

74313620 only in VII.1

74313621 only in VII.1

74313622 only in VII.1

74313623 only in VII.1

74313624 only in VII.1

74313625 only in VII.1

74313626 only in VII.1

74313627 only in VII.1

74313628 only in VII.1

74313629 only in VII.1

74313649 only in VII.1

74313800 only in VII.1

74313801 only in VII.1

74313802 only in VII.1

74313803 only in VII.1

74313804 only in VII.1

74313805 only in VII.1

74313806 only in VII.1

74313849 only in VII.1

$0.000000+\odot \quad 1.803850+2$

$\odot$

2
2

0

$\begin{array}{lll}617431 & 4 & 2\end{array}$

$\odot .000000+\odot \quad 1.803900+2$

$\odot$

0

0743142 


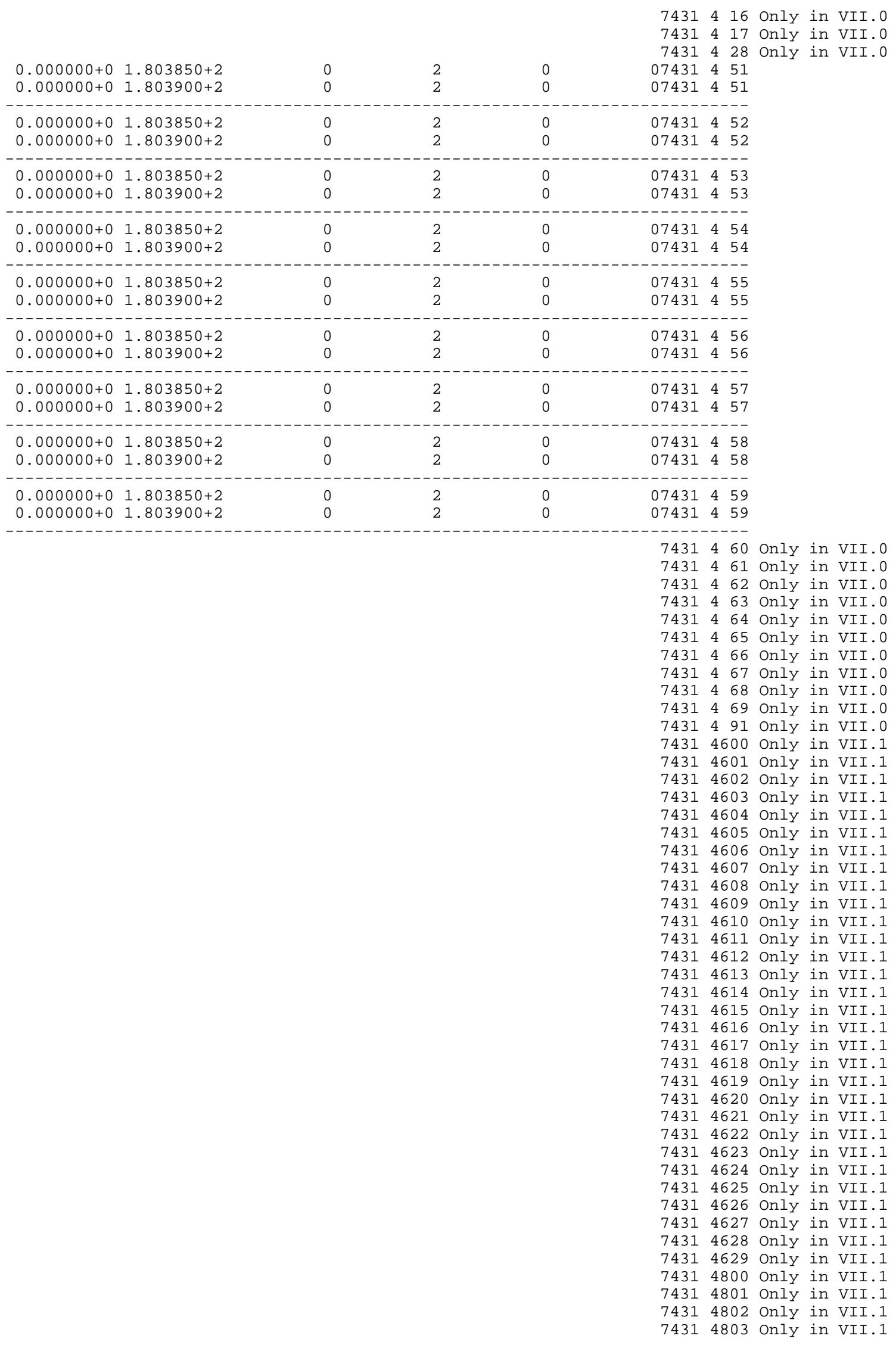




$\begin{array}{llll}1 & 1 & 2774316 & 6\end{array}$

7431616 only in VII.1

7431617 Only in VII.1

7431628 Only in VII.1

7431637 only in VII.1

7431641 only in VII.1

7431691 Only in VII.1

74316102 only in VII.1

74316649 only in VII.1

$74-W-183$

74316849 only in VII.1

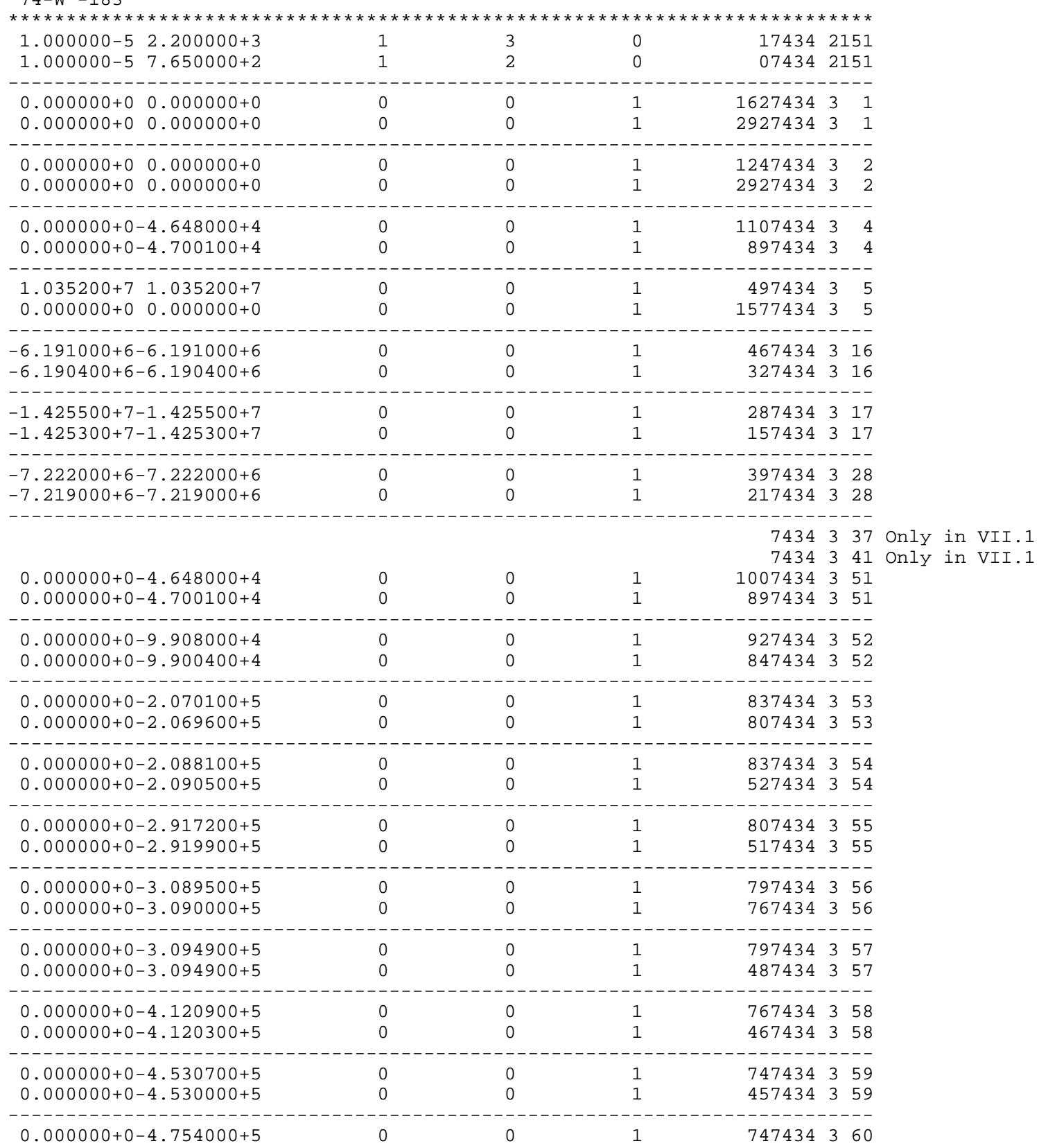




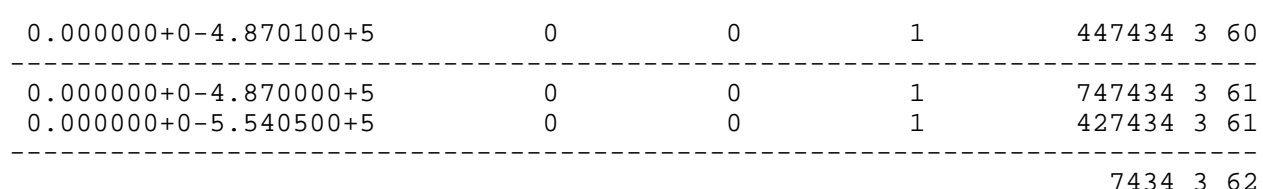

7434362 only in VII.O

7434363 only in VII.0

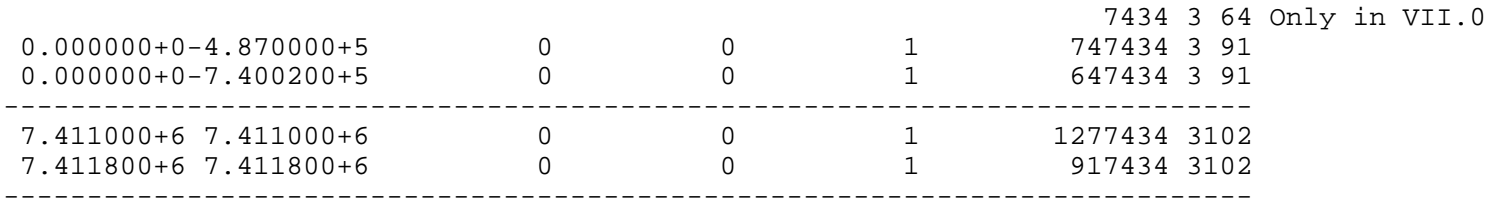

74343103 Only in VII.0

74343107 only in VII.0

74343600 only in VII.1

74343601 only in VII.1

74343602 only in VII.1

74343603 only in VII.1

74343604 only in VII.1

74343605 only in VII.1

74343606 only in VII.1

74343607 only in VII.1

74343608 only in VII.1

74343609 only in VII.1

74343610 only in VII.1

74343611 only in VII.1

74343612 only in VII.1

74343613 only in VII.1

74343614 only in VII.1

74343649 only in VII.1

74343800 only in VII.1

74343801 only in VII.1

74343802 only in VII.1

74343803 only in VII.1

74343804 only in VII.1

74343805 only in VII.1

74343806 only in VII.1

74343807 only in VII.1

74343808 only in VII.1

74343809 only in VII.1

74343810 only in VII.1

74343811 only in VII.1

74343812 only in VII.1

74343813 only in VII.1

74343814 only in VII.1

74343815 only in VII.1

74343816 only in VII.1

74343817 only in VII.1

74343818 only in VII.1

74343819 only in VII.1

74343820 only in VII.1

74343821 only in VII.1

74343822 only in VII.1

74343823 only in VII.1

74343824 only in VII.1

74343849 only in VII.1

$\begin{array}{cc}\odot .00000 \odot+\odot & 1.813790+2 \\ 0.0000 \odot \odot+\odot & 1.813800+2\end{array}$

0

2
2

0

$627434 \quad 4 \quad 2$

0743442

7434416 only in VII.O 7434417 only in VII.0 7434428 only in VII.0

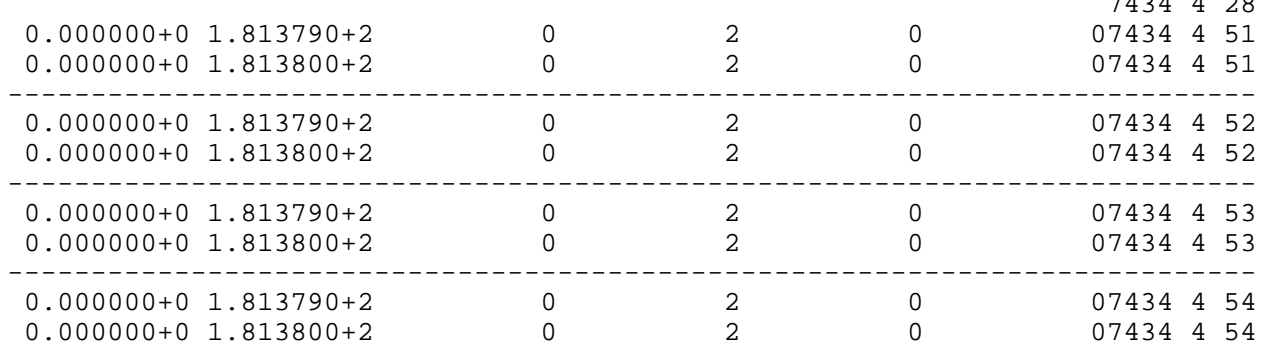




\begin{tabular}{|c|c|c|c|c|c|}
\hline $\begin{array}{l}\odot . \odot \odot \odot \odot \odot \odot+\odot ~ \\
\odot . \diamond \odot \odot \odot \odot \odot+\odot\end{array}$ & $\begin{array}{l}1.813790+2 \\
1.813800+2\end{array}$ & $\begin{array}{l}\odot \\
\odot\end{array}$ & $\begin{array}{l}2 \\
2\end{array}$ & $\begin{array}{l}\odot \\
\odot\end{array}$ & $\begin{array}{lll}07434 & 4 & 55 \\
07434 & 4 & 55\end{array}$ \\
\hline-------- & 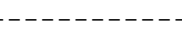 & - & $=-$ & -1 & 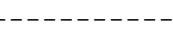 \\
\hline$\odot .000000+\odot$ & $1.813790+2$ & $\odot$ & 2 & $\odot$ & 07434456 \\
\hline $0.000000+0$ & $1.813800+2$ & $\odot$ & 2 & $\odot$ & 07434456 \\
\hline$------\cdot$ & --- & -- & - & - & 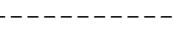 \\
\hline$\odot .0 \odot \odot \odot ० \odot+\odot$ & $1.813790+2$ & 0 & 2 & $\odot$ & $07434 \quad 4 \quad 57$ \\
\hline$\odot .000000+0$ & $1.813800+2$ & 0 & 2 & $\odot$ & $07434 \quad 4 \quad 57$ \\
\hline-------- & -------- & - & - & - & - - - - - - - \\
\hline$\odot .0 \odot \odot \odot \odot \odot+\odot$ & $1.813790+2$ & $\odot$ & 2 & $\odot$ & $07434 \quad 458$ \\
\hline $0.000000+0$ & $1.813800+2$ & 0 & 2 & $\odot$ & 07434458 \\
\hline----- & -------- & 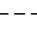 & - & - & --------- \\
\hline$\odot .000000+\odot$ & $1.813790+2$ & 0 & 2 & $\odot$ & $07434 \quad 4 \quad 59$ \\
\hline$\odot .0000 \bullet \odot+\odot$ & $1.813800+2$ & $\odot$ & 2 & 0 & $07434 \quad 4 \quad 59$ \\
\hline$=$ & --- & -- & -- & - & -------- \\
\hline$\odot . \diamond \odot \odot \odot \odot \odot+\odot$ & $1.813790+2$ & $\odot$ & 2 & $\odot$ & 07434460 \\
\hline $0.000000+0$ & $1.813800+2$ & $\odot$ & 2 & 0 & 07434460 \\
\hline - & -------- & & - & & ------- \\
\hline$\odot . \diamond \odot \odot \odot \odot \odot+\odot$ & $1.813790+2$ & $\odot$ & 2 & $\odot$ & $07434 \quad 4 \quad 61$ \\
\hline$\odot . \odot \odot \odot \odot \odot \odot+\odot$ & $1.813800+2$ & $\odot$ & 2 & $\odot$ & $07434 \quad 4 \quad 61$ \\
\hline
\end{tabular}

-

7434462 only in VII.0

7434463 only in VII.0

7434464 only in VII.0

7434491 only in VII.0

74344600 only in VII.1

74344601 only in VII.1

74344602 only in VII.1

74344603 only in VII.1

74344604 only in VII.1

74344605 only in VII.1

74344606 only in VII.1

74344607 only in VII.1

74344608 only in VII.1

74344609 only in VII.1

74344610 only in VII.1

74344611 only in VII.1

74344612 only in VII.1

74344613 only in VII.1

74344614 only in VII.1

74344800 only in VII.1

74344801 only in VII.1

74344802 only in VII.1

74344803 only in VII.1

74344804 Only in VII.1

74344805 only in VII.1

74344806 only in VII.1

74344807 only in VII.1

74344808 only in VII.1

74344809 only in VII.1

74344810 only in VII.1

74344811 only in VII.1

74344812 only in VII.1

74344813 only in VII.1

74344814 only in VII.1

74344815 only in VII.1

74344816 only in VII.1

74344817 only in VII.1

74344818 only in VII.1

74344819 only in VII.1

74344820 only in VII.1

74344821 only in VII.1

74344822 only in VII.1

74344823 only in VII.1

74344824 only in VII.1

7434516 only in VII.0

7434517 only in VII.0

7434528 only in VII.0

1

7434591 only in VII.0

1

$477434 \quad 6 \quad 5$

$277434 \quad 6 \quad 5$

7434616 only in VII.1

7434617 only in VII.1 
$74-W-184$

7434628 only in VII.1 7434637 Only in VII.1 7434641 only in VII.1 7434691 only in VII.1 74346102 only in VII.1 74346649 only in VII.1 74346849 only in VII.1

\begin{tabular}{|c|c|c|c|c|c|}
\hline $\begin{array}{ll}1.00 \odot \odot \odot \odot-5 ~ & 4.000 \odot \odot \odot+3 \\
1.000 \odot \odot \odot-5 & 2.6500 \odot \odot+3\end{array}$ & $\begin{array}{l}1 \\
1\end{array}$ & $\begin{array}{l}3 \\
2\end{array}$ & $\begin{array}{l}\odot \\
\odot\end{array}$ & $\begin{array}{l}17437 \\
07437\end{array}$ & $\begin{array}{l}2151 \\
2151\end{array}$ \\
\hline 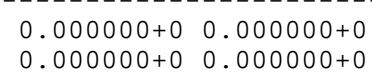 & $\begin{array}{l}\odot \\
\odot\end{array}$ & $\begin{array}{l}\odot \\
\odot\end{array}$ & $\begin{array}{l}1 \\
2\end{array}$ & $\begin{array}{l}1337437 \\
3927437\end{array}$ & $\begin{array}{ll}3 & 1 \\
3 & 1\end{array}$ \\
\hline 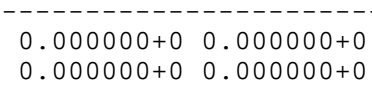 & $\begin{array}{l}\odot \\
\odot\end{array}$ & $\begin{array}{l}\odot \\
\odot\end{array}$ & $\begin{array}{l}1 \\
1\end{array}$ & $\begin{array}{l}1207437 \\
3867437\end{array}$ & $\begin{array}{ll}3 & 2 \\
3 & 2\end{array}$ \\
\hline $\begin{array}{l}\odot .0 \odot \odot \odot \odot \odot+\odot-1.11210 \odot+5 \\
\odot . \odot \odot \odot \odot \odot \odot+\odot-1.1119 \odot \odot+5\end{array}$ & $\begin{array}{l}\odot \\
\odot\end{array}$ & $\begin{array}{l}\odot \\
\odot\end{array}$ & $\begin{array}{l}1 \\
1\end{array}$ & 817437 & $\begin{array}{ll}3 & 4 \\
3 & 4\end{array}$ \\
\hline 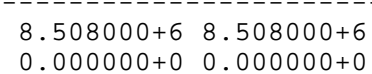 & $\begin{array}{l}\odot \\
\odot\end{array}$ & $\begin{array}{l}\odot \\
\odot\end{array}$ & $\begin{array}{l}1 \\
1\end{array}$ & $\begin{array}{r}457437 \\
1557437\end{array}$ & $\begin{array}{ll}3 & 5 \\
3 & 5\end{array}$ \\
\hline $\begin{array}{l}-7.411000+6-7.411000+6 \\
-7.411400+6-7.411400+6\end{array}$ & $\begin{array}{l}\odot \\
\odot\end{array}$ & $\begin{array}{l}\odot \\
\odot\end{array}$ & $\begin{array}{l}1 \\
1\end{array}$ & $\begin{array}{l}427437 \\
297437\end{array}$ & $\begin{array}{ll}3 & 16 \\
3 & 16\end{array}$ \\
\hline $\begin{array}{l}-1.360200+7-1 \cdot 360200+7 \\
-1.360200+7-1.360200+7\end{array}$ & $\begin{array}{l}\odot \\
\odot\end{array}$ & $\begin{array}{l}\odot \\
\odot\end{array}$ & $\begin{array}{l}1 \\
1\end{array}$ & $\begin{array}{l}277437 \\
167437\end{array}$ & $\begin{array}{ll}3 & 17 \\
3 & 17\end{array}$ \\
\hline $\begin{array}{l}-7.699000+6-7.699000+6 \\
-7.700000+6-7.700000+6\end{array}$ & $\begin{array}{l}\odot \\
\odot\end{array}$ & $\begin{array}{l}\ominus \\
\ominus\end{array}$ & $\begin{array}{l}1 \\
1\end{array}$ & $\begin{array}{l}357437 \\
197437\end{array}$ & $\begin{array}{ll}3 & 28 \\
3 & 28\end{array}$ \\
\hline
\end{tabular}

-

7437337 only in VII.1

\begin{tabular}{|c|c|c|c|c|}
\hline $\begin{array}{l}\odot .0000 \odot \odot+\odot-1.112100+5 \\
\odot .000 \odot \odot \odot+\odot-1.111900+5\end{array}$ & $\begin{array}{l}\odot \\
\odot\end{array}$ & $\begin{array}{l}\odot \\
\odot\end{array}$ & $\begin{array}{l}1 \\
1\end{array}$ & $\begin{array}{rrr}7437 & 3 & 41 \\
927437 & 3 & 51 \\
807437 & 3 & 51\end{array}$ \\
\hline $\begin{array}{l}0.000000+0-3.640600+5 \\
\odot .000000+\odot-3.640500+5\end{array}$ & $\begin{array}{l}\odot \\
\odot\end{array}$ & $\begin{array}{l}\odot \\
\odot\end{array}$ & $\begin{array}{l}1 \\
1\end{array}$ & $\begin{array}{lll}797437 & 3 & 52 \\
777437 & 3 & 52\end{array}$ \\
\hline $\begin{array}{l}\odot .000000+\odot-7.483100+5 \\
\odot .0000 \odot \odot+\odot-7.483300+5\end{array}$ & $\begin{array}{l}\odot \\
\odot\end{array}$ & $\begin{array}{l}\odot \\
\odot\end{array}$ & $\begin{array}{l}1 \\
1\end{array}$ & $\begin{array}{lll}687437 & 3 & 53 \\
457437 & 3 & 53\end{array}$ \\
\hline $\begin{array}{l}\odot .0 \odot \odot \odot \odot \odot+\odot-9.03280 \odot+5 \\
\odot .0 \odot \odot \odot \odot \odot+\odot-9.0329 \odot \odot+5\end{array}$ & $\begin{array}{l}\ominus \\
\ominus\end{array}$ & $\begin{array}{l}\odot \\
\odot\end{array}$ & $\begin{array}{l}1 \\
1\end{array}$ & $\begin{array}{lll}667437 & 3 & 54 \\
427437 & 3 & 54\end{array}$ \\
\hline $\begin{array}{l}\odot .000000+\odot-1.002480+6 \\
\odot .000000+\odot-1.002500+6\end{array}$ & $\begin{array}{l}\odot \\
\odot\end{array}$ & $\begin{array}{l}\odot \\
\odot\end{array}$ & $\begin{array}{l}1 \\
1\end{array}$ & $\begin{array}{lll}647437 & 3 & 55 \\
407437 & 3 & 55\end{array}$ \\
\hline $\begin{array}{l}\odot . \odot \odot \odot \odot \odot \odot+\odot-1.0 \odot 597 \odot+6 \\
\odot . \odot \odot \odot \odot \odot \odot+\odot-1 . \odot \odot 65 \odot \odot+6\end{array}$ & $\begin{array}{l}\odot \\
\odot\end{array}$ & $\begin{array}{l}\odot \\
\odot\end{array}$ & $\begin{array}{l}1 \\
1\end{array}$ & $\begin{array}{lll}647437 & 3 & 56 \\
397437 & 3 & 56\end{array}$ \\
\hline
\end{tabular}

7437357 only in VII.0 7437358 only in VII.0 7437359 only in VII.0 7437360 only in VII.0 7437361 only in VII.0 7437362 only in VII.0 7437363 only in VII.0 7437364 only in VII.0 7437365 only in VII.0 7437366 only in VII.0 7437367 only in VII.0 7437368 only in VII.0

\begin{tabular}{|c|c|c|c|c|c|c|c|}
\hline $\begin{array}{l}\odot . \odot \odot \odot \odot \odot \odot+\odot-6.892210+5 \\
\odot . \odot \odot \odot \odot \odot \odot+\odot-4.9727 \odot \odot+5\end{array}$ & $\begin{array}{l}\odot \\
\odot\end{array}$ & $\begin{array}{l}\odot \\
\odot\end{array}$ & $\begin{array}{l}1 \\
1\end{array}$ & $\begin{array}{r}7437 \\
707437 \\
757437\end{array}$ & $\begin{array}{ll}3 & 68 \\
3 & 91 \\
3 & 91\end{array}$ & only i & \\
\hline $\begin{array}{ll}5.753000+6 & 5.753000+6 \\
5.753780+6 & 5.753780+6\end{array}$ & $\begin{array}{l}\odot \\
\odot\end{array}$ & $\begin{array}{l}\odot \\
\odot\end{array}$ & $\begin{array}{l}1 \\
2\end{array}$ & $\begin{array}{r}1207437 \\
877437\end{array}$ & $\begin{array}{l}3102 \\
3102\end{array}$ & & \\
\hline & & & & $\begin{array}{l}7437 \\
7437 \\
7437 \\
7437\end{array}$ & $\begin{array}{l}3103 \\
3107 \\
3600 \\
3649\end{array}$ & $\begin{array}{l}\text { Only in } \\
\text { only in } \\
\text { only in } \\
\text { Only in }\end{array}$ & $\begin{array}{l}\text { VII.0 } \\
\text { VII.0 } \\
\text { VII.1 } \\
\text { VII.1 }\end{array}$ \\
\hline
\end{tabular}


74373800 only in VII.1

74373801 0nly in VII.1

74373802 only in VII.1

74373803 only in VII.1

74373804 Only in VII.1

74373805 only in VII.1

74373806 only in VII.1

74373807 only in VII.1

74373849 only in VII.1

$\begin{array}{ll}\odot .000000+\odot & 1.823710+2 \\ \odot .000000+\odot & 1.823700+2\end{array}$

$\odot$

$2 \quad 0$

$617437 \quad 4 \quad 2$

7437416 only in VII.0

7437417 only in VII.0

7437428 only in VII. 0

$\begin{array}{ll}\odot .000000+\odot & 1.823710+2 \\ \odot .000000+\odot & 1.823700+2\end{array}$

0
0

2

07437451

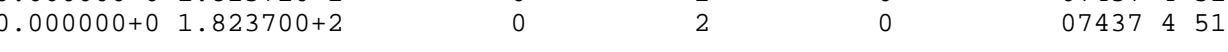

$0.000000+01.823710+2$

$\odot .000000+\odot 1.823700+2$

$0 \quad 2507437452$

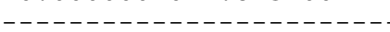

$\odot \quad 2$

$\odot .000000+\odot 1.823710+2$

$0.000000+01.823700+2$

0

$\odot .000000+\odot 1.823710+2 \quad 0 \quad 0$

$0.000000+01.823700+2 \quad 0 \quad 2$

-

$\begin{array}{lll}0.000000+\odot & 1.823710+2 & 0 \\ 0.000000+\odot & 1.823700+2 & 0\end{array}$

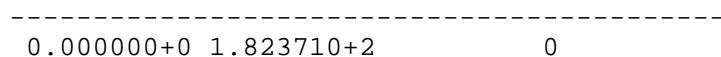

$\odot .000 \odot \odot \odot+\odot 1.823700+2$

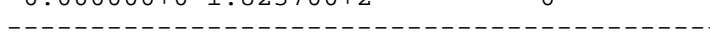

2

$0 \quad 07437455$

$0 \quad 07437455$

07437452

07437453

07437453

07437454

07437454

07437455

07437456

07437456

7437457 only in VII.0

7437458 only in VII.0

7437459 only in VII.0

7437460 only in VII.0

7437461 only in VII. 0

7437462 only in VII. 0

7437463 only in VII.0

7437464 only in VII. 0

7437465 only in VII.0

7437466 only in VII.0

7437467 only in VII.0

7437468 only in VII. 0

7437491 only in VII. 0

74374600 only in VII.1

74374800 Only in VII.1

74374801 only in VII.1

74374802 Only in VII.1

74374803 only in VII.1

74374804 only in VII.1

74374805 only in VII.1

74374806 only in VII.1

74374807 only in VII.1

7437516 only in VII.0

7437517 only in VII. 0

7437528 only in VII.0

$1.000000+\odot \quad 1.000000+\odot$

$\odot$

1
1

7437591 Only in VII. $\odot$

$1.000000+01.000000+0$

$1 \quad 1$

$\begin{array}{lll}457437 & 6 & 5 \\ 277437 & 6 & 5\end{array}$

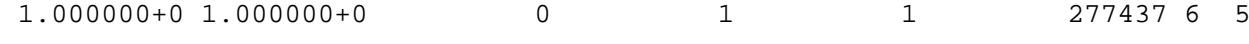

7437616 only in VII.1

7437617 Only in VII.1

7437628 only in VII.1

7437637 Only in VII.1

7437641 only in VII.1

7437691 only in VII.1

74376102 only in VII.1

74376649 only in VII.1

$74-W-186$

74376849 only in VII.1

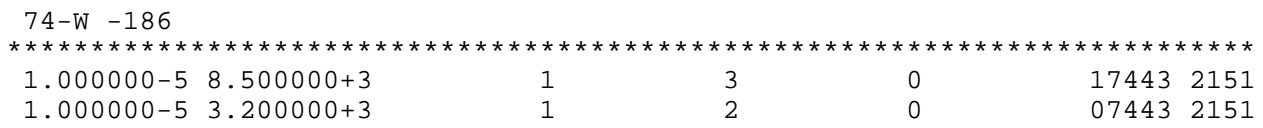




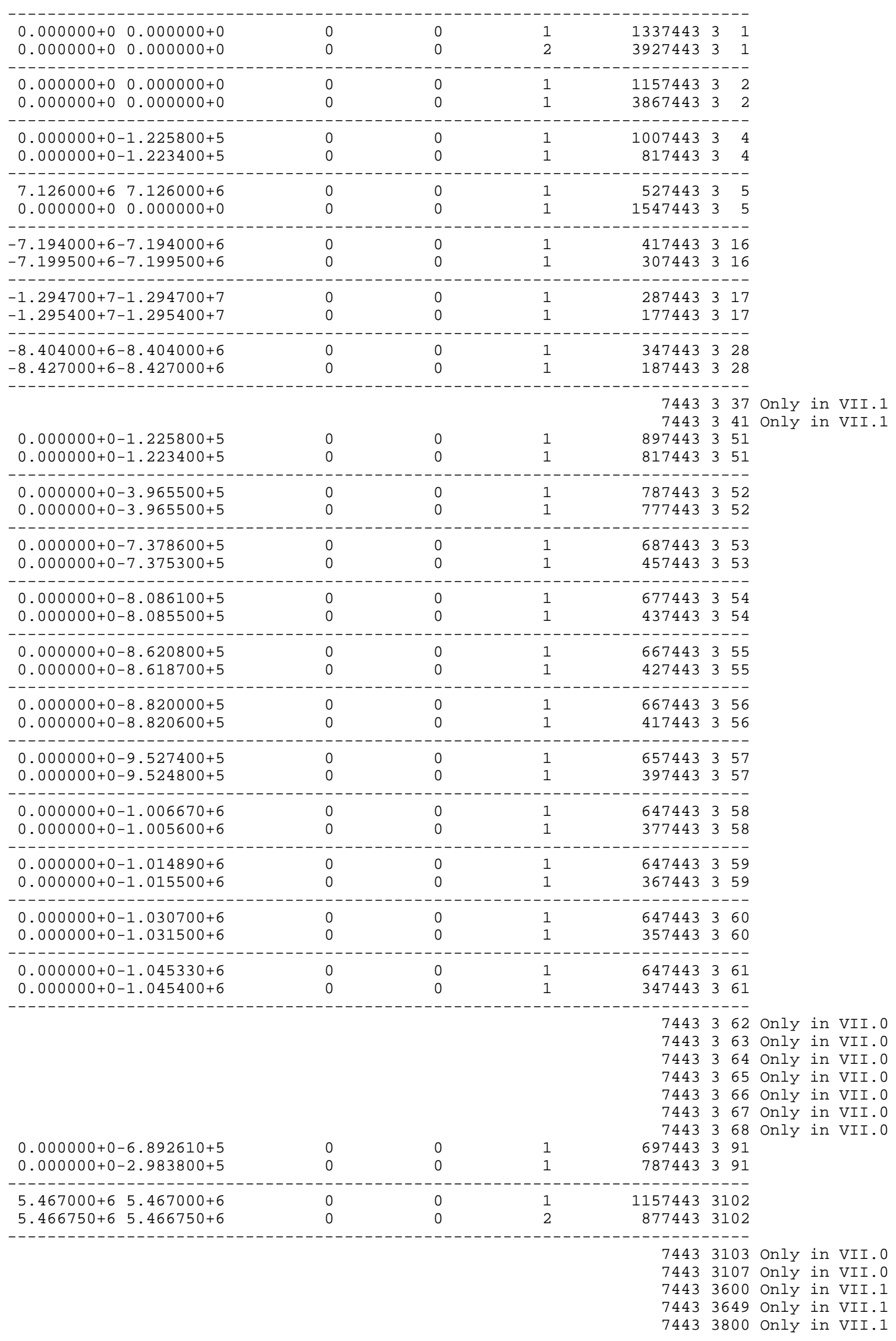




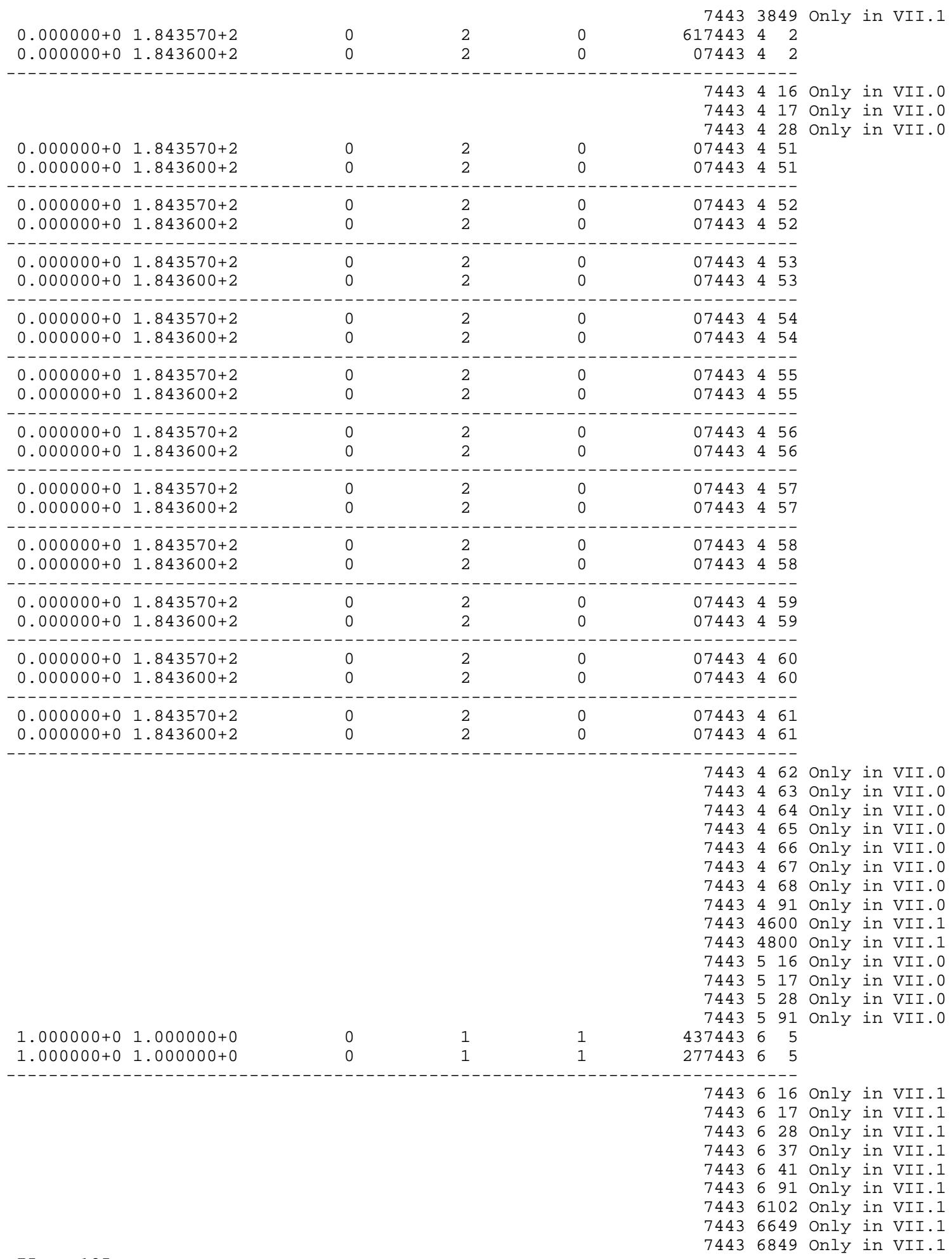

75-Re-185

\begin{tabular}{|c|c|c|c|c|c|}
\hline 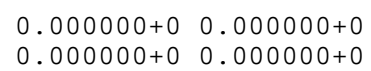 & $\begin{array}{l}\odot \\
\odot\end{array}$ & $\begin{array}{l}\odot \\
\odot\end{array}$ & $\begin{array}{l}1 \\
3\end{array}$ & $\begin{array}{l}10775253 \\
11475253\end{array}$ & $\begin{array}{l}1 \\
1\end{array}$ \\
\hline$\odot .0 \odot \odot \odot \odot \odot+\odot \quad \odot . \diamond 0 \odot \odot \odot \odot+\odot$ & $\odot$ & 0 & 1 & 10775253 & 2 \\
\hline$\odot .000000+\odot \quad 0.000000+\odot$ & 0 & 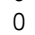 & 3 & 11475253 & 2 \\
\hline
\end{tabular}

752534 only in VII.0 


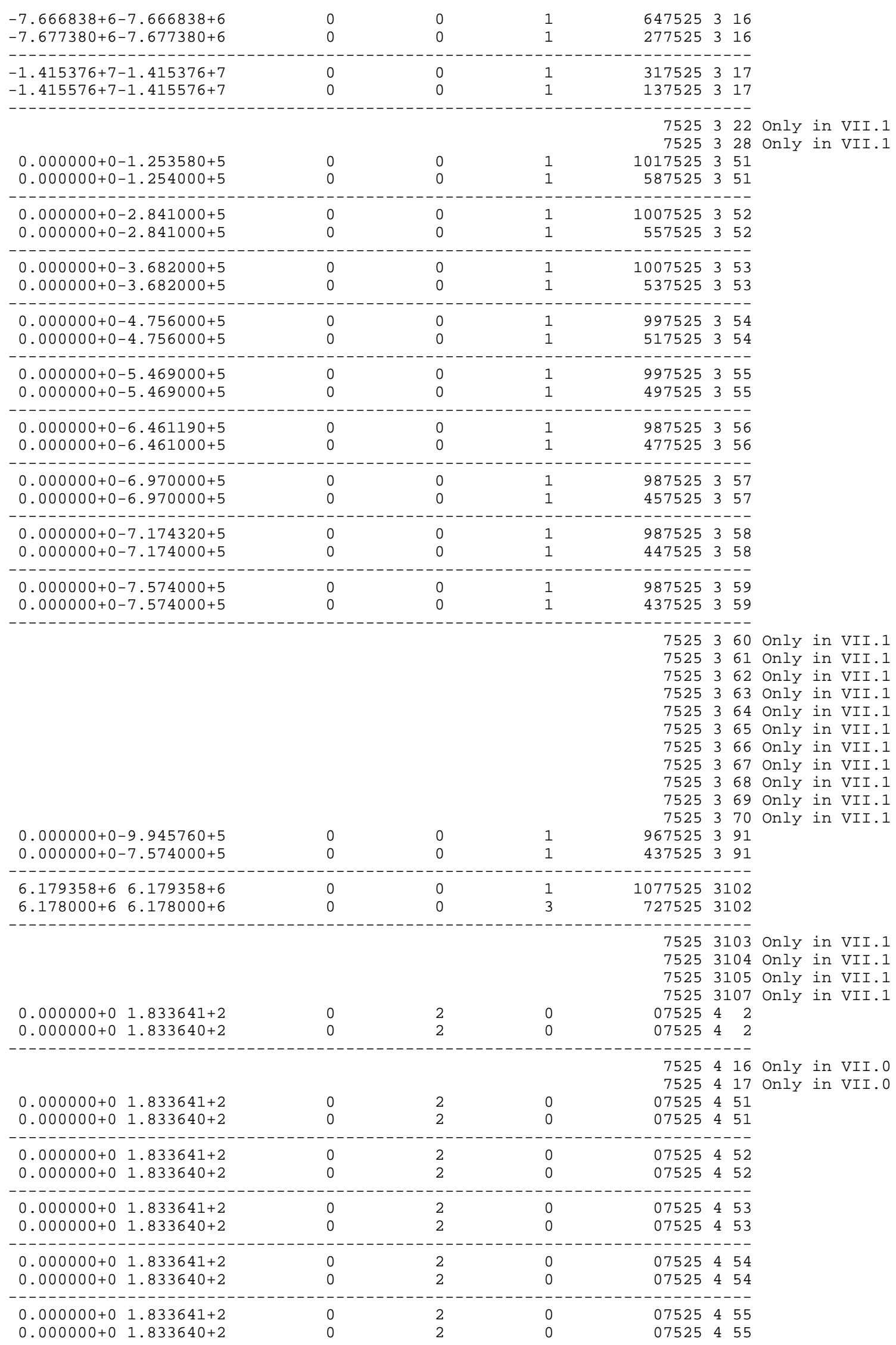




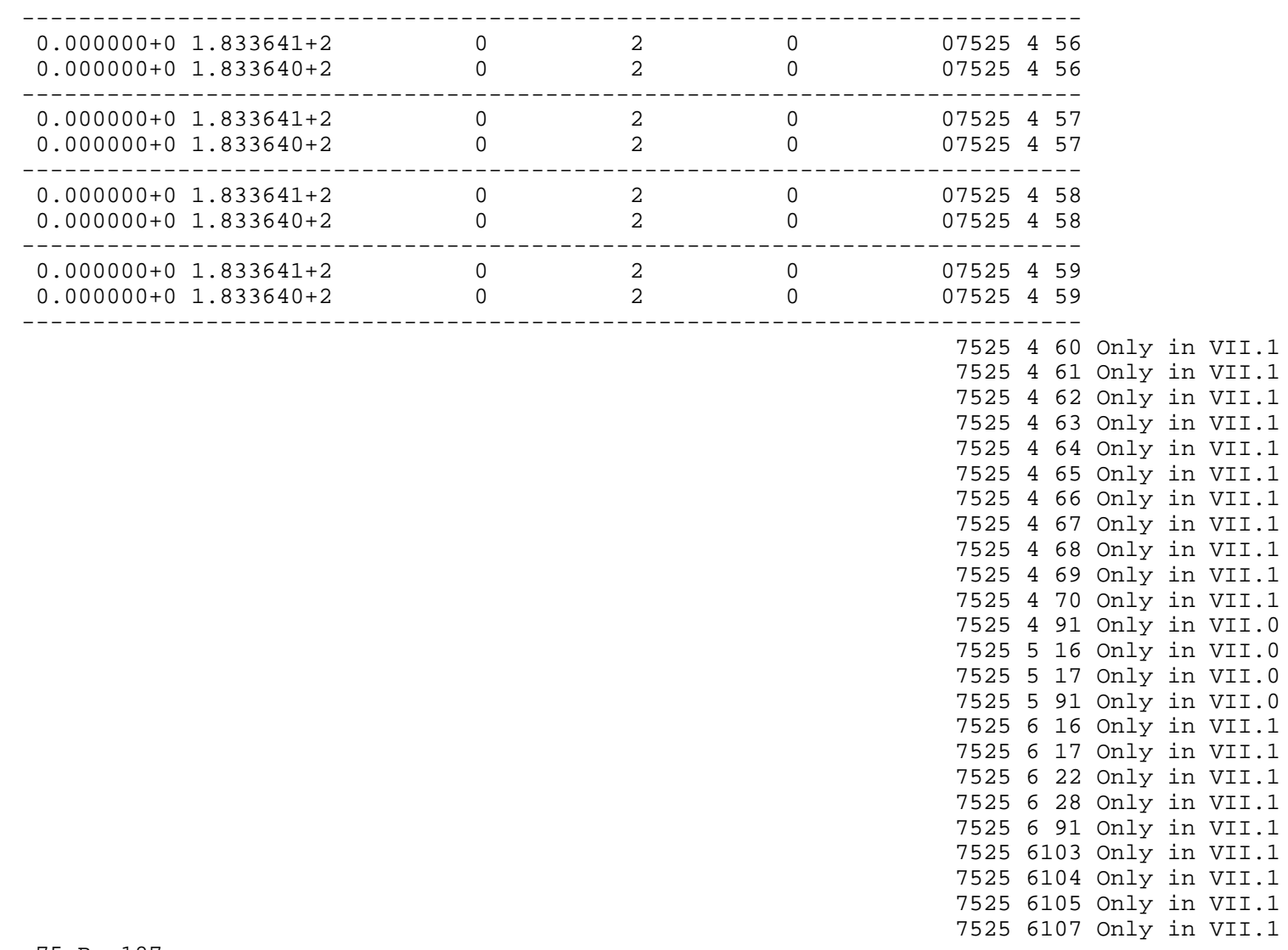

75-Re-187

\begin{tabular}{|c|c|c|c|c|c|}
\hline 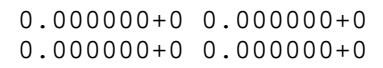 & $\begin{array}{l}\odot \\
\odot\end{array}$ & $\begin{array}{l}\odot \\
\odot\end{array}$ & $\begin{array}{l}1 \\
3\end{array}$ & $\begin{array}{lll}1077531 & 3 & 1 \\
1217531 & 3 & 1\end{array}$ & \\
\hline 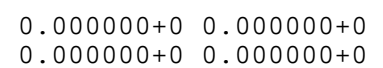 & $\begin{array}{l}\odot \\
\odot\end{array}$ & $\begin{array}{l}\odot \\
\odot\end{array}$ & $\begin{array}{l}1 \\
3\end{array}$ & $\begin{array}{lll}1077531 & 3 & 2 \\
1217531 & 3 & 2\end{array}$ & \\
\hline $\begin{array}{l}-7.356838+6-7.356838+6 \\
-7.360380+6-7.360380+6\end{array}$ & $\begin{array}{l}\odot \\
\odot\end{array}$ & $\begin{array}{l}\odot \\
\odot\end{array}$ & $\begin{array}{l}1 \\
1\end{array}$ & $\begin{array}{rrr}7531 & 3 & 4 \\
667531 & 3 & 16 \\
287531 & 3 & 16\end{array}$ & Only in VII.o \\
\hline $\begin{array}{l}-1.353620+7-1.353620+7 \\
-1.353876+7-1.353876+7\end{array}$ & $\begin{array}{l}\odot \\
\odot\end{array}$ & $\begin{array}{l}\odot \\
\odot\end{array}$ & $\begin{array}{l}1 \\
1\end{array}$ & $\begin{array}{lll}337531 & 3 & 17 \\
147531 & 3 & 17\end{array}$ & \\
\hline $\begin{array}{l}\odot .000000 \odot+\odot-1.342430+5 \\
\odot .000 \odot \odot \odot+\odot-1.34200 \odot+5\end{array}$ & $\begin{array}{l}\odot \\
\odot\end{array}$ & $\begin{array}{l}\odot \\
\odot\end{array}$ & $\begin{array}{l}1 \\
1\end{array}$ & $\begin{array}{rrr}7531 & 3 & 22 \\
7531 & 3 & 28 \\
1017531 & 3 & 51 \\
657531 & 3 & 51\end{array}$ & $\begin{array}{l}\text { Only in VII.1 } \\
\text { Only in VII.1 }\end{array}$ \\
\hline $\begin{array}{l}\odot .000 \odot \odot \odot+\odot-2.062440+5 \\
\odot .000 \odot \odot \odot+\odot-2.06200 \odot+5\end{array}$ & $\begin{array}{l}\odot \\
\odot\end{array}$ & $\begin{array}{l}\odot \\
\odot\end{array}$ & $\begin{array}{l}1 \\
1\end{array}$ & $\begin{array}{rrr}1007531 & 3 & 52 \\
627531 & 3 & 52\end{array}$ & \\
\hline $\begin{array}{l}0.000000+\odot-3.031300+5 \\
0.000000+\odot-3.027000+5\end{array}$ & $\begin{array}{l}\odot \\
\odot\end{array}$ & $\begin{array}{l}\odot \\
\odot\end{array}$ & $\begin{array}{l}1 \\
1\end{array}$ & $\begin{array}{rrr}1007531 & 3 & 53 \\
607531 & 3 & 53\end{array}$ & \\
\hline $\begin{array}{l}0.000000+0-3.9000000+5 \\
0.000000+0-3.900000+5\end{array}$ & $\begin{array}{l}\odot \\
\odot\end{array}$ & $\begin{array}{l}\ominus \\
\ominus\end{array}$ & $\begin{array}{l}1 \\
1\end{array}$ & $\begin{array}{rrr}1007531 & 3 & 54 \\
597531 & 3 & 54\end{array}$ & \\
\hline 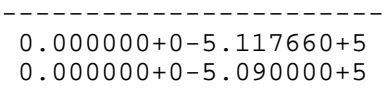 & $\begin{array}{l}\odot \\
\odot\end{array}$ & $\begin{array}{l}\odot \\
\odot\end{array}$ & $\begin{array}{l}1 \\
1\end{array}$ & $\begin{array}{lll}997531 & 3 & 55 \\
567531 & 3 & 55\end{array}$ & \\
\hline $\begin{array}{l}\odot . \odot \odot \odot \odot \odot \odot+\odot-5.8199 \odot \odot+5 \\
\odot . \odot \odot \odot \odot \odot \odot+\odot-5.1160 \odot \odot+5\end{array}$ & $\begin{array}{l}\odot \\
\odot\end{array}$ & $\begin{array}{l}\odot \\
\odot\end{array}$ & $\begin{array}{l}1 \\
1\end{array}$ & $\begin{array}{lll}997531 & 3 & 56 \\
557531 & 3 & 56\end{array}$ & \\
\hline $\begin{array}{l}\odot .0 \odot \odot \odot \odot \odot+\odot-5.891360+5 \\
\odot . \odot \odot \odot \odot \odot \odot+\odot-5.89 \odot \odot \odot \odot+5\end{array}$ & $\begin{array}{l}\odot \\
\odot\end{array}$ & $\begin{array}{l}\odot \\
\odot\end{array}$ & $\begin{array}{l}1 \\
1\end{array}$ & $\begin{array}{lll}997531 & 3 & 57 \\
547531 & 3 & 57\end{array}$ & \\
\hline
\end{tabular}




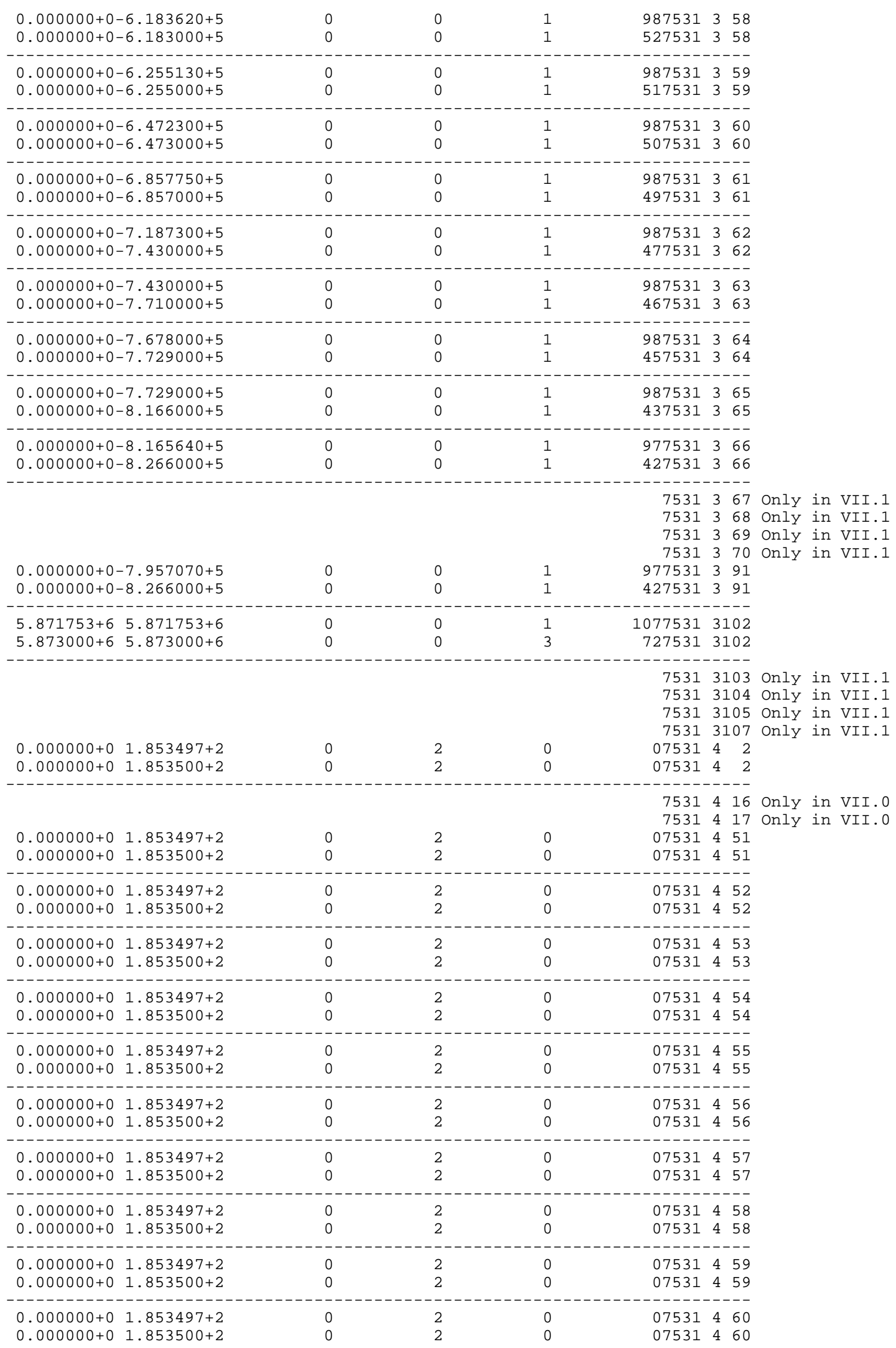




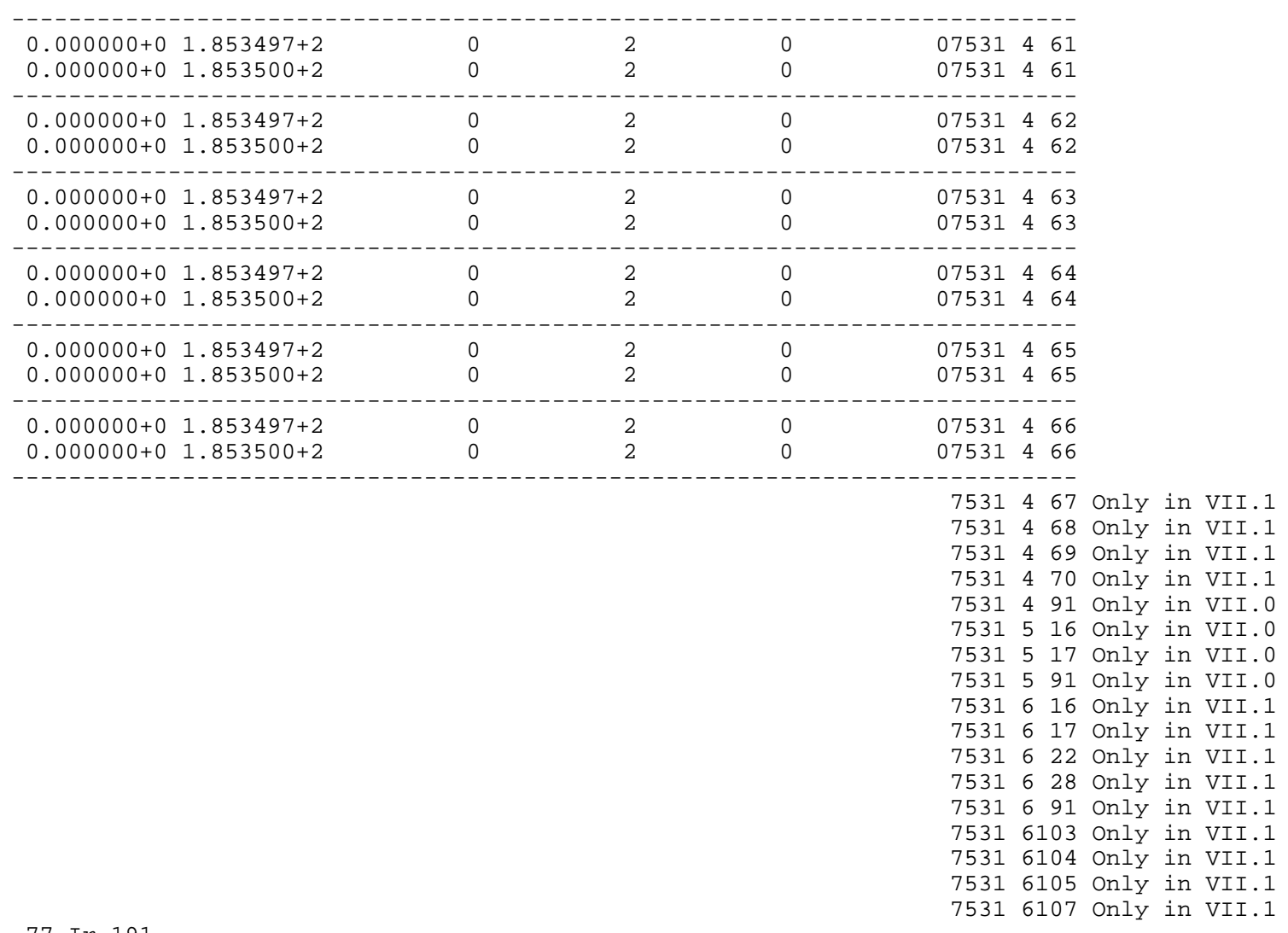

$77-\operatorname{Ir}-191$

-9.835711-4-6.465490-3 2.787750-3 7.048050-3-6.516570-3-7.813980-37725 4 58 $-9.835710-4-6.465490-3 \quad 2.787750-3 \quad 7.048050-3-6.516570-3-7.813980-37725458$

$0.000000+09.612251-7 \quad 6.20970-152.176700+5 \quad 1.571530-62.86127-157725628$

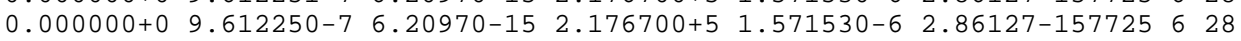

-

$77-\operatorname{Ir}-193$

$* * * * * * * * * * * * * * * * * * * * * * * * * * * * * * * * * * * * * * * * * * * * * * * * * * * * * * * * * * * * * * * * * * * * * * * * * * *$

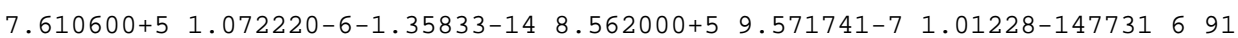
$7.610600+5 \quad 1.072220-6-1.35833-14 \quad 8.562000+5 \quad 9.571740-7 \quad 1.01228-147731691$

-

$79-\mathrm{Au}-197$

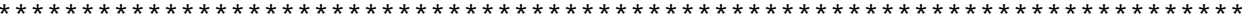
$80-\mathrm{Hg}-196$

-9.945219-1 9.772202-4-9.902681-1 5.801228-4-9.848078-1 3.966870-48025 42 -9.945219-1 9.772203-4-9.902681-1 5.801228-4-9.848078-1 3.966870-48025 42

(.

$\odot .000 \odot \odot \odot+\odot \quad 1.232180-1 \quad \odot .000 \odot \odot \odot+\odot \quad 2.334700-2 \quad 0.0000 \odot \odot+\odot \quad 9.927941-48025469$ $\odot .000000+\odot 1.232180-1 \quad 0.0000000+\odot \quad 2.334700-2 \quad 0.000000+\odot \quad 9.927940-48025469$

$0.000000+\odot \quad 9.813569-7 \quad 1.000500+6 \quad 2.710970-8 \quad 1.667500+6 \quad 1.01014-108025522$ $\odot .000 \odot \odot \odot+\odot \quad 9.813570-7 \quad 1.000500+6 \quad 2.710970-8 \quad 1.667500+6 \quad 1.01014-108025522$

$5.618709+69.75584-416.243010+60.000000+0 \quad 802565$

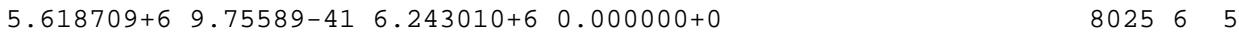

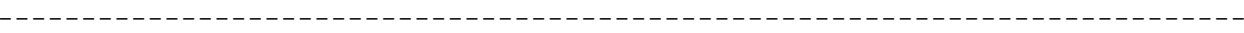

$80-\mathrm{Hg}-198$

$* * * * * * * * * * * * * * * * * * * * * * * * * * * * * * * * * * * * * * * * * * * * * * * * * * * * * * * * * * * * * * * * * * * * * * * * * * *$

-4.067366-1 9.941901-7-3.746066-1 1.925906-6-3.420201-1 2.481473-68031 42 -4.067366-1 9.941902-7-3.746066-1 1.925906-6-3.420201-1 2.481473-68031 42

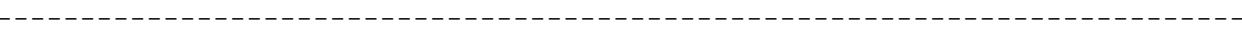
$2.487337+6 \quad 8.52358-13 \quad 1.687000-4 \quad 3.482272+6 \quad 7.48192-11 \quad 9.867999-48031 \quad 6 \quad 5$ $2.487337+6 \quad 8.52358-13 \quad 1.687000-4 \quad 3.482272+6 \quad 7.48192-11 \quad 9.868000-48031 \quad 6 \quad 5$ 10-1 $80-\mathrm{Hg}-199$

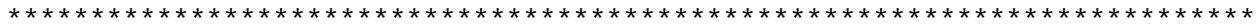


$\begin{array}{llllll}1.000000-5 & 9.594100-7 & 2.530000-2 & 9.592970-7 & 1.000000+\odot & 9.549969-780343107\end{array}$ $1.000000-5$ 9.594100-7 2.530000-2 9.592970-7 $1.000000+\odot \quad 9.549970-780343107$

-8.090170-1 9.943413-4-7.880108-1 9.978116-4-7.660444-1 $1.053366-3803442$ -8.090170-1 9.943413-4-7.880108-1 9.978115-4-7.660444-1 1.053366-38034 42

$0.000000+0-5.750460-40.000000+0-9.832371-40000000+0-3.217170-48034463$ $0.000000+0-5.750460-4 \quad 0.000000+0-9.832370-4 \quad 0.000000+0-3.217170-48034463$

$7.337642+75.62668-400.000000+0 \quad 503465$

$7.337642+7 \quad 5.62667-40 \quad 0.0000000+\odot \quad 803465$

(1)

$80-\mathrm{Hg}-200$

$* * * * * * * * * * * * * * * * * * * * * * * * * * * * * * * * * * * * * * * * * * * * * * * * * * * * * * * * * * * * * * * * * * * * * * * * * * *$

1.045285-1 7.703236-4 1.391731-1 8.803268-4 $1.736482-1 \quad 9.843440-48037442$

1.045285-1 7.703236-4 1.391731-1 8.803268-4 1.736482-1 9.843441-48037 42

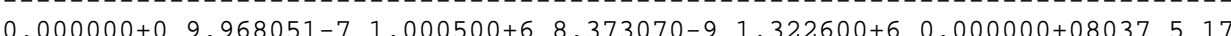

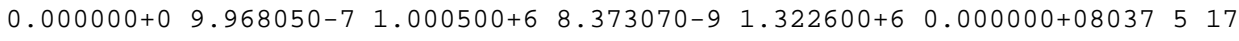

$0.000000+09.869721-$ $\odot .000000+\odot \quad 9.869720-7 \quad 1.000500+6 \quad 1.879220-8 \quad 1.667500+6 \quad 0.000000+08037522$

(

$\begin{array}{llllll}7.338202+7 & 6.91148-41 & 0.0000000+0 & 8037 & 6 & 5\end{array}$

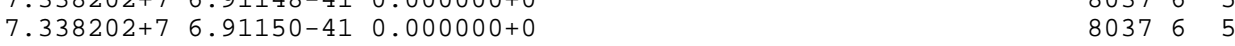

$80-\mathrm{Hg}-201$

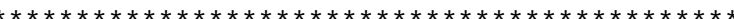

$6.000000+6 \quad 9.823859-4 \quad 8.000000+6 \quad 5.203440-5 \quad 1.0000000+7 \quad 1.688600-68040359$ $6.000000+6 \quad 9.823860-4 \quad 8.000000+6 \quad 5.203440-5 \quad 1.000000+7 \quad 1.688600-68040359$

$3.090170-19.083974-43.420201-11.322084-33.746066-11.767220-380404$ $3.090170-1$ 9.983973-4 3.420201-1 $1.322084-3 \quad 3.746066-1 \quad 1.767220-380404 \quad 2$ 3. $090170-1.9 .983973-43.420201-11.322$

3.373420-3-9.918601-4-1.074490-4-9.875390-5-4.109810-7-1.801250-58040 452 3.373420-3-9.918600-4-1.074490-4-9.875390-5-4.109810-7-1.801250-58040 4 52

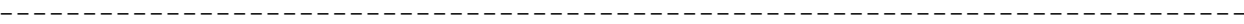

$1.079586+81.12104-440.000000+\odot \quad 804065$ $1.079586+8 \quad 1.11010-440.000000+0 \quad 8040 \quad 6 \quad 5$

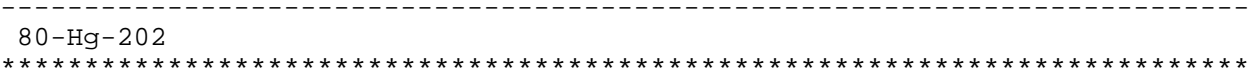

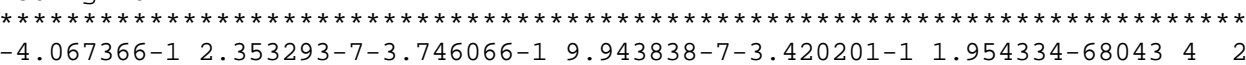
-4.067366-1 2.353293-7-3.746066-1 9.943837-7-3.420201-1 1.954334-68043 42

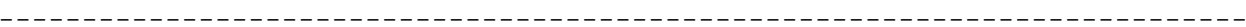
9.929599-4-1.072450-3-5.161110-5-5.923050-5-7.322550-6-3.003070-68043 455 9.929600-4-1.072450-3-5.161110-5-5.923050-5-7.322550-6-3.003070-68043 455 . $0.000000+0 \quad 9.704989-7 \quad 1.000500+6 \quad 3.711510-8 \quad 1.667500+6 \quad 5.257050-98043 \quad 5 \quad 22$ $\odot .0000 \odot \odot+\odot \quad 9.704990-7 \quad 1.000500+6 \quad 3.711510-8 \quad 1.667500+6 \quad 5.257050-98043522$

$4.301815+62.59989-404.779794+60.000000+0 \quad 804365$ $4.301815+6 \quad 2.59988-40 \quad 4.779794+6 \quad 0.000000+0 \quad 804365$

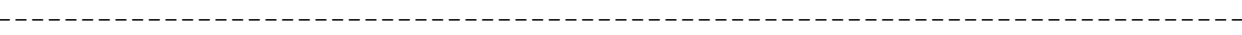
$80-\mathrm{Hg}-204$

1.045285-1 7.703236-4 1.391731-1 8.803268-4 $1.736482-1 \quad 9.843440-4804942$ 1.045285-1 7.703236-4 1.391731-1 8.803268-4 1.736482-1 9.843441-48049 4 2

$\begin{array}{lllll}-5.841320-6-5.859780-7 & 9.922099-7-1.779000-7 & 2.601100-9 & 2.109980-98049 & 4\end{array} 68$ -5.841320-6-5.859780-7 9.922100-7-1.779000-7 2.601100-9 2.109980-98049 468

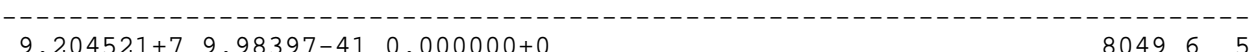

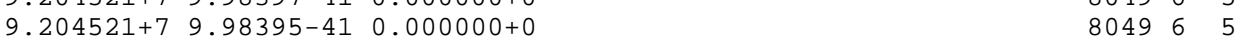

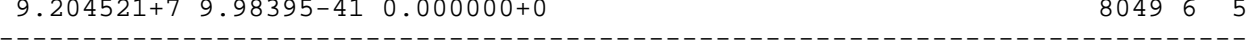

$82-\mathrm{Pb}-204$

$* * * * * * * * * * * * * * * * * * * * * * * * * * * * * * * * * * * * * * * * * * * * * * * * * * * * * * * * * * * * * * * * * * * * * * * * * * *$

$82-\mathrm{Pb}-206$

$* * * * * * * * * * * * * * * * * * * * * * * * * * * * * * * * * * * * * * * * * * * * * * * * * * * * * * * * * * * * * * * * * * * * * * * * * * *$

$\odot .000000+\odot 1.000000-4 \quad 000823142$

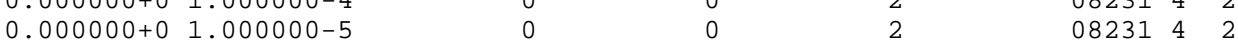

$82-\mathrm{Pb}-207$

$* * * * * * * * * * * * * * * * * * * * * * * * * * * * * * * * * * * * * * * * * * * * * * * * * * * * * * * * * * * * * * * * * * * * * * * * * * *$
$0.000000+01.000000-4$
$\odot$
$\odot$
2
0823442 
$82-\mathrm{Pb}-208$

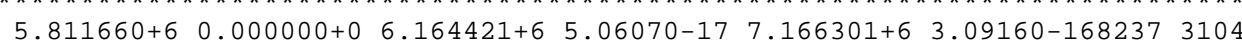
$\begin{array}{llllllll}5.811660+6 & 0.000000+\odot & 6.164422+6 & 5.06070-13 & 7.166298+6 & 1.34258-118237 & 3104\end{array}$

$5.811660+60.000000+08.750000+64.21530-16 \quad 9.000000+61.17090-1282373650$ $5.811660+6 \quad 0.000000+0 \quad 8.750000+6 \quad 4.21532-12 \quad 9.000000+6 \quad 1.170860-882373650$ $6.164422+60.000000+0.750000+62.96330-169.000000+6$. $\begin{array}{lllllll}6.164422+6 & 0.000000+\odot & 8.750000+6 & 2.96330-16 & 9.000000+6 & 1.50400-158237 & 3651\end{array}$

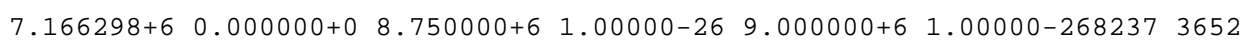
$7.166298+6 \quad 0.000000+\odot \quad 8.750000+6 \quad 1.00000-20 \quad 9.000000+6 \quad 1.00000-2082373652$

5.877853-1 9.921344-4 6.156615-1 $1.314650-36.427876-1 \quad 1.896842-382374 \quad 2$ 5.877853-1 9.921345-4 6.156615-1 $1.314650-3 \quad 6.427876-1 \quad 1.896842-38237 \quad 4 \quad 2$

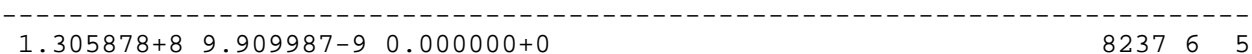
$1.305878+8 \quad 9.80592-510.000000+\odot \quad 8237 \quad 6 \quad 5$

$2.487934+59.650335-71.123846-23.483107+51.054284-61.458150-28237616$ $2.487934+5$ 9.650334-7 $1.123846-2 \quad 3.483107+5 \quad 1.054284-6 \quad 1.458150-28237616$

(20 $9.454148+4 \quad 8.903161-7 \quad 3.868335-2 \quad 1.094691+5 \quad 9.907588-7 \quad 3.941522-28237622$ 9.454148+4 8.903161-7 3.868335-2 $1.094691+5 \quad 9.907589-7 \quad 3.941522-28237622$ $\begin{array}{llllllll}4.478281+5 & 9.669297-7 & 2.086118-2 & 5.473454+5 & 9.309394-7 & 2.275852-28237 & 6 & 24\end{array}$ $\begin{array}{llllllll}4.478281+5 & 9.669298-7 & 2.086118-2 & 5.473454+5 & 9.309394-7 & 2.275852-28237 & 6 & 24\end{array}$ $0.000000+01.388497-63000000+544090476-7500000+59.775860-78237633$

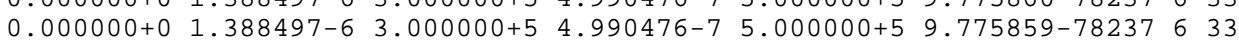

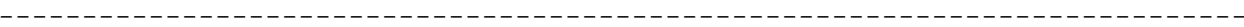
$\begin{array}{lllllll}2.587451+5 & 9.807123-7 & 1.193717-2 & 2.985521+5 & 1.038554-6 & 1.242066-28237 & 61\end{array}$ 2.587451+5 9.807124-7 1.193717-2 2.985521+5 $1.038554-6 \quad 1.242066-28237641$

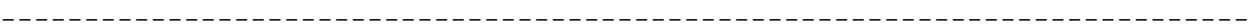
$\begin{array}{llllllll}2.487934+5 & 7.809822-7 & 5.648501-2 & 3.483107+5 & 9.584739-7 & 5.913704-28237 & 6 & 91\end{array}$ 2.487934+5 7.809822-7 5.648501-2 $3.483107+5 \quad 9.584740-7 \quad 5.913704-28237 \quad 691$ $3.500000+5 \quad 4.019961-7 \quad 4.5000000+5 \quad 9.726637-7 \quad 5.500000+5 \quad 8.244782-78237 \quad 6649$ $\begin{array}{lllllll}3.500000+5 & 4.019961-7 & 4.500000+5 & 9.726636-7 & 5.500000+5 & 8.244782-78237 & 6649\end{array}$

$8.328073+6 \quad 8.675260-7 \quad 7.647492-1 \quad 8.426630+6 \quad 9.747999-7 \quad 7.757668-182376749$ $8.328073+6 \quad 8.675260-7 \quad 7.647492-1 \quad 8.426630+6 \quad 9.748000-7 \quad 7.757668-18237 \quad 6749$

$0.000000+\odot \quad 0.000000+\odot \quad 0.000000+\odot \quad 4.806178+6 \quad 7.08020-410.000000+082376849$ $\odot .000000+\odot \quad 0.000000+\odot \quad 0.000000+\odot \quad 4.806178+6 \quad 7.08015-41 \quad 0.000000+08237 \quad 6849$

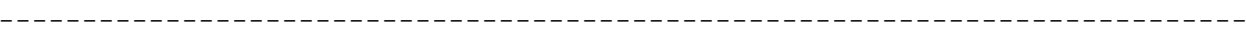
$83-\mathrm{Bi}-209$

$88-\mathrm{Ra}-223$

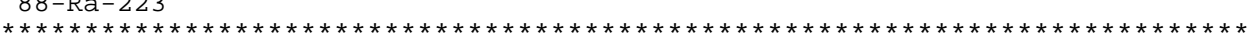

$88-\mathrm{Ra}-224$

$* * * * * * * * * * * * * * * * * * * * * * * * * * * * * * * * * * * * * * * * * * * * * * * * * * * * * * * * * * * * * * * * * * * * * * * * * * *$

$88-\mathrm{Ra}-225$

$* * * * * * * * * * * * * * * * * * * * * * * * * * * * * * * * * * * * * * * * * * * * * * * * * * * * * * * * * * * * * * * * * * * * * * * * * * *$

$88-\mathrm{Ra}-226$

$* * * * * * * * * * * *$
$89-\mathrm{AC}-225$

$89-\mathrm{Ac}-225$
$* * * * * * * * * * * * * * * * * * * * * * * * * * * * * * * * * * * * * * * * * * * * * * * * * * * * * * * * * * * * * * * * * * * * * * * * *$

89251452 only in VII.1 89251455 only in VII.1

1. $000000-5 \quad 4.500000-1$ 1. $000000-5$ 6.000000-1

$\odot .0 \odot \odot \odot \odot \odot+\odot \quad 0.0 \odot \odot \odot \odot \odot+\odot$ $0.000000+\odot \quad 0.000000+0$

$0.000000+0 \quad 0.000000+\Theta$ $0.000000+0 \quad 0.000000+0$

$\odot .00 \odot \odot \odot \odot+\odot-2.99400 \odot+4$ $0.000000+0-3.999990+4$

\begin{tabular}{|c|c|c|c|c|}
\hline $\begin{array}{l}\odot \\
\odot\end{array}$ & $\begin{array}{l}\odot \\
\odot\end{array}$ & $\begin{array}{l}\odot \\
\odot\end{array}$ & $\begin{array}{r}8925 \\
08925 \\
08925\end{array}$ & $\begin{array}{l}1456 \\
2151 \\
2151\end{array}$ \\
\hline- & - & - & ------ & -- \\
\hline$\odot$ & $\Theta$ & 1 & 1198925 & \\
\hline$\odot$ & $\Theta$ & 2 & 9489253 & \\
\hline-- & - & - & ------- & \\
\hline$\odot$ & $\odot$ & 1 & 1078925 & \\
\hline$\odot$ & $\odot$ & 2 & 388925 & \\
\hline-- & - & - & - - - - - - - & \\
\hline$\odot$ & $\odot$ & 1 & 828925 & \\
\hline$\odot$ & $\odot$ & 1 & 278925 & \\
\hline
\end{tabular}




$\begin{array}{lc}-6.667820+6-6.667820+6 & \odot \\ -6.664690+6-6.664690+6 & 0 \\ -1.233080+7-1.233080+7 & 0 \\ -1.234240+7-1.234240+7 & 0 \\ -\end{array}$

\begin{tabular}{|c|c|c|}
\hline$\odot$ & 1 & 288925316 \\
\hline$\odot$ & 1 & 13892531 \\
\hline- & & -- \\
\hline$\odot$ & 1 & 16892531 \\
\hline$\odot$ & 1 & 88925317 \\
\hline
\end{tabular}

\begin{tabular}{|c|c|c|c|c|c|c|}
\hline & & & & 8925 & 318 & Only in VII.1 \\
\hline & & & & 8925 & 319 & Only in VII.1 \\
\hline & & & & 8925 & 320 & only in VII.1 \\
\hline & & & & 8925 & 321 & only in VII.1 \\
\hline$-1 \cdot 919720+7-1 \cdot 919720+7$ & $\odot$ & $\odot$ & 1 & 28925 & 337 & \\
\hline$-1.920610+7-1.920610+7$ & 0 & 0 & 1 & 28925 & 337 & \\
\hline
\end{tabular}

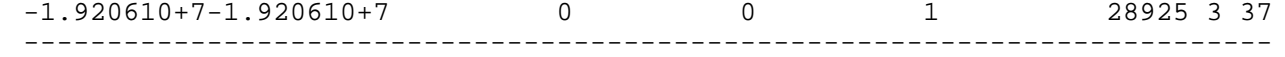

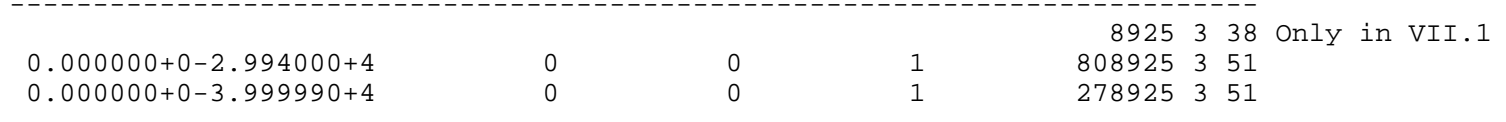

8925352 only in VII.1 8925353 only in VII.1 8925354 Only in VII.1 8925355 only in VII.1 8925356 only in VII.1 8925357 only in VII.1 8925358 only in VII.1 8925359 only in VII.1 8925360 Only in VII.1 8925361 only in VII.1 8925362 only in VII.1 8925363 only in VII.1 8925364 only in VII.1

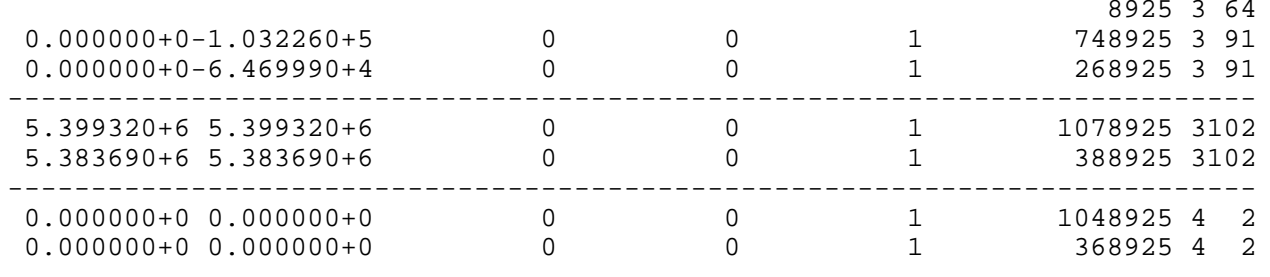

8925416 Only in VII.0 8925417 only in VII.0 8925418 only in VII.1 8925437 only in VII.0 8925451 only in VII.0 8925491 Only in VII.0 8925516 only in VII.0 8925517 only in VII.0 8925518 only in VII.1 8925537 only in VII.0 8925591 Only in VII.0 8925616 only in VII.1 8925617 only in VII.1 8925637 only in VII.1 8925651 only in VII.1 8925652 only in VII.1 8925653 only in VII.1 8925654 only in VII.1 8925655 only in VII.1 8925656 only in VII.1 8925657 only in VII.1 8925658 only in VII.1 8925659 only in VII.1 8925660 only in VII.1 8925661 Only in VII.1 8925662 only in VII.1 8925663 only in VII.1 8925664 only in VII.1 8925691 Only in VII.1 89256102 only in VII.1 


\begin{tabular}{|c|c|c|c|c|}
\hline 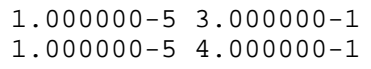 & $\begin{array}{l}\odot \\
\odot\end{array}$ & $\begin{array}{l}\odot \\
\odot\end{array}$ & $\begin{array}{l}\odot \\
\odot\end{array}$ & $\begin{array}{l}08928 \\
08928\end{array}$ \\
\hline 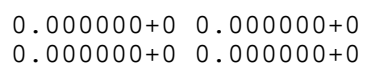 & $\begin{array}{l}\odot \\
\odot\end{array}$ & $\begin{array}{l}\odot \\
\odot\end{array}$ & $\begin{array}{l}1 \\
2\end{array}$ & $\begin{array}{r}1118928 \\
918928\end{array}$ \\
\hline 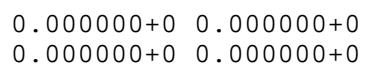 & $\begin{array}{l}\odot \\
\odot\end{array}$ & $\begin{array}{l}\odot \\
\odot\end{array}$ & $\begin{array}{l}1 \\
2\end{array}$ & $\begin{array}{r}1118928 \\
378928\end{array}$ \\
\hline $\begin{array}{l}\odot .00 \odot \odot \odot \odot+\odot-5.1000 \odot \odot+3 \\
\odot .000 \odot \odot \odot+\odot-2.9000 \odot \odot+5\end{array}$ & $\begin{array}{l}\odot \\
\odot\end{array}$ & $\begin{array}{l}\odot \\
\odot\end{array}$ & $\begin{array}{l}1 \\
1\end{array}$ & $\begin{array}{l}788928 \\
238928\end{array}$ \\
\hline $\begin{array}{l}-5 \cdot 399320+6-5 \cdot 399320+6 \\
-5.396690+6-5.396690+6\end{array}$ & $\begin{array}{l}\odot \\
\odot\end{array}$ & $\begin{array}{l}\odot \\
\odot\end{array}$ & $\begin{array}{l}1 \\
1\end{array}$ & $\begin{array}{l}308928 \\
148928\end{array}$ \\
\hline $\begin{array}{l}-1.206710+7-1.206710+7 \\
-1.206140+7-1.206140+7\end{array}$ & $\begin{array}{l}\odot \\
\odot\end{array}$ & $\begin{array}{l}\odot \\
\odot\end{array}$ & $\begin{array}{l}1 \\
1\end{array}$ & $\begin{array}{r}178928 \\
88928\end{array}$ \\
\hline
\end{tabular}

8928318 0nly in VII.1 8928319 0nly in VII.1 8928320 Only in VII.1

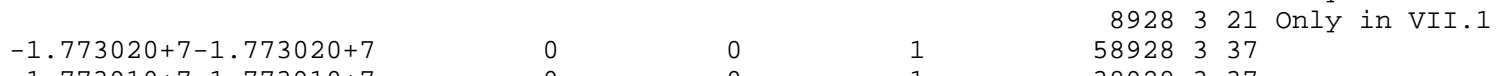

$\begin{array}{rrrrr}-1.773910+7-1.773910+7 & 0 & 0 & 1 & 38928337\end{array}$

r. 8928338 only in VII.1

8928351 Only in VII.1 8928352 only in VII.1 8928353 only in VII.1 8928354 only in VII.1 8928355 Only in VII.1 8928356 only in VII.1 8928357 only in VII.1

\begin{tabular}{|c|c|c|c|c|c|}
\hline $\begin{array}{l}\odot .0 \odot 0 \odot \odot \odot \odot+\odot-1.104910+5 \\
\odot . \odot \odot \odot \odot \odot \odot+\odot-2.90 \odot \odot \odot \odot+5\end{array}$ & $\begin{array}{l}\odot \\
\odot\end{array}$ & $\begin{array}{l}\odot \\
\odot\end{array}$ & $\begin{array}{l}1 \\
1\end{array}$ & $\begin{array}{r}8928 \\
648928 \\
238928\end{array}$ & $\begin{array}{ll}3 & 5 \\
3 & 9 \\
3 & 9\end{array}$ \\
\hline $\begin{array}{ll}6.530590+6 & 6.530590+6 \\
6.527690+6 & 6.527690+6\end{array}$ & $\begin{array}{l}\odot \\
\odot\end{array}$ & $\begin{array}{l}\odot \\
\odot\end{array}$ & $\begin{array}{l}1 \\
1\end{array}$ & $\begin{array}{r}1008928 \\
378928\end{array}$ & $\begin{array}{l}310 \\
310\end{array}$ \\
\hline 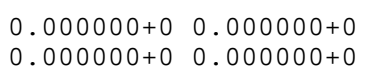 & $\begin{array}{l}\odot \\
\odot\end{array}$ & $\begin{array}{l}\odot \\
\odot\end{array}$ & $\begin{array}{l}1 \\
1\end{array}$ & $\begin{array}{l}978928 \\
368928\end{array}$ & $\begin{array}{l}4 \\
4\end{array}$ \\
\hline
\end{tabular}

8928416 only in VII.0 8928417 only in VII.0 8928418 only in VII.1 8928437 only in VII.0 8928491 Only in VII.0 8928516 Only in VII. 0 8928517 only in VII.0 8928518 0nly in VII.1 8928537 only in VII.0 8928591 only in VII.0 8928616 only in VII.1 8928617 only in VII.1 8928637 Only in VII.1 8928651 only in VII.1 8928652 only in VII.1 8928653 Only in VII.1 8928654 only in VII.1 8928655 Only in VII.1 8928656 only in VII.1 8928657 only in VII.1 8928691 Only in VII.1

$89-\mathrm{AC}-227$ 89286102 only in VII.1

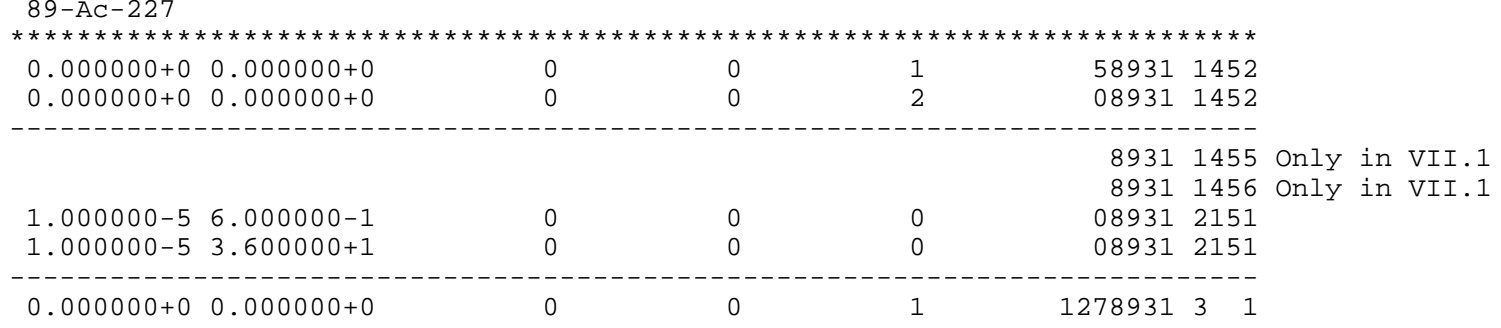




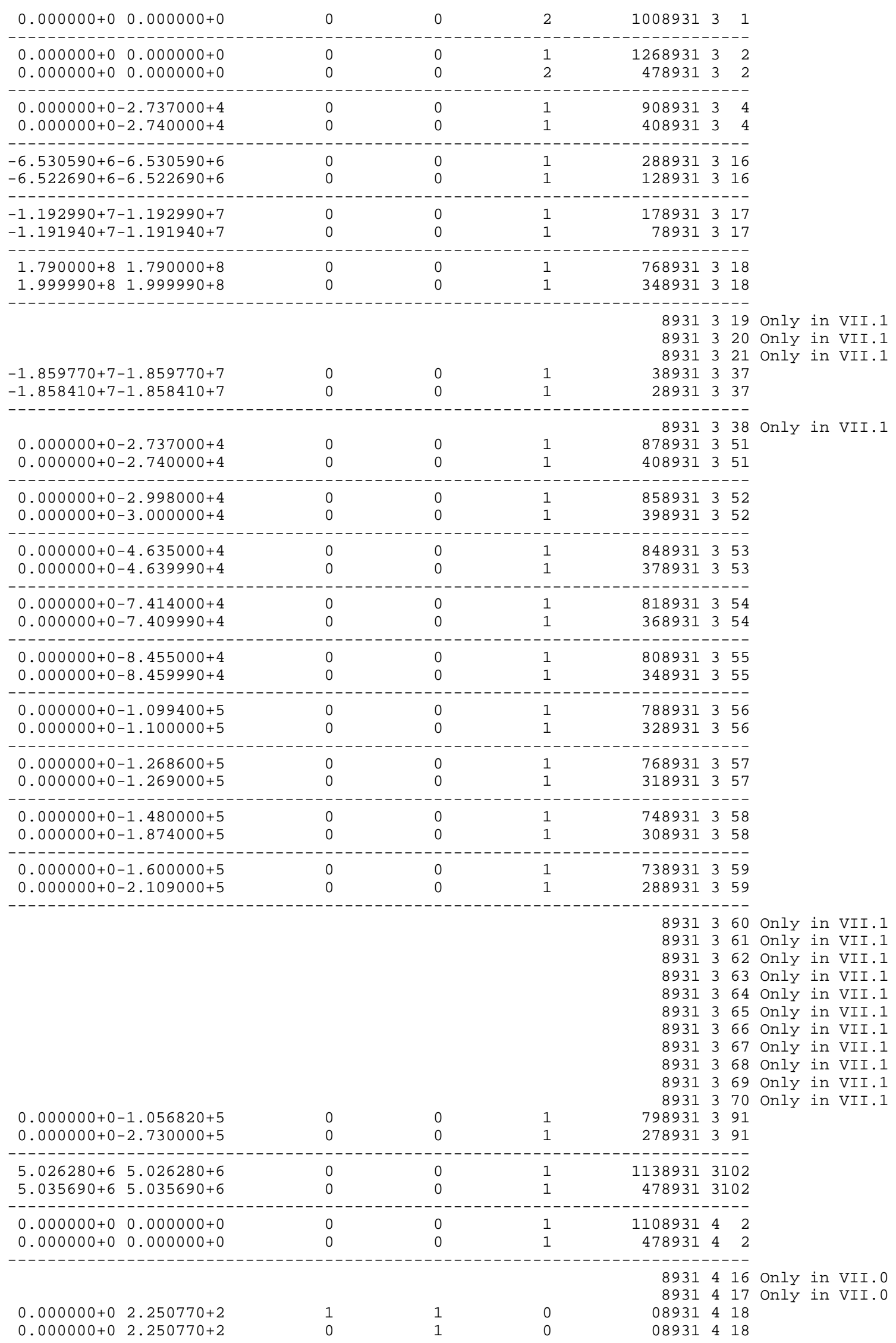




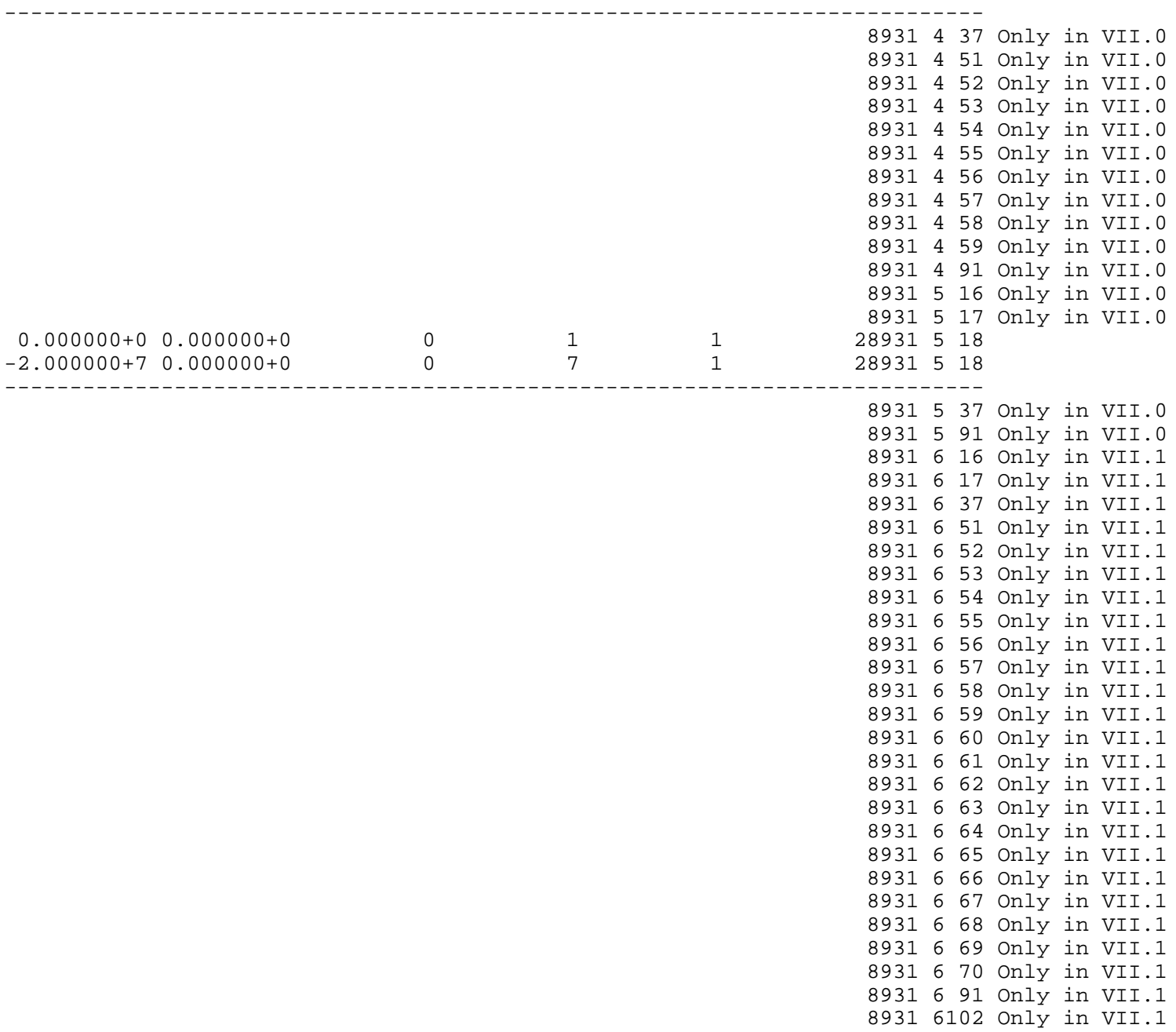

$90-$ Th- 227

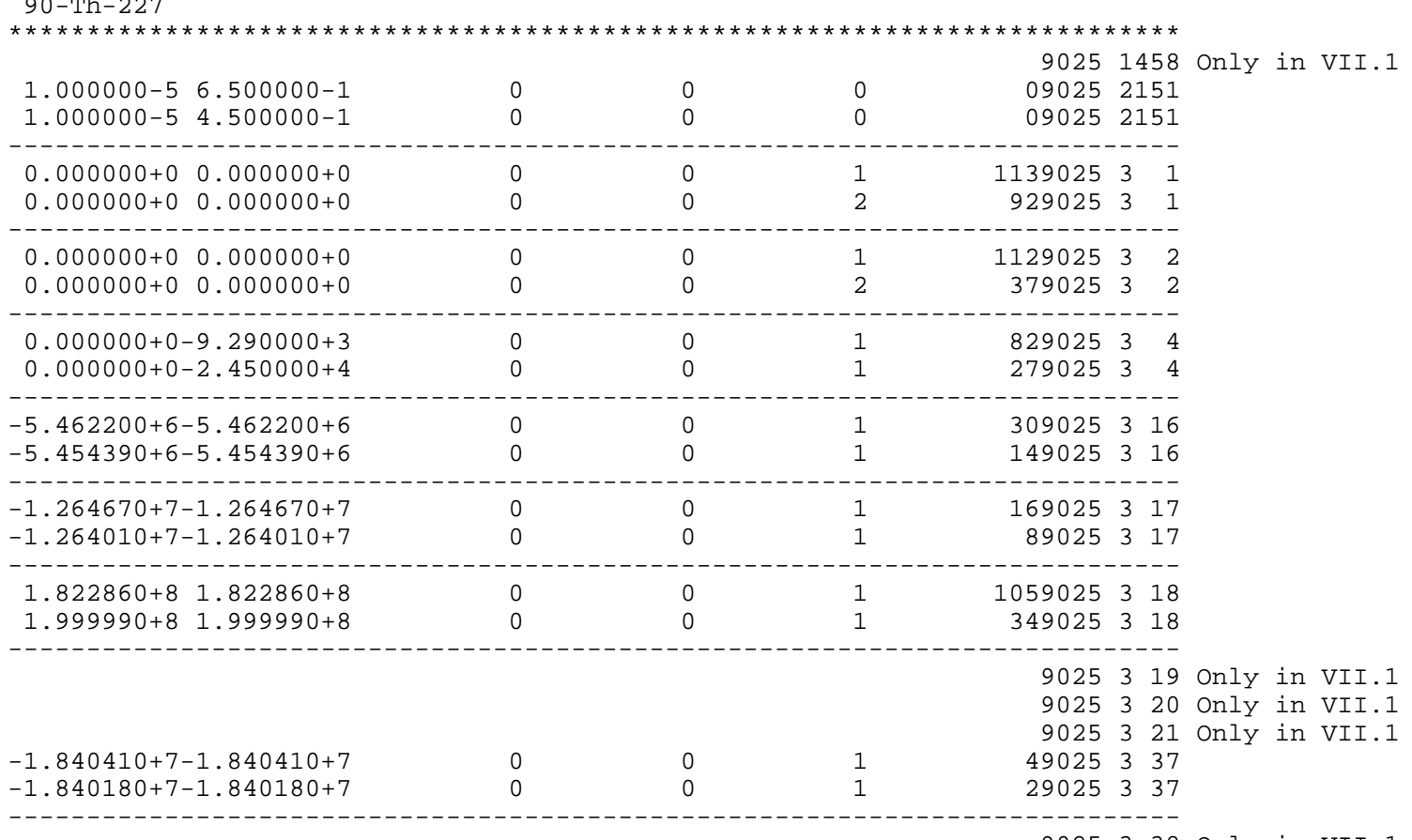

9025338 only in VII.1 


\begin{tabular}{|c|c|c|c|c|c|c|c|}
\hline $\begin{array}{l}\odot . \odot \odot \odot \odot \odot \odot+\odot-1.104890+5 \\
\odot . \odot \odot \odot \odot \odot \odot+\odot-2.450 \odot \odot \odot+4\end{array}$ & $\begin{array}{l}\odot \\
\odot\end{array}$ & $\begin{array}{l}\odot \\
\odot\end{array}$ & $\begin{array}{l}1 \\
1\end{array}$ & $\begin{array}{r}9025 \\
689025 \\
279025\end{array}$ & $\begin{array}{ll}3 & 03 \\
3 & 64 \\
3 & 91 \\
3 & 91\end{array}$ & Only in & VII.1 \\
\hline $\begin{array}{ll}7.105300+6 & 7.105300+6 \\
7.128690+6 & 7.128690+6\end{array}$ & $\begin{array}{l}\odot \\
\odot\end{array}$ & $\begin{array}{l}\odot \\
\odot\end{array}$ & $\begin{array}{l}1 \\
1\end{array}$ & $\begin{array}{r}1059025 \\
379025\end{array}$ & $\begin{array}{l}3102 \\
3102\end{array}$ & & \\
\hline 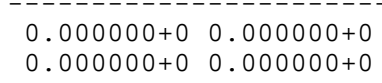 & $\begin{array}{l}\odot \\
\odot\end{array}$ & $\begin{array}{l}\odot \\
\odot\end{array}$ & $\begin{array}{l}1 \\
1\end{array}$ & $\begin{array}{r}1029025 \\
359025\end{array}$ & $\begin{array}{ll}4 & 2 \\
4 & 2\end{array}$ & & \\
\hline 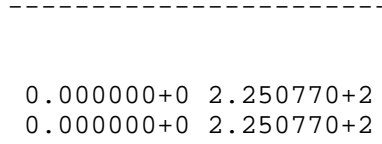 & $\begin{array}{l}1 \\
0\end{array}$ & $\begin{array}{l}1 \\
1\end{array}$ & $\begin{array}{l}\ominus \\
\odot\end{array}$ & $\begin{array}{r}9025 \\
9025 \\
09025 \\
09025\end{array}$ & $\begin{array}{ll}4 & 16 \\
4 & 17 \\
4 & 18 \\
4 & 18\end{array}$ & $\begin{array}{l}\text { Only in } \\
\text { Only in }\end{array}$ & $\begin{array}{l}\text { VII. } \odot \\
\text { VII. } \odot\end{array}$ \\
\hline $\begin{array}{rr}\odot .000 \odot \odot \odot \odot+\odot & \odot .00 \odot \odot \odot \odot+\odot \\
-2.0000 \odot \odot+7 & 0.0000 \odot \odot+\odot\end{array}$ & $\begin{array}{l}0 \\
\odot\end{array}$ & $\begin{array}{l}1 \\
7\end{array}$ & $\begin{array}{l}1 \\
1\end{array}$ & $\begin{array}{r}9025 \\
9025 \\
9025 \\
9025 \\
29025 \\
29025\end{array}$ & $\begin{array}{ll}4 & 37 \\
4 & 91 \\
5 & 16 \\
5 & 17 \\
5 & 18 \\
5 & 18\end{array}$ & $\begin{array}{l}\text { Only in } \\
\text { Only in } \\
\text { only in } \\
\text { Only in }\end{array}$ & $\begin{array}{l}\text { VII.0 } \\
\text { VII. } \\
\text { VII.0 } \\
\text { VII.0 }\end{array}$ \\
\hline & & & & $\begin{array}{l}9 \odot 25 \\
9 \odot 25 \\
9 \odot 25 \\
9 \odot 25 \\
9 \odot 25 \\
9 \odot 25 \\
9 \odot 25 \\
9 \odot 25 \\
9 \odot 25 \\
9 \odot 25 \\
9 \odot 25 \\
9 \odot 25 \\
9 \odot 25 \\
9 \odot 25 \\
9 \odot 25 \\
9 \odot 25 \\
9 \odot 25 \\
9 \odot 25 \\
9 \odot 25 \\
9 \odot 25 \\
9 \odot 25 \\
9 \odot 25\end{array}$ & $\begin{array}{ll}- & - \\
5 & 37 \\
5 & 91 \\
5455 \\
6 & 16 \\
6 & 17 \\
6 & 37 \\
6 & 51 \\
6 & 52 \\
6 & 53 \\
6 & 54 \\
6 & 55 \\
6 & 56 \\
6 & 57 \\
6 & 58 \\
6 & 59 \\
6 & 60 \\
6 & 61 \\
6 & 62 \\
6 & 63 \\
6 & 64 \\
6 & 91 \\
6102\end{array}$ & $\begin{array}{l}\text { Only in } \\
\text { Only in } \\
\text { Only in } \\
\text { Only in } \\
\text { Only in } \\
\text { Only in } \\
\text { Only in } \\
\text { Only in } \\
\text { Only in } \\
\text { Only in } \\
\text { Only in } \\
\text { Only in } \\
\text { Only in } \\
\text { Only in } \\
\text { Only in } \\
\text { Only in } \\
\text { Only in } \\
\text { Only in } \\
\text { Only in } \\
\text { Only in } \\
\text { Only in } \\
\text { Only in }\end{array}$ & $\begin{array}{l}\text { VII.0 } \\
\text { VII.0 } \\
\text { VII.1 } \\
\text { VII.1 } \\
\text { VII.1 } \\
\text { VII.1 } \\
\text { VII.1 } \\
\text { VII.1 } \\
\text { VII.1 } \\
\text { VII.1 } \\
\text { VII.1 } \\
\text { VII.1 } \\
\text { VII.1 } \\
\text { VII.1 } \\
\text { VII.1 } \\
\text { VII.1 } \\
\text { VII.1 } \\
\text { VII.1 } \\
\text { VII.1 } \\
\text { VII.1 } \\
\text { VII.1 }\end{array}$ \\
\hline
\end{tabular}

\begin{tabular}{|c|c|c|c|c|}
\hline 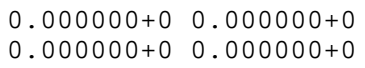 & $\begin{array}{l}\odot \\
\odot\end{array}$ & $\begin{array}{l}\odot \\
\odot\end{array}$ & $\begin{array}{l}1 \\
1\end{array}$ & $\begin{array}{ll}59028 & 1452 \\
79028 & 1452\end{array}$ \\
\hline 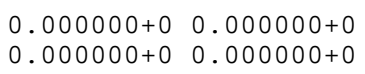 & $\begin{array}{l}\odot \\
\odot\end{array}$ & $\begin{array}{l}\odot \\
\odot\end{array}$ & $\begin{array}{l}1 \\
1\end{array}$ & $\begin{array}{ll}59028 & 1455 \\
49028 & 1455\end{array}$ \\
\hline 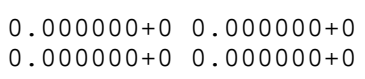 & $\begin{array}{l}0 \\
0\end{array}$ & $\begin{array}{l}\odot \\
\odot\end{array}$ & $\begin{array}{l}1 \\
1\end{array}$ & $\begin{array}{ll}29028 & 1456 \\
79028 & 1456\end{array}$ \\
\hline $\begin{array}{ll}9.022800+4 & 1.00000 \odot+\odot \\
9.022800+4 & 1.00000 \odot+\odot\end{array}$ & $\begin{array}{l}\odot \\
\odot\end{array}$ & $\begin{array}{l}\odot \\
\odot\end{array}$ & $\begin{array}{l}2 \\
1\end{array}$ & $\begin{array}{ll}09028 & 2151 \\
09028 & 2151\end{array}$ \\
\hline 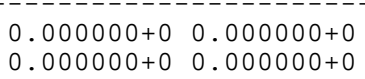 & $\begin{array}{l}\odot \\
\odot\end{array}$ & $\begin{array}{l}\ominus \\
\ominus\end{array}$ & $\begin{array}{l}1 \\
2\end{array}$ & $\begin{array}{rrr}1219028 & 3 & 1 \\
909028 & 3 & 1\end{array}$ \\
\hline$\odot .000 \odot \odot \odot+\odot \quad \odot .0 \odot \odot \odot \odot \odot+\odot$ & 0 & $\odot$ & 2 & $12090283 \quad 2$ \\
\hline
\end{tabular}




\begin{tabular}{|c|c|c|c|c|c|c|}
\hline $5.248790+65.248790+6$ & $\odot$ & $\odot$ & 2 & 509028 & 3102 & \\
\hline$\odot . \odot \odot \odot \odot \odot \odot+\odot \quad \odot . \diamond \odot \odot \odot \odot \odot+\odot$ & $\odot$ & $\odot$ & 1 & 1179028 & 4 & \\
\hline$\odot . \odot \odot \odot \odot \odot \odot+\odot \quad \odot . ๑ \odot \odot \odot \odot \odot+\odot$ & 0 & 0 & 1 & 309028 & 42 & \\
\hline & & & & 9028 & $\begin{array}{ll}4 & 16 \\
4 & 17\end{array}$ & $\begin{array}{l}\text { Only in VII.e } \\
\text { Only in VII.e }\end{array}$ \\
\hline$\odot .00000 \odot+\odot \quad 2.260700+2$ & 1 & 1 & $\odot$ & 09028 & 418 & \\
\hline
\end{tabular}

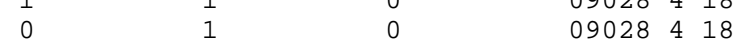

0

9028451 only in VII.0 9028452 only in VII.0 9028453 only in VII.0 9028454 only in VII.0 9028455 only in VII.0 9028456 only in VII.0 9028457 only in VII.0 9028458 only in VII.๑ 9028459 only in VII.0 9028460 only in VII.0 9028461 Only in VII. 0 9028462 only in VII.0 9028491 only in VII.0 9028516 only in VII.0 9028517 only in VII.0 $\begin{array}{rr}0.000000+\odot & \odot .0000000+\odot \\ -2.000000+7 & 0.000000+\odot\end{array}$ $\odot$

1
7

1

29028518

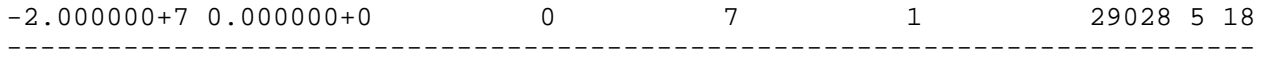

9028591 only in VII.0 9028616 only in VII.1 9028617 Only in VII.1 9028651 Only in VII.1 9028652 only in VII.1 9028653 Only in VII.1 9028654 Only in VII.1 9028655 only in VII.1 9028656 only in VII.1 9028657 only in VII.1 9028658 Only in VII.1 9028659 only in VII.1 9028660 only in VII.1 9028661 Only in VII.1 9028662 only in VII.1 9028663 only in VII.1 9028664 only in VII.1 9028665 only in VII.1 9028666 Only in VII.1 9028667 only in VII.1 9028668 only in VII.1 9028669 Only in VII.1 9028670 only in VII.1 9028671 only in VII.1 9028672 Only in VII.1 9028673 only in VII.1 9028674 Only in VII.1 9028675 Only in VII.1 9028676 only in VII.1 9028677 Only in VII.1 9028678 only in VII.1 9028679 only in VII.1 9028691 Only in VII.1

$90-$ Th- 229 90286102 only in VII.1

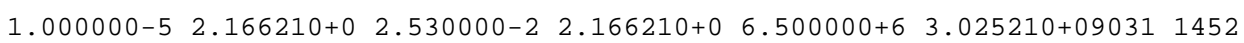
$\begin{array}{llllll}1.000000-5 & 2.087200+\odot & 2.530000-2 & 2.087200+\odot & 5.0000000+6 & 2.557200+09031 \\ 1452\end{array}$

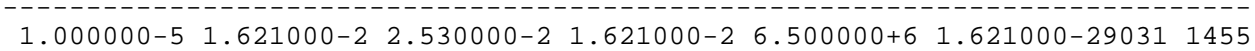
$1.000000-5 \quad 1.621000-2 \quad 2.530000-2 \quad 1.621000-2 \quad 5.000000+6 \quad 1.621000-290311455$

$1.000000-52.150000+02.000000+74.793000+0 \quad 0031456$

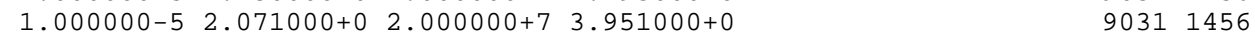

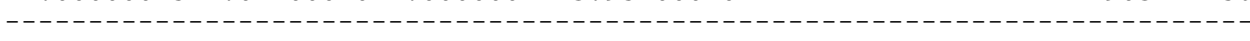

$\begin{array}{rrrrrr}9.022900+4 & 1.000000+\odot & 0 & 0 & 2 & 090312151\end{array}$
}




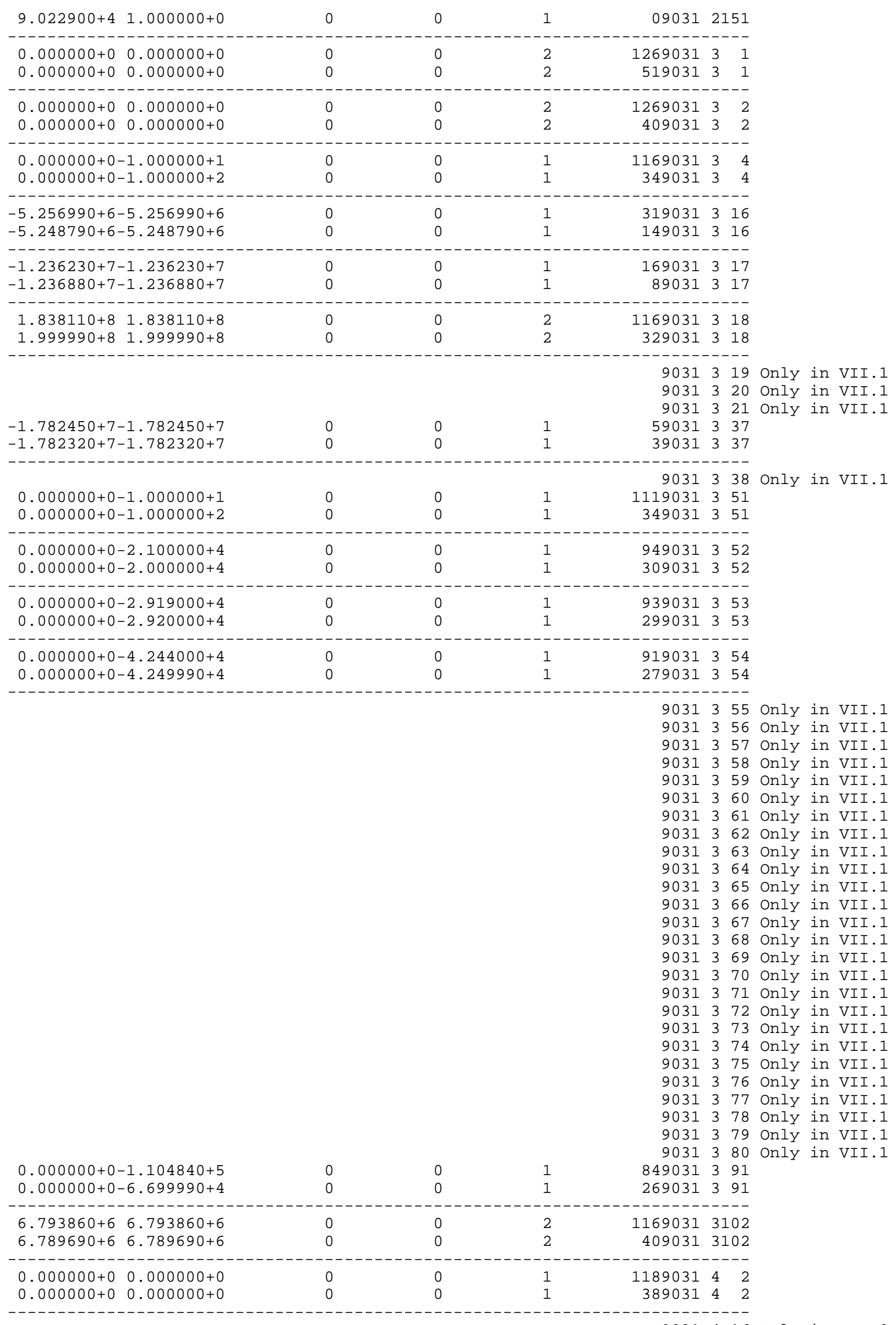

9031416 only in VII. 0 


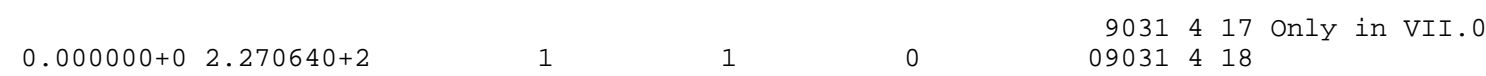

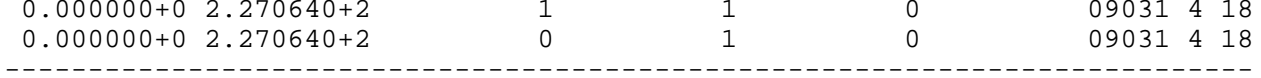

9031437 only in VII.0

9031451 Only in VII.0

9031452 only in VII.0

9031453 only in VII.0

9031454 only in VII. $\odot$

9031491 Only in VII.0

9031516 only in VII.0

9031517 only in VII.0

$0.000000+0 \quad 0.0000000+0$ $-2.000000+7 \quad 0.000000+0$

$\odot$

1
7

1

29031518

29031518

-

9031537 only in VII.0

9031591 only in VII.0

90315455 Only in VII.1

9031616 only in VII.1

9031617 only in VII.1

9031637 Only in VII.1

9031651 only in VII.1

9031652 only in VII.1

9031653 only in VII.1

9031654 only in VII.1

9031655 Only in VII.1

9031656 only in VII.1

9031657 only in VII.1

9031658 only in VII.1

9031659 only in VII.1

9031660 Only in VII.1

9031661 only in VII.1

9031662 only in VII.1

9031663 only in VII.1

9031664 only in VII.1

9031665 only in VII.1

9031666 only in VII.1

9031667 only in VII.1

9031668 only in VII.1

9031669 only in VII.1

9031670 only in VII.1

9031671 only in VII.1

9031672 only in VII.1

9031673 only in VII.1

9031674 Only in VII.1

9031675 only in VII.1

9031676 only in VII.1

9031677 only in VII.1

9031678 only in VII.1

9031679 Only in VII.1

9031680 only in VII.1

9031691 only in VII.1

90316102 Only in VII.1

$90-\mathrm{Th}-230$

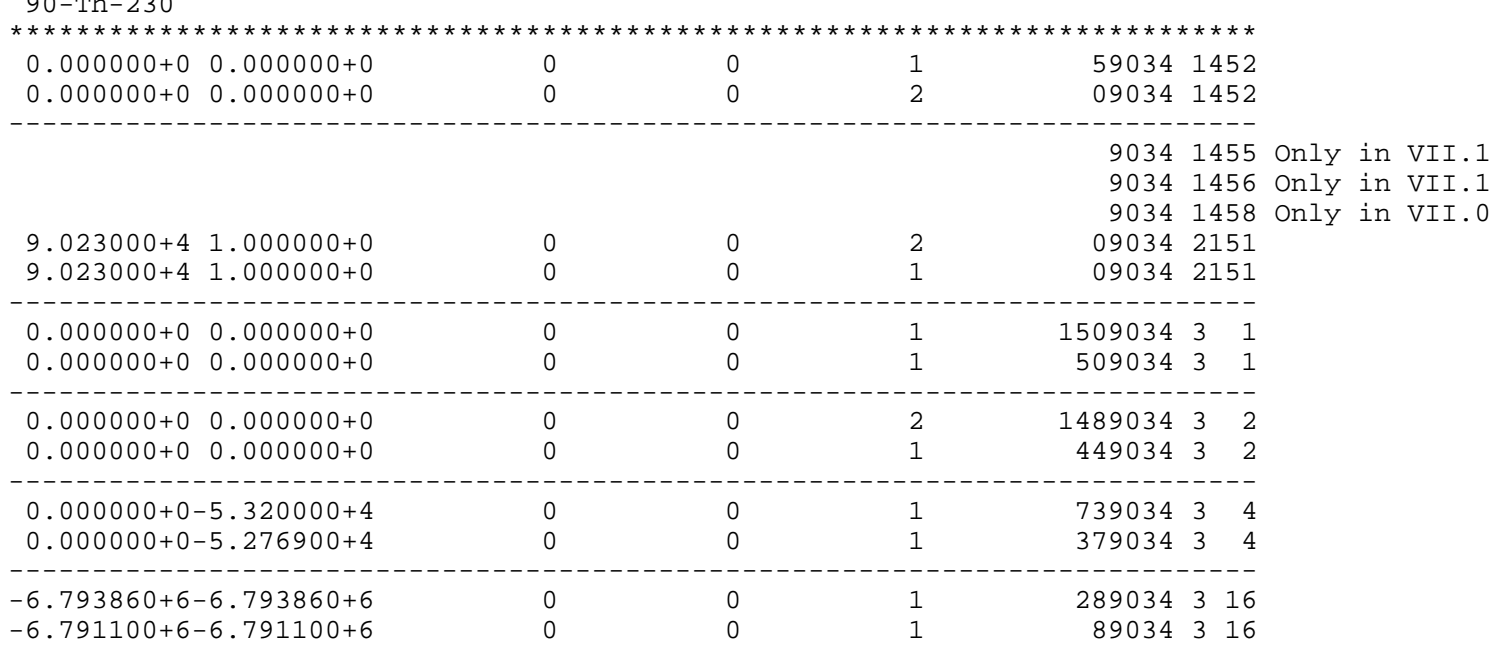




\begin{tabular}{|c|c|}
\hline $\begin{array}{l}-1.205080+7-1.205080+7 \\
-1.204000+7-1.204000+7\end{array}$ & $\begin{array}{l}\odot \\
\odot\end{array}$ \\
\hline \multicolumn{2}{|c|}{ 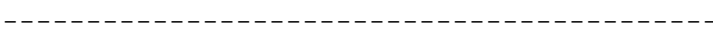 } \\
\hline $1.837000+8 \quad 1.837000+8$ & $\odot$ \\
\hline $1.905965+8 \quad 1.905965+8$ & $\odot$ \\
\hline
\end{tabular}

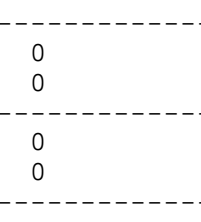

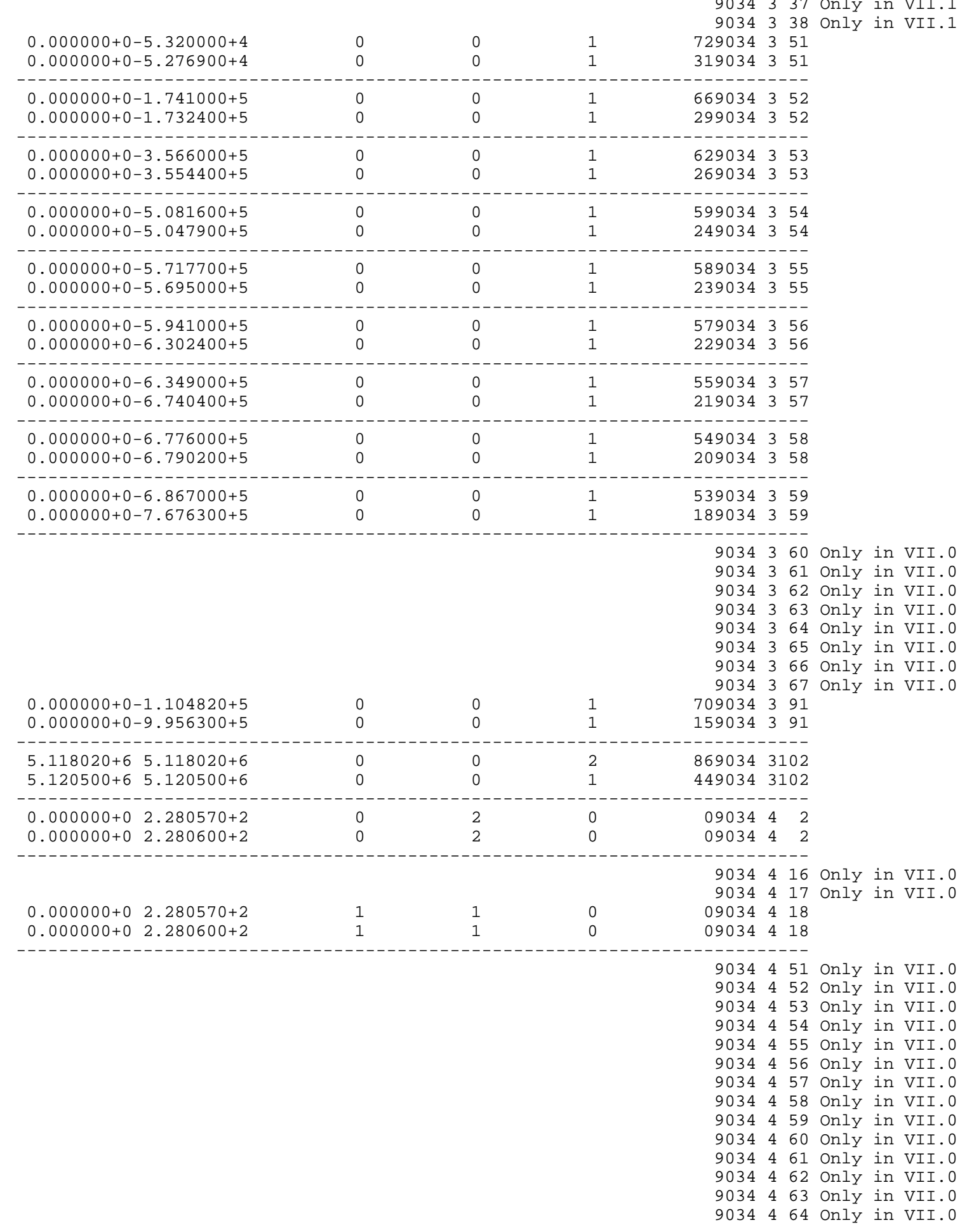


9034465 only in VII.๑ 9034466 only in VII.0 9034467 only in VII.0 9034491 only in VII.0 9034516 Only in VII.0

$0.000000+\odot \quad 0.0000000+\odot$ $-3.0000000+7 \quad 0.0000000+\odot$ $\stackrel{0}{0}$ 9034517 only in VII.0 29034518 29034518 - . - . . . -

9034591 only in VII.๑ 9034616 only in VII.1 9034617 Only in VII.1 9034637 Only in VII.1 9034651 only in VII.1 9034652 only in VII.1 9034653 only in VII.1 9034654 only in VII.1 9034655 Only in VII.1 9034656 only in VII.1 9034657 only in VII.1 9034658 Only in VII.1 9034659 only in VII.1 9034691 Only in VII.1

90-Th-231 Evaluation Only in VII.1 90346102 only in VII.1

$90-\mathrm{Th}-232$

$* * * * * * * * * * * * * * * * * * * * * * * * * * * * * * * * * * * * * * * * * * * * * * * * * * * * * * * * * * * * * * * * * * * * * * * * * * *$

(1) 4.948537-3 2.743675-2 $0.000000+\odot \quad 0.000000+$

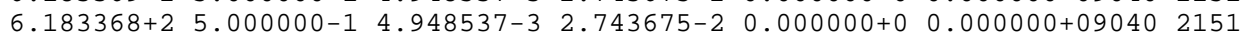

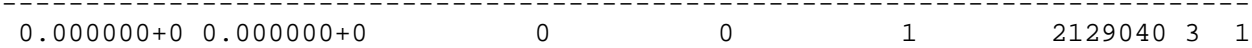

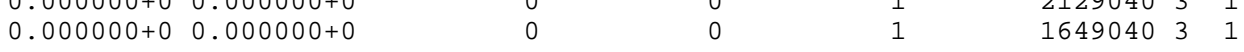

-...-.

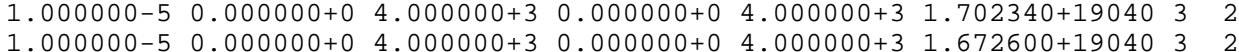

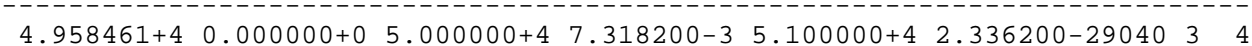
$\begin{array}{llllllll}4.958460+4 & 0.000000+\odot & 5.000000+4 & 7.386080-3 & 5.100000+4 & 2.363990-29040 & 3 & 4\end{array}$

$\begin{array}{ccccc}-3.922000+6-3.922000+6 & 0 & 0 & 1 & 2890403 \\ 1.407000+61.407000+6 & 0 & 0 & 1 & 3790403\end{array}$

$6.464980+6 \quad 0.000000+0 \quad 6.800000+6 \quad 7.644680-2 \quad 7.000000+6 \quad 2.498850-19040316$ $6.464980+6 \quad 0.00000 \odot+\odot \quad 6.800000+6 \quad 7.657270-2 \quad 7.00000 \odot+6 \quad 2.504650-19040316$

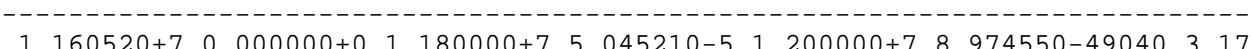

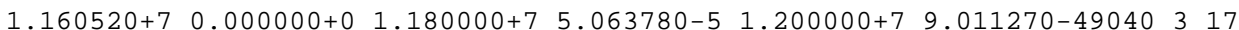

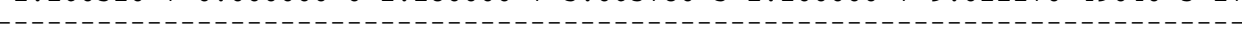

$\begin{array}{llllllll}1.000000-5 & 0.000000+0 & 4.000000+3 & 0.000000+0 & 4.000000+3 & 3.799060-69040 & 3 & 18\end{array}$

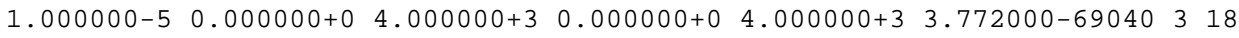

-

$\begin{array}{cccccc}4.083000+6 & 4.083000+6 & 0 & 0 & 1 & 55904032 \\ 4.083000+6 & 4.083000+6 & 0 & 0 & 1 & 579040322\end{array}$

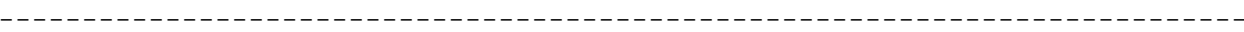

$2.233670+6 \quad \odot . \odot \odot \odot \odot \odot \odot+\odot \quad 1.180 \odot \odot \odot+7 \quad \odot . \odot \odot \odot \odot \odot \odot+\odot \quad 1.20 \odot \odot \odot \odot+7 \quad 3.57113-14904 \odot \quad 3 \quad 24$ $2.233668+6 \quad 0.000000+\odot \quad 1.180000+7 \quad 0.000000+\odot \quad 1.200000+7 \quad 3.56136-149040 \quad 324$

(1.

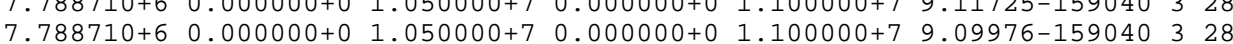

$1.353160+7 \quad 0.000000+\odot \quad 1.650000+7 \quad 0.000000+\odot \quad 1.700000+7 \quad 7.13681-159040341$ $\begin{array}{lllllllllll}1.353157+7 & 0.000000+\odot & 1.650000+7 & 0.00000 \odot+\odot & 1.700000+7 & 7.08939-159040 & 3 & 41\end{array}$ - - $4.958460+4 \quad 0.000000+0 \quad 5.000000+4 \quad 7.318190-3 \quad 5.100000+4 \quad 2.336200-29040 \quad 3 \quad 51$ $4.958460+4 \quad \odot .00 \odot \odot \odot \odot+\odot \quad 5.00 \odot \odot \odot \odot+4 \quad 7.386080-3 \quad 5.100 \odot \odot \odot+4 \quad 2.363990-29040 \quad 351$

$1.628250+5 \quad 0.000000+0 \quad 1.750000+5 \quad 8.498980-4 \quad 1.900000+51.791470-39040352$ $1.628247+5 \quad 0.000000+0 \quad 1.750000+5 \quad 8.603450-4 \quad 1.900000+5 \quad 1.833970-39040352$

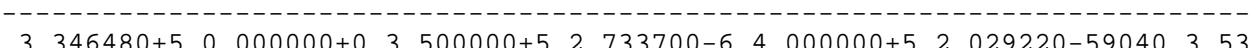
$\begin{array}{lllllllll}3.346480+5 & 0.000000+\odot & 3.500000+5 & 2.782700-6 & 4.000000+5 & 2.067350-59040 & 3 & 53\end{array}$

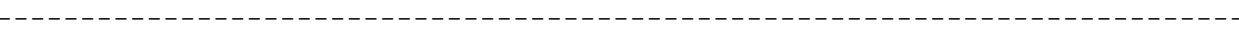

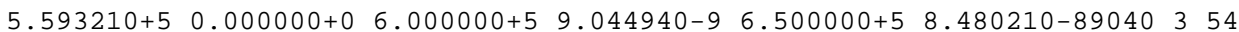

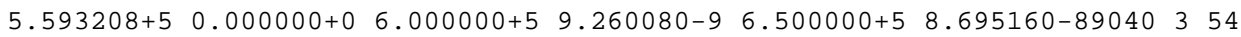


$7.173550+5 \quad 0.000000+\odot \quad 7.500000+5 \quad 6.583260-2 \quad 8.000000+5 \quad 1.409790-19040355$ $\begin{array}{llllll}7.173548+5 & 0.0000000+\odot & 7.500000+5 & 6.778220-2 & 8.000000+5 & 1.454040-19 \odot 4 \odot 355\end{array}$

$7.335250+5 \quad 0.000000+07.500000+51.543450-28.000000+5 \quad 5.042140-29040356$ $7.335248+5 \quad 0.000000+07.500000+5 \quad 1.589160-2 \quad 8.000000+5 \quad 5.200400-29040356$

$7.774650+50.000000+08.000000+53.588850-28.500000+59.115350-29040357$

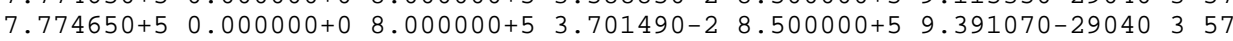

$7.777660+5 \quad 0.000000+0 \quad 8.000000+5 \quad 1.855330-2 \quad 8.500000+5 \quad 5.739300-29040358$ $7.777660+5 \quad 0.000000+08.000000+5 \quad 1.913560-2 \quad 8.500000+5 \quad 5.912900-29040358$

$7.887140+5 \quad \odot .000000+\odot \quad 8.000000+5 \quad 1.831790-2 \quad 8.500000+5 \quad 7.752570-29040359$ $7.887137+5 \quad 0.000000+\odot \quad 8.000000+5 \quad 1.889290-2 \quad 8.500000+5 \quad 7.987070-29040359$

$8.305950+5 \quad 0.000000+\odot \quad 8.500000+5 \quad 1.81384-12 \quad 9.000000+5 \quad 2.53617-119040360$ $8.305949+5 \quad 0.0000000+\odot \quad 8.5000000+5 \quad 1.86871-12 \quad 9.000000+5 \quad 2.61000-119040360$

$8.332060+5 \quad 0.000000+\odot \quad 8.5000000+5 \quad 1.332910-2 \quad 9.0000 \odot 0+5 \quad 4.327230-29040361$ $8.332060+5 \quad 0.0000000+0 \quad 8.5000000+5 \quad 1.343450-2 \quad 9.000000+5 \quad 4.392670-29040361$

$8.767950+50000000+09.000000+53.743860-39.500000+51.313100-29040362$ $8.767949+5 \quad 0.000000+0 \quad 9.000000+5 \quad 3.800480-3 \quad 9.500000+5 \quad 1.342570-29040362$

(1) $8.874410+5 \quad 0.000000+0 \quad 9.000000+5 \quad 3.304620-4 \quad 9.500000+5 \quad 1.846150-39040363$ $8.874410+5 \quad 0.000000+\odot \quad 9.0000000+5 \quad 3.354600-4 \quad 9.5000000+5 \quad 1.887580-39040363$ $8.939690+50.000000+09.000000+59.781640-49.500000+59.459290-390403$ $8.939690+5 \quad 0.000000+\odot \quad 9.000000+5 \quad 9.929570-4 \quad 9.500000+5 \quad 9.671550-39040364$

$9.645750+5 \quad 0.000000+\odot \quad 1.000000+6 \quad 1.943140-3 \quad 1.100000+6 \quad 8.906040-39040365$ $9.645748+5 \quad 0.000000+\odot \quad 1.000000+6 \quad 2.001170-3 \quad 1.100000+6 \quad 9.153900-39040365$ $1.027550+6 \quad 0.000000+\odot \quad 1.100000+6 \quad 1.413200-4 \quad 1.140000+6 \quad 2.819320-49040366$ $\begin{array}{lllllll}1.027547+6 & 0.000000+\odot & 1.100000+6 & 1.452530-4 & 1.140000+6 & 2.895500-49040 & 3\end{array} 66$ $1.047430+6 \quad 0.000 \odot \odot \odot+\odot \quad 1.100 \odot \odot \odot+6 \quad 6.402250-6 \quad 1.1400 \odot \odot+6 \quad 1.577030-59040367$ $1.047430+6 \quad 0.000000+\odot \quad 1.100000+6 \quad 6.580430-6 \quad 1.140000+6 \quad 1.619640-59040367$ $1.054460+6 \quad 0.000000+\odot \quad 1.100000+6 \quad 7.354120-5 \quad 1.140000+6 \quad 1.853980-49040368$ $1.054460+6 \quad 0.000000+\odot \quad 1.100000+6 \quad 7.558790-5 \quad 1.140000+6 \quad 1.904080-49040368$

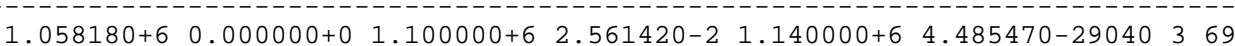
$1.058180+6 \quad 0.000000+\odot \quad 1.100000+6 \quad 2.632710-2 \quad 1.140000+6 \quad 4.606670-29040369$

$1.077560+60.000000+01.100000+61.378810-21.140000+63.384090-29040370$ $1.077560+6 \quad 0.000000+\odot \quad 1.100000+6 \quad 1.417180-2 \quad 1.140000+6 \quad 3.475530-29040370$ $1.082180+60.000000+\odot 1.100000+6 \quad 1.178520-2 \quad 1.140000+63.411610-29040371$

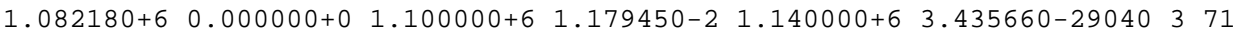

1. $1.083390+6 \quad 0.000000+0 \quad 1.100000+6 \quad 5.212090-3 \quad 1.140000+6 \quad 1.537540-29040372$ $\begin{array}{llllllll}1.083389+6 & 0.000000+\odot & 1.100000+6 & 5.216220-3 & 1.140000+6 & 1.548380-29040 & 3 & 72\end{array}$ $1.099160+6 \quad 0.000000+\odot \quad 1.140000+6 \quad 1.404360-2 \quad 1.200000+6 \quad 3.359360-29040373$ $\begin{array}{lllllll}1.099157+6 & 0.000000+\odot & 1.140000+6 & 1.414260-2 & 1.200000+6 & 3.415070-29040 & 3\end{array}$ $1.110510+6 \quad 0.000000+0 \quad 1.140000+6 \quad 9.924710-3 \quad 1.200000+6 \quad 2.912470-29040374$ $\begin{array}{llllllll}1.110507+6 & 0.000000+0 & 1.140000+6 & 9.994670-3 & 1.200000+6 & 2.960770-29040 & 3 & 74\end{array}$ $1.126680+6 \quad 0.000 \odot \odot \odot+\odot \quad 1.140000+6 \quad 7.354170-3 \quad 1.2000 \odot \odot+6 \quad 3.333500-29040375$ $1.126676+6 \quad 0.000000+01.140000+6 \quad 7.406010-3 \quad 1.200000+6 \quad 3.388780-29040375$

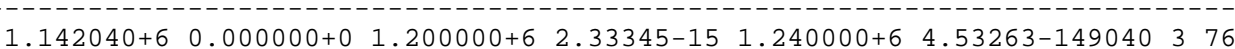
$\begin{array}{llllllll}1.142040+6 & 0.000000+\odot & 1.200000+6 & 2.37215-15 & 1.240000+6 & 4.63707-149040 & 3 & 76\end{array}$

$1.148270+6 \quad 0.000000+\odot \quad 1.200000+6 \quad 7.563760-3 \quad 1.240000+6 \quad 1.272770-29040377$ $1.148270+6 \quad 0.000000+\odot \quad 1.200000+6 \quad 7.689200-3 \quad 1.240000+6 \quad 1.302100-29040377$ $1.150980+6 \quad 0.000000+\Theta \quad 1.200000+6 \quad 3.201620-5 \quad 1.240000+6 \quad 7.383080-59040378$ $1.150980+6 \quad 0.000000+\odot \quad 1.200000+6 \quad 3.254710-5 \quad 1.240000+6 \quad 7.553190-59040378$

1. $1.153290+6 \quad 0.000000+\odot \quad 1.200000+6 \quad 5.159410-3 \quad 1.240000+6 \quad 1.059550-29040379$ 
$1.187640+6 \quad 0.000000+0 \quad 1.200000+6 \quad 3.529490-3 \quad 1.240000+6 \quad 1.392280-29040 \quad 380$ $1.187640+6 \quad 0.000000+\odot \quad 1.200000+6 \quad 3.588020-3 \quad 1.240000+6 \quad 1.424360-29040388$

$1.214160+6 \quad 0.000000+0 \quad 1.240000+6 \quad 4.402680-4 \quad 1.300000+6 \quad 1.807960-39040381$ $1.214155+6 \quad 0.000000+\odot \quad 1.240000+6 \quad 4.504120-4 \quad 1.300000+6 \quad 1.853300-39040381$

$1.223400+6 \quad 0.000000+01.240000+62.089070-3 \quad 1.300000+68.719840-39040382$

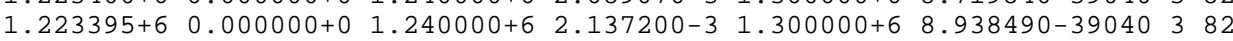

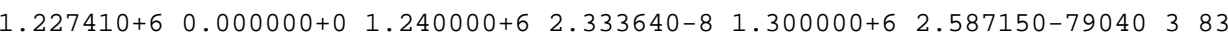

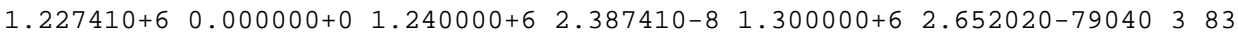

$1.255030+6 \quad \odot .000000+\odot \quad 1.300000+6 \quad 9.404840-9 \quad 1.350000+6 \quad 3.456270-89040384$ $1.255030+6 \quad 0.000000+0 \quad 1.3000000+6 \quad 9.640670-9 \quad 1.350000+6 \quad 3.538530-89040384$

$1.264170+6 \quad 0.000000+\odot \quad 1.300000+6 \quad 6.976220-8 \quad 1.350000+6 \quad 3.951400-79040335$ $1.264170+6 \quad 0.0000000+\odot \quad 1.3000000+6 \quad 7.151150-8 \quad 1.350000+6 \quad 4.045450-79040385$

$1.298820+6 \quad 0.000000+\odot \quad 1.350000+6 \quad 1.591810-2 \quad 1.4000 \odot \odot+6 \quad 3.065440-290403 \quad 86$ $1.298820+6 \quad 0.000000+\odot \quad 1.350000+6 \quad 1.629700-2 \quad 1.400000+6 \quad 3.134500-29040386$

$1.335180+60.000000+01350000+62.174600-51.400000+61.257640-49040387$

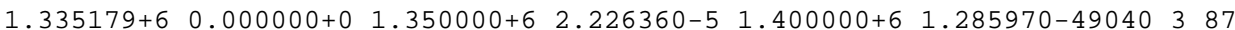

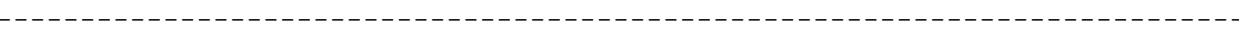

$\begin{array}{lllllll}1.375960+6 & 0.000000+\odot & 1.400000+6 & 3.589470-5 & 1.460000+6 & 1.800960-49040 & 388\end{array}$ $1.375955+6 \quad 0.000000+\odot \quad 1.400000+6 \quad 3.670340-5 \quad 1.460000+6 \quad 1.838790-49040388$

$1.393230+60.000000+01.400000+61.004570-51.460000+61.306480-49040389$ $1.393230+6 \quad 0.000000+\odot 1.400000+6 \quad 1.027200-5 \quad 1.460000+6 \quad 1.333920-49040389$

$4.455000+5 \quad 0.000000+04.500000+56.248020-5 \quad 5.000000+51.126060-49040301$

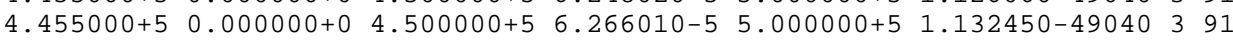

烈 $\begin{array}{llllll}1.000000-5 & 0.000000+0 & 4.000000+3 & 0.000000+0 & 4.000000+3 & 1.321810+090403102\end{array}$ $1.000000-5 \quad 0.000000+\odot \quad 4.000000+3 \quad 0.000000+0 \quad 4.000000+3 \quad 1.183070+090403102$

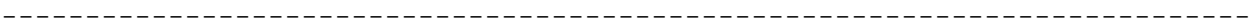
90403112 only in VII.1

$2.930680+6 \quad 0.000000+0 \quad 5.750000+6 \quad 0.0000000+0 \quad 6.100000+6 \quad 4.71082-159040 \quad 3600$ $2.930680+6 \quad 0.000000+\odot \quad 5.750000+6 \quad 0.000000+0 \quad 6.100000+6 \quad 4.71161-159040 \quad 3600$ $2.930680+60.000000+\odot 5.750000+6 \quad 0.000000+06.100000+6 \quad 1.30887-1490403649$ $2.930680+6 \quad 0.0000000+\odot \quad 5.750000+6 \quad 0.000000++\odot \quad 6.1000000+6 \quad 1.30909-1490403649$

(2.

$\begin{array}{llllll}8.655000+6 & 8.655000+6 & 0 & 0 & 1 & 8890403800\end{array}$

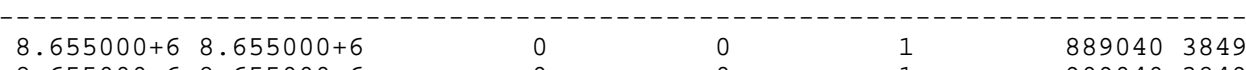

$\begin{array}{rrrrrr}8.655000+6 & 8.655000+6 & 0 & 0 & 1 & 9090403849\end{array}$

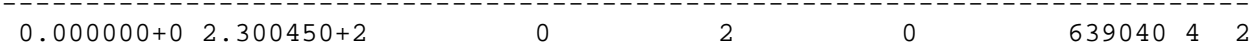

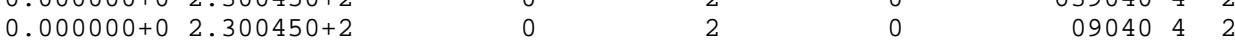

(4.

$4.568890-2-2.344780-2-1.309000-23.095110-3 \quad 9040451$ $4.568900-2-2.344780-2-1.309000-23.095110-3 \quad 9040451$

$8.267510-2-5.956630-3-4.941050-30.000000+0 \quad 9040452$ $8.267510-2-5.956640-3-4.941050-30.000000+\odot \quad 9040452$

$1.476250-14.981510-2-8.510160-5-5.778520-3 \quad 9040453$ $1.476250-14.981510-2-8.510150-5-5.778520-3 \quad 9040453$

$-8.171520-35.121470-3$
$-8.171520-35.121460-3$

$-8.171520-35.121460-3 \quad 9040454$

$-5.059820-40.000000+0$
$-5.059810-40.000000+0$
$-5.059810-40.000000+0 \quad 9040455$

$3.238360-3 \quad 1.158240-1 \quad 1.685290-2 \quad 2.429430-2-7.915440-3 \quad 1.449480-29040456$ 3.238360-3 1.158240-1 1.685290-2 2.429430-2-7.915430-3 $1.449480-29040456$

$\begin{array}{rr}7.595890-4-5.990100-3 & 904040457\end{array}$

1.

$1.350790-2-3.003530-25.954940-3-1.323090-3-1.076770-2-7.610340-39040458$ 
1.350790-2-3.003530-2 5.954940-3-1.323100-3-1.076770-2-7.610340-39040 4 58 $1.184360-3-9.779560-3 \quad 9040459$ $\begin{array}{lr}1.184350-3-9.779560-3 & 9040459\end{array}$ 
$-9.820030-4 \quad 4.221780-3$

9040483

$2.687500-3,2.5726$

9040484

$-2.687490-3 \quad 2.572610-3$

9040484

$8.060270-44.116630-2 \quad 9040485$

8.060260-4 4.116630-2 9040485

$1.603630-3-3.329860-37.245740-4-2.406090-3 \quad 9040486$

$1.603630-3-3.329860-37.245750-4-2.406090-3 \quad 9040486$

$2.922510-26.769290-29.805710-40.000000+\odot \quad 9040487$

$2.922510-2 \quad 6.769300-2 \quad 9.805710-4 \quad 0.000000+\odot \quad 9040487$

$2.335830-27.113720-2$
$2.335830-27.113710-2$

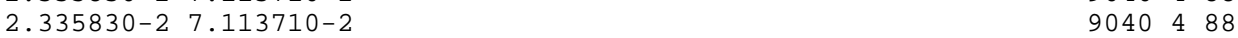

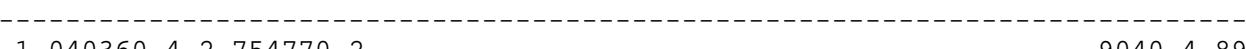

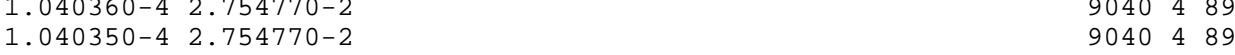

0.00000+9

$\begin{array}{llllll}0.000000+0 & 0 & 0 & 1 & 5790404800\end{array}$

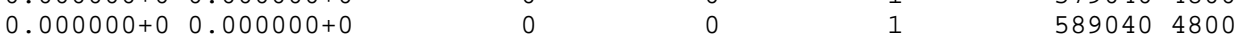

$\begin{array}{llllllll}1.000000+\odot & 1.000000+\odot & 0 & 1 & 1 & 289040 & 6 & 5\end{array}$

$\begin{array}{lllllll}1.000000+\odot & 1.000000+\odot & 0 & 1 & 1 & 3690406 & 5\end{array}$

(1)

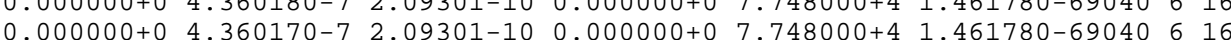
0.000000+0 3.567370-6 $7.17859-101.090800+55.343040-62.191220-99040617$

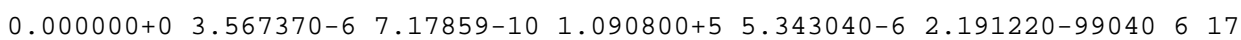

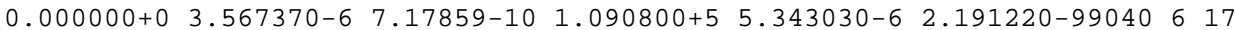

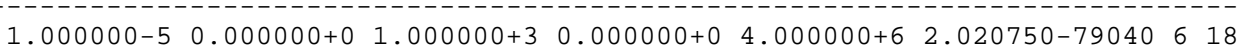
$1.000000-5-5.550760-6 \quad 1.000000+3-5.550760-6 \quad 4.000000+6 \quad 2.020750-79040618$

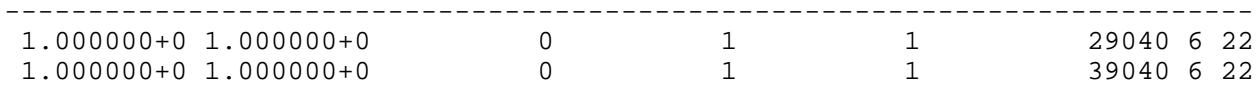

$\begin{array}{llllllll}0.000000+0 & 2.783420-7 & 8.83523-11 & 1.157300+5 & 1.658490-6 & 1.106590-99040 & 6 & 24\end{array}$ $0.000000+0 \quad 2.783420-7 \quad 8.83523-11 \quad 1.157300+5 \quad 1.658490-6 \quad 1.106580-99040 \quad 6 \quad 24$

- . -

$0.000000+0 \quad 1.547120-8 \quad 2.069240-9 \quad 1.517680-9-2.89150-10 \quad 4.36544-119040628$ $0.000000+\odot 1.547120-8 \quad 2.069240-9 \quad 1.517680-9-2.89150-10 \quad 4.36545-119040628$

$7.54496-130.000000+02.458550+66.88917-115.07155-130.000000+00040641$ $7.54496-13 \quad 0.000000+\odot \quad 2.458550+6 \quad 6.88916-11 \quad 5.07155-13 \quad 0.000000+09040 \quad 6 \quad 41$

1.

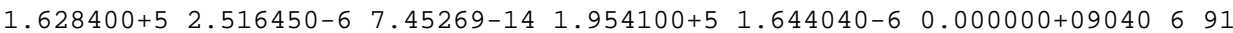
$1.628400+5 \quad 2.516450-6 \quad 7.45268-14 \quad 1.954100+5 \quad 1.644040-6 \quad 0.0000 \odot \odot+09 \odot 40691$

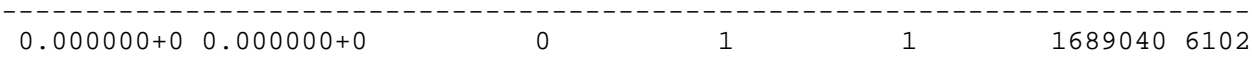

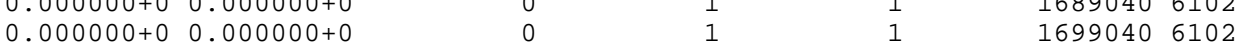

_.

$\begin{array}{llllll} & & \end{array}$ 2.58106-10 2.25961-11 2.944230+6 9.292310-9 5.30952-10 4.68649-119040 6649

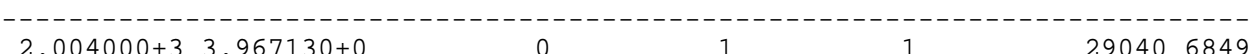

$\begin{array}{llllll}2.004000+3 & 3.967130+0 & 0 & 1 & 1 & 390406849\end{array}$

2.

$90-\mathrm{Th}-233$

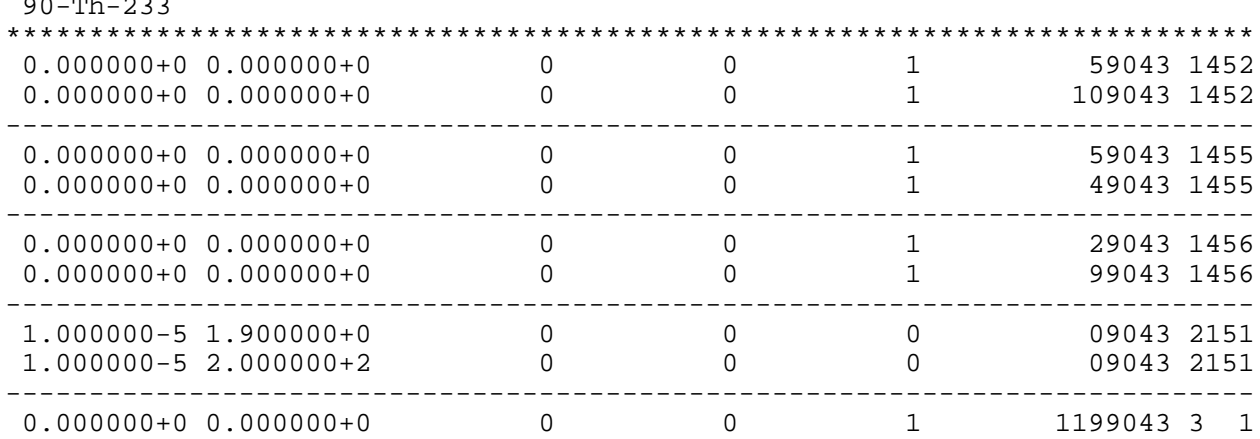




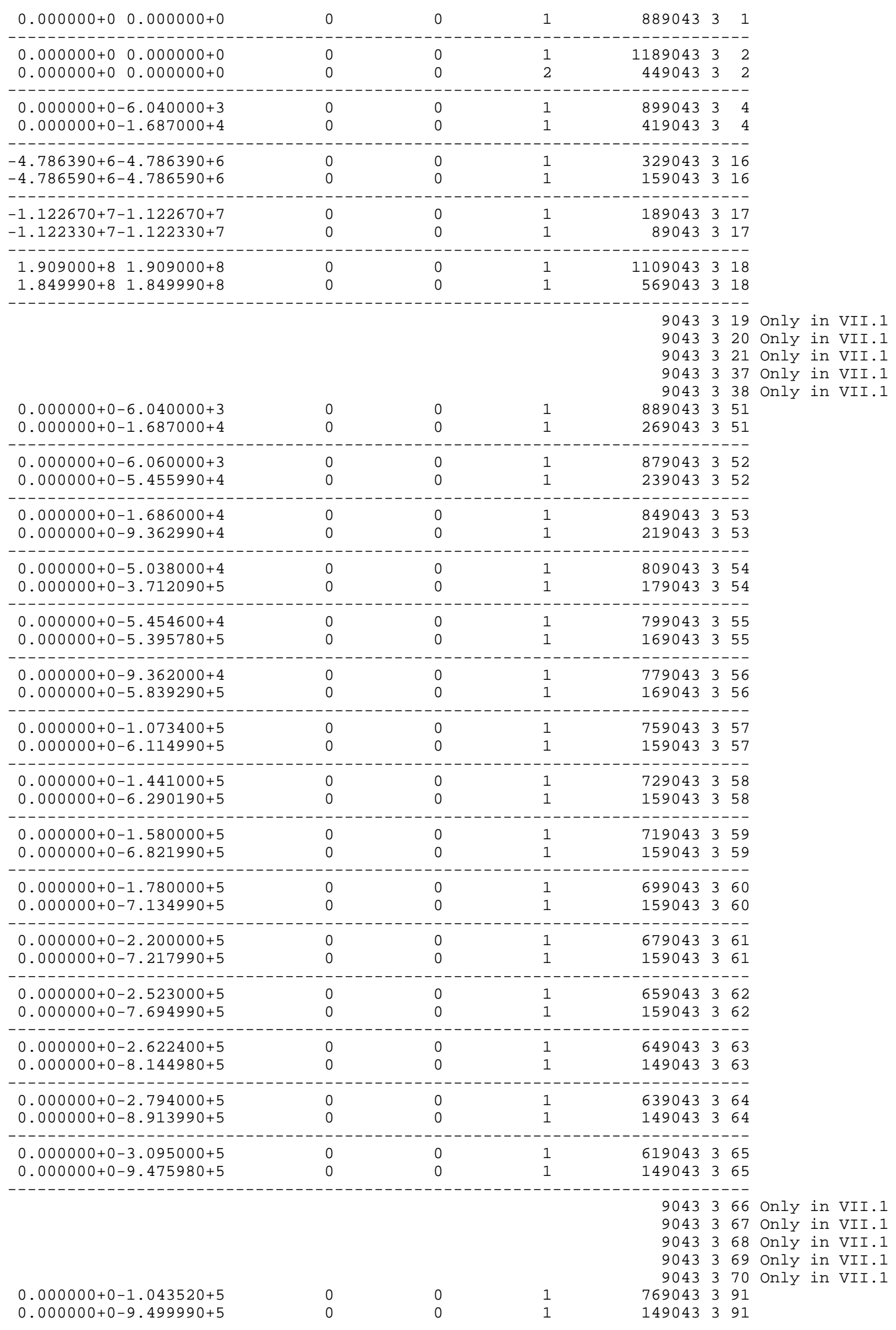




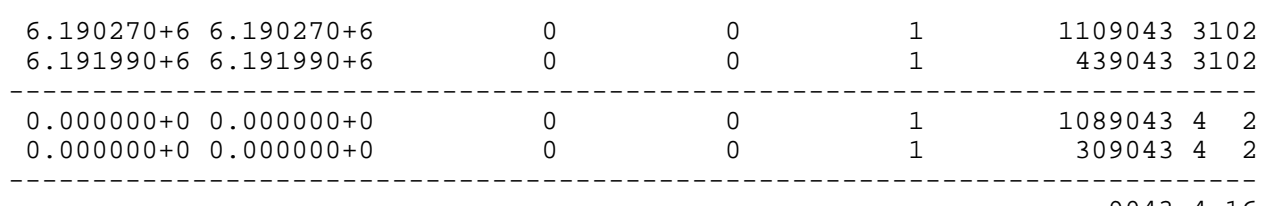

\begin{tabular}{|c|c|c|c|c|}
\hline & & & & $\begin{array}{llll}9043 & 4 & 16 & \text { Only in VII.0 } \\
9043 & 4 & 17 & \text { Only in VII.0 }\end{array}$ \\
\hline $0.000000+0 \quad 2.310400+2$ & 1 & 1 & $\odot$ & 09043418 \\
\hline$\odot .0 \odot \odot \odot \odot \odot+\odot \quad 2.31040 \odot+2$ & $\odot$ & 1 & $\odot$ & 09043418 \\
\hline
\end{tabular}

$$
\begin{aligned}
& 9043451 \text { only in VII. } 0 \\
& 9043452 \text { Only in VII.0 } \\
& 9043453 \text { only in VII.0 } \\
& 9043454 \text { only in VII.0 } \\
& 9043455 \text { only in VII.0 } \\
& 9043456 \text { only in VII.0 } \\
& 9043457 \text { only in VII.0 } \\
& 9043458 \text { only in VII.0 } \\
& 9043459 \text { Only in VII.0 } \\
& 9043460 \text { Only in VII.0 } \\
& 9043461 \text { Only in VII.0 } \\
& 9043462 \text { only in VII.0 } \\
& 9043463 \text { only in VII.0 } \\
& 9043464 \text { only in VII.0 } \\
& 9043465 \text { only in VII.0 }
\end{aligned}
$$

$\begin{array}{llllll}0 & 1 & 1 & 29043 & 5 & 18 \\ \odot & 7 & 1 & 29043 & 5 & 18\end{array}$

9043591 only in VII.0 9043616 only in VII.1 9043617 only in VII.1 9043637 only in VII.1 9043651 only in VII.1 9043652 only in VII.1 9043653 only in VII.1 9043654 only in VII.1 9043655 only in VII.1 9043656 only in VII.1 9043657 only in VII.1 9043658 only in VII.1 9043659 only in VII.1 9043660 only in VII.1 9043661 only in VII.1 9043662 only in VII.1 9043663 only in VII.1 9043664 only in VII.1 9043665 only in VII.1 9043666 only in VII.1 9043667 only in VII.1 9043668 only in VII.1 9043669 only in VII.1 9043670 only in VII.1 9043691 only in VII.1 90436102 only in VII.1

$90-\mathrm{Th}-234$

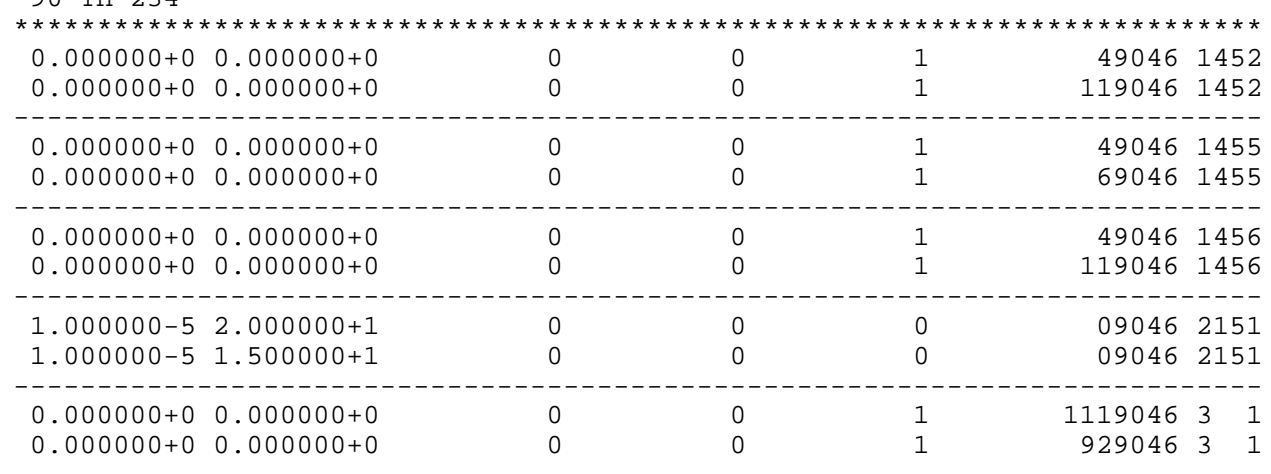




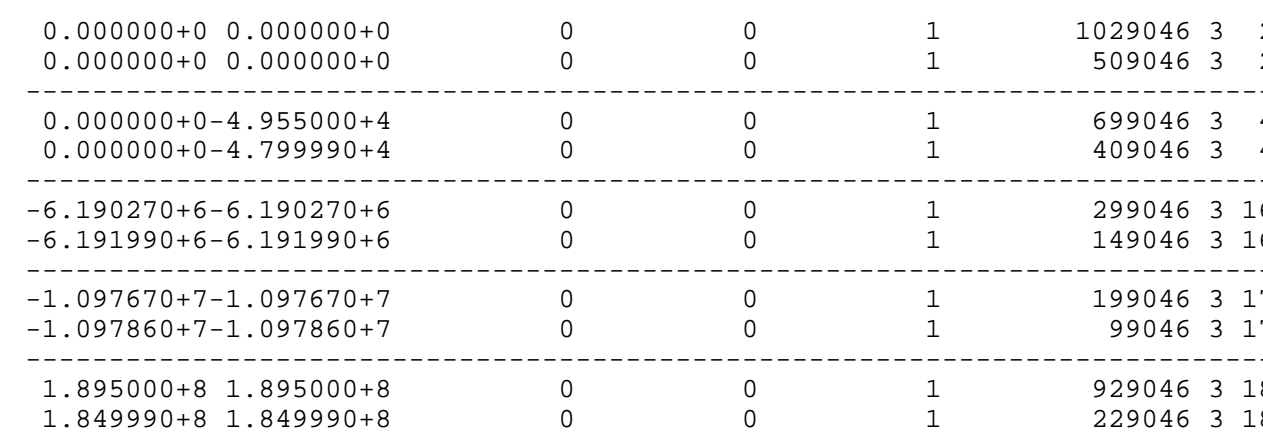

$1.849990+81.849990+8$
$\ldots$

9046319 Only in VII.1 9046320 only in VII.1 9046321 only in VII.1 9046337 only in VII.1 9046338 only in VII.1

$\odot .0 \odot \odot \odot \odot \odot+\odot-4.955 \odot \odot \odot+4$ $0.000000+0-4.799990+4$

๑. $0 \odot \odot \odot \odot \odot+\odot-1.630 \odot \odot \odot+5$

$0.000000+0-1.630000+5$
$\odot .000000+0-1.600000+5$

4

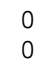

- - - - - - - - - - - - - - -

$\odot .000000+0-3.365000+5$

(1)

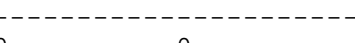

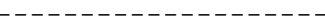

๑. $000000+\odot-5.648000+5$

$0.000000+0-5.759990+5$

0
0
0

$0 \quad 0$

$1 \quad 199046352$

$689046 \quad 3 \quad 51$

239046351

$29046 \quad 3 \quad 52$

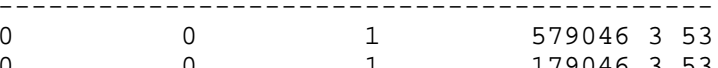

$\begin{array}{rrr}0 & 1 & 179046353\end{array}$

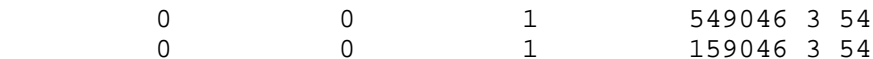

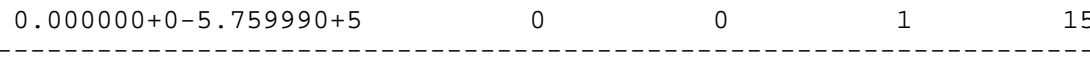

9046355 only in VII.0 9046356 only in VII.0 9046357 only in VII.0 9046358 only in VII.0 9046359 only in VII.0 9046360 only in VII.0 9046361 only in VII.0 9046362 only in VII.0 9046363 only in VII.0 9046364 only in VII.0 9046365 Only in VII.0 9046366 only in VII.0 9046367 only in VII.0 $\odot .00000 \odot+0-1.104740+5$
$0.000000+0-1.060000+6$

5
6

$4.430250+64.430250+6$

$4.430250+6 \quad 4.430250+6$ $4.533690+6 \quad 4.533690+6$

$0 \quad 0 \quad 11659046 \quad 391$
$\begin{array}{lll}659046 & 3 & 91 \\ 139046 & 3 & 91\end{array}$ 139046 3 91

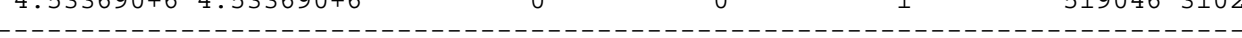

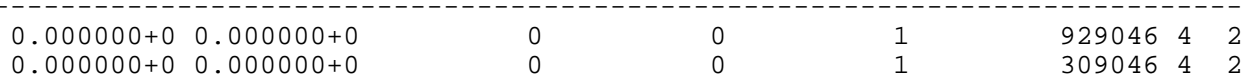

$0.000000+0 \quad 0.000000+0$

$309046 \quad 4 \quad 2$

9046416 only in VII.0 9046417 only in VII.0

๑. $000000+0 \quad 2.320330+2$ $\odot .000000+\odot \quad 2.320330+2$

$\begin{array}{ll}1 & 1 \\ 0 & 1\end{array}$

09046418

09046418

9046451 only in VII.0 9046452 only in VII.0 9046453 only in VII.0 9046454 only in VII.0 9046455 only in VII.0 9046456 only in VII.0 9046457 only in VII.0 9046458 only in VII.0 9046459 only in VII.0 9046460 only in VII.0 9046461 only in VII.0 9046462 only in VII.0 9046463 only in VII.0 9046464 only in VII.0 9046465 only in VII.0 9046466 Only in VII.0 
๑. $000000+\odot \quad \odot .000000+\odot$ $-2.000000+7 \quad 0.000000+0$
9046467 only in VII.0 9046491 only in VII.0 9046516 only in VII.0 9046517 only in VII.0 29046518 29046518

9046591 only in VII.0 9046616 only in VII.1 9046617 0nly in VII.1 9046637 only in VII.1 9046651 only in VII.1 9046652 only in VII.1 9046653 only in VII.1 9046654 only in VII.1 9046691 0nly in VII.1 90466102 only in VII.1

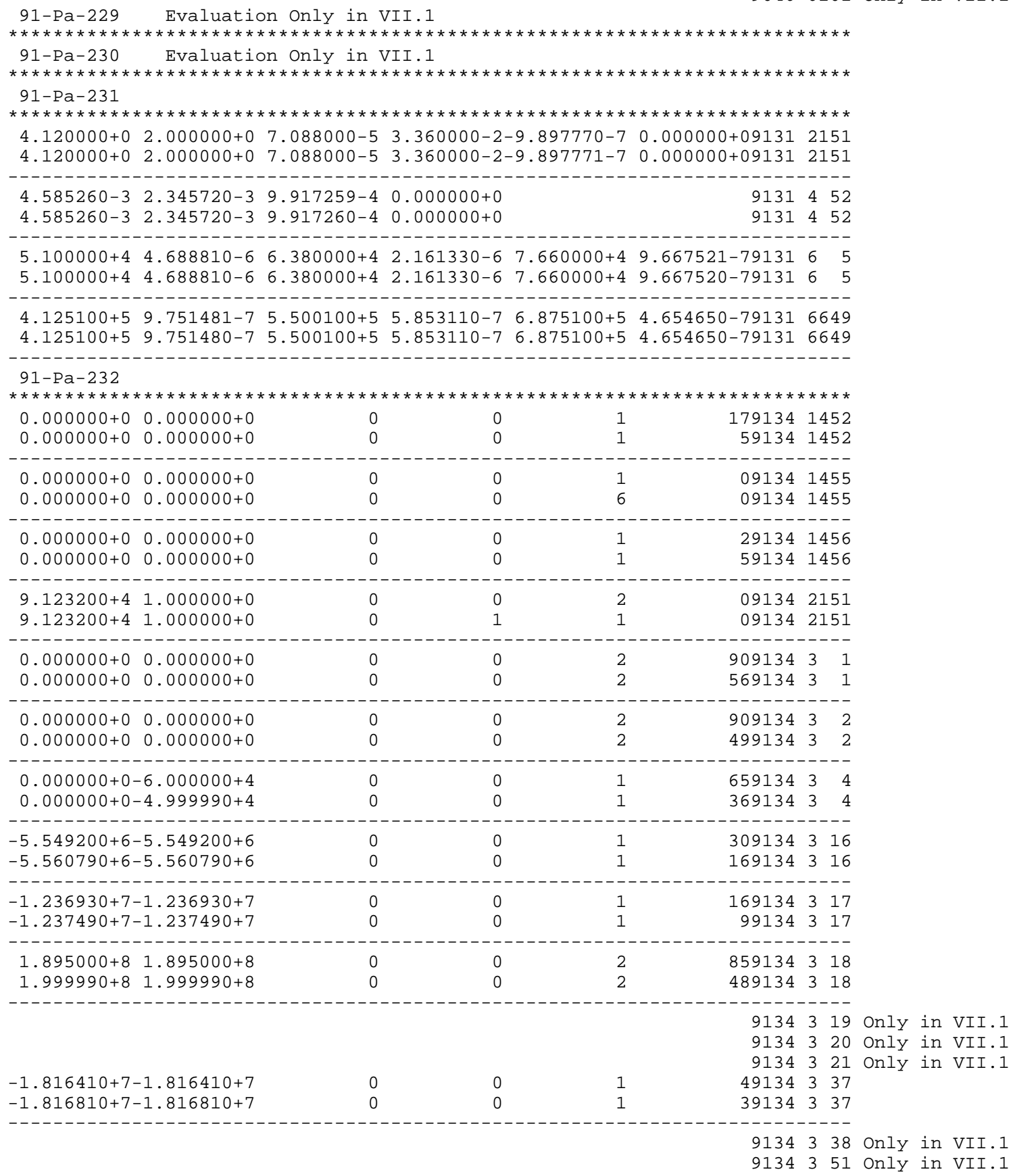




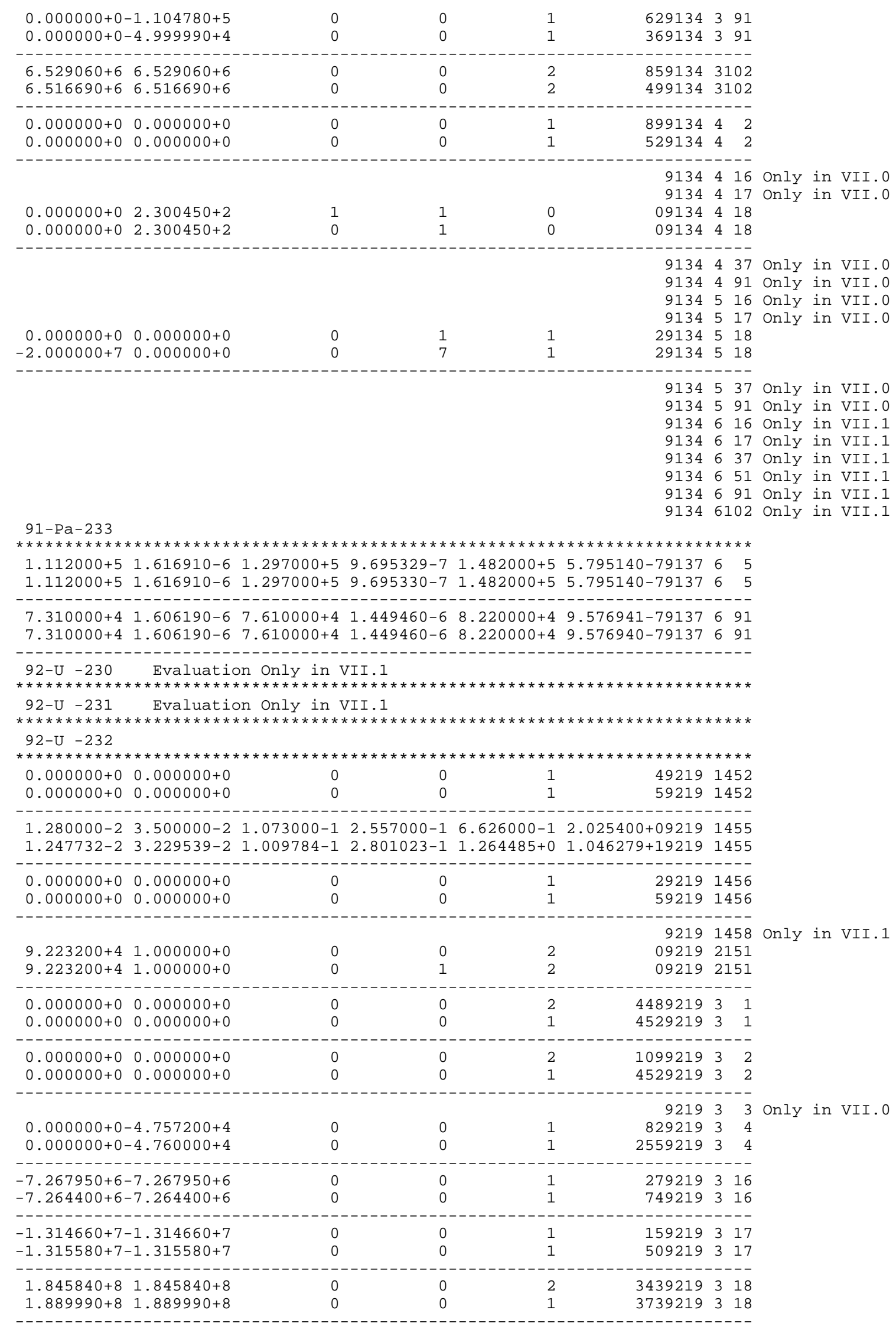




\begin{tabular}{|c|c|c|c|c|c|c|}
\hline $\begin{array}{ll}1.845840+8 & 1.845840+8 \\
1.889990+8 & 1.889990+8\end{array}$ & $\begin{array}{l}\odot \\
\odot\end{array}$ & $\begin{array}{l}\odot \\
\odot\end{array}$ & $\begin{array}{l}2 \\
1\end{array}$ & $\begin{array}{l}3369219 \\
3739219\end{array}$ & $\begin{array}{ll}3 & 19 \\
3 & 19\end{array}$ & \\
\hline $\begin{array}{ll}1.845840+8 & 1.845840+8 \\
1.889990+8 & 1.889990+8\end{array}$ & $\begin{array}{l}\odot \\
\odot\end{array}$ & $\begin{array}{l}\odot \\
\odot\end{array}$ & $\begin{array}{l}1 \\
1\end{array}$ & $\begin{array}{r}419219 \\
2879219\end{array}$ & $\begin{array}{ll}3 & 20 \\
3 & 20\end{array}$ & \\
\hline $\begin{array}{ll}1.845840+8 & 1.845840+8 \\
1.889990+8 & 1.889990+8\end{array}$ & $\begin{array}{l}\odot \\
\odot\end{array}$ & $\begin{array}{l}\odot \\
\odot\end{array}$ & $\begin{array}{l}1 \\
1\end{array}$ & $\begin{array}{r}189219 \\
1429219\end{array}$ & $\begin{array}{ll}3 & 21 \\
3 & 21\end{array}$ & \\
\hline $\begin{array}{ll}1.845840+8 & 1.845840+8 \\
1.889990+8 & 1.889990+8\end{array}$ & $\begin{array}{l}\odot \\
\odot\end{array}$ & $\begin{array}{l}\odot \\
\odot\end{array}$ & $\begin{array}{l}1 \\
1\end{array}$ & $\begin{array}{r}9219 \\
109219 \\
879219\end{array}$ & $\begin{array}{ll}3 & 37 \\
3 & 38 \\
3 & 38\end{array}$ & Only in VII.0 \\
\hline $\begin{array}{l}\odot .0 \odot \odot \odot \odot \odot+\odot-4.75720 \odot+4 \\
\odot . \odot \odot \odot \odot \odot \odot+\odot-4.760 \odot \odot \odot+4\end{array}$ & $\begin{array}{l}0 \\
0\end{array}$ & $\begin{array}{l}0 \\
\odot\end{array}$ & $\begin{array}{l}1 \\
1\end{array}$ & $\begin{array}{r}819219 \\
2559219\end{array}$ & $\begin{array}{ll}3 & 51 \\
3 & 51\end{array}$ & \\
\hline $\begin{array}{l}\odot .0 \odot \odot \odot \odot \odot+\odot-1.56570 \odot+5 \\
\odot .0 \odot \odot \odot \odot \odot+\odot-1.5660 \odot \odot+5\end{array}$ & $\begin{array}{l}\odot \\
\odot\end{array}$ & $\begin{array}{l}0 \\
0\end{array}$ & $\begin{array}{l}1 \\
1\end{array}$ & $\begin{array}{r}759219 \\
2459219\end{array}$ & $\begin{array}{ll}3 & 52 \\
3 & 52\end{array}$ & \\
\hline $\begin{array}{l}\odot .00 \odot \odot \odot \odot+\odot-3.2260 \odot \odot+5 \\
\odot .00 \odot \odot \odot \odot+\odot-3.2230 \odot \odot+5\end{array}$ & $\begin{array}{l}0 \\
\odot\end{array}$ & $\begin{array}{l}0 \\
\odot\end{array}$ & $\begin{array}{l}1 \\
1\end{array}$ & $\begin{array}{r}709219 \\
2349219\end{array}$ & $\begin{array}{ll}3 & 53 \\
3 & 53\end{array}$ & \\
\hline $\begin{array}{l}\odot .000 \odot \odot \odot+\odot-5.410 \odot \odot \odot+5 \\
\odot .00 \odot \odot \odot \odot+\odot-5.40700 \odot+5\end{array}$ & $\begin{array}{l}0 \\
\odot\end{array}$ & $\begin{array}{l}\odot \\
\odot\end{array}$ & $\begin{array}{l}1 \\
1\end{array}$ & $\begin{array}{r}679219 \\
2209219\end{array}$ & $\begin{array}{ll}3 & 54 \\
3 & 54\end{array}$ & \\
\hline $\begin{array}{l}\odot . \odot 0 \odot \odot \odot \odot+\odot-5.631940+5 \\
\odot . \odot \odot \odot \odot \odot \odot+\odot-5.6320 \odot \odot+5\end{array}$ & $\begin{array}{l}0 \\
\odot\end{array}$ & $\begin{array}{l}\odot \\
\odot\end{array}$ & $\begin{array}{l}1 \\
1\end{array}$ & $\begin{array}{r}669219 \\
2179219\end{array}$ & $\begin{array}{ll}3 & 55 \\
3 & 55\end{array}$ & \\
\hline 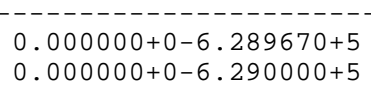 & $\begin{array}{c}--\cdot \\
\odot \\
0\end{array}$ & $\begin{array}{l}\odot \\
\odot\end{array}$ & $\begin{array}{l}1 \\
1\end{array}$ & $\begin{array}{r}649219 \\
2139219\end{array}$ & $\begin{array}{ll}3 & 56 \\
3 & 56\end{array}$ & \\
\hline $\begin{array}{l}\odot . \odot \odot \odot \odot \odot \odot+\odot-6.91210 \odot+5 \\
\odot . \odot \odot \odot \odot \odot \odot+\odot-6.9110 \odot \odot+5\end{array}$ & $\begin{array}{l}0 \\
\odot\end{array}$ & $\begin{array}{l}0 \\
\odot\end{array}$ & $\begin{array}{l}1 \\
1\end{array}$ & $\begin{array}{r}639219 \\
2089219\end{array}$ & $\begin{array}{ll}3 & 57 \\
3 & 57\end{array}$ & \\
\hline $\begin{array}{l}\odot .00 \odot \odot \odot \odot+\odot-7.34560 \odot+5 \\
\odot . \odot \odot \odot \odot \odot \odot+\odot-7.346 \odot \odot \odot+5\end{array}$ & $\begin{array}{l}--\cdot \\
0 \\
0\end{array}$ & $\begin{array}{l}0 \\
\odot\end{array}$ & $\begin{array}{l}1 \\
1\end{array}$ & $\begin{array}{r}619219 \\
2059219\end{array}$ & $\begin{array}{ll}-1 & - \\
3 & 58 \\
3 & 58\end{array}$ & \\
\hline $\begin{array}{l}\odot .0 \odot \odot \odot \odot \odot+\odot-7.4690 \odot \odot+5 \\
\odot . \odot \odot \odot \odot \odot \odot+\odot-8.0550 \odot \odot+5\end{array}$ & $\begin{array}{l}0 \\
0\end{array}$ & $\begin{array}{l}0 \\
\odot\end{array}$ & $\begin{array}{l}1 \\
1\end{array}$ & $\begin{array}{r}609219 \\
1999219\end{array}$ & $\begin{array}{ll}3 & 59 \\
3 & 59\end{array}$ & \\
\hline $\begin{array}{l}\odot . \odot \odot \odot \odot \odot \odot+\odot-8.058 \odot \odot \odot+5 \\
\odot . \odot \odot \odot \odot \odot \odot+\odot-8.33 \odot \odot \odot \odot+5\end{array}$ & $\begin{array}{l}0 \\
0\end{array}$ & $\begin{array}{l}0 \\
0\end{array}$ & $\begin{array}{l}1 \\
1\end{array}$ & $\begin{array}{r}589219 \\
1979219\end{array}$ & $\begin{array}{ll}3 & 60 \\
3 & 60\end{array}$ & \\
\hline $\begin{array}{l}\odot . \odot \odot \odot \odot \odot \odot+\odot-8.33 \odot 7 \odot \odot+5 \\
\odot . \diamond \odot \odot \odot \odot \odot+\odot-8.668 \odot \odot \odot+5\end{array}$ & $\begin{array}{l}0 \\
\odot\end{array}$ & $\begin{array}{l}0 \\
\odot\end{array}$ & $\begin{array}{l}1 \\
1\end{array}$ & $\begin{array}{r}579219 \\
1939219\end{array}$ & $\begin{array}{ll}3 & 61 \\
3 & 61\end{array}$ & \\
\hline $\begin{array}{l}\odot . \odot \odot \odot \odot \odot \odot+\odot-8.667920+5 \\
\odot . \odot \odot \odot \odot \odot \odot+\odot-9.114 \odot \odot \odot+5\end{array}$ & $\begin{array}{l}0 \\
\odot\end{array}$ & $\begin{array}{l}0 \\
\odot\end{array}$ & $\begin{array}{l}1 \\
1\end{array}$ & $\begin{array}{r}569219 \\
1909219\end{array}$ & $\begin{array}{ll}3 & 62 \\
3 & 62\end{array}$ & \\
\hline $\begin{array}{l}\odot . \odot \odot \odot \odot \odot \odot+\odot-9.11420 \odot+5 \\
\odot . \odot \odot \odot \odot \odot \odot+\odot-9.7070 \odot \odot+5\end{array}$ & $\begin{array}{l}0 \\
0\end{array}$ & $\begin{array}{l}0 \\
\odot\end{array}$ & $\begin{array}{l}1 \\
1\end{array}$ & $\begin{array}{r}549219 \\
1859219\end{array}$ & $\begin{array}{ll}3 & 63 \\
3 & 63\end{array}$ & \\
\hline 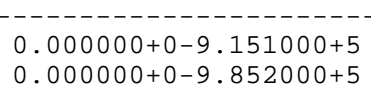 & $\begin{array}{l}0 \\
\odot\end{array}$ & $\begin{array}{l}0 \\
\odot\end{array}$ & $\begin{array}{l}1 \\
1\end{array}$ & $\begin{array}{r}539219 \\
1839219\end{array}$ & $\begin{array}{ll}3 & 64 \\
3 & 64\end{array}$ & \\
\hline $\begin{array}{l}\odot . \odot \odot \odot \odot \odot \odot+\odot-9.70680 \odot+5 \\
\odot .00 \odot \odot \odot \odot+\odot-1.016900+6\end{array}$ & $\begin{array}{l}0 \\
0\end{array}$ & $\begin{array}{l}0 \\
\odot\end{array}$ & $\begin{array}{l}1 \\
1\end{array}$ & $\begin{array}{r}529219 \\
1809219\end{array}$ & $\begin{array}{ll}3 & 65 \\
3 & 65\end{array}$ & \\
\hline 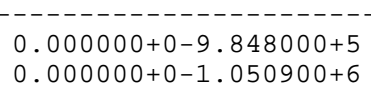 & $\begin{array}{l}0 \\
0\end{array}$ & $\begin{array}{l}0 \\
\odot\end{array}$ & $\begin{array}{l}1 \\
1\end{array}$ & $\begin{array}{r}519219 \\
1769219\end{array}$ & $\begin{array}{ll}3 & 66 \\
3 & 66\end{array}$ & \\
\hline $\begin{array}{l}\odot .00 \odot \odot \odot \odot+\odot-1.016850+6 \\
\odot . \odot \odot \odot \odot \odot \odot+\odot-1.097200+6\end{array}$ & $\begin{array}{l}\odot \\
\odot\end{array}$ & $\begin{array}{l}\odot \\
\odot\end{array}$ & $\begin{array}{l}1 \\
1\end{array}$ & $\begin{array}{r}499219 \\
1729219\end{array}$ & $\begin{array}{ll}3 & 67 \\
3 & 67\end{array}$ & \\
\hline & & & & $\begin{array}{l}9219 \\
9219 \\
9219 \\
9219 \\
9219 \\
9219 \\
9219 \\
9219 \\
9219 \\
9219 \\
9219\end{array}$ & $\begin{array}{ll}3 & 68 \\
3 & 69 \\
3 & 70 \\
3 & 71 \\
3 & 72 \\
3 & 73 \\
3 & 74 \\
3 & 75 \\
3 & 76 \\
3 & 77 \\
3 & 78\end{array}$ & 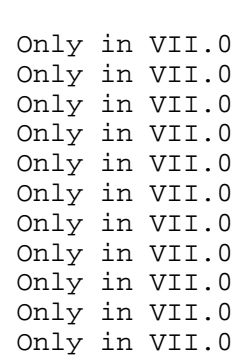 \\
\hline
\end{tabular}


9219379 only in VII. $\odot$ 9219380 only in VII.0 9219381 only in VII. 0 9219382 only in VII.0 9219383 only in VII.0 9219384 only in VII.0 9219385 only in VII.0 9219386 only in VII.0 9219387 only in VII.0 9219388 only in VII.0 9219389 only in VII.0 9219390 only in VII.0

$0.000000+0-1.104780+5$ $0.000000+0-1.050000+6$

5

$5.762090+6 \quad 5.762090+6$

$5.743400+6 \quad 5.743400+6$

$\begin{array}{lll}789219 & 3 & 91\end{array}$ 1779219391

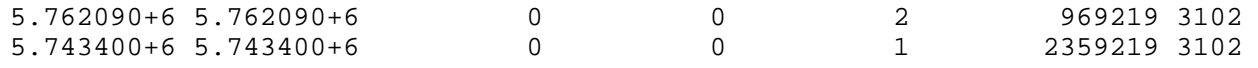

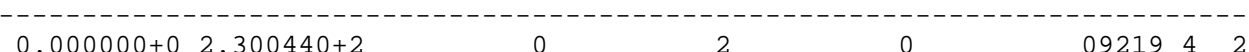

$\begin{array}{rrrrrrr}0.000000+0 & 2.300440+2 & 0 & 2 & 0 & 09219 & 4 \\ 0.000000+\odot & 2.300438+2 & 0 & 2 & 0 & 09219 & 4\end{array}$

0.

$\begin{array}{rrrrrrr}0.000000+0 & 2.300440+2 & 1 & 1 & 0 & 09219418 \\ 0.000000+0 & 2.320304+2 & 1 & 1 & 0 & 09219418\end{array}$

_.

9219419 only in VII.0 9219420 Only in VII.0 9219421 only in VII.0 9219438 only in VII.0 9219451 only in VII.0 9219452 only in VII. 0 9219453 only in VII.0 9219454 only in VII.0 9219455 only in VII.0 9219456 only in VII.0 9219457 only in VII.0 9219458 only in VII.e 9219459 Only in VII.0 9219460 only in VII.0 9219461 only in VII.0 9219462 only in VII.0 9219463 only in VII.0 9219464 Only in VII.0 9219465 only in VII.0 9219466 only in VII.0 9219467 only in VII.0 9219468 only in VII.0 9219469 only in VII.0 9219470 only in VII.0 9219471 Only in VII.0 9219472 Only in VII.0 9219473 only in VII.0 9219474 only in VII.0 9219475 only in VII. $\odot$ 9219476 only in VII.0 9219477 only in VII.0 9219478 only in VII.0 9219479 only in VII. 0 9219480 Only in VII.0 9219481 only in VII.0 9219482 only in VII.e 9219483 only in VII.0 9219484 only in VII.0 9219485 Only in VII.0 9219486 only in VII.0 9219487 only in VII.0 9219488 Only in VII.0 9219489 only in VII.0 9219490 only in VII.0

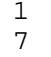

1

29219518 29219518

9219519 only in VII.0 9219520 only in VII.0 9219521 Only in VII.0 9219538 only in VII. $\odot$ 
$\begin{array}{llll}7.299540+6 & 2.000000+\odot \quad 2.000000+7 & 2.000000+\odot\end{array}$ $7.295958+6 \quad 2.00000 \odot+\odot \quad 3.000 \odot \odot \odot+7 \quad 2.000 \odot \odot \odot+\odot$

92195455 only in VII.1

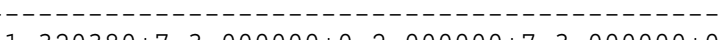

9219616

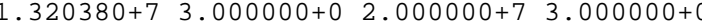
9219616

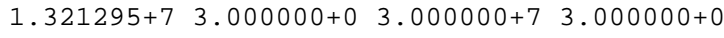

9219617

$9219 \quad 6 \quad 17$

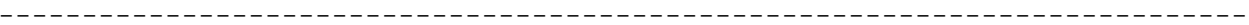

9219637 only in VII.0

9219651 only in VII.1

9219652 only in VII.1

9219653 only in VII.1

9219654 only in VII.1

9219655 only in VII.1

9219656 only in VII.1

9219657 only in VII.1

9219658 only in VII.1

9219659 Only in VII.1

9219660 only in VII.1

9219661 only in VII.1

9219662 only in VII.1

9219663 only in VII.1

9219664 only in VII.1

9219665 only in VII.1

9219666 only in VII.1

$\begin{array}{lll}1.109580+5 & 1.000000+\odot \quad 2.000000+7 & 1.000000+\odot\end{array}$

9219667 only in VII.1

$\begin{array}{llll}1.054564+6 & 1.000000+0 & 3.000000+7 & 1.000000+0\end{array}$

9219691

9219691

$1.054564+6 \quad 1.000000+03.000000+7 \quad 1.000000+0 \quad 9219691$

$92-U-233$

$1.291100-2 \quad 3.473800-21.192800-12.861700-17.877000-12.441700+0922$

1.247938-2 3.227449-2 1.046448-1 2.941518-1 $1.242443+01.022323+192221455$

$4.200000+6 \quad 9.615540-7 \quad 4.300000+6 \quad 1.850741-6 \quad 4.400000+6 \quad 2.801157-69222320$

$\begin{array}{llllllllll}4.2000 \odot \odot+6 & 9.615539-7 & 4.300000+6 & 1.850741-6 & 4.400000+6 & 2.801157-69222 & 3 & 20\end{array}$

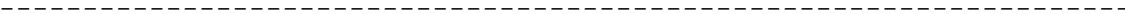

$\odot .000 \odot \odot \odot+\odot \quad 1.287773-3 \quad 0.000 \odot \odot \odot+\odot \quad 9.999553-4 \quad 0.00 \odot \odot \odot \odot+\odot \quad 5.004197-49222473$ $\odot .000000+\odot \quad 1.287773-3 \quad 0.000000+\odot \quad 9.999554-4 \quad 0.000000+\odot \quad 5.004197-49222473$

(-

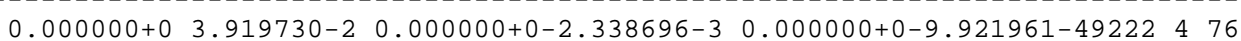
๑. $000000+\odot 3.919730-2 \quad 0.000000+0-2.338696-3 \quad 0.000000+0-9.921962-49222476$

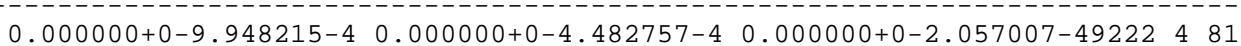
$\odot .0 \odot \odot \odot \odot \odot+\odot-9.948216-4 \quad \odot .0 \odot \odot \odot \odot \odot+\odot-4.482757-4 \quad \odot .0 \odot \odot \odot \odot \odot+\odot-2.0570 \odot 7-49222481$

$1.000000-58.593243-22.000000+78.593243-2 \quad 92225455$

$1.000000-58.675847-23.000000+78.675847-2 \quad 92225455$

- -

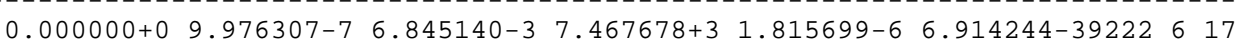
$0.000000+\odot \quad 9.976306-7 \quad 6.845140-3 \quad 7.467678+3 \quad 1.815699-6 \quad 6.914244-3922266$

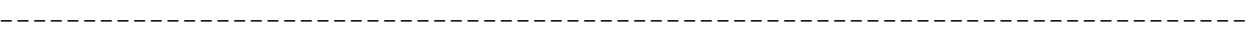

$92-U-234$

$* * * * * * * * * * * * * * * * * * * * * * * * * * * * * * * * * * * * * * * * * * * * * * * * * * * * * * * * * * * * * * * * * * * * * * * * * * *$

1.308200-2 3.368400-2 $1.209500-1 \quad 2.951700-1 \quad 8.136300-1 \quad 2.572100+092251455$ 1.248240-2 3.129530-2 1.074630-1 3.032230-1 $1.288730+01.044220+192251455$

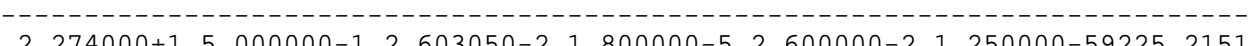
2. 274000+1 5.000000-1 2.603000-2 $1.800000-5 \quad 2.600000-2$ 1.250000-59225 2151

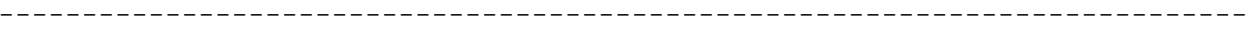

$1.480000+6 \quad 9.509682-4 \quad 1.500000+6 \quad 9.861999-4 \quad 1.520000+6 \quad 1.021398-39225354$ $1.480000+6 \quad 9.509682-4 \quad 1.500000+6 \quad 9.862000-4 \quad 1.520000+6 \quad 1.021398-39225354$

$2.650000+71.033863-32.700000+71.015322-32.750000+79.977483-49225361$

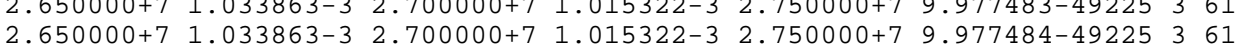
- - - - - - - - - - - - - - - - - - - - - - - - - - - - - - - - - - - - -

$2.750000+7 \quad 9.977483-4 \quad 2.800000+7 \quad 9.812100-4 \quad 2.850000+7 \quad 9.658632-49225 \quad 3 \quad 62$ $2.750000+7 \quad 9.977484-4 \quad 2.800000+7 \quad 9.812100-4 \quad 2.850000+7 \quad 9.658632-49225362$ $2.7500 \odot \odot+7 \quad 9.977483-4 \quad 2.80 \odot \odot \odot \odot+7 \quad 9.81210 \odot-4 \quad 2.850 \odot \odot \odot+7 \quad 9.658632-49225 \quad 3 \quad 64$ $2.750000+7 \quad 9.977484-4 \quad 2.800000+7 \quad 9.812100-4 \quad 2.850000+7 \quad 9.658632-49225364$

$9.866999-4-1.580000-4-1.986000-40.000000+0 \quad 9225452$ $9.867000-4-1.580000-4-1.986000-40.000000+\odot \quad 9225452$ 
1.207000-1-1.111000-2-1.818000-2-5.383000-3 4.023000-3-9.861001-49225 453 1.207000-1-1.111000-2-1.818000-2-5.383000-3 4.023000-3-9.861000-49225 453

7.499000-2 3.372000-3 $1.095000-4 \quad 9.945001-7 \quad 1.877000-6 \quad 8.917000-69225460$ 7.499000-2 3.372000-3 1.095000-4 9.945000-7 $1.877000-6 \quad 8.917000-69225460$

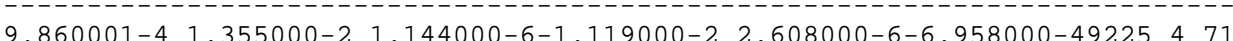
$9.860000-4$ 1.355000-2 $1.144000-6-1.119000-2 \quad 2.608000-6-6.958000-49225471$

(6. $6.692000-4 \quad 3.344000-3 \quad 9.712001-7 \quad 2.593000-6-7.279000-8-3.602000-89225476$ 6.692000-4 3.344000-3 9.712000-7 2.593000-6-7.279000-8-3.602000-89225 476

$1.000000-55.496524$. $1.000000-55.623233-23.000000+75.623233-2 \quad 92255455$

(5) $\begin{array}{lllllllll}5.476398+4 & 9.822145-8 & 9.865809-4 & 6.472107+4 & 1.060994-7 & 1.004135-39225 & 6 & 16\end{array}$ 5.476398+4 9.822145-8 9.865808-4 6.472107+4 $1.060994-7 \quad 1.004135-39225616$ $2.364808+4 \quad 9.724685-7 \quad 1.222921-1 \quad 2.738199+4 \quad 1.035056-6 \quad 1.415931-19225691$ $2.364808+4 \quad 9.724684-7 \quad 1.222921-1 \quad 2.738199+4 \quad 1.035056-6 \quad 1.415931-19225691$ $92-U-235$

(1)

$\begin{array}{llllll}1.333600-2 & 3.273900-2 & 1.207800-1 & 3.027800-1 & 8.494900-1 & 2.853000+09228 \\ 14455\end{array}$ $1.249056-2$ 3.182406-2 $1.093753-1 \quad 3.169898-1 \quad 1.353983+08.636377+092281455$

$1.830000+2 \quad 1.673394-3 \quad 1.840000+2 \quad 1.823250-3 \quad 1.850000+2 \quad 9.815576-492281460$ $1.830000+21.673394-3 \quad 1.840000+2 \quad 1.823250-3 \quad 1.850000+2 \quad 9.815577-492281460$

$6.911070+2 \quad 4.00000 \odot+\odot \quad 9.916601-4 \quad 4.319600-2-2.550100-2-2.599400-292282151$ $6.911070+2 \quad 4.000000+\odot \quad 9.916600-4 \quad 4.319600-2-2.550100-2-2.599400-292282151$

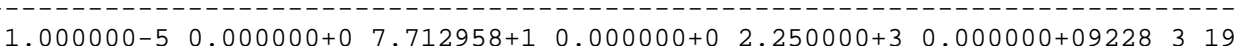

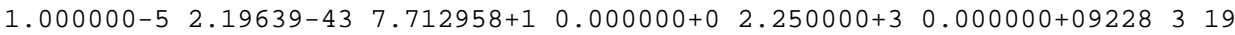

$-2.123213-2-5.575145-2 \quad 4.503431-2-4.007026-2 \quad 8.664596-4 \quad 9.875596-49228476$ $-2.123213-2-5.575145-2$ 4.503431-2-4.007026-2 8.664596-4 9.875595-49228 476

$-5.501023-6-5.653619-6-9.676723-7-1.311852-7-1.349319-8-1.592145-99228480$ $-5.501023-6-5.653619-6-9.676724-7-1.311852-7-1.349319-8-1.592145-99228480$

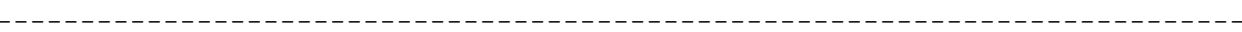
- $1.130429-2-9.546876-3-9.897758-4 \quad 1.520279-2 \quad 1.188212-2 \quad 4.399529-39228489$ -1.130429-2-9.546876-3-9.897757-4 1.520279-2 1.188212-2 4.399529-39228 489

0.00000+0 $1.000000-5$

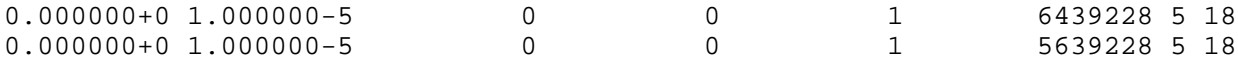

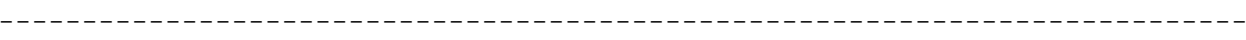

$1.000000-5 \quad 3.500755-22.000000+7 \quad 3.500755-2 \quad 92285455$

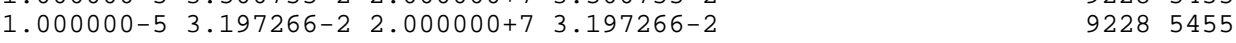

$92-U-236$

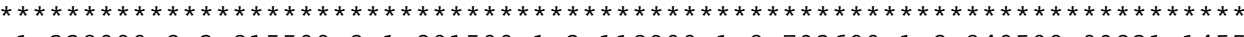

$\begin{array}{llllll}1.338000-2 & 3.215500-2 & 1.201500-1 & 3.112900-1 & 8.793600-1 & 2.840500+09231 \\ 1.4455\end{array}$ 1.248999-2 3.068560-2 $1.091980-1 \quad 3.224705-1 \quad 1.378583+01.021014+192311455$

$6.556000+2 \quad 5.000000-1 \quad 1.164570-1 \quad 9.310700-2 \quad 2.300000-2 \quad 3.500000-492312151$ $6.556000+2$ 5. $500000-1 \quad 1.164600-1 \quad 9.310700-2 \quad 2.300000-2 \quad 3.500000-492312151$ $\begin{array}{llllllll}1.500000+3 & 1.568000-2 & 4.539315+4 & 8.692747-3 & 4.750000+4 & 8.357362-39231 & 3 & 2\end{array}$ $1.500000+3 \quad 2.120000-2 \quad 4.539315+4 \quad 1.175295-2 \quad 4.750000+4 \quad 1.129949-2923132$ $\begin{array}{llllllll}1.944900+8 & 1.944900+8 & 0 & 0 & 1 & 3069231 & 3 & 3\end{array}$ $\begin{array}{rrrrrrr}5.125900+6 & 5.125900+6 & 0 & 0 & 1 & 30792313 & 3\end{array}$

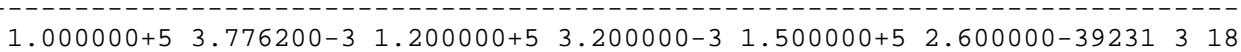
$\begin{array}{lllllllll}1.000000+5 & 2.168180-3 & 1.200000+5 & 2.138383-3 & 1.500000+5 & 2.126137-39231 & 3 & 18\end{array}$

$1.000000+5 \quad 3.776200-3 \quad 1.200000+5 \quad 3.200000-3 \quad 1.500000+5 \quad 2.600000-39231319$ $\begin{array}{llllllll}1.000000+5 & 2.168180-3 & 1.200000+5 & 2.138383-3 & 1.500000+5 & 2.126137-39231 & 3 & 19\end{array}$

$4.600000+6 \quad 9.847264-4 \quad 4.700000+6 \quad 2.035577-3 \quad 4.800000+6 \quad 3.477558-39231320$

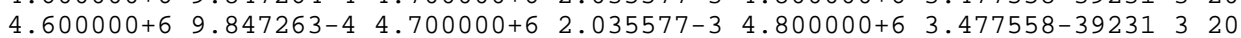

1. $1.900000+7$ 1.022200-3 $1.920000+7$ 1..009736-3 $1.940000+7$ 9.977207-49231 369 


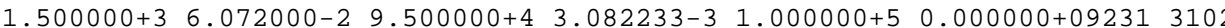
$1.500000+3 \quad 5.520000-2 \quad 9.500000+42.802030-3 \quad 1.000000+50.000000+092313102$

$-9.135455-19.527130-4-8.987940-10.749775-4-8.829476-19.781579-4923$ -9.135455-1 9.527130-4-8.987940-1 9.749775-4-8.829476-1 9.781578-492314 2 4.373092-6 9.695495-7 1.079881-7 8.610352-9 9.32573-10 7.31001-119231 451 4.373092-6 9.695496-7 1.079881-7 8.610352-9 9.32573-10 7.31001-119231 451 $0.000000+\odot-3.930517-2 \quad 0.000000+\odot \quad 9.808810-4 \quad 0.000000+\odot 1.002357-39231468$ $0.000000+\odot-3.930517-2 \quad 0.000000+\odot 9.808811-4 \quad 0.000000+\odot$ 1.002357-392314 68

$6.692000-4 \quad 3.344000-3 \quad 9.712001-7 \quad 2.593000-6-7.279000-8-3.602000-89231476$ $6.692000-4 \quad 3.344000-3 \quad 9.712000-7 \quad 2.593000-6-7.279000-8-3.602000-89231476$

$1.0000 \odot \odot-53.015174-22.000000+73.015174-2 \quad 92315455$ $1.000000-5 \quad 2.556350-2 \quad 3.000000+7 \quad 2.556350-2 \quad 92315455$

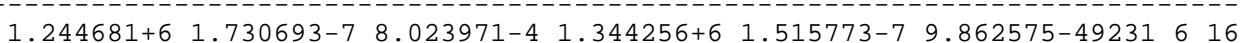
1.244681+6 1.730693-7 8.023971-4 1.344256+6 $1.515773-7 \quad 9.862574-49231616$

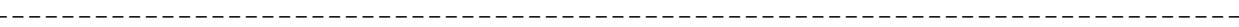
$92-U-237$

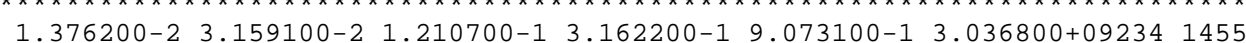
1.249577-2 3.037978-2 1.068975-1 3.240317-1 $1.334253+09.544175+092341455$

\begin{tabular}{|c|c|c|c|c|c|}
\hline $\begin{array}{ll}1.000 \odot \odot \odot-5 ~ & 2.000 \odot \odot \odot+2 \\
1.000 \odot \odot \odot-5 & 1.02500 \odot+2\end{array}$ & $\begin{array}{l}1 \\
1\end{array}$ & $\begin{array}{l}2 \\
1\end{array}$ & $\begin{array}{l}\odot \\
\odot\end{array}$ & $\begin{array}{l}\odot 9234 \\
09234\end{array}$ & $\begin{array}{l}2151 \\
2151\end{array}$ \\
\hline 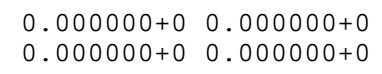 & $\begin{array}{l}\odot \\
\odot\end{array}$ & $\begin{array}{l}\odot \\
\odot\end{array}$ & $\begin{array}{l}1 \\
3\end{array}$ & $\begin{array}{l}1179234 \\
1509234\end{array}$ & $\begin{array}{ll}3 & 1 \\
3 & 1\end{array}$ \\
\hline 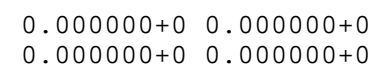 & $\begin{array}{l}\odot \\
\odot\end{array}$ & $\begin{array}{l}\odot \\
\odot\end{array}$ & $\begin{array}{l}1 \\
1\end{array}$ & $\begin{array}{l}1179234 \\
1509234\end{array}$ & $\begin{array}{l}3 \\
3\end{array}$ \\
\hline 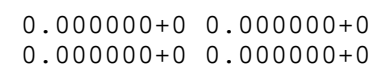 & $\begin{array}{l}\odot \\
\odot\end{array}$ & $\stackrel{\odot}{\ominus}$ & $\begin{array}{l}1 \\
3\end{array}$ & $\begin{array}{l}1179234 \\
1509234\end{array}$ & $\begin{array}{l}3 \\
3\end{array}$ \\
\hline $\begin{array}{ll}1.8000 \odot \odot+8 & 1.800 \odot \odot \odot+8 \\
1.8000 \odot \odot+8 & 1.800000+8\end{array}$ & $\begin{array}{l}\odot \\
\odot\end{array}$ & $\begin{array}{l}\odot \\
\odot\end{array}$ & $\begin{array}{l}1 \\
3\end{array}$ & $\begin{array}{r}829234 \\
1059234\end{array}$ & $\begin{array}{ll}3 & 18 \\
3 & 18\end{array}$ \\
\hline $\begin{array}{ll}6.153800+6 & 6.153800+6 \\
6.153800+6 & 6.153800+6\end{array}$ & $\begin{array}{l}\odot \\
\odot\end{array}$ & $\begin{array}{l}\odot \\
\odot\end{array}$ & $\begin{array}{l}1 \\
3\end{array}$ & $\begin{array}{l}679234 \\
919234\end{array}$ & $\begin{array}{l}3102 \\
3102\end{array}$ \\
\hline
\end{tabular}

$7.662000-2 \quad 3.530000-3 \quad 1.148000-4 \quad 5.537000-6 \quad 9.747999-7-2.788000-69234454$ 7.662000-2 3.530000-3 1.148000-4 5.537000-6 9.748000-7-2.788000-69234 454

$1.000000-51.784102-22.000000+7 \quad 1.784102-2 \quad 92345455$ $1.000000-51.522158-23.000000+7 \quad 1.522158-2 \quad 92345455$

$1.692797+6 \quad 9.693127-8 \quad 6.088917-4 \quad 1.891950+6 \quad 7.382647-8 \quad 9.935336-4923466$ $1.692797+6 \quad 9.693127-8 \quad 6.088917-4 \quad 1.891950+6 \quad 7.382647-8 \quad 9.935337-49234616$

$1.493644+59.130957-72062296-31.692797+59.685172-72.159910-39234637$ $1.493644+5 \quad 9.130957-7 \quad 2.062296-3 \quad 1.692797+5 \quad 9.685171-7 \quad 2.159910-39234637$

_. . .

$\begin{array}{lllllll}2.117647+4 & 1.265218-6 & 2.205882+4 & 9.540925-7 & 2.294118+4 & 6.900423-79234 & 6\end{array}$ $2.117647+4 \quad 1.265218-6 \quad 2.205882+4 \quad 9.540924-7 \quad 2.294118+4 \quad 6.900423-79234 \quad 691$

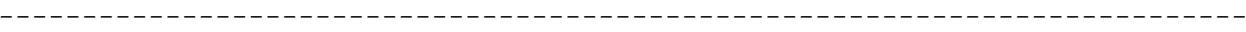

$92-U-238$

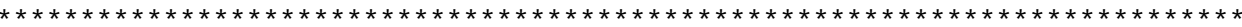

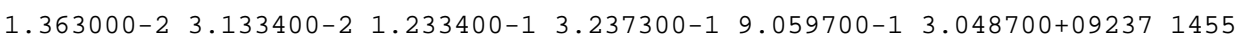
1.249423-2 3.025520-2 1.159376-1 3.414764-1 1.318630+@ 9.979027+09237 1455

$1.450000+67.478600-41.500000+6 \quad 8.679900-4 \quad 1.550000+69.893799-49237362$ $1.450000+6 \quad 7.478600-4 \quad 1.500000+6 \quad 8.679900-4 \quad 1.550000+6 \quad 9.893800-49237362$

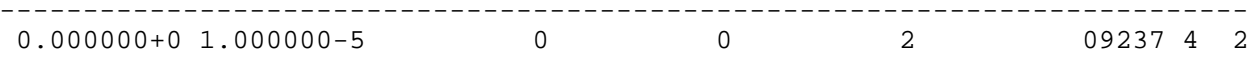

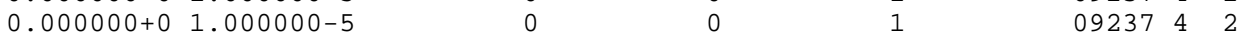

$7.499000-2 \quad 3.372000-3 \quad 1.095000-4 \quad 9.945001-7 \quad 1.877000-6 \quad 8.917000-69237465$ $\begin{array}{lllllll}7.499000-2 & 3.372000-3 & 1.095000-4 & 9.945000-7 & 1.877000-6 & 8.917000-69237 & 4\end{array}$

$9.860001-41.355000-2$ 1.144000-6-1.119000-2 2.608000-6-6.958000-492374 71 
$9.860000-4$ 1.355000-2 1.144000-6-1.119000-2 2.608000-6-6.958000-49237471 -2.123213-2-5.575145-2 4.503431-2-4.007026-2 8.664596-4 9.875596-492374 76 -2.123213-2-5.575145-2 4.503431-2-4.007026-2 8.664596-4 9.875595-49237476 (1) $-5.501023-6-5.653619-6-9.676723-7-1.311852-7-1.349319-8-1.592145-99237480$ $-5.501023-6-5.653619-6-9.676724-7-1.311852-7-1.349319-8-1.592145-99237480$

-1.130429-2-9.546876-3-9.897758-4 $1.520279-2 \quad 1.188212-2 \quad 4.399529-39237489$ -1.130429-2-9.546876-3-9.897757-4 1.520279-2 1.188212-2 4.399529-392374 89

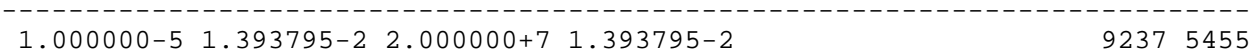
$1.000000-51.034128-23.000000+71.034128-2 \quad 92375455$

$2.489452+59.729187-76.176682-33.485232+59.581910-78.405816-39237617$ $2.489452+5 \quad 9.729186-7 \quad 6.176682-3 \quad 3.485232+5 \quad 9.581910-7 \quad 8.405816-39237 \quad 6 \quad 17$

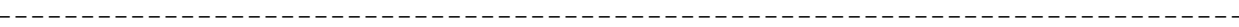

$\begin{array}{llllllll}5.601266+5 & 9.995942-7 & 7.343512-4 & 5.850212+5 & 9.759401-7 & 7.577155-49237 & 6 & 91\end{array}$ 5.601266+5 9.995943-7 $7.343512-4 \quad 5.850212+5 \quad 9.759401-7 \quad 7.577155-49237 \quad 691$

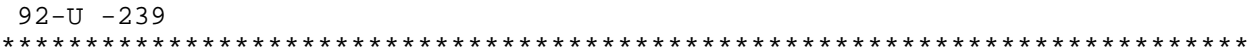

$\begin{array}{cccccc}9.223900+4 & 1.000000+\odot & 0 & 0 & 2 & 092402151\end{array}$

0

$1 \quad 2 \quad 092402151$

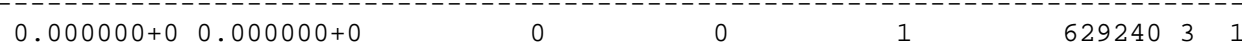

\begin{tabular}{c}
$0.000000+0$ \\
\hline
\end{tabular}

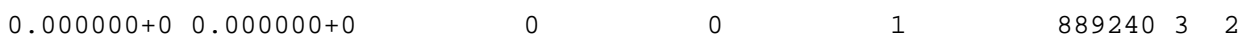

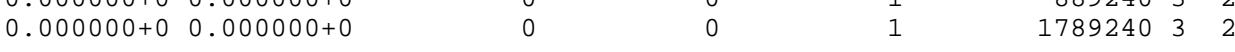

$4.826662+6 \quad 0.000000+05.000000+6 \quad 2.972386-2 \quad 5.250000+6 \quad 3.402231-19240316$ $4.826577+6 \quad 0.000000+\odot \quad 5.000000+6 \quad 3.024480-2 \quad 5.250000+6 \quad 3.462489-19240316$

(1.

$\begin{array}{llllll}-1.095900+7-1.095900+7 & 0 & 0 & 1 & 329240317\end{array}$ $\begin{array}{rrrrrr}-1.095930+7-1.095930+7 & 0 & 0 & 1 & 329240317\end{array}$

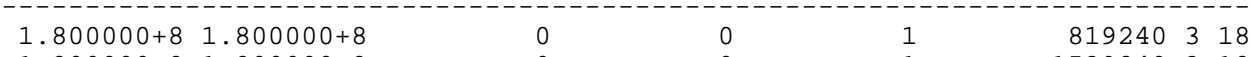

$\begin{array}{rrrrrr}1.800000+8 & 1.800000+8 & 0 & 0 & 1 & 1539240318\end{array}$

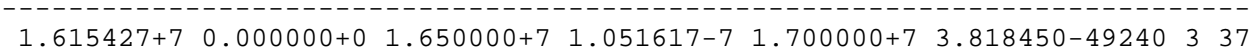
$\begin{array}{lllllllll}1.615387+7 & 0.000000+\odot & 1.650000+7 & 8.474438-8 & 1.700000+7 & 2.994790-49240 & 3 & 37\end{array}$

$4.267932+4 \quad 0.0000 \odot \odot+\odot \quad 4.500 \odot \odot \odot+4 \quad 9.896425-2 \quad 5.000 \odot \odot \odot+4 \quad 1.573151-19240351$ $4.267932+4 \quad 0.0000000+0 \quad 4.500000+4 \quad 8.618800-2 \quad 5.000000+4 \quad 1.381900-19240351$

$9.901603+40.000 \odot \odot \odot+\Theta 1.00 \odot \odot \odot \odot+5 \quad 2.575827-3 \quad 1.200 \odot \odot \odot+54.041081-29240352$ $9.901603+4 \quad 0.000000+0 \quad 1.000000+5 \quad 2.374600-3 \quad 1.200000+5 \quad 3.802200-29240352$

-

$1.343646+5 \quad 0.000000+\odot \quad 1.400000+5 \quad 5.328146-3 \quad 1.464152+5 \quad 1.114856-29240353$ $1.343646+5 \quad \odot .000000+\odot 1.400000+5 \quad 5.131689-3 \quad 1.464152+5 \quad 1.081951-29240353$

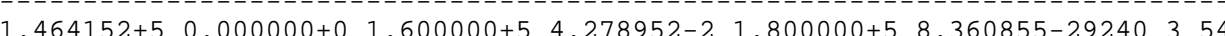
$1.464152+5 \quad 0.000000+0 \quad 1.600000+5 \quad 4.221600-2 \quad 1.800000+5 \quad 8.414698-29240354$

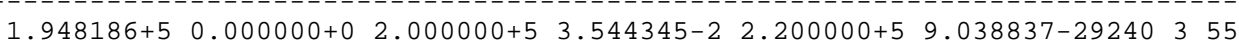
$1.948186+5 \quad \odot .000000+\odot \quad 2.000000+5 \quad 3.614500-2 \quad 2.200000+5 \quad 9.293400-29240355$

. $2.232380+5 \quad \odot .000000+\odot \quad 2.272548+5 \quad 1.166036-2 \quad 2.400000+5 \quad 4.334412-29240356$

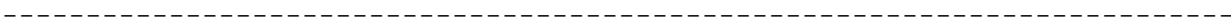

$2.272548+5 \quad 0.000000+\odot \quad 2.400000+5 \quad 1.314223-2 \quad 2.500000+5 \quad 2.255282-29240357$

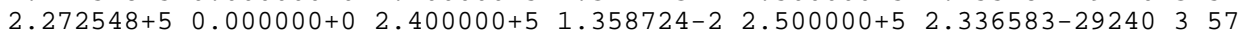

$2.938346+5 \quad 0.000000+\odot \quad 3.000000+5 \quad 2.225977-2 \quad 3.030734+5 \quad 2.731991-29240358$ $2.938346+5 \quad 0.000000+\odot \quad 3.000000+5 \quad 2.314400-2 \quad 3.030734+5 \quad 2.840629-29240358$

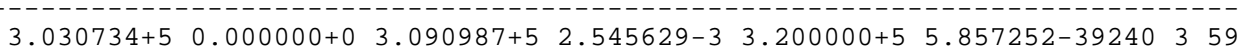

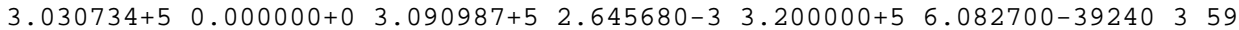
$3.090987+50.000000+\Theta 3.200000+5 \quad 8.739802-3 \quad 3.400000+5 \quad 2.074665-29240360$ $\begin{array}{llllllll}3.090987+5 & 0.000000+0 & 3.200000+5 & 9.076200-3 & 3.400000+5 & 2.147720-29240 & 3 & 60\end{array}$

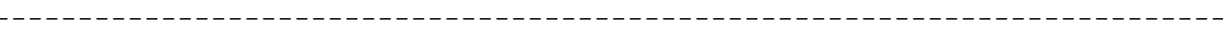

$3.742726+5 \quad 0.000000+\odot \quad 3.800000+5 \quad 7.343651-4 \quad 4.0000000+5 \quad 3.488479-39240 \quad 3 \quad 61$ $3.742726+5 \quad 0.000000+0 \quad 3.800000+5 \quad 7.535392-4 \quad 4.000000+5 \quad 3.559900-39240361$ 
$4.318143+5 \quad 0.000000+0 \quad 4.500000+5 \quad 1.715085-5 \quad 5.000000+5 \quad 6.709235-59240 \quad 362$ $4.318143+5 \quad 0.000000+\odot \quad 4.500000+5 \quad 1.723309-5 \quad 5.000000+5 \quad 6.669588-59240362$

$5.523207+5 \quad 0.000000+06.000000+5 \quad 9.121577-5 \quad 6.527198+51.637260-49240363$

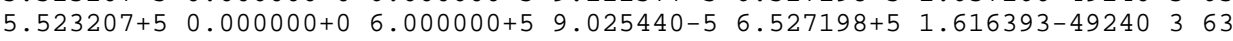

$6.527198+50.000000+07.000000+52.679465-47.732489+57.882143-49240364$ $6.527198+5 \quad 0.000000+\odot \quad 7.000000+5 \quad 2.640802-4 \quad 7.732489+5 \quad 7.758656-49240364$

$7.732489+5 \quad 0.000000+\odot \quad 8.000000+5 \quad 1.093671-4 \quad 9.000000+5 \quad 6.766953-49240365$ $7.732489+5 \quad 0.000000+08.000000+5 \quad 1.076151-4 \quad 9.0000000+5 \quad 6.655269-49240365$

$9.037975+5 \quad 0.000000+\odot \quad 1.000000+6 \quad 4.376700-4 \quad 1.066983+6 \quad 7.479008-49240366$ $9.037975+5 \quad 0.000000+\odot \quad 1.000000+6 \quad 4.306732-4 \quad 1.066983+6 \quad 7.369347-49240366$

$1.066983+6 \quad \odot .000 \odot \odot \odot+\odot \quad 1.174937+6 \quad 8.839994-4 \quad 1.2000 \odot \odot+6 \quad 1.059683-39240367$ $1.066983+6 \quad 0.0000000+\odot \quad 1.174937+6 \quad 8.728328-4 \quad 1.200000+6 \quad 1.046763-39240367$

$1.174937+6 \quad 0.000000+\odot \quad 1.200000+6 \quad 1.930001-4 \quad 1.255274+6 \quad 5.698038-49240368$ $1.174937+6 \quad 0.000000+\odot \quad 1.200000+6 \quad 1.906468-4 \quad 1.255274+6 \quad 5.636577-49240368$

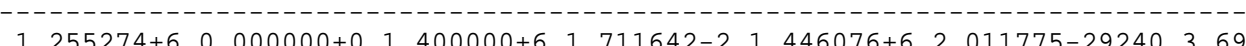
$1.255274+6 \quad \odot .000000+\odot \quad 1.400000+6 \quad 1.692496-2 \quad 1.446076+6 \quad 1.989316-29240369$

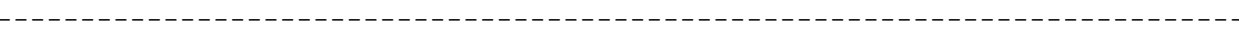

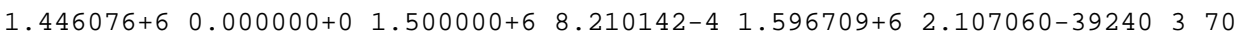
$1.446076+6 \quad 0.000000+\odot \quad 1.500000+6 \quad 8.119760-4 \quad 1.596709+6 \quad 2.084306-39240370$

$1.596709+60.000000+01.600000+63.078136-51.757384+61.231788-39240371$ $1.596709+6 \quad 0.000000+\odot \quad 1.600000+6 \quad 3.044924-5 \quad 1.757384+6 \quad 1.218887-39240371$

$1.757384+6 \quad 0.000000+0 \quad 1.800000+6 \quad 4.734083-41.857806+6 \quad 9.982595-49240372$

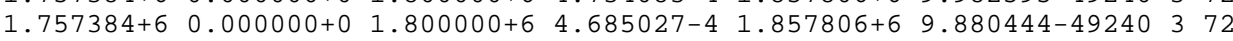

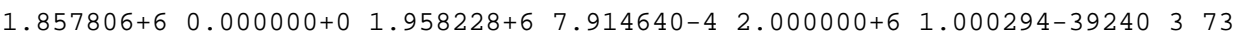

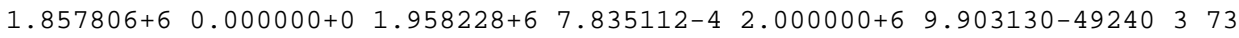

$1.958228+6 \quad \odot .000000+\odot \quad 2.000000+6 \quad 4.182199-4 \quad 2.159072+6 \quad 1.755398-39240374$ $1.958228+6 \quad 0.000000+\odot \quad 2.000000+6 \quad 4.140471-4 \quad 2.159072+6 \quad 1.742684-39240374$

$2.159072+6 \quad 0.000000+\odot \quad 2.309705+6 \quad 2.278092-3 \quad 2.400084+6 \quad 3.204483-39240375$ $2.159072+6 \quad 0.000000+\odot \quad 2.309705+6 \quad 2.270274-3 \quad 2.400084+6 \quad 3.200884-39240 \quad 3 \quad 75$

$2.309705+6 \quad 0.000 \odot \odot \odot+\odot \quad 2.400 \odot 84+6 \quad 1.876465-3 \quad 2.5000 \odot 0+6 \quad 3.296627-39240376$ $2.309705+6 \quad 0.0000000+\odot \quad 2.4000084+6 \quad 1.874357-3 \quad 2.500000+6 \quad 3.301327-39240376$

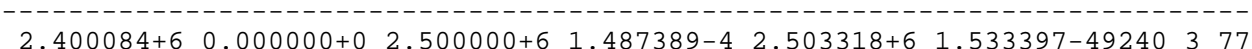
$2.400084+6 \quad 0.000000+0 \quad 2.500000+6 \quad 1.489507-4 \quad 2.503318+6 \quad 1.535757-49240377$ $2.503318+60.000000+\Theta 2.952405+6 \quad 1.003608-2 \quad 3.000000+6 \quad 1.034094-29240378$ $2.503318+6 \quad \odot .0000000+\odot \quad 2.952405+6 \quad 1.010698-2 \quad 3.0000000+6 \quad 1.041341-2924 \odot 378$ $2.952405+6 \quad 0.000000+0 \quad 3.000000+6 \quad 1.350909-3 \quad 3.202456+6 \quad 5.838520-39240379$ $2.952405+6 \quad \odot .000000+\odot \quad 3.000000+6 \quad 1.360376-3 \quad 3.202456+6 \quad 5.885106-39240379$ (2024

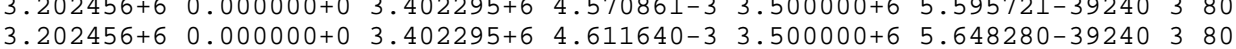

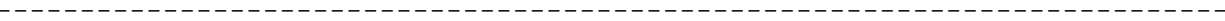
$3.402295+6 \quad 0.000000+\odot \quad 3.500000+6 \quad 1.646956-3 \quad 3.552928+6 \quad 2.432630-39240381$ $\begin{array}{llllllll}3.402295+6 & 0.000000+\odot & 3.500000+6 & 1.662425-3 & 3.552928+6 & 2.455744-39240 & 3 & 81\end{array}$ $3.552928+6 \quad 0.000000+\odot \quad 3.652346+6 \quad 7.133156-4 \quad 3.752768+6 \quad 1.279391-39240382$

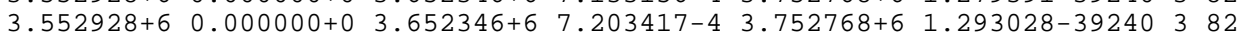
$3.652346+6 \quad 0.000000+\odot \quad 3.752768+6 \quad 8.359367-4 \quad 3.853190+6 \quad 1.440079-39240333$ $3.652346+6 \quad 0.000000+\odot \quad 3.752768+6 \quad 8.448473-4 \quad 3.853190+6 \quad 1.456610-39240383$

$3.752768+6 \quad 0.000000+\odot \quad 3.853190+6 \quad 8.651377-4 \quad 3.925494+6 \quad 1.283678-39240384$ $3.752768+6 \quad 0.000000+\odot \quad 3.853190+6 \quad 8.750694-4 \quad 3.925494+6 \quad 1.299176-39240384$ $3.853190+6 \quad 0.000 \odot \odot \odot+\Theta 3.925494+64.188816-4 \quad 4.000 \odot \odot \odot+6 \quad 6.845223-49240385$ $3.853190+6 \quad 0.000000+\odot \quad 3.925494+6 \quad 4.239386-4 \quad 4.000000+6 \quad 6.932080-49240385$

3.

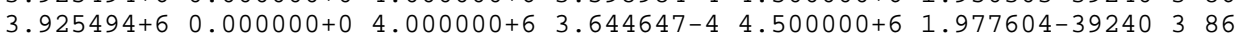




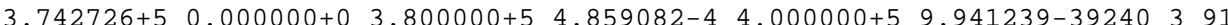
$3.742726+5 \quad \odot .0000000+\odot \quad 3.800000+5 \quad 4.985948-4 \quad 4.0000000+5 \quad 1.014477-29240391$

$\begin{array}{rrrrrr}5.930800+6 & 5.930800+6 & 0 & 0 & 1 & 9692403102\end{array}$

$\begin{array}{rrrrrr}5.930800+6 & 5.930800+6 & 0 & 0 & 1 & 15392403102\end{array}$

$92-U-240$

$\begin{array}{lllllllll}1.078221+7 & 0.000000+\odot & 1.080000+7 & 1.64512-42 & 1.100000+7 & 1.622900-49243 & 3 & 17\end{array}$ $\begin{array}{llllllll}1.078221+7 & 0.000000+0 & 1.080000+7 & 1.64581-42 & 1.100000+7 & 1.622900-49243 & 3 & 17\end{array}$

(1.002633-39243 357 4.200000+6 9.727297-4 $4.300000+6 \quad 9.878623-4 \quad 4.400000+6 \quad 1.002633-39243 \quad 357$

$1.133858-23.681401-3 \quad 9.783953-42.340624-45.143415-59.969714-6924344$ 1.133858-2 3.681401-3 9.783954-4 2.340624-4 5.143415-5 9.969714-69243 42

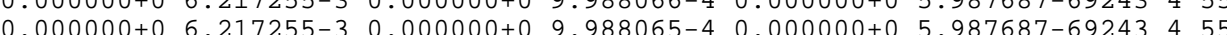
$0.000000+0-9.857893-7 \quad 0.000000+0-2.835380-8 \quad 0.000000+\odot-1.350161-99243457$ $\odot .000000+\odot-9.857892-7 \quad 0.000000+\odot-2.835380-8 \quad 0.000000+0-1.350161-99243457$

$\odot .00000 \odot+\odot \quad 9.794074-7 \quad 0.000000+\odot \quad 2.172264-8 \quad 0.000000+\odot 2.660714-99243463$ $0.000000+\odot \quad 9.794075-7 \quad 0.000000+\odot \quad 2.172264-8 \quad 0.000000+\odot \quad 2.660714-99243463$

$\odot .0 \odot \odot \odot \odot \odot+\odot ~ 4.563329-5 \quad 0.0 \odot \odot \odot \odot \odot+\odot ~ 9.566922-7 \quad 0.0 \odot \odot \odot \odot \odot+\odot-2.855296-69243470$ $\odot .000000+\odot \quad 4.563329-5 \quad 0.0000000+\odot \quad 9.566923-7 \quad 0.000000+0-2.855296-69243470$

$6.692000-4 \quad 3.344000-39.712001-7 \quad 2.593000-6-7.279000-8-3.602000-89243476$ $6.692000-4 \quad 3.344000-3 \quad 9.712000-7 \quad 2.593000-6-7.279000-8-3.602000-89243476$

7.095188+5 $1.098172-69.027065-3 \quad 7.344141+59.899715-7 \quad 4.951523-29243691$ 7.095188+5 $1.098172-6 \quad 9.027065-3 \quad 7.344141+5 \quad 9.899714-7 \quad 4.951523-29243661$

-

$92-U-241$

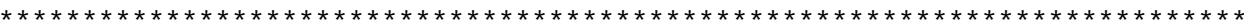

9.866999-4-1.066000-3-4.006000-7 $0.0000000+0 \quad 9246451$ $9.867000-4-1.066000-3-4.006000-70.000000+0 \quad 9246451$

-

-2.123213-2-5.575145-2 4.503431-2-4.007026-2 8.664596-4 9.875596-49246 4 63 -2.123213-2-5.575145-2 4.503431-2-4.007026-2 8.664596-4 9.875595-49246463 $-5.501023-6-5.653619-6-9.676723-7-1.311852-7-1.349319-8-1.592145-99246467$ $-5.501023-6-5.653619-6-9.676724-7-1.311852-7-1.349319-8-1.592145-99246467$ 1. -1.130429-2-9.546876-3-9.897757-4 $1.520279-2 \quad 1.188212-2 \quad 4.399529-39246476$ $\begin{array}{llllllll}5.477082+4 & 9.578685-7 & 7.681016-5 & 6.472916+4 & 1.034695-6 & 7.958543-59246 & 691\end{array}$ 5.477082+4 9.578684-7 7.681016-5 6.472916+4 1.034695-6 7.958543-59246 691 93-Np-234 Evaluation Only in VII.1

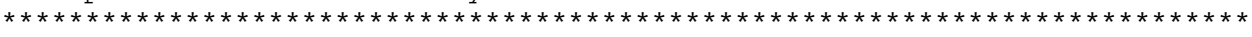
$93-\mathrm{Np}-235$

\begin{tabular}{|c|c|c|c|c|c|c|}
\hline $\begin{array}{l}\odot . \odot \odot \odot \odot \odot \odot+\odot \\
\odot . \odot \odot \odot \odot \odot \odot+\odot ~\end{array}$ & 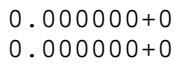 & $\begin{array}{l}\odot \\
\odot\end{array}$ & $\begin{array}{l}\odot \\
\odot\end{array}$ & $\begin{array}{l}1 \\
1\end{array}$ & $\begin{array}{r}179340 \\
49340\end{array}$ & $\begin{array}{l}1452 \\
1452\end{array}$ \\
\hline $\begin{array}{l}\odot .0 \odot \odot \odot \odot \odot+\odot \\
\odot . \odot \odot \odot \odot \odot \odot+\odot\end{array}$ & $\begin{array}{l}\odot .00 \odot \odot \odot \odot+\odot \\
\odot . \diamond \odot \odot \odot \odot \odot+\odot\end{array}$ & $\begin{array}{l}\odot \\
\odot\end{array}$ & $\begin{array}{l}\odot \\
\odot\end{array}$ & $\begin{array}{l}1 \\
1\end{array}$ & $\begin{array}{r}179340 \\
49340\end{array}$ & $\begin{array}{l}1455 \\
1455\end{array}$ \\
\hline $\begin{array}{l}1.000000-5 \\
1.000000-5\end{array}$ & $\begin{array}{l}2.632300+\odot \\
2.659000+\odot\end{array}$ & $\begin{array}{l}2.000000+7 \\
2.000000+7\end{array}$ & $\begin{array}{l}5.430500+\odot \\
5.259000+\odot\end{array}$ & & $\begin{array}{l}9340 \\
9340\end{array}$ & $\begin{array}{l}1456 \\
1456\end{array}$ \\
\hline $\begin{array}{l}1.000 \odot \odot \odot-5 \\
1.000000-5\end{array}$ & $\begin{array}{l}\text { 2. } .0000 \odot \odot-1 \\
\text { 4. } 0000 \odot \odot-1\end{array}$ & $\begin{array}{l}\odot \\
\odot\end{array}$ & $\begin{array}{l}\odot \\
\odot\end{array}$ & $\stackrel{\odot}{\odot}$ & $\begin{array}{l}\odot 9340 \\
09340\end{array}$ & $\begin{array}{l}2151 \\
2151\end{array}$ \\
\hline $\begin{array}{l}\odot .0 \odot \odot \odot \odot \odot+\odot \\
\odot .0 \odot \odot \odot \odot \odot+\odot\end{array}$ & $\begin{array}{l}\odot . \odot \odot \odot \odot \odot \odot+\odot ~ \\
\odot . \diamond \odot \odot \odot \odot \odot+\odot\end{array}$ & $\begin{array}{l}\odot \\
\odot\end{array}$ & $\begin{array}{l}\odot \\
\odot\end{array}$ & $\begin{array}{l}1 \\
1\end{array}$ & $\begin{array}{l}1049340 \\
1239340\end{array}$ & $\begin{array}{ll}3 & 1 \\
3 & 1\end{array}$ \\
\hline $\begin{array}{l}\odot .000000+\odot \\
\odot .0 \odot \odot \odot \odot \odot+\odot\end{array}$ & $\begin{array}{l}\odot .00000 \odot+\odot \\
\odot .00 \odot \odot \odot \odot+\odot\end{array}$ & $\begin{array}{l}\odot \\
\odot\end{array}$ & $\begin{array}{l}\odot \\
\odot\end{array}$ & $\begin{array}{l}1 \\
1\end{array}$ & $\begin{array}{r}989340 \\
1169340\end{array}$ & $\begin{array}{l}3 \\
3\end{array}$ \\
\hline
\end{tabular}




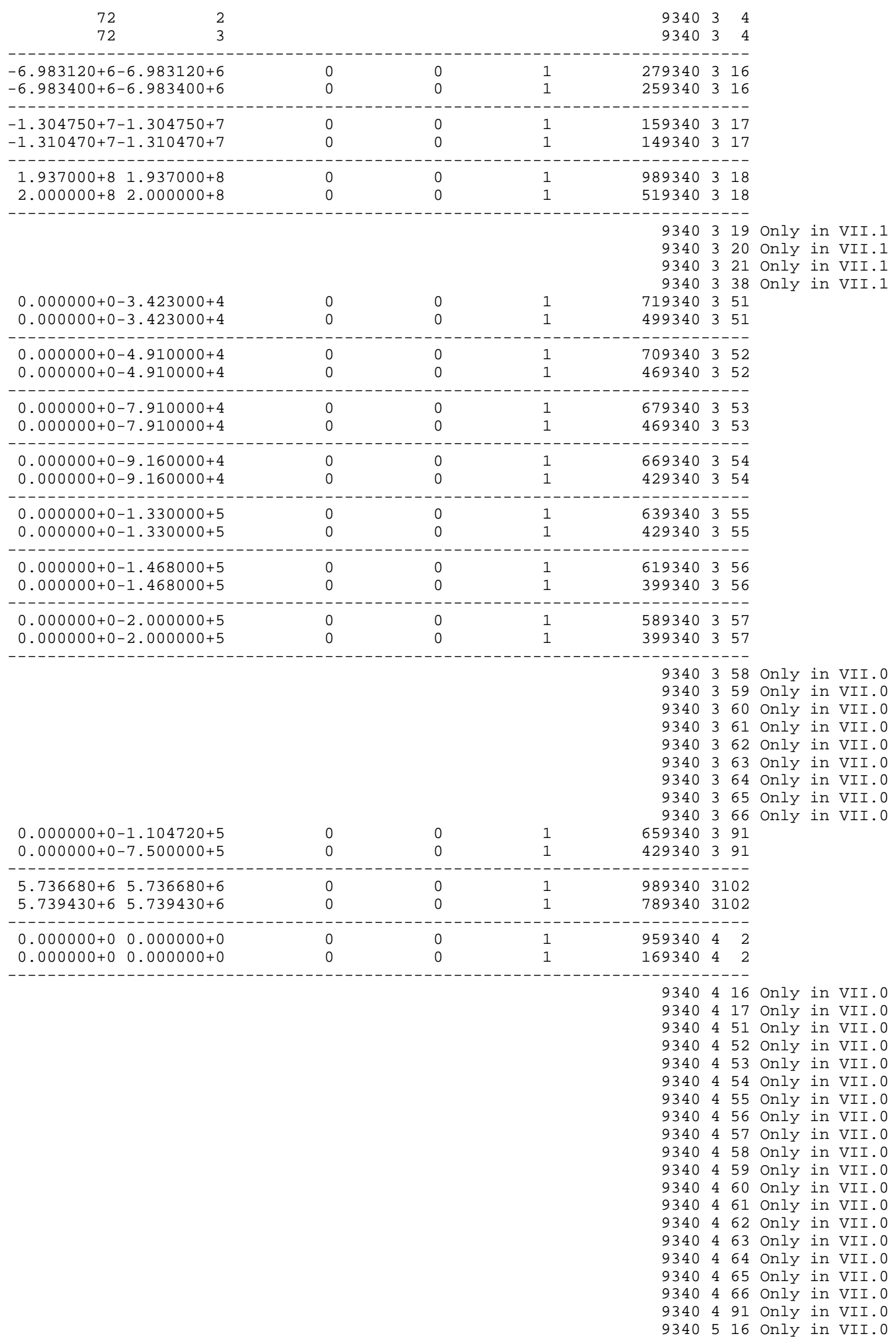




\begin{tabular}{|c|c|c|c|c|c|c|}
\hline $\begin{array}{rr}\odot .000 \odot \odot \odot+\odot & \odot .00 \odot \odot \odot \odot+\odot \\
-2.00 \odot \odot \odot \odot+8 & \odot . \odot \odot \odot \odot \odot \odot+\odot\end{array}$ & $\begin{array}{ll}\odot & 1 \\
\odot & 7\end{array}$ & $\begin{array}{l}1 \\
1\end{array}$ & $\begin{array}{r}9340 \\
29340 \\
29340\end{array}$ & $\begin{array}{ll}5 & 17 \\
5 & 18 \\
5 & 18\end{array}$ & only in & VII.O \\
\hline - - - - - - & - - - - & & $\begin{array}{l}9340 \\
9340 \\
9340 \\
9340 \\
9340 \\
9340 \\
9340 \\
9340 \\
9340 \\
9340 \\
9340 \\
9340\end{array}$ & $\begin{array}{ll}5 & 91 \\
6 & 16 \\
6 & 17 \\
6 & 51 \\
6 & 52 \\
6 & 53 \\
6 & 54 \\
6 & 55 \\
6 & 56 \\
6 & 57 \\
6 & 91 \\
6102\end{array}$ & $\begin{array}{l}\text { Only in } \\
\text { Only in } \\
\text { Only in } \\
\text { Only in } \\
\text { Only in } \\
\text { Only in } \\
\text { Only in } \\
\text { Only in } \\
\text { Only in } \\
\text { Only in } \\
\text { Only in } \\
\text { Only in }\end{array}$ & $\begin{array}{l}\text { VII. } 0 \\
\text { VII.1 } \\
\text { VII.1 } \\
\text { VII.1 } \\
\text { VII.1 } \\
\text { VII.1 } \\
\text { VII.1 } \\
\text { VII.1 } \\
\text { VII.1 } \\
\text { VII.1 } \\
\text { VII.1 } \\
\text { VII.1 }\end{array}$ \\
\hline
\end{tabular}

$93-\mathrm{Np}-236$

\begin{tabular}{|c|c|c|c|c|}
\hline 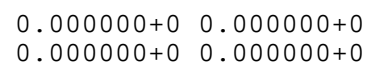 & $\begin{array}{l}\odot \\
\odot\end{array}$ & $\stackrel{\ominus}{\ominus}$ & $\begin{array}{l}1 \\
1\end{array}$ & $\begin{array}{rr}179343 & 1452 \\
49343 & 1452\end{array}$ \\
\hline 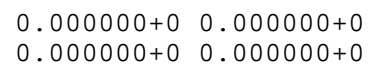 & $\begin{array}{l}\odot \\
\odot\end{array}$ & $\begin{array}{l}\odot \\
\odot\end{array}$ & $\begin{array}{l}1 \\
1\end{array}$ & $\begin{array}{rr}179343 & 1455 \\
49343 & 1455\end{array}$ \\
\hline 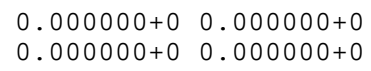 & $\begin{array}{l}\odot \\
\odot\end{array}$ & $\begin{array}{l}\odot \\
\odot\end{array}$ & $\begin{array}{l}1 \\
1\end{array}$ & $\begin{array}{ll}29343 & 1456 \\
49343 & 1456\end{array}$ \\
\hline $\begin{array}{ll}9.323600+4 & 1.000000+\odot \\
9.323600+4 & 1.000000+\odot\end{array}$ & $\begin{array}{l}\odot \\
\odot\end{array}$ & $\begin{array}{l}1 \\
0\end{array}$ & $\begin{array}{l}2 \\
1\end{array}$ & $\begin{array}{ll}09343 & 2151 \\
09343 & 2151\end{array}$ \\
\hline 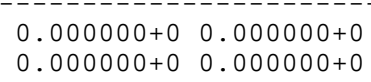 & $\begin{array}{l}\odot \\
\odot\end{array}$ & $\begin{array}{l}\ominus \\
\ominus\end{array}$ & $\begin{array}{l}2 \\
2\end{array}$ & $\begin{array}{lll}949343 & 3 & 1 \\
829343 & 3 & 1\end{array}$ \\
\hline 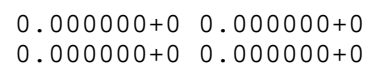 & $\begin{array}{l}\odot \\
\odot\end{array}$ & $\begin{array}{l}\odot \\
\odot\end{array}$ & $\begin{array}{l}2 \\
2\end{array}$ & $\begin{array}{lll}909343 & 3 & 2 \\
809343 & 3 & 2\end{array}$ \\
\hline $\begin{array}{l}\odot .000 \odot \odot \odot+\odot-5.6000 \odot \odot+4 \\
\odot .000 \odot \odot \odot+\odot-5.999990+4\end{array}$ & $\begin{array}{l}\odot \\
\odot\end{array}$ & $\begin{array}{l}\odot \\
\odot\end{array}$ & $\begin{array}{l}1 \\
1\end{array}$ & $\begin{array}{lll}709343 & 3 & 4 \\
349343 & 3 & 4\end{array}$ \\
\hline $\begin{array}{l}-5.736680+6-5.736680+6 \\
-5.729790+6-5.729790+6\end{array}$ & $\begin{array}{l}\odot \\
\odot\end{array}$ & $\begin{array}{l}\odot \\
\odot\end{array}$ & $\begin{array}{l}1 \\
1\end{array}$ & $\begin{array}{lll}309343 & 3 & 16 \\
269343 & 3 & 16\end{array}$ \\
\hline $\begin{array}{l}-1.271980+7-1.271980+7 \\
-1.270900+7-1.270900+7\end{array}$ & $\begin{array}{l}\odot \\
\odot\end{array}$ & $\begin{array}{l}\odot \\
\odot\end{array}$ & $\begin{array}{l}1 \\
1\end{array}$ & $\begin{array}{lll}159343 & 3 & 17 \\
149343 & 3 & 17\end{array}$ \\
\hline $\begin{array}{ll}1.950000+8 & 1.950000+8 \\
1.999990+8 & 1.999990+8\end{array}$ & $\begin{array}{l}\odot \\
\odot\end{array}$ & $\begin{array}{l}\odot \\
\ominus\end{array}$ & $\begin{array}{l}2 \\
2\end{array}$ & $\begin{array}{lll}909343 & 3 & 18 \\
439343 & 3 & 18\end{array}$ \\
\hline
\end{tabular}

9343319 only in VII.1 9343320 Only in VII.1 9343321 only in VII.1 9343337 only in VII.1

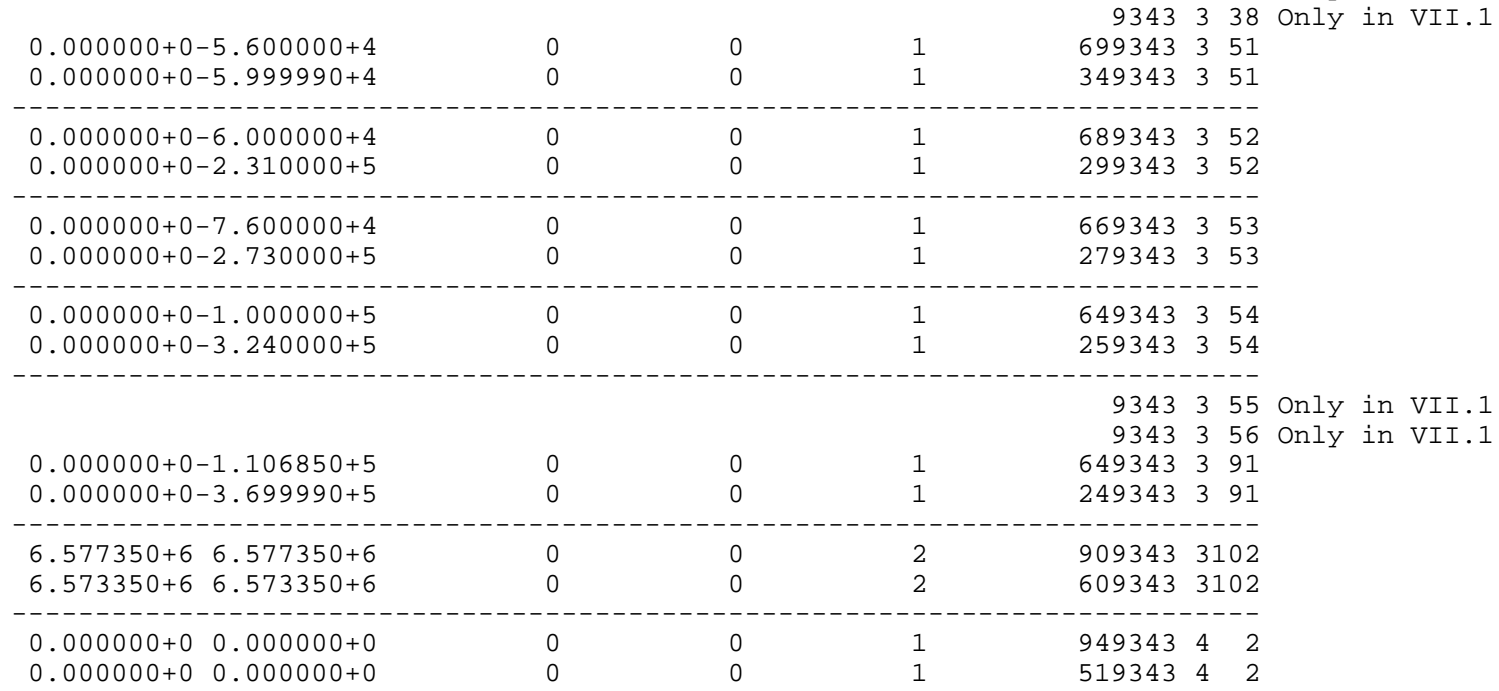




\begin{tabular}{|c|c|c|c|c|c|c|c|}
\hline & & & & $\begin{array}{l}9343 \\
9343\end{array}$ & $\begin{array}{ll}4 & 16 \\
4 & 17\end{array}$ & $\begin{array}{l}\text { Only in } \\
\text { Only in }\end{array}$ & $\begin{array}{l}\text { VII. } 0 \\
\text { VII.0 }\end{array}$ \\
\hline$\odot . \odot \odot \odot \odot \odot \odot+\odot \quad 2.340190+2$ & 1 & 1 & $\odot$ & 09343 & 418 & & \\
\hline$\odot .000000+\odot \quad 2.340190+2$ & 0 & 1 & 0 & 09343 & 418 & & \\
\hline-- & & & & $\begin{array}{r}9343 \\
9343 \\
9343 \\
9343 \\
9343 \\
9343 \\
9343\end{array}$ & $\begin{array}{ll}4 & 51 \\
4 & 52 \\
4 & 53 \\
4 & 54 \\
4 & 91 \\
5 & 16 \\
5 & 17\end{array}$ & $\begin{array}{l}\text { Only in } \\
\text { Only in } \\
\text { Only in } \\
\text { Only in } \\
\text { Only in } \\
\text { Only in } \\
\text { Only in }\end{array}$ & $\begin{array}{l}\text { VII. } 0 \\
\text { VII.0 } \\
\text { VII.0 } \\
\text { VII.0 } \\
\text { VII. } \\
\text { VII. } \\
\text { VII.0 }\end{array}$ \\
\hline $\begin{array}{rr}0.0 \odot \odot \odot \odot \odot+\odot & 0.00 \odot \odot \odot \odot \odot+\odot \\
-2.00 \odot \odot \odot \odot+7 & 0.00 \odot \odot \odot \odot+\odot\end{array}$ & $\begin{array}{l}\odot \\
\odot\end{array}$ & $\begin{array}{l}1 \\
7\end{array}$ & $\begin{array}{l}1 \\
1\end{array}$ & $\begin{array}{l}29343 \\
29343\end{array}$ & $\begin{array}{ll}5 & 18 \\
5 & 18\end{array}$ & & \\
\hline & & & & $\begin{array}{l}9343 \\
9343 \\
9343 \\
9343 \\
9343 \\
9343 \\
9343 \\
9343 \\
9343 \\
9343 \\
9343 \\
9343\end{array}$ & $\begin{array}{ll}5 & 91 \\
6 & 16 \\
6 & 17 \\
6 & 37 \\
6 & 51 \\
6 & 52 \\
6 & 53 \\
6 & 54 \\
6 & 55 \\
6 & 56 \\
6 & 91 \\
6102\end{array}$ & $\begin{array}{l}\text { Only in } \\
\text { Only in } \\
\text { Only in } \\
\text { Only in } \\
\text { Only in } \\
\text { Only in } \\
\text { Only in } \\
\text { Only in } \\
\text { Only in } \\
\text { Only in } \\
\text { Only in } \\
\text { Only in }\end{array}$ & $\begin{array}{l}\text { VII.0 } \\
\text { VII.1 } \\
\text { VII.1 } \\
\text { VII.1 } \\
\text { VII.1 } \\
\text { VII.1 } \\
\text { VII.1 } \\
\text { VII.1 } \\
\text { VII.1 } \\
\text { VII.1 } \\
\text { VII.1 } \\
\text { VII.1 }\end{array}$ \\
\hline
\end{tabular}

$93-\mathrm{Np}-237$

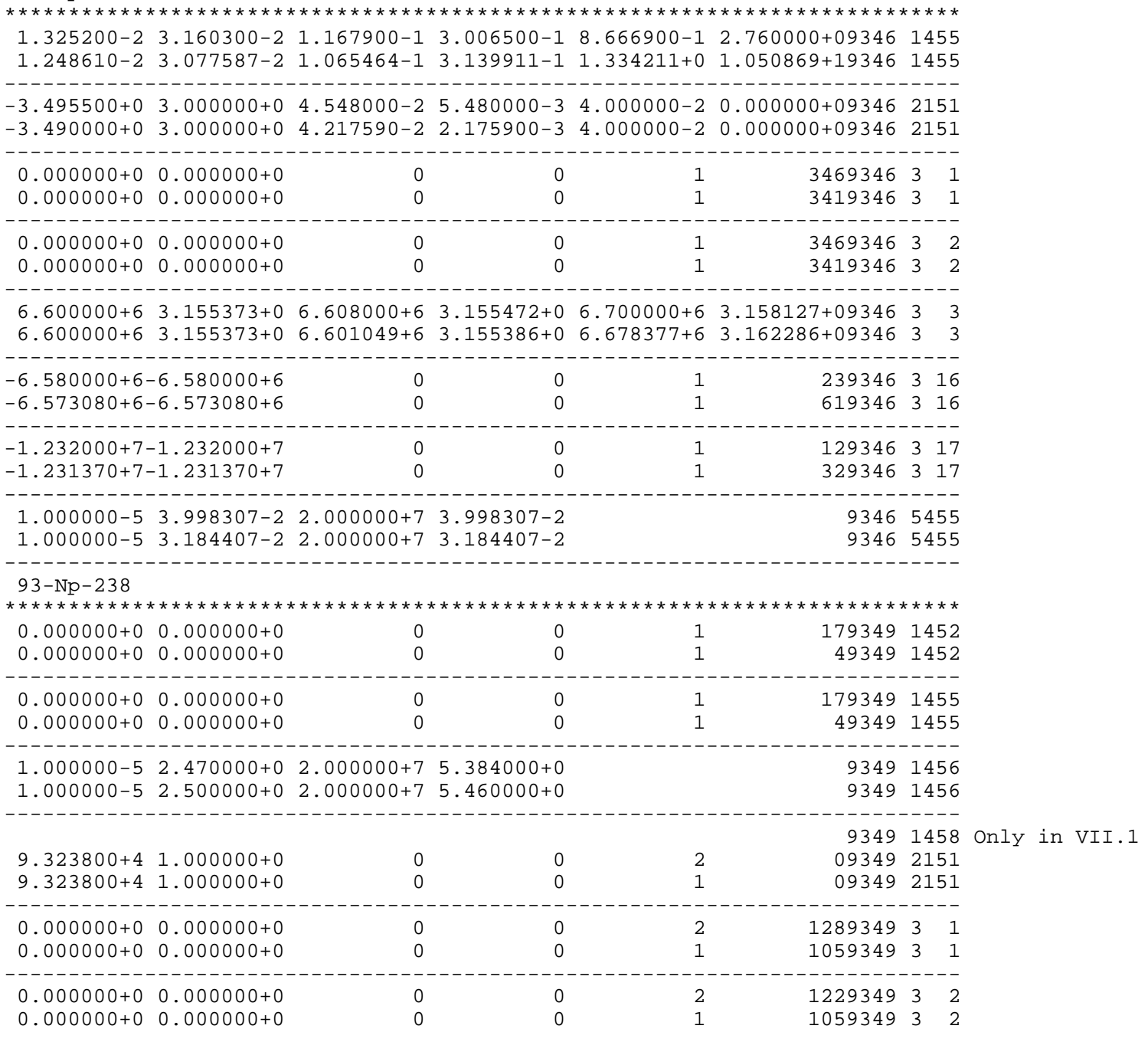




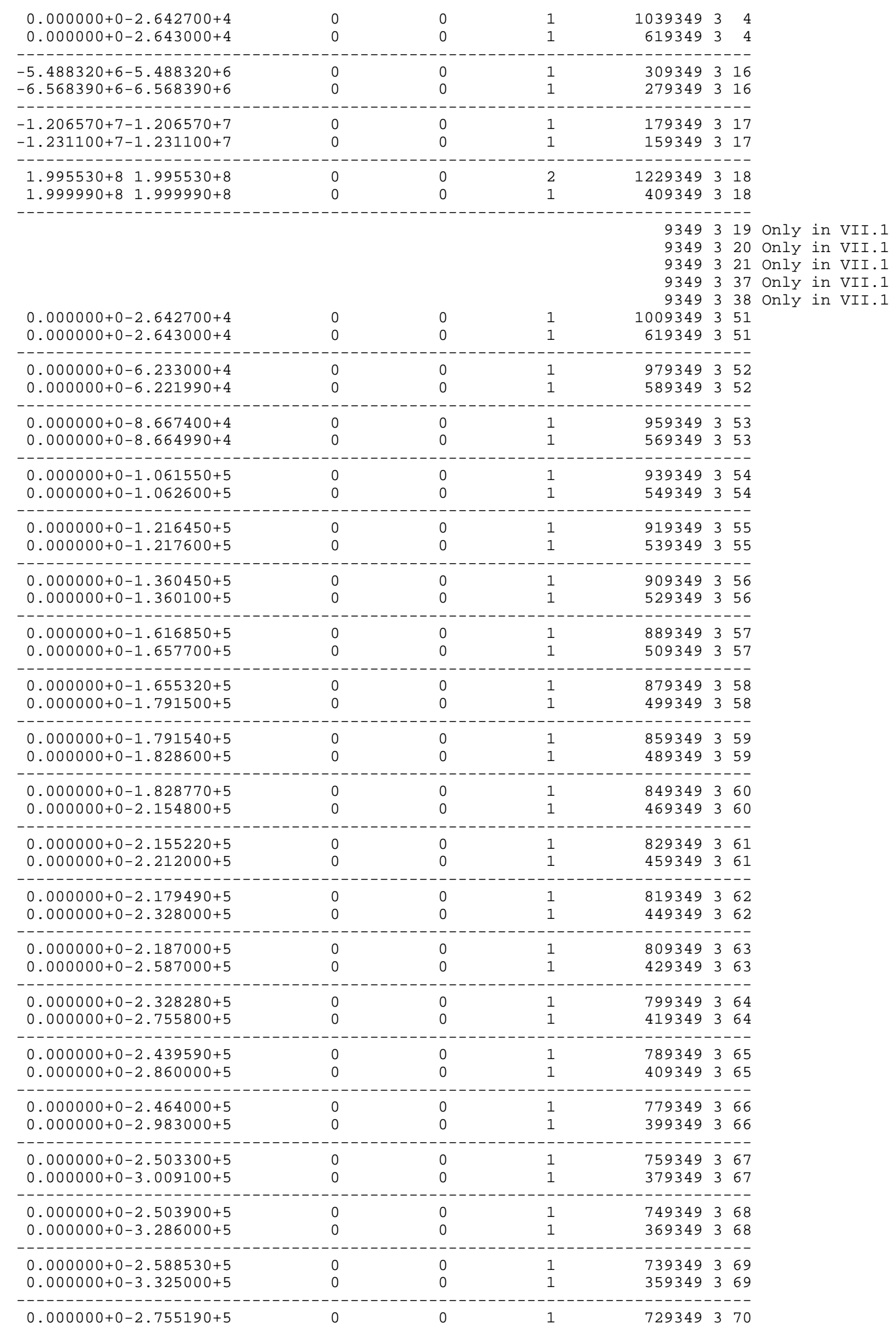




\begin{tabular}{|c|c|c|c|c|}
\hline $0.000000+0-3.423800+5$ & $\odot$ & $\odot$ & 1 & 349349370 \\
\hline \multicolumn{5}{|c|}{ 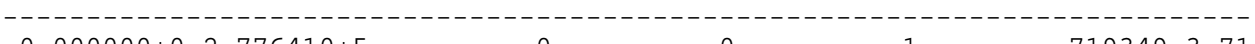 } \\
\hline$\odot .000000+0-2.776410+5$ & $\odot$ & $\odot$ & 1 & 719349371 \\
\hline $0.000000+0-3.501000+5$ & $\odot$ & $\odot$ & 1 & 339349371 \\
\hline \multicolumn{5}{|c|}{ 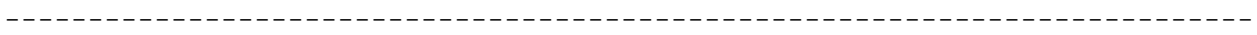 } \\
\hline $0.000000+0-2.858000+5$ & $\odot$ & $\odot$ & 1 & 709349372 \\
\hline $0.000000+0-3.684090+5$ & $\odot$ & $\odot$ & 1 & 329349372 \\
\hline \multicolumn{5}{|c|}{ 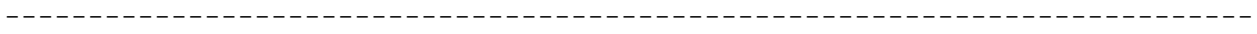 } \\
\hline$\odot .000 \odot \odot \odot+\odot-2.970300+5$ & $\odot$ & $\odot$ & 1 & 699349373 \\
\hline $0.000000+0-3.740990+5$ & $\odot$ & $\odot$ & 1 & 319349373 \\
\hline \multicolumn{5}{|c|}{ - - - - - - - - - - - - - - - - - - - - - - - - - - - - - - - - - - - - - - - - - - - - - - - - - - - - - - - - - } \\
\hline $0.000000+\odot-2.983680+5$ & $\odot$ & $\odot$ & 1 & 689349374 \\
\hline$\odot . \odot \odot \odot \odot \odot \odot+\odot-4.08499 \odot+5$ & $\odot$ & $\odot$ & 1 & 309349374 \\
\hline \multicolumn{5}{|c|}{ - - - - - - - - - - - - - - - - - - - - - - - - - - - - - - - - - - - - - - - - - - - - - - } \\
\hline $0.000000+0-2.992300+5$ & $\odot$ & $\odot$ & 1 & 669349375 \\
\hline$\odot .000000+0-4.312990+5$ & $\odot$ & $\odot$ & 1 & 299349375 \\
\hline \multicolumn{5}{|c|}{ - - - - - - - - - - - - - - - - - - - - - - - - - - - - - - - - - - - - - - - - - - - } \\
\hline $0.000000+0-2.997880+5$ & $\odot$ & $\odot$ & 1 & 659349376 \\
\hline $0.000000+0-4.403990+5$ & $\odot$ & $\odot$ & 1 & 289349376 \\
\hline \multicolumn{5}{|c|}{ - } \\
\hline $0.000000+0-3.006800+5$ & $\odot$ & $\odot$ & 1 & 649349377 \\
\hline $0.000000+0-4.562990+5$ & $\odot$ & $\odot$ & 1 & 279349377 \\
\hline \multicolumn{5}{|c|}{ 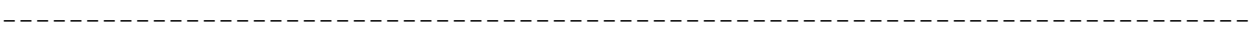 } \\
\hline $0.000000+0-3.007430+5$ & $\odot$ & $\odot$ & 1 & 639349378 \\
\hline$\odot .0 \odot \odot \odot \odot \odot+\odot-4.625990+5$ & $\odot$ & $\odot$ & 1 & 269349378 \\
\hline \multicolumn{5}{|c|}{ 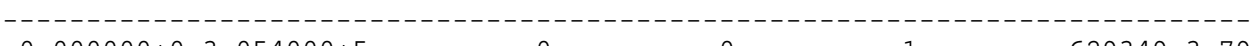 } \\
\hline $0.000000+0-3.054000+5$ & $\odot$ & $\odot$ & 1 & 629349379 \\
\hline$\odot .000000+0-5.241990+5$ & $\odot$ & $\odot$ & 1 & 249349379 \\
\hline
\end{tabular}

9349380 Only in VII.1 9349381 only in VII.1 9349382 only in VII.1 9349383 only in VII.1 9349384 only in VII.1

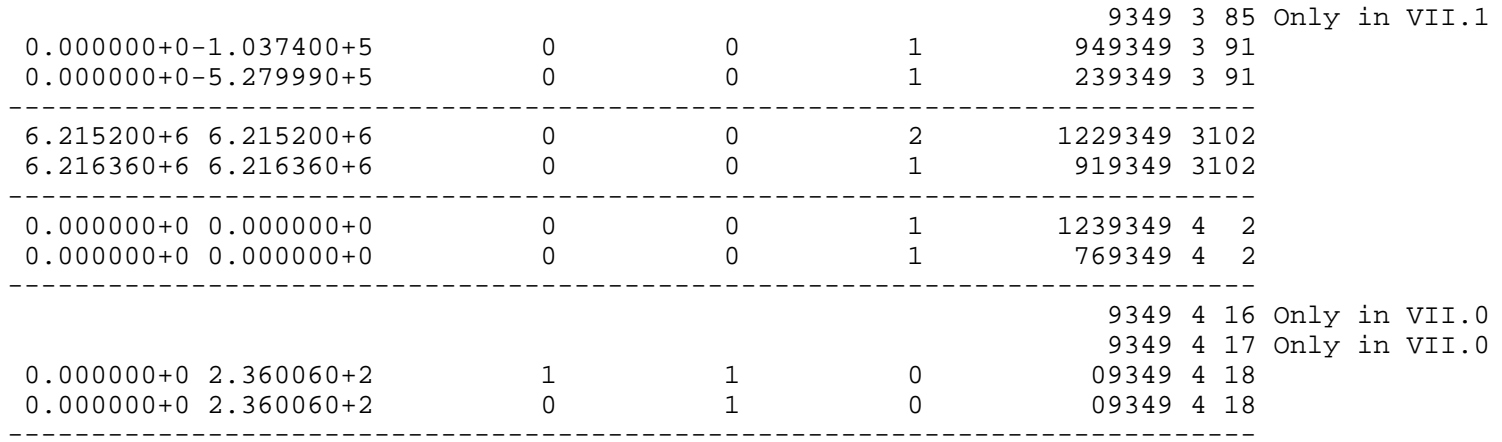

9349451 Only in VII.0 9349452 only in VII.0 9349453 only in VII.0 9349454 only in VII.0 9349455 only in VII.0 9349456 only in VII.0 9349457 only in VII.0 9349458 only in VII.0 9349459 only in VII.0 9349460 only in VII.0 9349461 only in VII.0 9349462 only in VII.0 9349463 only in VII.0 9349464 only in VII.0 9349465 only in VII.0 9349466 only in VII.0 9349467 only in VII.0 9349468 only in VII.0 9349469 only in VII.0 9349470 only in VII.0 9349471 only in VII.0 9349472 only in VII.0 9349473 only in VII.0 9349474 only in VII.0 9349475 only in VII.0 9349476 only in VII.0 


\begin{tabular}{|c|c|c|c|}
\hline $\begin{array}{rr}0.000 \odot \odot \odot+\odot & 0.00 \odot \odot \odot \odot+\odot \\
-2.000 \odot \odot \odot+7 & 0.00 \odot \odot \odot \odot+\odot\end{array}$ & $\begin{array}{l}\odot \\
\odot\end{array}$ & $\begin{array}{l}1 \\
7\end{array}$ & $\begin{array}{l}1 \\
1\end{array}$ \\
\hline
\end{tabular}

9349477 only in VII. $\odot$ 9349478 only in VII.0 9349479 only in VII.0 9349491 only in VII.0 9349516 only in VII.0 9349517 only in VII.0 29349518 29349518

9349591 only in VII.0 93495455 only in VII.1 9349616 only in VII.1 9349617 only in VII.1 9349637 only in VII.1 9349651 0nly in VII.1 9349652 only in VII.1 9349653 only in VII.1 9349654 only in VII.1 9349655 only in VII.1 9349656 only in VII.1 9349657 only in VII.1 9349658 only in VII.1 9349659 only in VII.1 9349660 only in VII.1 9349661 only in VII.1 9349662 only in VII.1 9349663 only in VII.1 9349664 only in VII.1 9349665 only in VII.1 9349666 only in VII.1 9349667 only in VII.1 9349668 only in VII.1 9349669 only in VII.1 9349670 only in VII.1 9349671 only in VII.1 9349672 only in VII.1 9349673 only in VII.1 9349674 only in VII.1 9349675 only in VII.1 9349676 only in VII.1 9349677 only in VII.1 9349678 only in VII.1 9349679 only in VII.1 9349680 only in VII.1 9349681 only in VII.1 9349682 only in VII.1 9349683 only in VII.1 9349684 only in VII.1 9349685 only in VII.1 9349691 0nly in VII.1

$93-\mathrm{Np}-239$ 93496102 only in VII.1

\begin{tabular}{|c|c|c|c|c|c|}
\hline 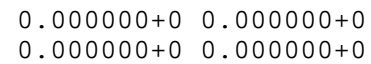 & $\begin{array}{l}\odot \\
\odot\end{array}$ & $\begin{array}{l}\odot \\
\odot\end{array}$ & $\begin{array}{l}1 \\
2\end{array}$ & $\begin{array}{rr}1079352 & 1452 \\
09352 & 1452\end{array}$ & \\
\hline..-- & - & & & 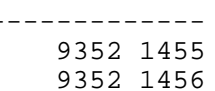 & $\begin{array}{l}\text { Only in VII.1 } \\
\text { Only in VII.1 }\end{array}$ \\
\hline 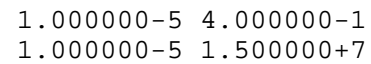 & $\begin{array}{l}\odot \\
\odot\end{array}$ & $\begin{array}{l}\odot \\
\odot\end{array}$ & $\begin{array}{l}\odot \\
\odot\end{array}$ & $\begin{array}{ll}09352 & 2151 \\
09352 & 2151\end{array}$ & \\
\hline 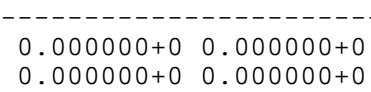 & $\odot$ & 0 & $\frac{1}{2}$ & $\begin{array}{lll}1289352 & 3 & 1 \\
1409352 & 3 & 1\end{array}$ & \\
\hline 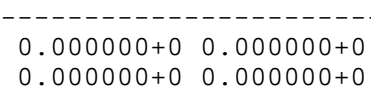 & $\odot$ & 0 & 1 & $\begin{array}{rrr}1119352 & 3 & 2 \\
549352 & 3 & 2\end{array}$ & \\
\hline $\begin{array}{l}0.000000+0-3.113100+4 \\
\odot .000000+0-3.114000+4\end{array}$ & $\odot$ & 0 & 1 & $\begin{array}{lll}869352 & 3 & 4 \\
449352 & 3 & 4\end{array}$ & \\
\hline $\begin{array}{l}-6.215200+6-6.215200+6 \\
-6.227020+6-6.227020+6\end{array}$ & $\odot$ & $\begin{array}{l}\odot \\
\odot\end{array}$ & $\begin{array}{l}1 \\
1\end{array}$ & $\begin{array}{lll}299352 & 3 & 16 \\
199352 & 3 & 16\end{array}$ & \\
\hline $\begin{array}{l}-1 \cdot 170350+7-1 \cdot 170350+7 \\
-1 \cdot 170560+7-1 \cdot 170560+7\end{array}$ & $\odot$ & $\begin{array}{l}\odot \\
\odot\end{array}$ & $\begin{array}{l}1 \\
1\end{array}$ & $\begin{array}{rrr}189352 & 3 & 17 \\
99352 & 3 & 17\end{array}$ & \\
\hline
\end{tabular}




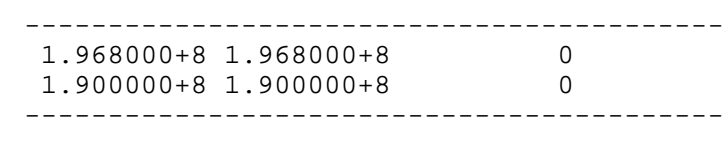

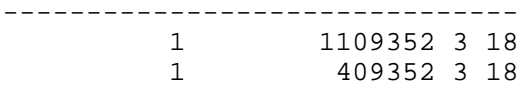

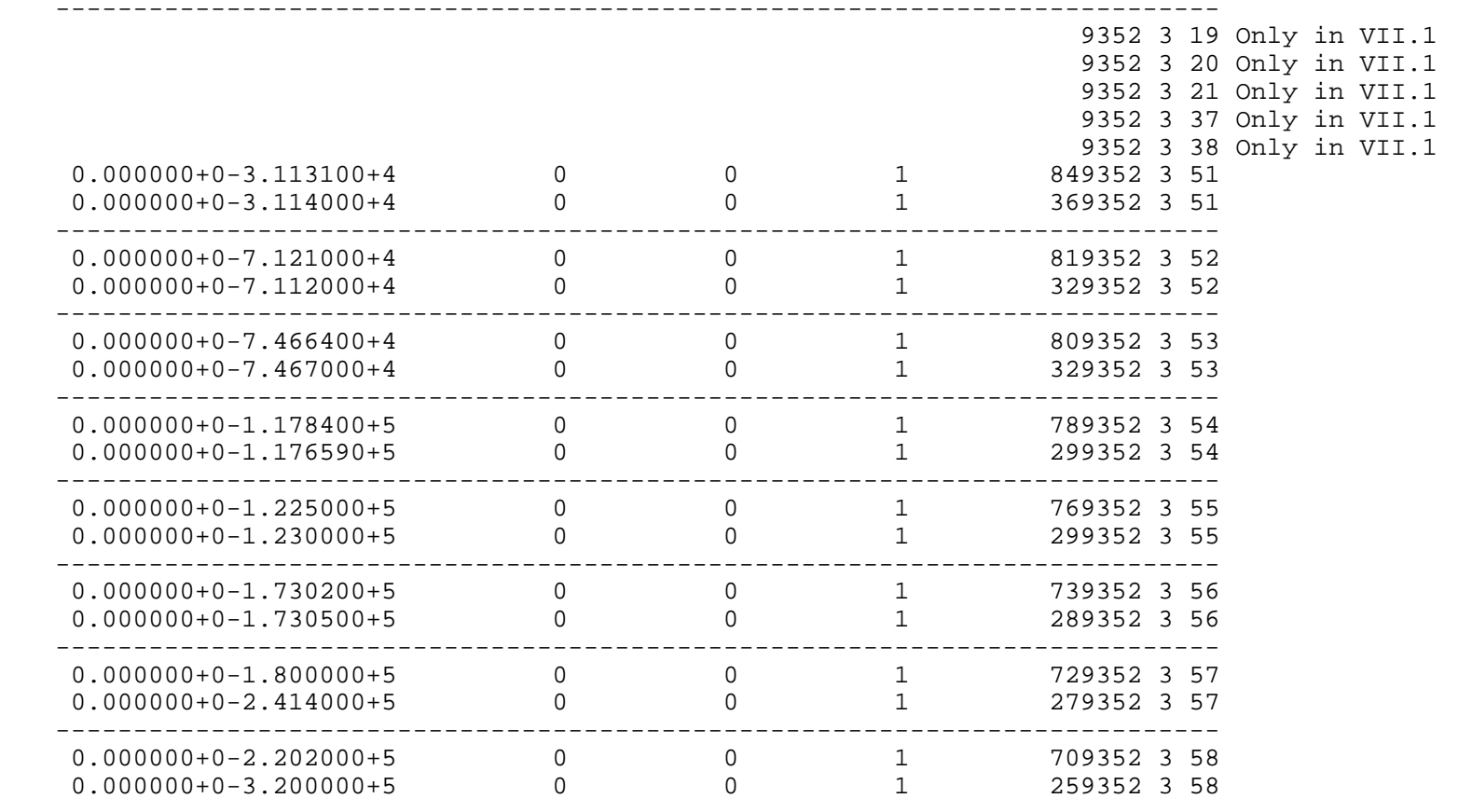

9352 359 Only in VII. 1

9352360 only in VII.1 9352361 only in VII.1 9352362 Only in VII.1 9352363 only in VII.1 9352364 only in VII.1 9352365 only in VII.1 9352366 only in VII.1 9352367 only in VII.1 9352368 only in VII.1 9352369 Only in VII.1

\begin{tabular}{|c|c|c|c|c|c|c|}
\hline $\begin{array}{l}\odot . \odot \odot \odot \odot \odot \odot+\odot-1.09629 \odot+5 \\
\odot . \odot \odot \odot \odot \odot \odot+\odot-4.30 \odot \odot \odot \odot+5\end{array}$ & $\begin{array}{l}\odot \\
\odot\end{array}$ & $\begin{array}{l}\odot \\
\odot\end{array}$ & $\begin{array}{l}1 \\
1\end{array}$ & $\begin{array}{r}9352 \\
799352 \\
249352\end{array}$ & $\begin{array}{ll}3 & 70 \\
3 & 91 \\
3 & 91\end{array}$ & Only in VII.1 \\
\hline \multicolumn{7}{|c|}{ - } \\
\hline $5.068970+65.068970+6$ & $\odot$ & $\odot$ & 1 & 1119352 & \multicolumn{2}{|c|}{3102} \\
\hline $5.168100+65.168100+6$ & $\odot$ & $\odot$ & 1 & 929352 & \multicolumn{2}{|l|}{3102} \\
\hline \multicolumn{7}{|c|}{ - - - - - - - - - - - - - - - - - - - - - - - - - - - - - - - - - - - - - - - - - - - - - - } \\
\hline $0.000000+\odot \quad 0.00000 \odot+\odot$ & $\odot$ & 0 & 1 & 1089352 & 42 & \\
\hline $0.000000+0 \quad 0.000000+0$ & $\odot$ & $\odot$ & 1 & 519352 & 2 & \\
\hline \multicolumn{7}{|c|}{ 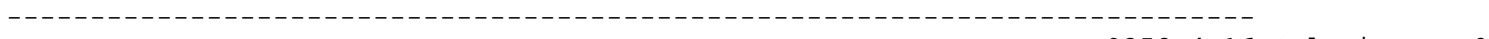 } \\
\hline & & & & 9352 & & only in VII.O \\
\hline & & & & 9352 & 417 & only in VII.0 \\
\hline$\odot .0 \odot \odot \odot \odot \odot+\odot \quad 2.369990+2$ & 1 & 1 & $\odot$ & 09352 & 418 & \\
\hline$\odot .0 \odot \odot \odot \odot \odot+\odot \quad 2.369990+2$ & $\odot$ & 1 & $\odot$ & 09352 & 418 & \\
\hline
\end{tabular}
r. 9352451 Only in VII. 0 9352452 only in VII.0 9352453 only in VII.0 9352454 only in VII.0 9352455 only in VII.0 9352456 only in VII.0 9352457 only in VII.0 9352458 only in VII.0 9352491 only in VII.0 9352516 only in VII.0 9352517 Only in VII.0

$0.000000+0 \quad 0.000000+0$

0
0

1
7

1

29352518

29352518

_.

9352591 Only in VII.0 9352616 only in VII.1 


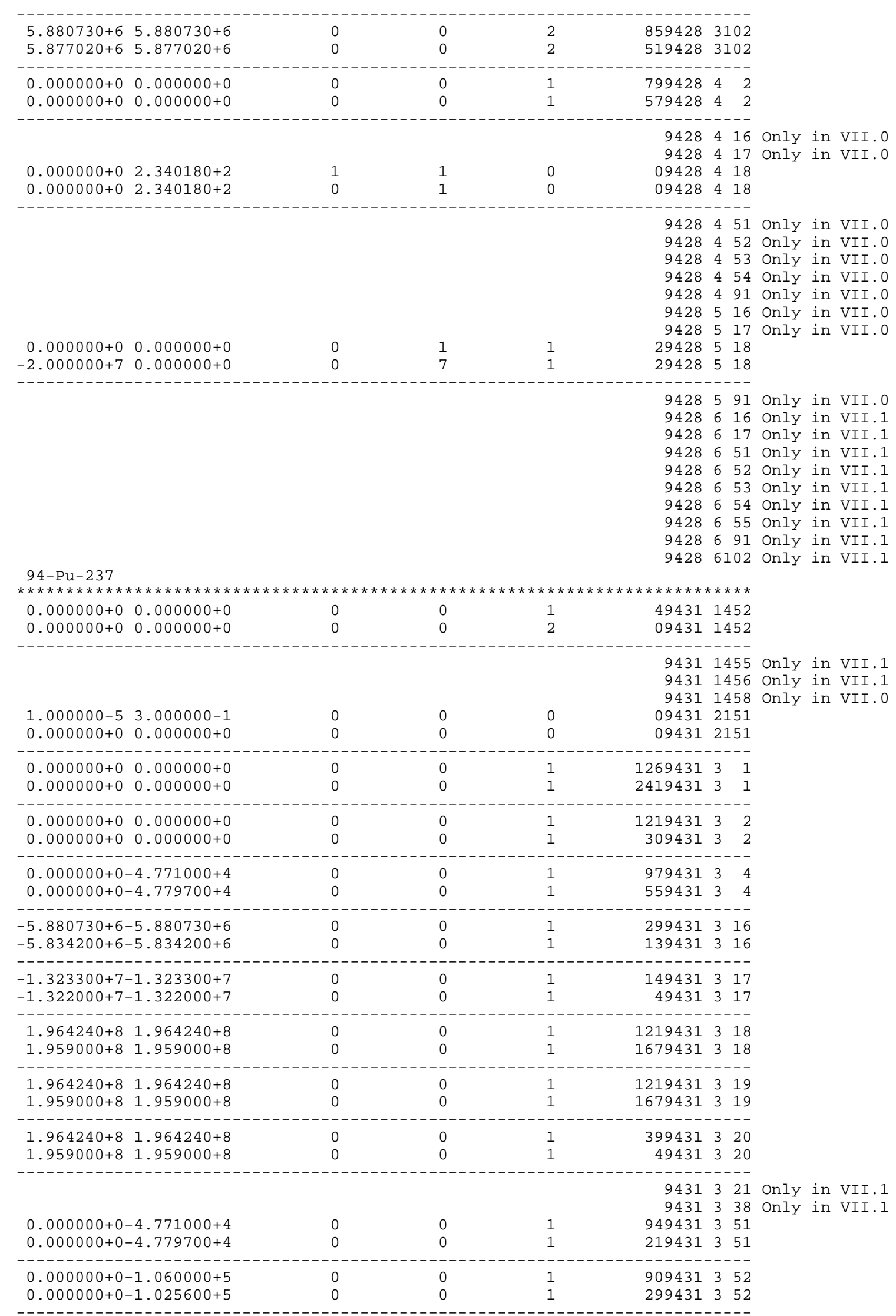




\begin{tabular}{|c|c|c|c|c|}
\hline $\begin{array}{l}\odot . \odot \odot \odot \odot \odot \odot+\odot-1.455440+5 \\
\odot . \odot \odot \odot \odot \odot \odot+\odot-1.45380 \odot+5\end{array}$ & $\begin{array}{l}\odot \\
\odot\end{array}$ & $\begin{array}{l}\odot \\
\odot\end{array}$ & $\begin{array}{l}1 \\
1\end{array}$ & $\begin{array}{lll}879431 & 3 & 53 \\
229431 & 3 & 53\end{array}$ \\
\hline $\begin{array}{l}\odot . \odot \odot \odot \odot \odot \odot+\odot-1.55450 \odot+5 \\
\odot . \odot \odot \odot \odot \odot \odot+\odot-1.56330 \odot+5\end{array}$ & $\begin{array}{l}\odot \\
\odot\end{array}$ & $\begin{array}{l}\odot \\
\odot\end{array}$ & $\begin{array}{l}1 \\
1\end{array}$ & $\begin{array}{lll}869431 & 3 & 54 \\
299431 & 3 & 54\end{array}$ \\
\hline $\begin{array}{l}0.000000+0-1.750000+5 \\
0.000000+0-1.702800+5\end{array}$ & $\begin{array}{l}\odot \\
\odot\end{array}$ & $\begin{array}{l}\odot \\
\odot\end{array}$ & $\begin{array}{l}1 \\
1\end{array}$ & $\begin{array}{lll}849431 & 3 & 55 \\
249431 & 3 & 55\end{array}$ \\
\hline $\begin{array}{l}\odot .0 \odot \odot \odot \odot \odot+\odot-2.01180 \odot+5 \\
\odot .0 \odot \odot \odot \odot \odot+\odot-1.9915 \odot \odot+5\end{array}$ & $\begin{array}{l}\odot \\
\odot\end{array}$ & $\begin{array}{l}\odot \\
\odot\end{array}$ & $\begin{array}{l}1 \\
1\end{array}$ & $\begin{array}{lll}829431 & 3 & 56 \\
279431 & 3 & 56\end{array}$ \\
\hline $\begin{array}{l}\odot . \odot \odot \odot \odot \odot \odot+\odot-2.2425 \odot \odot+5 \\
\odot . \odot \odot \odot \odot \odot \odot+\odot-2.28 \odot 3 \odot \odot+5\end{array}$ & $\begin{array}{l}\odot \\
\odot\end{array}$ & $\begin{array}{l}\odot \\
\odot\end{array}$ & $\begin{array}{l}1 \\
1\end{array}$ & $\begin{array}{lll}819431 & 3 & 57 \\
289431 & 3 & 57\end{array}$ \\
\hline $\begin{array}{l}0.0000000+0-2.570000+5 \\
0.000000+0-2.788100+5\end{array}$ & $\begin{array}{l}\odot \\
\odot\end{array}$ & $\begin{array}{l}\odot \\
\odot\end{array}$ & $\begin{array}{l}1 \\
1\end{array}$ & $\begin{array}{lll}799431 & 3 & 58 \\
229431 & 3 & 58\end{array}$ \\
\hline $\begin{array}{l}\odot .00 \odot \odot \odot \odot+\odot-2.802200+5 \\
\odot .00 \odot \odot \odot \odot+\odot-3.09680 \odot+5\end{array}$ & $\begin{array}{l}\odot \\
\odot\end{array}$ & $\begin{array}{l}\odot \\
\odot\end{array}$ & $\begin{array}{l}1 \\
1\end{array}$ & $\begin{array}{lll}789431 & 3 & 59 \\
189431 & 3 & 59\end{array}$ \\
\hline $\begin{array}{l}\odot .00 \odot \odot \odot \odot+\odot-3.0400 \odot \odot+5 \\
\odot .00 \odot \odot \odot \odot+\odot-3.19640 \odot+5\end{array}$ & $\begin{array}{l}\odot \\
\odot\end{array}$ & $\begin{array}{l}\odot \\
\odot\end{array}$ & $\begin{array}{l}1 \\
1\end{array}$ & $\begin{array}{lll}769431 & 3 & 60 \\
169431 & 3 & 60\end{array}$ \\
\hline
\end{tabular}

9431361 only in VII.1 9431362 Only in VII.1 9431363 only in VII.1 9431364 only in VII.1 9431365 only in VII.1 9431366 only in VII.1 9431367 only in VII.1 9431368 only in VII.1 9431369 only in VII.1 9431370 Only in VII.1 9431371 only in VII.1 9431372 Only in VII.1 9431373 only in VII.1 9431374 Only in VII.1 9431375 only in VII.1 9431376 only in VII.1 9431377 only in VII.1 9431378 only in VII.1 9431379 Only in VII.1 9431380 only in VII.1

$0.000000+0-1.036650+5$ $\odot .000000+\odot-3.196400+5$

$\odot$
0
-

$6.999880+6 \quad 6.999880+6$

$6.998000+6 \quad 6.998000+6$

(0) - n

. . . . . . . . . . . . . . . .

0

$0.000000+\odot \quad 0.0000000+\odot$ $0.000000+0 \quad 0.000000+0$

0

$\odot$

-

9431416 only in VII.๑ 9431417 Only in VII.0 9431419 Only in VII.0 9431420 only in VII.0 9431451 Only in VII.0 9431452 only in VII.0 9431453 Only in VII. 0 9431454 only in VII.0 9431455 only in VII.0 9431456 only in VII.0 9431457 only in VII.0 9431458 only in VII.0 9431459 Only in VII.0 9431460 only in VII.0 9431491 Only in VII.0 9431516 only in VII.0

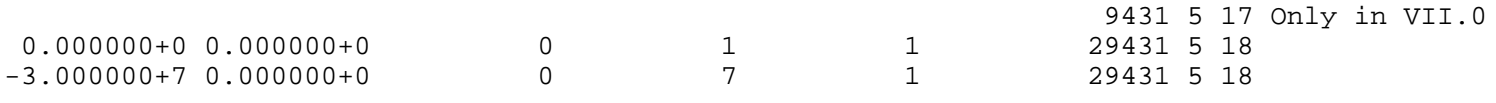


9431591 only in VII. 0 9431616 only in VII.1 9431617 only in VII.1 9431651 Only in VII.1 9431652 Only in VII.1 9431653 only in VII.1 9431654 Only in VII.1 9431655 Only in VII.1 9431656 only in VII.1 9431657 only in VII.1 9431658 only in VII.1 9431659 Only in VII.1 9431660 Only in VII.1 9431661 only in VII.1 9431662 only in VII.1 9431663 Only in VII.1 9431664 only in VII.1 9431665 only in VII.1 9431666 only in VII.1 9431667 Only in VII.1 9431668 only in VII.1 9431669 only in VII.1 9431670 Only in VII.1 9431671 Only in VII.1 9431672 only in VII.1 9431673 only in VII.1 9431674 only in VII.1 9431675 Only in VII.1 9431676 only in VII.1 9431677 only in VII.1 9431678 only in VII.1 9431679 Only in VII.1 9431680 only in VII.1 9431691 Only in VII.1

$94-\mathrm{Pu}-238$ 94316102 only in VII.1

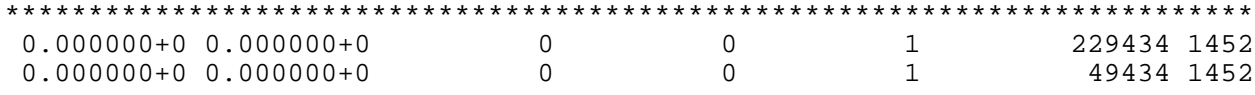

(.

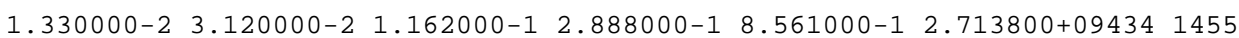
$1.332500-2$ 3.117100-2 1.161500-1 2.888400-1 8.561400-1 2.713800+09434 1455

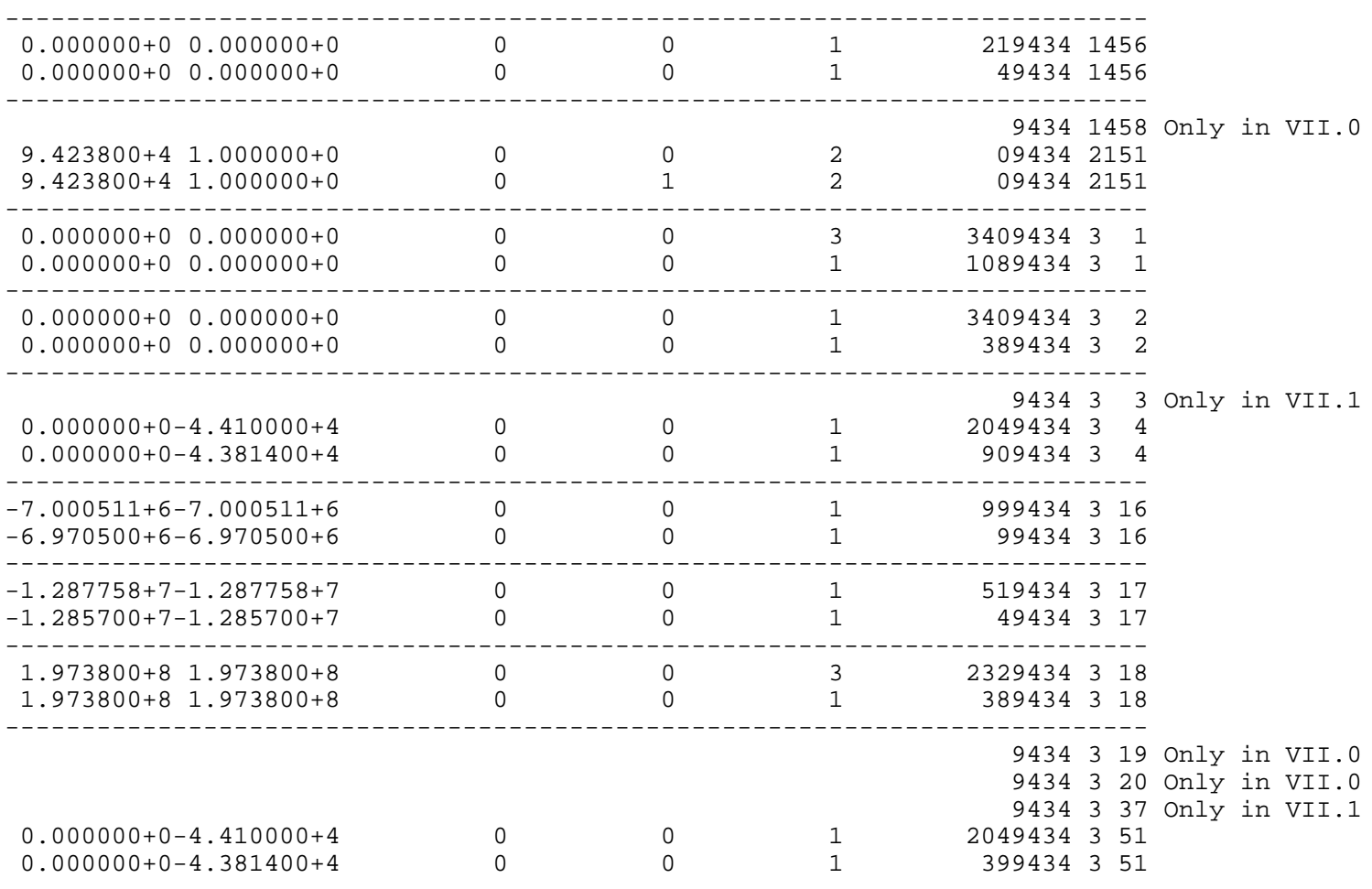




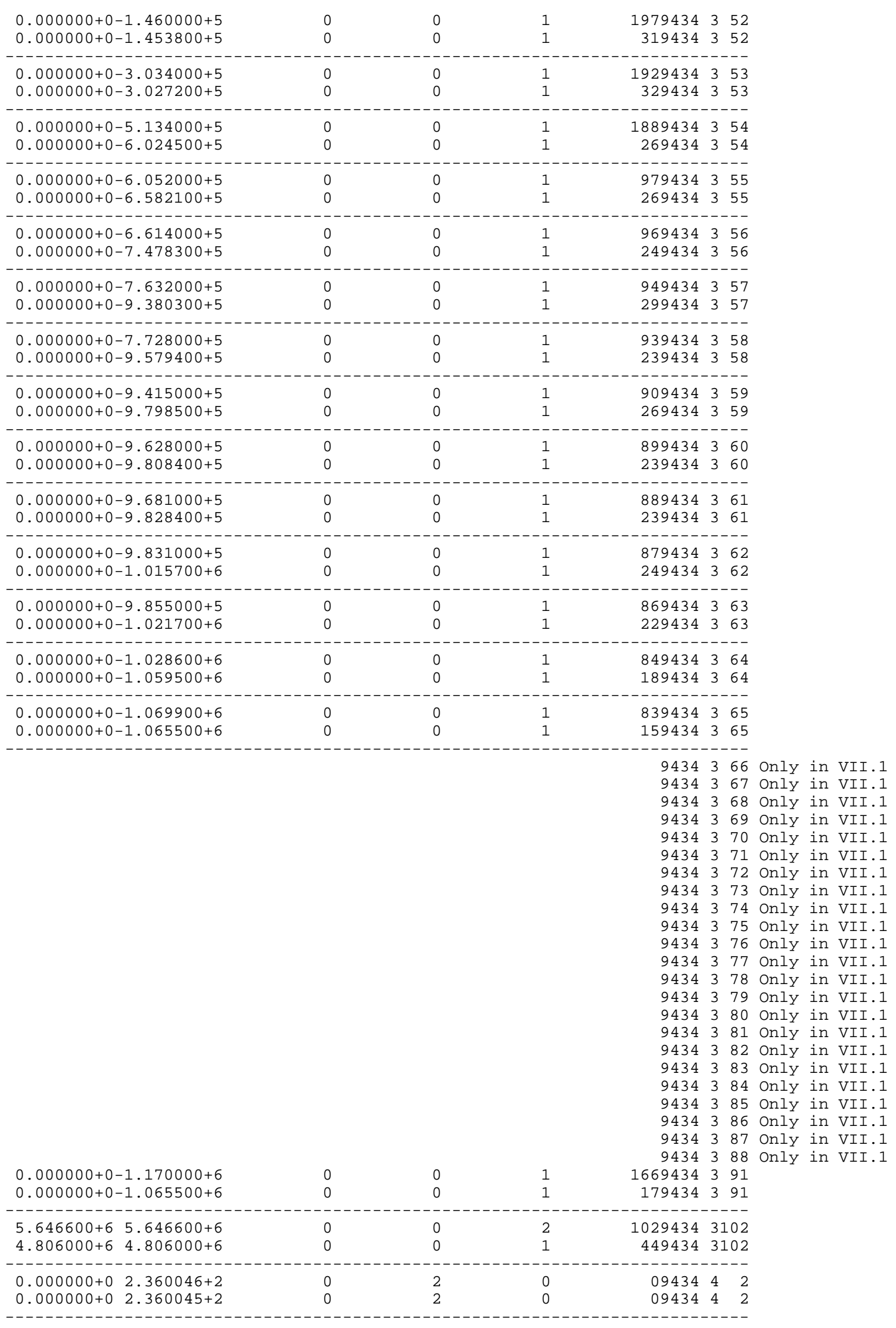

9434416 only in VII. 0 


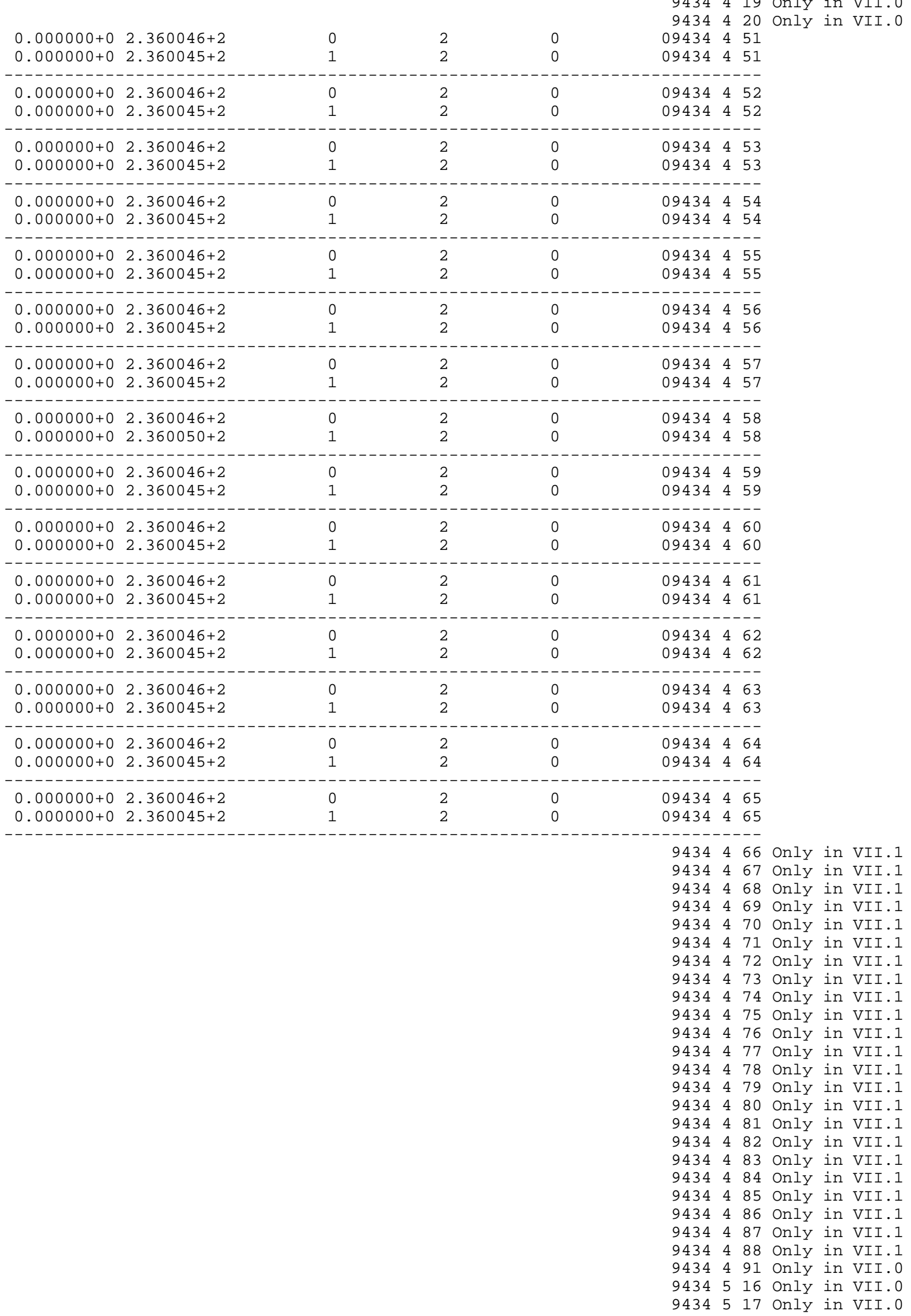


$0.000 \odot \odot \odot+\odot \quad 0.000 \odot \odot \odot+\odot$ $-3.000000+7 \quad 0.000000+0$
0
0
1

1
29434518

29434518

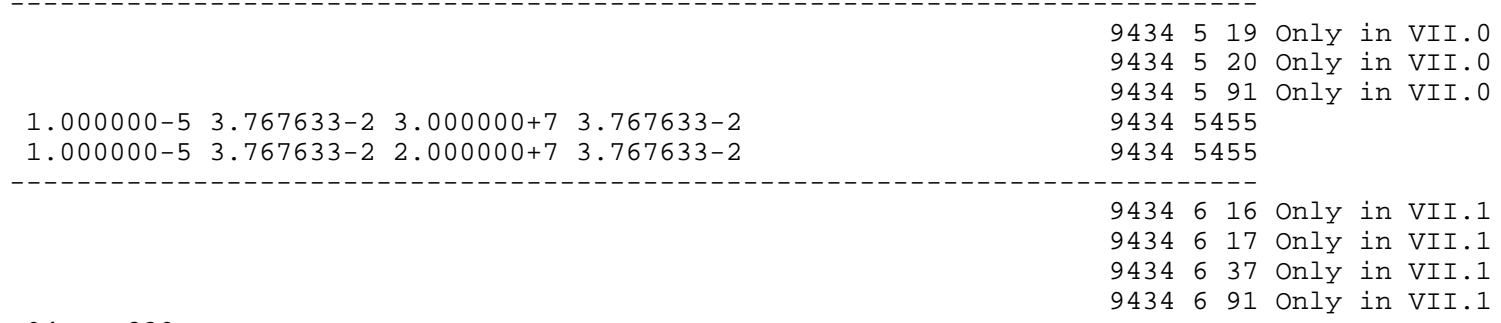

$94-\mathrm{Pu}-239$

1.248110-2 2.994667-2 1.071553-1 3.176193-1 $1.352380+01.069116+194371455$ $1.500000+1 \quad 9.871808-4 \quad 1.600000+1 \quad 9.452257-4 \quad 1.700000+1 \quad 1.073559-394371460$

-

3. $0000 \odot \odot+4 \quad 8.474100+\odot \quad 0.0000 \odot \odot+\odot \quad 9.47400 \odot-4 \quad 4.07000 \odot-2 \quad 2.697000+094372151$ $2.950000+4 \quad 8.474100+\odot \quad 0.000000+0 \quad 9.474000-4 \quad 4.070000-2 \quad 2.697000+094372151$

-2.123213-2-5.575145-2 4.503431-2-4.007026-2 8.664596-4 9.875596-494374 76 -2.123213-2-5.575145-2 4.503431-2-4.007026-2 8.664596-4 9.875595-49437476

$-5.501023-6-5.653619-6-9.676723-7-1.311852-7-1.349319-8-1.592145-99437480$ $-5.501023-6-5.653619-6-9.676724-7-1.311852-7-1.349319-8-1.592145-99437480$

$-1.130429-2-9.546876-3-9.897758-41.520279-21.188212-24.399529-39437489$ -1.130429-2-9.546876-3-9.897757-4 1.520279-2 1.188212-2 4.399529-39437489

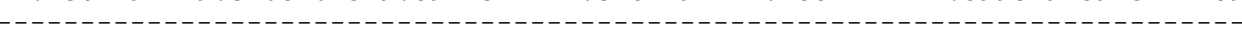

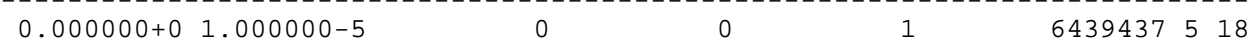

$0.000000+0$ 1.000000-5 $\quad 00018$

-

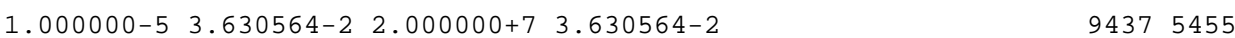

$1.000000-53.308424-2 \quad 2.000000+73.308424-2 \quad 94375455$

$94-\mathrm{Pu}-240$

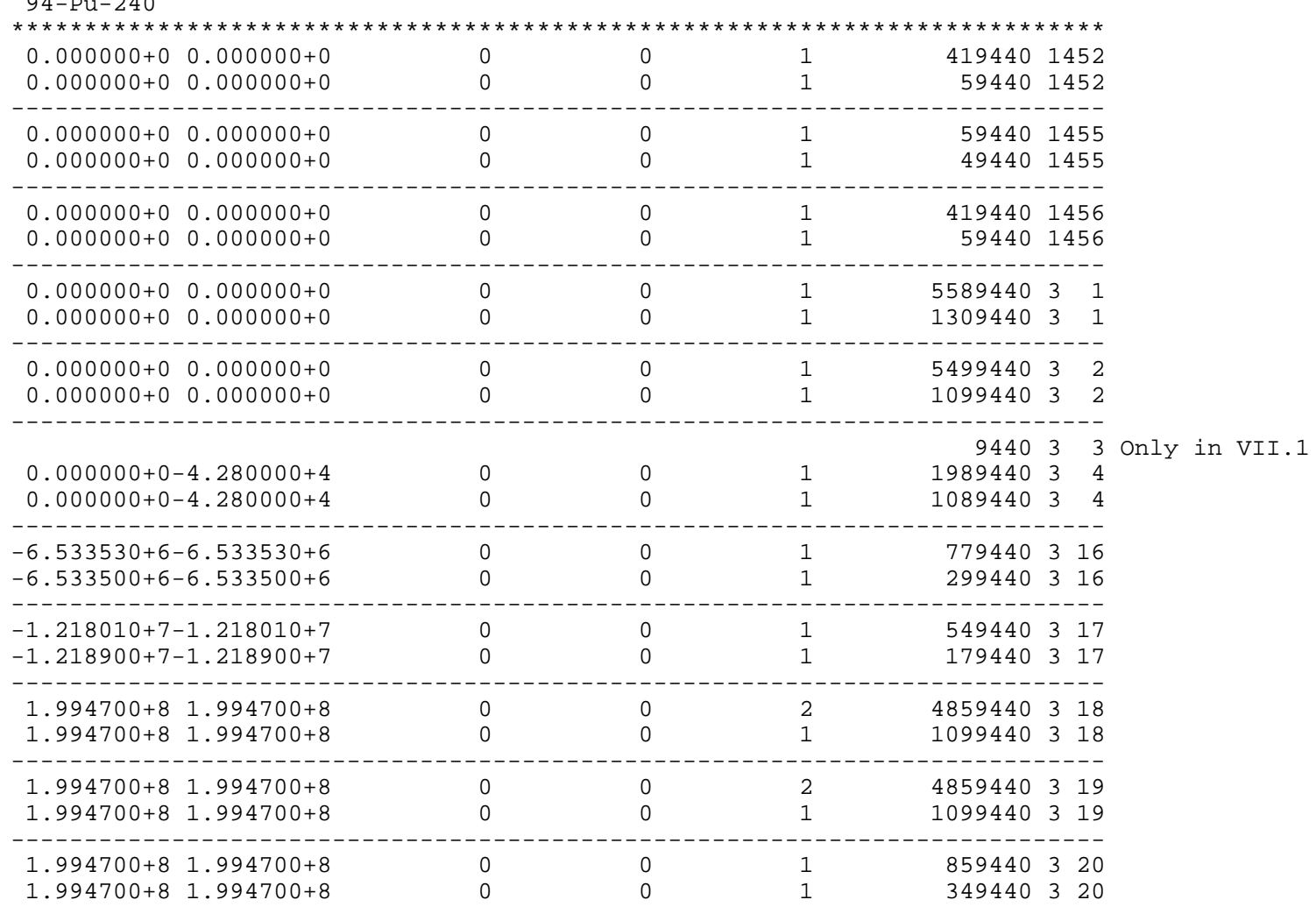




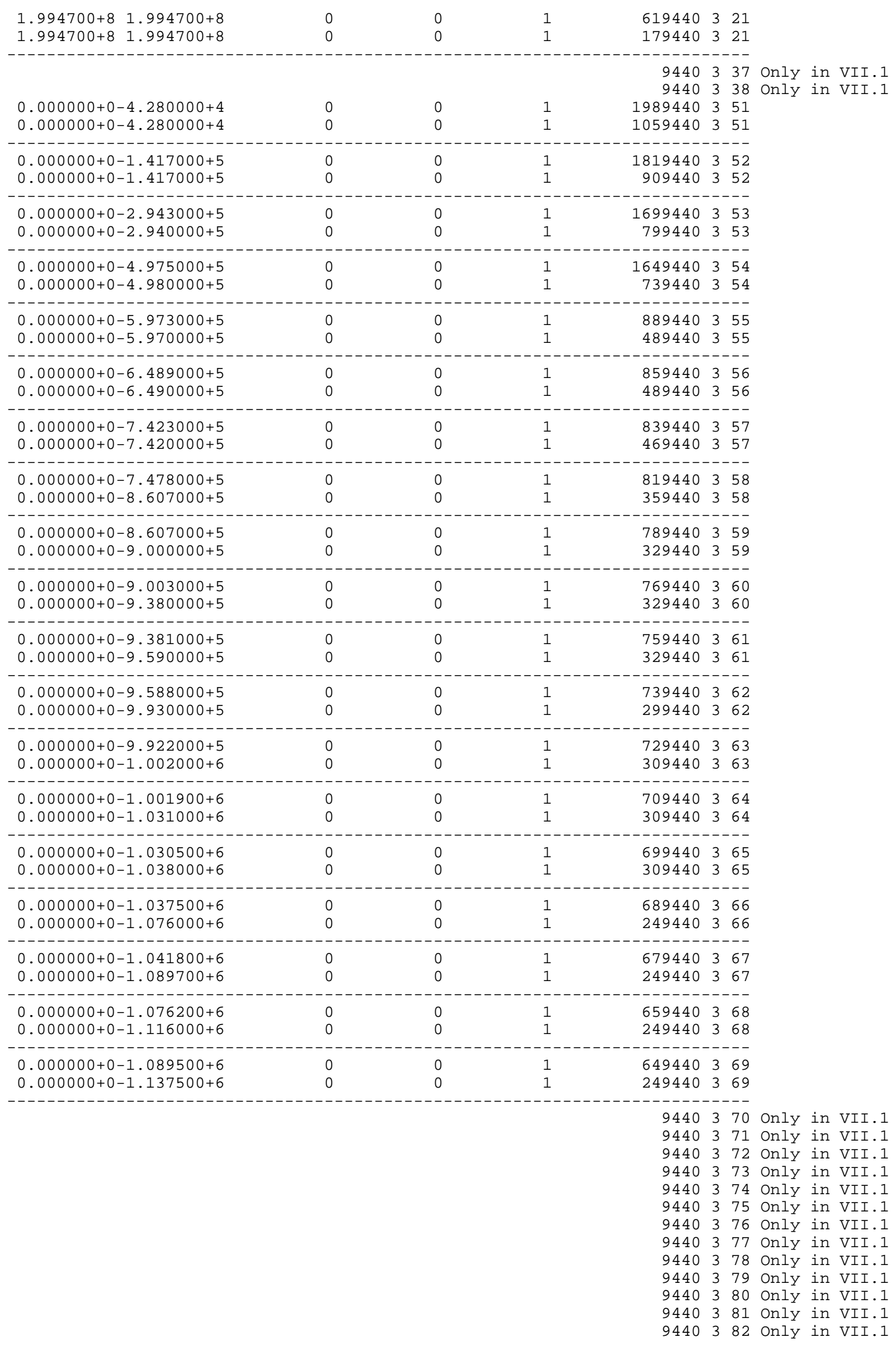




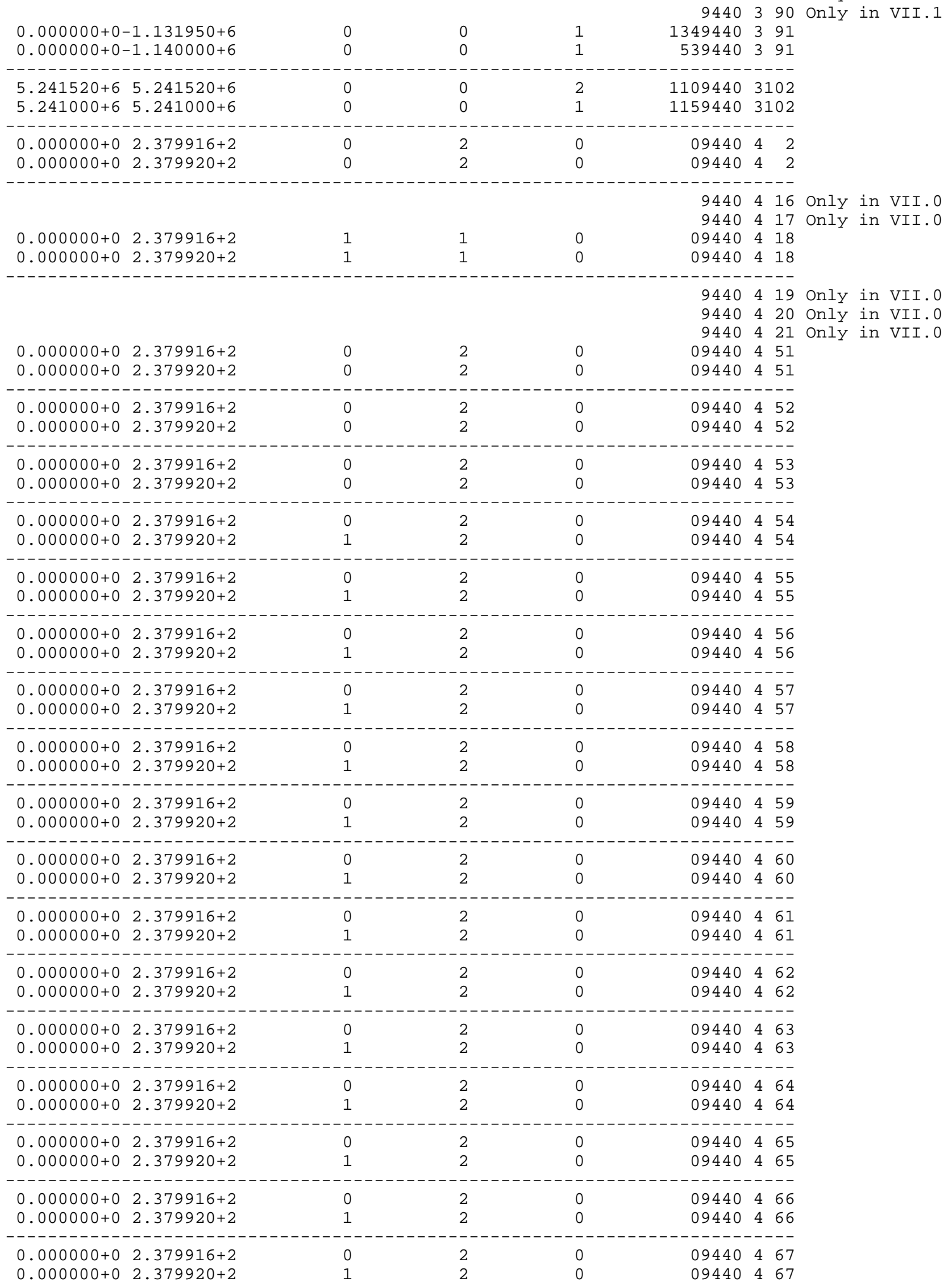




\begin{tabular}{|c|c|c|c|c|c|c|c|c|c|}
\hline $\begin{array}{ll}\odot . \odot \odot \odot \odot \odot \odot+\odot ~ & 2.379916+2 \\
\odot . \odot \odot \odot \odot \odot \odot+\odot) & 2.379920+2\end{array}$ & $\begin{array}{l}\odot \\
1\end{array}$ & $\begin{array}{l}2 \\
2\end{array}$ & $\begin{array}{l}\odot \\
\odot\end{array}$ & $\begin{array}{l}09440 \\
09440\end{array}$ & $\begin{array}{l}4 \\
4\end{array}$ & $\begin{array}{l}68 \\
68\end{array}$ & & & \\
\hline \multicolumn{10}{|c|}{ - - - - - - - - - - - - - - - - - - - - - - - - - - - - - - - - - - - - - - - - - - - - - - - - - - - - - - } \\
\hline$\odot .0 \odot \odot \odot \odot \odot+\odot 2.379916+2$ & $\odot$ & 2 & $\odot$ & 09440 & 4 & 69 & & & \\
\hline $0.000000+02.379920+2$ & 1 & 2 & $\odot$ & 09440 & 4 & 69 & & & \\
\hline \multicolumn{10}{|c|}{ 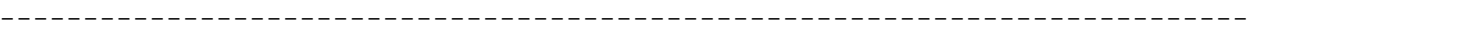 } \\
\hline & & & & 9440 & 4 & 70 & only & in & VII. 1 \\
\hline & & & & 9440 & 4 & 71 & Only & in & VII . 1 \\
\hline & & & & 9440 & 4 & 72 & Only & in & VII.1 \\
\hline & & & & 9440 & 4 & 73 & Only & in & VII.1 \\
\hline & & & & 9440 & 4 & 74 & Only & in & VII . 1 \\
\hline & & & & 9440 & 4 & 75 & Only & in & VII.1 \\
\hline & & & & 9440 & 4 & 76 & Only & in & VII . 1 \\
\hline & & & & 9440 & 4 & 77 & only & in & VII. 1 \\
\hline & & & & 9440 & 4 & 78 & Only & in & VII. 1 \\
\hline & & & & 9440 & 4 & 79 & Only & in & VII.1 \\
\hline & & & & 9440 & 4 & 80 & Only & in & VII. 1 \\
\hline & & & & 9440 & 4 & 81 & Only & in & VII . 1 \\
\hline & & & & 9440 & 4 & 82 & Only & in & VII.1 \\
\hline & & & & 9440 & 4 & 83 & only & in & VII. 1 \\
\hline & & & & 9440 & 4 & 84 & only & in & VII. 1 \\
\hline & & & & 9440 & 4 & 85 & Only & in & VII. 1 \\
\hline & & & & 9440 & 4 & 86 & Only & in & VII. 1 \\
\hline & & & & 9440 & 4 & 87 & Only & in & VII. 1 \\
\hline & & & & 9440 & 4 & 88 & Only & in & VII.1 \\
\hline & & & & 9440 & 4 & 89 & Only & in & VII.1 \\
\hline & & & & 9440 & 4 & 90 & Only & in & VII. 1 \\
\hline & & & & 9440 & 4 & 91 & Only & in & VII. 0 \\
\hline & & & & 9440 & 5 & 16 & Only & in & VII. $\odot$ \\
\hline & & & & 9440 & 5 & 17 & Only & in & VII. $\odot$ \\
\hline $0.000000+0 \quad 0.000000+0$ & $\odot$ & 1 & 1 & 29440 & 5 & 18 & & & \\
\hline$-3.000000+7 \quad 0.000000+0$ & $\odot$ & 7 & 1 & 29440 & 5 & 18 & & & \\
\hline \multicolumn{10}{|c|}{ 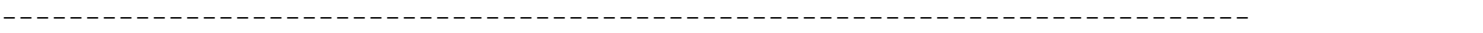 } \\
\hline & & & & 9440 & 5 & 19 & Only & in & VII . $\odot$ \\
\hline & & & & 9440 & 5 & 20 & only & in & VII. $\odot$ \\
\hline & & & & 9440 & 5 & 21 & Only & in & VII. $\odot$ \\
\hline & & & & 9440 & 5 & 91 & Only & in & VII. 0 \\
\hline & & & & 9440 & 6 & 16 & Only & in & VII. 1 \\
\hline & & & & 9440 & 6 & 17 & Only & in & VII.1 \\
\hline & & & & 9440 & 6 & 37 & Only & in & VII.1 \\
\hline & & & & 9440 & 6 & 91 & Only & in & VII. 1 \\
\hline
\end{tabular}

94 - Pu - 241

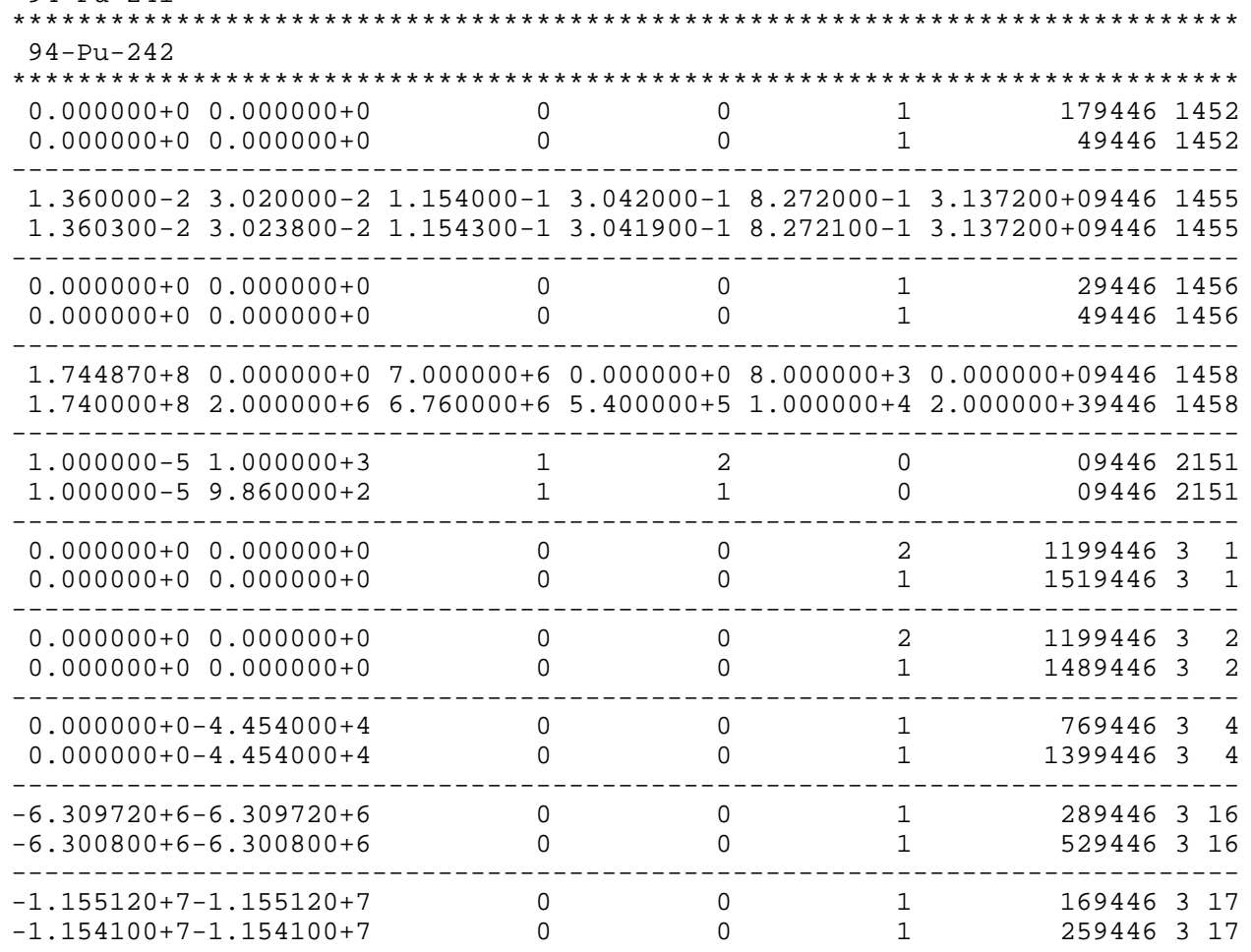




\begin{tabular}{|c|c|c|c|c|c|}
\hline $\begin{array}{ll}2.027780+8 & 2.027780+8 \\
2.015800+8 & 2.015800+8\end{array}$ & 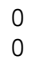 & $\stackrel{\odot}{\odot}$ & $\begin{array}{l}2 \\
1\end{array}$ & $\begin{array}{lll}1119446 & 3 & 18 \\
1329446 & 3 & 18\end{array}$ & \\
\hline 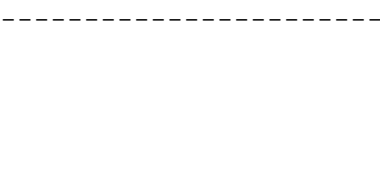 & -- & - & - & $\begin{array}{lll}9446 & 3 & 19 \\
9446 & 3 & 20 \\
9446 & 3 & 21 \\
9446 & 3 & 37 \\
9446 & 3 & 38\end{array}$ & $\begin{array}{l}\text { Only in VII.1 } \\
\text { only in VII.1 } \\
\text { Only in VII.1 } \\
\text { Only in VII.1 } \\
\text { Only in VII.1 }\end{array}$ \\
\hline$\odot . \odot \odot \odot \odot \odot \odot+\odot-4.454 \odot \odot \odot+4$ & $\odot$ & $\odot$ & 1 & 759446351 & \\
\hline $0.000000+0-4.454000+4$ & $\odot$ & $\odot$ & 1 & 1399446351 & \\
\hline $\begin{array}{l}\odot .0 \odot \odot \odot \odot \odot+\odot-1.473 \odot \odot \odot+5 \\
\odot .0 \odot \odot \odot \odot \odot+\odot-1.472 \odot \odot \odot+5\end{array}$ & 0 & $\odot$ & 1 & $\begin{array}{rrr}709446 & 3 & 52 \\
1319446 & 3 & 52\end{array}$ & \\
\hline $\begin{array}{l}\odot .0 \odot \odot \odot \odot \odot+\odot-3.064 \odot \odot \odot+5 \\
\odot .0 \odot \odot \odot \odot \odot+\odot-3.059 \odot \odot \odot+5\end{array}$ & 0 & $\odot$ & 1 & 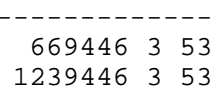 & \\
\hline $\begin{array}{l}\odot .0 \odot \odot \odot \odot \odot+\odot-5.1810 \odot \odot+5 \\
\odot . \odot \odot \odot \odot \odot \odot+\odot-5.176 \odot \odot \odot+5\end{array}$ & $\begin{array}{l}-- \\
0\end{array}$ & $\odot$ & 1 & $\begin{array}{rrr}639446 & 3 & 54 \\
1149446 & 3 & 54\end{array}$ & \\
\hline $\begin{array}{l}\odot . \odot \odot \odot \odot \odot \odot+\odot-7.786 \odot \odot \odot+5 \\
\odot . \odot \odot \odot \odot \odot \odot+\odot-7.787 \odot \odot \odot+5\end{array}$ & $\begin{array}{c}- \\
\odot \\
\odot\end{array}$ & $\odot$ & 1 & $\begin{array}{lll}609446 & 3 & 55 \\
769446 & 3 & 55\end{array}$ & \\
\hline $\begin{array}{l}\odot . \odot \odot \odot \odot \odot \odot+\odot-7.8 \odot 45 \odot \odot+5 \\
\odot . \odot \odot \odot \odot \odot \odot+\odot-7.8 \odot 3 \odot \odot \odot+5\end{array}$ & $\begin{array}{c}- \\
\odot \\
\odot\end{array}$ & $\odot$ & 1 & $\begin{array}{lll}599446 & 3 & 56 \\
779446 & 3 & 56\end{array}$ & \\
\hline $\begin{array}{l}\odot .0 \odot \odot \odot \odot \odot+\odot-8.323000+5 \\
\odot .0 \odot \odot \odot \odot \odot+\odot-8.3290 \odot \odot+5\end{array}$ & $\begin{array}{c}--- \\
\odot \\
\odot\end{array}$ & $\odot$ & 1 & $\begin{array}{rrr}579446 & 3 & 57 \\
759446 & 3 & 57\end{array}$ & \\
\hline $\begin{array}{l}\odot .0 \odot \odot \odot \odot \odot+\odot-8.6500 \odot \odot+5 \\
\odot .0 \odot \odot \odot \odot \odot+\odot-8.650 \odot \odot \odot+5\end{array}$ & $\begin{array}{l}- \\
\odot \\
\odot\end{array}$ & $\odot$ & 1 & $\begin{array}{lll}569446 & 3 & 58 \\
739446 & 3 & 58\end{array}$ & \\
\hline 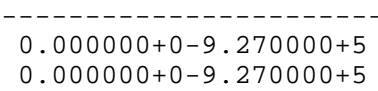 & $\begin{array}{c}- \\
\odot \\
\odot\end{array}$ & $\odot$ & $\begin{array}{l}1 \\
1\end{array}$ & $\begin{array}{lll}549446 & 3 & 59 \\
719446 & 3 & 59\end{array}$ & \\
\hline 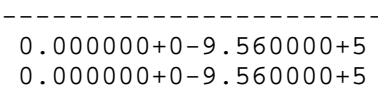 & 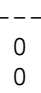 & $\odot$ & 1 & $\begin{array}{lll}539446 & 3 & 60 \\
699446 & 3 & 60\end{array}$ & \\
\hline $\begin{array}{l}\odot . \odot \odot \odot \odot \odot \odot+\odot-9.925 \odot \odot \odot+5 ~ \\
\odot .0 \odot \odot \odot \odot \odot+\odot-9.856 \odot \odot \odot+5 ~\end{array}$ & $\begin{array}{c}- \\
\odot \\
\odot\end{array}$ & $\begin{array}{l}\odot \\
\odot\end{array}$ & $\begin{array}{l}1 \\
1\end{array}$ & $\begin{array}{lll}529446 & 3 & 61 \\
689446 & 3 & 61\end{array}$ & \\
\hline $\begin{array}{l}\odot .0 \odot \odot \odot \odot \odot+\odot-1.01950 \odot+6 \\
\odot .0 \odot \odot \odot \odot \odot+\odot-9.9500 \odot \odot+5\end{array}$ & $\begin{array}{ll}- \\
\odot\end{array}$ & $\odot$ & 1 & $\begin{array}{lll}509446 & 3 & 62 \\
679446 & 3 & 62\end{array}$ & \\
\hline$\odot .0 \odot \odot \odot \odot \odot+\odot-1.0392 \odot \odot+6$ & 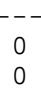 & $\odot$ & 1 & $\begin{array}{lll}499446 & 3 & 63 \\
659446 & 3 & 63\end{array}$ & \\
\hline $\begin{array}{l}\odot .0 \odot \odot \odot \odot \odot+\odot-1.064 \odot \odot \odot+6 \\
\odot .0 \odot \odot \odot \odot \odot+\odot-1.04 \odot \odot \odot \odot+6\end{array}$ & $\begin{array}{c}- \\
\odot \\
\odot\end{array}$ & $\odot$ & 1 & $\begin{array}{rrr}489446 & 3 & 64 \\
649446 & 3 & 64\end{array}$ & \\
\hline $\begin{array}{l}\odot .0 \odot \odot \odot \odot \odot+\odot-1.08440 \odot+6 \\
0.0 \odot \odot \odot \odot \odot+\odot-1.0640 \odot \odot+6\end{array}$ & 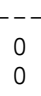 & $\odot$ & 1 & $\begin{array}{lll}479446 & 3 & 65 \\
629446 & 3 & 65\end{array}$ & \\
\hline $\begin{array}{l}\odot .0 \odot \odot \odot \odot \odot+\odot-1.09210 \odot+6 \\
\odot .0 \odot \odot \odot \odot \odot+\odot-1.0870 \odot \odot+6\end{array}$ & $\begin{array}{c}-\cdot- \\
\odot \\
\odot\end{array}$ & $\odot$ & 1 & $\begin{array}{lll}469446 & 3 & 66 \\
559446 & 3 & 66\end{array}$ & \\
\hline 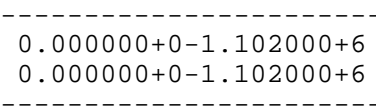 & $\begin{array}{c}-- \\
0 \\
0\end{array}$ & $\begin{array}{l}\odot \\
\odot\end{array}$ & 1 & $\begin{array}{lll}459446 & 3 & 67 \\
599446 & 3 & 67\end{array}$ & \\
\hline 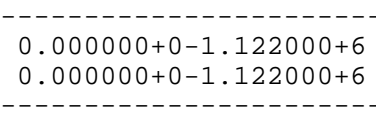 & $\begin{array}{l}-1 \\
0 \\
0\end{array}$ & $\begin{array}{l}\odot \\
\odot\end{array}$ & $\begin{array}{l}1 \\
1\end{array}$ & $\begin{array}{lll}449446 & 3 & 68 \\
589446 & 3 & 68\end{array}$ & \\
\hline $\begin{array}{l}\odot \cdot \odot \odot \odot \odot \odot \odot+\odot-1 \cdot 1510 \odot \odot+6 \\
\odot \cdot 0 \odot \odot \odot \odot \odot+\odot-1 \cdot 152 \odot \odot \odot+6\end{array}$ & $\begin{array}{l}- \\
0 \\
0\end{array}$ & $\odot$ & 1 & $\begin{array}{rrr}439446 & 3 & 69 \\
569446 & 3 & 69\end{array}$ & \\
\hline \multicolumn{5}{|c|}{ 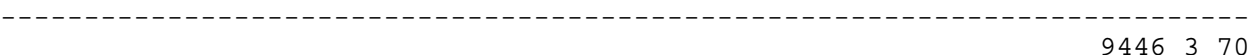 } & Only in VII.1 \\
\hline \multirow{2}{*}{$\begin{array}{l}\odot . \odot \odot \odot \odot \odot \odot+\odot-1.15479 \odot+5 \\
\odot . \odot \odot \odot \odot \odot \odot+\odot-4.2324 \odot \odot+5\end{array}$} & $\stackrel{\odot}{\odot}$ & $\begin{array}{l}\odot \\
\odot\end{array}$ & $\begin{array}{l}1 \\
1\end{array}$ & $\begin{array}{rll}729446 & 3 & 91 \\
1189446 & 3 & 91\end{array}$ & \\
\hline & \multicolumn{5}{|c|}{$\begin{array}{l}- \\
-\end{array}$} \\
\hline $\begin{array}{ll}5.03420 \odot+6 & 5.03420 \odot+6 \\
5.07100 \odot+6 & 5.07100 \odot+6\end{array}$ & $\begin{array}{l}\odot \\
\odot\end{array}$ & $\begin{array}{l}\odot \\
\odot\end{array}$ & $\begin{array}{l}2 \\
3\end{array}$ & $\begin{array}{rr}869446 & 3102 \\
1329446 & 3102\end{array}$ & \\
\hline 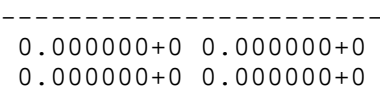 & $\begin{array}{l}-1 \\
0 \\
0\end{array}$ & $\odot$ & 1 & $\begin{array}{lll}949446 & 4 & 2 \\
219446 & 4 & 2\end{array}$ & \\
\hline
\end{tabular}




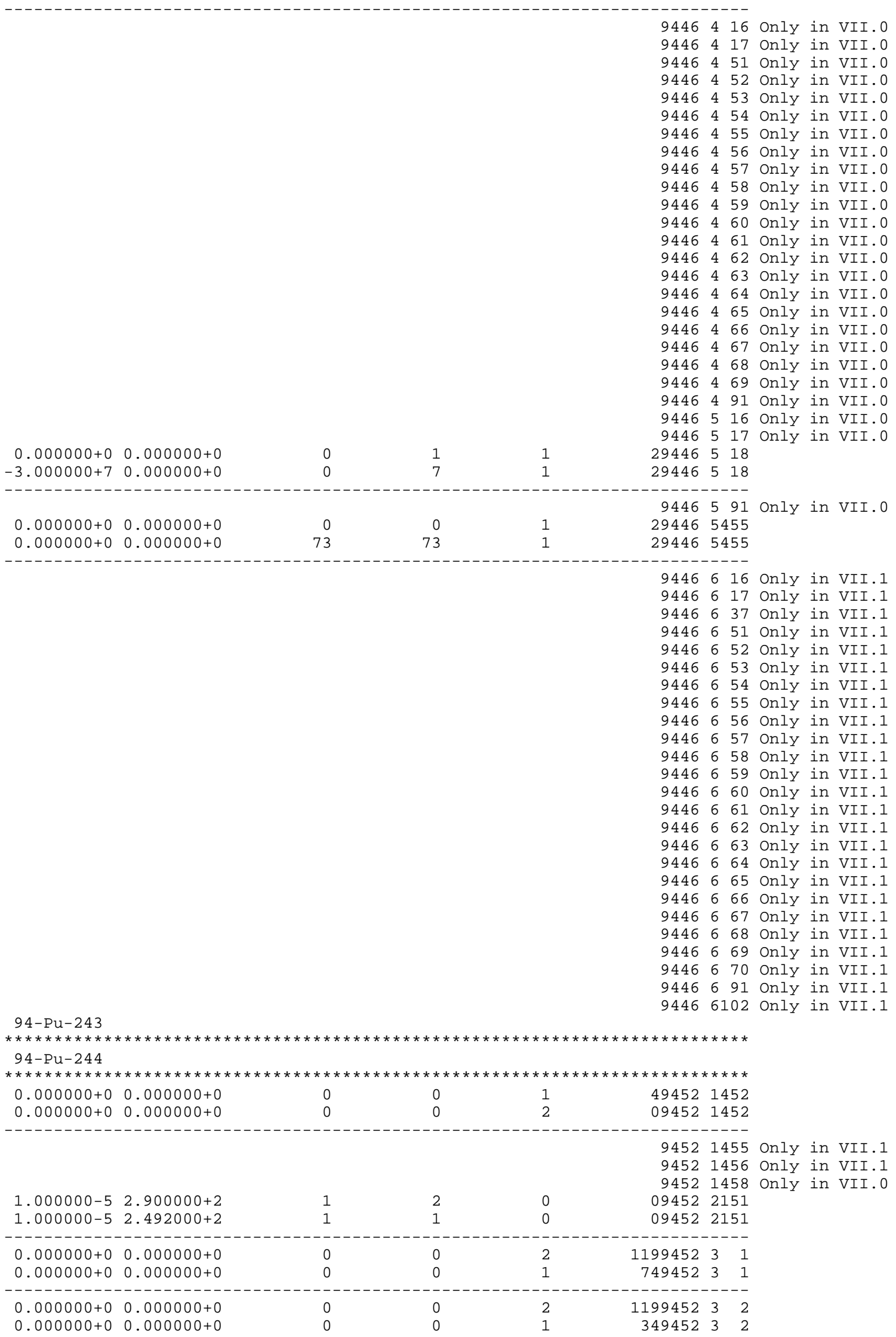




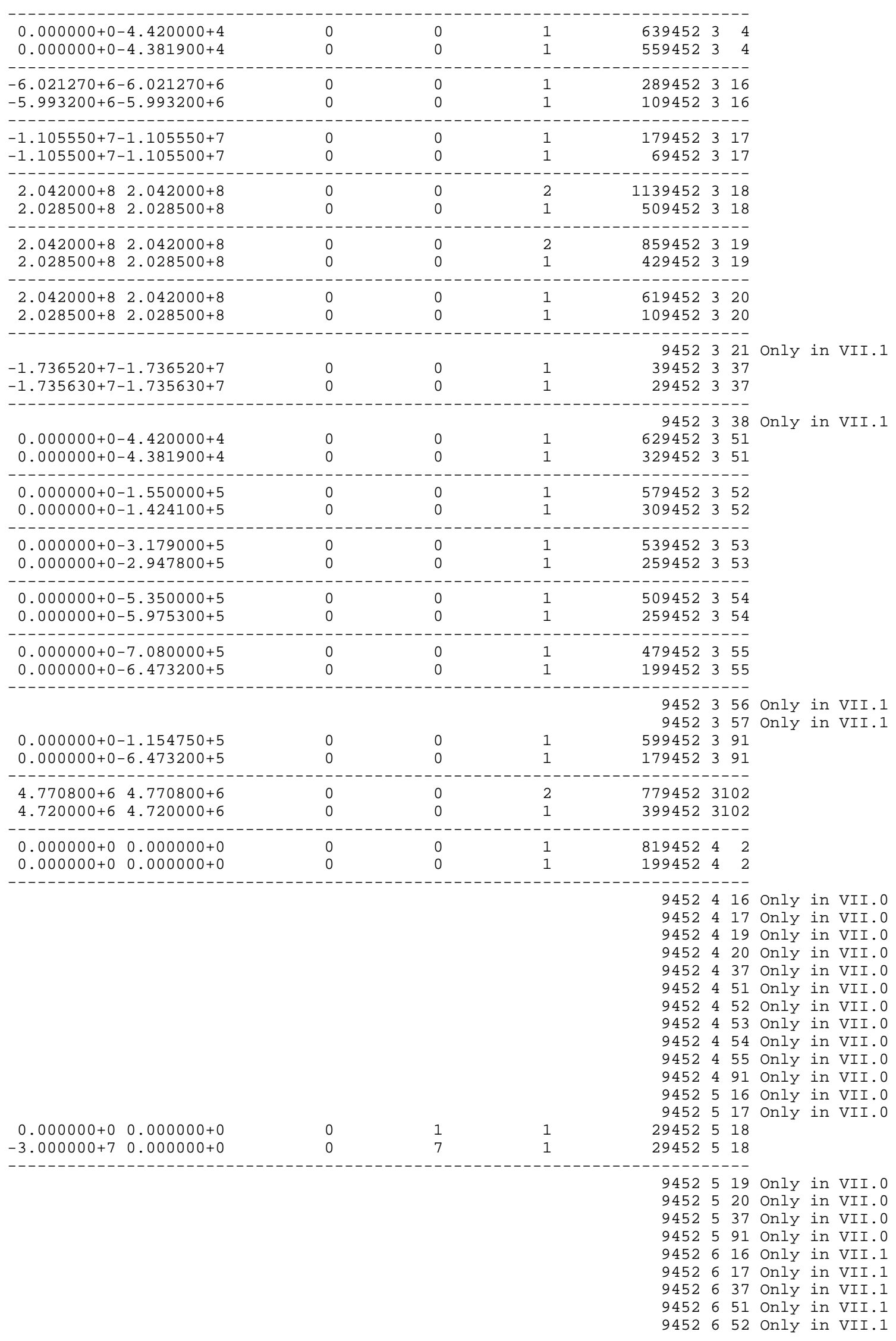


$94-\mathrm{Pu}-246$

9452653 only in VII.1 9452654 Only in VII.1 9452655 only in VII.1 9452656 only in VII.1 9452657 Only in VII.1 9452691 only in VII.1 94526102 Only in VII.1

\begin{tabular}{|c|c|c|c|c|c|}
\hline $\begin{array}{ll}1.000 \odot \odot \odot-5 ~ & 2.678200+\odot \\
1.000 \odot \odot \odot-5 & 3.038200+\odot\end{array}$ & $\begin{array}{l}\text { 4. } 00000 \odot+6 \\
4.00000 \odot+6\end{array}$ & $\begin{array}{l}3.462200+\Theta \\
3.822200+0\end{array}$ & $\begin{array}{l}7.000000+6 \\
7.000000+6\end{array}$ & $\begin{array}{ll}-6 & 4.018200+09458 \\
-6 & 4.378200+09458\end{array}$ & $\begin{array}{l}1452 \\
1452\end{array}$ \\
\hline $\begin{array}{ll}1 . \odot \odot \odot \odot \odot \odot-5 ~ & 2.58 \odot \odot \odot \odot+\odot \\
1.0 \odot \odot \odot \odot \odot-5 ~ & 2.940 \odot \odot \odot+\odot\end{array}$ & $\begin{array}{l}2.0 \odot \odot \odot \odot \odot+7 \\
2.0 \odot \odot \odot \odot \odot+7\end{array}$ & $\begin{array}{l}6.500000+0 \\
6.860000+0\end{array}$ & & $\begin{array}{r}9458 \\
9458\end{array}$ & $\begin{array}{l}1456 \\
1456\end{array}$ \\
\hline 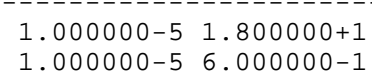 & $\begin{array}{l}\odot \\
\odot\end{array}$ & $\begin{array}{l}\odot \\
\ominus\end{array}$ & & $\begin{array}{l}\odot 9458 \\
09458\end{array}$ & $\begin{array}{l}2151 \\
2151\end{array}$ \\
\hline 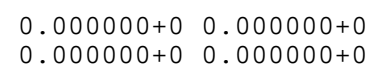 & $\stackrel{\odot}{\odot}$ & $\begin{array}{l}\odot \\
\odot\end{array}$ & & $\begin{array}{l}1049458 \\
1019458\end{array}$ & $\begin{array}{ll}3 & 1 \\
3 & 1\end{array}$ \\
\hline 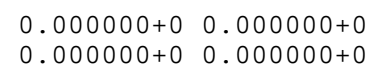 & 0 & $\begin{array}{l}\odot \\
\odot\end{array}$ & & $\begin{array}{r}959458 \\
1029458\end{array}$ & $\begin{array}{ll}3 & 2 \\
3 & 2\end{array}$ \\
\hline $\begin{array}{l}\odot .00000 \odot+\odot-4.6000 \odot \odot+4 \\
\odot .000000+\odot-4.600000+4\end{array}$ & $\begin{array}{l}\odot \\
\odot\end{array}$ & $\begin{array}{l}\odot \\
\odot\end{array}$ & & $\begin{array}{l}679458 \\
589458\end{array}$ & $\begin{array}{ll}3 & 4 \\
3 & 4\end{array}$ \\
\hline $\begin{array}{l}-5.782200+6-5.782200+6 \\
-5.880400+6-5.880400+6\end{array}$ & $\begin{array}{l}\odot \\
\odot\end{array}$ & $\begin{array}{l}\odot \\
\odot\end{array}$ & & $\begin{array}{l}309458 \\
289458\end{array}$ & $\begin{array}{ll}3 & 16 \\
3 & 16\end{array}$ \\
\hline$-1.055300+7-1.05530 \odot+7$ & $\begin{array}{l}\odot \\
\odot\end{array}$ & $\stackrel{\odot}{\odot}$ & & $\begin{array}{l}199458 \\
199458\end{array}$ & $\begin{array}{ll}3 & 17 \\
3 & 17\end{array}$ \\
\hline $2.071000+8 \quad 2.071000+8$ & $\odot$ & 0 & & 669458 & 318 \\
\hline
\end{tabular}

\begin{tabular}{rrrrrr}
$2.000000+8$ & $2.000000+8$ & 0 & 0 & 2 & 4994583 \\
\hline
\end{tabular}

-

9458319 only in VII.1 9458320 only in VII.1 9458321 Only in VII.1 9458337 only in VII.1 9458338 only in VII.1

\begin{tabular}{ccccr}
$0.0000000+0-4.600000+4$ & 0 & 0 & 1 & 669458351 \\
$0.000000+0-4.600000+4$ & 0 & 0 & 1 & 369458351 \\
\hline $0.000000+0-1.550000+5$ & 0 & 0 & 1 & 619458352
\end{tabular}

$\begin{array}{llllll}\odot .00000 \odot \odot+\odot-1.55000 \odot+5 & 0 & 0 & 1 & 619458352 \\ 0.0000 \odot \odot+\odot-1.55000 \odot+5 & \odot & \odot & 1 & 309458352\end{array}$

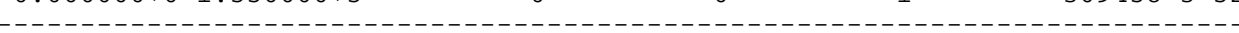

9458353 only in VII.1 9458354 only in VII.1 9458355 only in VII.1 9458356 only in VII.1 9458357 only in VII.1 9458358 only in VII.1 9458359 only in VII.1

\begin{tabular}{|c|c|}
\hline $\begin{array}{l}\odot . \odot \odot \odot \odot \odot \odot+\odot-1.154710+5 \\
\odot . \odot \odot \odot \odot \odot \odot+\odot-3 . \odot \odot \odot \odot \odot \odot+5\end{array}$ & $\begin{array}{l}\odot \\
0\end{array}$ \\
\hline $\begin{array}{ll}4.470510+6 & 4.470510+6 \\
5.969640+6 & 5.969640+6\end{array}$ & $\begin{array}{l}\odot \\
\odot\end{array}$ \\
\hline 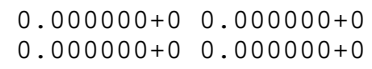 & $\begin{array}{l}\odot \\
\odot\end{array}$ \\
\hline
\end{tabular}

$\odot$
0
0
0
0

$1 \quad 639458391$

639458391 469458391

1

$1 \quad 8794583102$

$1 \quad 6494583102$

90945842

1
1

9458416 only in VII.0 9458417 only in VII.e 9458451 Only in VII.0 9458452 only in VII.0 9458491 only in VII.0 9458516 only in VII.0 9458517 only in VII. 0

$\odot .000000+\odot \quad 0.0000000+\odot$ $-2.000000+8 \quad 0.000000+0$

\begin{tabular}{ll}
0 & 1 \\
$\odot$ & 7 \\
\hline
\end{tabular}

29458518

29458518

129458518

9458591 only in VII.0 9458616 only in VII.1 
9458617 only in VII.1 9458637 0nly in VII.1 9458651 only in VII.1 9458652 only in VII.1 9458653 Only in VII.1 9458654 only in VII.1 9458655 only in VII.1 9458656 only in VII.1 9458657 only in VII.1 9458658 only in VII.1 9458659 only in VII.1 9458691 Only in VII.1 95-Am-240 Evaluation Only in VII.1 94586102 only in VII.1

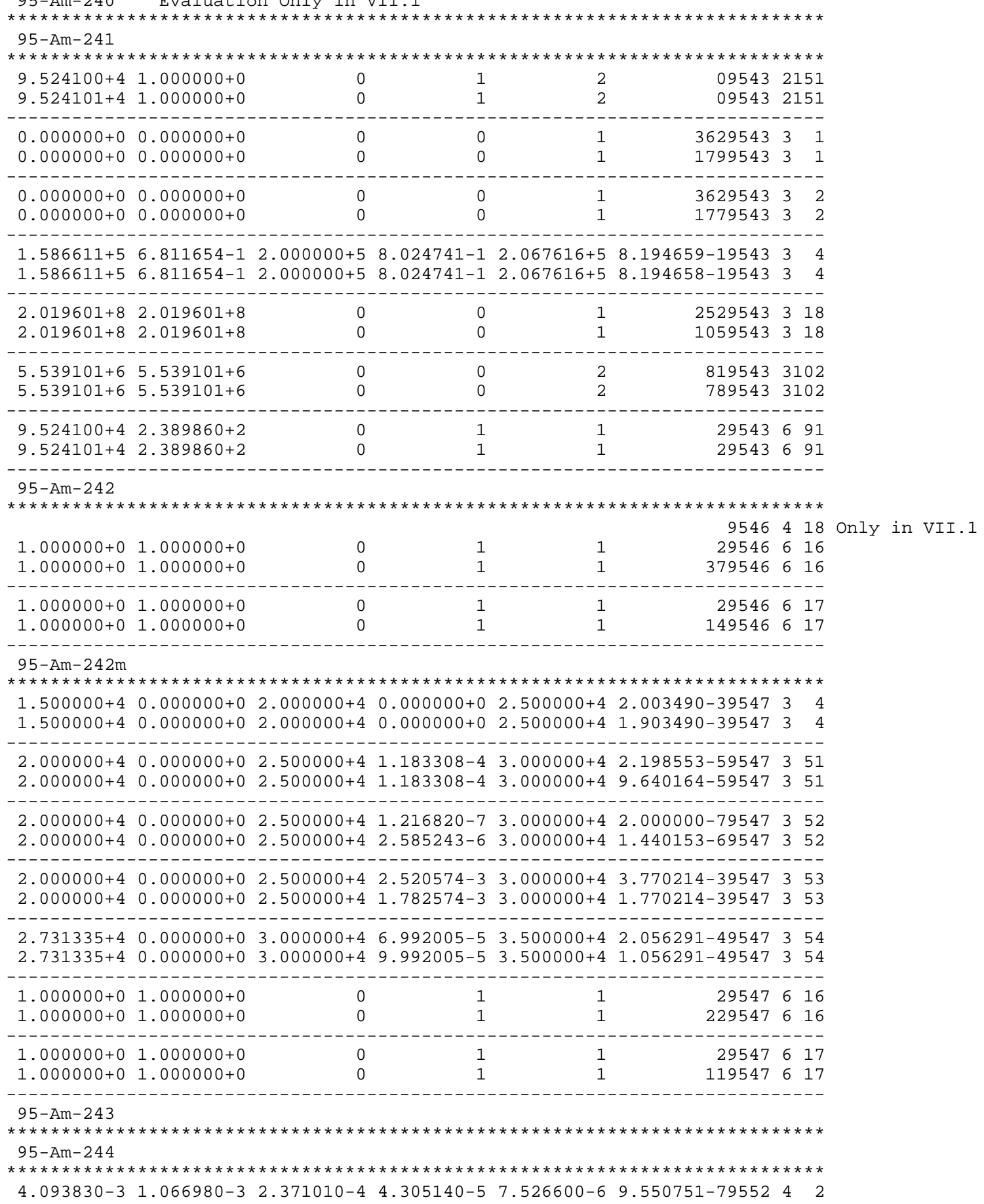


4.093830-3 $1.066980-3 \quad 2.371010-4 \quad 4.305140-5 \quad 7.526600-6 \quad 9.550750-79552442$

$\odot .000000+0-9.927639-7 \quad 9552472$

$\odot .000000+\odot-9.927640-7 \quad 9552472$

$0.000000+0-9.916261-7 \quad 9552473$

$0.000000+0-9.916260-7 \quad 9552473$

$95-\mathrm{Am}-244 \mathrm{~m}$

$2.095200+5 \quad 6.525000-4 \quad 2.357210+5 \quad 9.306930-4 \quad 2.400000+5 \quad 9.788109-49553356$ $2.095200+5 \quad 6.525000-4 \quad 2.357210+5 \quad 9.306930-4 \quad 2.400000+5 \quad 9.788110-49553356$

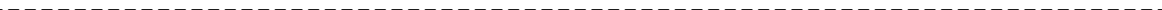

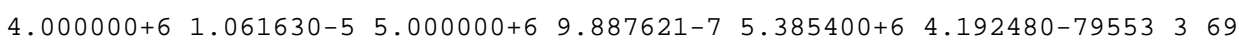
4.000000+6 $1.061630-5 \quad 5.0000000+6 \quad 9.887620-7 \quad 5.385400+6 \quad 4.192480-79553369$

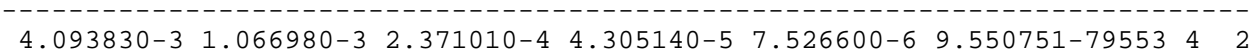
4.093830-3 $1.066980-3 \quad 2.371010-4 \quad 4.305140-5 \quad 7.526600-6 \quad 9.550750-79553442$

$\odot .0 \odot \odot \odot \odot \odot+\odot ~ 9.977171-7 \quad 0.0 \odot \odot \odot \odot \odot+\odot-2.207510-5 \quad 0.0 \odot \odot \odot \odot \odot+\odot-8.782030-69553463$ $0.000000+\odot \quad 9.977170-7 \quad 0.0000000+0-2.207510-5 \quad 0.000000+0-8.782030-69553463$

2.

$\begin{array}{llllll}2.020000+8 & 2.020000+8 & 0 & 0 & 1 & 959628318\end{array}$

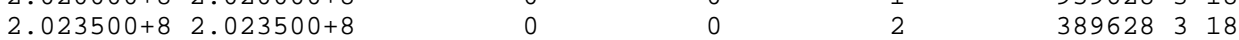

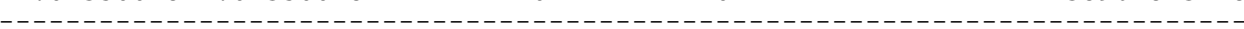

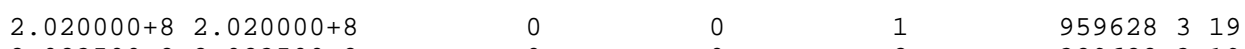

$2.023500+8 \quad 2.023500+8 \quad 000389628319$

0

\begin{tabular}{|c|c|}
\hline $200 \odot 0+8 \quad 2.0200 \odot \odot+8$ & $\odot$ \\
\hline
\end{tabular}

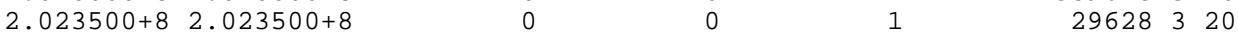

9628 321 only in VII.1

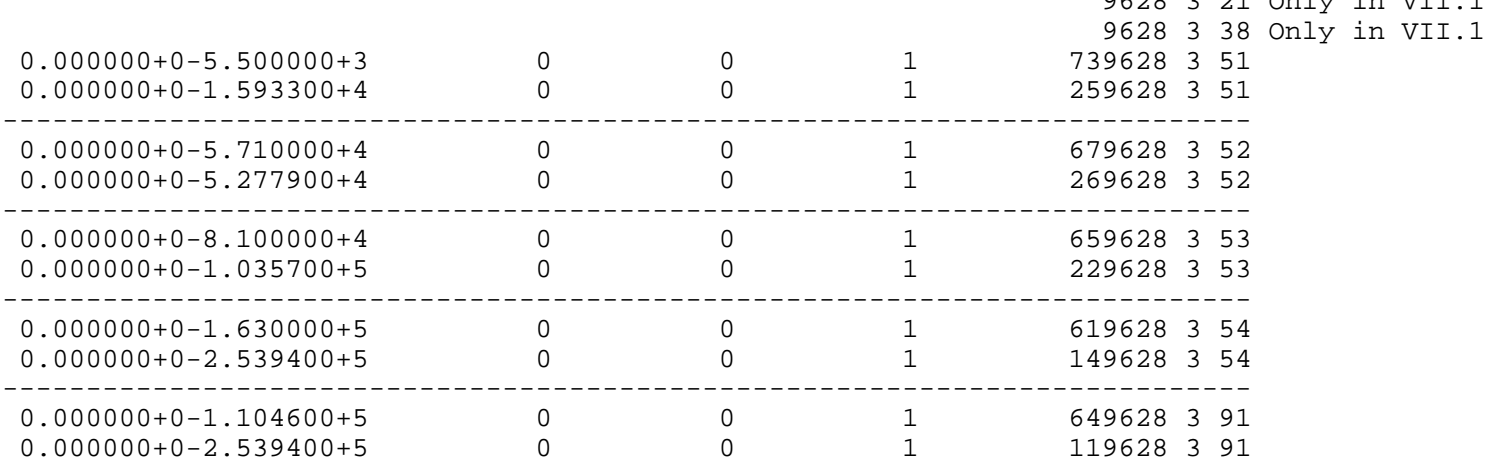




\begin{tabular}{|c|c|c|}
\hline $\begin{array}{l}6.969520+6 \\
6.968000+6\end{array}$ & $\begin{array}{l}6.969520+6 \\
6.968000+6\end{array}$ & $\begin{array}{l}\odot \\
\odot\end{array}$ \\
\hline \multicolumn{3}{|c|}{$-\mathrm{C}_{-1}$} \\
\hline$\odot . \odot \odot \odot \odot \odot \odot+\odot$ & $\odot .0 \odot \odot \odot \odot \odot+\odot$ & $\odot$ \\
\hline $0.000000+0$ & $0.000000+0$ & $\odot$ \\
\hline
\end{tabular}

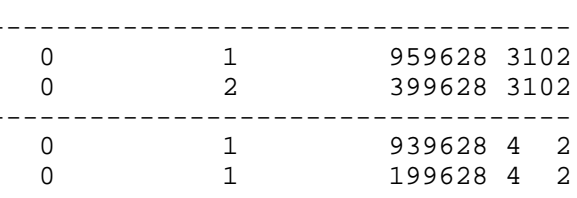

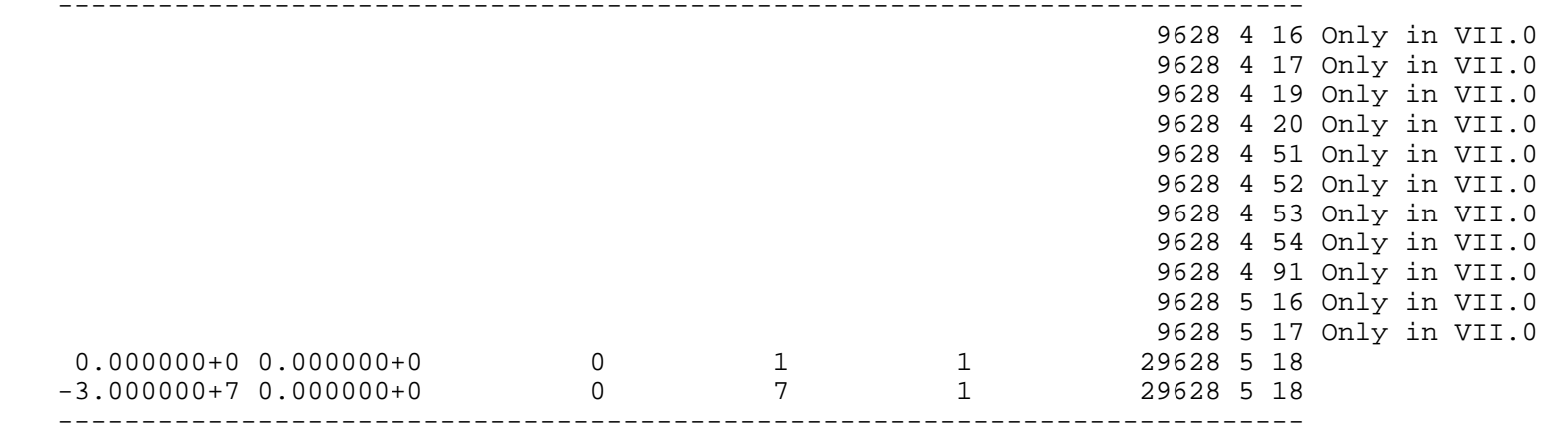
9628519 Only in VII. 0 9628520 only in VII.0 9628591 Only in VII.0 9628616 Only in VII.1 9628617 only in VII.1 9628651 Only in VII.1 9628652 only in VII.1 9628653 only in VII.1 9628654 Only in VII.1 9628691 only in VII.1

$96-\mathrm{Cm}-242$ 96286102 Only in VII.1

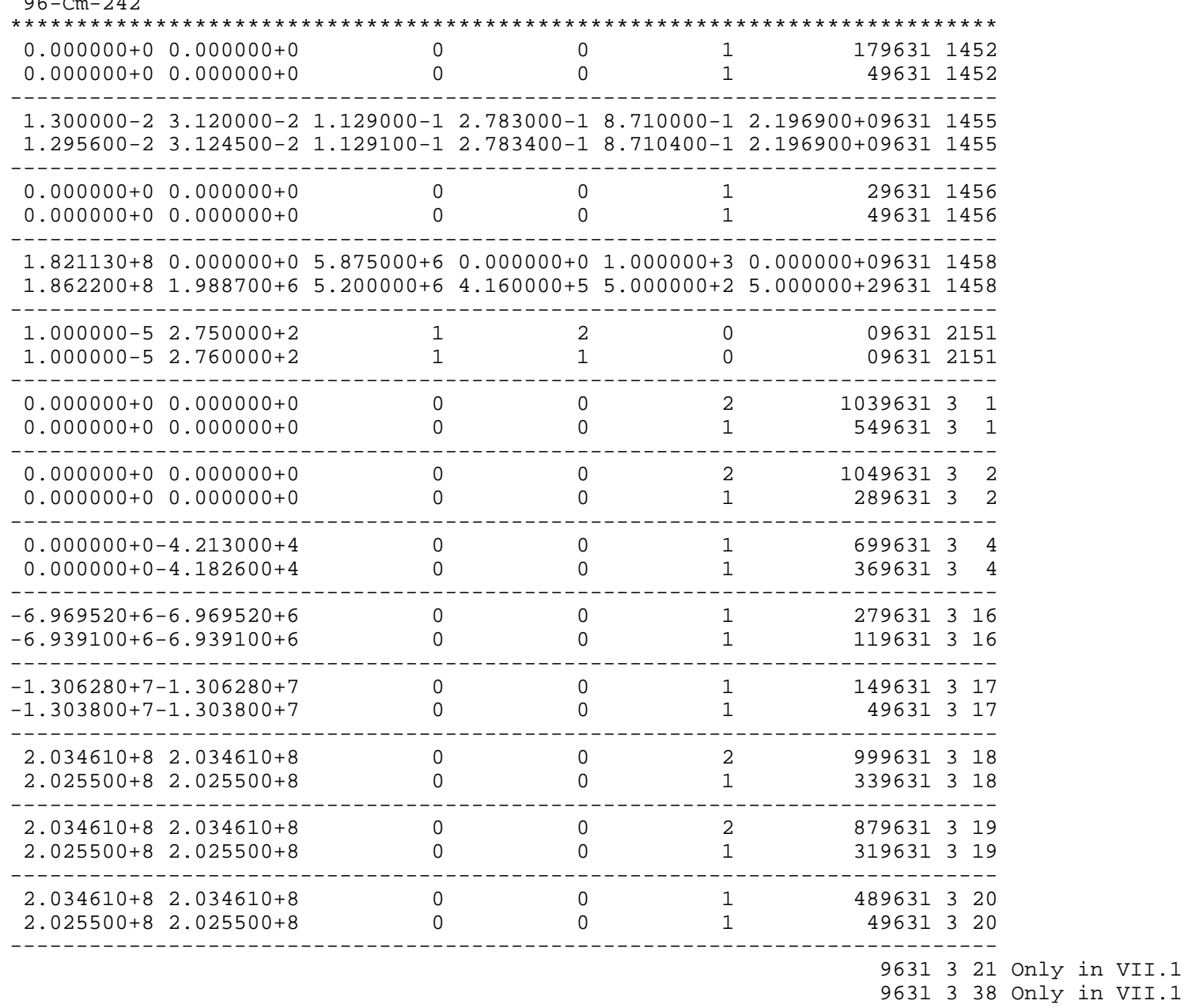




\begin{tabular}{|c|c|c|c|c|}
\hline $\begin{array}{l}\odot .0 \odot \odot \odot \odot \odot+\odot-4.2130 \odot \odot+4 \\
\odot .0 \odot \odot \odot \odot \odot+\odot-4.18260 \odot+4\end{array}$ & 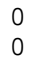 & $\stackrel{\odot}{\odot}$ & $\begin{array}{l}1 \\
1\end{array}$ & $\begin{array}{lll}689631 & 3 & 5 \\
309631 & 3 & 5\end{array}$ \\
\hline \multicolumn{5}{|c|}{ 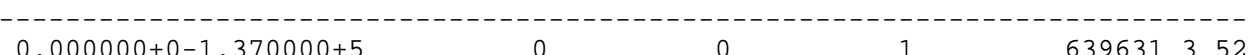 } \\
\hline $0.000000+0-1.370000+5$ & $\odot$ & $\odot$ & 1 & 6396313 \\
\hline $0.000000+0-1.384200+5$ & $\odot$ & $\odot$ & 1 & 2596313 \\
\hline \multicolumn{5}{|c|}{ - - - - - - - - - - - - - - - - - - - - - - - - - - - - - - - - - - - - - - - - - } \\
\hline $0.000000+0-2.880000+5$ & $\odot$ & $\odot$ & 1 & 58963135 \\
\hline $0.000000+0-2.838200+5$ & $\odot$ & $\odot$ & 1 & 14963135 \\
\hline \multicolumn{5}{|c|}{ 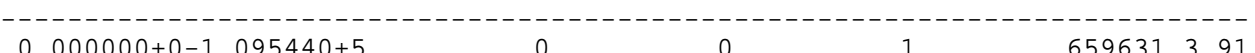 } \\
\hline$\odot .000000+0-1.095440+5$ & $\odot$ & $\odot$ & 1 & 6596313 \\
\hline $0.000000+0-2.838200+5$ & $\odot$ & $\odot$ & 1 & 2096313 \\
\hline 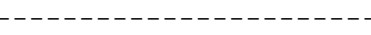 & - & - & & $-----1--$ \\
\hline $5.692940+65.692940+6$ & $\odot$ & $\odot$ & 2 & \\
\hline $5.703 \odot \odot \odot+6 \quad 5.7 \odot 3 \odot \odot \odot+6$ & $\odot$ & $\odot$ & 1 & 349631316 \\
\hline \multirow{2}{*}{\multicolumn{5}{|c|}{$\begin{array}{ccccc}0.000000+0 & 0.000000+0 & 0 & 0 & 9296314\end{array}$}} \\
\hline & & & 1 & \\
\hline $0.000000+0 \quad 0.000000+0$ & $\odot$ & $\odot$ & 1 & 1996314 \\
\hline
\end{tabular}

9631416 only in VII.0 9631417 only in VII.0 9631419 only in VII.0 9631420 only in VII.0 9631451 only in VII.0 9631452 only in VII.0 9631453 only in VII.0 9631491 Only in VII.0 9631516 only in VII.0 9631517 only in VII.0 $0.000000+0 \quad 0.000000+0$ $-3.000000+7 \quad 0.000000+0$

$\begin{array}{lll}0 & 1 & 1 \\ 0 & 7 & 1\end{array}$

29631518 29631518

9631519 Only in VII. 0 9631520 only in VII.0 9631591 Only in VII.0 9631616 only in VII.1 9631617 Only in VII.1 9631651 only in VII.1 9631652 only in VII.1 9631653 only in VII.1 9631691 only in VII.1 $96-\mathrm{Cm}-243$ 96316102 only in VII.1

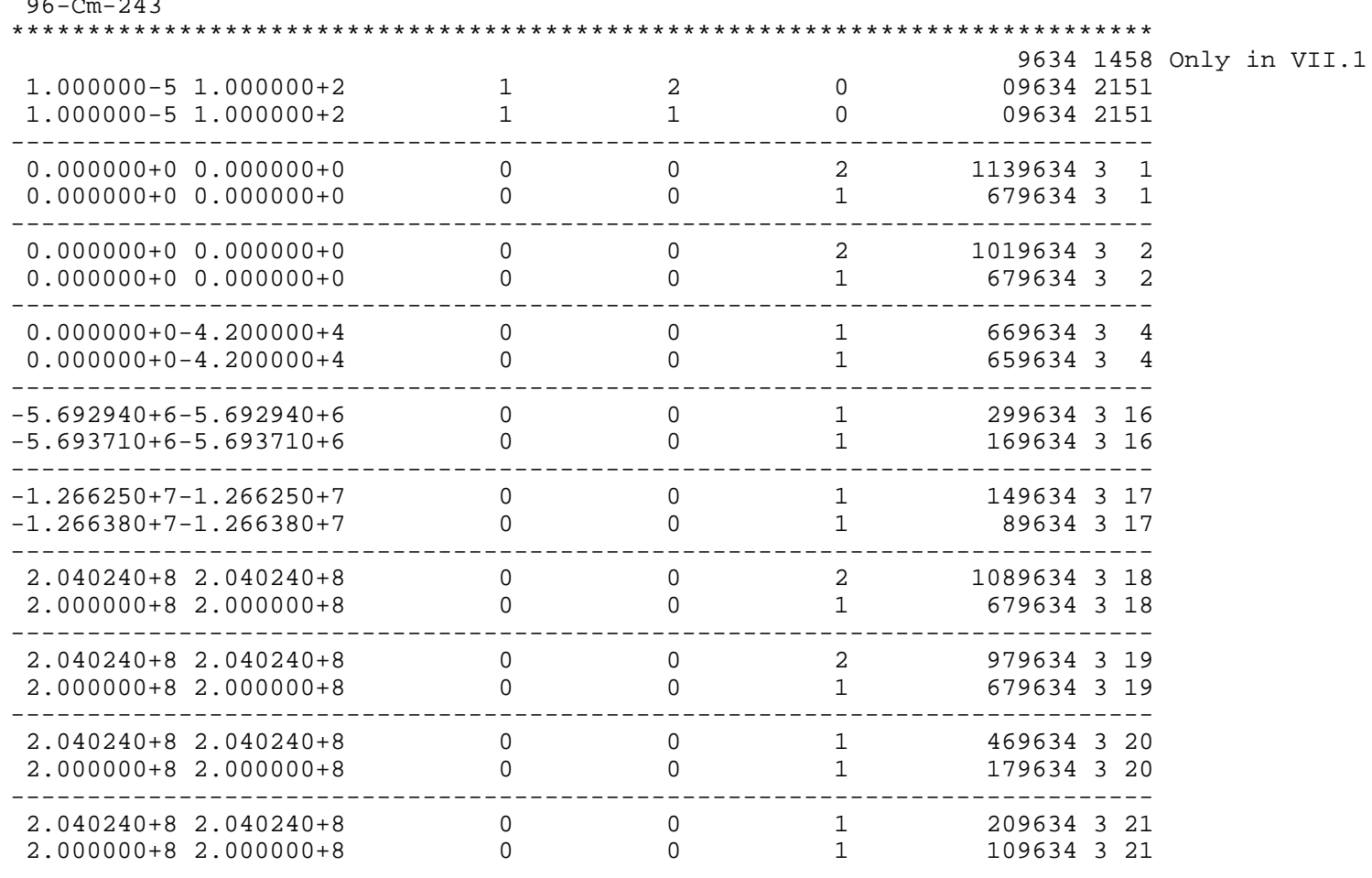




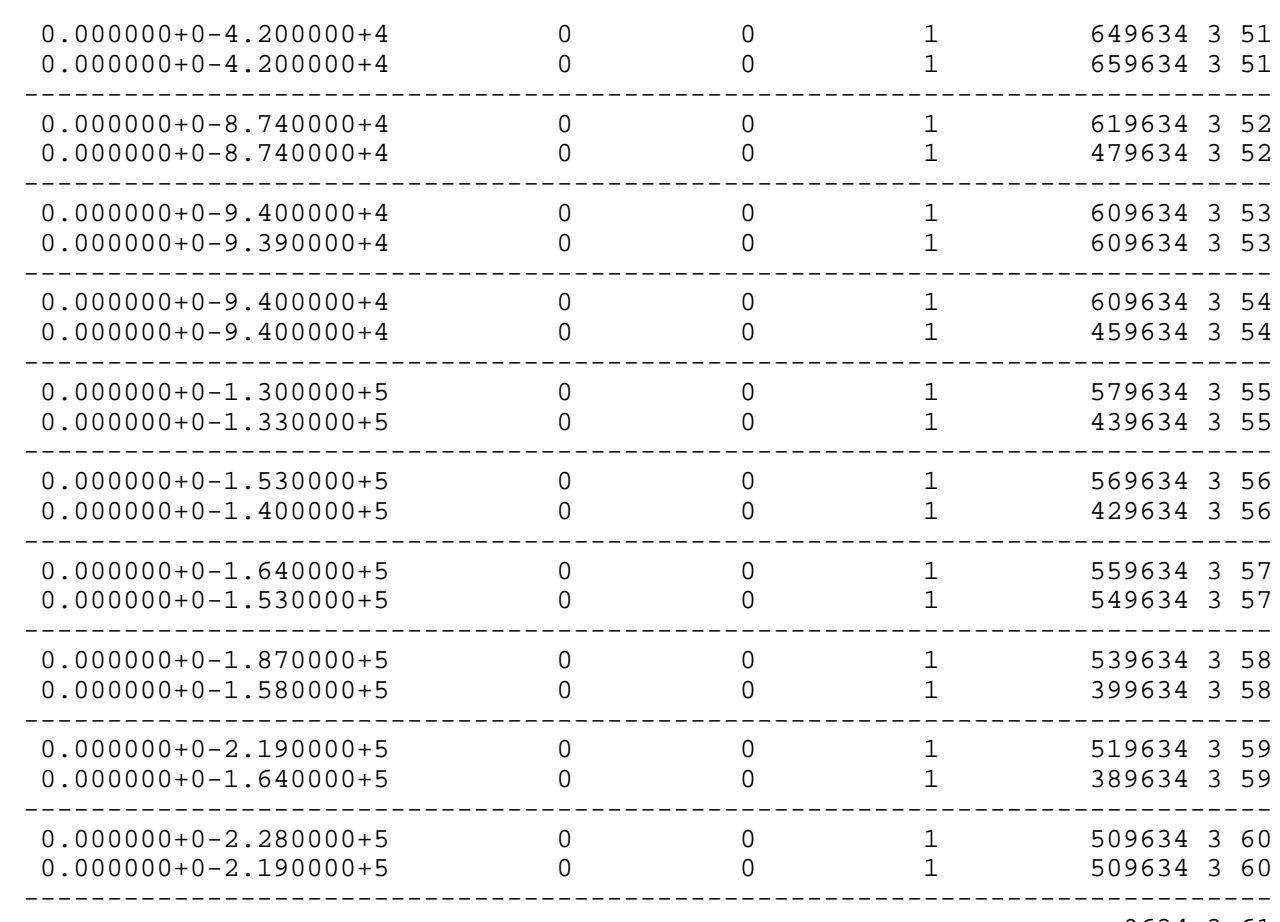

9634361 only in VII.0 9634362 only in VII.0 9634363 only in VII.0 9634364 only in VII.0 9634365 only in VII.0 9634366 only in VII.0 9634367 only in VII.0 9634368 only in VII.0 9634369 only in VII.0 9634370 only in VII.0 9634371 only in VII.0 9634372 only in VII.0 9634373 only in VII.0 9634374 only in VII.0

$\odot .0 \odot \odot \odot \odot \odot+\odot-1.154770+5$ $\odot .00 \odot \odot \odot \odot+0-4.0100 \odot \odot+5$

5

$6.801260+6 \quad 6.801260+6$

6. $799500+6 \quad 6.799500+6$

$\begin{array}{lll}599634 & 3 & 91\end{array}$ 319634391

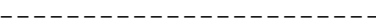

$\odot .0 \odot \odot \odot \odot \odot+\odot \quad \odot .00 \odot \odot \odot \odot+\odot$ $\odot .00 \odot \odot \odot \odot+\odot \quad \odot .000 \odot \odot \odot+\odot$

$\begin{array}{ccc}6 & 0 & 0 \\ 0 & 0 & 0 \\ 0 & 0 & 0 \\ 0 & 0 & 0\end{array}$

$2 \quad 8096343102$

$1 \quad 5296343102$

1

$\begin{array}{lll}799634 & 4\end{array}$

$179634 \quad 4 \quad 2$

9634416 only in VII.0 9634417 only in VII.0 9634419 only in VII.0 9634420 only in VII.0 9634421 only in VII.0 9634451 only in VII.0 9634452 only in VII.0 9634453 only in VII.0 9634454 only in VII.0 9634455 only in VII.0 9634456 only in VII.0 9634457 only in VII.0 9634458 only in VII.0 9634459 only in VII.0 9634460 only in VII.0 9634461 only in VII.0 9634462 only in VII.0 9634463 only in VII.0 9634464 only in VII.0 9634465 only in VII.0 9634466 only in VII.0 9634467 only in VII.0 


$\odot$

1

1
1

9634468 only in VII.0 9634469 Only in VII.0 9634470 only in VII. $\odot$ 9634471 only in VII.e 9634472 Only in VII.0 9634473 only in VII.0 9634474 only in VII.0 9634491 Only in VII.0 9634516 only in VII.0 9634517 only in VII.0 29634518 29634518

9634519 only in VII.0 9634520 only in VII.0 9634521 Only in VII.0 9634591 only in VII.0 9634616 Only in VII.1 9634617 only in VII.1 9634651 Only in VII.1 9634652 Only in VII.1 9634653 only in VII.1 9634654 only in VII.1 9634655 only in VII.1 9634656 only in VII.1 9634657 0nly in VII.1 9634658 only in VII.1 9634659 Only in VII.1 9634660 only in VII.1 9634691 Only in VII.1

$96-\mathrm{Cm}-244$

96346102 only in VII.1

\begin{tabular}{|c|c|c|c|c|c|c|}
\hline $\begin{array}{l}\odot .000000+ \\
\odot .00000 \odot+\end{array}$ & $\begin{array}{l}0.000000+\odot \\
0.000 \odot \odot \odot+\odot\end{array}$ & $\begin{array}{l}\odot \\
\odot\end{array}$ & $\begin{array}{l}\odot \\
\odot\end{array}$ & $\begin{array}{l}1 \\
1\end{array}$ & $\begin{array}{r}179637 \\
49637\end{array}$ & $\begin{array}{l}1452 \\
1452\end{array}$ \\
\hline $\begin{array}{l}\odot .0 \odot \odot \odot \odot \odot++ \\
\odot . \diamond \odot \odot \odot \odot \odot++\end{array}$ & $\begin{array}{l}\odot .0 \odot \odot \odot \odot \odot+\odot \\
\odot . \diamond \odot \odot \odot \odot \odot+\odot\end{array}$ & $\begin{array}{l}\odot \\
\odot\end{array}$ & $\begin{array}{l}\odot \\
\odot\end{array}$ & $\begin{array}{l}1 \\
1\end{array}$ & $\begin{array}{r}179637 \\
49637\end{array}$ & $\begin{array}{l}1455 \\
1455\end{array}$ \\
\hline $\begin{array}{l}1.000 \odot \odot \odot- \\
1.00 \odot \odot \odot ०-\end{array}$ & $\begin{array}{l}3.00000 \odot+\odot \\
3.2400 \odot \odot+\odot\end{array}$ & $\begin{array}{l}+7 \\
+7\end{array}$ & & & $\begin{array}{l}9637 \\
9637\end{array}$ & $\begin{array}{l}1456 \\
1456\end{array}$ \\
\hline
\end{tabular}

$-6.650000+0 \quad 5.000000-1 \quad 4.760000-2 \quad 4.200000-3 \quad 3.700000-2 \quad 6.40000096371458$ only in VII.1

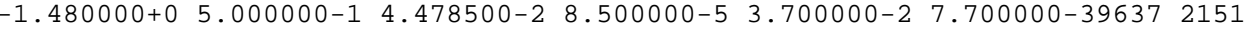

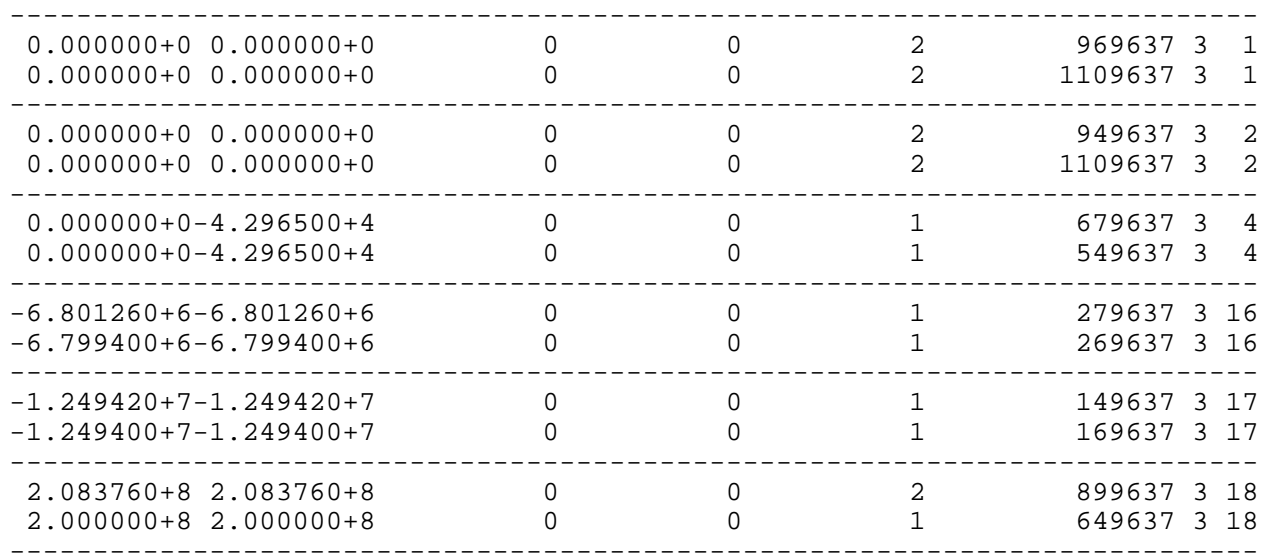

9637319 only in VII.1 9637320 Only in VII.1 9637321 Only in VII.1

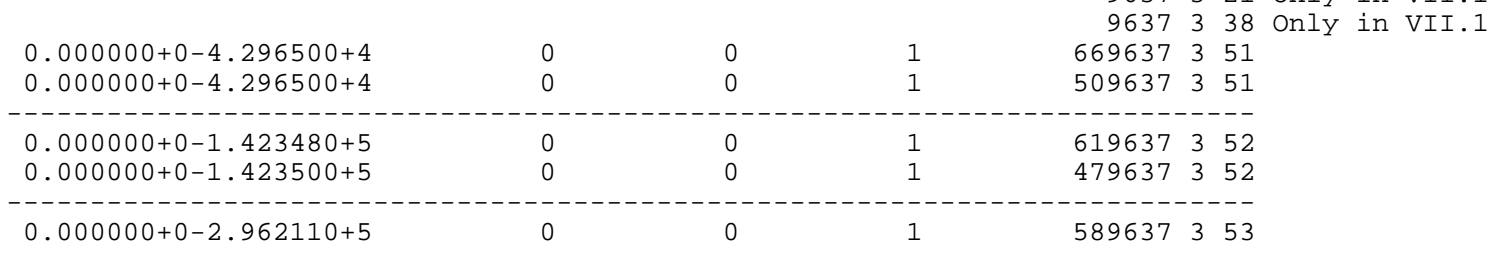




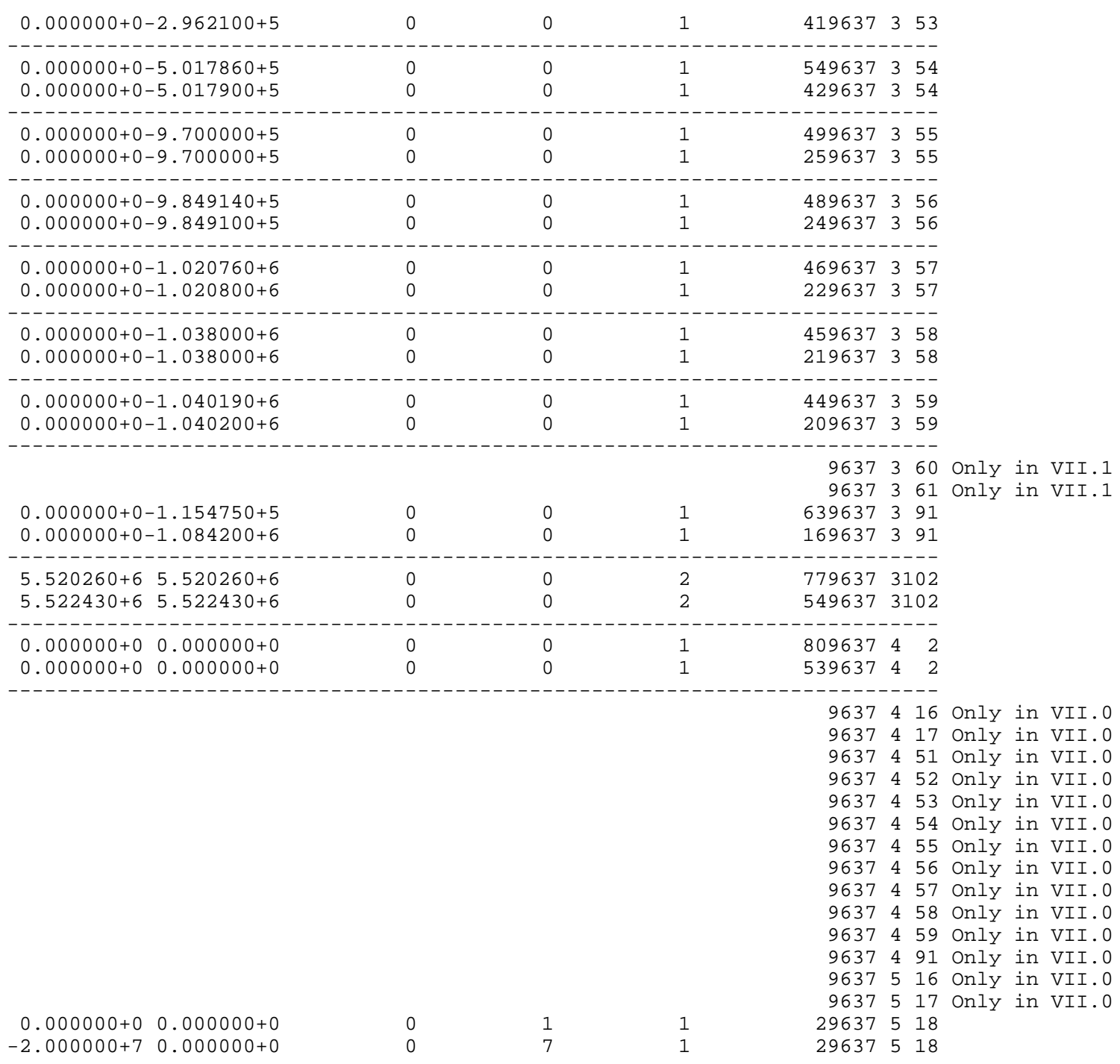

r. 9637591 only in VII.0
9637616 only in VII.1 9637617 only in VII.1 9637651 only in VII.1 9637652 only in VII.1 9637653 only in VII.1 9637654 only in VII.1 9637655 only in VII.1 9637656 only in VII.1 9637657 Only in VII.1 9637658 only in VII.1 9637659 only in VII.1 9637660 only in VII.1 9637661 only in VII.1 9637691 Only in VII.1

$96-\mathrm{Cm}-245$ 96376102 only in VII.1

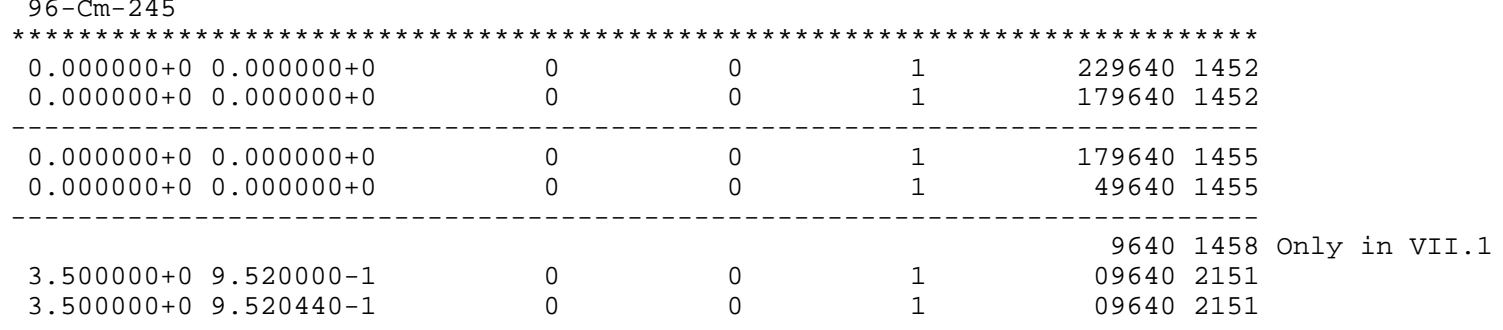




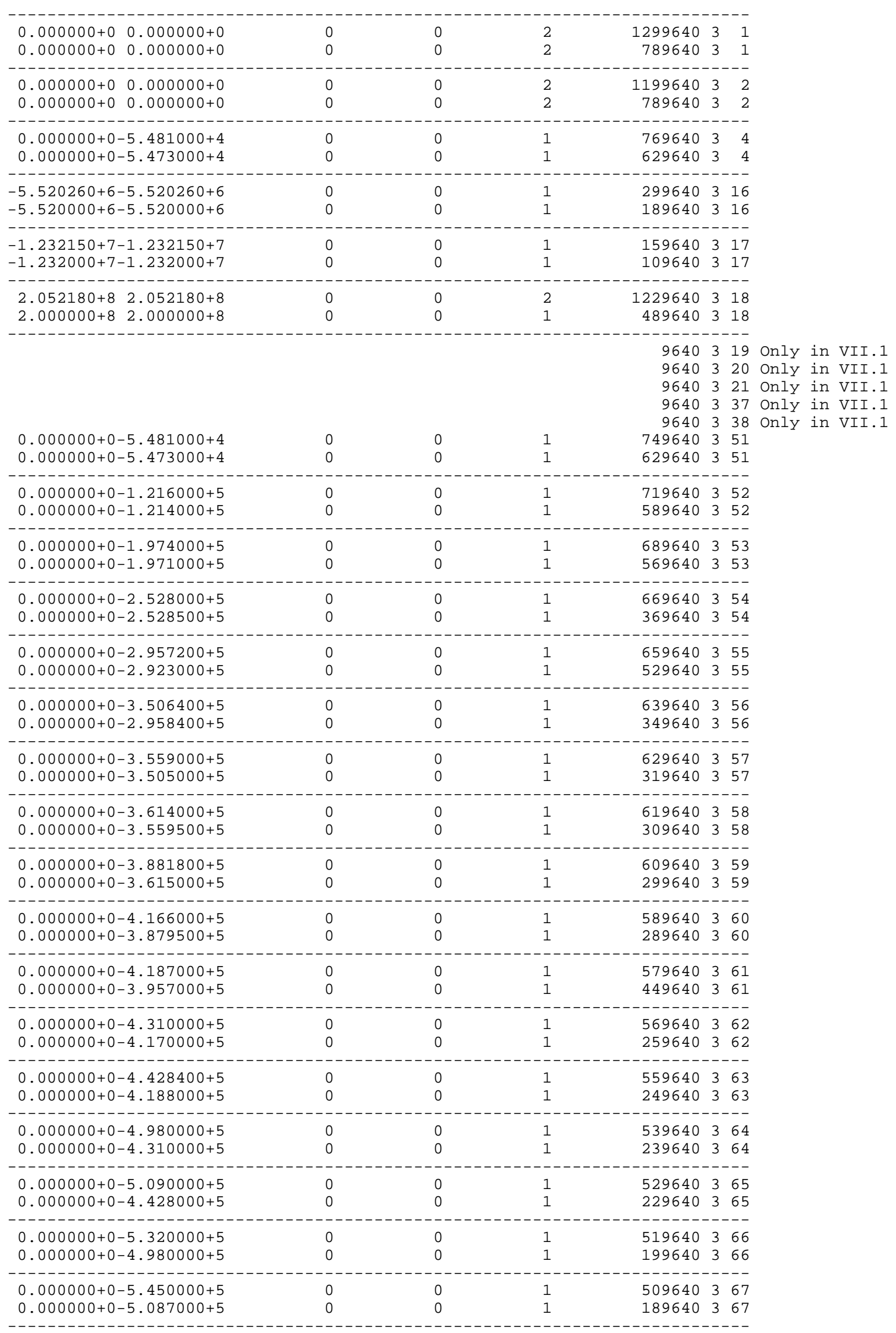


9640368 only in VII.1 9640369 Only in VII.1

\begin{tabular}{|c|c|}
\hline $\begin{array}{l}\odot .00 \odot \odot \odot \odot+\odot-1.11507 \odot+5 \\
\odot .00 \odot \odot \odot \odot+\odot-5.1100 \odot \odot+5\end{array}$ & $\begin{array}{l}\odot \\
\odot\end{array}$ \\
\hline $\begin{array}{ll}6.457580+6 & 6.457580+6 \\
6.450000+6 & 6.450000+6\end{array}$ & $\begin{array}{l}\odot \\
\odot\end{array}$ \\
\hline 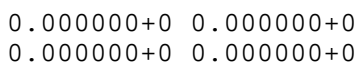 & $\begin{array}{l}\odot \\
\odot\end{array}$ \\
\hline
\end{tabular}

9640370 Only in VII.1 729640391

349640391

9196403102

6996403102

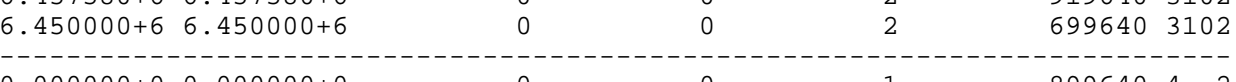

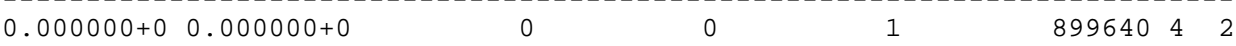

1
1

9640416 only in VII.0 9640417 only in VII.0 9640451 only in VII.0 9640452 Only in VII.0 9640453 only in VII.0 9640454 Only in VII.0 9640455 only in VII.0 9640456 only in VII.0 9640457 only in VII.0 9640458 only in VII.0 9640459 only in VII.0 9640460 only in VII.0 9640461 only in VII.0 9640462 only in VII.0 9640463 only in VII.0 9640464 Only in VII.0 9640465 only in VII.0 9640466 only in VII.0 9640467 only in VII.0 9640491 only in VII.0 9640516 only in VII.0 9640517 only in VII.0

\section{$\odot$}

1 209640518

$\begin{array}{lllll}1.000 \odot \odot \odot-5 ~ & 2.216223-2 & 2.0000 \odot \odot \odot+7 & 2.216223-2\end{array}$

9640591 only in VII. $\odot$ $1.000000-52.216220-2 \quad 2.000000+7 \quad 2.216220-2$

96405455

96405455

-

9640616 only in VII.1 9640617 only in VII.1 9640637 Only in VII.1 9640651 only in VII.1 9640652 only in VII.1 9640653 Only in VII.1 9640654 Only in VII.1 9640655 only in VII.1 9640656 Only in VII.1 9640657 only in VII.1 9640658 only in VII.1 9640659 only in VII.1 9640660 only in VII.1 9640661 Only in VII.1 9640662 only in VII.1 9640663 only in VII.1 9640664 Only in VII.1 9640665 only in VII.1 9640666 only in VII.1 9640667 only in VII.1 9640668 only in VII.1 9640669 Only in VII.1 9640670 only in VII.1 9640691 only in VII.1 $96-\mathrm{Cm}-246$

96406102 only in VII.1

\begin{tabular}{|c|c|c|c|c|c|c|}
\hline $\begin{array}{l}\odot . \odot \odot \odot \odot \odot \odot+\odot \\
\odot . \diamond \odot \odot \odot \odot \odot+\odot\end{array}$ & $\begin{array}{l}\odot . \odot \odot \odot \odot \odot \odot+\odot \\
\odot . \odot \odot \odot \odot \odot \odot+\odot\end{array}$ & $\begin{array}{l}\ominus \\
\odot\end{array}$ & $\begin{array}{l}\odot \\
\odot\end{array}$ & $\begin{array}{l}1 \\
1\end{array}$ & $\begin{array}{r}49643 \\
159643\end{array}$ & $\begin{array}{l}1452 \\
1452\end{array}$ \\
\hline $\begin{array}{l}\odot .00000 \odot+\odot \\
\odot .0000 \odot \odot+\odot\end{array}$ & $\begin{array}{l}\odot .0000 \odot \odot+\odot \\
\odot .00 \odot \odot \odot \odot+\odot\end{array}$ & $\begin{array}{l}\odot \\
\odot\end{array}$ & $\begin{array}{l}\odot \\
\odot\end{array}$ & $\begin{array}{l}1 \\
6\end{array}$ & $\begin{array}{l}09643 \\
09643\end{array}$ & $\begin{array}{l}1455 \\
1455\end{array}$ \\
\hline $0.000000+\odot$ & $0.0000000+\odot$ & $\odot$ & $\odot$ & 1 & 49643 & 1456 \\
\hline
\end{tabular}




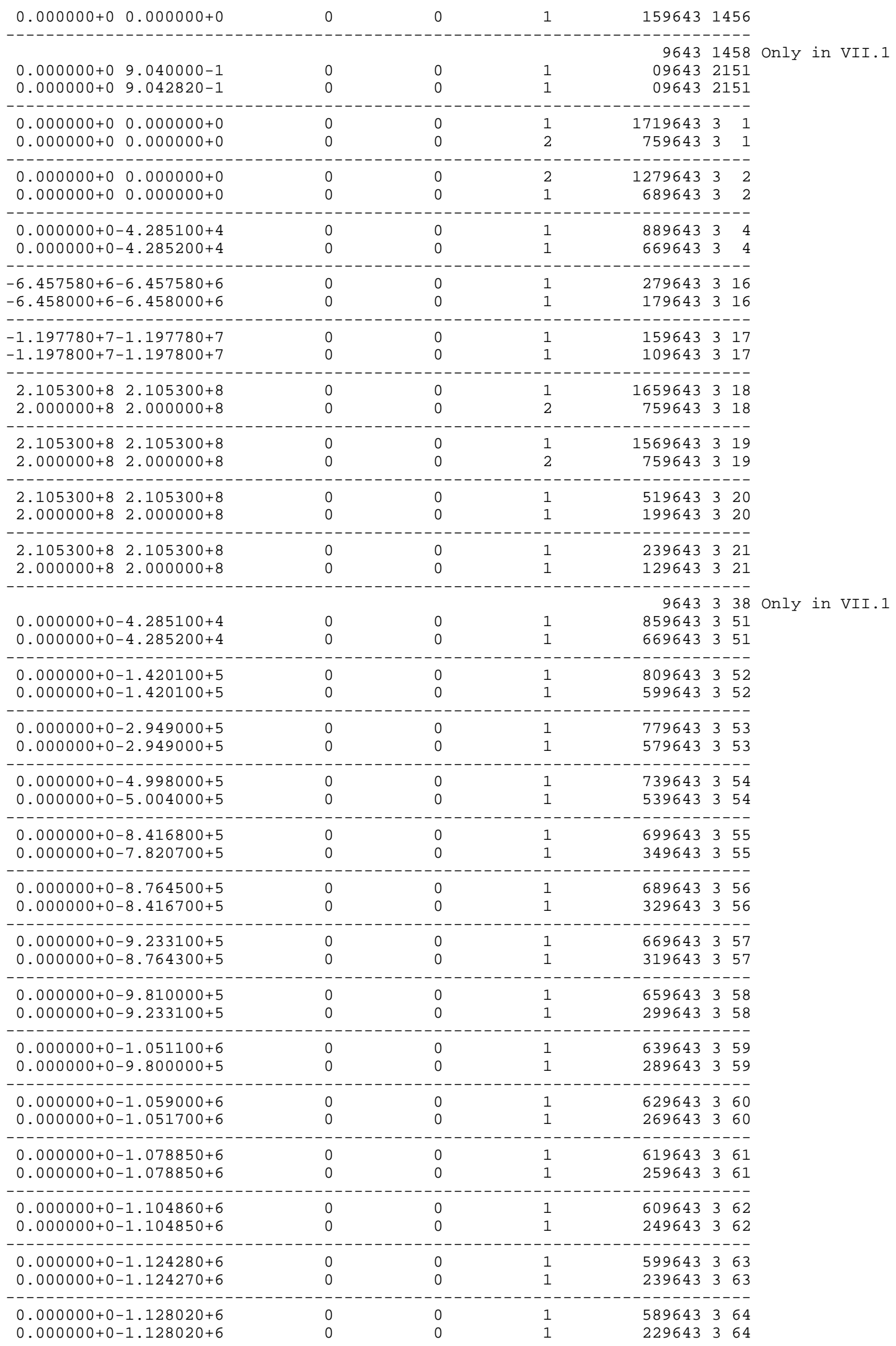




\begin{tabular}{|c|c|c|c|c|}
\hline $\begin{array}{l}\odot .000 \odot \odot \odot+\odot-1.128800+6 \\
\odot .0 \odot \odot \odot \odot \odot+\odot-1.12940 \odot+6\end{array}$ & $\begin{array}{l}\odot \\
\odot\end{array}$ & $\begin{array}{l}\odot \\
\odot\end{array}$ & $\begin{array}{l}1 \\
1\end{array}$ & $\begin{array}{lll}579643 & 3 & 65 \\
219643 & 3 & 65\end{array}$ \\
\hline $\begin{array}{l}\odot . \odot \odot \odot \odot \odot \odot+\odot-1.165490+6 \\
\odot .00 \odot \odot \odot \odot+\odot-1.165490+6\end{array}$ & $\begin{array}{l}\odot \\
\odot\end{array}$ & $\begin{array}{l}0 \\
\odot\end{array}$ & 1 & 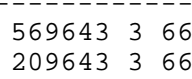 \\
\hline $\begin{array}{l}\odot . \odot \odot \odot \odot \odot \odot+\Theta-1.174740+6 \\
\odot . \odot \odot \odot \odot \odot \odot+\odot-1.174740+6\end{array}$ & $\begin{array}{l}\odot \\
\odot\end{array}$ & $\begin{array}{l}\odot \\
\odot\end{array}$ & $\begin{array}{l}1 \\
1\end{array}$ & $\begin{array}{lll}559643 & 3 & 67 \\
199643 & 3 & 67\end{array}$ \\
\hline $\begin{array}{l}\odot .0000 \odot \odot+\odot-1.178600+6 \\
\odot . \odot \odot \odot \odot \odot \odot+\odot-1.17920 \odot+6\end{array}$ & $\stackrel{\odot}{\odot}$ & $\begin{array}{l}\odot \\
\odot\end{array}$ & $\begin{array}{l}1 \\
1\end{array}$ & 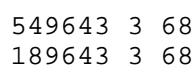 \\
\hline $\begin{array}{l}\odot .000 \odot \odot \odot+\odot-1.210530+6 \\
\odot .0 \odot \odot \odot \odot \odot+\odot-1.19590 \odot+6\end{array}$ & $\begin{array}{l}\odot \\
\odot\end{array}$ & $\begin{array}{l}\odot \\
\odot\end{array}$ & $\begin{array}{l}1 \\
1\end{array}$ & $\begin{array}{lll}539643 & 3 & 69 \\
169643 & 3 & 69\end{array}$ \\
\hline $\begin{array}{l}\odot .0 \odot \odot \odot \odot \odot+\odot-1.219980+6 \\
\odot .0 \odot \odot \odot \odot \odot+\odot-1.210530+6\end{array}$ & $\begin{array}{l}\odot \\
\odot\end{array}$ & $\begin{array}{l}\odot \\
\odot\end{array}$ & $\begin{array}{l}1 \\
1\end{array}$ & $\begin{array}{lll}529643 & 3 & 70 \\
159643 & 3 & 70\end{array}$ \\
\hline
\end{tabular}

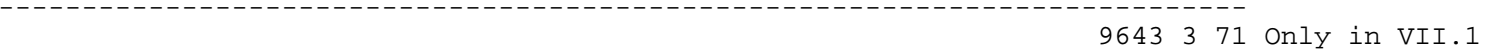

9643372 Only in VII.1

9643373 only in VII.1

9643374 Only in VII.1

9643375 only in VII.1

9643376 only in VII.1

9643377 Only in VII.1

9643378 only in VII.1

9643379 only in VII.1

9643380 Only in VII.1

\begin{tabular}{|c|c|c|c|c|c|}
\hline $\begin{array}{l}\odot . \odot \odot \odot \odot \odot \odot+\odot-1.145310+5 \\
\odot . \odot \odot \odot \odot \odot \odot+\odot-1.2200 \odot \odot+6\end{array}$ & $\begin{array}{l}\odot \\
\odot\end{array}$ & $\begin{array}{l}\odot \\
\odot\end{array}$ & $\begin{array}{l}1 \\
1\end{array}$ & $\begin{array}{l}829643 \\
309643\end{array}$ & $\begin{array}{ll}3 & 91 \\
3 & 91\end{array}$ \\
\hline $\begin{array}{ll}5.155860+6 & 5.155860+6 \\
5.160000+6 & 5.160000+6\end{array}$ & $\begin{array}{l}\odot \\
\odot\end{array}$ & $\begin{array}{l}\odot \\
\odot\end{array}$ & $\begin{array}{l}2 \\
1\end{array}$ & $\begin{array}{l}999643 \\
529643\end{array}$ & \\
\hline 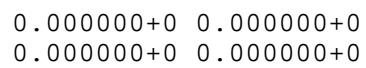 & $\begin{array}{l}\odot \\
\odot\end{array}$ & $\begin{array}{l}\odot \\
\odot\end{array}$ & $\begin{array}{l}1 \\
1\end{array}$ & $\begin{array}{l}999643 \\
219643\end{array}$ & \\
\hline
\end{tabular}

9643416 only in VII.0 9643417 Only in VII.0 9643419 only in VII.0 9643420 only in VII.0 9643421 only in VII.0 9643451 only in VII.0 9643452 only in VII.0 9643453 only in VII.0 9643454 only in VII.0 9643455 only in VII.0 9643456 only in VII.0 9643457 only in VII.0 9643458 only in VII.0 9643459 only in VII.0 9643460 only in VII.0 9643461 only in VII.0 9643462 only in VII.0 9643463 only in VII.0 9643464 Only in VII.0 9643465 only in VII.0 9643466 only in VII.0 9643467 only in VII.0 9643468 only in VII.0 9643469 only in VII.0 9643470 only in VII.0 9643491 Only in VII.0 9643516 only in VII.0 9643517 Only in VII.0 
$96-\mathrm{Cm}-247$

9643651 only in VII.1 9643652 0nly in VII.1 9643653 only in VII.1 9643654 Only in VII.1 9643655 only in VII.1 9643656 only in VII.1 9643657 only in VII.1 9643658 only in VII.1 9643659 only in VII.1 9643660 Only in VII.1 9643661 only in VII.1 9643662 Only in VII.1 9643663 only in VII.1 9643664 only in VII.1 9643665 Only in VII.1 9643666 only in VII.1 9643667 only in VII.1 9643668 only in VII.1 9643669 only in VII.1 9643670 Only in VII.1 9643671 Only in VII.1 9643672 Only in VII.1 9643673 only in VII.1 9643674 Only in VII.1 9643675 only in VII.1 9643676 only in VII.1 9643677 only in VII.1 9643678 Only in VII.1 9643679 only in VII.1 9643680 only in VII.1 9643691 Only in VII.1 96436102 Only in VII.1

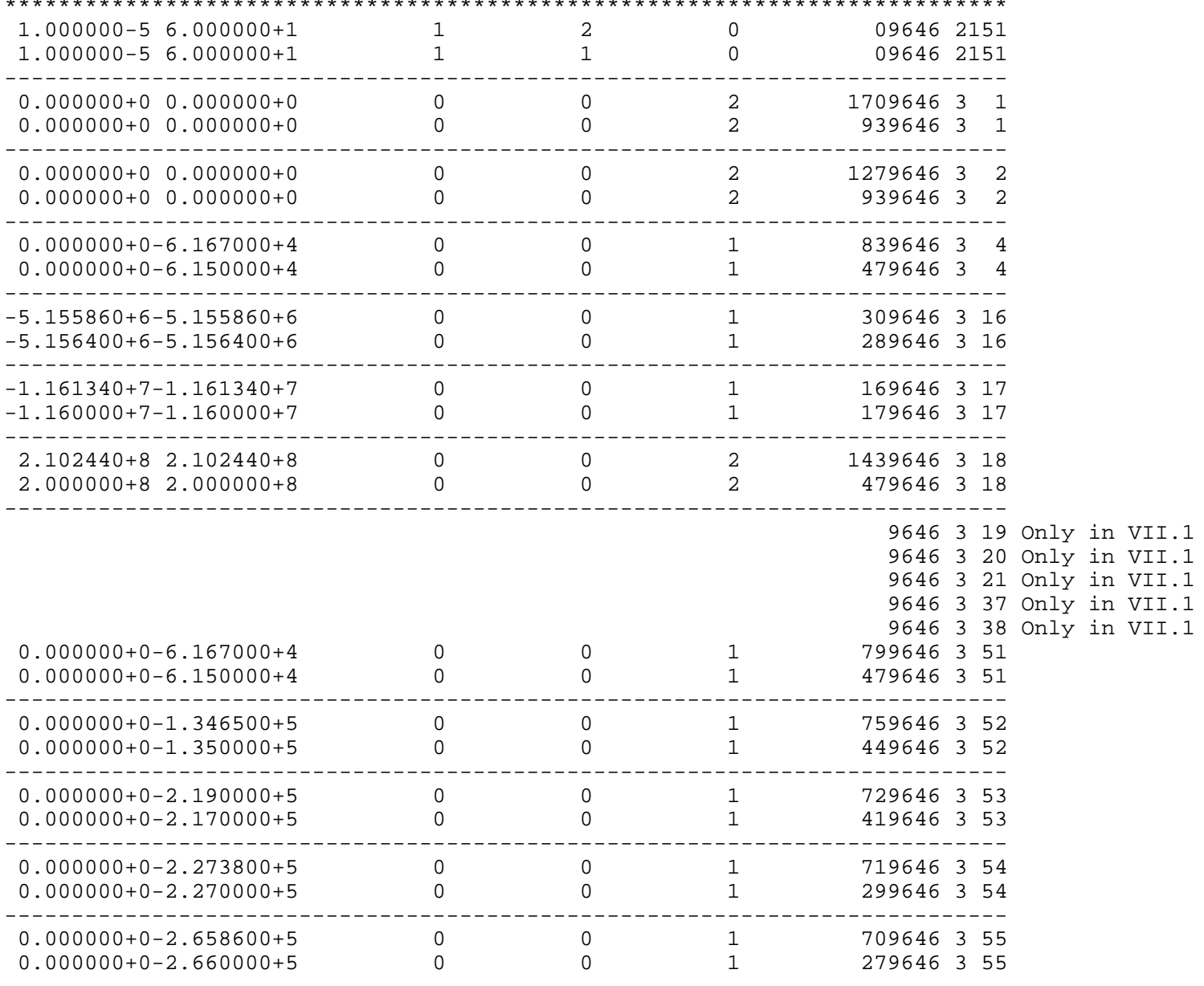


$\begin{array}{lllll}0.0 \odot \odot \odot \odot \odot+\odot-2.85410 \odot+5 & 0 & 0 & 1 & 699646356 \\ 0.0 \odot \odot \odot \odot \odot+\odot-2.850 \odot \odot \odot+5 & \odot & \odot & 1 & 269646356\end{array}$

9646357 only in VII.1

9646358 only in VII.1

9646359 only in VII.1

9646360 only in VII.1

9646361 only in VII.1

9646362 only in VII.1

9646363 only in VII.1

9646364 only in VII.1

9646365 only in VII.1

9646366 only in VII.1

9646367 only in VII.1

9646368 only in VII.1

9646369 only in VII.1

9646370 only in VII.1

9646371 0nly in VII.1

9646372 only in VII.1

9646373 only in VII.1

9646374 only in VII.1

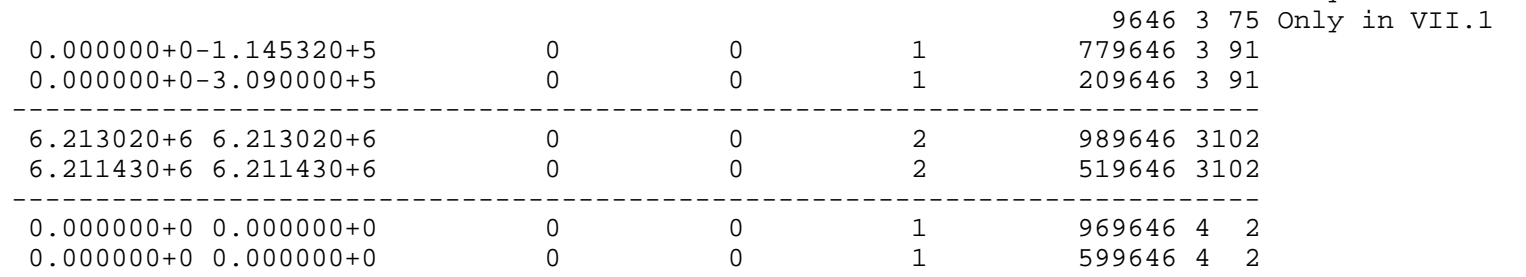

9646 4 Only in VII. 0

9646417 only in VII.0

9646451 only in VII.0

9646452 only in VII.0

9646453 only in VII.0

9646454 only in VII.0

9646455 only in VII.0

9646456 only in VII.0

9646491 only in VII.0

9646516 only in VII.0

9646517 only in VII.0

$0.000000+\odot \quad 0.000000+0$

$\odot$

1

1

29646518

$-2.000000+7 \quad 0.000000+0$

$\begin{array}{lllll}1 & 7 & 1 & 29646 & 5\end{array}$

129645

9646591 Only in VII.0 9646616 only in VII.1 9646617 only in VII.1 9646637 only in VII.1 9646651 only in VII.1 9646652 only in VII.1 9646653 only in VII.1 9646654 only in VII.1 9646655 only in VII.1 9646656 only in VII.1 9646657 only in VII.1 9646658 only in VII.1 9646659 only in VII.1 9646660 only in VII.1 9646661 only in VII.1 9646662 only in VII.1 9646663 only in VII.1 9646664 only in VII.1 9646665 only in VII.1 9646666 only in VII.1 9646667 only in VII.1 9646668 only in VII.1 9646669 only in VII.1 9646670 only in VII.1 9646671 only in VII.1 9646672 only in VII.1 9646673 only in VII.1 9646674 only in VII.1 9646675 0nly in VII.1 9646691 Only in VII.1 


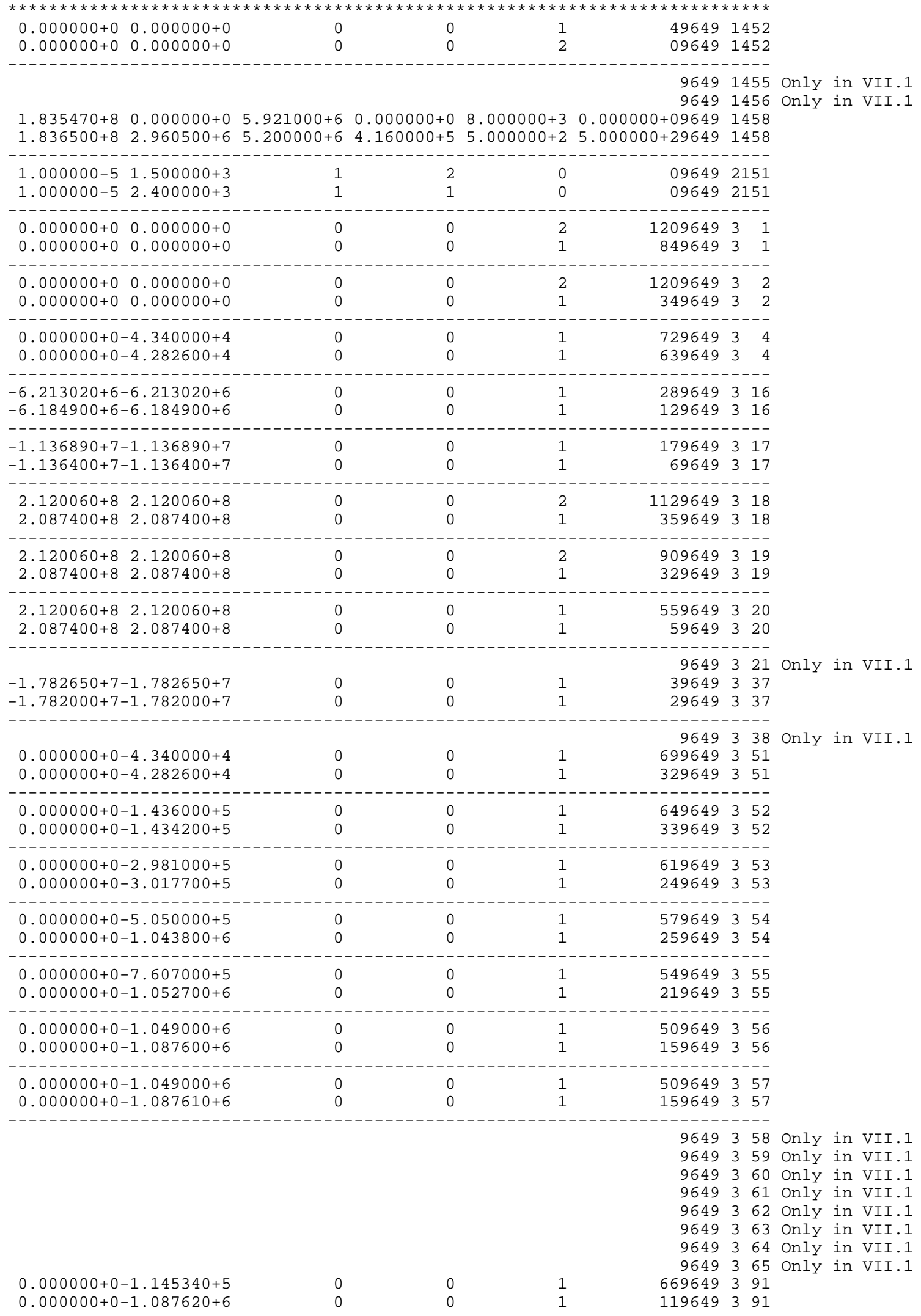




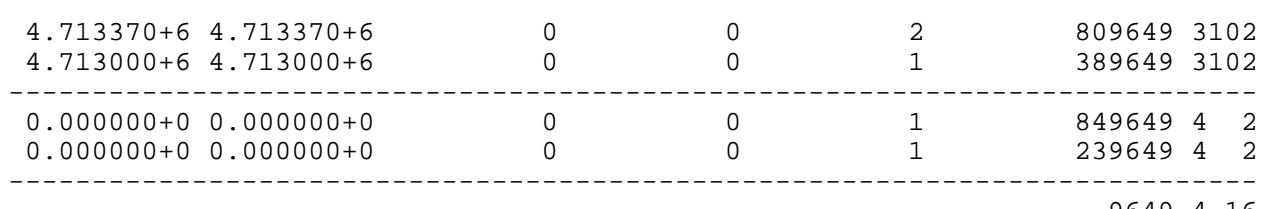

9649416 only in VII.0 9649417 Only in VII.0 9649419 only in VII.0 9649420 Only in VII.0 9649437 Only in VII.0 9649451 only in VII.0 9649452 only in VII.0 9649453 only in VII.0 9649454 only in VII.0 9649455 only in VII.0 9649456 only in VII.0 9649457 only in VII.0 9649491 Only in VII.0 9649516 only in VII.0 $0.000000+0 \quad 0.000000+0$ $\odot$
0

\begin{tabular}{rrr}
0 & 1 & 1 \\
0 & 7 & 1 \\
\hline & - & -
\end{tabular}
29649518 $-3.000000+7 \quad 0.000000+0$

$96-\mathrm{Cm}-249$

\begin{tabular}{|c|c|c|c|c|c|c|}
\hline 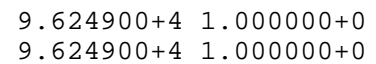 & 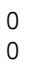 & $\begin{array}{l}\odot \\
1\end{array}$ & $\begin{array}{l}1 \\
2\end{array}$ & \multicolumn{2}{|c|}{096522151} & \\
\hline \multicolumn{7}{|c|}{ 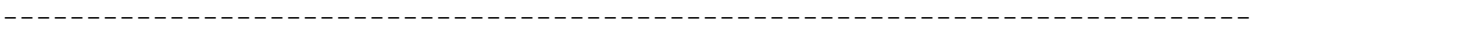 } \\
\hline $0.000000+\odot \quad 0.00000 \odot+\odot$ & $\odot$ & $\odot$ & 1 & 11796523 & 1 & \\
\hline $0.000000+0 \quad 0.000000+0$ & ○ & $\odot$ & 2 & 9996523 & 1 & \\
\hline \multicolumn{7}{|c|}{ 年 } \\
\hline \multirow{2}{*}{ 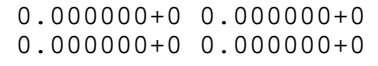 } & $\odot$ & $\odot$ & 1 & 10896523 & 2 & \\
\hline & $\odot$ & $\odot$ & 2 & 9996523 & 2 & \\
\hline \multicolumn{7}{|c|}{ 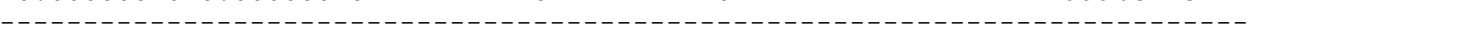 } \\
\hline \multirow{2}{*}{$\begin{array}{l}\odot .0 \odot \odot \odot \odot \odot+\odot-2.62280 \odot+4 \\
\odot .0 \odot \odot \odot \odot \odot+\odot-2.62340 \odot+4\end{array}$} & $\odot$ & $\odot$ & 1 & 8196523 & 4 & \\
\hline & $\odot$ & $\odot$ & 1 & 6596523 & 4 & \\
\hline \multicolumn{7}{|c|}{ 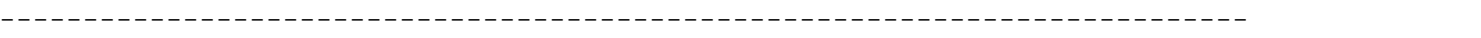 } \\
\hline \multirow{2}{*}{$\begin{array}{l}-4.713370+6-4.713370+6 \\
-4.713400+6-4.713400+6\end{array}$} & $\odot$ & $\odot$ & 1 & 3296523 & 16 & \\
\hline & $\odot$ & $\odot$ & 1 & 3096523 & 16 & \\
\hline \multicolumn{7}{|c|}{ 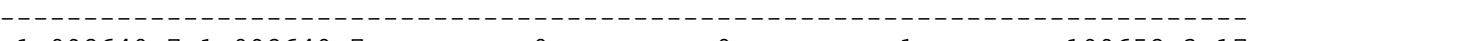 } \\
\hline \multirow{2}{*}{$\begin{array}{l}-1.092640+7-1.092640+7 \\
-1.092480+7-1.092480+7\end{array}$} & $\odot$ & $\odot$ & 1 & 1996523 & 17 & \\
\hline & $\odot$ & $\odot$ & 1 & 1896523 & 17 & \\
\hline \multicolumn{7}{|c|}{ - - - - - - - - - - - - - - - - - - - - - - - - - - - - - - - - - - - - - - - - - - - - - - - - - - } \\
\hline \multirow{2}{*}{$\begin{array}{ll}2.1300 \odot \odot+8 & 2.1300 \odot \odot+8 \\
2.00 \odot \odot \odot \odot+8 & 2.00 \odot \odot \odot \odot+8\end{array}$} & $\odot$ & $\odot$ & 1 & 10196523 & 18 & \\
\hline & $\odot$ & $\odot$ & 2 & 4596523 & 18 & \\
\hline \multicolumn{7}{|c|}{ 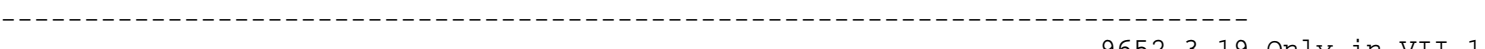 } \\
\hline & & & & 96523 & 19 & Only in VII.1 \\
\hline & & & & 96523 & 20 & Only in VII.1 \\
\hline & & & & $\begin{array}{ll}9652 & 3 \\
9652 & 3\end{array}$ & 21 & Only in VII.1 \\
\hline & & & & 96523 & 31 & 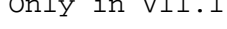 \\
\hline
\end{tabular}

9649519 only in VII.0 9649520 only in VII.e 9649537 only in VII.0 9649591 Only in VII.0 9649616 Only in VII.1 9649617 Only in VII.1 9649637 only in VII.1 9649651 Only in VII.1 9649652 only in VII.1 9649653 only in VII.1 9649654 only in VII.1 9649655 only in VII.1 9649656 Only in VII.1 9649657 only in VII.1 9649658 only in VII.1 9649659 Only in VII.1 9649660 only in VII.1 9649661 only in VII.1 9649662 only in VII.1 9649663 only in VII.1 9649664 Only in VII.1 9649665 Only in VII.1 9649691 only in VII.1 96496102 only in VII.1 


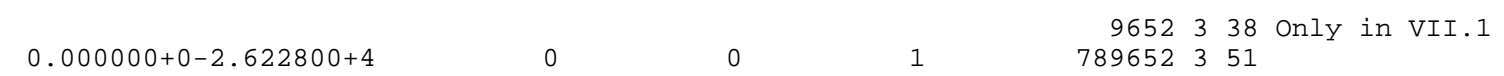

\begin{tabular}{|c|c|c|c|c|}
\hline $\begin{array}{l}\odot . \odot \odot \odot \odot \odot \odot+\odot-2.6228 \odot \odot+4 \\
\odot . \odot \odot \odot \odot \odot \odot+\odot-2.62340 \odot+4\end{array}$ & $\begin{array}{l}\odot \\
0\end{array}$ & $\begin{array}{l}\odot \\
\odot\end{array}$ & $\begin{array}{l}1 \\
1\end{array}$ & $\begin{array}{lll}789652 & 3 & 51 \\
629652 & 3 & 51\end{array}$ \\
\hline \multicolumn{5}{|c|}{ 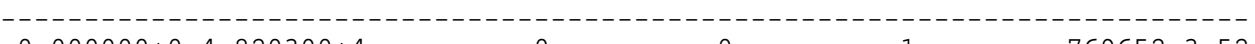 } \\
\hline$\odot .00 \odot \odot \odot \odot+\odot-4.820300+4$ & $\odot$ & $\odot$ & 1 & 769652352 \\
\hline $0.000000+0-4.820300+4$ & $\odot$ & 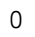 & 1 & 609652352 \\
\hline \multicolumn{5}{|c|}{ 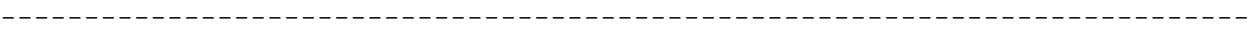 } \\
\hline $0.000000+0-4.875800+4$ & $\odot$ & $\odot$ & 1 & 759652353 \\
\hline $0.000000+0-4.874300+4$ & $\odot$ & $\odot$ & 1 & 5996523 \\
\hline \multicolumn{5}{|c|}{ - - - - - - - - - - - - - - - - - - - - - - - - - - - - - - - - - - - - - - - - - - - - - } \\
\hline $0.000000+0-1.100000+5$ & $\odot$ & $\odot$ & 1 & 719652354 \\
\hline $0.000000+0-1.090000+5$ & $\odot$ & $\odot$ & 1 & 5896523 \\
\hline \multicolumn{5}{|c|}{ 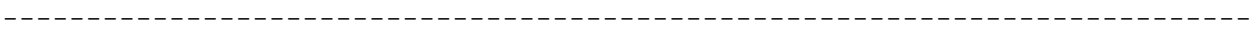 } \\
\hline $0.000000+0-1 \cdot 101540+5$ & $\odot$ & $\odot$ & 1 & 709652355 \\
\hline $0.000000+0-1.101600+5$ & $\odot$ & $\odot$ & 1 & 439652355 \\
\hline \multicolumn{5}{|c|}{ 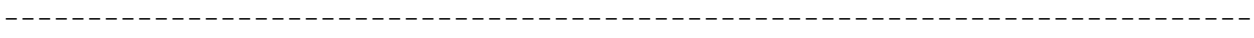 } \\
\hline$\odot .0 \odot \odot \odot \odot \odot+\odot-1.460 \odot \odot \odot+5$ & $\odot$ & $\odot$ & 1 & 679652356 \\
\hline$\odot .000000+\odot-1.460000+5$ & $\odot$ & $\odot$ & 1 & 429652356 \\
\hline \multicolumn{5}{|c|}{ 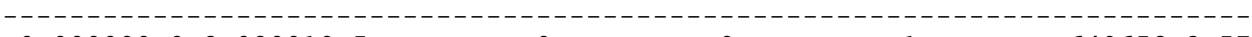 } \\
\hline$\odot .0 \odot \odot \odot \odot \odot+\odot-2.08 \odot \odot 10+5$ & $\odot$ & $\odot$ & 1 & 649652357 \\
\hline$\odot .0000 \odot \odot+\odot-2.079900+5$ & $\odot$ & $\odot$ & 1 & 399652357 \\
\hline \multicolumn{5}{|c|}{ 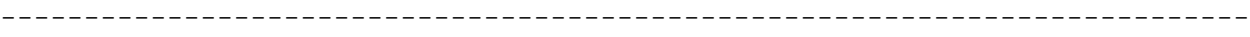 } \\
\hline $0.000000+0-2.200000+5$ & $\odot$ & $\odot$ & 1 & 639652358 \\
\hline $0.000000+0-2.200000+5$ & $\odot$ & $\odot$ & 1 & 389652358 \\
\hline \multicolumn{5}{|c|}{ - - - - - - - - - - - - - - - - - - - - - - - - - - - - - - - - - - - - - - - - - - - - - - - - - } \\
\hline $0.000000+0-2.420080+5$ & $\odot$ & $\odot$ & 1 & 629652359 \\
\hline$\odot .0 \odot \odot \odot \odot \odot+\odot-2.420100+5$ & $\odot$ & $\odot$ & 1 & 379652359 \\
\hline \multicolumn{5}{|c|}{ 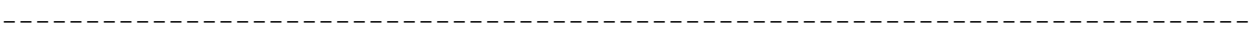 } \\
\hline$\odot .00000 \odot+\odot-2.421700+5$ & $\odot$ & $\odot$ & 1 & 619652360 \\
\hline$\odot .0 \odot \odot \odot \odot \odot+\odot-2.88990 \odot+5$ & $\odot$ & $\odot$ & 1 & 359652360 \\
\hline \multicolumn{5}{|c|}{ 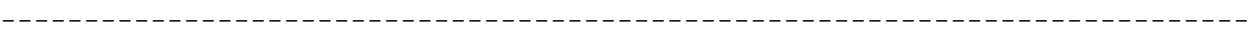 } \\
\hline $0.000000+0-2.890060+5$ & $\odot$ & $\odot$ & 1 & 599652361 \\
\hline$\odot . \odot \odot \odot \odot \odot \odot+\odot-3 . \odot \odot \odot \odot \odot \odot+5$ & $\odot$ & $\odot$ & 1 & 339652361 \\
\hline \multicolumn{5}{|c|}{ 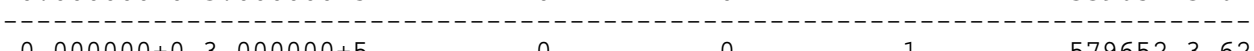 } \\
\hline$\odot .0 \odot \odot \odot \odot \odot+\odot-3 . \odot \odot \odot \odot \odot \odot+5$ & $\odot$ & $\odot$ & 1 & 5796523 \\
\hline$\odot . \odot \odot \odot \odot \odot \odot+\odot-3.5 \odot \odot \odot \odot \odot+5$ & $\odot$ & $\odot$ & 1 & 3296523 \\
\hline
\end{tabular}

9652363 only in VII.0 9652364 only in VII.0 9652365 only in VII.0 9652366 only in VII.0 9652367 only in VII.0 9652368 only in VII.0 9652369 only in VII.0

$\odot . \odot \odot \odot \odot \odot \odot+\odot-1 . \odot 47980+5$ $0.000000+0-6.880000+5$

$5.832430+65.832430+6$

$\begin{array}{ll}5.832430+6 & 5.832430+6 \\ 5.832430+6 & 5.832430+6\end{array}$

5

- . - . - . . . . . . . . . .

$\odot .0 \odot \odot \odot \odot \odot+\odot \quad \odot .0 \odot \odot \odot \odot \odot+\odot$

$\odot .000 \odot \odot \odot+\odot \quad \odot .0000 \odot \odot+\odot$

0
0
-
0
0

$\odot$
0
$---\cdot-$
0
0
-----
0
0

729652391 189652391

$\begin{array}{lr}1 & 189652391\end{array}$

$1 \quad 10196523102$

26696523102

9652416 only in VII.0 9652417 only in VII.0 9652451 only in VII.0 9652452 only in VII.0 9652453 only in VII.0 9652454 only in VII.0 9652455 only in VII.0 9652456 only in VII.0 9652457 only in VII.0 9652458 only in VII.0 9652459 only in VII.0 9652460 Only in VII.0 9652461 only in VII.0 9652462 only in VII.0 9652463 only in VII.0 9652464 only in VII.0 9652465 only in VII.0 9652466 only in VII.0 9652467 only in VII.0 9652468 only in VII.0 9652469 only in VII.0 9652491 Only in VII.0 


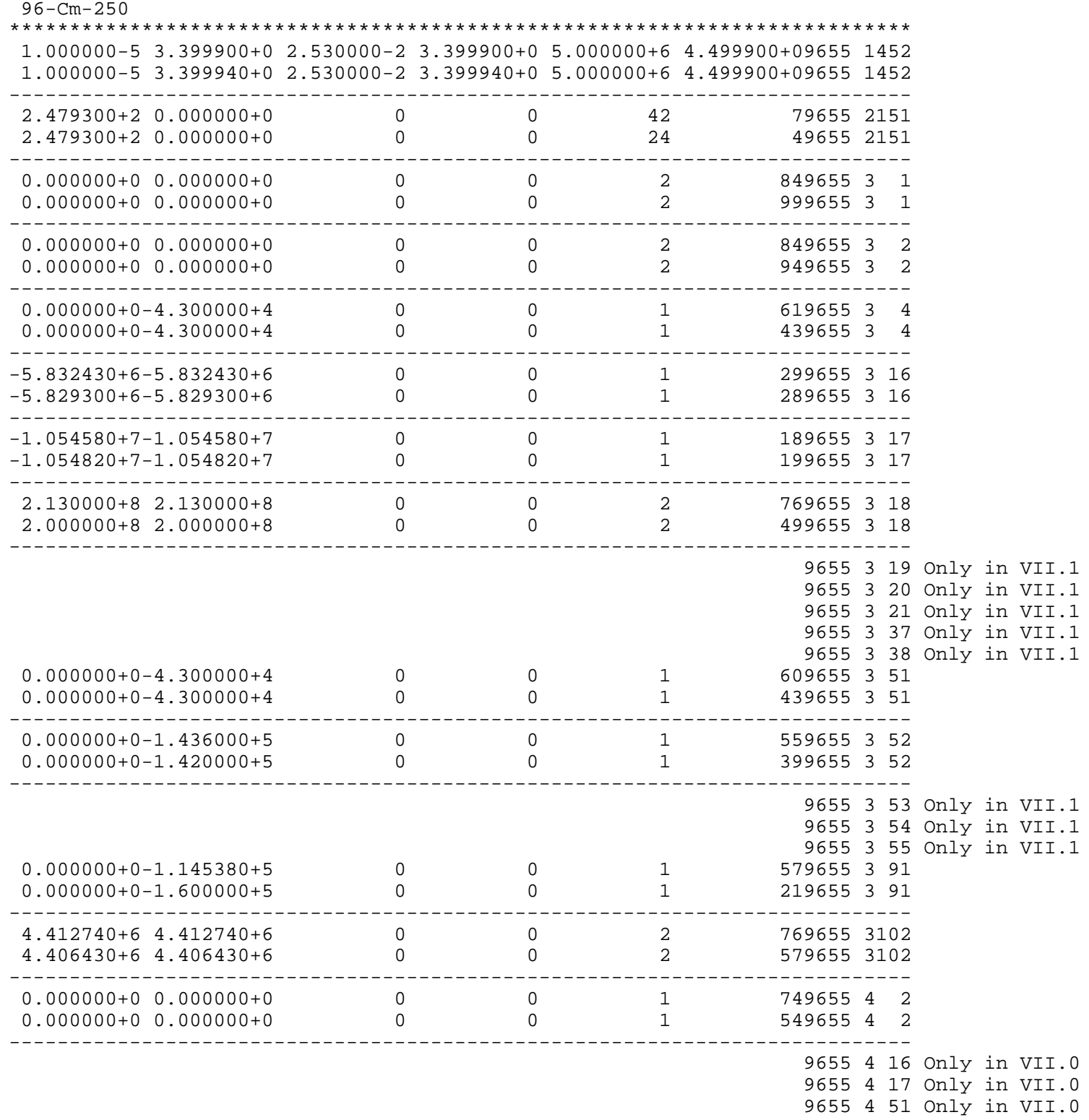


$0.000000+\odot \quad \odot, 000000+\odot$ $-2.000000+7 \quad 0.000000+0$
9655452 only in VII.0 9655491 Only in VII.0 9655516 only in VII.0 9655517 only in VII.0 29655518 29655518

9655591 Only in VII.0 9655616 only in VII.1 9655617 only in VII.1 9655637 Only in VII.1 9655651 Only in VII.1 9655652 only in VII.1 9655653 only in VII.1 9655654 only in VII.1 9655655 only in VII.1 9655691 only in VII.1 96556102 Only in VII.1

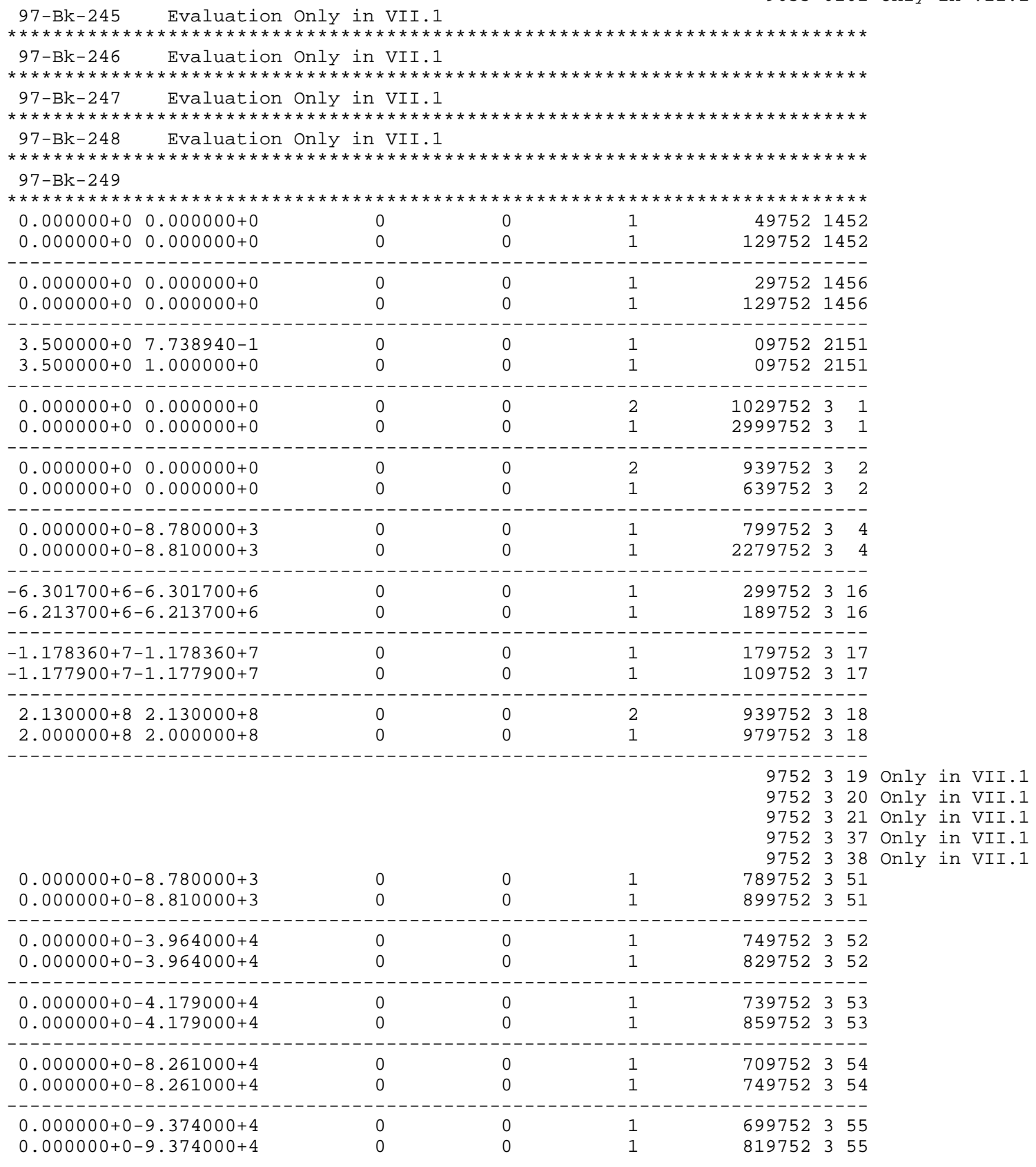




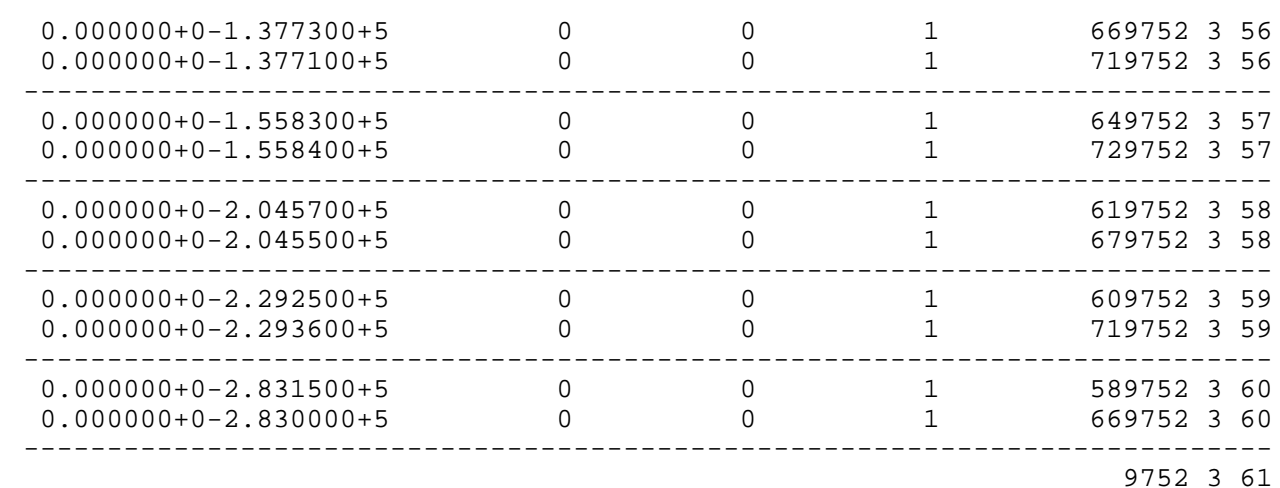

9752361 only in VII.O 9752362 only in VII.0 9752363 only in VII.0 9752364 only in VII.0 9752365 only in VII.0 9752366 only in VII.0 9752367 only in VII.0 9752368 only in VII.0 $\odot .000000+0-1.104450+5$
$\odot .00000 \odot+\odot-5.190000+5$ $\odot$ $\odot$ 1 689752391 $0.000000+0-5.190000+5$

\begin{tabular}{|c|}
\hline 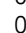 \\
\hline
\end{tabular}

$4.969570+6 \quad 4.969570+6$ $4.970000+6 \quad 4.970000+6$

0

$\begin{array}{rrr}2 & 939752 & 3102 \\ 1 & 1239752 & 3102\end{array}$

$0 \quad 0 \quad 0 \quad 1 \quad 12397523102$

\section{$\odot .00000 \odot+\odot \quad 2.469350+2$ $0.000000+02.469400+2$}

.....

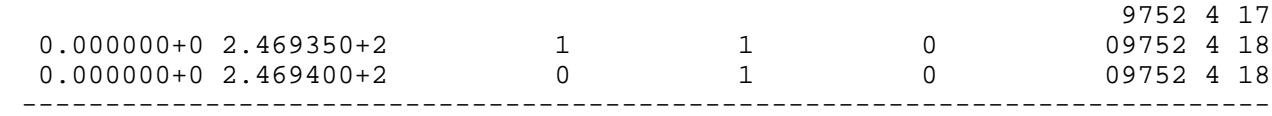

0
0

97523103 only in VII.0 97523107 only in VII.0 0975242 0975242

9752416 only in VII.0 9752417 Only in VII.0 09752418 09752418

9752451 Only in VII.0 9752452 only in VII.0 9752453 only in VII.0 9752454 Only in VII.0 9752455 only in VII.0 9752456 only in VII.0 9752457 only in VII.0 9752458 only in VII.0 9752459 only in VII.0 9752460 only in VII.0 9752461 only in VII.0 9752462 only in VII.0 9752463 only in VII.0 9752464 only in VII.0 9752465 only in VII.0 9752466 only in VII.0 9752467 only in VII.0 9752468 only in VII.0 9752491 Only in VII.0 9752516 only in VII.0 9752517 Only in VII.0

$0.000000+0 \quad 0.000000+0$ $\begin{array}{lll}-2.000000+7 & 0.000000+0\end{array}$

\begin{abstract}
$\odot$
\end{abstract}
0

- . - . - . - . - . - . . . .
29752518

29752518

9752591 only in VII. 0 9752616 only in VII.1 9752617 only in VII.1 9752637 only in VII.1 9752651 only in VII.1 9752652 only in VII.1 9752653 only in VII.1 9752654 only in VII.1 9752655 only in VII.1 9752656 only in VII.1 9752657 only in VII.1 9752658 Only in VII.1 
$97-\mathrm{Bk}-250$

9752659 only in VII.1 9752660 0nly in VII.1 9752691 only in VII.1 97526102 only in VII.1

\begin{tabular}{|c|c|c|c|c|c|}
\hline 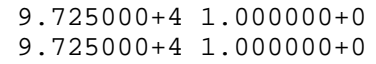 & $\begin{array}{l}\odot \\
\odot\end{array}$ & $\begin{array}{l}0 \\
1\end{array}$ & $\begin{array}{l}1 \\
2\end{array}$ & $\begin{array}{ll}09755 & 2151 \\
09755 & 2151\end{array}$ & \\
\hline 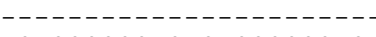 & --- & --- & -- & -------- & \\
\hline$\odot .000000+\odot \quad \odot .00000 \odot+0$ & $\odot$ & $\odot$ & 1 & 12097553 & \\
\hline $0.000000+0 \quad 0.000000+0$ & 0 & $\odot$ & 2 & $\begin{array}{lll}669755 & 3 & 1\end{array}$ & \\
\hline 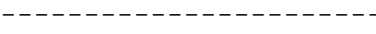 & -- & -- & -- & --------- & \\
\hline $0.000000+0 \quad 0.000000+0$ & 0 & $\odot$ & 1 & 12097553 & \\
\hline$\odot . \odot \odot \odot \odot \odot \odot+\odot \quad \odot . \odot \odot \odot \odot \odot \odot+\odot$ & $\odot$ & $\odot$ & 2 & 6697553 & \\
\hline 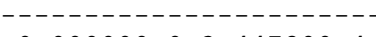 & -- & --- & -- & $-2---1-1$ & \\
\hline$\odot .0 \odot \odot \odot \odot \odot+\odot-3.447200+4$ & 0 & $\odot$ & 1 & 8697553 & \\
\hline$\odot . \odot \odot \odot \odot \odot \odot+\odot-3.4 \odot \odot \odot \odot \odot+4$ & $\odot$ & $\odot$ & 1 & 5897553 & \\
\hline-------------------- & -- & - & -- & ---------- & \\
\hline$-4.969570+6-4.969570+6$ & 0 & $\odot$ & 1 & 319755316 & \\
\hline$-4.969690+6-4.969690+6$ & 0 & $\odot$ & 1 & 179755316 & \\
\hline - - - - - - - - - - - - - - - - - - & -- & $\ldots$ & -- & --------- & \\
\hline$-1 \cdot 127130+7-1 \cdot 127130+7$ & ○ & $\odot$ & 1 & $\begin{array}{lll}189755 & 3 & 17\end{array}$ & \\
\hline$-1 \cdot 118340+7-1 \cdot 118340+7$ & 0 & $\odot$ & 1 & 109755317 & \\
\hline------------------- & -- & - & -- & --------- & \\
\hline $2.144000+8 \quad 2.144000+8$ & $\odot$ & $\odot$ & 1 & 1129755318 & \\
\hline $1.899990+8 \quad 1.899990+8$ & $\odot$ & $\odot$ & 2 & 449755318 & \\
\hline--------------- & -- & -- & -- & ---------- & \\
\hline & & & & 9755319 & Only in VII.1 \\
\hline & & & & 9755320 & Only in VII.1 \\
\hline & & & & 9755321 & Only in VII.1 \\
\hline$-1.675320+7-1.675320+7$ & $\odot$ & $\odot$ & 1 & $\begin{array}{lll}79755 & 3 & 37\end{array}$ & \\
\hline$-1.674910+7-1.674910+7$ & $\odot$ & $\odot$ & 1 & $\begin{array}{lll}59755 & 3 & 37\end{array}$ & \\
\hline----------------- & -- - & -1 & -- & --------- & \\
\hline & & & & 9755338 & Only in VII.1 \\
\hline $0.000000+0-3.447200+4$ & $\odot$ & $\odot$ & 1 & 859755351 & \\
\hline$\odot . \odot \odot \odot \odot \odot \odot+\odot-3.4 \odot \odot \odot \odot \odot+4$ & $\odot$ & $\odot$ & 1 & 589755351 & \\
\hline - - - - - - - - - - - - - - - - - & -- & - & - & 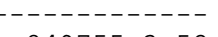 & \\
\hline$\odot . \odot \odot \odot \odot \odot \odot+\odot-3.55870 \odot+4$ & $\odot$ & $\odot$ & 1 & 849755352 & \\
\hline$\odot . \odot \odot \odot \odot \odot \odot+\odot-3.55999 \odot+4$ & $\odot$ & $\odot$ & 1 & 579755352 & \\
\hline - - - - - - - - - - - - - - - - - - & -- & -- & - & 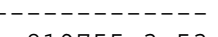 & \\
\hline$\odot .0 \odot \odot \odot \odot \odot+\odot-7.8326 \odot \odot+4$ & $\odot$ & $\odot$ & 1 & 819755353 & \\
\hline$\odot .0 \odot \odot \odot \odot \odot+\odot-7.809990+4$ & $\odot$ & $\odot$ & 1 & 549755353 & \\
\hline - - - - - - - - - - - - - - - - - & -- & -- & - & $2-1-2-1$ & \\
\hline$\odot . \odot \odot \odot \odot \odot \odot+\odot-8 . \odot 258 \odot \odot+4$ & $\odot$ & $\odot$ & 1 & 809755354 & \\
\hline$\odot . \odot \odot \odot \odot \odot \odot+\odot-8.63999 \odot+4$ & $\odot$ & $\odot$ & 1 & 529755354 & \\
\hline - - - - - - - - - - - - - - - & -- & - & - & 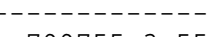 & \\
\hline$\odot . \odot \odot \odot \odot \odot \odot+\odot-8.64 \odot \odot \odot \odot+4$ & $\odot$ & $\odot$ & 1 & 799755355 & \\
\hline$\odot . \odot \odot \odot \odot \odot \odot+\odot-9.699990+4$ & $\odot$ & $\odot$ & 1 & 519755355 & \\
\hline - - - - - - - - - - - - - - - - - & -- & - & -- & 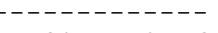 & \\
\hline$\odot .000 \odot \odot \odot+\odot-9.749300+4$ & $\odot$ & $\odot$ & 1 & 789755356 & \\
\hline$\odot .0 \odot \odot \odot \odot \odot+\odot-1.0410 \odot \odot+5$ & $\odot$ & $\odot$ & 1 & 499755356 & \\
\hline------------------- & -- & - & -- & --------- & \\
\hline$\odot . \odot \odot \odot \odot \odot \odot+\odot-1 . \odot 38280+5$ & $\odot$ & $\odot$ & 1 & 769755357 & \\
\hline$\odot . \odot \odot \odot \odot \odot \odot+\odot-1.2540 \odot \odot+5$ & $\odot$ & $\odot$ & 1 & 489755357 & \\
\hline 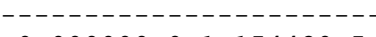 & -- & - & -- & 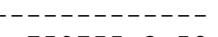 & \\
\hline$\odot . \odot \odot \odot \odot \odot \odot+\odot-1.15442 \odot+5$ & $\odot$ & $\odot$ & 1 & 759755358 & \\
\hline ๑ . $000000+0-1.290000+5$ & $\odot$ & $\odot$ & 1 & 479755358 & \\
\hline - - - - - - - - - - - - - - - - - - & & -- & -- & ----------- & \\
\hline$\odot .0 \odot \odot \odot \odot \odot+\odot-1.250 \odot 70+5$ & $\odot$ & $\odot$ & 1 & 739755359 & \\
\hline 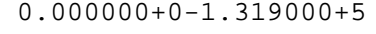 & ○ & $\odot$ & 1 & 469755359 & \\
\hline - - - - - - - - - - - - & -- & - & - & 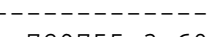 & \\
\hline$\odot . \odot \odot \odot \odot \odot \odot+\odot-1.3 \odot 492 \odot+5$ & 0 & $\odot$ & 1 & 729755360 & \\
\hline$\odot .0 \odot \odot \odot \odot \odot+\odot-1.570 \odot \odot \odot+5$ & $\odot$ & $\odot$ & 1 & 449755360 & \\
\hline - - - - - - - - - - - - - - - - - - & -- & - & - & $-1---1$ & \\
\hline$\odot . \odot \odot \odot \odot \odot \odot+\odot-1.37320 \odot+5$ & $\odot$ & $\odot$ & 1 & 719755361 & \\
\hline$\odot . \odot \odot \odot \odot \odot \odot+\odot-1.67 \odot \odot \odot \odot+5$ & $\odot$ & $\odot$ & 1 & 439755361 & \\
\hline------------------ & -- & $-\ldots$ & - & -------- & \\
\hline$\odot . \odot \odot \odot \odot \odot \odot+\odot-1.464720+5$ & 0 & $\odot$ & 1 & 699755362 & \\
\hline$\odot . \odot \odot \odot \odot \odot \odot+\odot-1.7540 \odot \odot+5$ & $\odot$ & $\odot$ & 1 & 429755362 & \\
\hline - - - - - - - - - - - - - - - - - & -- & - & -- & 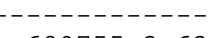 & \\
\hline$\odot .0 \odot \odot \odot \odot \odot+\odot-1.485950+5$ & $\odot$ & $\odot$ & 1 & 689755363 & \\
\hline$\odot .0 \odot \odot \odot \odot \odot+\odot-1.910 \odot \odot \odot+5$ & $\odot$ & $\odot$ & 1 & 419755363 & \\
\hline 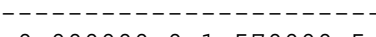 & ? & - & -- & 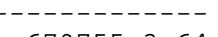 & \\
\hline $0.000000+0-1.570000+5$ & ○ & $\odot$ & 1 & 679755364 & \\
\hline$\odot .000000+0-2.118000+5$ & ○ & $\odot$ & 1 & 399755364 & \\
\hline
\end{tabular}




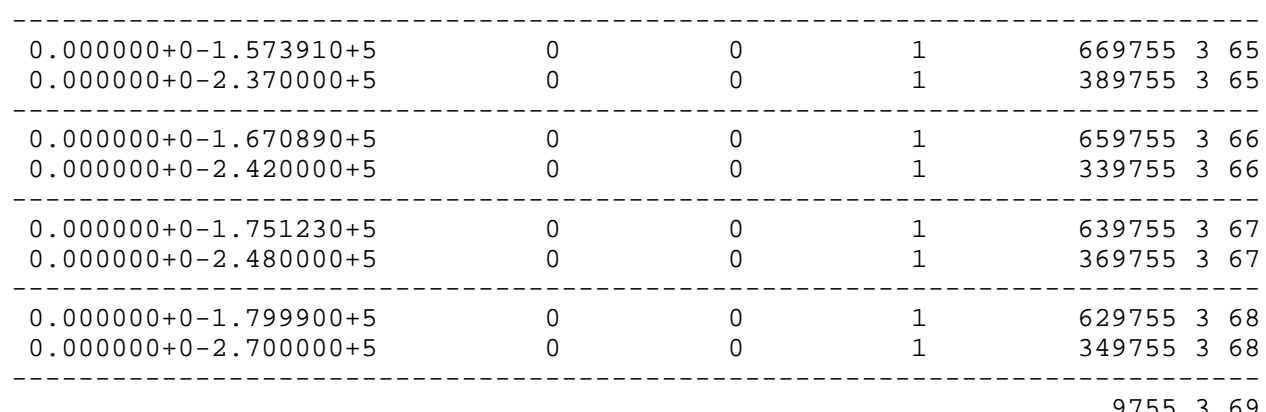

\begin{tabular}{|c|c|c|c|c|c|c|}
\hline & & & & 9755 & 37 & Only in VII.1 \\
\hline$\odot .0 \odot \odot \odot \odot \odot+\odot-1.025350+5$ & $\odot$ & $\odot$ & 1 & 779755 & 39 & \\
\hline $0.000000+0-2.960000+5$ & $\odot$ & $\odot$ & 1 & 339755 & & \\
\hline \multicolumn{7}{|c|}{ 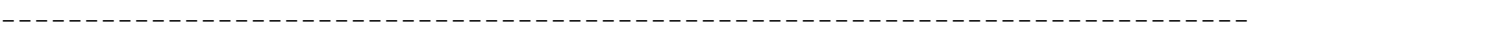 } \\
\hline $5.795070+65.795070+6$ & 0 & $\odot$ & 1 & 1139755 & 310 & \\
\hline $8.649990+58.649990+5$ & 0 & $\odot$ & 2 & 629755 & 310 & \\
\hline \multicolumn{7}{|c|}{ - - - - - - - - - - - - - - - - - - - - - - - - - - - - - - - - - - - - - - - - - - - - } \\
\hline $0.000000+0 \quad 0.000000+0$ & 0 & $\odot$ & 1 & 1099755 & 4 & 2 \\
\hline $0.000 \odot \odot \odot+\odot \quad \odot .00 \odot \odot \odot \odot+\odot$ & $\odot$ & $\odot$ & 1 & 709755 & 4 & 2 \\
\hline \multicolumn{7}{|c|}{ - - - - - - - - - - - - - - - - - - - - - - - - - - - - - - - - - - - - - - - - - - - - - - - } \\
\hline & & & & 9755 & & 6 only in VII.0 \\
\hline & & & & 9755 & & 7 only in VII.0 \\
\hline $0.000000+02.479300+2$ & 1 & 1 & $\odot$ & ๑9755 & 418 & \\
\hline $.000000+02$. & 0 & 1 & $\theta$ & & & \\
\hline
\end{tabular}

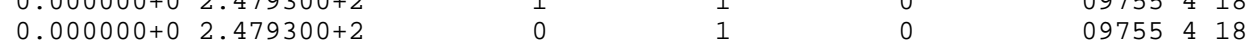

9755437 only in VII.0 9755451 only in VII.0 9755452 only in VII.0 9755453 only in VII.0 9755454 only in VII.0 9755455 only in VII.0 9755456 only in VII.0 9755457 only in VII.0 9755458 only in VII.0 9755459 only in VII.0 9755460 Only in VII.0 9755461 only in VII.0 9755462 only in VII.0 9755463 only in VII.0 9755464 only in VII.0 9755465 only in VII.0 9755466 only in VII.0 9755467 only in VII.0 9755468 Only in VII.0 9755491 only in VII.0 9755516 only in VII.0

$\odot .00000 \odot+\odot \quad 0.000 \odot \odot \odot+\odot$ $-2.000000+7 \quad 0.000000+0$
0

0

1
1
9755517 only in VII.0 $29755 \quad 518$

29755518
9755537 only in VII. $\odot$ 9755591 Only in VII.0 9755616 only in VII.1 9755617 only in VII.1 9755637 only in VII.1 9755651 Only in VII.1 9755652 only in VII.1 9755653 only in VII.1 9755654 only in VII.1 9755655 only in VII.1 9755656 only in VII.1 9755657 only in VII.1 9755658 only in VII.1 9755659 only in VII.1 9755660 only in VII.1 9755661 only in VII.1 9755662 only in VII.1 9755663 only in VII.1 9755664 only in VII.1 9755665 only in VII.1 9755666 Only in VII.1 
9755667 only in VII.1 9755668 0nly in VII.1 9755669 only in VII.1 9755670 only in VII.1 9755691 Only in VII.1

98-Cf-246 Evaluation only in VII.1

97556102 only in VII.1

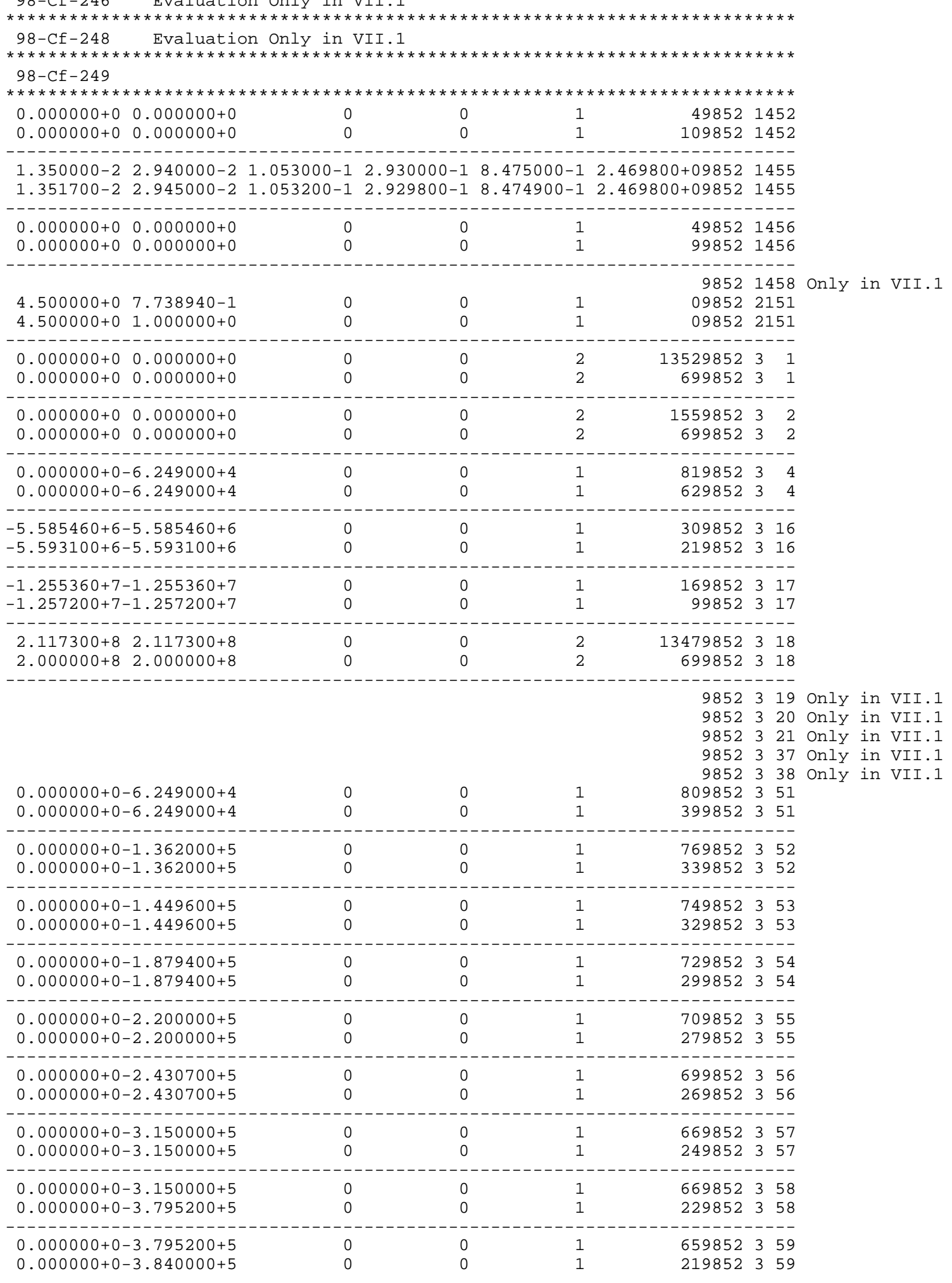




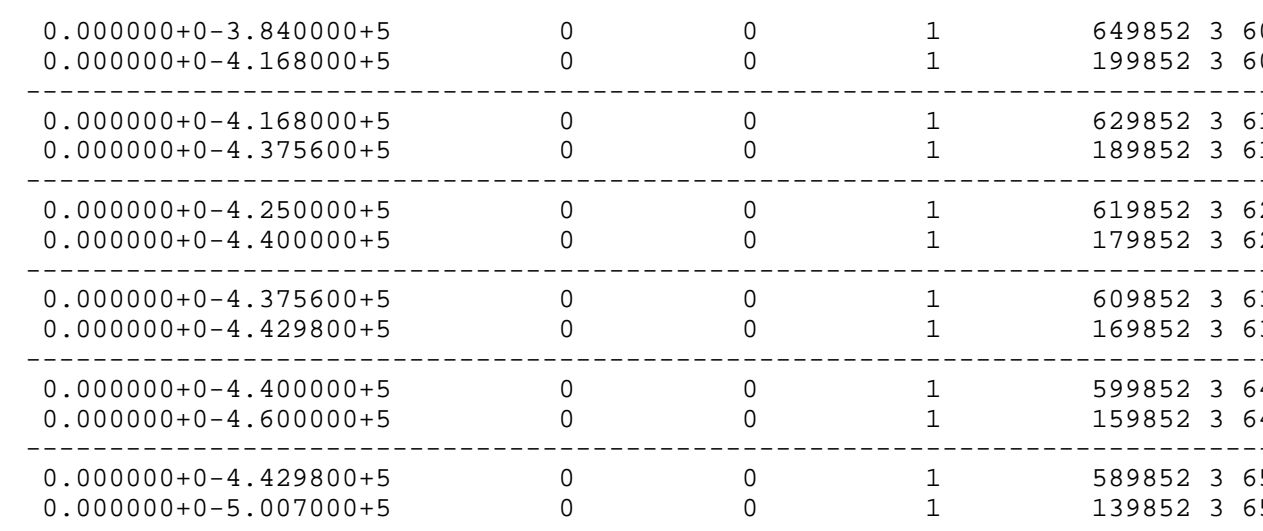

9852366 Only in VII. 1
VII. 1

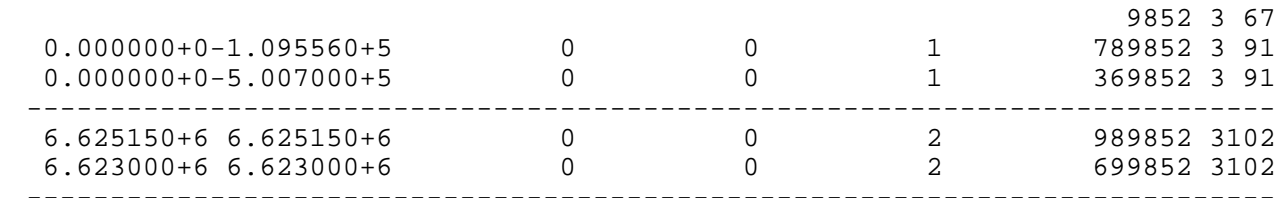

9852 3103 Only in VII. 0
9852 3107 Only in VII. 0

\begin{tabular}{|c|c|c|}
\hline$\odot .0000 \odot \odot+\odot \quad 2.469350+2$ & $\odot$ & 2 \\
\hline $0.000000+02.469400+2$ & $\odot$ & 2 \\
\hline
\end{tabular}
98523107 Only in VII.0 0985242 0985242

\begin{tabular}{|c|c|c|}
\hline$\odot . \odot \odot \odot \odot \odot \odot+\odot \quad 2.46935 \odot+2$ & 1 & 1 \\
\hline $0.000000+0 \quad 2.469400+2$ & $\odot$ & 1 \\
\hline
\end{tabular}
9852416 only in VII.O 9852417 Only in VII.0 09852418 09852418 9852451 only in VII. 0 9852452 only in VII.0 9852453 only in VII.0 9852454 only in VII.0 9852455 only in VII.0 9852456 only in VII.0 9852457 only in VII.0 9852458 only in VII.0 9852459 only in VII.0 9852460 Only in VII.0 9852461 Only in VII.0 9852462 only in VII.0 9852463 only in VII.0 9852464 only in VII.0 9852465 only in VII.0 9852491 only in VII.0 9852516 only in VII.0 9852517 only in VII.0 29852518 29852518 $0.000000+0 \quad 0.000000+0$ $\odot$
0 $-2.000000+7 \quad 0.000000+0$ $\begin{array}{ll}1 & 1 \\ 7 & 1\end{array}$

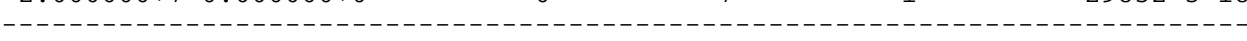

9852591 Only in VII.0 9852616 only in VII.1 9852617 only in VII.1 9852637 only in VII.1 9852651 only in VII.1 9852652 only in VII.1 9852653 only in VII.1 9852654 only in VII.1 9852655 only in VII.1 9852656 only in VII.1 9852657 only in VII.1 9852658 only in VII.1 9852659 only in VII.1 9852660 only in VII.1 9852661 only in VII.1 9852662 only in VII.1 9852663 only in VII.1 9852664 Only in VII.1 
9852665 only in VII.1 9852666 Only in VII.1 9852667 only in VII.1 9852691 only in VII.1

98526102 only in VII.1

98-Cf-250

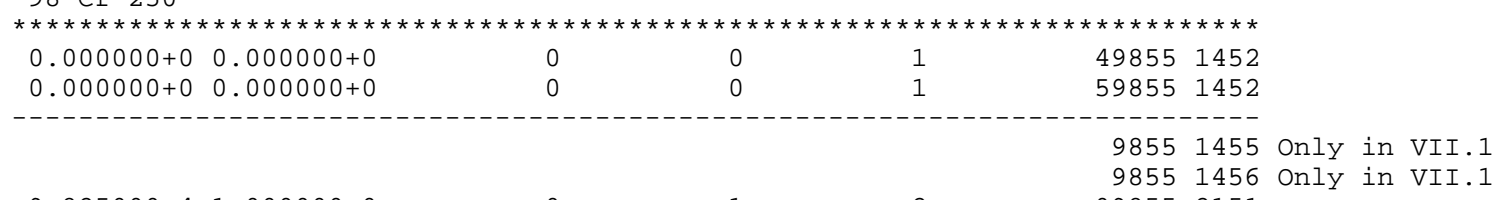

\begin{tabular}{|c|c|c|c|c|c|c|}
\hline $\begin{array}{ll}9.82500 \odot+4 & 1.0000 \odot \odot+\odot \\
9.82500 \odot+4 & 1.0000 \odot \odot+\odot\end{array}$ & $\begin{array}{l}\odot \\
\odot\end{array}$ & $\begin{array}{l}1 \\
0\end{array}$ & $\begin{array}{l}2 \\
2\end{array}$ & $\begin{array}{l}09855 \\
09855\end{array}$ & $\begin{array}{l}1 \\
21 \\
21\end{array}$ & $\begin{array}{l}151 \\
151\end{array}$ \\
\hline 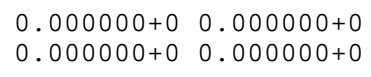 & $\begin{array}{l}0 \\
0\end{array}$ & $\begin{array}{l}\odot \\
\odot\end{array}$ & $\begin{array}{l}2 \\
4\end{array}$ & $\begin{array}{r}959855 \\
1709855\end{array}$ & $\begin{array}{l}3 \\
3\end{array}$ & $\begin{array}{l}1 \\
1\end{array}$ \\
\hline 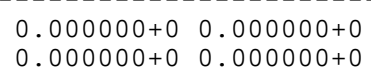 & $\begin{array}{l}0 \\
0\end{array}$ & $\begin{array}{l}\odot \\
\odot\end{array}$ & $\begin{array}{l}2 \\
1\end{array}$ & $\begin{array}{l}909855 \\
759855\end{array}$ & $\begin{array}{l}3 \\
3\end{array}$ & $\begin{array}{l}2 \\
2\end{array}$ \\
\hline $\begin{array}{l}\odot .0 \odot 0 \odot \odot \odot+\odot-4.272200+4 \\
\odot .0 \odot \odot \odot \odot \odot+\odot-4.4819 \odot \odot+4\end{array}$ & $\begin{array}{l}\odot \\
\odot\end{array}$ & $\begin{array}{l}\odot \\
\odot\end{array}$ & $\begin{array}{l}1 \\
1\end{array}$ & $\begin{array}{l}709855 \\
209855\end{array}$ & $\begin{array}{l}3 \\
3\end{array}$ & $\begin{array}{l}4 \\
4\end{array}$ \\
\hline $\begin{array}{l}-6.625150+6-6.625150+6 \\
-6.610000+6-6.610000+6\end{array}$ & $\begin{array}{l}0 \\
\odot\end{array}$ & $\begin{array}{l}\odot \\
\odot\end{array}$ & $\begin{array}{l}1 \\
1\end{array}$ & $\begin{array}{l}289855 \\
199855\end{array}$ & $\begin{array}{l}3 \\
3\end{array}$ & $\begin{array}{l}16 \\
16\end{array}$ \\
\hline $\begin{array}{l}-1.221060+7-1.221060+7 \\
-1.221000+7-1.221000+7\end{array}$ & $\begin{array}{l}0 \\
0\end{array}$ & $\begin{array}{l}\odot \\
\odot\end{array}$ & $\begin{array}{l}1 \\
1\end{array}$ & $\begin{array}{l}169855 \\
129855\end{array}$ & $\begin{array}{l}3 \\
3\end{array}$ & $\begin{array}{l}17 \\
17\end{array}$ \\
\hline $\begin{array}{ll}2.14400 \odot+8 & 2.144000+8 \\
2.0000 \odot \odot+8 & 2.0000 \odot \odot+8\end{array}$ & $\begin{array}{l}0 \\
\odot\end{array}$ & $\begin{array}{l}\odot \\
\odot\end{array}$ & $\begin{array}{l}2 \\
1\end{array}$ & $\begin{array}{l}909855 \\
259855\end{array}$ & & $\begin{array}{l}18 \\
18\end{array}$ \\
\hline
\end{tabular}

r. 9855319 only in VII.1

9855320 Only in VII.1 9855321 only in VII.1 $-1.917880+7-1.917880+7$ $-1.859000+7-1.859000+7$

$\begin{array}{lll}0 & 0 & 1 \\ 0 & 0 & 1\end{array}$

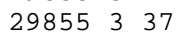
59855337

-

9855338 only in VII.1 9855351 only in VII.1 9855352 Only in VII.1 9855353 only in VII.1 9855354 only in VII.1

$0.000000+0-1.095580+5$ $0.000000+0-4.481920+4$

_......

$5.108490+6 \quad 5.108490+6$

$5.110000+6 \quad 5.110000+6$

\begin{tabular}{|c|c|}
\hline & $\begin{array}{l}\odot \\
\odot\end{array}$ \\
\hline & $\begin{array}{l}\odot \\
\odot\end{array}$ \\
\hline
\end{tabular}

$0.000000+0 \quad 2.479280+2$

$0.000000+\odot \quad 2.478600+2$

$\begin{array}{cccc}0.000000+0 & 2.479280+2 & 0 & 2 \\ 0.000000+0 & 2.478600+2 & 0 & 2\end{array}$

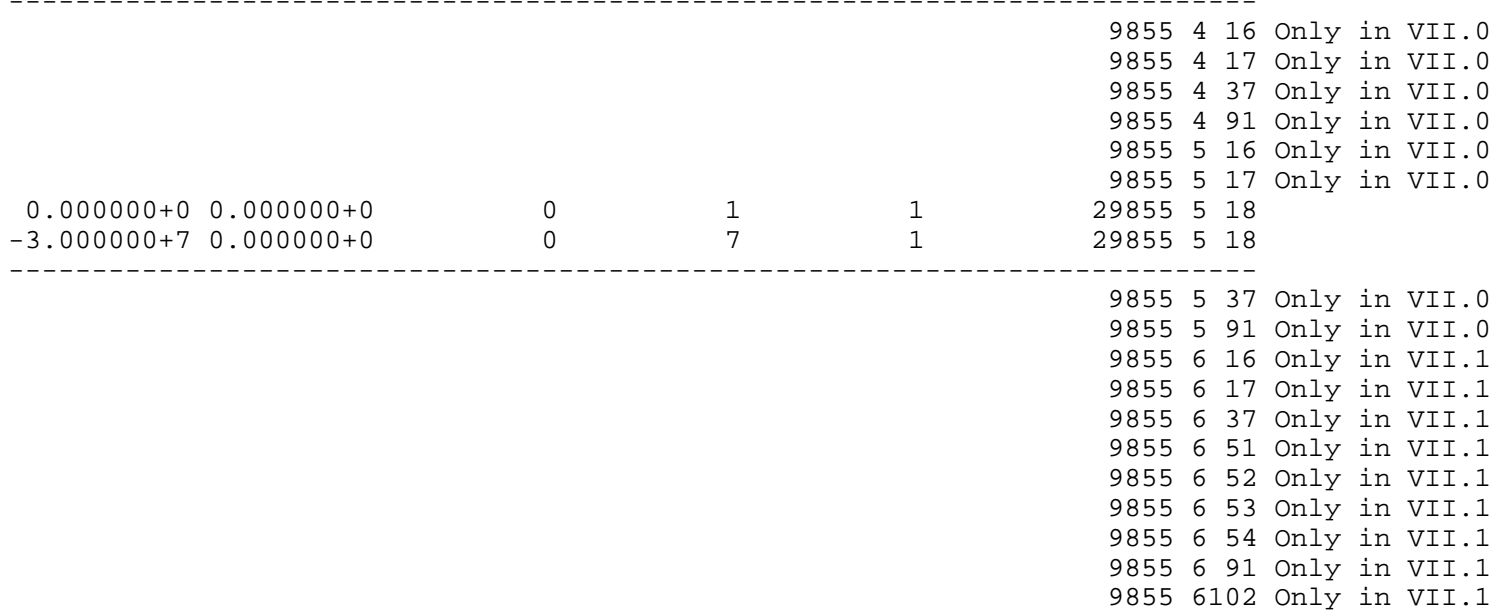




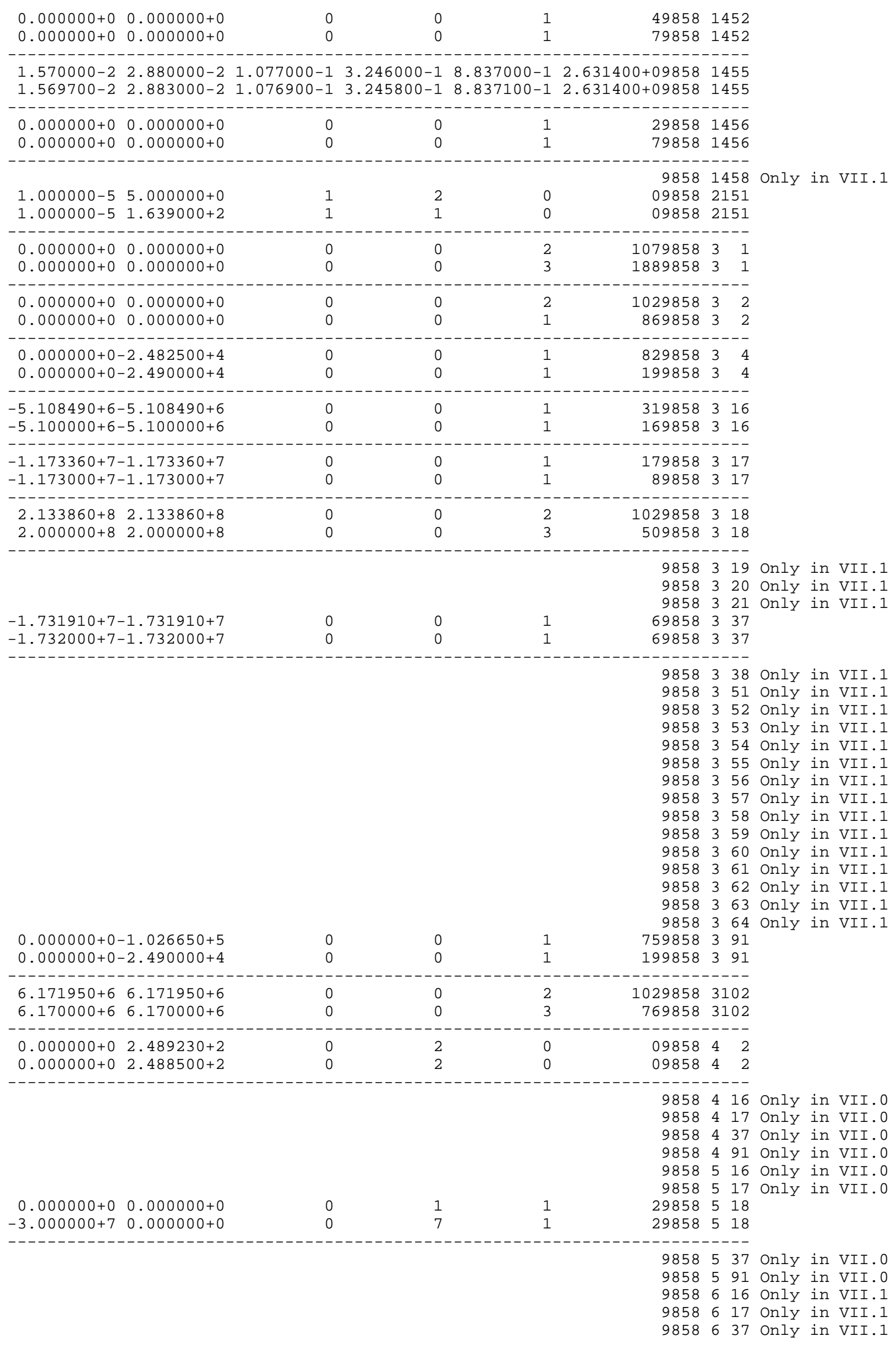


98-Cf -252

9858651 only in VII.1 9858652 0nly in VII.1 9858653 only in VII.1 9858654 Only in VII.1 9858655 Only in VII.1 9858656 only in VII.1 9858657 only in VII.1 9858658 only in VII.1 9858659 only in VII.1 9858660 Only in VII.1 9858661 only in VII.1 9858662 Only in VII.1 9858663 Only in VII.1 9858664 only in VII.1 9858691 Only in VII.1 98586102 Only in VII.1

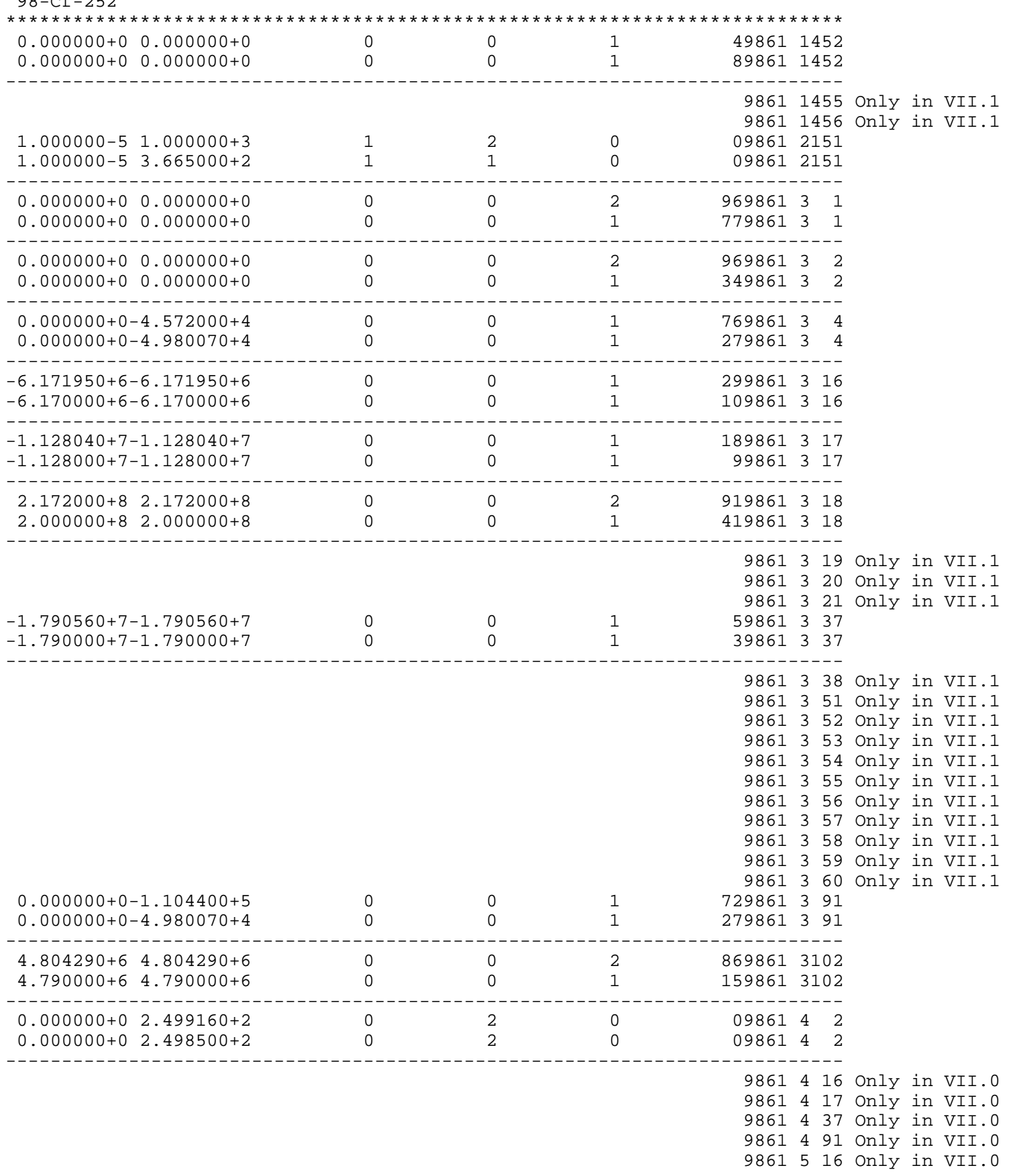




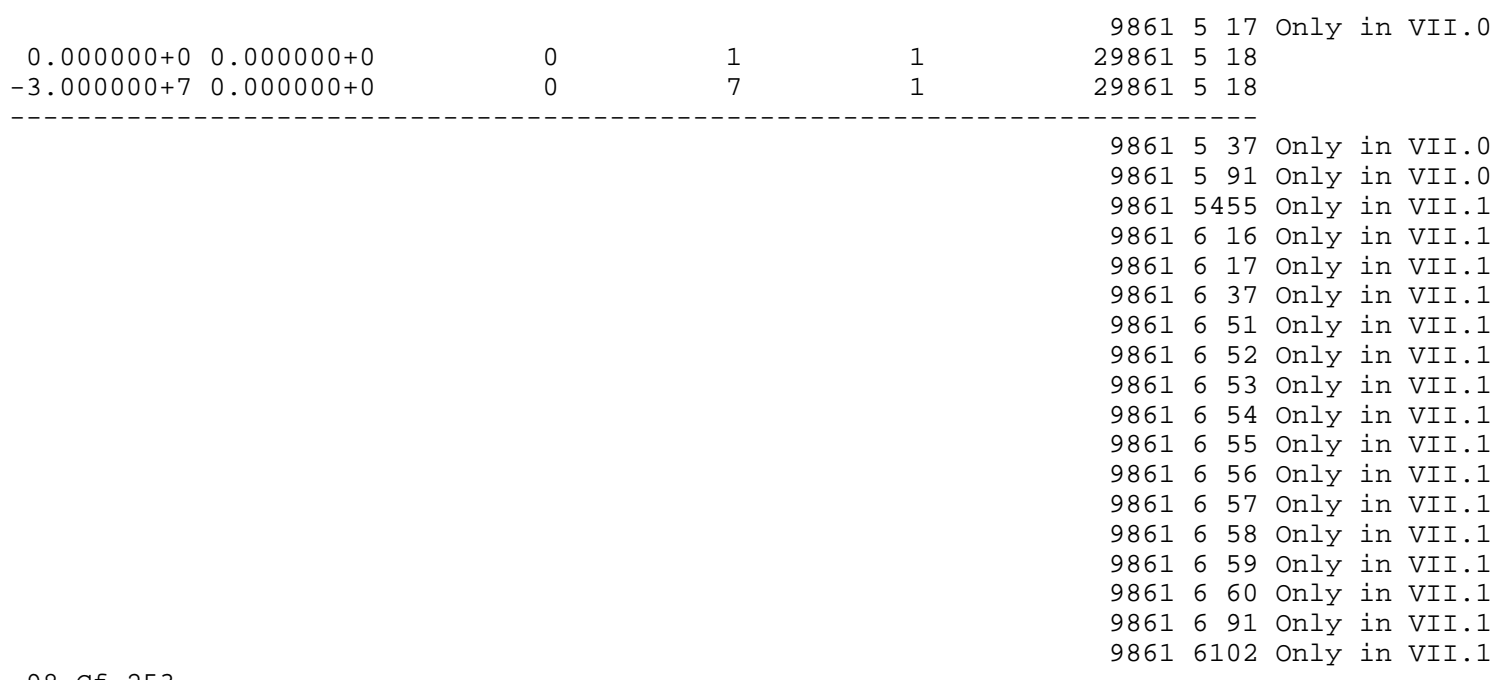

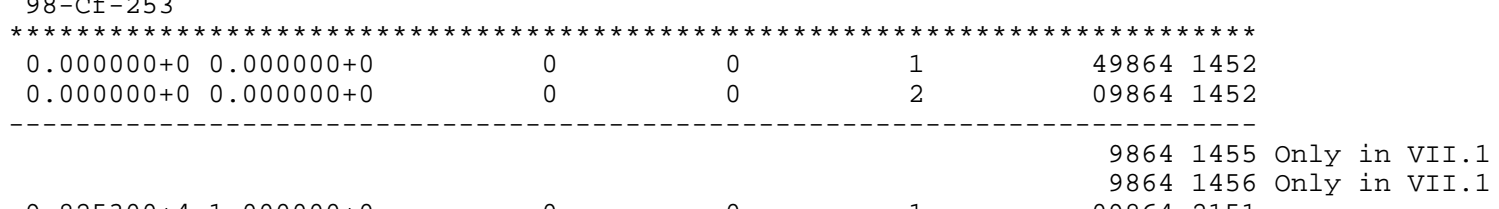

\begin{tabular}{|c|c|c|c|c|c|c|}
\hline 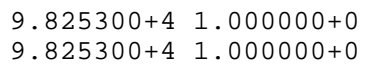 & $\begin{array}{l}\odot \\
\odot\end{array}$ & $\begin{array}{l}\odot \\
1\end{array}$ & $\begin{array}{l}1 \\
2\end{array}$ & $\begin{array}{l}09864 \\
09864\end{array}$ & $\begin{array}{l}2 \\
2\end{array}$ & 151 \\
\hline 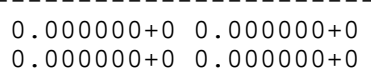 & $\begin{array}{l}0 \\
0\end{array}$ & $\begin{array}{l}\odot \\
\odot\end{array}$ & $\begin{array}{l}1 \\
1\end{array}$ & $\begin{array}{r}1089864 \\
79864\end{array}$ & & $\begin{array}{l}1 \\
1\end{array}$ \\
\hline $\begin{array}{ll}\odot . \diamond \odot \odot \odot \odot \odot+\odot & \odot . \diamond \odot \odot \odot \odot \odot+\odot \\
\odot . \diamond \odot \odot \odot \odot \odot+\odot & \odot . \diamond \odot \odot \odot \odot \odot+\odot\end{array}$ & $\begin{array}{l}\odot \\
\ominus\end{array}$ & $\begin{array}{l}\ominus \\
\ominus\end{array}$ & $\begin{array}{l}1 \\
1\end{array}$ & $\begin{array}{r}1089864 \\
69864\end{array}$ & & 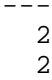 \\
\hline
\end{tabular}

986434 only in VII.1 9864316 only in VII.1

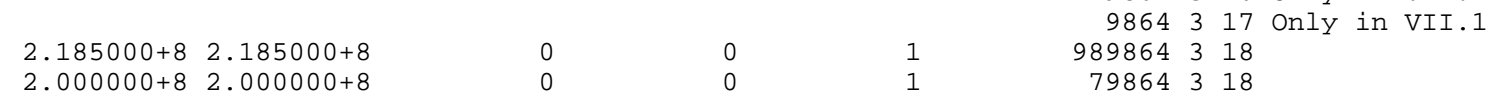

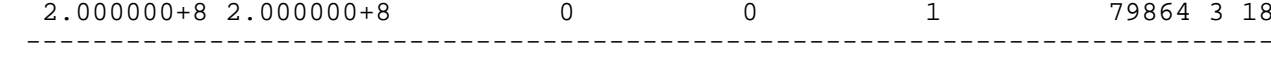

9864319 0nly in VII.1 9864320 Only in VII.1 9864321 Only in VII.1 9864337 Only in VII.1 9864338 only in VII.1 9864351 Only in VII.1 9864352 only in VII.1 9864353 Only in VII.1 9864354 Only in VII.1 9864355 Only in VII.1 9864356 Only in VII.1

\begin{tabular}{|c|c|c|c|c|c|c|c|}
\hline $\begin{array}{ll}6.031560+6 & 6.031560+6 \\
5.980000+6 & 5.980000+6\end{array}$ & $\begin{array}{l}\odot \\
\odot\end{array}$ & $\begin{array}{l}\ominus \\
\ominus\end{array}$ & $\begin{array}{l}1 \\
1\end{array}$ & $\begin{array}{r}9864 \\
989864 \\
79864\end{array}$ & $\begin{array}{l}31 \\
31\end{array}$ & $\begin{array}{l}91 \\
02 \\
.02\end{array}$ & Only i \\
\hline 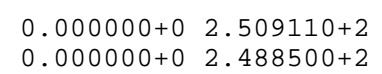 & $\begin{array}{l}0 \\
\odot\end{array}$ & $\begin{array}{l}2 \\
2\end{array}$ & $\begin{array}{l}\ominus \\
\ominus\end{array}$ & $\begin{array}{l}09864 \\
09864\end{array}$ & $\begin{array}{l}4 \\
4\end{array}$ & $\begin{array}{l}2 \\
2\end{array}$ & \\
\hline $\begin{array}{ll}0.000000+\odot & 2.509110+2 \\
\odot .000000+\odot & 2.509100+2\end{array}$ & $\begin{array}{l}1 \\
1\end{array}$ & $\begin{array}{l}1 \\
1\end{array}$ & $\begin{array}{l}\odot \\
\odot\end{array}$ & $\begin{array}{l}09864 \\
09864\end{array}$ & $\begin{array}{l}4 \\
4\end{array}$ & $\begin{array}{l}18 \\
18\end{array}$ & \\
\hline $\begin{array}{rr}0.0000 \odot \odot+\odot & 0.00000 \odot \odot+\odot \\
-2.0000 \odot \odot+7 & 0.0000 \odot \odot+\odot\end{array}$ & $\begin{array}{l}\odot \\
\odot\end{array}$ & $\begin{array}{l}1 \\
7\end{array}$ & $\begin{array}{l}1 \\
1\end{array}$ & $\begin{array}{l}29864 \\
29864\end{array}$ & $\begin{array}{l}5 \\
5\end{array}$ & $\begin{array}{l}18 \\
18\end{array}$ & \\
\hline & & & & $\begin{array}{l}9864 \\
9864 \\
9864 \\
9864 \\
9864 \\
9864 \\
9864\end{array}$ & $\begin{array}{l}6 \\
6 \\
6 \\
6 \\
6 \\
6 \\
6\end{array}$ & $\begin{array}{l}16 \\
17 \\
37 \\
51 \\
52 \\
53 \\
54\end{array}$ & $\begin{array}{l}\text { Only in VII.1 } \\
\text { Only in VII.1 } \\
\text { Only in VII.1 } \\
\text { Only in VII.1 } \\
\text { Only in VII.1 } \\
\text { Only in VII.1 } \\
\text { Only in VII.1 }\end{array}$ \\
\hline
\end{tabular}


$98-\mathrm{Cf}-254$

9864655 only in VII.1 9864656 Only in VII.1 9864691 only in VII.1 98646102 only in VII.1

\begin{tabular}{|c|c|}
\hline 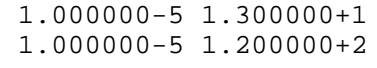 & $\begin{array}{l}\odot \\
\odot\end{array}$ \\
\hline 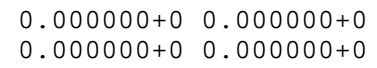 & $\begin{array}{l}\odot \\
\odot\end{array}$ \\
\hline 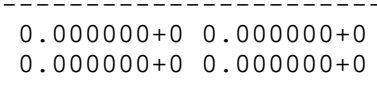 & $\begin{array}{l}\odot \\
\odot\end{array}$ \\
\hline $\begin{array}{l}\odot .0 \odot \odot \odot \odot \odot+\odot-4.5720 \odot \odot+4 \\
\odot .0 \odot \odot \odot \odot \odot+\odot-4.499990+4\end{array}$ & $\begin{array}{l}\ominus \\
\ominus\end{array}$ \\
\hline $\begin{array}{l}-6.031560+6-6.031560+6 \\
-6.028690+6-6.028690+6\end{array}$ & $\begin{array}{l}\ominus \\
\ominus \\
\ominus\end{array}$ \\
\hline $\begin{array}{l}-1.083580+7-1.083580+7 \\
-1.083240+7-1.083240+7\end{array}$ & $\begin{array}{l}\odot \\
\odot\end{array}$ \\
\hline $\begin{array}{ll}2.199000+8 & 2.199000+8 \\
1.999990+8 & 1.999990+8\end{array}$ & $\odot$ \\
\hline
\end{tabular}

$0 \quad 10106986731$

$\begin{array}{llrl}0 & 1 & 6898673 & 1\end{array}$

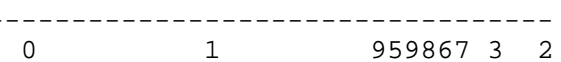

$\begin{array}{lllll}0 & 1 & 539867 & 3 & 2\end{array}$

0

$\begin{array}{lllll}0 & 1 & 4098673 & 3\end{array}$

(9-15

$\begin{array}{lllll}0 & 1 & 299867 & 3 & 16 \\ 0 & 1 & 159867 & 3 & 16\end{array}$

$\begin{array}{lllll}0 & 1 & 199867 & 3 & 17\end{array}$

\begin{tabular}{rrrrr}
0 & 1 & 199867 & 3 & 17 \\
0 & 1 & 119867 & 3 & 17 \\
\hline
\end{tabular}

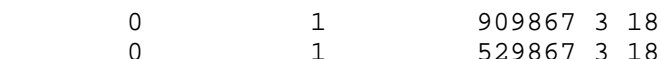

$\begin{array}{lllll}1 & 529867318\end{array}$

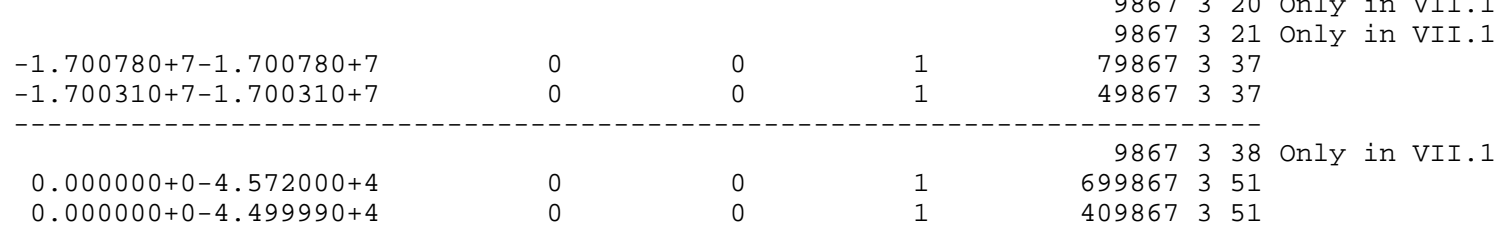

9867352 only in VII.1 9867353 Only in VII.1 9867354 only in VII.1

\begin{tabular}{|c|c|c|c|c|}
\hline $\begin{array}{l}\odot . \odot \odot \odot \odot \odot \odot+\odot-1.104370+5 \\
\odot . \odot \odot \odot \odot \odot \odot+\odot-1.400 \odot \odot \odot+5\end{array}$ & $\begin{array}{l}\odot \\
\odot\end{array}$ & $\begin{array}{l}\odot \\
\odot\end{array}$ & $\begin{array}{l}1 \\
1\end{array}$ & $\begin{array}{l}669867 \\
369867\end{array}$ \\
\hline $\begin{array}{ll}4.603080+6 & 4.603080+6 \\
4.449990+6 & 4.449990+6\end{array}$ & $\begin{array}{l}0 \\
\odot\end{array}$ & $\begin{array}{l}\odot \\
\odot\end{array}$ & $\begin{array}{l}1 \\
1\end{array}$ & $\begin{array}{l}909867 \\
539867\end{array}$ \\
\hline 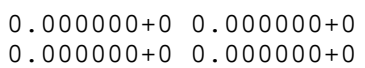 & $\begin{array}{l}\odot \\
\odot\end{array}$ & $\begin{array}{l}\odot \\
\odot\end{array}$ & $\begin{array}{l}1 \\
1\end{array}$ & $\begin{array}{l}939867 \\
539867\end{array}$ \\
\hline
\end{tabular}

r. 9867416 only in VII.0
$0.000000+0 \quad 2.519050+2 \quad 0987417$ only in VII.0

$\begin{array}{rrrrrr}1 & 1 & 0 & 098674 & 48\end{array}$

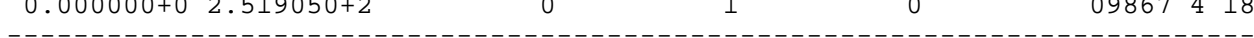

9867437 only in VII.0 9867451 Only in VII.0 9867491 Only in VII.0 9867516 Only in VII.0 9867517 only in VII. 0

$0.000000+\odot \quad \odot .000000+\odot$ $-2.000000+7 \quad 0.000000+0$

0
0
0

29867518

29867518

9867537 only in VII.0 9867591 Only in VII.0 9867616 only in VII.1 9867617 Only in VII.1 9867637 only in VII.1 9867651 only in VII.1 9867652 Only in VII.1 9867653 only in VII.1 9867654 Only in VII.1 9867691 Only in VII.1 98676102 only in VII.1 
99-Es-252 Evaluation Only in VII.1

$99-\mathrm{ES}-253$

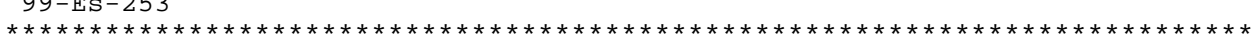

99131452 Only in VII.1

99131455 Only in VII.1

99131456 only in VII.1

$9.925300+4 \quad 1.000000+0$

$9.925300+41.000000+0$

099132151

$\begin{array}{rrrr}0 & 0 & 2 & 099132151\end{array}$

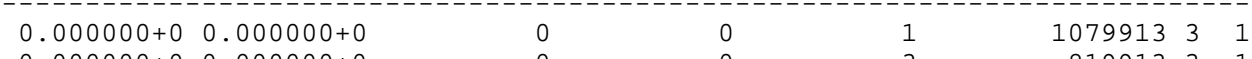

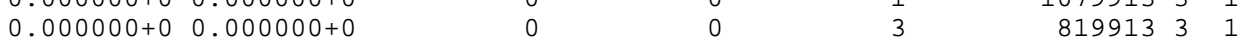

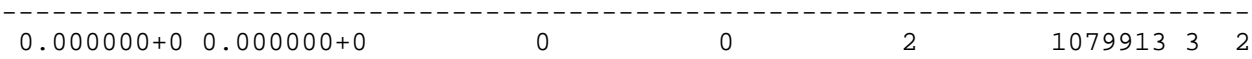

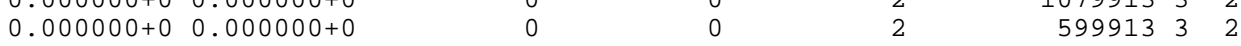

9913 39 Only in VII. 1

9913316 only in VII.1

9913317 only in VII.1

9913318 Only in VII.1

9913319 only in VII.1

9913320 Only in VII.1

9913321 only in VII.1

9913337 only in VII.1

9913338 only in VII.1

9913351 only in VII.1

9913352 Only in VII.1

9913353 only in VII.1

9913354 only in VII.1

9913355 only in VII.1

9913391 only in VII.1

$5.093030+6 \quad 5.093030+6$

$5.092000+65.092000+6$

$\odot$

9699133102

$0.000000+0 \quad 2.509110+2$

$0.000000+0 \quad 2.488500+2$

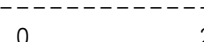

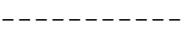

$488500+2$

2

0991342

0991342

9913418 Only in VII.1 9913518 only in VII.1 9913616 only in VII.1 9913617 Only in VII.1 9913637 only in VII.1 9913651 only in VII.1 9913652 only in VII.1 9913653 only in VII.1 9913654 only in VII.1 9913655 only in VII.1 9913691 only in VII.1

$99-$ ES -254

99136102 only in VII.1

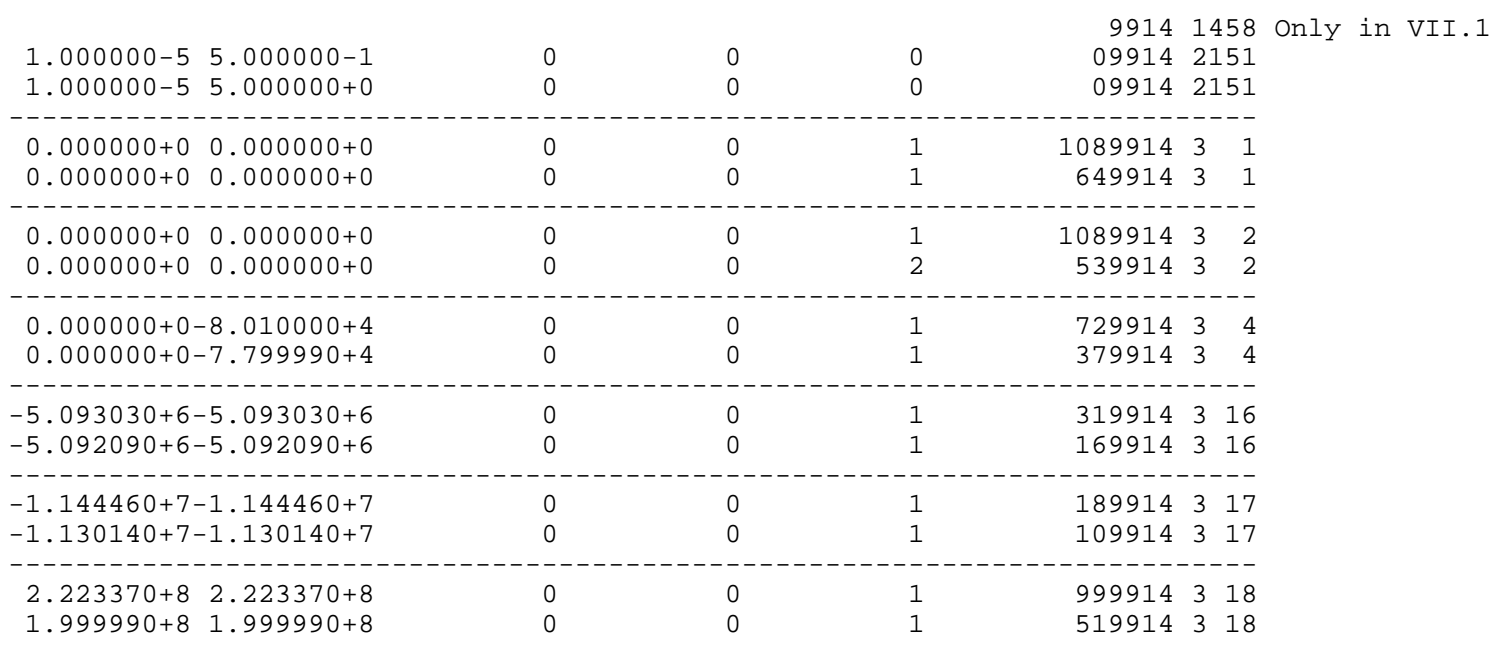

9914319 Only in VII.1

9914320 Only in VII.1 


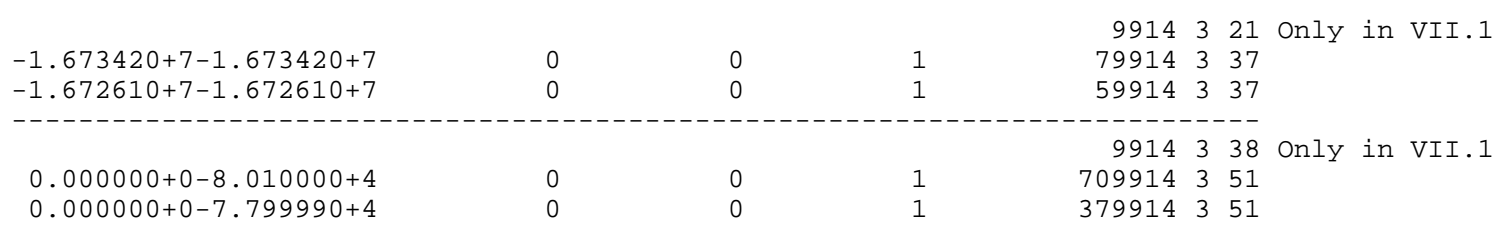

9914352 only in VII.1 9914353 only in VII.1 9914354 only in VII.1 9914355 Only in VII.1 9914356 Only in VII.1

\begin{tabular}{|c|c|}
\hline $\begin{array}{l}\odot . \odot \odot \odot \odot \odot \odot+\odot-1.077530+5 \\
\odot . \odot \odot \odot \odot \odot \odot+\odot-5 . \odot 29990+5\end{array}$ & $\begin{array}{l}\odot \\
\odot\end{array}$ \\
\hline 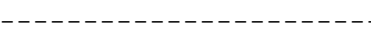 & 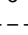 \\
\hline $\begin{array}{ll}5.974430+6 & 5.974430+6 \\
5.982690+6 & 5.982690+6\end{array}$ & $\begin{array}{l}\odot \\
\odot\end{array}$ \\
\hline - - - - - - - - - - - - - - - & - \\
\hline 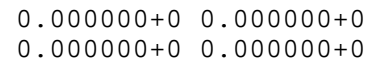 & $\begin{array}{l}\odot \\
\odot\end{array}$ \\
\hline
\end{tabular}
9914357 only in VII.1 689914391 289914391

\begin{tabular}{|c|c|c|c|c|}
\hline $\begin{array}{l}\odot . \odot \odot \odot \odot \odot \odot+\odot-1 . \odot 77530+5 \\
\odot . \odot \odot \odot \odot \odot \odot+\odot-5 . \odot 2999 \odot+5\end{array}$ & $\begin{array}{l}\odot \\
\odot\end{array}$ & $\begin{array}{l}\odot \\
\odot\end{array}$ & $\begin{array}{l}1 \\
1\end{array}$ & $\begin{array}{lll}689914 & 3 & 91 \\
289914 & 3 & 91\end{array}$ \\
\hline $.974430+6 \quad 5.974430+6$ & $\odot$ & $\odot$ & 1 & 999914 \\
\hline
\end{tabular}

$\begin{array}{rrrrrr}5.982690+6 & 5.982690+6 & 0 & 0 & 1 & 5399143102\end{array}$

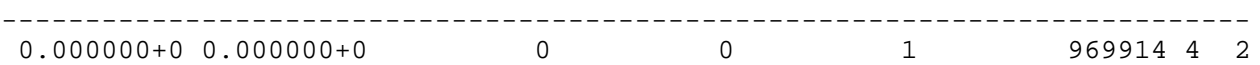

$0 \quad 1 \quad 519914-42$

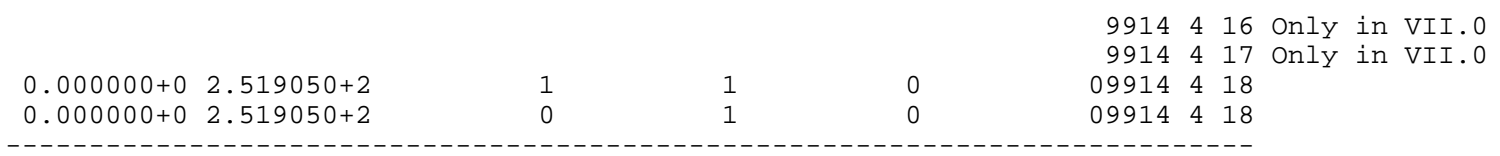

9914437 only in VII.0 9914451 Only in VII.0 9914491 only in VII.0 9914516 only in VII.0 9914517 only in VII.0 $0.000000+0 \quad 0.000000+0$ $-2.000000+7 \quad 0.000000+0$

\begin{tabular}{ccc}
0 & 1 & 1 \\
0 & 7 & 1 \\
\hline & $\ldots$ & $\ldots$
\end{tabular}

29914518 29914518

_.

9914537 only in VII.0 9914591 only in VII. 0 99145455 only in VII.1 9914616 Only in VII.1 9914617 Only in VII.1 9914637 only in VII.1 9914651 only in VII.1 9914652 only in VII.1 9914653 only in VII.1 9914654 Only in VII.1 9914655 only in VII.1 9914656 only in VII.1 9914657 only in VII.1 9914691 0nly in VII.1

99-Es-254m Evaluation Only in VII.1 99146102 Only in VII.1

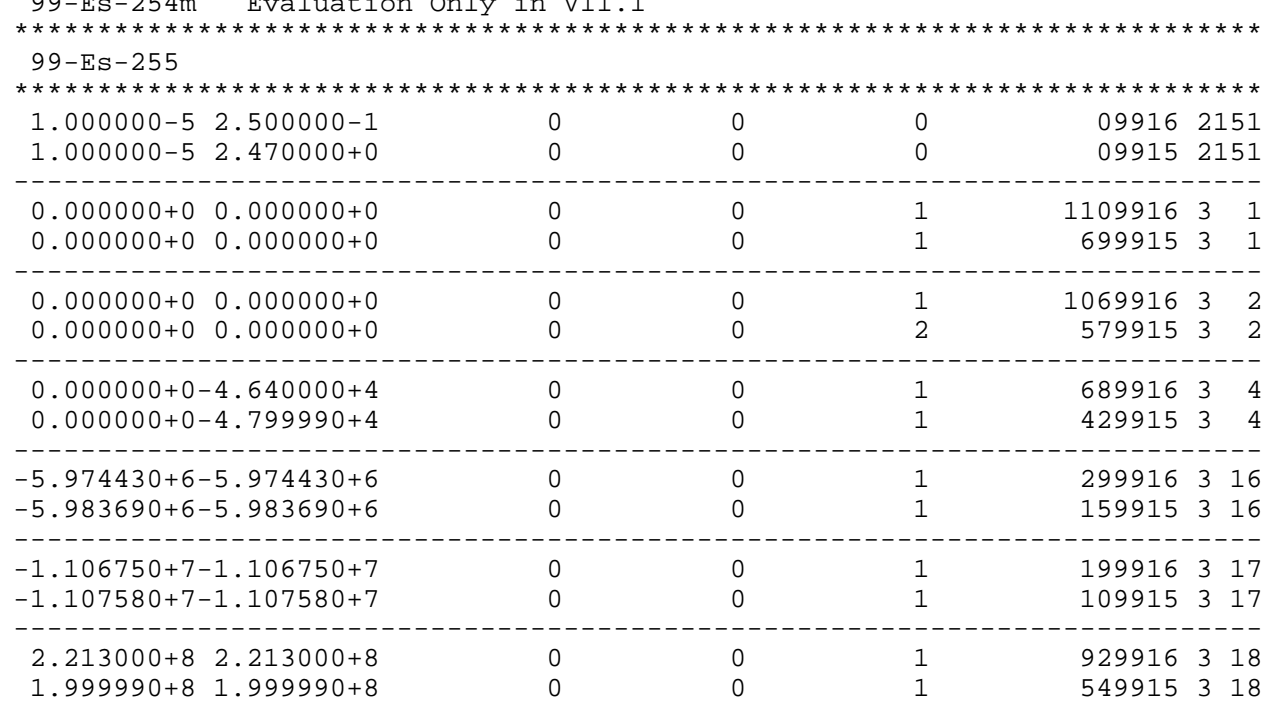




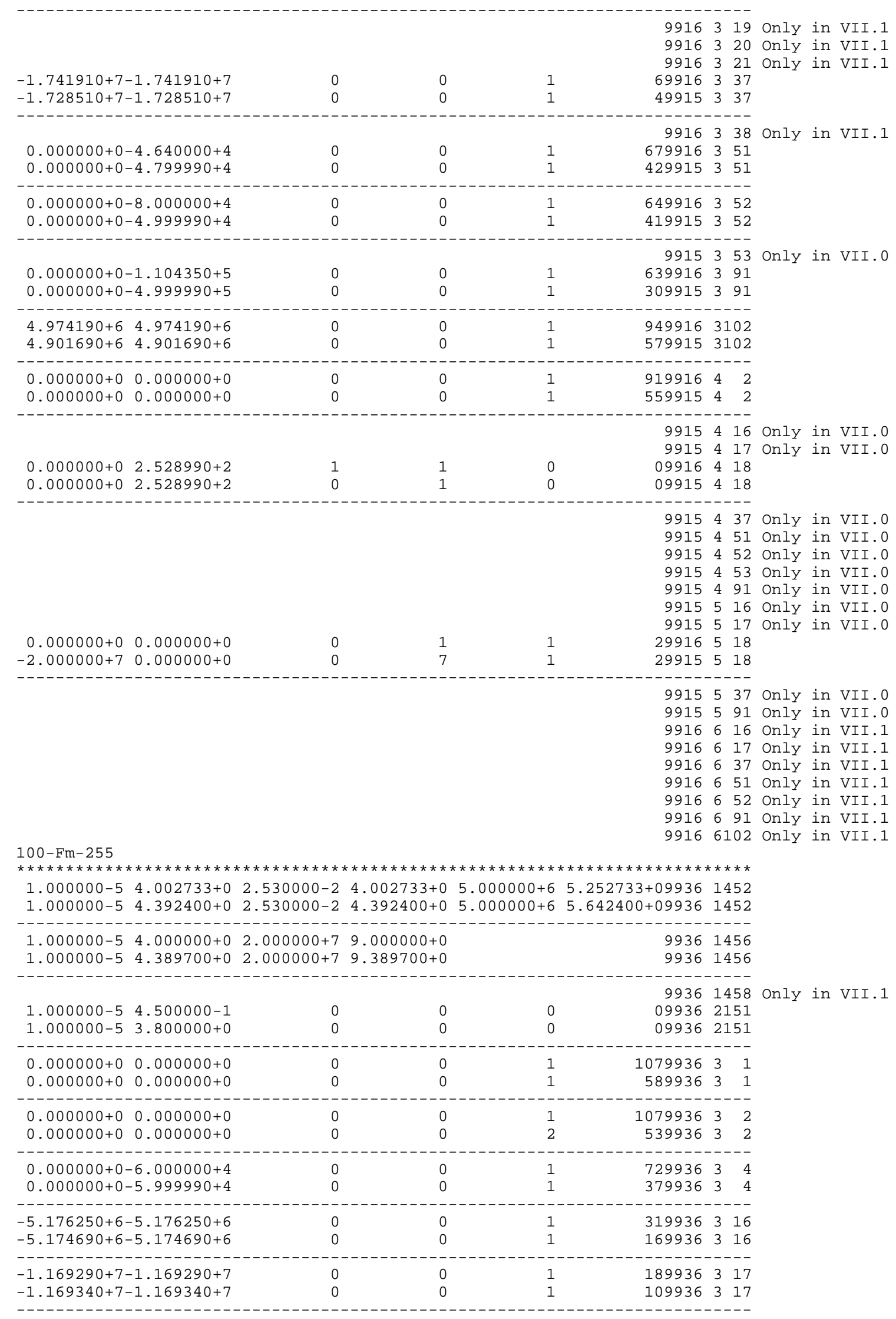




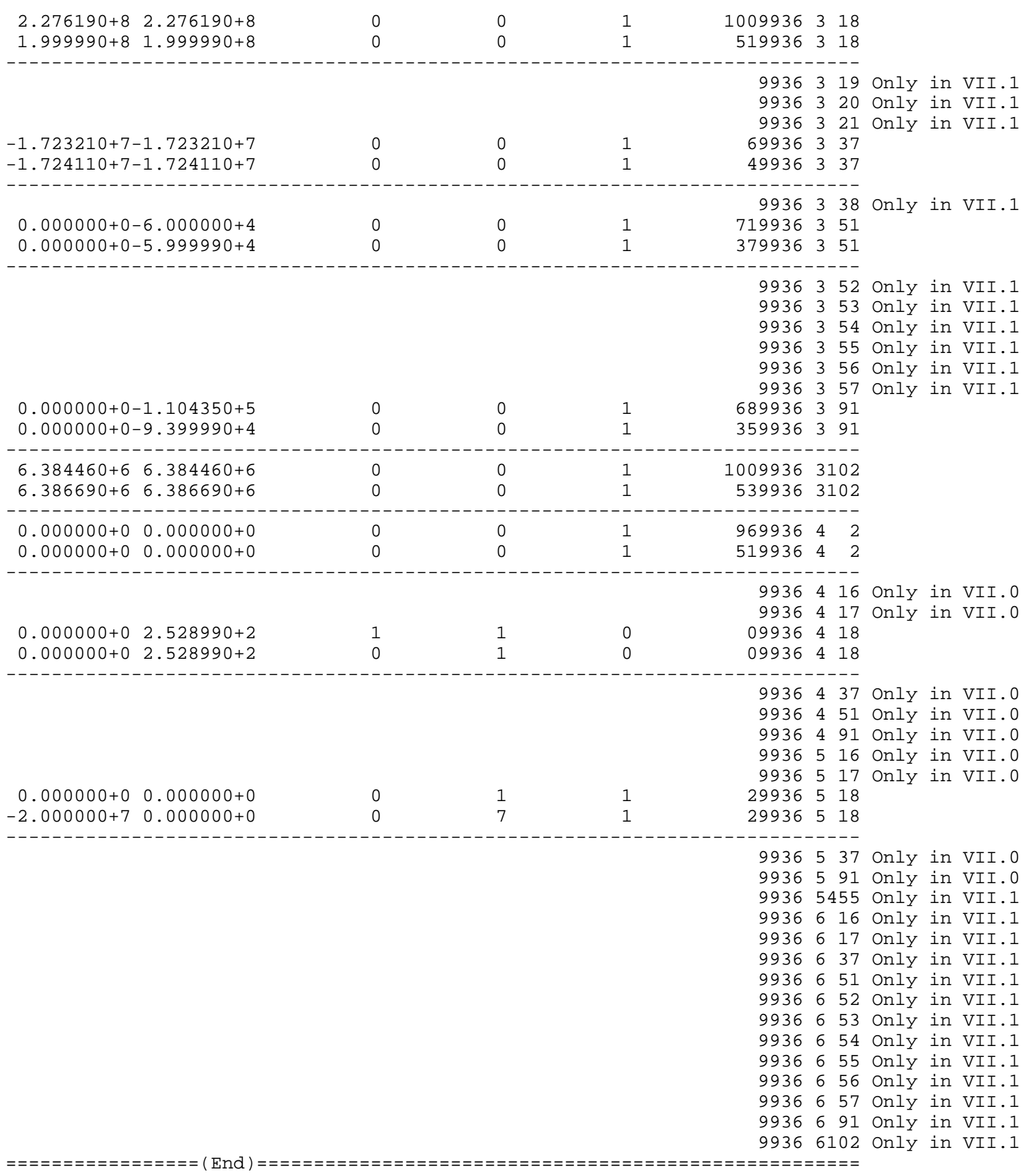

